\title{
GEOHYDROLOGY AND WATER QUALITY OF STRATIFIED-DRIFT AQUIFERS IN THE SACO AND OSSIPEE RIVER BASINS, EAST-CENTRAL NEW HAMPSHIRE
}

\section{By Richard Bridge Moore and Laura Medalie}

U.S. GEOLOGICAL SURVEY

Water-Resources Investigations Report 94-4182

Prepared in cooperation with

NEW HAMPSHIRE DEPARTMENT OF ENVIRONMENTAL SERVICES,

WATER RESOURCES DIVISION

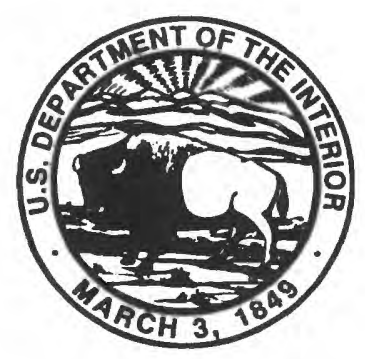

Bow, New Hampshire 1995 


\section{U.S. DEPARTMENT OF THE INTERIOR BRUCE BABBITT, Secretary}

U.S. GEOLOGICAL SURVEY

Gordon P. Eaton, Director

District Chief,

U.S. Geological Survey, WRD

New Hampshire-Vermont District

525 Clinton Street

Bow, NH 03304
U.S. Geological Survey

Earth Science Information Center

Open-File Reports Section

Federal Center

Box 25286, MS 517

Denver, CO 80225 


\section{CONTENTS}

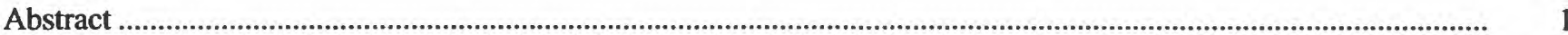

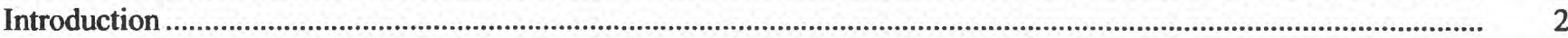

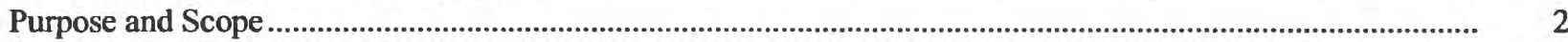

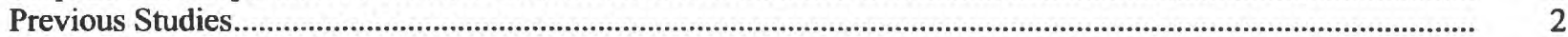

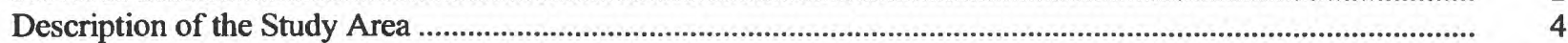

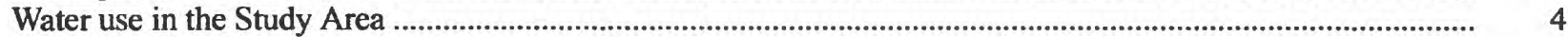

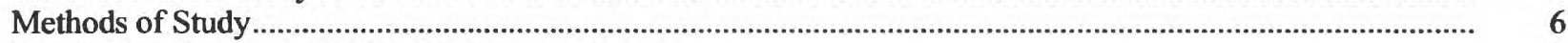

Numbering System for Wells, Borings, and Springs .......................................................................................... 8

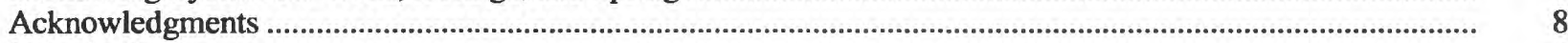

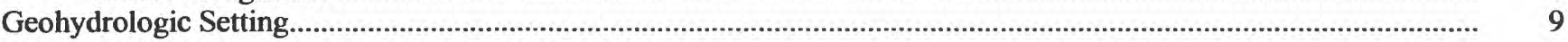

Postglacial Redistribution of Glacial Deposits ........................................................................................... 9

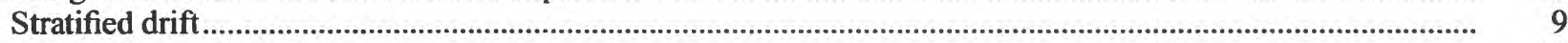

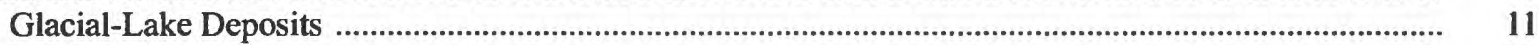

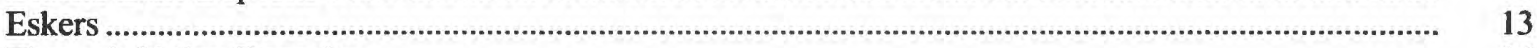

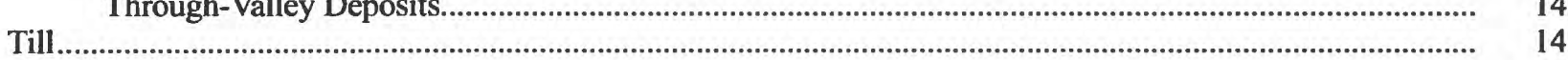

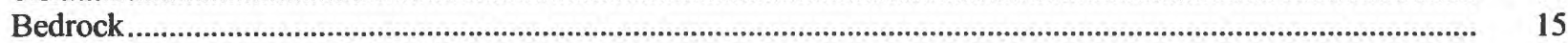

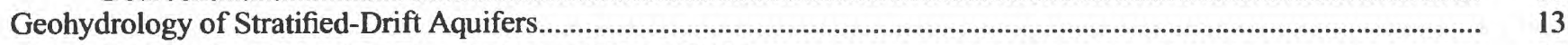

Delineation of Aquifer Boundaries and Water Table ......................................................................................... 16

Areal Extent of Stratified-Drift Aquifers ............................................................................................... 16

Stratigraphic Position of Lithologic Units and Altitude of Water Table ................................................... 16

Ground-Water-Site Inventory .................................................................................................... 16

Seismic Refraction............................................................................................................. 16

Seismic-Reflection Surveys at Eight Selected Lakes or Ponds ................................................... 17

Recharge, Discharge, and Direction of Ground-Water Flow............................................................................. 17

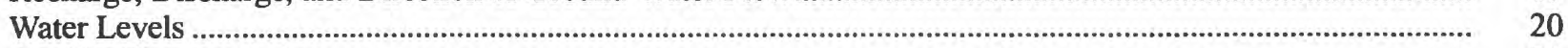

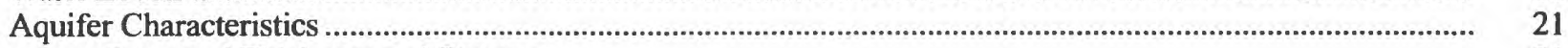

Saturated Thickness and Storage............................................................................................................ 21

Relation of Aquifer Thickness to Geohydrologic Setting …....................................................... 22

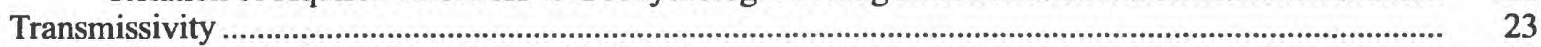

Characteristics of Selected Stratified-Drift Aquifers .......................................................................... 24

North Chatham Aquifer in Chatham, N.H............................................................................... 25

Chatham Aquifer in Chatham, N.H. ......................................................................................... 25

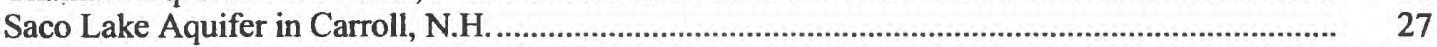

Dry River Aquifer in Harts Location, N.H. ............................................................................. 27

Bartlett Aquifer in Bartlett, N.H. ....................................................................................................... 27

Saco River Aquifer in Conway and Bartlett, N.H. ................................................................... 27

Weeks Brook Aquifer in Chatham and Conway, N.H............................................................... 28

Passaconaway Aquifer in Albany and Waterville Valley, N.H.................................................... 28

Pequawket Brook Aquifer in Madison, Albany, and Conway, N.H............................................... 28

Bearcamp River Aquifer in Sandwich and Tamworth, N.H....................................................... 29

Ossipee Lake Aquifer in Tamworth, Madison, Ossipee, Freedom, and Effingham, N.H. ............. 29

Pine River Aquifer in Ossipee, Effingham, and Wakefield, N.H. ................................................ 29

Estimation of Ground-Water Availability in the Ossipee Lake Aquifer.................................................................. 30

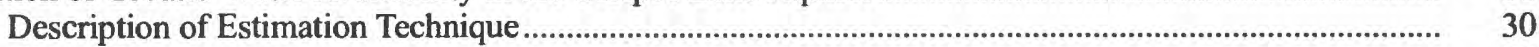

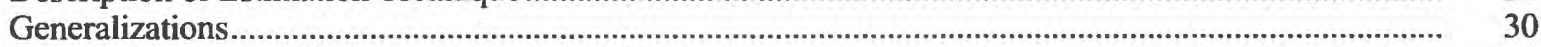

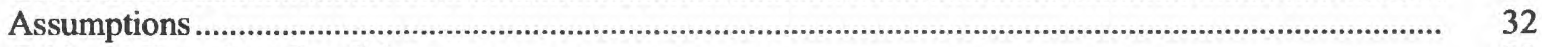

Grid and Boundary Conditions .............................................................................................................. 33

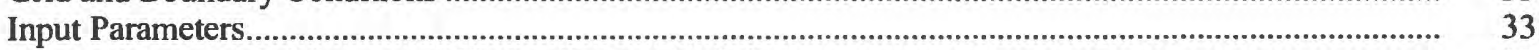

Saturated Thickness and Hydraulic Conductivity .......................................................................... 33

Specific Yield.......................................................................................................................... 33

Infiltration from the Streams, Ponds, and Lakes.......................................................................

Ground-Water Availability ........................................................................................................... 33

Sensitivity Analysis of the Ground-Water Availability Estimate............................................................... 


\section{Contents--Continued}

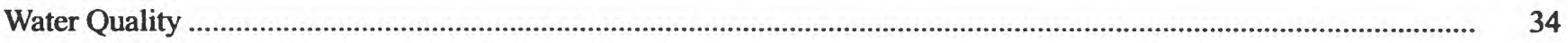

pH

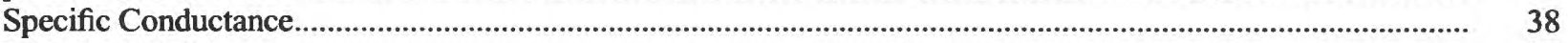

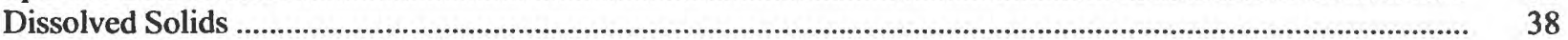

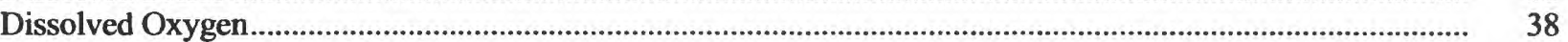

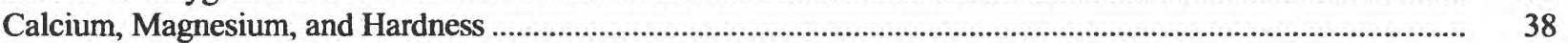

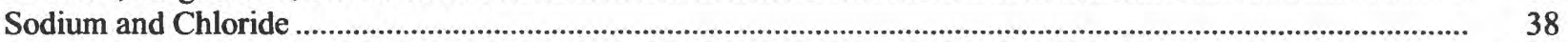

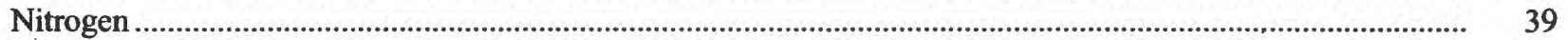

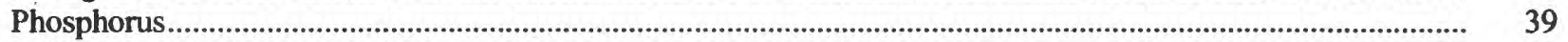

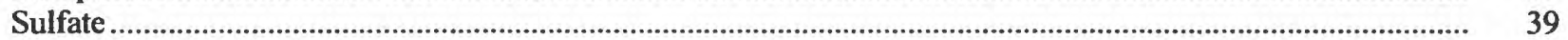

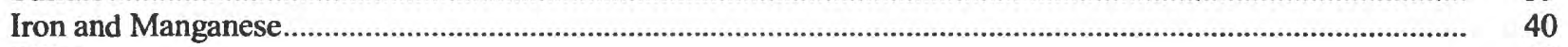

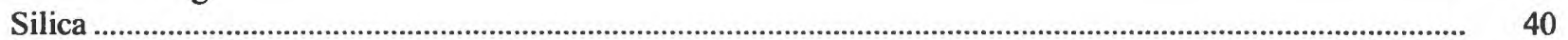

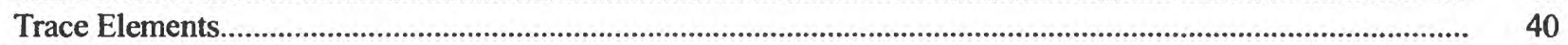

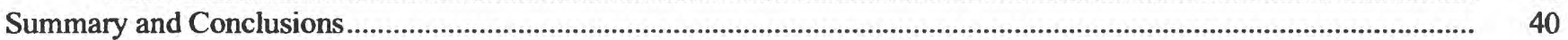

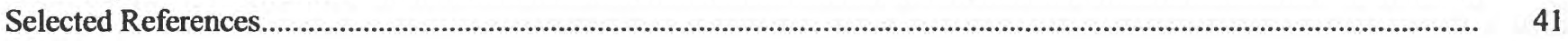

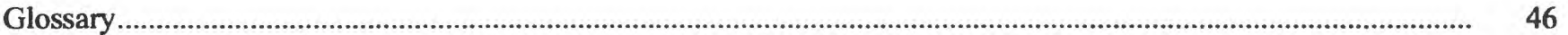

Appendixes

1. Description of selected wells, borings, and springs in the Saco and Ossipee River Basins, east-central New Hampshire

2. Stratigraphic logs of selected wells and borings in the Saco and Ossipee River Basins, east-central New Hampshire

3. Geohydrologic sections interpreted from seismic-refraction data in the Saco and Ossipee River Basins, east-central New Hampshire

4. Low-flow streamflow measurements at miscellaneous sites in the Saco and Ossipee River Basins; east-central New Hampshire.

5. Chemical analyses of ground-water samples from the Saco and Ossipee River Basins, east-central New Hampshire

\section{FIGURES}

1. Map showing location of the Saco and Ossipee River Basins study area

2. Map showing location of major lakes, rivers, and surface-water divides in the Saco and

Ossipee River Basins study area in east-central New Hampshire

3. Map showing location of stratified-drift aquifers underlying the Saco and Ossipee River Basin study area east-central New Hampshire.

4. Map showing the Ossipee Lake Quadrangle showing the location of three "through valleys," east-central New Hampshire

5. Map showing location of geologic sections interpreted from seismic-reflection data in the Ossipee River Basin, east-central New Hampshire

6-8. Geologic sections interpreted from seismic-reflection data in the Ossipee River Basin east-central New Hampshire for:

6. Freedom, A-A'.

7. Madison, B-B'

8. Effingham, C-C'

9. Graph showing relation between estimated hydraulic conductivity, median grain size, and degree of sorting of stratified drift in New Hampshire...

10. Map showing selected aquifers in the Saco and Ossipee River Basins study area, east-central New Hampshire...

11. Map showing location of Ossipee Lake ground-water availability model area, east-central New Hampshire .......

12. Map showing water-quality-sampling locations in the Saco and Ossipee River Basins study area, east-central New Hampshire. 


\section{Contents-Continued}

\section{TABLES}

1. Registered municipal water-supply systems and ground- and surface-water-source data for towns in the Saco and Ossipee River Basins, east-central New Hampshire

2. Two-letter town codes used as prefixes in the numbering system for wells, borings, and springs in the Saco and Ossipee River Basins, east-central New Hampshire.

3. Relation of mean hydraulic conductivity to median grain size and degree of sorting of stratified drift

in New Hampshire ................................................................................................................ ground-water availability estimates, east-central New Hampshire

5. Statistical summary of water-quality analyses of ground-water samples from the Saco and Ossipee River Basins, east-central New Hampshire

\section{PLATES (Plates are in map jacket)}

1-2. Maps showing altitude of water table, data collection locations, and materials for stratified-drift aquifers in the Saco and Ossipee River Basins, east-central New Hampshire:

1. northern plate.

2. southern plate.

3-4. Maps showing saturated thickness and transmissivity of stratified drift in the Saco and Ossipee River Basins, east-central New Hampshire:

3. northern plate.

4. southern plate. 


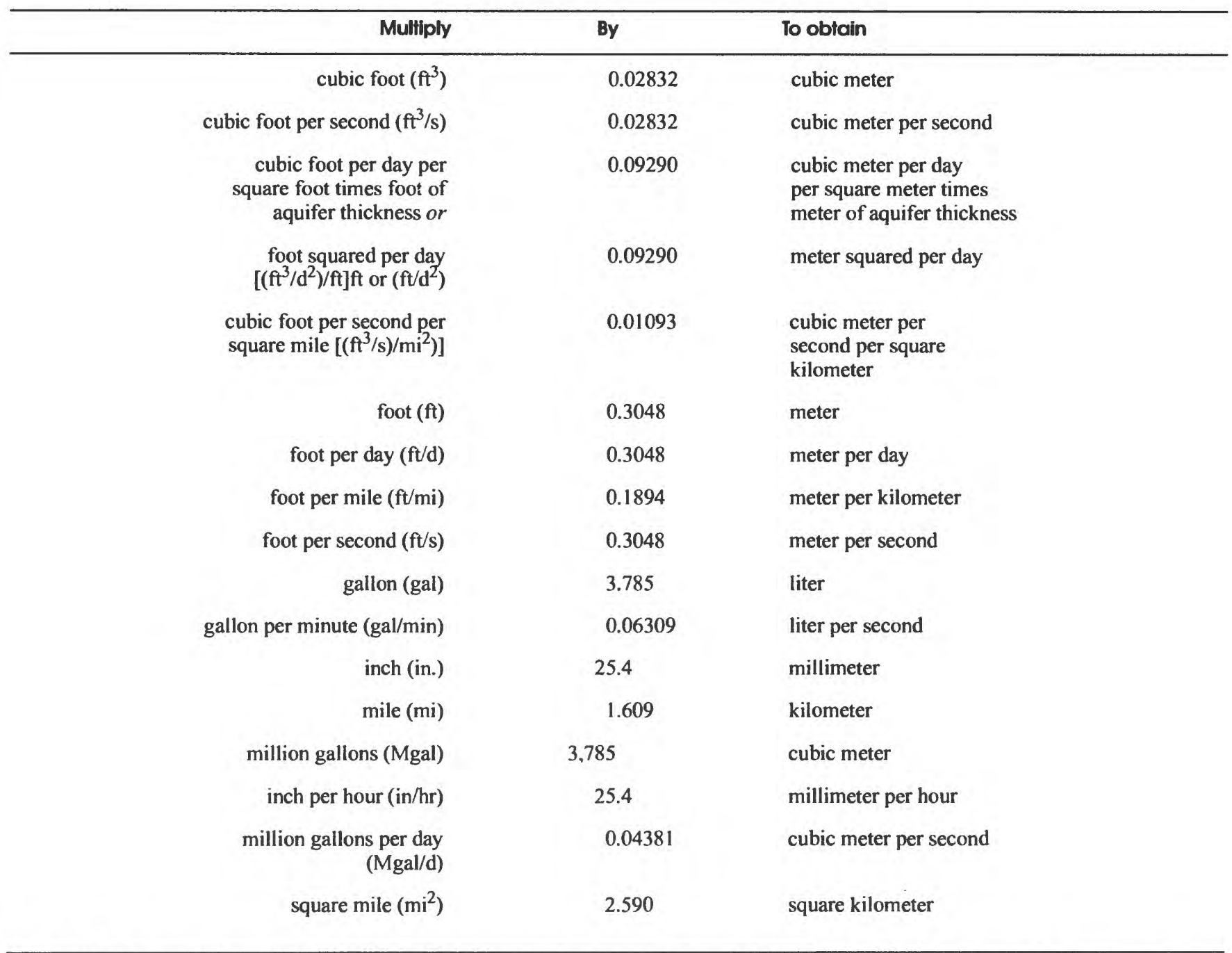

Sea level: In this report, "sea level" refers to the National Geodetic Vertical Datum of 1929 -a geodetic datum derived from a general adjustment of the first-order level nets of both the United States and Canada, formerly called Sea Level Datum of 1929.

\section{ABBREVIATED WATER-QUALITY UNITS USED IN REPORT}

In this report, chemical concentration in water is expressed in milligrams per liter $(\mathrm{mg} / \mathrm{L})$ or micrograms per liter ( $\mu \mathrm{g} / \mathrm{L})$. Mitligrams per liter is a unit expressing the concentration of chemical constituents in solution as weight (milligrams) of solute per unit volume (liter) of water; $1,000 \mu \mathrm{g} / \mathrm{L}$ (micrograms per liter) is equivalent to $1 \mathrm{mg} / \mathrm{L}$ (milligrams per liter). Water temperature in degrees Celsius $\left({ }^{\circ} \mathrm{C}\right.$ ) can be converted to degrees Fahrenheit $\left({ }^{\circ} \mathrm{F}\right)$ by use of the following equation:

$$
{ }^{\circ} \mathrm{F}=1.8\left({ }^{\circ} \mathrm{C}\right)+32
$$

Specific conductance of water is expressed in microsiemens per centimeter at 25 degrees Celsius ( $\mu \mathrm{S} / \mathrm{cm})$. This unit is equivalent to micromhos per centimeter at 25 degrees Celsius ( $\mu \mathrm{mho} / \mathrm{cm}$ ), formerly used by the U.S. Geological Survey.

The ionic concentration in water is expressed in milliequivalents per liter (meq/L). Milliequivalents per liter is the concentration in $\mathrm{mg} / \mathrm{L}$ of an ion multiplied by the charge of the ion and divided by the atomic weight of the ion. 


\title{
GEOHYDROLOGY AND WATER QUALITY OF STRATIFIED-DRIFT AQUIFERS IN THE SACO AND OSSIPEE RIVER BASINS, EAST-CENTRAL NEW HAMPSHIRE
}

\author{
By Richard Bridge Moore and Laura Medalie
}

\section{Abstract}

The U.S. Geological Survey, in cooperation with the New Hampshire Department of Environmental Services, Water Resources Division, has assessed the geohydrology and water quality of stratified-drift aquifers in the Saco and Ossipee River Basins in east-central New Hampshire. Stratified-drift aquifers discontinuously underlie 152.5 square miles of the Saco and Ossipee River Basins, which drains 869.4 square miles and consists of sorted coarse-grained sediments deposited by glaciation. Saturated thicknesses of stratified drift in the study area are locally greater than 280 feet but generally are less. Transmissivity locally exceeds 8,000 feet squared per day but is generally less. Approximately 93.6 square miles (or 10.8 percent of the study area) have transmissivities greater than 1,000 feet squared per day.

Characteristics of stratified-drift materials that affect ground-water storage and flow are related to the original glaciofluvial or glaciolacustrine environments in which they were deposited. Deglaciation by a systematic process of stagnation zone retreat resulted in the formation of deltas and other lake deposits, primarily along the central parts of the Saco and Ossipee River Valleys. Glacial outwash and alluvium covered much of the earlier valley-fill deposits. Glacial lakes were present at the time of deglaciation in the Saco and Ossipee River Basins and their presence greatly affected deglaciation and the depositional processes that formed many of the stratified-drift aquifers in the study area.
The geohydrologic investigation of stratified-drift aquifers focused on basic aquifer properties including aquifer boundaries; recharge, discharge, and direction of ground-water flow; saturated thickness and storage; and transmissivity. Surficial geologic mapping assisted in the determination of aquifer boundaries. Data from 1,848 wells, test borings, and springs were collected, and stored in the U.S. Geological Survey's Ground Water Site Inventory (GWSI) data base. Over 1,130 of these sites were from areas covered by stratified drift. These data were used to produce maps of water-table configuration, saturated thickness, and transmissivity of stratified drift. A total of 13.3 miles of seismic-refraction profiling (at 61 locations) and 13.7 miles of seismic-reflection profiling were used to aid in preparing the water-table and saturated-thickness maps. Seismicreflection data also were collected and interpreted along 13.7 miles of the shores of Ossipee Lake and 7 other water bodies in the study area. These data aided in the preparation of the saturated-thickness maps.

The Ossipee Lake aquifer in the towns of Ossipee, Freedom, Effingham, Madison, and Tamworth was analyzed by use of transient simulations and a two-dimensional, finite-difference flow model to determine ground-water availability. The available water, after a 180-day period of pumping from four hypothetical wells open to the aquifer, was 7.72 million gallons per day, 31.6 percent of the water pumped came from ground-water storage and 68.4 percent came from combined induced infiltration and ground water captured before discharge to surface-water bodies.

Results of the analysis of water samples from 25 test wells and 4 springs indicate that water in the stratified-drift aquifers generally meets U.S. Environmental 
Protection Agency primary and secondary drinkingwater regulations. Sites of known ground-water contamination in the aquifers were not sampled. Elevated concentrations above background levels of chloride and sodium, iron and manganese, and fluoride were the exceptions.

\section{INTRODUCTION}

The population of communities in the Saco and Ossipee River Basins increased by about 93 percent between 1970 and 1990 (U.S. Bureau of the Census, 1991). Recreational and second-home development has been especially rapid in this area, which includes parts of the Lakes Region in east-central New Hampshire and the White Mountains. This growth has steadily increased demands for water. In 1990, the total withdrawal from stratified-drift aquifers for municipal supply was about $2.7 \mathrm{Mgal} / \mathrm{d}$ (F. H. Chormann Jr., New Hampshire Department of Environmental Services, Water Resources Division, written commun., 1991). In addition, large ski areas in the mountains rely heavily on water resources to make snow to augment the natural snow cover. Also, stringent U.S. Environmental Protection Agency (USEPA) primary and secondary drinkingwater regulations on the treatment requirements of surface-water supplies have prompted municipalities to look closely at the ground-water resources. Of the 19 towns in the Saco and Ossipee River Basins, 6 have municipal-water systems of which 2 use only ground water, 3 use surface water from the basin, and 1 uses ground and surface water. Most of these towns need to expand their water-supply capabilities. The rural areas of the towns in the study area rely on private wells.

The U.S. Geological Survey (USGS), in cooperation with the New Hampshire Department of Environmental Services, Water Resources Division (NHDESWRD), has done a series of ground-water studies in New Hampshire that provide the detailed geohydrologic information necessary for determining optimal use of available water supplies and for the development of new water supplies. The study described in this report encompasses the Saco and Ossipee River Basins (fig. 1). For most of the studies, watershed basin divides were selected as study-area boundaries because they are the natural subdivision of the hydrologic system; only a few stratified-drift aquifers in New Hampshire extend across major surface-water divides. Completed studies and reports include the Nashua Regional Planning
Commission area (Toppin, 1987); the Exeter, Lamprey, and Oyster River Basins (Moore, 1990); the lower Merrimack and coastal River Basins (Stekl and Flanagan, 1992); the Bellamy, Cocheco, and Salmon Falls River Basins (Mack and Lawlor, 1992); the middle Merrimack River Basin (Ayotte and Toppin, 1995); the lower Connecticut River Basin (Moore and others, 1994); the Contoocook River Basin (Harte and Johnson, 1995) and the Pemigewasset River Basin (Cotton and Olimpio, in press).

\section{Purpose and Scope}

The purpose of this report is to (1) describe the hydrologic and geologic characteristics of the stratifieddrift aquifers in the Saco and Ossipee River Basins, including areal extent of the stratified-drift aquifers, ground-water levels, general directions of ground-water flow, saturated thickness, and transmissivities; (2) present a technique for the evaluation and estimation of ground-water availability, and (3) assess the general quality of water in the stratified-drift aquifers.

The study generally was limited to the collection, compilation, and evaluation of data from the stratifieddrift aquifers underlying the study area. Yield of a large portion of the largest of the aquifers, in the area surrounding Ossipee Lake, was evaluated with a drawdown modeling-simulation technique to illustrate how this technique can be used to provide estimates of ground-water availability after 180 days of pumping.

\section{Previous Studies}

Previous studies in the area include a basic data report of geohydrologic and surface-water data for the Saco River valley-fill aquifer from Bartlett, New Hampshire to Fryeburg, Maine by Johnson and others (1987). An accompanying interpretive report on the hydrogeology, water quality, and effects of pumping was written by Tepper and others (1990). A reconnaissance map of the availability of ground water in the Saco River Basin was presented by Cotton (1975) on a map at a scale of 1:125,000. Surficial-geologic maps in the study area had been produced at a scale of 1:62,500 with accompanying reports. Goldthwait (1968) mapped and reported on surficial deposits in the Wolfeboro 15-minute quadrangle, and Newton (1974) mapped and reported on surficial deposits in the Ossipee Lake 15-minute quadrangle. 


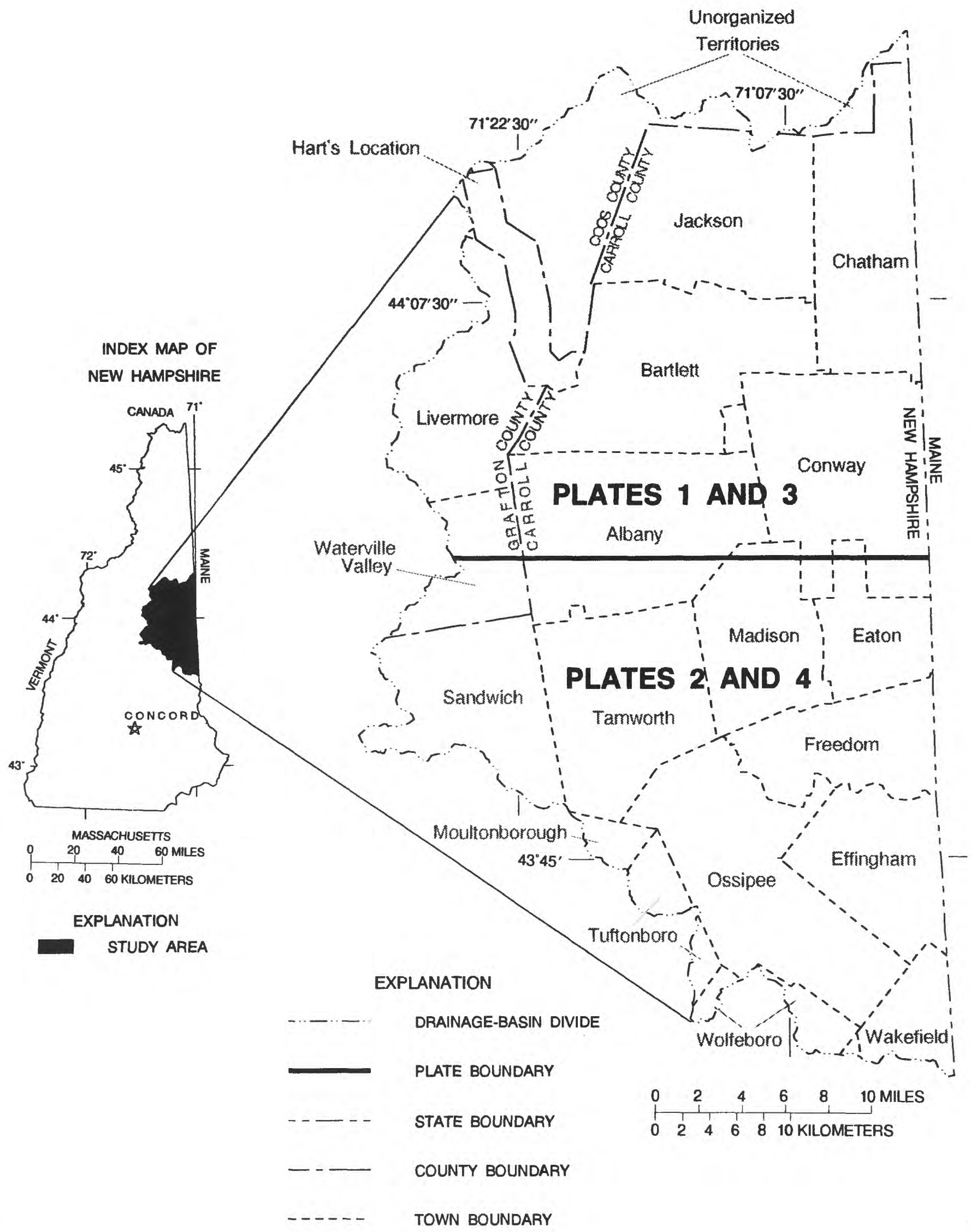

Figure 1. Location of the Saco and Ossipee River Basin study area. 


\section{Description of the Study Area}

The Saco and Ossipee River Basins encompass about $869 \mathrm{mi}^{2}$ in the glaciated Appalachian groundwater region in east-central New Hampshire. The two basins cover an area $48.5 \mathrm{mi}$ long north to south and about 27.4 mi wide east to west (fig. 2). The mountainous northern part is in the White Mountain section of the New England physiographic province and the southern part is in the New England upland section (Feneman, 1938; Raiz, 1954). There are 19 towns and 9 grants or unorganized territories that are either partly or entirely in the study area.

Surface altitude of the basins ranges from just under $400 \mathrm{ft}$ above sea level, at two sites where the Saco and Ossipee Rivers flow into the State of Maine, to $6,288 \mathrm{ft}$ above sea level at the summit of Mount Washington, the highest point in the Northeastern United States.

The Ossipee River originates at Ossipee Lake, $407 \mathrm{ft}$ above sea level, and drains eastward into the State of Maine. Major tributaries to Ossipee Lake are the north-flowing Pine River, the east-flowing Bearcamp River, and the south flowing Chocorua River. The Ossipee River is named from the Native American word "Awoss-sebi" meaning river on the other side (Piotrowski, 1977). The Saco River originates at a small water body called Saco Lake, $1,887 \mathrm{ft}$ above sea level in Crawford Notch. Major tributaries of the Saco River, in the study area, are the north-flowing Pequawket Brook, the east-flowing Swift River, and the south-flowing Rocky Brook, Ellis River, and East Branch of the Saco River (fig. 2). The word "Saco" is derived from the Native American word "Sokook" meaning at the southern place (or from the south side) (Piotrowski, 1977).

Average annual precipitation ranges from about 40 in. in the southern part of the basin to more than $60 \mathrm{in}$. in the northern headwaters. Average annual runoff in the basin ranges from about 20 to $50 \mathrm{in}$. (Knox and Nordenson, 1955). Average annual runoff for the Ossipee River Basin upstream of the gage on the Ossipee River at Effingham, is about $28 \mathrm{in} /$ year. Average annual runoff for the Saco River Basin, upstream of the gage on the Saco River at Conway, is about $33 \mathrm{in} /$ year (Toppin and others, 1991).

\section{Water Use in the Study Area}

Eight municipal water-supply systems in the Saco and Ossipee River Basins are water users registered with the NHDES-WRD because they withdraw more than 20,000 gal of water on an average day (table 1). All water-use data discussed here is from Frederick $\mathrm{H}$. Chormann Jr., New Hampshire Department of Environmental Services, Water Resources Division (written commun., 1994). The largest system, Wolfeboro Water Works, provides water service to over 5,000 residents of Wolfeboro. This system is noteworthy because withdrawals from Upper Beech Pond, in the southwestern corner of the Ossipee River Basin (pl. 2), are exported for use in the Winnipesaukee River Basin. Thus, over $0.7 \mathrm{Mgal}$ are exported or lost from the study area on an average day. Water that is withdrawn from the remaining sources listed in table 1 is almost entirely used within the study area.

Four of the water-supply systems use surfacewater sources, three of the water-supply systems withdraw water from stratified-drift aquifers, and one of the water-supply systems (in Madison) withdraws water from both stratified-drift and bedrock aquifers. The Lower Bartlett Water Precinct, the Conway Village Fire District, and the North Conway Water Precinct all withdraw water from the Saco River Valley stratified-drift aquifer. In 1990, total withdrawal by these three systems was $1.68 \mathrm{Mgal} / \mathrm{d}$. This amount of withdrawal decreased slightly during 1991-93. In addition to the large systems listed in table 1 , several small community water-supply systems (for mobile-home parks, developments, and condominium associations) in Albany, Bartlett, Conway, Freedom, Madison, Ossipee, Tamworth, and Wakefield pump water from stratified-drift aquifers. These small water-supply systems do not normally install meters on wells, so withdrawals must be estimated on the basis of the number of people served.

In addition to water-supply system data, the State water-use data base contains information on other registered water users in the basin. In 1993, total withdrawals from surface water for snowmaking in Bartlett, Conway, Jackson (1990 data), and Madison were $1.330 \mathrm{Mgal} / \mathrm{d}$. Some ground water $(0.003 \mathrm{Mgal} / \mathrm{d})$ was also withdrawn in Bartlett for snowmaking. Groundwater withdrawal in Tamworth for biomass power production was $0.360 \mathrm{Mgal} / \mathrm{d}$ in 1993 . Surface-water withdrawal for mining operations in Ossipee and Madison was $0.283 \mathrm{Mgal} / \mathrm{d}$, for golf-course irrigation in Conway 


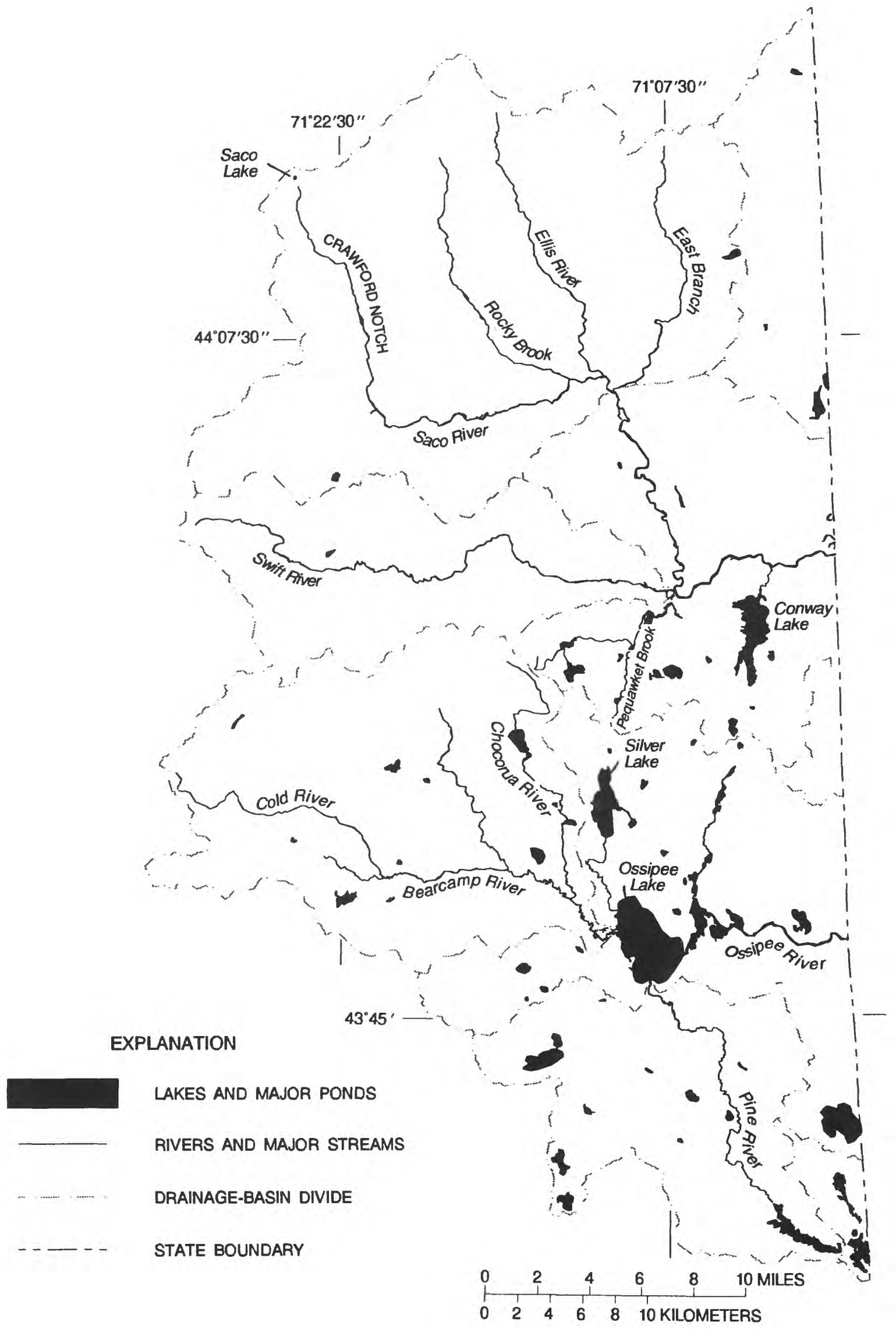

Figure 2. Location of major lakes, rivers, and surface-water divides in the Saco and Ossipee River Basins study area, east-central New Hampshire. 
Table 1. Registered municipal water-supply systems and ground-and surface-water source data for towns in the Saco and Ossipee River Basins, east-central New Hampshire

[Source and withdrawal data from the New Hampshire Department of Environmental Services, Water Resources Division (1990); population data from the New Hampshire Department of Environmental Services, Water Supply and Pollution Control Division (Rene Pelletier, written commun., 1993); Mgal/d, million gallons per day; --, not applicable; GPW, gravel-packed well; BRW, bedrock well]

\begin{tabular}{|c|c|c|c|c|c|c|}
\hline Town & $\begin{array}{l}\text { Water-system } \\
\text { name }\end{array}$ & $\begin{array}{l}\text { Surface-water body or } \\
\text { local well name }\end{array}$ & $\begin{array}{l}\text { Well } \\
\text { number }\end{array}$ & $\begin{array}{l}\text { Type of } \\
\text { well }\end{array}$ & $\begin{array}{c}\text { Average } \\
\text { daily } \\
\text { withdrawals } \\
\text { in Mgal/d }\end{array}$ & $\begin{array}{l}\text { Population } \\
\text { served }\end{array}$ \\
\hline Bartlett & $\begin{array}{l}\text { Bartlett Village } \\
\text { Water Precinct }\end{array}$ & Albany Brook & -- & -- & 0.099 & 625 \\
\hline Bartlett & $\begin{array}{l}\text { Lower Bartlett } \\
\text { Water Precinct }\end{array}$ & $\begin{array}{l}\text { Saco River Intervale \#1 } \\
\text { Saco River Intervale \#2 }\end{array}$ & $\begin{array}{l}\text { BCW } 17 \\
\text { BCW } 114\end{array}$ & $\begin{array}{l}\text { GPW } \\
\text { GPW }\end{array}$ & $\begin{array}{l}.055 \\
.052\end{array}$ & 1,000 \\
\hline Conway & $\begin{array}{l}\text { Conway Village } \\
\text { Fire District }\end{array}$ & Swift River Well & CWW 102 & GPW & .680 & 1,937 \\
\hline Conway & $\begin{array}{l}\text { North Conway } \\
\text { Water Precinct }\end{array}$ & $\begin{array}{l}\text { GPW \#1 } \\
\text { GPW \#2 } \\
\text { GPW \#3 }\end{array}$ & $\begin{array}{l}\text { CWW } 99 \\
\text { CWW } 100 \\
\text { CWW } 101\end{array}$ & $\begin{array}{l}\text { GPW } \\
\text { GPW } \\
\text { GPW }\end{array}$ & $\begin{array}{l}.026 \\
.311 \\
.557\end{array}$ & 4,000 \\
\hline Jackson & $\begin{array}{l}\text { Jackson Water } \\
\text { Precinct }\end{array}$ & Ellis River & -- & -- & .037 & 500 \\
\hline Madison & $\begin{array}{l}\text { Village District } \\
\text { of Eidelweiss }\end{array}$ & $\begin{array}{l}\text { Grachen Well Field } \\
\text { Muddy Beach Well Field } \\
\text { New gravel-packed well }\end{array}$ & $\begin{array}{l}\text { MBW } 72^{1} \\
\text { MBW } 58^{2} \\
\text { MBW } 145^{3} \\
\text { MBW } 249^{4}\end{array}$ & $\begin{array}{l}\text { GPW } \\
\text { BRW } \\
\text { BRW } \\
\text { GPW }\end{array}$ & $\begin{array}{l}.027 \\
.052\end{array}$ & 725 \\
\hline Ossipee & $\begin{array}{l}\text { Ossipee Water } \\
\text { Department }\end{array}$ & Danhole River & - & - & .087 & 850 \\
\hline Wolfeboro & $\begin{array}{l}\text { Wolfeboro Water } \\
\text { Works }\end{array}$ & Upper Beach Pond & - & - & .725 & 5,405 \\
\hline Total & & & & & 2.692 & 15,042 \\
\hline
\end{tabular}

${ }^{1}$ MBW 72 represents five gravel-packed wells, four of which are inactive.

${ }^{2} \mathrm{MBW} 58$ represents two bedrock wells, both of which are inactive because of high radioactivity levels in the water

${ }^{3} \mathrm{MBW} 145$ represents two bedrock wells.

${ }^{4} \mathrm{MBW} 249$ was drilled in 1993 to supplement pumpage from existing well fields.

was $0.060 \mathrm{Mgal} / \mathrm{d}$, and for fish farming in Ossipee was $0.044 \mathrm{Mgal} / \mathrm{d}$ (1990 data). Industrial withdrawals in 1993 were $0.001 \mathrm{Mgal} / \mathrm{d}$ each in Madison (ground water) and in Ossipee (surface water). The amount of water used for snowmaking, mining, irrigation, and fish farming are average daily withdrawals based on an entire calendar year; the values would be larger if they were presented as averages only during the season of actual use. In addition, instream use for hydroelectric power generation in Ossipee was $8.105 \mathrm{Mgal} / \mathrm{d}$ (on the basis of 8 months of reported data in 1993).

\section{Methods of Study}

The following methods were used in this study: 
1. Areal extent of stratified-drift deposits was mapped. Soil maps (Diers and Vieira, 1977; and digitized U.S. Soil Conservation Service data on file with Complex Systems Research Center, University of New Hampshire, Durham, N.H.) and surficial-geologic maps (Goldthwait, 1968, and Newton, 1974) were used as an aid in determining the contact between stratified drift and till. These data were supplemented by field mapping done specifically for this study.

2. Available subsurface data on ground-water levels, saturated thickness, and stratigraphy of stratified-drift aquifers were compiled and data were reviewed for deficiencies. These data were obtained from published and unpublished sources of the USGS and the NHDES-WRD. Additional data were obtained from bridge borings of the New Hampshire Department of Transportation (NHDOT), well-drilling contractors, engineering consultants, municipalities, and local residents.

3. The location of production wells, test wells, and test borings were plotted on base maps at a scale of 1:24,000 and the data was added to the USGS Ground-Water Site-Inventory (GWSI) data base. Wells and borings were cross-referenced by an identification number and owner, as well as other locational and well construction information, water levels, and lithologic logs. All latitudes and longitudes are referenced to the North American Datum of 1927. The site locations are shown on plates 1 and 2 and information from these sites appears in appendixes 1 and 2 .

4. Seismic-refraction profiling, a surface-geophysical technique, was applied at 61 locations in the study area to determine depths to the water table and depths to the bedrock surface. These surveys ranged from 350 to $4,100 \mathrm{ft}$ in length. Locations of the profiles are shown on plates 1 and 2. Results are given in appendix 3. A 12channel, signal-enhancement seismograph was used to measure time-of-travel for a sound wave from a shot point to 12 geophone locations. Altitudes of geophones and shot points were determined by leveling to a common datum. The seismic-refraction data were interpreted by use of a time-delay, ray-tracing computer program developed by Scott and others (1972). Data from nearby wells and test holes were used to verify the interpretations.

5. Seismic-reflection profiling, another surfacegeophysical technique, was used to determine depths to bedrock and to infer the sediment type of the aquifers that lie beneath Ossipee Lake and seven other surface-water bodies in the study area. The methods used in data collection and interpretation are discussed in detail by Haeni (1986 and 1988b), Reynolds and Williams (1988), and Morrissey and others (1985).

6. Test borings were made at 38 locations to improve definition of the thickness and geohydrologic characteristics of the stratified-drift aquifers. Locations of the test borings are shown on plates 1 and 2. Split-spoon samples of the subsurface sediments collected at specific depths were used to evaluate the grain-size characteristics and identify the stratigraphic sequence of materials composing the aquifers. Thirty-two test borings were finished as observation wells with a 2 -inch inside-diameter polyvinyl chloride casing and slotted well screen. Wells were developed by use of a cycling-pneumatic pump. Water levels were measured periodically in these wells, and water samples were collected from selected wells.

7. Data collected as described in items 1 through 6 were used to prepare maps showing the watertable altitudes and saturated thicknesses of the stratified-drift aquifers.

8. Hydraulic conductivities of aquifer materials were estimated from field descriptions of the grainsize-distributions of samples from the test borings. Field descriptions were compared to descriptions of 454 sieved samples from previous ground-water studies in New Hampshire (Moore and others, 1994; Ayotte and Toppin, 1995). Transmissivities were estimated from the logs of test borings by assigning hydraulic conductivities to specific intervals, multiplying the hydraulic conductivities by the saturated thickness of the interval, and summing the results. Additional transmissivities were obtained from reports by geohydrologic consultants or from analysis of 
unpublished aquifer-test data. This information was used to prepare maps showing the distribution of transmissivity of the stratifieddrift aquifers (pls. 3 and 4). Drillers logs from wells and test borings not drilled by the USGS, in the areas of stratified drift, were examined during the preparation of these maps.

9. The aquifer boundary, water table, saturated thickness, and transmissivity maps were digitized from 1:24,000 and 1:25,000 scale-stable mylar quadrangle maps and entered into a Geographic Information System (GIS) computer data base. The digitized quadrangle maps then were merged into one study-wide GIS coverage for each map feature. GIS coverages include well, test boring, and spring locations; seismicrefraction and seismic-reflection profile locations; water-table configurations; saturated thicknesses; and transmissivities of the stratified drift.

10. Surface-water-discharge measurements (appendix 4) were made at 36 sites during low flow when the surface water is primarily ground-water discharge. These low-flow measurements indicate quantities of ground water potentially available from aquifers.

11. A large part of the aquifer surrounding Ossipee Lake was selected to demonstrate a technique for estimating water availability by use of a groundwater-flow model developed by McDonald and Harbaugh (1988) and modified by Orzol and McGrath (1992). Model-input data were generated by use of the GIS computer data-base coverages discussed in method number 9 .

12. Samples of ground water from 25 test wells and 4 springs were collected and analyzed for physical characteristics (specific conductivity, $\mathrm{pH}$, temperature) and concentrations of selected organic and inorganic constituents. The data provided by these analyses (appendix 5) were used to assess the water quality of the stratifieddrift aquifers. The data were also entered and stored in the National Water Information System (NWIS) computerized data base.

\section{Numbering System for Wells, Borings, and Springs}

Local numbers assigned to wells, test borings, and springs entered into GWSI consist of a two-letter town code (table 2), a supplemental letter designation ("A" for borings done for hydrologic purposes with no casing set, "B" for borings done primarily for constructional purposes, "S" for springs, and "W" for all wells in which a casing was set), and a sequential number in each town. For example, the first well in the town of Albany is ADW 1.

\section{Acknowledgments}

The authors thank the State and Federal agencies, municipalities, well-drilling contractors, and consulting firms who provided data for this study. Included are personnel from the New Hampshire Department of Environmental Services, Water Resources Division, Kenneth Stern, Frederick Chormann Jr., Richard Schofield and Kevin Riel. Appreciation is also expressed to Complex Systems Research Center at the University of New Hampshire for providing digitized soils and hydrography data. Thanks are also given to the residents and land owners in the study area who graciously allowed access to their land for purposes of data collection, and to Robert Newton, Fredrick Chormann, Jr. and Richard Schofield for providing reviews of this report.

Table 2. Two-letter town codes used as prefixes in the numbering system for wells, borings, and springs in the Saco and Ossipee River Basins, east-central New Hampshire

\begin{tabular}{lc|lc}
\hline Town & $\begin{array}{c}\text { Two- } \\
\text { letter } \\
\text { Code }\end{array}$ & Town & $\begin{array}{c}\text { Two- } \\
\text { letter } \\
\text { Code }\end{array}$ \\
\hline Albany & AD & Jackson & JA \\
Bartlett & BC & Madison & MB \\
Chatham & CK & Ossipee & OX \\
Conway & CW & Sandwich & SE \\
Eaton & EC & Tamworth & TA \\
Effingham & EF & Tuftonboro & TZ \\
Freedom & FL & Wakefield & WA \\
Harts & HJ & Wolfeboro & WR \\
Location & & & \\
\hline
\end{tabular}




\section{GEOHYDROLOGIC SETTING}

Ground water underlies the land surface throughout the Saco and Ossipee River Basins. Subsurface formations that have sufficient saturated permeable materials that yield significant quantities of water to wells are termed aquifers. Even aquifers with low productivity may yield enough water to wells for domestic users.

The three types of aquifers present in the study area are: (1) stratified drift, which is the major source of ground water for municipalities (fig. 3); (2) till, which locally can supply ground water for domestic use; and (3) bedrock, which yields a variable supply but is a major source of ground water for private domestic needs and small businesses (U.S. Geological Survey, 1985).

\section{Postglacial Redistribution of Glacial Deposits}

Modification and redistribution of glacial deposits in the study area has been significant. These processes include erosion and redeposition of material by rivers, by streams, and, to a lesser extent, perhaps by wind. Postglacial down cutting by the Saco and Ossipee Rivers and their tributaries has resulted in erosional channels, alluvial fans, stream terraces, flood-plain deposits, and modern deltas.

Alluvial fans are an integral part of many stratifieddrift-aquifer systems because fan deposits can increase potential yield of the aquifers by increasing the infiltration or recharge from tributary streams. Typically, alluvial-fan deposits form under extreme flood conditions, and where a stream emerges from the till uplands, flows out onto flatter areas of stratified drift, and deposits coarse material (largely gravel and sand). Under conditions of low streamflow, alluvial fans enhance infiltration from streams as they flow out onto the areas of stratified drift, which can dry up the river. The Dry River, in Hart's Location (plate 1), is named for this reason. The formation of alluvial-fan deposits may have been most active immediately following deglaciation from redeposition of the newly exposed land surface. Deposition of alluvial fans has continued until the present day.

Alluvial fans are found at numerous sites throughout the study areas. Fans are especially prevalent in the northern more mountainous part of the study area. A partial list of alluvial-fan sites include: a site along the west side of Province Lake in Effingham (Goldthwait, 1968); the west side of Crystal Lake in Eaton, and where the Chocorua River emerges from the steep slopes of Mt. Chocorua in Albany (Newton, 1974); where streams emerge onto the valley fill, where the Mill, Charles, Chandler, McDonough, Langdon and Weeks Brooks emerge in Chatham; where Avalanche, Davis and Nancy Brooks, and the Sawyer and Dry Rivers emerge in Hart's Location where the Razor, Meadow, Albany, Bartlett, and Mountain Brooks and Rocky Branch, Ellis, and East Branch Rivers emerge in Bartlett; the Miles Brook and the Ellis and Wildcat Rivers in Jackson; Oliverian Brook in Albany; Swift River in Livermore; Whiteface River at Whiteface Intervale, Wonalancet River at Wonalancet Intervale, and the Cold River in Sandwich (pls. 1 and 2).

Postglacial redeposition of sediments also includes modern deltas built out into Ossipee Lake, such as the Bearcamp River delta, the Pine River delta, the Lovell River delta, and the Chocorua River delta. Along the southeast shore of Ossipee Lake, post-glacial migration of the lake outlet is the result of redeposition of sediment by beach processes and long-shore currents (Newton, 1974, p. 38).

\section{Stratified Drift}

Stratified-drift aquifers, the focus of this study, consist of stratified, sorted, dominantly coarse-grained sediments (sands and gravels) deposited by glacial meltwater at the time of deglaciation. Alluvial and windblown deposits, younger than the stratified drift, in contact with stratified drift, are included as part of the stratified-drift aquifers. Distributions of the stratifieddrift aquifers in the study area are shown in figure 3. Hydraulic characteristics of these sediments that affect ground-water storage and flow are related to the glaciofluvial and glaciolacustrine environments in which the sediments were deposited. Stratified-drift deposits are composed of distinct layers of sediments with different grain-size distributions, sorted according to the depositional environment. For example, fastmoving meltwater streams are apt to deposit coarsegrained sediments with large pore spaces between grains. If saturated, these materials generally form aquifers that transmit ground water readily. Slowmoving meltwater in streams, lakes, and ponds are apt to deposit fine-grained materials (which consist of very 


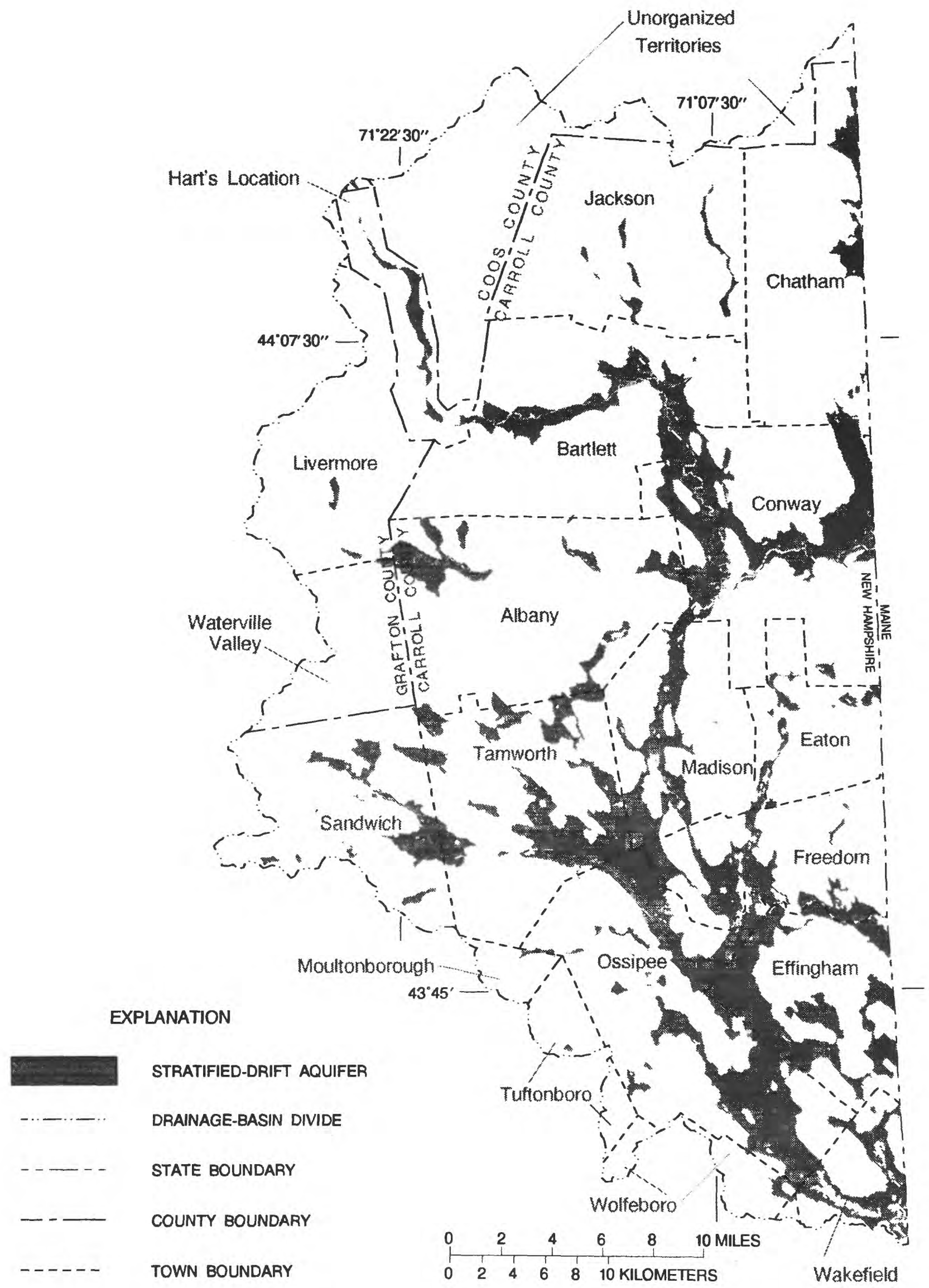

Figure 3. Location of stratified-drift aquifers underlying the Saco and Ossipee River Basins study area, east-central New Hampshire. 
fine sand, silt and clay). These fine-grained deposits do not transmit water as readily as the coarse-grained deposits.

The size and arrangement of voids or pore spaces between sediment particles determine the capacity of the aquifer material to store and transmit ground water. Large, interconnected pore spaces readily transmit ground water and provide a large volume of groundwater storage. The ratio of total volume of pore space to the total volume of sediment is a measure of the porosity or space available for ground-water storage. Specific yield, the ratio of the volume of water that can be drained by gravity to the total volume of the sediment, is used to measure the amount of water stored in an aquifer. Total volume of pore space per total volume of sediment and specific yield are not equal because some water is held on the grain surfaces by tensional forces and will not drain by gravity. These characteristics are related to the original depositional environment of the sediments, and, thus, the term "stratified-drift aquifer" refers to several different types of aquifers, depending on the mode of deposition.

The deglaciation process, and the location of glacial lakes during deglaciation, had a pronounced effect on the types of stratified-drift aquifers in the study area. Throughout New England, deglaciation is believed to have occurred by a systematic process of retreat with minor localized readvances (Koteff and Pessl, 1981). As the active glacial-ice margin receded to the north, zones of stagnant ice were left in contact with the active ice margin. Numerous features throughout the southern part of the study area are associated with stagnant ice and characterize this northward retreat of a stagnant-ice zone in front of the active glacier (Newton, 1974). Examples of segmented eskers and deltas fed by eskers support this style of deglaciation and are among the most potentially productive aquifers.

Stratified-drift aquifers underlying the study area include ice-contact eskers and deltas, outwash deltas deposited by meltwater streams flowing in front of the glacier, collapsed outwash, kames, deposits from glacial-ice-dam-break flood deposits (alluvial fans and deltas), and alluvial fans and deltas fed by streams resulting from precipitation falling directly on the initially barren land surface. In certain cases, exposed till and other glacial sediments were eroded and redeposited.
The stratified-drift aquifers in the Saco and Ossipee River Valleys and in valleys of the major tributaries include a through valley that connects the two basins. Fine-grained, stratified-drift deposits in parts of these valleys typically are overlain by thick deposits of sand and gravel. Some potentially productive stratifieddrift aquifers are discontinuous; however, others in the study area are connected and form the largest potentially productive stratified-drift aquifer in New Hampshire. This continuous stratified-drift aquifer extends $37.3 \mathrm{mi}$ northward from Wakefield to Bartlett N.H. and eastward into Maine.

Three valleys, called "through valleys", cut north to south across the hilly zone between the Saco and Ossipee River drainage basins (Newton, 1974) (fig. 4). The forms of the valleys are maintained as one through valley crosses a low divide such that streams south of the divide flow south and streams north of the divide flow north. These valleys are filled with glacial-icestagnation features such as eskers, kames, kame terraces, and kame fields. The three through valleys are referred to as the Eaton, Silver Lake, and Chocorua through valleys (Newton, 1974). These valleys are the result of erosion by several glaciations during the Pleistocene Epoch, however, the stratified-drift aquifers in the Saco and Ossipee River Basins were deposited by the most recent glaciation.

\section{Glacial-Lake Deposits}

During the earliest stages of deglaciation of the study area, the natural drainage to the north in the Pine River Basin, in the southern part of the study area, was obstructed by the ice margin, forcing the melt water to drain south. The drainage divide acted as a dam and formed a series of lakes between it and the glacier, similar to the Contoocook River Basin, another northwardly draining basin in New Hampshire, (Moore, 1993; Moore, in Harte and Johnson, 1995; Hildreth and Moore, in press). At least one and perhaps two spillways formed in the sand and gravel of the divide between Pine River Pond and Stump Pond (altitude $600 \mathrm{ft}$ above sea level) (pl. 2). As the ice margin continued to melt back to the north, the first lake expanded until a lower lake outlet was exposed northwest of White Pond and the first lake drained rapidly to the levels of the newly exposed outlet of the second lake (altitude $560 \mathrm{ft}$ above sea level). Further melting back of the ice margin caused the lake to merge with a third 


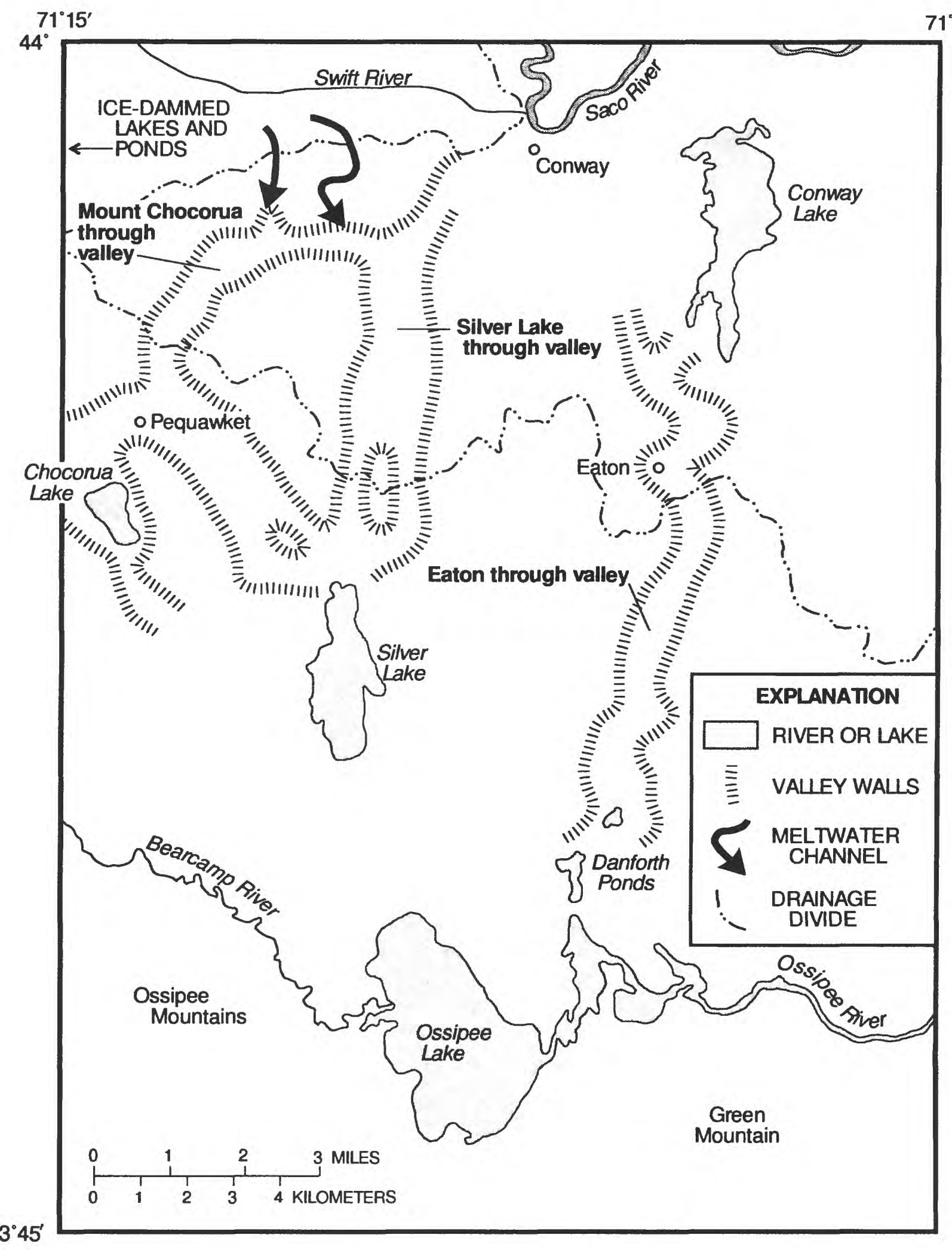

Figure 4. Ossipee Lake Quadrangle showing the location of the three "through valleys," east-central New Hampshire (Modified from Newton, 1974). 
lake, only slightly lower in altitude, that extended beyond the study area into Maine. Continued melting northward of the ice margin exposed another, lower outlet to the north of Green Mountain (altitude $460 \mathrm{ft}$ above sea level) and the lake drained rapidly to the levels of the newly exposed outlet. Next the outlet shifted to a position $0.6 \mathrm{mi}$ to the north where the Ossipee River is presently.

In the Saco River Basin, lake deposits are also found in the Conway area. These may have been deposited in a single large lake (Leavitt and Perkins, 1935, p. 94; Thompson, 1986; Tepper and others, 1990, p. $10-$ 11 ), or it is also possible that with the rapid infilling of sediment, dams formed and several smaller lakes were created instead of one large one (Tepper and others, 1990, p. 10-11).

The formation of glacial lakes was enhanced by the underlying bedrock surface - scoured and plucked by glacial ice during multiple glaciations during the Pleistocene Epoch. For example, the underlying bedrock surface surrounding Ossipee Lake was deeply eroded into a trough-shaped basin. The center of the glacial lake, where Ossipee Lake is presently, was at least $280 \mathrm{ft}$ deeper than the lowest point of its rim in the eastern part of the lake where the Ossipee River now flows eastward from Berry Bay. This natural "bowl" in the bedrock surface was an important factor in the formation of a glacial lake during deglaciation. Sediment deposition during deglaciation rapidly filled in most of this lake, and post-glacial lowering of the lake level was the result of erosion at the outlet. Ossipee Lake is essentially a remnant of the previous glacial lake.

Glacial-lake environments thus dominated the central portions of the Ossipee and the Saco River Basins during deglaciation. The largest amounts of stratified drift were deposited in glacial lakes that were being filled with outwash and fine-grained sediments. The coarsest stratified-drift deposits formed in these areas are ice-contact eskers and deltas. Some of the deltas may have been fed by sediment in meltwater flowing from the esker channels in or beneath the glacial ice. Locally, the coarse-grained stratified drift, deposited as subaqueous fans and deltas, is found beneath finegrained stratified drift. Typically the deltas prograded out over fine-grained deposits and resulted in a lithologic sequence that coarsens upward. The eskers and deltas constitute the primary aquifers found in areas where glacial lakes were present.
Confined aquifers beneath glacial-lake silts and clays are not well documented within the study area. This may be a result of the absence of confined aquifers or may simply reflect a limitation of the available documentation. When installing a bedrock well, local well drillers may not notice a layer of aquifer material between fine sediment and the bedrock. Alternatively, when installing a well in stratified drift, drillers often find an adequate source of water above the silts and clays and have no reason to drill further.

The surfaces defining previous levels of glacial lakes throughout New Hampshire have been uplifted in response to the removal of the glacial ice (isostatic rebound). The uplift is greater further to the northnorthwest. Projections of these previous lake-level surfaces now slope to the south-southeast. The exact slope of these projected lake surfaces in the Ossipee River Basin have not yet been determined. In western New Hampshire in the Connecticut River Valley, a stabilized lake-level surface of glacial Lake Hitchcock is indicated by numerous ice-contact deltas and numerous other deltas that have not been modified by collapse and have topset-foreset contacts along a single plane. This stabilized lake-level surface indicates that the postglacial uplift of New England, which resulted from the melting of the continental ice sheet, was delayed by at least 5,000 years (Koteff and Larsen, 1989). After the postglacial uplift the lake-level surface now slopes about $4.8 \mathrm{ft} / \mathrm{mi}$ downward in the direction of about S. $21^{\circ} \mathrm{E}$. (Koteff and Larsen, 1989). The projected lake-level surfaces of glacial lakes to the east in the Merrimack River Valley (glacial Lakes Tyngsboro, Merrimack, and Hooksett) now slope 4.7-4.9 $\mathrm{ft} / \mathrm{mi}$ downward to the south-southeast (Koteff and others, 1984). Similarly to the southeast, the projected sea-level surface, onto which numerous glaciomarine deltas were deposited, now slopes about $4.5 \mathrm{ft} / \mathrm{mi}$ downward in the direction of S. $28.5^{\circ}$ E. (Koteff and others, 1993). From this information, glacial lakes in the study area also are assumed to have had previous lake-level surfaces that now slope to the south-southeast.

\section{Eskers}

Eskers are long ridges of sand and gravel deposited either in meltwater channels in the zone of ice stagnation during deglaciation, or at the ice margin where the glacier retreats in contact with a standing water body. In the second case, sand and gravel is deposited where a 
meltwater channel empties into a water body. Steady retreat of the ice margin causes deposition at locations progressively further up the meltwater channel. Thus, a ridge that follows the course of the previous channel is created. Eskers are numerous in the southern part of the study area (Goldthwait, 1968; Newton, 1974). Eskers are also found in Tamworth, Albany, Madison, Freedom, Eaton, and Conway (Newton, 1974). In the northern part of the study area, however, eskers are short, scarce, and not well documented.

The most notable group of esker segments is the Pine River Esker, which contains the largest esker deposits in New Hampshire. These esker segments extend from Wakefield northward through Ossipee, and Effingham. Train loads of sand and gravel from this esker are transported, on a daily basis, to Massachusetts. Much of the esker in Ossipee has already been mined above the water table. Many subparallel lower ridges are found on either side of a main esker and specific ridges become braided, suggesting that different water courses were melted in the ice during different periods of time (Goldthwait, 1968, p. 33). The segmented nature of the eskers, with relatively narrow ridges leading up to broader areas, indicate that the esker system was developed in sequential stages. The broader areas, such as northwest of Pine River Pond and at White Pond, are probably areas where the ice channels opened up at or near the temporary ice margins.

Eskers in the study area have also been differentiated into two groups (Newton, 1974), on the basis of their orientation to the valleys - valley-side eskers and valley-bottom eskers. Valley-side eskers are oriented approximately at right angles to the valley walls, whereas valley-bottom eskers are parallel to the axis of the valley walls.

Eskers with significant saturated thickness easily store and readily transmit water. In New Hampshire, eskers often formed where an ice margin melted into a glacial lake in front of the glacier. In these areas, eskers formed before, or simultaneously with, the removal of ice from that area and the simultaneous inundation by the glacial lake. As a result, coarse-grained stratified drift (the esker deposit) locally is buried beneath finegrained stratified drift (the lake-bottom sediments). Given this history and mode of deglaciation, subaqueous fans and distal ends of deltas are also locally buried beneath fine-grained lake-bottom deposits. The location of confined aquifers composed of these buried deposits, may be undetected in areas lacking subsurface data.

\section{Through-Valley Deposits}

Through-valley deposits in the three through valleys in the Saco and Ossipee River Basins (fig. 4) grade to the level of the glacial lakes. Deglaciation resulted in the formation of eskers, kames, kame terraces, and outwash deposits. These deposits compose the stratifieddrift aquifers of these areas. Examples of these aquifers are found in Albany, Conway, Eaton, Freedom, and Madison. On the north and west sides of the Chocorua through valley and the Silver Lake through valley, glacial meltwater formed channels that slope down to the valley fill (fig. 4) (Newton, 1974). Given the severity of the erosion and the large size of the boulders carried by the meltwater, these channels were likely caused by glacial-ice-dam-break floods from water ponded between the ice and the drainage divide for the Swift River Basin (fig. 4). Similar features have been observed in southcentral New Hampshire, in the Contoocook River Basin (Moore, 1993; Moore, in Harte and Johnson, 1995; Hildreth and Moore, in press). Sediment-laden meltwater was carried in these channels to the valleys below, and, thus was a source for some sediment of the throughvalley deposits. Meltwater emerging directly from the glacial ice, as evidenced by the eskers, was another source of sediments.

\section{Till}

Till is an unsorted mixture of rock debris ranging in size from clay to boulders that was deposited directly by glacial ice. Within the study area, till is the dominant surficial material. Beneath flowing ice, compact lodgement till was deposited discontinuously on the bedrock surface. In some areas, a large amount of lodgement till was deposited on north- and northwest-facing bedrockhill slopes that locally blocked the flow of the debrisladen ice. As a result, the thickness of till can exceed $200 \mathrm{ft}$. Much of this thick lodgement till is believed to be from the previous Illinoian glaciation over 133,000 years ago, rather than from the most recent glaciation, the Wisconsinan (B. Stone, in Davis and others, 1993, p. EE-4). This lower till, of the Illinoian glaciation, is typically much less permeable than the upper till deposited during the Wisconsinan glaciation. 
Ablation till, which discontinuously overlies compact till or bedrock, formed as residual deposits on the melting (wasting) ice surface and gradually settled on the underlying surface as the ice melted. Ablation till is less compact than lodgement till, is slightly stratified in places, and can contain lenses of stratified material. Because ablation till with stratified lenses may grade laterally into stratified drift, the distinction between till and stratified drift is uncertain. Generally, till directly overlies bedrock. In the Saco and Ossipee River Basins, however, till directly overlies stratified drift in Chatham at well CKW 35 (pl. 1). The mode of deposition of the stratified drift, buried beneath till, has not been determined.

Till is not considered to be a major source of ground water because of generally low hydraulic conductivity. Nonetheless, till, composing an aquifer, is important for domestic water supply. Water-level fluctuations in till can be large enough (several feet) to dry shallow dug wells during dry seasons.

\section{Bedrock}

Bedrock in the Saco and Ossipee River Basins consists of metamorphic, plutonic, and volcanic rocks. The metamorphic bedrock underlies about 27 percent of the study area (Lyons and others, 1986). Metasediments of Silurian age comprise about 16.4 percent of the total study area; whereas younger metasediments of Devonian age, largely of the Littleton Formation, comprise about 10.5 percent of the total study area. Plutonic and associated volcanic bedrock underlies the remaining 73 percent of the study area (Lyons and others, 1986). From oldest to youngest, the Devonian bedrock, of the New Hampshire Plutonic Suite, constitute about 9 percent of the study area. Located in the more mountainous northern half, Mississippian bedrock compose about 22 percent, and Jurassic bedrock, of the White Mountain Plutonic Suite, compose about 35.4 percent of the study area. The Cretaceous bedrock compose about 5.8 percent of the study area and underlies two of the highest areas in the southern half of the study area. These rocks constitute the classic ring-dike complex of the Ossipee Mountains (Wilson, 1969) and they underlie Green Mountain.

Metamorphic and plutonic rocks are generally hard and compact and contain recoverable water only in open fractures (secondary porosity). The size, distribu- tion, and degree of interconnection of these open fractures are highly variable and their numbers generally decrease with depth. Thus, the capacity of bedrock to store water is variable but generally small. Bedrock wells commonly yield dependable supplies of acceptable water quality for domestic and small commercial uses, however, there have been instances where bedrock wells could not supply domestic needs.

Zones where bedrock is extensively fractured can yield large quantities of water. For example, a municipal well open to bedrock in Madison provides an average daily yield of $0.052 \mathrm{Mgal} / \mathrm{d}$ (table 1). Six municipalities in New Hampshire, all outside the study area, have wells open to crystalline bedrock and three of these are reported to yield approximately $0.5 \mathrm{Mgal} / \mathrm{d}$. Systematic exploration may allow location of production wells in fractured zones (Cotton and Hammond, 1985).

Weathered bedrock, perhaps remnant from Tertiary weathering or at least weathering before the last glaciation, was identified in numerous well logs in the study area, especially in Albany, Bartlett, Conway, and Jackson (appendix 2). Deeply weathered granite, called "rotten rock", on the lee side of hills, was apparently protected from the glacial erosion that scoured the bedrock surface down to fresh solid rock (Davis and others, 1993, p. EE-13). In these areas where weathering of granitic gneiss has produced numerous small fractures between mineral grains, the weathered rock can be water bearing. There are numerous bedrock wells in these areas of rotten rock with reported well yields in excess of $40 \mathrm{gal} / \mathrm{min}(0.058 \mathrm{Mgal} / \mathrm{d})$ (appendixes 1 and 2).

\section{GEOHYDROLOGY OF STRATIFIED-DRIFT AQUIFERS}

The geohydrology of the stratified-drift aquifers was described by identifying (1) aquifer boundaries, (2) direction of ground-water flow from recharge to discharge areas, (3) aquifer thickness and storage, and (4) aquifer transmissivity. Data sources in this investigation include surficial-geologic maps, records of wells, test borings and springs, seismic-refraction profiles, and seismic-reflection profiles. Results of the geohydrologic investigation are presented on plates 1-4 
and in the text that follows. Plates 1 and 3 are for the northern half of the study area, and plates 2 and 4 are for the southern half.

\section{Delineation of Aquifer Boundaries and Water Table}

Stratified-drift aquifers underlying the study area are composed of fine- to coarse-grained sands and gravels deposited by glacial meltwaters; these deposits, in part, are now sufficiently saturated to yield significant quantities of water to wells and springs. Locations of the lateral boundaries of the aquifers are defined as the contacts between the stratified drift and till and (or) bedrock. The position of the contact was determined by use of surficial geologic maps, soil maps, test-boring logs, and field mapping done specifically for this study. The bottom boundary is the contact of the stratified drift with the till and (or) bedrock surface and was determined by use of data from seismic refraction, seismic reflection, test borings, and domestic water wells. Water-table altitudes were also determined from wells, surface-water bodies, and geophysical data.

\section{Areal Extent of Stratified-Drift Aquifers}

The areal extent of coarse-grained stratified-drift aquifers is shown on plates 1 and 2 and in figure 3 . Also shown are the locations of glaciolacustrine lake-bottom sediments. A source of data for delineating aquifer boundaries was 1:62,500-scale, surficial-geologic maps (Goldthwait, 1968; Newton, 1974), and previous 1:24,000 scale aquifer maps for the Conway area (Tepper and others, 1990). Aquifer boundaries for the majority of the study area were updated or specifically mapped at the 1:24,000-scale. The aquifer-boundary maps shown on plates 1 and 2 are simplified surficialgeologic maps. These aquifer maps show the areal extent of the stratified-drift deposits that contain sufficient saturated permeable material to yield significant quantities of water to wells and springs. Surficialgeologic maps show several additional features not included on the aquifer-boundary maps, such as swamps that can conceal aquifer boundaries.

Aquifer boundaries are shown in plates 1 and 2 as solid, dashed, or dotted lines. In the explanation for the plates, solid lines represent boundaries that are "approximately located," dashed lines represent "inferred" boundaries, and dotted lines represent "concealed" boundaries. A solid line contact on a 1:25,000-scale USGS map implies about a \pm 80 -feet horizontal accuracy. In most areas, the solid-line boundaries probably are plotted with nearly this accuracy.

\section{Stratigraphic Position of Lithologic Units and Altitude of Water Table}

The stratigraphic position of lithologic units and altitude of water table were determined by use of data stored in the GWSI data base and from the seismicrefraction and seismic-reflection surveys.

\section{Ground-Water-Site Inventory}

Subsurface data from wells, bore holes, and springs were inventoried and plotted on plates 1 and 2 . Geohydrologic data for 1,848 locations have been added to the USGS computerized GWSI data base (appendixes 1 and 2). Data on 1,473 new wells (drilled since 1984) in the study area came from the New Hampshire Water Well Board data base. About 1,130 of the 1,848 total sites are in the areas mapped as stratified drift.

The data assembled in the GWSI data base were used to produce the plates that accompany this report. The following sections of this report present the methods used in plate production. Data and contoured interpretive information also can be transferred automatically to the State of New Hampshire's GIS data base and the data analyzed relative to other geographic features. Applications of the USGS GWSI data base are discussed by Mercer and Morgan (1981).

\section{Seismic Refraction}

Seismic-refraction surveys, totaling $13.3 \mathrm{mi}$, were completed at 61 locations in the study area to determine depths to the water table and bedrock (pls. 1 and 2). A 12-channel, signal-enhancement seismograph was used to record the arrival times of compressional waves generated by a sound source. The data were collected according to procedures described by Haeni (1988b). Interpretive seismic-refraction profiles, made by use of a computer program (Scott and others, 1972), are given in appendix 3 . Seismic velocities estimated for the materials under investigation and used in the seismic interpretations are 900 to $1,500 \mathrm{ft} / \mathrm{s}$ for unsaturated stratified drift, about $5,000 \mathrm{ft} / \mathrm{s}$ for saturated stratified 
drift, and about 10,000 to $20,000 \mathrm{ft} / \mathrm{s}$ for bedrock. Estimated depths were generally in agreement with data for nearby wells and observations of outcrops, springs and brooks, and with shallow auger-hole data collected during the refraction surveys. Vertical scales of the seismic-refraction cross sections (appendix 3 ) are exaggerated and non-uniform.

Altitude of the land surface (appendix 3) in feet above sea level, was estimated by leveling the geophone locations relative to one another, and tying that information to sea level datum by use of information from USGS topographic quadrangle maps. Altitudes of the land surface shown in appendix 3 are assumed to be accurate to half a contour interval or about $10 \mathrm{ft}$.

Estimated altitude of the water table in stratified drift, as determined by interpretation of the seismicrefraction data, is shown in appendix 3 . The altitude is accurate to within a little over $10 \mathrm{ft}$ and represents the water table at the time the seismic-refraction data were collected in the summer and fall of 1991 and 1992.

Estimated altitude of the bedrock surface is shown in appendix 3. The estimation of the altitude of the bedrock surface is not as accurate as that of the water table because errors in the interpretation are cumulative. If the depths to the water table, estimated by the interpretation of the seismic-refraction data, are erroneously shallow, the estimated position of the bedrock will be low by an even greater margin than the water table. Additional error will result if the relief of the bedrock surface differs considerably over distances less than the 50 - or 100 -feet geophone spacing used in profiling, or if a thick layer of till overlies the bedrock. Where till is present in significant thickness and is not accounted for in the interpretation of seismic data, the computed depth to the bedrock is slightly less than the actual depth. In general, accuracy of the altitude of the bedrock surface probably averages plus or minus 10 percent of the depth to bedrock but may be greater in some places.

\section{Seismic-Reflection Surveys at Eight Selected Lakes or Ponds}

High-resolution, continuous seismic-reflection surveys were completed and interpreted along $13.7 \mathrm{mi}$ of 8 lakes or ponds in the study area. Seismic-reflection data were used to map the depth to the bedrock below the water surface and to define the bulk aquifer stratig- raphy. This information was used to interpolate between the survey locations beneath the water bodies and points on land.

During data collection, a sound source and array of hydrophone receivers were towed behind or along side a boat that traveled slowly along the lake shoreline. Compressional sound waves, generated by a source, traveled through the water column and penetrated the materials beneath the lake and were reflected back to the water surface in response to the physical differences in geologic strata. The reflected sound waves were received by the array of hydrophones, converted to an electrical signal, amplified, filtered, and displayed on a graphic recorder.

Locations where the seismic-reflection surveys provided useable data are shown on plate 2. Also, the locations of three selected surveys are shown in figure 5. The accompanying seismic-reflection records and interpreted geology from these three selected surveys are shown in figures 6-8. The lake bottom, the bedrock or till surface, and the type of unconsolidated deposits are indicated.

\section{Recharge, Discharge, and Direction of Ground-Water Flow}

Ground-water recharge is the water that is added to the saturated zone of an aquifer. Natural recharge is the difference between total precipitation and the amount of water that is lost, before reaching the water table, as surface runoff and evapotranspiration. Vegetation and soil permeability affect the quantity of recharge to aquifers. Sandy soil covered by mature forests can absorb up to about 1 in. of rainfall per hour and silty, clayey soil covered by mature forests may absorb less than $0.1 \mathrm{in} / \mathrm{hr}$ (Heath, 1983). In August through October 1977, Hill $(1979$, p. 87) estimated that one third of the rainfall that fell on the surface of the Newmarket Plains aquifer in southeastern New Hampshire reached the water table. In other parts of the Northeast, estimated recharge to stratified-drift aquifers has been as much as one-half of the annual precipitation (MacNish and Randall, 1982; Plukowski and Kantrowitz, 1964).

Recharge to the stratified-drift aquifers also comes from the adjacent till-covered bedrock uplands. Morrissey (1983) estimated that the average annual lateral inflow of ground water from upland areas to a stratified- 


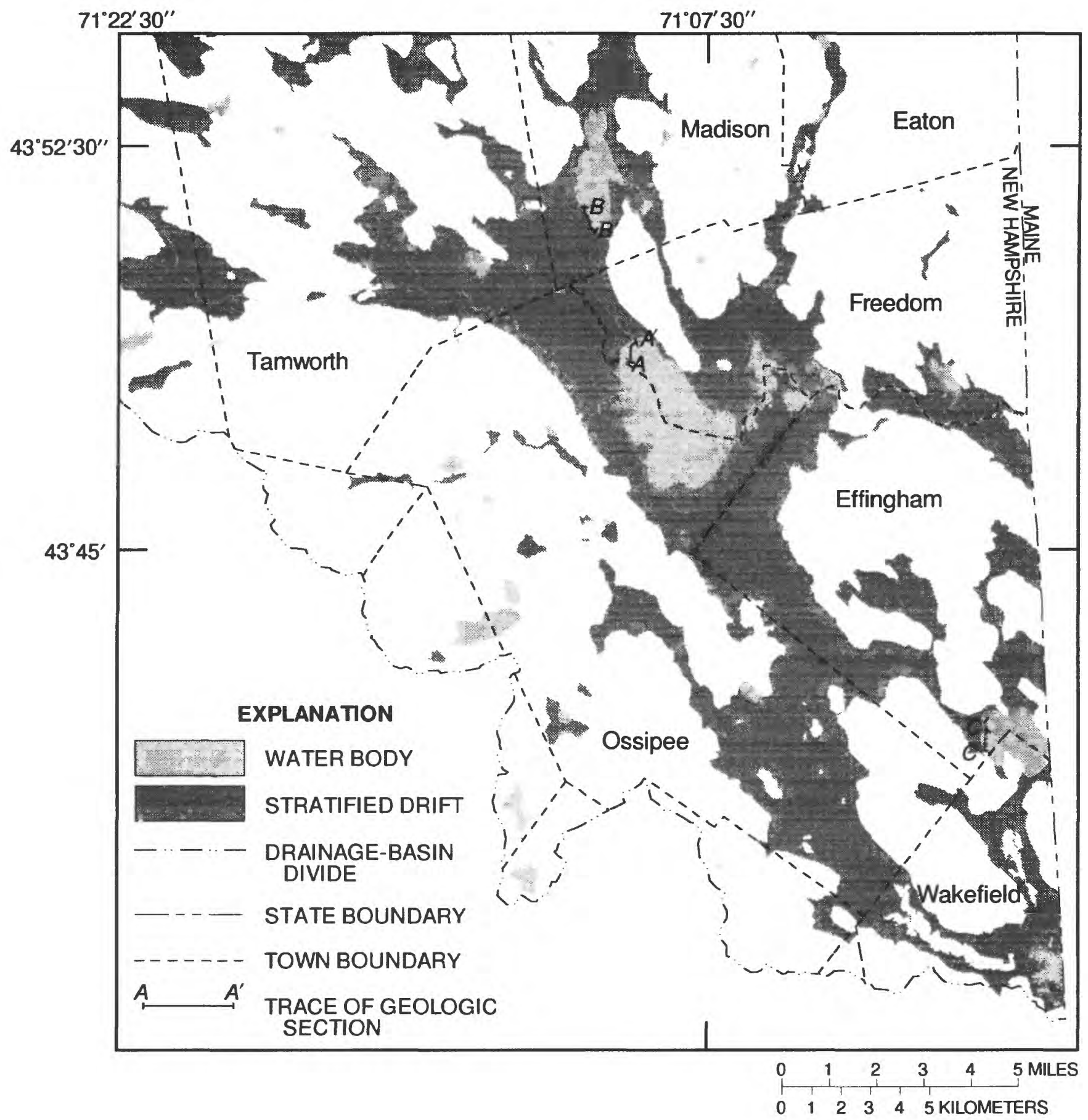

Figure 5. Location of geologic sections interpreted from seismic-reflection data in the Ossipee River Basin, east-central New Hampshire. 


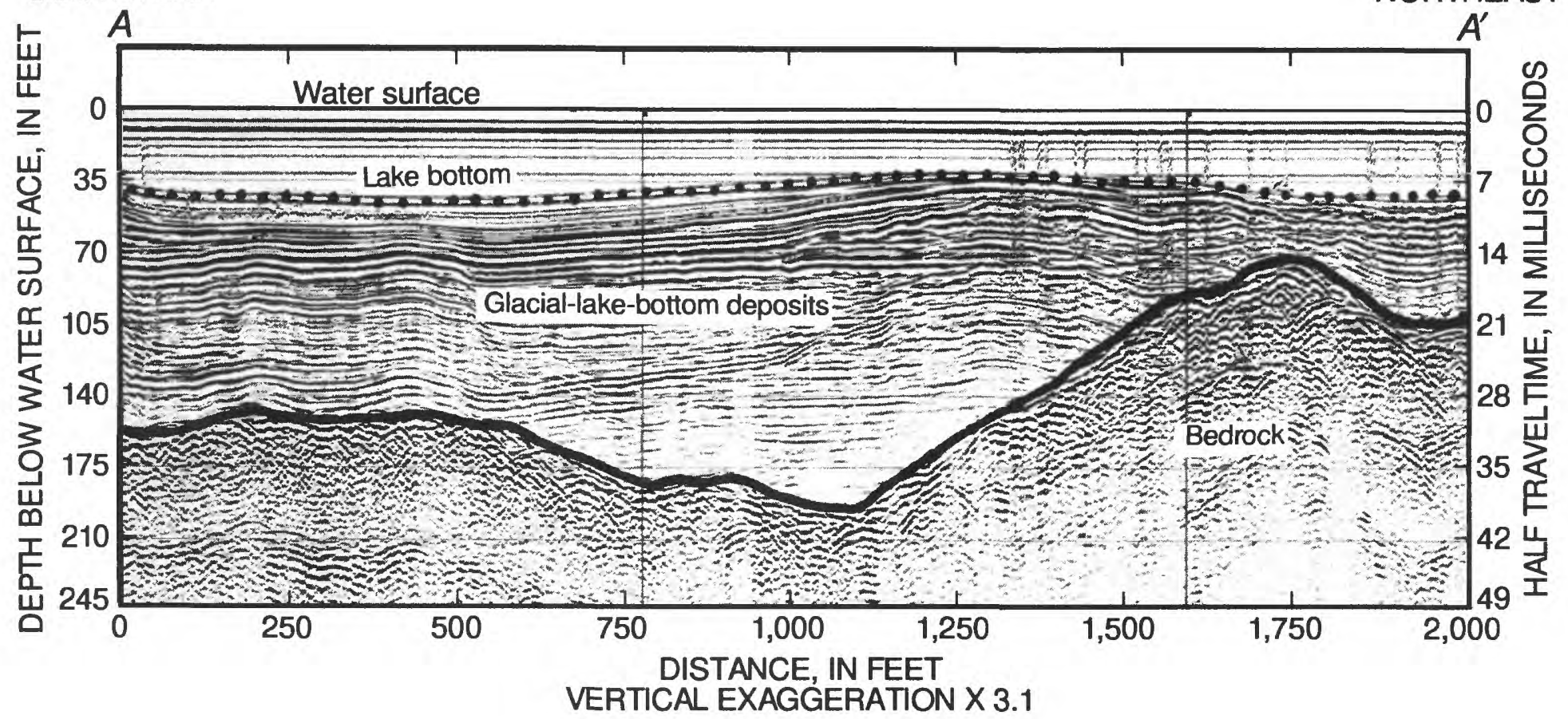

Figure 6. Geologic section interpreted from seismic-reflection data for Freedom line $A-A^{\prime}$ in the Ossipee River Basin. Location of section shown on figure 5.

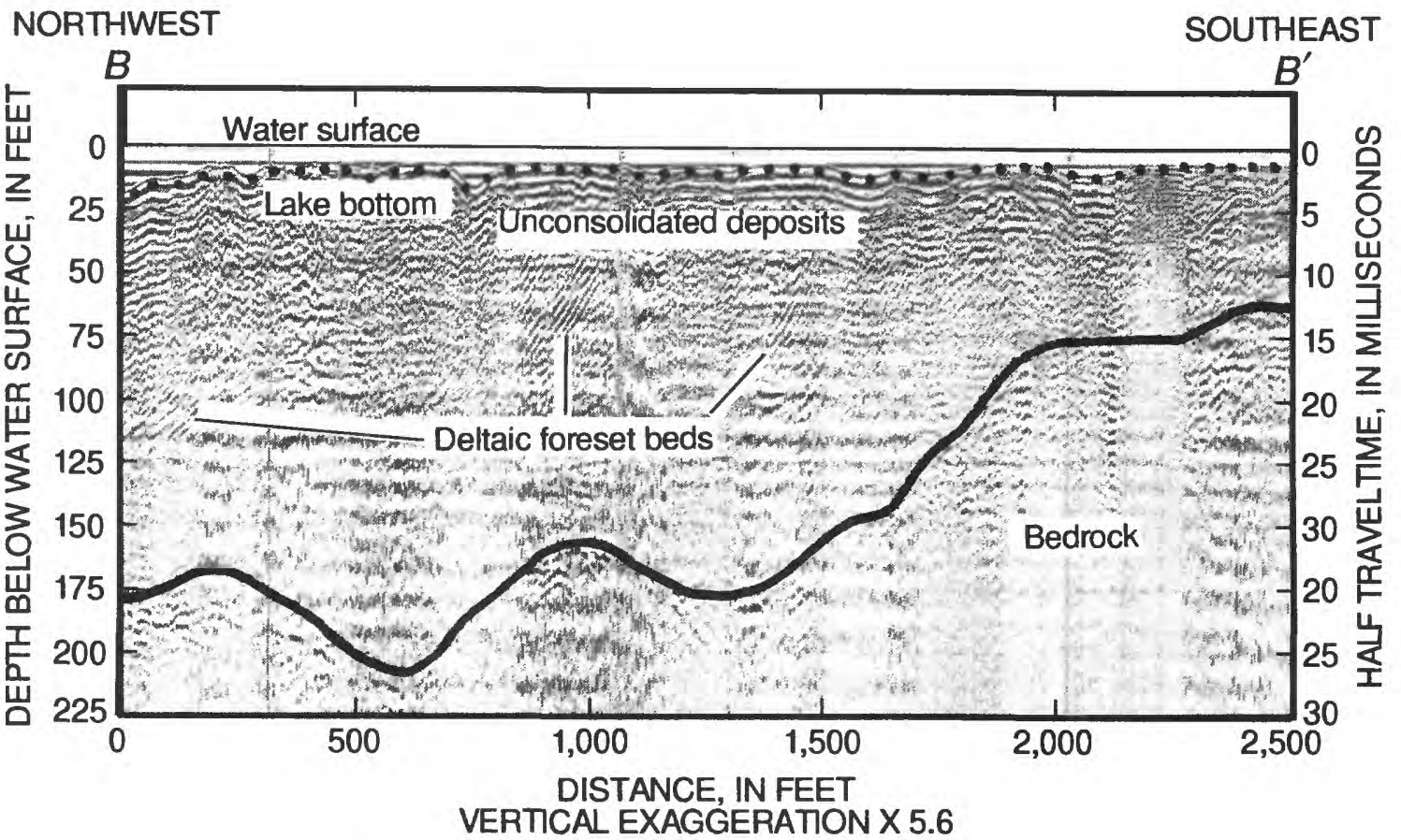

Figure 7. Geologic section interpreted from seismic-reflection data for Madison line B-B' in the Ossipee River Basin, east-central New Hampshire. Location of section shown of figure 5. 
screened in till tend to have larger fluctuations than water levels in wells screened in coarse-grained stratified drift.

The altitude of the water table in the stratified-drift aquifers is shown on plates 1 and 2. Water-table contours were drawn to represent water levels during the summer season. Maps of the water-table altitude are based on water-level data derived from (1) altitudes of streams, ponds, and rivers as shown on USGS 1:24,000scale topographic maps; (2) well records in the GWSI data base (including the 29 observation wells); and (3) geophysical seismic-refraction profiles (appendix 3).

Water-level altitudes were mapped for unconfined aquifers in the stratified drift but not for confined aquifers. The altitude of the water table is controlled locally by the stratigraphy, which differs from place to place. In areas where the gradient is steep, the water table was difficult to contour. In some areas, water-level data were not available, thus contours were not drawn.

Water-table contours generally indicate natural sources of recharge to and discharge from aquifers. A water-table contour that intersects a gaining stream forms a "V" with the pointed end facing upstream and water is flowing from the aquifer to the stream. In some locations, the opposite occurs. This is especially true where small tributary streams enter areas underlain by stratified drift along steep till slopes and during dry periods when the water table is low. A water-table contour that intersects a losing stream forms a "V" that points downstream and water is flowing from the stream to the aquifer. The water-table maps (pls. 1 and 2) were drawn in a manner where streams initially flowing over the stratified drift are drawn as losing streams, but in all other locations the streams are depicted as gaining streams.

\section{Aquifer Characteristics}

The geohydrology of stratified-drift aquifers shown on plates 3 and 4 is based on aquifer characteristics that include saturated thickness, storage, and hydraulic conductivity. Estimates of saturated thickness and hydraulic conductivity were used to calculate transmissivity (pls. 3 and 4). These characteristics can be used to assess the water-supply potential of stratifieddrift aquifers. The saturated thicknesses are related to the hydrogeologic setting, and characteristics of selected aquifers in the Saco and Ossipee River Basins are discussed in the section "Characteristics of Selected Aquifers."

\section{Saturated Thickness and Storage}

Saturated thickness of an unconfined, stratifieddrift aquifer is the vertical distance between the water table and the base of the aquifer. For many stratifieddrift aquifers, the base is the contact between the stratified-drift deposits and till or bedrock. For other aquifers, the bottom is the contact between upper coarsegrained deposits and underlying fine-grained deposits. However, saturated thickness of underlying finegrained stratified drift commonly is unknown, so that the thickness contours shown on plates 3 and 4 include the total stratified-drift thickness. The saturated thickness and specific yield of an unconfined aquifer determines the amount of ground water that can be released from storage.

The storage coefficient of an aquifer is defined as the volume of water released from or taken into storage per unit surface area of the aquifer per unit change in head (Theis, 1938, Heath, 1983). In an unconfined aquifer, the storage coefficient approximately equals the specific yield-the amount of water that can be obtained by gravity drainage from a unit volume of the aquifer. Laboratory tests on 13 unconsolidated sediment samples from southern New Hampshire that ranged from fine-grained lacustrine sands to coarsegrained sands and gravels indicate that specific yields range from 0.14 to 0.34 with an average of 0.26 (Weigle and Kranes, 1966). A value of 0.2 commonly is used to estimate specific yield in stratified-drift aquifers in New England.

Saturated-thickness maps can be used to estimate the total amount of ground water stored in an aquifer. The saturated volume of an unconfined aquifer is approximately equal to the sum of the products of the areas between successive pairs of saturated-thickness contours multiplied by the average saturated thickness for each area. The volume of ground water stored in the aquifer can then be estimated as the product of the saturated volume multiplied by the storage coefficient.

Saturated-thickness maps shown on plates 3 and 4 were prepared by use of data from surficial-geologic maps, seismic-refraction profiles, seismic-reflection profiles, test drilling, and records from the GWSI data 
base. A 40-foot-contour interval was used to show saturated thicknesses of stratified drift. Saturated thicknesses exceeded $280 \mathrm{ft}$ and may not be entirely aquifer material. Layers of saturated silts and clays that lie above, below, or interfinger with the aquifer are included in the saturated thicknesses contours shown. Areas of contact between stratified drift and till, bedrock outcrops, and areas adjacent to the outcrops indicate zero saturated thickness.

Seismic-refraction profiles show the depth to the water table and depth to bedrock, but usually do not show depth to till deposits unless the till is thick and has a seismic velocity that is significantly faster than the seismic velocity of saturated stratified drift. For the purpose of preparing the maps presented in this study, till was assumed to be thin (less than $15 \mathrm{ft}$ thick) unless there was evidence to the contrary. The assumption that till, if present beneath stratified drift, is generally thin was tested by use of the GWSI data base. Statewide, 220 sites were identified as having known thickness of till overlying bedrock and underlying stratified drift. Saturated thickness of these 220 sites ranged from 0.5 to $140 \mathrm{ft}$, the median was $7 \mathrm{ft}$, and the third quartile was $15 \mathrm{ft}$ (Moore and others, 1994).

In some areas, saturated thickness of subsurface materials was determined from test-boring and splitspoon sampling data. Samples were usually collected every 5 or $10 \mathrm{ft}$. Values for saturated thickness are the distances from points where the test borings first encountered the water table down to the top of the till or, in cases where till was not encountered, down to the top of the bedrock.

Well and test-hole data stored in the GWSI data base were the final source of information used in the preparation of the saturated-thickness maps (pls. 3 and 4). Unfortunately, many sites lacked information on the depth to the bottom of the stratified drift and depth to the water table. For example, logs of wells penetrating bedrock usually provide data about the bottom of the stratified drift but not depth to the water table. Conversely, logs of shallow dug wells usually provide information about the depth to the water table but not the depth to the bottom of the stratified drift. At specific sites where depth to water table was unavailable from direct measurements, the water-table altitude was obtained from the water-table maps (pls. 1 and 2). Depths to the bottom of stratified-drift deposits were assumed to be indicated by one of the following factors in the priority shown.

1. Depth to top of till, if known. Till is assumed not to overlie stratified drift except in a few areas where evidence is available to the contrary;

2. depth to top of bedrock, if known;

3. length of well casing minus $10 \mathrm{ft}$, if the well is known to penetrate bedrock. Depth to bedrock is assumed to be about $10 \mathrm{ft}$ above the bottom of the casing. This approximation applies to wells that penetrate crystalline bedrock in New Hampshire.

If a well penetrated stratified drift and was terminated by refusal before till or bedrock was clearly reached, the depth to the bottom of the stratified drift was equal to or greater than the depth to refusal. Saturated thickness was then assumed to be greater than or equal to the difference between the water-table depth and the refusal depth. If a well penetrated stratified drift, and neither till, bedrock, nor refusal was reached, the depth to the bottom of the well was assumed to be above the bottom of the stratified drift. Saturated thickness was assumed to be greater than the difference between the water-table depth and the depth to the bottom of the well (pls. 3 and 4).

\section{Relation of Aquifer Thickness to Geoydrologic Setting}

The relation of aquifer thickness to geohydrologic setting involves the underlying bedrock. Areas where the saturated thickness is greatest are where the surface of the bedrock has been the most deeply eroded. Overdeepened pockets of valleys eroded by glacial plucking and scouring of the bedrock surface, subsequently were filled with stratified drift. Glaciers must have been the primary source of this erosion because the bedrock in the valleys was locally eroded to altitudes lower than any bedrock-controlled base level that would limit the downward erosion by rivers and streams. The largest and deepest of these pockets is centered around Ossipee Lake.

Certain types of bedrock were eroded more than other types and underlie the thickest parts of the stratified-drift aquifers in the study area. For example, the Mississippian-aged binary granite of the Effingham pluton (Lyons and others, 1986) was eroded the most, 
resulting in saturated thicknesses that exceed $200 \mathrm{ft}$ near and under Ossipee Lake (pl. 4). Other geologic formations that were locally eroded deeply and subsequently filled in with stratified drift with saturated thicknesses greater than $160 \mathrm{ft}$ include the Conway Granite in the North Conway area; the Mount Osceola Granite; and the Littleton Formation. In contrast, Mount Washington, the highest peak in the study area (and the highest in the Northeastern United States) is also underlain by the Littleton Formation (Lyons and others, 1986). This illustrates the wide areal variation in the competence or resistance to erosion of the Littleton Formation.

\section{Transmissivity}

Transmissivity is defined as the rate at which water at the prevailing kinematic viscosity can be transmitted through a unit width of an aquifer under a unit hydraulic gradient (Lohman and others, 1972). The transmissivity $(T)$ of an aquifer is equal to the saturated thickness (b), in feet, multiplied by the horizontal hydraulic conductivity ( $\mathrm{K}$, a directional measure of the permeability), in $\mathrm{ft} / \mathrm{d}$, and is expressed in feet squared per day $\left(\mathrm{ft}^{2} / \mathrm{d}\right)$; thus,

$$
\mathrm{T}=\mathrm{K}(\mathrm{b}) \text {. }
$$

Transmissivity at a specific site was derived from estimates of hydraulic conductivity of lithologic units in the aquifers. Hydraulic conductivity, in turn, was estimated from grain-size distributions of samples of aquifer materials by use of the regression equation developed by Olney (1983). Hydraulic conductivity, however, which has a vertical and a horizontal vector component, is not accounted for by this equation. In this relation, an effective grain size $\left(D_{10}\right.$, in phi units) was used to estimate hydraulic conductivity $(\mathrm{K}$, in feet per day) with the equation:

$$
K=2,100 \times 10^{-0.655\left(D_{10}\right)} \text {. }
$$

The effective grain size $\left(D_{10}\right)$ is a controlling factor for the hydraulic conductivity of stratified drift in New Hampshire and is defined as that grain size where 10 percent of the sample consists of smaller grains and 90 percent of the sample consists of larger grains. Olney (1983) developed this relation from results of permeameter tests of stratified-drift samples from Massachusetts. Moore (1990) found that this relation yielded results that fall within the range of results from other relations that have been developed between grainsize distribution and hydraulic conductivity (Krumbein and Monk, 1942; Bedinger, 1961; and Masch and Denney, 1966). Comparisons with aquifer-test data, however, indicate that applying equation 2 may not give accurate results for very coarse-grained sand and (or) gravel. Estimates of hydraulic conductivity for aquifers comprised of coarse sands and gravels were, in part, based on comparisons to aquifer-test data for similar deposits. Hydraulic conductivity (and transmissivity) based on grain-size relations are only estimates and may differ from results of aquifer-test analyses. Additionally, transmissivities calculated from aquifer-test data may be affected by hydrologic boundaries such as rivers or valley walls.

Hydraulic conductivity was estimated for 454 samples of stratified drift from southern New Hampshire by means of equation 2. The samples were collected in the Exeter and Lamprey River Basins (Moore, 1990); in the seacoast area and the Lower Merrimack River Basin (Flanagan and Stekl, 1990); in the Bellamy, Cocheco, Salmon Falls River Basins (Mack and Lawlor, 1992); in the Lower Connecticut River Basin (Moore and others, 1994); in the Contoocook River Basin (Harte and Johnson, 1995), and in the Middle Merrimack River Basin (Ayotte and Toppin, 1995). The grain-size distribution and the effective grain size $\left(D_{10}\right)$ were determined for these 454 samples.

Hydraulic conductivities calculated from equation 2 were plotted with median grain size in phi groups, and the resultant plot was divided into three categories of degree of sorting (fig. 9). These relative categories are used to describe the types of stratified-driftaquifer deposits found in New Hampshire. The degree of sorting was based on the standard deviation of each specific sample.

If standard deviations were greater than 1.75 phi, the stratified-drift samples were considered poorly sorted; if standard deviations were 1.25 phi to 1.75 phi, the samples were considered moderately sorted; and if standard deviations were less than 1.25 phi, the samples were considered well sorted. A regression equation was developed for each of the three categories to determine the relation between hydraulic conductivity and median grain size (fig. 9). The coefficient of determination $\left(\mathrm{R}^{2}\right)$ was 0.93 for the well-sorted samples, 0.72 for the moderately sorted samples, and 0.54 for the poorly sorted samples. The calculated hydraulic conductivity, 


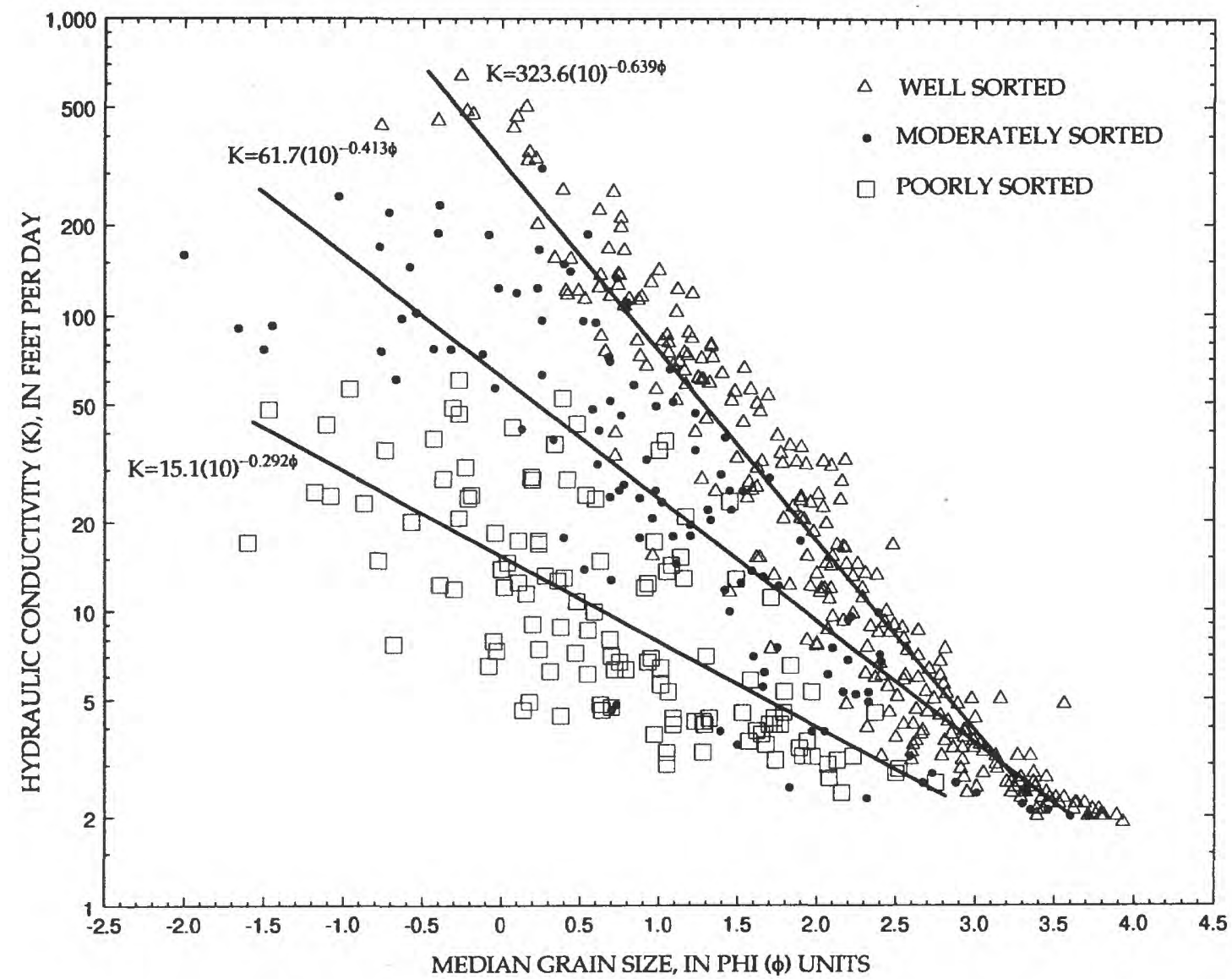

Figure 9. Relation between estimated hydraulic conductivity, median grain size, and degree of sorting of stratified drift in New Hampshire (from Ayotte and Toppin. 1995).

grouped by ranges of median grain size and by ranges of standard deviation (degree of sorting), is shown in table 3.

Grain-size distribution descriptions were used to characterize the transmissivities (pls. 3 and 4) at the test wells drilled for this study from the stratigraphic logs (appendix 2) and data from table 3. Transmissivity at each test hole was calculated by multiplying the estimated horizontal hydraulic conductivity and the saturated thickness of the corresponding interval of the stratigraphic log and summing the products.

The transmissivities shown on plates 3 and 4 are based on saturated thicknesses and estimates of hydraulic conductivity and rely mainly on grain-size-distribution data from geologic and drillers' logs from test borings and wells. Saturated silts and clays were excluded from the transmissivity estimates because their contribution to transmissivity was assumed to be negligible. Also rough estimates of transmissivity were calculated from logs of drillers, where the grain-size distributions were not described in detail. In a few cases transmissivities were estimated from results of aquifer tests. Several test wells for which grain-size distributions were determined in New Hampshire are adjacent to wells where aquifer tests were conducted. Transmissivities computed by grain-size distributions at these wells were within 50 percent of values computed by use of the aquifer-test data but usually were lower than values computed with the aquifer-test data.

Other factors that affect aquifer transmissivities include viscosity of the water, compaction of the sediment, and the interconnection of pore spaces. Transmissivity can be extremely variable over short distances because of the heterogeneous nature of stratified-drift deposits. Because of the variability associated with estimates of transmissivity, the values shown on plates 3 and 4 should be considered generalized estimates.

\section{Characteristics of Selected Stratified-Drift Aquifers}

The most extensive and most productive (or poten- 
tially productive) aquifers are discussed below. The stratified-drift aquifers encompass $152.5 \mathrm{mi}^{2}$, or 17.5 percent of the study area and underlie valleys throughout the study area. Aquifers are discussed from north to south.

Table 3. Relation of mean hydraulic conductivity to median grain size and degree of sorting of stratified drift in New Hampshire

$[<$, less than; >, greater than; --, no data. Data from Ayotte and Toppin, 1995, and Moore and others, 1994]

\begin{tabular}{|c|c|c|c|c|}
\hline \multirow[t]{2}{*}{$\begin{array}{l}\text { Median } \\
\text { grain } \\
\text { size (phi } \\
\text { units) }\end{array}$} & \multirow[t]{2}{*}{$\begin{array}{l}\text { Median grain } \\
\text { description }\end{array}$} & $\begin{array}{l}\text { WELL } \\
\text { SORTED } \\
\text { (standard } \\
\text { deviation } \\
\text { <1.25 phi) }\end{array}$ & $\begin{array}{l}\text { MODER- } \\
\text { ATELY } \\
\text { SORTED } \\
\text { (standard } \\
\text { deviation } \\
1.25 \text { phi to } \\
1.75 \text { phi) }\end{array}$ & $\begin{array}{c}\text { POORLY } \\
\text { SORTED } \\
\text { (standard } \\
\text { deviation } \\
>1.75 \text { phi) }\end{array}$ \\
\hline & & \multicolumn{3}{|c|}{$\begin{array}{l}\text { Mean hydraulic conductivity (K), } \\
\text { in feet per day }{ }^{1}\end{array}$} \\
\hline-1.75 & Granules & - & 320 & 49 \\
\hline-1.25 & Granules & - & 200 & 35 \\
\hline-.75 & $\begin{array}{l}\text { Very coarse } \\
\text { sand }\end{array}$ & 970 & 120 & 25 \\
\hline-.25 & $\begin{array}{l}\text { Very coarse } \\
\text { sand }\end{array}$ & 470 & 78 & 18 \\
\hline .25 & Coarse sand & 220 & 48 & 13 \\
\hline .75 & Coarse sand & 110 & 30 & 9 \\
\hline 1.25 & $\begin{array}{l}\text { Medium } \\
\text { sand }\end{array}$ & 51 & 19 & 7 \\
\hline 1.75 & $\begin{array}{l}\text { Medium } \\
\text { sand }\end{array}$ & 25 & 12 & 5 \\
\hline 2.25 & Fine sand & 12 & 7 & 3 \\
\hline 2.75 & Fine sand & 6 & 4 & 2 \\
\hline 3.25 & $\begin{array}{l}\text { Very fine } \\
\text { sand }\end{array}$ & 3 & 3 & -- \\
\hline 3.75 & $\begin{array}{l}\text { Very fine } \\
\text { sand }\end{array}$ & 2 & 2 & -- \\
\hline
\end{tabular}

${ }^{1}$ Hydraulic conductivity calculated by use of methods described by Olney (1983).

\section{North Chatham Aquifer in Chatham, N.H.}

North Chatham aquifer (fig. 10) underlies the valley of the Cold River in the Saco River Basin in the northern part of Chatham and extends beyond the study area into Maine (pl. 1). The stratified drift in the valley was deposited from several sources including deposition from meltwater during the melting of the last con- tinental glacier. A braided esker ridge of sand and gravel extends eastward out of a cirque at the northern end of this aquifer, perhaps indicating that some lateWisconsinan alpine-glacial ice was present in the cirque. Additional sources of sediment include extensive, more recent, alluvial deposits from the Charles and Mill Brooks (pl. 1).

Water-table altitudes in the North Chatham aquifer (fig. 10) range from about 420 to above $720 \mathrm{ft}$ above sea level (pl. 1). Areas where the gradients are steep indicate areas of low transmissivity (or at least low vertical hydraulic conductivity) within the alluvial-fan deposits. Areas of low transmissivity may be the result of low hydraulic conductivity in the aquifer or a small saturated thickness of an upper aquifer overlying material of low conductivity, such as till-covered bedrock. Saturated thicknesses of stratified drift for the North Chatham aquifer range from 0 to over $120 \mathrm{ft}$. The thickest sections of aquifer are near the Cold River in the southern part of the aquifer. The underlying bedrock type beneath this thick section is the binary granite of the Sebago and Effingham plutons (Lyons and others, 1986). Transmissivities range from 0 to between $4,000 \mathrm{ft}^{2} / \mathrm{d}$ and $8,000 \mathrm{ft}^{2} / \mathrm{d}$ with the highest transmissivity in the southern part where the aquifer is thickly saturated.

\section{Chatham Aquifer in Chatham, N.H.}

The Chatham aquifer (fig. 10) underlies the valley of the Cold River in the Saco River Basin in the central part of Chatham near the village center and extends beyond the study area into Maine (pl. 1). The stratified drift in this area was deposited mainly during deglaciation of the last continental glacier. Additional sources of sediment include alluvial deposits from Langdon and McDonough Brooks (pl. 1). Sand and gravel overlying silt and clay layers are found in the aquifer and indicate that a water body was present during deglaciation and filled with sediment. Silt and clay layers, for example, are found at depths between 68 and $84 \mathrm{ft}$ below the land surface at well CKW 11 .

Water-table altitudes at the Chatham aquifer (fig. 10) range from slightly less than $460 \mathrm{ft}$ to greater than $580 \mathrm{ft}$ above sea level (pl. 1). Saturated thicknesses of stratified drift for the Chatham aquifer range from 0 to greater than $40 \mathrm{ft}$. The thickest sections of 


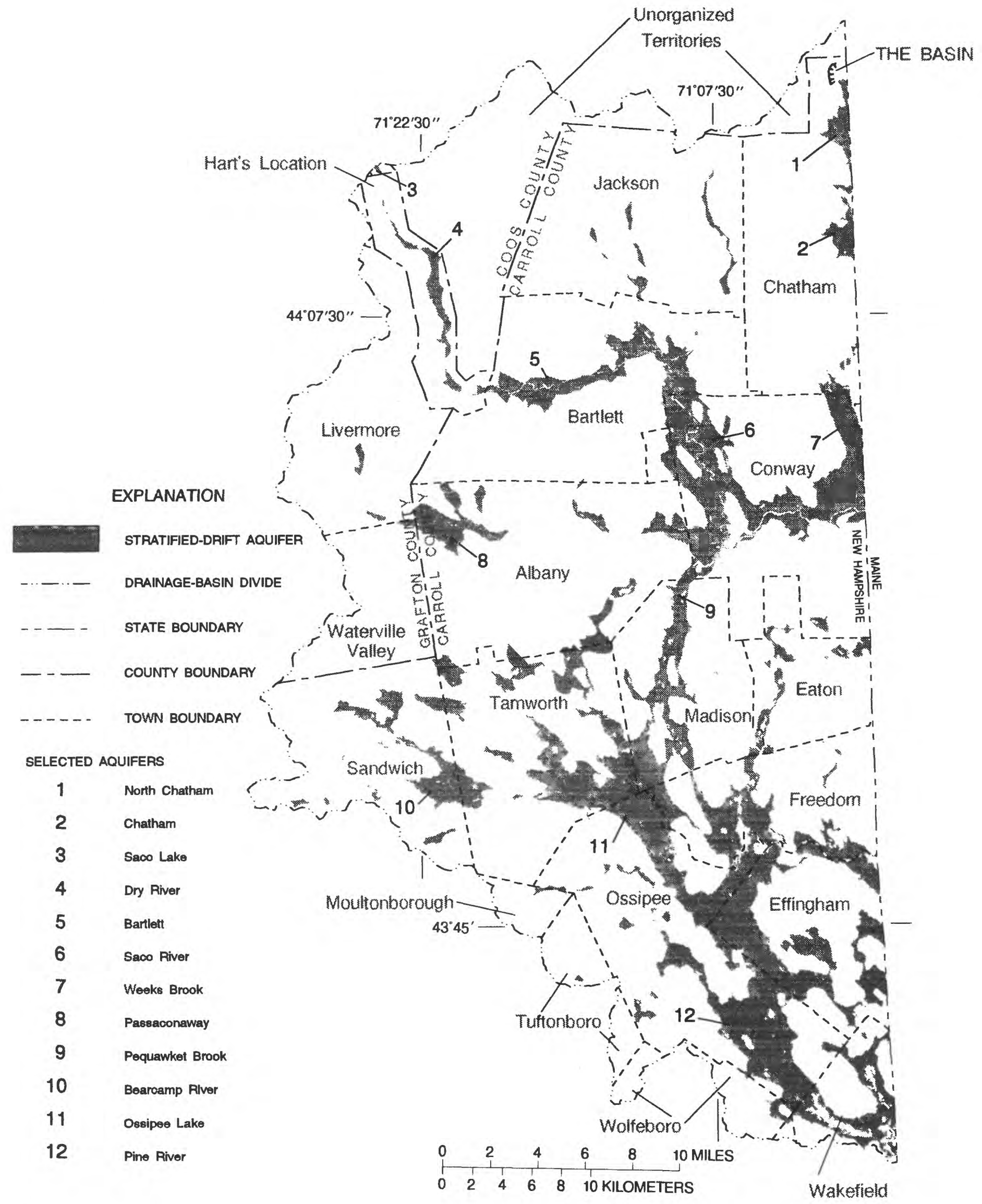

Figure 10. Selected aquifers in the Saco and Ossipee River Basins study area, east-central New Hampshire. 
stratified drift are near the village center with transmissivities that range from 0 to between $1,000 \mathrm{ft}^{2} / \mathrm{d}$ and $2,000 \mathrm{ft}^{2} / \mathrm{d}$.

\section{Saco Lake Aquifer in Carroll, N.H.}

Saco Lake aquifer (fig. 10) is at the head of the Saco River in Carroll, N.H. The drainage divide in the central part of the valley is composed of stratified drift that was deposited at the ice margin. Outwash from the glacier completely filled in the valley between the glacier and the previous till and bedrock divide $0.6 \mathrm{mi}$ to the south. Stratified-drift deposits grade to a V-shaped notch carved out of the bedrock at the southern end of the aquifer. Meltwater from the glacier carved this notch into a previously carved glacial U-shaped valley located at the previous divide. As a result, the drainage divide shifted to the north by deposition of sediments at the ice margin and slightly enlarged the Saco River Basin (see cover illustration).

Water-table altitudes in the Saco Lake aquifer (fig. 10, pl.1) are from 1,880 and 1,900 ft above sea level (pl. 1). Currently, water levels in the Saco River headwaters and the aquifer are controlled by the same $V$-shaped notch that controlled the glacial meltwater during deglaciation. Saturated thicknesses of stratified drift for the Saco Lake aquifer exceed $80 \mathrm{ft}$ as indicated by seismic-refraction profiles (appendix 3 ). Transmissivities range from 0 to between $4,000 \mathrm{ft}^{2} / \mathrm{d}$ and $8,000 \mathrm{ft}^{2} / \mathrm{d}$ (pl. 4).

\section{Dry River Aquifer in Hart's Location, N.H.}

Dry River aquifer (fig. 10) underlies the main valley of the Saco River in Crawford Notch in Hart's Location. In addition to the glacially derived sediments, a huge alluvial-fan deposit is present in the northern part of this aquifer. The alluvial fan formed under extreme flood conditions where the Dry River emerges from the till uplands, and flows out onto flat areas of stratified drift. Very coarse material (largely gravel and sand including large boulders) were deposited. The alluvial fan enhances infiltration from the aptly named Dry River, which dries up much of the year where it flows onto the valley fill.

Water-table altitudes at the Dry River aquifer (fig. 10) range from about 840 to $1,280 \mathrm{ft}$ above sea level (pl. 1). Absence of subsurface data in the area of the Dry River alluvial fan prevented the contouring of the saturated thickness and transmissivity values. Just to the south, however, saturated thicknesses of stratified drift exceed $160 \mathrm{ft}$, in the center of the valley (pl. 3). The underlying bedrock beneath the thickest section is the Conway Granite (Lyons and others, 1986). Transmissivities are greater than $8,000 \mathrm{ft}^{2} / \mathrm{d}$ in the southern part where the aquifer is comprised of thick, saturated deposits.

\section{Bartlett Aquifer in Bartlett, N.H.}

Bartlett aquifer (fig. 10) underlies the Saco River Valley in Bartlett. It is the northern part of a large stratified-drift aquifer that extends $37.3 \mathrm{mi}$ from Bartlett to Wakefield N.H. and into Maine. The stratified drift in this area was deposited from several sources including deposition from meltwater during the melting of the last continental glacier. Alluvial-fan deposits from Albany, Bartlett, Meadow, and Razor Brooks (pl. 1) added to the deposits composing the aquifer, as well as considerable deposition of alluvial sands and gravels by the Saco River. A boulder gravel from this deposition by previous channels of the Saco River is found near the surface of much of the central part of the valley.

Water-table altitudes in the Bartlett aquifer (fig. 10) range from about 580 to $760 \mathrm{ft}$ above sea level (pl. 1). Saturated thicknesses of stratified drift for the Bartlett aquifer exceed $80 \mathrm{ft}$ near the center of the valley. The underlying bedrock beneath the thickest section is the Conway Granite (Lyons and others, 1986). Transmissivities range from 0 to between $4,000 \mathrm{ft}^{2} / \mathrm{d}$ and $8,000 \mathrm{ft}^{2} / \mathrm{d}$ with the highest transmissivity occurring in the central and western part of the aquifer (pl. 3).

\section{Saco River Aquifer in Conway and Bartlett, N.H.}

The Saco River aquifer (fig. 10) is in the main Saco River Valley in Conway and in the eastern part of Bartlett. The aquifer continues down valley from the Bartlett aquifer, and is part of the large stratified-drift aquifer that extends from Bartlett to Wakefield. The stratified drift that fills the valley here includes glaciallake deposits and outwash. Alluvial-fan deposits from the Rocky Branch, the East Branch, and the Ellis River (pl. 1) added to the deposits composing the aquifer. The Saco River has also deposited and reworked the deposits of sands and gravels. A boulder gravel is found near 
the surface in the northern part of the aquifer, especially where previous channels of the Ellis River enter the main valley of the Saco River.

Water-table altitudes in the Saco River aquifer (fig. 10, pl. 1) range from less than $420 \mathrm{ft}$ to greater than $640 \mathrm{ft}$ above sea level (pl. 1). Saturated thicknesses of stratified drift for the Saco River aquifer exceed $180 \mathrm{ft}$ near the confluence of the Saco and Ellis Rivers in the northern part of the aquifer. The underlying bedrock type beneath the thickest stratified drift is the Mount Osceola Granite, whereas other thick sections in Conway are underlain by the Conway Granite (Lyons and others, 1986). Transmissivities exceed $8,000 \mathrm{ft}^{2} / \mathrm{d}$ in the central and northern parts where the aquifer is coarse grained and thickly saturated. More detailed description of this aquifer is given in Tepper and others (1990).

\section{Weeks Brook Aquifer in Chatham and Conway, N.H.}

Weeks Brook aquifer (fig. 10) is in a tributary valley to the Saco River in the southern part of Chatham and northeastern part of Conway (pl. 1). The stratified drift composing this aquifer was deposited mainly during deglaciation of the last continental glacier and includes glacial-ice-contact deposits, glacial-lake deposits, deltaic deposits, and alluvial fans. An ice-contact ridge of sand and gravel deposits and alluvial fan at Weeks Brook lead into southerly dipping deltaic beds about $400 \mathrm{ft}$ west of wells CWW 69 and 70 (pl. 1). Interfingering of the deltaic sands and gravels with glaciolacustrine silts and clays are apparent from the logs of CWW 67, 69 and 70 (appendix 2). Weeks Brook also deposited alluvial sediments over the glacial-lake sediments.

Water-table altitudes in the Weeks Brook aquifer (fig. 10) range from less than $400 \mathrm{ft}$ to greater than $520 \mathrm{ft}$ above sea level (pl. 1). Areas where the gradients are steep reflect areas of low transmissivity (or at least low vertical hydraulic conductivity) in the alluvial fan. Saturated thicknesses of stratified drift for the Weeks Brook aquifer exceed $120 \mathrm{ft}$ near the Cold River in the north-central part of the valley. The bedrock type underlying the entire aquifer is Conway Granite (Lyons and others, 1986). Transmissivities range from 0 to between $4,000 \mathrm{ft}^{2} / \mathrm{d}$ and $8,000 \mathrm{ft}^{2} / \mathrm{d}$ with the highest transmissivity in the north-central part where the aquifer is coarse grained and thickly saturated (pl. 3 ).

\section{Passaconaway Aquifer in Albany and Waterville Valley, N.H.}

Passaconaway aquifer (fig. 10) underlies the valley of the Swift River in the northwestern part of Albany and the northeastern corner of Waterville Valley (pl. 1). The stratified-drift aquifer fills a pocket in the bedrock surface that was glacially eroded. Very fine sand and silt layers at well ADW 14 indicate some ponding of glacial meltwater during deglaciation. However, the aquifer is composed of predominantly coarse-grained ice-contact, outwash, and alluvial deposits. The Swift River and Oliverian Brook are the source of most of the alluvial deposition.

Water-table altitudes in the Passaconaway aquifer (fig. 10) range from less than 1,220 to slightly greater than $1,280 \mathrm{ft}$ above sea level (pl. 1). Saturated thicknesses of stratified drift for the Passaconaway aquifer exceed $80 \mathrm{ft}$ (pl. 3). The bedrock type underlying this aquifer is the Conway Granite (Lyons and others, 1986). Transmissivities range from 0 to between $2,000 \mathrm{ft}^{2} / \mathrm{d}$ and $4,000 \mathrm{ft}^{2} / \mathrm{d}$ with the highest transmissivity in the central parts where the aquifer is coarse-grained and thickly saturated (pl. 3).

\section{Pequawket Brook Aquifer in Madison, Albany, and Conway, N.H.}

Pequawket Brook aquifer (fig. 10) is in the through valley that extends from the southwest corner of Conway, through the southeast corner of Albany and south to Silver Lake in Madison (pls. 1 and 2). The stratified drift composing this aquifer was deposited mainly from meltwater during the melting of the last continental glacier. Depositional ice-contact features include eskers, kames, and kame terraces. A meltwater channel on the slopes of the valley is at an altitude higher than the altitude of the valley fill and leads down to a kame terrace on the western side of the valley fill in the northern part of Madison (Newton, 1974). Glacial-ice-dam-break floods, from water ponded between the ice and the drainage divide for the Swift River Basin, probably emerged onto an alluvial fan and kame terrace with some deltaic bedding. Prominent esker ridges of sand and gravel are present in the through valley including the northernmost esker that extends as a peninsula into Pequawket Pond in the southeast corner of Conway (pl. 2). A similar ridge of sand and gravel is to the east of Ledge Pond. Ledge Pond in Madison is on the western side of the valley between the valley wall of till and 
bedrock. This ridge of sand and gravel broadens into a large deposit towards the southeast end of the pond. The lithologic log for well MBW 56 indicates that this deposit may be deltaic with coarse-grained beds interfingering at depth with very fine sands and silts. Ledge Pond is approximately $57 \mathrm{ft}$ higher in altitude than Pequawket Brook about $1,000 \mathrm{ft}$ to the east. Additional esker ridges are present throughout the central part of the valley and extend southward to the north end of Silver Lake. There the ridges split into two series of eskers; one series, including Big Island, is along the east shore and the other series is along the west shore.

Water-table altitudes in the Pequawket Brook aquifer (fig. 10, pls. 1 and 2) range from 457 to $520 \mathrm{ft}$ above sea level. Saturated thicknesses of stratified drift for the Pequawket Brook aquifer exceed $80 \mathrm{ft}$ southeast of Ledge Pond and near the center of the aquifer (pl. 3). The bedrock type underlying the thickest stratified drift is the binary granite of the Sebago and Effingham plutons (Lyons and others, 1986). Transmissivities exceed $8,000 \mathrm{ft}^{2} / \mathrm{d}$ in the northern part of the aquifer where there is thickly saturated sand and gravel in the center of the valley (pl. 4).

\section{Bearcamp River Aquifer in Sandwich and Tamworth, N.H.}

Bearcamp River aquifer (fig. 10) underlies the valley of the Bearcamp River in eastern Sandwich and western Tamworth (pl. 2). The stratified drift in this valley was deposited in a glacial lake. A major source of the sediment deposition was from meltwater approximately following the course of the Cold River. The large boulders (the maximum size of the gravel carried by the meltwater) indicate erosion and redeposition caused by glacial-ice-dam-break floods. The coarsegrained deposits in the northern part of the aquifer grade into finer glacial-lake deposits in the southern part of the aquifer. Flow in the Bearcamp and Cold Rivers have reworked some of the deposits. Alluvial deposits cover most of the central part of the aquifer.

Water-table altitudes in the Bearcamp River aquifer (fig. 10) range from less than 580 to greater than $720 \mathrm{ft}$ above sea level (pl. 2). Saturated thicknesses of stratified drift for the Bearcamp River aquifer exceed $120 \mathrm{ft}$ just east of the Cold River in the southern part of the aquifer. The bedrock type underlying this aquifer is the Winnipesaukee Quartz Diorite of the New Hampshire Plutonic Suite. Transmissivities range from 0 to between $4,000 \mathrm{ft}^{2} / \mathrm{d}$ and $8,000 \mathrm{ft}^{2} / \mathrm{d}$ with the highest transmissivity in the central part of the aquifer where it is thickly saturated near the source of coarse-grained sediment (pl. 4).

\section{Ossipee Lake Aquifer in Tamworth, Madison, Ossipee, Freedom, and Effingham, N.H.}

Ossipee Lake aquifer (fig. 10) is in the main valley of the Bearcamp, Ossipee, and Pine Rivers in the towns of Tamworth, Madison, Ossipee, Freedom, and Effingham (pl. 2). It is the central part of the largest stratifieddrift aquifer in the State that extends from Bartlett to Wakefield. Stratified drift in the main valley was primarily deposited into a glacial-lake basin during deglaciation. Depositional features in the valley include eskers, kames, deltas, outwash (largely topset beds), fine-grained glacial-lake-bottom beds, alluvial, and beach deposits. The lake basin, eroded out of bedrock, was greater than $280 \mathrm{ft}$ below the present surface of Ossipee Lake and greater than $300 \mathrm{ft}$ below present land surface at its deepest point. The basin filled rapidly with sediment as the ice margin receded northward. Silver and Ossipee Lakes are relicts of the previous glacial lake. The lakes are located where ice blocks of the former glacier persisted the longest and prevented deposition. A large outwash fan is present just southwest of Silver Lake (Newton, 1974). Seismic-reflection data from the southwest shore of the lake, however, indicate northwest-dipping deltaic foreset beds (fig. 8). Presumably, these foreset beds were fed by the sediment-laden meltwater flowing through the esker system on the east side of the lake.

Water-table altitudes in the Ossipee Lake aquifer (fig. 10) range from above 407 to above $580 \mathrm{ft}$ above sea level (pl. 2). Saturated thicknesses of stratified drift for the Ossipee Lake aquifer exceed $280 \mathrm{ft}$ just northwest of Ossipee Lake (pl. 4). The bedrock type underlying the thickest stratified drift is the binary granite of the Sebago and Effingham plutons (Lyons and others, 1986). Transmissivities exceed $8,000 \mathrm{ft}^{2} / \mathrm{d}$ in the thickly saturated ice-contact esker, kame, and deltaic deposits (pl. 4).

\section{Pine River Aquifer in Ossipee, Effingham, and Wakefield, N.H.}

Pine River aquifer (fig. 10) underlies the main valley of the Pine River in the towns of Ossipee, Effingham, and Wakefield (pl. 2). The Pine River aquifer is 
the southern part of the large stratified-drift aquifer that extends from Bartlett to Wakefield. The stratified drift that fills the valley here was also deposited into a glacial lake but at a higher altitude than the Ossipee Lake aquifer. The outlet to this lake was controlled by spillways cut into the sand and gravel at the divide between the Pine River subbasin and the Little Ossipee River subbasin (pl. 2). Depositional features in the subbasins include the largest esker system in the State (the Pine River Esker) as well as kames, deltas, outwash, finegrained glacial-lake-bottom beds, and alluvial deposits. At White Pond and just north of Pine River Pond (pl. 2), the Pine River Esker merges with areas of broad sand and gravel deposition (presumably deltaic). These areas indicate ice-marginal positions consistent with the morphosequence concept presented by Koteff and Pessl (1981).

Water-table altitudes in the Pine River aquifer (fig. 10) are from below 420 to above $680 \mathrm{ft}$ above sea level (pl. 2). Saturated thicknesses of stratified drift for the Pine River aquifer exceed $80 \mathrm{ft}$ in the central part of the area (pl. 4). The bedrock type underlying the thickest stratified drift is the binary granite of the Sebago and Effingham plutons (Lyons and others, 1986). Transmissivities exceed $8,000 \mathrm{ft}^{2} / \mathrm{d}$ in the thickly saturated parts of the Pine River Esker and associated deposits (pl. 4).

\section{Estimation of Ground-Water Availability in the Ossipee Lake Aquifer}

The Ossipee Lake aquifer was selected to estimate potential water availability by use of a numerical flow model to simulate ground-water flow. Parts of Effingham, Freedom, Ossipee, Madison, and Tamworth are included in the modeled area (fig. 11). Ice-contact, outwash, and glacial-lake deposits including eskers and deltaic deposits constitute the areally extensive aquifer. The model area of the aquifer encompasses $47.3 \mathrm{mi}^{2}$, parts of 5 towns, and the maximum saturated thickness of the aquifer exceeds $280 \mathrm{ft}$. The saturated volume of the aquifer in the model area is about 105 billion $\mathrm{ft}^{3}$ $\left(0.71 \mathrm{mi}^{3}\right)$. A relatively large model area was selected because the Ossipee Lake aquifer is extensive, and pumpage from any part of the aquifer can possibly affect the outflow to the Ossipee River. The Ossipee River is the eventual discharge point for all the water in the aquifer minus the water that is returned to the atmosphere by evaporation and transpiration.

\section{Description of Estimation Technique}

In this study, a numerical finite-difference model, MODFLOW (McDonald and Harbaugh, 1988), was used to determine water availability from the Ossipee Lake aquifer. The numerical model was used to simulate more closely the geometry and hydraulics of the aquifer (pl. 4) than possible with an analytical model. This simulation is a modification of techniques presented by Lapham (1988) for similar aquifers in Massachusetts and by Moore (1990) for similar aquifers in southeastern New Hampshire. The numerical model allows simulation of the areal variation of the saturated thickness and hydraulic conductivity of the aquifer and the location and characteristics of rivers and lakes overlying the aquifer. Ground-water availability was simulated by simultaneously pumping from hypothetical production wells.

\section{Generalizations}

To numerically simulate this aquifer, it was necessary to make generalizations and assumptions. Five major generalizations of this aquifer include the following.

1. The principle of superposition is applied by reducing the complex ground-water-flow equations into simpler components. Drawdown in the aquifer is approximated by the drawdown calculated for a simpler hypothetical aquifer with characteristics similar to the actual aquifer but initially simulated with a flat water table and no recharge. Initially, all heads in the hypothetical aquifer are set equal to 0 , so that the aquifer is simulated with a flat water table and no flow. Heads change only in response to simulated pumping stresses. Sources of water during these pumping simulations include water from storage and induced infiltration of streamflow. The principle of superposition is thus applied. Water flowing in the actual aquifer, which is captured by pumping wells before reaching the stream, is included in the simulation as part of the induced infiltration.

2. Ground-water availability was estimated over 180 days of continuous pumping at constant rates. Recharge from induced infiltration from the streams was simulated. In studies by Mazzaferro and others (1979) and Toppin 


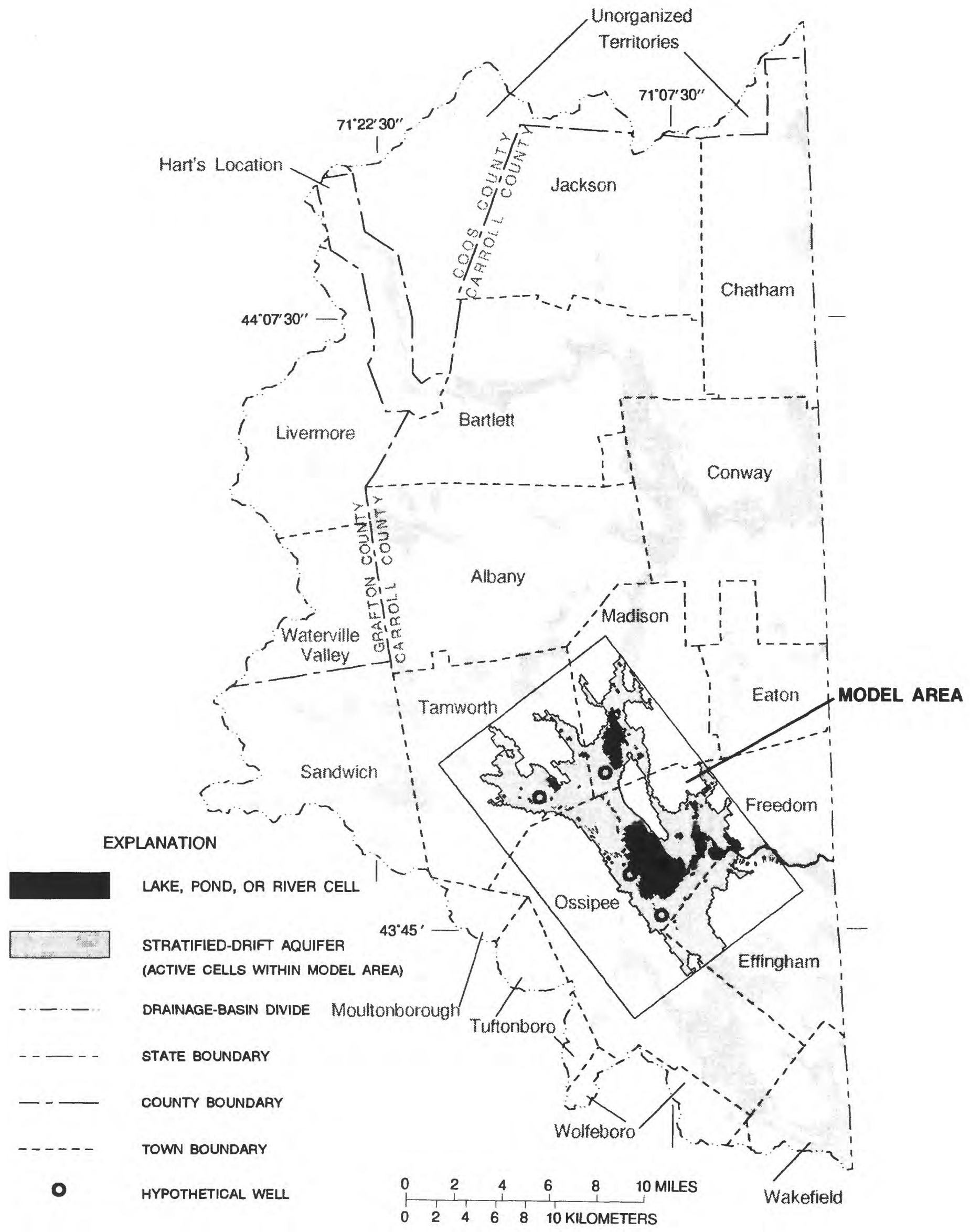

Figure 11. Location of Ossipee Lake ground-water availability model area, east-central New Hampshire. 
(1987), a pumping period of 180-days with no areal recharge was used because the period approximates the growing season during which evapotranspiration is high and areal recharge to the aquifers is assumed to be low. It is also assumed that areal recharge to the aquifer during the rest of the year is sufficient to allow continuous pumping at the simulated pumping rate. During this 180 -day period, water may recharge the aquifer areally and from adjacent till or bedrock. However, additional areal recharge or lateral recharge from adjacent till or bedrock, caused by the pumping, will not be simulated in the model.

\section{Assumptions}

The following simplifying assumptions about the ground-water-flow system were made in developing the model.

1. Aquifer and streambed properties are representative of the natural ground-waterflow system. Transmissivity and saturated thickness, shown on plate 4, and the streambed properties used are assumed to be a reasonable representation of the natural system.

2. Two-dimensional flow is adequate to represent the flow system. In the actual system, groundwater flow is horizontal and vertical but predominantly horizontal. Vertical gradients are generally downward in areas of groundwater recharge and upward in areas of groundwater discharge, such as to rivers. Strong vertical gradients result near discharging wells, but for unconfined aquifers, the magnitude of the vertical-flow gradients near a pumped well decreases rapidly with distance from the well. The error associated with simulating groundwater availability by considering only twodimensional horizontal flow is negligible.

\section{Ground water is withdrawn from wells that are} fully penetrating and $\mathbf{1 0 0}$-percent efficient. Wells used for supply are generally not fully penetrating and are commonly screened in the bottom 25 percent of the aquifer. In addition, these wells are not 100 -percent efficient. Increased drawdown in the well results from energy loss between the aquifer and the well.
This increased drawdown is a function of well design and construction. The effect of this simplifying assumption is that less drawdown is simulated in the well than would be found in a real well.

4. There is no additional flow of ground water between till and (or) bedrock and the stratified-drift aquifer induced by pumping. The model area includes a stratified-drift aquifer in a till-covered bedrock valley. In an aquifer where horizontal and vertical gradients result between the stratified drift and the underlying till and (or) bedrock, ground water flows between the aquifer and the surrounding geologic units. For application of the model, it is assumed that the ground-water flow between till and (or) bedrock and the stratified-drift aquifer will not be increased in response to the pumping from the stratified-drift aquifer.

5. Finite-difference approximation of the nonlinear, partial-differential equations governing three-dimensional ground-water flow result in reasonable estimates of yield for an aquifer. Flow simulated in the numerical model is described by linear differential equations that are solved numerically on the basis of a finite-difference approximation. The aquifers are discretized in space or divided into discrete blocks (cells) in which hydraulic properties are assumed to be constant. For unconfined aquifers, the linear equations are not strictly applicable because changes in the potentiometric surface (water table) affect the transmissivity and changes in the transmissivity with time result in a non-linear aquifer response. This non-linearity is accounted for in the model, in part, and values of transmissivity are readjusted as a result of the declining water table for each of the 20 time steps (specified by the user) in the simulation. Because the changes in transmissivity are small throughout most of the aquifer, inaccuracies that result from this approximation are small. Exact solutions to the linear differential equations are impossible; therefore, solutions are determined by solving the series of linear equations through iteration until the greatest change in the solution is less than some specified limit. For example, a limit 
of $0.01 \mathrm{ft}$ was used to end the iteration process in this model. When head changes between iterations for all cells in the model no longer exceed $0.01 \mathrm{ft}$, the simulation for a given time step is complete.

\section{Grid and Boundary Conditions}

The grid used to discretize the aquifer consists of 264 rows and 184 columns of variably spaced cells. Cell widths at the four hypothetical well sites are $1 \mathrm{ft}$ on each side to approximate the size of a well. Cell widths were doubled in width for each cell along columns or rows away from the well site until a distance of $250 \mathrm{ft}$ from the well was reached. At this point, a uniform spacing of $100 \mathrm{ft}$ was used for the next $1,000 \mathrm{ft}$. Beyond $1,250 \mathrm{ft}$ from the well, for the remainder of the model, the widths of the columns and rows were set to $500 \mathrm{ft}$. All numerical calculations were between nodes centered in each of the 26,190 active cells. Model boundaries on the east and west sides, and most of the northern boundary, coincided with the stratified drift-till boundary and were simulated as "no-flow" boundaries. With these boundaries, it is assumed that pumping will not induce additional flow across the boundaries. The southern and the remainder of the northern boundary were also simulated as "no-flow" boundaries because the effects of pumping do not extend that far.

\section{Input Parameters}

Model input parameters consist of data on (1) the altitude of the water table, (2) the saturated thickness, (3) the hydraulic conductivity, (4) the specific yield, (5) and streambed hydraulic conductivity. Data was entered into the model by use of GIS files, and the MODFLOWARC program (Orzol and McGrath, 1992).

\section{Saturated Thickness and Hydraulic Conductivity}

The saturated-thickness data for the Ossipee Lake aquifer were taken directly from plate 4 . The saturatedthickness contours were overlain on the model grid and the appropriate saturated thickness (b) was assigned to each cell. The saturated thickness was discretized by averaging saturated thickness when node centers were between contours. For example, cells with node centers that fell between the aquifer boundary and the 40 -foot contour were assigned a saturated thickness of $20 \mathrm{ft}$; cells with node centers that fell between the 40- and 80 foot contours were assigned a thickness of $60 \mathrm{ft}$.

Transmissivity was discretized by assigning the average transmissivity for a given zone shown on plate 4 . Cells with node centers that fell in a given transmissivity zone were assigned the average value $(\mathrm{T})$ for that zone. Hydraulic conductivity $(\mathrm{K})$ at each cell was then calculated by the equation $K=T / b$.

\section{Specific Yield}

The specific yield of the Ossipee Lake aquifer was not measured for the model area. Johnson (1967) summarized the results of specific yield for sediments from many studies. He reported that the average specific yield for fine sands was 0.21 , for coarse sands was 0.27 , and for coarse gravel was 0.22 . Ayotte and Toppin (1995) found that for data collected in a shallow, coarsegrained sand and gravel aquifer in south-central New Hampshire, specific yield ranged from 0.21 to 0.29 . A value of 0.2 was used for the aquifer simulated in this model as a conservative estimate of specific yield.

\section{Infiitration From the Streams, Ponds, and Lakes}

Infiltration from the streams and other surfacewater bodies is simulated with the leaky streambed module of the MODFLOW model (McDonald and Harbaugh, 1988). Cells with node centers that fell on surface-water bodies were simulated as river (or lake) nodes covering the entire area of the cell. Intermittent streams were not simulated in the model. The vertical hydraulic conductivity of all streambed and lakebottom material was assumed to be $2 \mathrm{ft} / \mathrm{d}$, a value similar to that used by numerous other studies (Haeni, 1978; Lapham,1988; Harte and Mack, 1992; Ayotte and Toppin, 1995). The rivers were simulated with a uniform average depth of $3 \mathrm{ft}$ and Silver and Ossipee Lakes were assigned a uniform average depth of $20 \mathrm{ft}$ (on the basis of field observations). Stream and lake beds were assigned a thickness of $2 \mathrm{ft}$.

\section{Ground-Water Availability}

Ground-water availability was determined with four hypothetical wells (fig. 11) in high transmissivity zones (pl. 4). The rate of withdrawal was adjusted so that the total drawdown at the well was not more than 
70 percent of the total saturated thickness of the aquifer at each well site. At the end of 180 days, 31.6 percent or $7.72 \mathrm{Mgal} / \mathrm{d}\left(11.9 \mathrm{ft}^{3} / \mathrm{s}\right)$ of the water withdrawn from the wells came from ground-water storage. The remaining 68.4 percent or $5.28 \mathrm{Mgal} / \mathrm{d}\left(8.17 \mathrm{ft}^{3} / \mathrm{s}\right)$ of water withdrawn from wells came from induced infiltration and ground water captured before discharging to surface-water bodies. This amount of induced infiltration and captured ground water is below $11 \mathrm{ft}^{3} / \mathrm{s}$, the minimum daily flow for the period of record (1943-90) of the Ossipee River and below the 99.9-percent flow duration of $82 \mathrm{ft}^{3} / \mathrm{s}$; the 95.0 -percent flow duration of $157 \mathrm{ft}^{3} / \mathrm{s}$, or the 90.0 -percent flow duration of $179 \mathrm{ft}^{3} / \mathrm{s}$. Flow in the Ossipee River should be reduced, for example, by less than 10 percent 99.9 percent of the time at the simulated level of withdrawal. Of the $7.72 \mathrm{Mgal} / \mathrm{d}$ withdrawn by the hypothetical wells, $1.2 \mathrm{Mgal} / \mathrm{d}$ was withdrawn from the well in Tamworth, $2.17 \mathrm{Mgal} / \mathrm{d}$ was withdrawn from the well in Madison, $1.28 \mathrm{Mgal} / \mathrm{d}$ was withdrawn from the northern well in Ossipee, and $3.06 \mathrm{Mgal} / \mathrm{d}$ was withdrawn from the southern well in Ossipee.

\section{Sensitivity Analysis of the Ground-Water Availability Estimate}

Sensitivity analyses were performed to assess the effects of uncertainty of the ground-water availability estimate in the following model parameters: horizontal hydraulic conductivity, streambed (or lake bed) vertical hydraulic conductivity, and stream (or lake) depth. Input data for these parameters were doubled, increased by one half, and reduced by one half, in a series of model runs. The same drawdown criteria were used for each model run, such that drawdown at each well was not more than 70 percent of the total saturated thickness of the aquifer at each well site.

The effects of changes in the input data on the model results, in terms of combined withdrawal from the four wells are shown in table 4 . Results indicate that combined withdrawal from the four hypothetical wells is not sensitive to the stream (or lake) depths or streambed vertical hydraulic conductivity. However, the combined withdrawals are sensitive to the estimated horizontal hydraulic conductivity of the aquifer.

\section{WATER QUALITY}

Samples of water from 25 test wells and 4 springs, col- lected in August and September 1992, were analyzed for inorganic and organic compounds in order to evaluate the water quality of the stratified-drift aquifers in the Saco and Ossipee River Basins study area (fig. 12). During the sampling phase of this study, areas with known ground-water contamination were generally avoided. Sample analyses from an additional 49 sites in the study area, in Bartlett and Conway, are presented by Johnson and others (1987).

Table 4. Results of sensitivity tests with combined withdrawal of four hypothetical wells in stratified drift for the ground-water availability estimates, east-central New Hampshire

[All withdrawals are reported in million gallons per day]

\begin{tabular}{cccc}
\hline $\begin{array}{c}\text { Amount of } \\
\text { change of input } \\
\text { parameters } \\
\text { (in percent) }\end{array}$ & $\begin{array}{c}\text { Hydraulic } \\
\text { conductivity }\end{array}$ & $\begin{array}{c}\text { Streambed } \\
\text { conductivity }\end{array}$ & $\begin{array}{c}\text { Stream } \\
\text { depth }\end{array}$ \\
\hline-50 & 3.96 & 7.68 & 7.71 \\
50 & 11.4 & 7.74 & 7.72 \\
100 & 15.1 & 7.75 & 7.72 \\
\hline
\end{tabular}

All water samples were analyzed by the USGS National Water Quality Laboratory (NWQL) in Arvada, Colo. Samples were collected and analyzed according to procedures described by Fishman and Friedman (1989).

Results of the analysis of water samples are presented in appendix 5. A statistical summary of the chemical analysis of the ground-water samples, (table 5) is included for comparison with the USEPA (1991a, 1991b, 1992) drinking-water regulations. Results of the sample analyses indicate that water from the stratified-drift aquifers is generally suitable for drinking and other domestic or municipal uses with the following exceptions.

1. One well (CKW 11) contained water with elevated concentrations of chloride and sodium;

2. four of the wells contained water with elevated concentrations of iron and manganese (a common and presumably natural phenomenon in New Hampshire). Another four wells contained water with elevated manganese concentrations without the elevated iron; and 


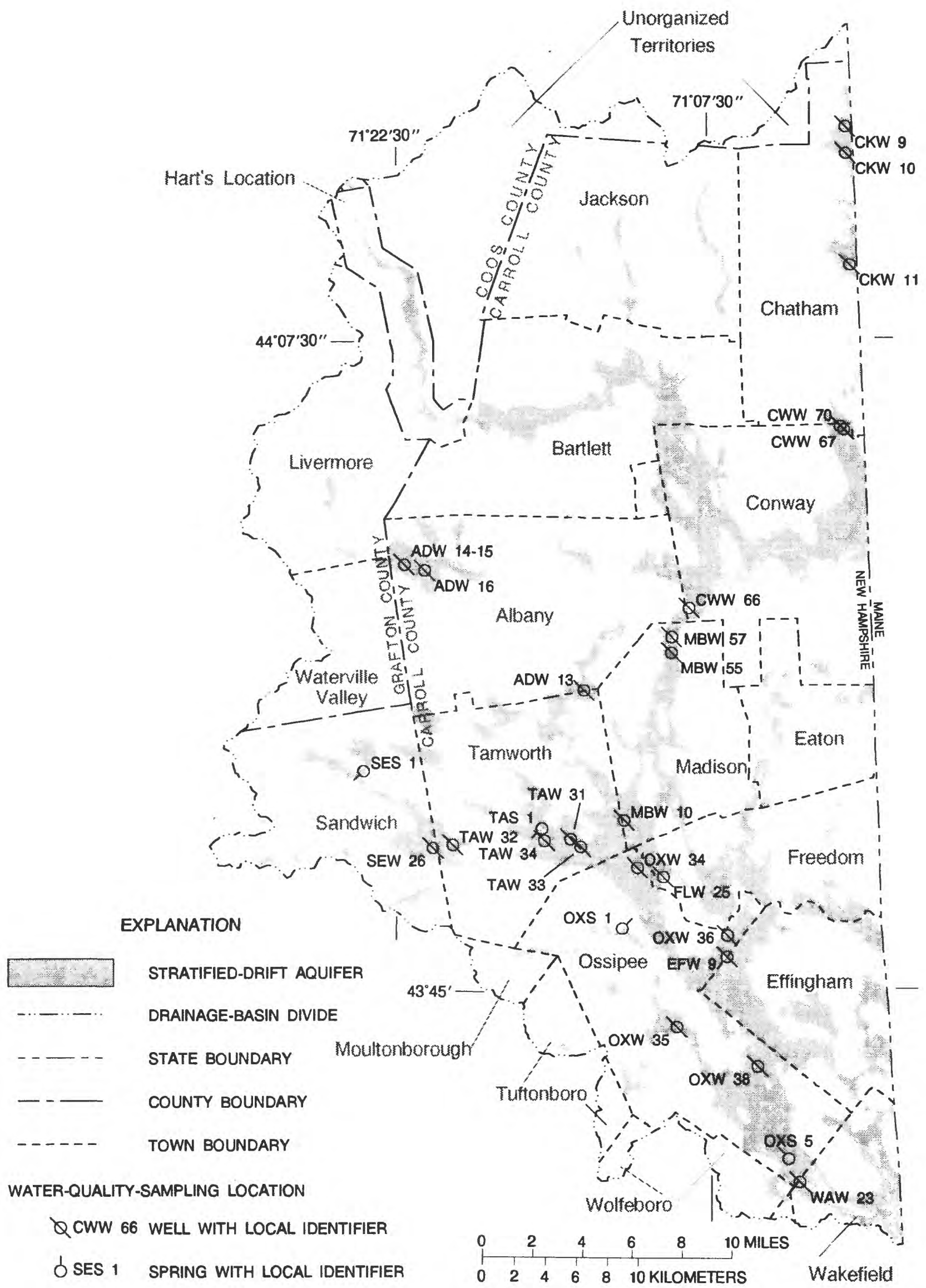

Figure 12. Water-quality-sampling location in the Saco and Ossipee River Basins study area, east-central New Hampshire. 
Table 5. Statistical summary of water-quality analyses of ground-water samples from the Saco and Ossipee River Basins, east-central New Hampshire

$[\mu \mathrm{S} / \mathrm{cm}$, microsiemens per centimeter at 25 degrees Celsius; A less than symbol " $<$ " precedes a value whenever the concentrattion was below reporting level; $\mathrm{mg} / \mathrm{L}$, milligrams per liter; $\mathrm{NO}_{2}$, nitrite; $\mathrm{NO}_{3}$, nitrate; $\mu \mathrm{g} / \mathrm{L}$, micrograms per liter; ,-- not applicable]

\begin{tabular}{|c|c|c|c|c|c|c|c|c|}
\hline Water-quality constituent & $M^{\prime} L^{1}$ & $\mathrm{SMCL}^{2}$ & $\begin{array}{l}\text { Number of } \\
\text { samples }\end{array}$ & $\begin{array}{l}\text { Mini- } \\
\text { mum }\end{array}$ & $\begin{array}{c}\text { First } \\
\text { quartile }\end{array}$ & Median & $\begin{array}{l}\text { Third } \\
\text { quartile }\end{array}$ & $\begin{array}{l}\text { Maxi- } \\
\text { mum }\end{array}$ \\
\hline $\begin{array}{l}\text { Specific conductance, field } \\
\text { ( } \mu \mathrm{S} / \mathrm{cm} \text { at } 25 \text { degrees Celsius) }\end{array}$ & -- & -- & 29 & 16 & 31.5 & 48 & 66 & 1,190 \\
\hline $\mathrm{pH}$, field (standard units) & -- & $6.5-8.5$ & 29 & 5.3 & 5.8 & 6.0 & 6.45 & 7.3 \\
\hline $\begin{array}{l}\text { Temperature, water, in degrees } \\
\text { Celsius }\end{array}$ & -- & -- & 29 & 7.0 & 7.75 & 9.0 & 10.0 & 14.5 \\
\hline Dissolved Oxygen, (mg/L as $\left.\mathrm{O}_{2}\right)$ & -- & -- & 25 & 0 & 3.3 & 9.5 & 11.3 & 11.8 \\
\hline Hardness, total (mg/L as $\mathrm{CaCO}_{3}$ ) & -- & -- & 29 & 3 & 7 & 12 & 19 & 33 \\
\hline Acidity, total (mg/L as $\mathrm{H})$ & -- & - & 29 & $<.1$ & $<.1$ & .1 & .2 & .9 \\
\hline Calcium, dissolved (mg/L as $\mathrm{Ca}$ ) & -- & -- & 29 & .8 & 2.0 & 3.8 & 5.6 & 11 \\
\hline $\begin{array}{l}\text { Magnesium, dissolved } \\
\quad(\mathrm{mg} / \mathrm{L} \text { as } \mathrm{Mg})\end{array}$ & -- & -- & 29 & .11 & .30 & .47 & .90 & 2.7 \\
\hline Sodium, dissolved (mg/L as $\mathrm{Na}$ ) & -- & $20-250$ & 29 & 1.5 & 2.85 & 4.4 & 6.6 & 220 \\
\hline Potassium, dissolved (mg/L as $\mathrm{K}$ ) & -- & -- & 29 & .3 & .5 & .9 & 1.25 & 3.6 \\
\hline Sulfate, dissolved $\left(\mathrm{mg} / \mathrm{L}\right.$ as $\left.\mathrm{SO}_{4}\right)$ & -- & 250 & 29 & $<.1$ & 1.65 & 2.6 & 3.6 & 55 \\
\hline Chloride, dissolved (mg/L as $\mathrm{Cl}$ ) & -- & 250 & 29 & .3 & .5 & 2.2 & 9.1 & 300 \\
\hline Fluoride, dissolved (mg/L as F) & 4 & 2 & 29 & $<.1$ & $<.1$ & .2 & 1.0 & 2.9 \\
\hline $\begin{array}{l}\text { Solids, sum of constituents, } \\
\text { dissolved }(\mathrm{mg} / \mathrm{L})\end{array}$ & -- & -- & 28 & 18 & 30 & 42 & 50.5 & 612 \\
\hline $\begin{array}{l}\text { Nitrogen, Nitrite, dissolved } \\
\quad(\mathrm{mg} / \mathrm{L} \text { as } \mathrm{N})\end{array}$ & 1 & -- & 21 & $<.01$ & $<.01$ & $<.01$ & $<.01$ & $<.01$ \\
\hline $\begin{array}{l}\text { Nitrogen, } \mathrm{NO}_{2}+\mathrm{NO}_{3} \text {, dissolved } \\
\quad(\mathrm{mg} / \mathrm{L} \text { as } \mathrm{N})\end{array}$ & 10 & -- & 21 & $<.05$ & $<.05$ & .18 & .365 & 1.30 \\
\hline $\begin{array}{l}\text { Nitrogen, Ammonia, dissolved } \\
\quad(\mathrm{mg} / \mathrm{L})\end{array}$ & -- & -- & 21 & $<.01$ & $<.01$ & .01 & .01 & .09 \\
\hline $\begin{array}{l}\text { Nitrogen, Ammonia-plus-Organic, } \\
\text { dissolved (mg/L) }\end{array}$ & -- & -- & 21 & $<.2$ & $<.2$ & $<.2$ & $<.2$ & $<.2$ \\
\hline $\begin{array}{l}\text { Phosphorus, dissolved } \\
\text { (mg/L as } \mathrm{P})\end{array}$ & -- & -- & 21 & $<.01$ & $<.01$ & .01 & .02 & .17 \\
\hline $\begin{array}{l}\text { Phosphorus, ortho, dissolved } \\
\text { (mg/L as P) }\end{array}$ & -- & -- & 21 & $<.01$ & $<.01$ & $<.01$ & $<.01$ & .04 \\
\hline
\end{tabular}


Table 5. Statistical summary of water-quality analyses of ground-water samples from the Saco and Ossipee River Basins, east-central New Hampshire--Continued

\begin{tabular}{|c|c|c|c|c|c|c|c|c|}
\hline Water-quality constituent & $\mathrm{MCL}^{1}$ & $\mathrm{SMCL}^{2}$ & $\begin{array}{c}\text { Number of } \\
\text { samples }\end{array}$ & $\begin{array}{l}\text { Mini- } \\
\text { mum }\end{array}$ & $\begin{array}{c}\text { First } \\
\text { quartile }\end{array}$ & Median & $\begin{array}{c}\text { Third } \\
\text { quartile }\end{array}$ & $\begin{array}{l}\text { Maxi- } \\
\text { mum }\end{array}$ \\
\hline
\end{tabular}

\begin{tabular}{|c|c|c|c|c|c|c|c|c|}
\hline Silica, dissolved $\left(\mathrm{mg} / \mathrm{L}\right.$ as $\mathrm{SiO}_{2}$ ) & -- & -- & 29 & 6.4 & 9.75 & 12 & 16 & 40 \\
\hline Barium, dissolved ( $\mu \mathrm{g} / \mathrm{L}$ as $\mathrm{Ba})$ & 2,000 & -- & 29 & 5 & 6 & 7 & 10.5 & 40 \\
\hline $\begin{array}{l}\text { Beryllium, dissolved } \\
\qquad(\mu \mathrm{g} / \mathrm{L} \text { as } \mathrm{Be})\end{array}$ & -- & 4 & 29 & $<.5$ & $<.5$ & $<.5$ & $<.5$ & .9 \\
\hline $\begin{array}{l}\text { Cadmium, dissolved } \\
(\mu \mathrm{g} / \mathrm{L} \text { as } \mathrm{Cd})\end{array}$ & 5 & -- & 29 & $<1$ & $<1$ & $<1$ & $<1$ & 4 \\
\hline Cobalt, dissolved ( $\mu \mathrm{g} / \mathrm{L}$ as $\mathrm{Co})$ & -- & -- & 29 & $<3$ & $<3$ & $<3$ & $<3$ & 4 \\
\hline Copper, dissolved $(\mu \mathrm{g} / \mathrm{L}$ as $\mathrm{Cu})$ & -- & 1,000 & 29 & $<10$ & $<10$ & $<10$ & $<10$ & $<10$ \\
\hline Iron, dissolved $(\mu \mathrm{g} / \mathrm{L}$ as $\mathrm{Fe})$ & -- & 300 & 29 & $<3$ & $<3$ & 4 & 33 & 8,700 \\
\hline Lead, dissolved ( $\mu \mathrm{g} / \mathrm{L}$ as $\mathrm{Pb})$ & 50 & 0 & 29 & $<10$ & $<10$ & $<10$ & $<10$ & $<10$ \\
\hline Lithium, dissolved ( $\mu \mathrm{g} / \mathrm{L}$ as $\mathrm{Li})$ & -- & -- & 29 & $<4$ & $<4$ & $<4$ & $<4$ & 17 \\
\hline $\begin{array}{l}\text { Manganese, dissolved } \\
\quad(\mu \mathrm{g} / \mathrm{L} \text { as } \mathrm{Mn})\end{array}$ & -- & 50 & 29 & $<1$ & 3 & 20 & 62.5 & 720 \\
\hline $\begin{array}{l}\text { Molybdenum, dissolved } \\
\quad(\mu \mathrm{g} / \mathrm{L} \text { as } \mathrm{Mo})\end{array}$ & -- & -- & 29 & $<10$ & $<10$ & $<10$ & $<10$ & 10 \\
\hline $\begin{array}{l}\text { Strontium, dissolved } \\
(\mu \mathrm{g} / \mathrm{L} \text { as } \mathrm{Sr})\end{array}$ & -- & -- & 29 & 8 & 18 & 27 & 41.5 & 81 \\
\hline Vanadium, dissolved ( $\mu \mathrm{g} / \mathrm{L}$ as $\mathrm{V})$ & -- & -- & 29 & $<6$ & $<6$ & $<6$ & $<6$ & 10 \\
\hline Zinc, dissolved ( $\mu \mathrm{g} / \mathrm{L}$ as $\mathrm{Zn})$ & -- & 5,000 & 29 & $<3$ & $<3$ & 4 & 5.5 & 50 \\
\hline
\end{tabular}

${ }^{1}$ MCL (Maximum Contaminant Level) is an enforceable, health-based maximum level (concentration) for contaminants in public drinking-water supplies as defined in the national primary and secondary drinking-water regulations established by the U.S. Environmental Protection Agency (1992).

${ }^{2}$ SMCL (Secondary Maximum Contaminat Level) is a non-enforceable, maximum level (concentration) for contaminants in public drinking-water supplies as defined in the national primary and secondary drinking-water regulations established by the U.S. Environmental Protection Agency (1992).

3. one well (ADW 14) contained water with an elevated concentration of fluoride.

The sampling procedure varied with the source of the water sampled. The four springs maintain constant flow, therefore, additional evacuation before sampling was unnecessary. The USGS wells were developed with a pneumatic pump several weeks before sampling. At the time of sampling, the USGS wells were pumped until at least three times the volume of water in the well was evacuated and until the temperature, specific con- ductance, and dissolved oxygen stabilized. These procedures ensured that the water sampled was water from the aquifer and not stagnant.

\section{pH}

The $\mathrm{pH}$ of water is a measure of the hydrogen ion concentration $\left(\mathrm{H}^{+}\right)$in solution. Water with a $\mathrm{pH}$ of seven is considered to be neutral, less than seven is acidic, and greater than seven is basic. The $\mathrm{pH}$ of most ground water in the United States ranges from about 6.0 to 8.5 (Hem 1985). The range of $\mathrm{pH}$ in stratified-drift 
aquifers sampled across New Hampshire (Moore and others, 1994) varied from 5.26 to 8.48 with a median value of 6.12. In this study, the most acidic water with a value of 5.3 was obtained from well $\mathrm{ADW} 15$. The most acidic water was below the recommended regulation shown in table 5. The most basic water was taken from spring SES 1 with a near neutral $\mathrm{pH}$ of 7.3.

\section{Specific Conductance}

Specific conductance - a measure of the ability of water to conduct electrical current-ranged from $16 \mu \mathrm{S} / \mathrm{cm}$ in water from well CKW 9 to $1,190 \mu \mathrm{S} / \mathrm{cm}$ from well CKW 11 (appendix 5). The water from CKW 11 was the only sample that exceeded the recommended limit of $500 \mu \mathrm{S} / \mathrm{cm}$ established by the New Hampshire Water Supply and Pollution Control Commission (1984) for public drinking water. The median for all water samples in the study $(48 \mu \mathrm{S} / \mathrm{cm})$ was less than the median $(132 \mu \mathrm{S} / \mathrm{cm})$ for public supply wells completed in stratified-drift aquifers for the entire State (Morrissey and Regan,1987).

\section{Dissolved Solids}

Dissolved solids in water include all ionized and un-ionized dissolved solids in solution. The concentrations of all water samples from the stratified-drift aquifers ranged from 18 to $612 \mathrm{mg} / \mathrm{L}$ with a median of $42 \mathrm{mg} / \mathrm{L}$. Only water from well, CKW 11, was above the recommended limit for drinking water of $500 \mathrm{mg} / \mathrm{L}$ established by the New Hampshire Water Supply Engineering Bureau (written commun., 1990) for public drinking water. The relatively low concentration of dissolved solids in stratified-drift aquifers is attributed to the low solubility of the aquifer matrix and the relatively short time water is in contact with the aquifer (Morrissey and Regan, 1987). The well with the highest concentration of dissolved solids (CKW 11) also had the highest concentration of chloride and sodium.

\section{Dissolved Oxygen}

Oxygen is supplied to ground water through recharge and by movement of air through unsaturated material above the water table. The concentration of dissolved oxygen (DO) in water that is in contact with air is a function of temperature and pressure, and to a lesser degree, the concentration of other solutes (Hem, 1985 , p. 155). Dissolved-oxygen concentrations in water from 25 samples ranged from $0 \mathrm{mg} / \mathrm{L}$ (well
TAW 31) to $11.8 \mathrm{mg} / \mathrm{L}$ (well WAW 23); the median was $9.5 \mathrm{mg} / \mathrm{L}$ (table 5 ). Water containing measurable amounts of dissolved oxygen may flow long distances in the ground-water system, if little reaction results with aquifer material.

\section{Calcium, Magnesium, and Hardness}

Calcium and magnesium are common elements of alkaline-earth minerals. Calcium and magnesium are also the predominate cations in most natural ground water (Hem, 1985). Concentrations of dissolved calcium in 29 samples ranged from 0.8 to $11 \mathrm{mg} / \mathrm{L}$; the median was $3.8 \mathrm{mg} / \mathrm{L}$. Concentrations of dissolved magnesium in 29 water samples from the stratified-drift aquifers ranged from 0.11 to $2.7 \mathrm{mg} / \mathrm{L}$; the median was $0.47 \mathrm{mg} / \mathrm{L}$. The USEPA has not assigned a drinkingwater regulation for calcium or magnesium concentrations.

Hardness of water-a property of water that produces a residue when mixed with soap and (or) when heated - is the result of the presence of several different cations, particularly calcium and magnesium, and is expressed in an equivalent quantity of milligrams per liter $\mathrm{CaCO}_{3}$ (calcium carbonate) (Hem 1985). The hardness of water in all 29 samples ranged from $3 \mathrm{mg} / \mathrm{L}$ to $33 \mathrm{mg} / \mathrm{L} \mathrm{CaCO}{ }_{3}$. Water that is less than $60 \mathrm{mg} / \mathrm{L} \mathrm{CaCO}{ }_{3}$ is considered soft (Hem, 1985). The median for all the samples was $12 \mathrm{mg} / \mathrm{L}$ and the median for public supply wells completed in stratified-drift aquifers in the entire State of New Hampshire (Morrissey and Regan, 1987) was $37 \mathrm{mg} / \mathrm{L}$.

\section{Sodium and Chloride}

Sodium $\left(\mathrm{Na}^{+}\right)$and chloride $\left(\mathrm{Cl}^{-}\right)$can be introduced into ground water from natural and manmade sources. The major natural source of chloride is atmospheric precipitation and dry fallout, and contributes about $0.5 \mathrm{mg} / \mathrm{L}$ to the New Hampshire land surface (Hall, 1975). The major manmade source of both sodium and chloride is salt $(\mathrm{NaCl})$ used in road deicing. Based on limited data, Hall estimated that in 1975 towns and cities in New Hampshire used at least 33,000 tons of $\mathrm{NaCl}$ per year. Hall suggests that heavy metals such as hexavalent chromium, which is toxic to humans, may be released by the corrosion of car bodies. He also suggests that dissolved salt coupled with $\mathrm{pH}$ ranges of 5.5 to 7.5 could pose an additional health threat as a result of the corrosion of iron, zinc and cadmium from plumb- 
ing systems. During the period, from the winter of 1982-83 to the winter of 1992-93, the State of New Hampshire used an average of 152,000 tons/yr of $\mathrm{NaCl}$ to deice state highways and roads (Robert Hogan, New Hampshire Department of Transportation, oral commun., 1993) that contributed to an increase in concentrations of sodium and chloride in ground water statewide.

Water samples from well CKW 11 in the stratifieddrift aquifer had concentrations of sodium and chloride of $220 \mathrm{mg} / \mathrm{L}(8.5 \mathrm{meq} / \mathrm{L})$ and $300 \mathrm{mg} / \mathrm{L}(9.5 \mathrm{meq} / \mathrm{L})$, respectively. The ratio of these milliequivalents suggests that $\mathrm{NaCl}$ could be a major contributor to both constituents. The water from well CKW 11 was the only sample with concentrations higher than the U.S. Environmental Protection Agency (1992) Secondary Maximum Contaminant Level (SMCL) ${ }^{1}$ of $250 \mathrm{mg} / \mathrm{L}$ of chloride and higher than the Health Advisory Level of $20 \mathrm{mg} / \mathrm{L}$ for sodium. Well $\mathrm{CKW} 11$ is downgradient from a NHDOT road deicing salt storage site. The SMCL for chloride was established as a taste threshold and the Health Advisory Limit for sodium was established as a recommended limit for people with heart, hypertension or kidney problems who are on sodiumrestricted diets. The median concentrations of sodium $(6.0 \mathrm{mg} / \mathrm{L})$ and of chloride $(6.8 \mathrm{mg} / \mathrm{L})$ were below these regulations. The median concentration for sodium in ground water from public supply wells completed in stratified-drift aquifers in New Hampshire is $11.0 \mathrm{mg} / \mathrm{L}$ (Morrissey and Regan, 1987).

\section{Nitrogen}

Nitrogen is present in many forms in natural waters depending on the source of nitrogen and the degree of decomposition. Nitrogen is present in water as nitrite $\left(\mathrm{NO}_{2}{ }^{-}\right)$or nitrate $\left(\mathrm{NO}_{3}{ }^{-}\right)$anions, as ammonium $\left(\mathrm{NH}_{4}{ }^{+}\right)$ cations, and at intermediate oxidation states as a part of organic solutes (Hem, 1985, p. 124). The predominant form of inorganic nitrogen in natural water is nitrate, from the oxidation of nitrogeneous compounds. High levels of nitrate in ground water can originate from fertilizer application, leachate from sewage systems (such as septic tanks, sewage lagoons, or cesspools), or wastes

${ }^{1}$ SMCL (Secondary maximum contaminant level) is a nonenforceable, maximum level (concentration) for contaminants in public drinking-water supplies as defined in the national primary and secondary drinking-water regulations established by the U.S. Environmental Protection Agency (1992). from farm animals. High levels of nitrate (greater than $10 \mathrm{mg} / \mathrm{L}$ as nitrogen or greater than $44 \mathrm{mg} / \mathrm{L}$ as nitrate) in drinking water has been linked to methemoglobinemia in small children, or blue-baby syndrome (Hem, 1985 , p. 125). Dissolved nitrite-plus-nitrate (as N) concentrations in 21 water samples ranged from less than 0.05 to $1.3 \mathrm{mg} / \mathrm{L}$; the median was $0.18 \mathrm{mg} / \mathrm{L}$. Water from well MBW 55 in Madison had a concentration of nitrogen $(1.3 \mathrm{mg} / \mathrm{L})$, which is elevated from background levels but is below the USEPA's (1992) primary drinking-water standard $(10 \mathrm{mg} / \mathrm{L})$ for nitrogen (appen$\operatorname{dix} 5$ ).

\section{Phosphorus}

Although phosphorus is a common element in igneous rocks and is abundant in soils, concentrations of phosphorus in ground water are generally low. Many inorganic compounds of phosphorus have low solubility in water and can be used as fertilizer (Hem, 1985). Manmade sources of phosphorus in ground water can come from waste disposal through septic tanks and sewage lagoons, and from chemical fertilizer applications. Six of 21 samples analyzed for dissolved phosphorus and 18 of 21 water samples analyzed for dissolved orthophosphate had concentrations below the minimum reporting level. The highest concentration for dissolved phosphorus $(0.17 \mathrm{mg} / \mathrm{L})$ was detected in water from well CWW 67 in Conway. The highest concentration for dissolved orthophosphate $(0.04 \mathrm{mg} / \mathrm{L})$ was detected in water from spring SES 1 in Sandwich (appendix 5). At present, the USEPA has not set a drinking-water regulation concentration for phosphorus.

\section{Sulfate}

Sulfur is widely distributed in reduced form in igneous, sedimentary, and metamorphic rocks as metallic sulfide minerals such as pyrite (Hem, 1985). Evaporite minerals, such as gypsum and anhydrite, also contain sulfur, but these minerals are generally absent in stratified-drift aquifers in New Hampshire. However, oxidation of sulfur by human activities, such as combustion of fuels and smelting of ores and resultant atmospheric deposition, is a major source of sulfate $\left(\mathrm{SO}_{4}{ }^{-2}\right)$. Under reducing conditions, sulfate can be reduced by anaerobic bacteria to hydrogen sulfide $\left(\mathrm{H}_{2} \mathrm{~S}\right)$ gas, which can be detected by smell at concentrations of only a few tenths of a milligram per liter (Hem, 1985). Concentra- 
tions of dissolved sulfate in 28 water samples ranged from less than 0.1 to $55 \mathrm{mg} / \mathrm{L}$, and the median was $2.6 \mathrm{mg} / \mathrm{L}$ (table 5). None of the samples exceeded the USEPA's (1992) secondary drinking water regulation $(250 \mathrm{mg} / \mathrm{L})$ for sulfate.

\section{Iron and Manganese}

Elevated concentrations of iron and manganese were the most common water-quality problems found during this study. Iron is the fourth most abundant element in the Earth's outer crust and is an essential element to plant and animal metabolism. If present in high concentrations in residential water supplies, however, iron forms red oxyhydroxide precipitates that stain clothes and plumbing fixtures. Manganese, also an abundant metallic element, is an undesirable impurity in water as a result of its tendency to deposit black oxide stains (Hem, 1985). Both iron and manganese are common in minerals of bedrock and stratified drift in the study area. The water from 4 of the 29 sites sampled had iron concentrations that exceeded the SMCL of $300 \mu \mathrm{g} / \mathrm{L}$ and water from 8 sites had manganese concentrations that exceeded the SMCL of $50 \mu \mathrm{g} / \mathrm{L}$. Water from well OXW 36 had the highest concentrations of iron, $8,700 \mu \mathrm{g} / \mathrm{L}$ with other high concentrations as follows: CWW 67, 7,100 $\mu \mathrm{g} / \mathrm{L}$; TAW 33, $5,300 \mu \mathrm{g} / \mathrm{L}$; and OXW 34, $890 \mu \mathrm{g} / \mathrm{L}$. The median value for iron was $4 \mu \mathrm{g} / \mathrm{L}$. Water from these same wells, as well as four others, had high concentrations of manganese. The median manganese concentration of $20 \mu \mathrm{g} / \mathrm{L}$ is lower than the SMCL. Three of the four wells contained water with elevated iron concentrations, OXW 36, OXW 34 and TAW 33, also contained water with low concentrations of dissolved oxygen (appendix 5). Iron as $\mathrm{Fe}^{+2}$, is highly soluble in water with low concentrations of oxygen and elevated concentrations of iron can result.

\section{Silica}

The element silicon $\left(\mathrm{Si}^{+4}\right)$ is the second most abundant element (after oxygen) in the Earth's crust (Hem, 1985). Crystalline silica $\left(\mathrm{SiO}_{2}\right)$ as quartz is a major constituent of many igneous and metamorphic rocks. Feldspars, amphiboles, and other minerals that also contain silica, are widely distributed in igneous and metamorphic rocks. These silica-rich minerals are found in sediments from stratified-drift aquifers in New Hampshire. Concentrations of silica, however, are generally low in natural waters. Dissolved silica concentrations in water from the 29 sites ranged from 6.4 to $40 \mathrm{mg} / \mathrm{L}$ with a median of $12 \mathrm{mg} / \mathrm{L}$ (table 5). At present (1994), there is no USEPA drinking-water regulation for silica.

\section{Trace Elements}

Trace elements are elements that nearly always occur in concentrations less than $1.0 \mathrm{mg} / \mathrm{L}$ in natural waters (Hem, 1985). Water samples collected from USGS wells in the Saco and Ossipee River Basins and analyzed for fluoride, beryllium, boron, cadmium, cobalt, copper, molybdenum, zinc, lead, lithium, and vanadium generally contained concentrations that ranged from less than detection level to a few micrograms per liter. Trace elements with concentrations consistently above detection level, but less than $1.0 \mathrm{mg} / \mathrm{L}$, were barium (less than 5 to $40 \mu \mathrm{g} / \mathrm{L}$ with a median of $7 \mu \mathrm{g} / \mathrm{L}$ ) and strontium ( 8 to $81 \mu \mathrm{g} / \mathrm{L}$ with a median of $27 \mu \mathrm{g} / \mathrm{L})$. None of the water samples exceeded the USEPA's primary drinking-water standard of $2,000 \mu \mathrm{g} / \mathrm{L}$ for barium (1992). Strontium is a common element that replaces calcium or potassium in minor amounts in igneous-rock minerals, such as minerals in granite (Hem, 1985). There is no USEPA drinking-water regulation for strontium. Water from well ADW 14, had a concentration of fluoride $(2.9 \mathrm{mg} / \mathrm{L})$ in excess of the USEPA's secondary drinking-water standard of $2 \mathrm{mg} / \mathrm{L}$ (USEPA, 1992). Fluorine is an element found in several minerals including fluorite $\left(\mathrm{CaF}_{2}\right)$, apatite, $\mathrm{Ca}_{5}(\mathrm{Cl}, \mathrm{F}, \mathrm{OH})$, and amphiboles and may be present naturally in the water in a wide variety of geologic terranes (Hem, 1985).

\section{SUMMARY AND CONCLUSIONS}

Population growth and economic development have increased demands for water in the Saco and Ossipee River Basins in east-central New Hampshire. The Saco and Ossipee River Basins drain $869.4 \mathrm{mi}^{2}$ and contains $152.5 \mathrm{mi}^{2}$ of stratified-drift aquifers. In 1990 , 2.30 Mgal/d were withdrawn from the stratified-drift aquifers by the three largest users of ground water in the study area (Wolfeboro Water Works, North Conway Water Precinct, and Conway Village Fire District). As the need for water increases, the need for information increases to ensure optimal use of available resources. This report provides geohydrologic information on stratified-drift aquifers in the Saco and Ossipee River 
Basins drainage area. The report also describes geohydrologic characteristics, presents a technique for assessing ground-water availability, and the quality of water in the stratified-drift aquifers is assessed.

Most of the stratified-drift aquifers in the Saco and Ossipee River Basins consist of stratified, sorted, principally coarse-grained sediments (sands and gravels) deposited by glacial meltwater at the time of deglaciation. Interconnected voids or pore spaces between sediment particles provide space through which stored ground water can flow. Characteristics of the sediments that affect ground-water storage and flow are related to the glaciofluvial environment in which they were deposited. The presence of glacial lakes resulted in the formation of deltas and other lake deposits. The deglaciation process also resulted in eskers, kames, kame terraces, and outwash deposits. These coarse-grained stratified-drift deposits formed in contact with glacial ice tend to have a high capacity to store and transmit water.

Aquifer boundaries were delineated from available maps of surficial geology or were specifically mapped as part of this study. Thicknesses of saturated stratified drift were contoured with the aid of the surficial mapping, seismic-refraction profiling, seismic-reflection profiling, test drilling, and available well data. Saturated thickness is related to the geohydrologic setting in which the aquifers were formed. Layers of saturated silts and clays that lie above, below, or interfinger with the aquifer are included in the saturated thicknesses depicted. Saturated thicknesses of stratified drift in the study area are locally greater than $280 \mathrm{ft}$, but generally are less.

Hydraulic conductivity of the stratified drift was estimated from field descriptions of the grain-size distributions of samples collected during test drilling, and sieve analysis data. Aquifer transmissivity was calculated from the estimates of hydraulic conductivity and from aquifer-thickness data. Geologic and drillers' logs were examined during this process, and saturated silts and clays were excluded from the transmissivity estimates because their contribution is negligible. Estimated transmissivity values, in the study area, range from nearly 0 to greater than $8,000 \mathrm{ft}^{2} / \mathrm{d}$.

The Ossipee Lake aquifer in Ossipee, Freedom, Effingham, Madison, and Tamworth was selected for analysis of ground-water availability. A two- dimensional, numerical finite-difference flow model and transient simulations were used to simulate the aquifer. The objective was to estimate the yield to 4 hypothetical wells after a 180-day period of pumping. The results show that the Ossipee Lake aquifer supplied $7.72 \mathrm{Mgal} / \mathrm{d}$ to the 4 wells of which 31.6 percent of the water pumped came from ground-water storage and 68.4 percent came from combined induced infiltration and ground water captured before discharge to surfacewater bodies.

Ground-water quality in water from 25 test wells and 4 springs in the stratified-drift aquifers generally meets U.S. Environmental Protection Agency primary and secondary drinking-water regulations. Known areas of ground-water contamination were not sampled. Water samples from one well had elevated concentrations of chloride and sodium. Water samples from four sites had elevated iron and manganese concentrations (which occurs naturally in many parts of $\mathrm{New}$ Hampshire) and water samples from four other sampling sites had elevated manganese only. Water samples from one well had elevated concentrations of fluoride.

\section{SELECTED REFERENCES}

Ayotte, J.D. and Toppin, K.W., 1995, Geohydrology and water quality of stratified-drift aquifers in the middle Merrimack River Basin, south-central, New Hampshire: U.S. Geological Survey Water-Resources Investigations Report 92-4192, 52 p., 8 pls.

Bates, R.L., and Jackson, J.A.(eds.), 1987, Glossary of Geology (3d ed): Alexandria, Va., American Geological Institute, $788 \mathrm{p}$.

Bedinger, M.S., 1961, Relation between median grain size and permeability in the Arkansas River Valley, in Geological Survey Research, 1961, Short papers in the geologic and hydrologic sciences, articles 147-292: U.S. Geological Survey Professional Paper 424-C, p. C31C32.

Billings, M.P., 1956, The geology of New Hampshire, part II-Bedrock geology: Concord, New Hampshire State Planning and Development Commission, 203 p., 1 pl., scale: $1: 250,000$.

Boudette, E.L., Canney, F.C., Cotton, J.E., Davis, R.I., and others, 1985, High levels of arsenic in the ground waters of southeastern New Hampshire-a geochemical reconnaissance: U.S. Geological Survey Open-File Report 85-202, $25 \mathrm{p}$. 
Chapman, D.H., 1974, New Hampshire's landscape - how it was formed: New Hampshire Profiles, v. 23, no. 1, p. 41-56.

Cotton, J.E., 1975, Availability of ground water in the Saco River Basin, east-central New Hampshire: U.S. Geological Survey Water-Resources Investigations Report 39-74, scale: 1:125,000.

1987, Ground-water resources of the Lamprey River Basin, southeastern New Hampshire: U.S. Geological Survey Water-Resources Investigations Report 84-4252, $53 \mathrm{p}$.

Cotton, J.E., and Hammond, R.E., 1985, New Hampshire ground-water resources, in National water summary 1984: U.S. Geological Survey Water-Supply Paper 2275, p. 303-308.

Cotton, J.E., and Olimpio, J.R., in press, Geohydrology, yield, and water quality of stratified-drift aquifers in the Pemigewasset River Basin, central New Hampshire: U.S. Geological Survey, Water-Resources Investigations Report 94-4083, 258 p., 10 pls.

Diers, R.W., and Vieira, F.J., 1977, Soil survey of Carroll County, New Hampshire: U.S. Department of Agriculture, Soil Conservation Service, 161 p., 63 map sheets, scale 1:24,000.

Davis, P.T., Thompson, W.B., Stone, B.D., Newton, R.M., and Fowler, B.K., 1993, Multiple glaciations and deglaciation along a transect from Boston, Massachusetts, to the White Mountains, New Hampshire in Cheney, J.T., and Hepburn, J.P., 1993, Field trip guidebook for the Northeastern United States: 1993 Geological Society of America Annual Meeting and 85th Annual New England Intercollegiate Geological Conference, October 25-28, Boston, Mass., v. 2, p. EE-1-EE-27.

Fenneman, N.M., 1938, Physiography of Eastern United States: New York, McGraw-Hill Book Co., 714 p.

Ferris, J.G., Knowles, D.B., Brown, R.H., and Stallman, R.W., 1962, Theory of aquifer tests: U.S. Geological Survey Water-Supply Paper 1536-E, p. 69-174.

Fishman, M.J., and Friedman, L.C., 1989, Methods of determination of inorganic substances in water and fluvial sediments ( $3 \mathrm{~d}$ ed.): U.S. Geological Survey Techniques of Water-Resources Investigations, book 5, chap. Al, 545 p.

Flanagan, S.M., and Stekl, P.J., 1989, Geohydrologic, ground-water-quality, and streamflow data for the stratified-drift aquifers in the lower Merrimack and coastal river basins, southeastern New Hampshire: U.S. Geological Survey Open-File Report 89-390, 130 p., 3 pls.

Folk, R.L., 1974, Petrology of sedimentary rocks: Austin, Tex., Hemphill Publishing Co., 182 p.

Goldthwait, J.W., 1916, Glaciation in the White Mountains of New Hampshire: Geological Society of America Bulletin, v. 27, p. 263-294.

1925, The geology of New Hampshire: New Hampshire Academy of Science Handbook, no. 1, 86 p.

1938, The uncovering of New Hampshire by the last ice sheet: American Journal of Science, v. 36, p. 345372.

Goldthwait, J.W., Goldthwait, Lawrence, and Goldthwait, R.P., 1951, The geology of New Hampshire, part I, surficial geology: New Hampshire State Planning and Development Commission, 83 p., 1 pl., scale 1:250,000.

Goldthwait, R.P., 1940, Geology of the Presidential Range: New Hampshire Academy of Science, Bulletin no. 1, $43 \mathrm{p}$.

Goldthwait, R.P., 1968, Surficial geology of the WolfeboroWinnipesaukee area, New Hampshire: New Hampshire Department of Resources and Economic Development, 60 p., 1 pl., scale 1:62,500.

Haeni, F.P., 1978, Computer modeling of ground-water availability in the Pootatuck River Valley, Newtown, Connecticut: U.S. Geological Survey Water-Resources Investigations $78-77,64 \mathrm{p}$.

1986, Application of continuous seismic-reflection methods to hydrologic studies: Ground Water, v. 24, no. 1, p. 23-31.

1988a, Evaluation of the continuous seismic-reflection methods for determining the thickness and lithology of stratified drift in glaciated northeast in Randall, A.D., and Johnson, A.I. ,eds., 1988, Regional aquifer systems of the United States - the northeast glacial aquifers: American Water Resources Association Monograph Series no. 11, p. 63-82.

1988b, Application of seismic-refraction techniques to hydrologic studies: U.S. Geological Survey Techniques of Water-Resources Investigations, book 2, chap. D2, $86 \mathrm{p}$.

Hall, F.R., 1975, Chloride in natural waters of New Hampshire: Durham, N.H., University of New Hampshire, Agricultural Experiment Station Bulletin $504,25 \mathrm{p}$. 
Harte, P.T., and Johnson, William, 1995, Geohydrology and water quality of stratified-drift aquifers in the Contoocook River Basin, south-central New Hampshire, with a section on Geohydrologic setting, by Richard Bridge Moore: U.S. Geological Survey WaterResources Investigations Report 92-4154, 72 p., 4 pls.

Harte, P.T., and Mack, T.J., 1992, Geohydrology of, and simulation of ground-water flow in, the MilfordSouhegan glacial-drift aquifer, Milford, New Hampshire: U.S. Geological Survey Water-Resources Investigations Report 91-4177, 75 p.

Hazen, A., 1892, Some physical properties of sands and gravels with special reference to their use in filtration in Massachusetts State Board of Health, 24th Annual Report: p. 541-556.

Heath, R.C., 1983, Basic ground-water hydrology: U.S. Geological Survey Water-Supply Paper 2220, 84 p.

Hem, J.D., 1985, Study and interpretation of the chemical characteristics of natural water (3d ed.): U.S. Geological Survey Water-Supply Paper 2254, 263 p.

Hildreth, C.T., and Moore, R.B., in press, Late Wisconsinan deglaciation styles of parts of the Contoocook, Souhegan, and Piscataquog Drainage Basins, New Hampshire: U.S. Geological Survey Open-File Report 95-307, $56 \mathrm{p}$.

Hill, D.B., 1979, The hydrology of an ice-contact deposit at Newmarket Plains, New Hampshire: Durham, N.H., University of New Hampshire, [unpublished thesis] $71 \mathrm{p}$.

Johnson, A.I., 1967, Specific yield-compilation of specific yields for various materials: U.S. Geological Survey Water-Supply Paper 1662-D, 74 p.

Johnson, C.D., Tepper, D.H., and Morrissey, D.J., 1987, Geohydrologic and surface-water data for the Saco River Valley glacial aquifer from Bartlett, New Hampshire to Fryeburg, Maine: October 1983 through January 1986: U.S. Geological Survey Open-File Report 87-44, 80 p.

Knox, C.E., and Nordenson, T.J., 1955, Average annual runoff and precipitation in the New England-New York area: U.S. Geological Survey Hydrologic Investigations Atlas HA-7, 3 sheets.

Koteff, Carl, and Larsen, F.D., 1989, Postglacial uplift in western New England: geologic evidence for delayed rebound, in Gregersen S., and Basham P.W., eds., Earthquakes at North-Atlantic passive margins: neotectonics and postglacial rebound: Kluwer Academic Publishers, $693 \mathrm{p}$.
Koteff, Carl, and Pessl, F., Jr., 1981, Systematic ice retreat in New England: U.S. Geological Survey Professional Paper 1179, $20 \mathrm{p}$.

Koteff, Carl, Robinson, G.R., Goldsmith, Richard, Thompson, W.B. 1993, Delayed postglacial uplift and synglacial sea levels in coastal central New England: Quaternary Research, v. 40, p. 46-54.

Koteff, Carl, Stone, B.D., and Caldwell, D.W., 1984, Deglaciation of the Merrimack River Valley, southern New Hampshire, in Hanson, L.S., ed., Guidebook for the geology of the coastal lowlands, Boston to Kennebunk, Maine: New England Intercollegiate Geological Conference, 76th annual meeting, 1984, p. 381-393.

Krumbein, W.C., and Monk, G.D., 1942, Permeability as a function of the size parameters of unconsolidated sand: Transactions of the American Institute of Mineralogical and Metallurgical Engineers, v. 151, p. 153-163.

Langbein, W.B., and Iseri, K.T., 1960, Manual of hydrology, part 1, general surface-water techniques-general introduction and hydrologic definitions: U.S. Geological Survey Water-Supply Paper 1541-A, 29 p.

Lapham, W.W., 1988, Yield and quality of ground water from stratified-drift aquifers, Taunton River Basin, Massachusetts: U.S. Geological Survey WaterResources Investigations Report 86-4053, 69 p., 2 pls.

Lawlor, Sean, and Mack, T.J., 1992, Geohydrologic, groundwater-quality, and streamflow data for the stratified-drift aquifers in the Bellamy, Cocheco, and Salmon Falls River Basins, southeastern New Hampshire: U.S. Geological Survey Open-File Report 89-583, 137 p., 3 pls.

Leavitt, H.W., and Perkins, E.H., 1935, A survey of road materials and glacial geology of Maine, v. 2: Orono, Me., University Press, Maine Technology Experiment Station Bulletin 30, 232 p.

Lohman, S.W., 1972, Ground-water hydraulics: U.S. Geological Survey Professional Paper 708, 70 p.

Lohman, S.W., and others, 1972, Definitions of selected ground-water terms-Revisions and conceptual refinements: U.S. Geological Survey Water-Supply Paper 1988, $21 \mathrm{p}$.

Lougee, R.J., 1939, Geology of the Connecticut watershed, in Biological survey of the Connecticut watershed: New Hampshire Fish and Game Department, p. 131-149.

1940, Deglaciation of New England: Journal of Geomorphology, v. 3, p. 188-217. 
Lyons, J.B., Bothner, W.A., Moench, R.H., and Thompson, J.B., Jr., eds., 1986, Interim geologic map of New Hampshire: New Hampshire Department of Resources and Economic Development, Open-File Report 86-1, 1 map sheet, scale 1:250,000.

Mack, T.J., and Lawlor, Sean, 1992, Geohydrology and water quality of stratified-drift aquifers in the Bellamy, Cocheco, and Salmon Falls River Basins, southeastern New Hampshire: U.S. Geological Survey WaterResources Investigations Report 90-4161, 65 p., 6 pls.

MacNish, R.D. and Randall, A.D., 1982, Stratified-drift aquifers in the Susquehanna River Basin, New York: New York State Department of Environmental Conservation Bulletin 75, $68 \mathrm{p}$.

Masch F.O., and Denny, K.J., 1966, Grain size distribution and its effect on the permeability of unconsolidated sands: Water Resources Research, v. 2, no. 4, p. 665677.

Mazzaferro, D.L., Handman, E.H., and Thomas, M.P., 1979, Water resources inventory of Connecticut, part 8, Quinnipiac River Basin, Connecticut: Connecticut Water Resources Bulletin 27, 88 p., 5 pls.

McDonald, M.G., and Harbaugh, A.W., 1988, A modular three-dimensional finite-difference ground-water-flow model: U.S. Geological Survey Techniques of WaterResources Investigations, book 6, chap. Al, $586 \mathrm{p}$.

Mercer, M.W., and Morgan, C.O., 1981, Storage and retrieval of ground-water data at the U.S. Geological Survey: Groundwater, v. 19 , no. 5, p. 543-551.

Moore, R.B., 1990, Geohydrology and water quality of stratified-drift aquifers in the Exeter, Lamprey, and Oyster River Basins, southeastern New Hampshire: U.S. Geological Survey Water-Resources Investigations Report 88-4128, 61 p., 8 pls.

1993, Geologic evidence for catastrophic draining of glacial lakes in the Contoocook, Souhegan, and Piscataquog River Basins, south-central New Hampshire [abs.]: Geological Society of America Abstracts with Programs, 1993 Annual Meeting, v. 25, no. 6, p. A-157.

Moore, R.B., Johnson, C.D., and Douglas, E.M., 1994, Geohydrology and water quality of stratified-drift aquifers in the lower Connecticut River Basin, southwestern New Hampshire: U.S. Geological Survey Water-Resources Investigations Report 92-4013, 187 p., 4 pls.
Morrissey, D.J., 1983, Hydrology of the Little Androscoggin River Valley Aquifer, Oxford County, Maine: U.S. Geological Survey Water-Resources Investigations Report 83-4018, 79 p., 8 pls.

Morrissey, D.J., Haeni, F.P., and Tepper, D.H., 1985, Continuous seismic-reflection profiling of a glacial-drift deposit on the Saco River, Maine and New Hampshire, in National Water Well Association, Proceedings of the Association of Ground Water Scientists and Engineers, Eastern Regional Ground Water Conference: Worthington, Ohio, Water Well Journal Publishing Co., p. 277-296.

Morrissey, D.J., and Regan, J.M., 1987, New Hampshire ground-water quality in U.S. Geological Survey, 1987, National water summary 1986 -Hydrologic events and ground-water quality: U.S. Geological Survey WaterSupply Paper 2325, $560 \mathrm{p}$.

National Oceanic and Atmospheric Administration, 1988, Climatological data annual summary, New England, 1988: Asheville, N.C., v. 100, no. 13, 52 p.

New Hampshire Office of State Planning, 1985, New Hampshire population projections for cities and towns: $19 \mathrm{p}$.

New Hampshire Water Resources Board, 1984, Water resources management plan: $47 \mathrm{p}$.

New Hampshire Water Supply and Pollution Control Commission and U.S. Environmental Protection Agency, 1971, Water-quality standards summary (New Hampshire): New Hampshire Water Supply and Pollution Control Commission, $91 \mathrm{p}$.

New Hampshire Water Supply and Pollution Control Commission, 1982, Inventory of groundwater and surface water potential nonpoint pollution sources: $75 \mathrm{p}$.

1982, Public water supplies, facilities and policy summary 1981: $44 \mathrm{p}$.

1984, Drinking water regulations: Chapter Ws 300 , $85 \mathrm{p}$.

Newton, R.M., 1974, Surficial geology of the Ossipee Lake quadrangle, New Hampshire: New Hampshire Department of Resources and Economic Development, 52 p., 2 pls., scale 1:62,500.

Olney, S.L., 1983, An investigation of the relationship between the coefficient of permeability and effective grain size of unconsolidated sands: Boston, Mass., Boston University [unpublished master's thesis] $61 \mathrm{p}$. 
Orzol, L.L., and McGrath, T.S., 1992, Modifications of the U.S. Geological Survey modular, finite-difference, ground-water-flow model to read and write geographic information system files: U.S. Geological Survey OpenFile Report 92-50, $199 \mathrm{p}$.

Pettyjohn, W.A., Studlick, J.R., Bain, R.C., and Lehr, J.H., 1979, A ground-water-quality atlas of the United States: National Water Well Association for National Demonstration Water Project, $272 \mathrm{p}$.

Plukowski, E.J., and Kantrowitz, I.H., 1964, Hydrology of the Babylon-Islip area, Suffolk County, Long Island, New York: U.S. Geological Survey Water-Supply Paper $1768,119 \mathrm{p}$.

Piotrowski, T.M., 1977, History of the American indians in the Manchester, New Hampshire area: Manchester, N.H., University of New Hampshire, 104 p.

Randall, A.D., 1978, Infiltration from tributary streams in the Susquehanna River Basin, New York: U.S. Geological Survey Journal of Research, v. 6, no. 3, p. 285-297.

Randall, A.D., and Johnson, A.I., eds., 1988, Regional aquifer systems of the United States-the northeast glacial aquifers: American Water Resources Association Monograph Series No. 11, 156 p.

Raisz, Erwin, 1954, Physiographic diagram, in U.S. Geological Survey, National atlas of the United States, 1970: U.S. Geological Survey, 417 p.

Reilly, T.E., Franke, O.L., and Bennett, G.D., 1984, The principle of superposition and its application in groundwater hydraulics: U.S. Geological Survey Open-File Report 84-459, $36 \mathrm{p}$.

Reynolds, R.J., and Williams, J.H., 1988, Continuous seismic-reflection profiling of glacial drift along the Susquehanna, Chemung, and Chenango Rivers, southcentral New York and north-central Pennsylvania in Randall, A.D., and Johnson, A.I., eds., Regional aquifer systems of the United States - the northeast glacial aquifers: American Water Resources Association Monograph Series no. 11, p. 83-103.

Scott, J.H., Tibbetts, B.L., and Burdick, R.G., 1972, Computer analysis of seismic-refraction data: U.S. Bureau of Mines Report of Investigations RI 7595, 95 p.

Smith, A.P., Kingsley, Louise, and Quinn, Alonzo, 1939, The geology of the Mt. Chocorua quadrangle, New Hampshire: New Hampshire Planning and Development Commission, $24 \mathrm{p}$.
Spear, R.W., 1989, Late-quaternary history of high-elevation vegetation in the White Mountains of New Hampshire: Ecological Society of America, Ecological Monographs 59(2), p. 125-151.

Stekl, P.J., and Flanagan, S.M., 1992, Geohydrology and water quality of stratified-drift aquifers in the lower Merrimack and coastal River basins, southeastern New Hampshire: U.S. Geological Survey Water-Resources Investigations Report 90-4025, 93 p., 6 pls.

Tepper, D.H., Morrissey, D.J., Johnson, C.J., and Maloney, T.J., 1990, Hydrogeology, water quality, and effects of increased municipal pumpage on the Saco River valley glacial aquifer: Bartlett, New Hampshire to Fryeburg, Maine: U.S. Geological Survey Water-Resources Investigations Report 88-4179, 113 p.

Theis, C.V., 1938, The significance and nature of the cone of depression in ground-water bodies: Economic Geology, v. 33 , no. 8 , p. 889-902.

1963, Estimating the transmissibility of a water-table aquifer from the specific capacity of a well, in Bentall, Ray, compiler, Methods of determining permeability, transmissivity and drawdown: U.S. Geological Survey Water-Supply Paper 1536-I, p. 332-336.

Thompson, W.B., 1986, Glacial geology of the White Mountain foothills, southwestern Maine, in Newberg, D.W., ed., New England Intercollegiate Geologic Conference, 78th annual meeting, Bates College, Lewiston, Me., October 1986, Guidebook for field trips in southwestern Maine: Bates College, p. 275-288.

Thomas, M.P., 1966, Effect of glacial geology upon the time distribution of streamflow in eastern and southern Connecticut: U.S. Geological Survey Professional Paper 550-B, p. b209-b212.

Trescott, P.C., Pinder, G.F., and Larson, S.P., 1976, Finitedifference model for aquifer simulation in two dimensions with results of numerical experiments: U.S. Geological Survey Techniques of Water-Resources Investigations, book 7, chap. $\mathrm{C} 1,116 \mathrm{p}$.

Toppin, K.W., 1987, Hydrogeology of stratified-drift aquifers and water quality in the Nashua Regional Planning Commission area south-central New Hampshire: U.S. Geological Survey Water-Resources Investigations Report 86-4358, 101 p., 6 pls.

Toppin, K.W., McKenna, K.E., Cotton, J.E., and Denner, J.C., 1991, Water resources data New Hampshire and Vermont water year 1990: U.S. Geological Survey Water-Data Report NH-VT-90-1, 136 p. 
Upham, Warren, 1897, Modified drift in New Hampshire, in Hitchcock, C.H., ed., Geology of New Hampshire: Concord, N.H., v. 3, part II, 740 p.

U.S. Army Corps of Engineers, 1980, Magnitude and frequency of low streamflows in New Hampshire: U.S. Department of the Army, $70 \mathrm{p}$.

1990, New Hampshire water supply planning study, Bartlett, Conway, Jackson, U.S. Department of the Army, 23 p., 4 pls.

U.S. Bureau of the Census, 1980, 1980 census of population and housing, New Hampshire: U.S. Department of Commerce, PHC 80-p-31, 4 p.

1991, 1990 census of population and housing, summary population and housing characteristics $-\mathrm{New}$ Hampshire 1990: U.S. Department of Commerce, CPH$1-31,130 \mathrm{p}$.

U.S. Environmental Protection Agency, 1991a, Final rule, National primary and secondary drinking water regulations -Synthetic organic chemicals and inorganic chemicals (sections 141.11, 141.12, 141.32, 141.50, $141.51,141.61$, and 141.62 of part 141 and 143.3 of part 143): U.S. Federal Register, v. 56, no. 20, January 30 , 1991, p. 3526-3597.

1991b, Final rule, Maximum contaminant level goals and national primary drinking water regulations for lead and copper (sections 141.11, 141.32, and 141.51 of part 141): U.S. Federal Register, v. 56, no. 110, June 7, 1991, p. $26,460-26,564$.

1992, Final rule, National primary and secondary drinking-water regulations - Synthetic organic chemicals and inorganic chemicals (sections 141.12, 141.32, $141.50,141.51,141.61$, and 141.62 of part 141 and 143.3 of part 143): U.S. Federal Register, v. 57, no. 138, July 17,1992 , p. 31,776-31,849.

U.S. Geological Survey, 1985, National water summary 1984-Hydrologic events, selected water-quality trends, and ground-water resources: U.S. Geological Survey Water-Supply Paper 2275, $467 \mathrm{p}$.

1987, National water summary 1986-Hydrologic events and ground-water quality: U.S. Geological Survey Water-Supply Paper 2325, $560 \mathrm{p}$.

U.S. Soil Conservation Service, 1977, Soil survey of Carroll County, New Hampshire: Washington, D.C., U.S. Department of Agriculture, $161 \mathrm{p}$.
Weigle, J.M., and Kranes, Richard, 1966, Records of selected wells, springs, test holes, materials tests, and chemical analyses of water in the lower Merrimack River Valley in New Hampshire: U.S. Geological Survey Open-File Report, New Hampshire Basic Data Report 2, 44 p.

White, G.W., 1937, A sketch of the geology of the Androscoggin, Saco and coastal watersheds, in Hoover, E.E., Biological survey of the Androscoggin, Saco, and coastal watersheds: New Hampshire Fish and Game Department, p. 81-84.

Wilson, R.T., 1969, The geology of the Ossipee Lake quadrangle New Hampshire: New Hampshire Department of Resources and Economic Development, $166 \mathrm{p}$.

Wiltshire, D.A., Lyford, F.P., and Cohen, A.J., 1986, Bibliography on ground water in glacial-aquifer systems in the northeastern United States: U.S. Geological Survey Circular 972, $26 \mathrm{p}$.

\section{GLOSSARY}

Ablation Till: Loosely consolidated rock debris, formerly carried by glacial ice, that accumulated in places as the surface ice was removed by melting, evaporation, or other processes.

Aquifer: A geologic formation, group of formations, or part of a formation that contains sufficient saturated permeable materials to yield significant quantities of water to wells and springs. Where water only partly fills an aquifer, the upper surface of the saturated zone is free to rise and fall.

Aquifer boundary: A geologic or hydrologic feature that limits the extent of an aquifer.

Bedrock: Solid rock, locally called "ledge," that forms the earth's crust. It may be exposed at the surface but more commonly is buried beneath a few inches to more than $100 \mathrm{ft}$ of unconsolidated deposits.

Cone of depression: A depression produced in a water table or other potentiometric surface by the withdrawal of water from an aquifer; in cross section, shaped like an inverted cone with its apex at the pumped well.

Confined Aquifer: An aquifer saturated with water and bounded above and below by material having a distinctly lower hydraulic conductivity than the aquifer.

Contact: A plane or irregular surface between two different types or ages of rocks or unconsolidated sediments. 
Cubic feet per second $\left(\mathrm{ft}^{3} / \mathrm{s}\right)$ : A unit expressing rate of discharge. One cubic foot per second is equal to the discharge of a stream 1-foot wide and 1-foot deep flowing at an average velocity of 1 foot per second.

Cubic feet per second per square mile $\left[\left(\mathrm{ft}^{3} / \mathrm{s}\right) / \mathrm{mi}^{\mathbf{2}}\right]$ : A unit expressing average number of cubic feet of water flowing per second from each square mile of area drained.

Darcy's Law: An equation relating the factors controlling ground-water flow. Darcy's law is $\mathrm{Q}=\mathrm{KA}(\mathrm{dh} / \mathrm{dl})$, where $Q$ is the quantity of water per unit of time; $K$ is the hydraulic conductivity, which depends on the size and arrangement of the water-transmitting openings (pores and fractures) and on the dynamic characteristics of the fluid (water) such as kinematic viscosity, density, and the strength of the gravitational field; $A$ is the cross-sectional area, at a right angle to the flow direction, through which the flow occurs; and $\mathrm{dh} / \mathrm{dl}$ is the hydraulic gradient.

Deposit: Earth material that has accumulated by natural processes.

Dissolved solids: The residue from a clear sample of water after evaporation and drying for 1 hour at $180^{\circ} \mathrm{C}$; consists primarily of dissolved mineral constituents, but may also contain organic matter and water of crystallization.

Drainage area: The area or tract of land, measured in a horizontal plane, that gathers water and contributes it ultimately to some point on a stream channel, lake, reservoir, or other water body.

Drawdown: The lowering of the water table or potentiometric surface caused by the withdrawal of water from an aquifer by pumping; equal to the difference between the static water level and the pumping water level.

Drumlin: A low, smoothly rounded, elongated oval-shaped hill of glacial till built under the margin of glacial ice and shaped by its flow; its longer axis is parallel to the direction of flow of the ice.

Effective grain size: The grain size at which 10 percent of the sample consists of smaller grains and 90 percent consists of larger grains.

Esker: A long ridge of sand and gravel that was deposited by water flowing in tunnels within or beneath glacial ice.

First quartile: For a set of measurements arranged in order of magnitude, that value where 25 percent of the measurements are lower in magnitude and 75 percent of the measurements are higher in magnitude.
Flow duration (of a stream): The percentage of time during which specified daily discharges are equaled or exceeded within a given time period.

Fluvial: Pertaining to the flow of liquid water in the natural environment.

Fracture: A break, crack, or opening in bedrock along which water may move.

Glacial lake: A body of water that formed from the melting of glacial ice.

Glaciofluvial: Pertaining to the flow of meltwater streams from glacial ice and to the landforms produced by such streams, including kames, kame terraces, and outwash plains.

Glaciolacustrine: Deposits in glacial lakes; especially deposits such as deltas and varved sediments, composed of material deposited by meltwater streams flowing into lakes adjacent to the glacier.

Gneiss: A coarse-grained metamorphic rock with alternating bands of granular and micaceous minerals.

Granite: A coarse-grained, light-colored, igneous rock.

Gravel: Unconsolidated rock debris composed principally of particles larger than 2 millimeters in diameter.

Ground water: Water beneath the water table in soils or geologic formations that are fully saturated and under pressure equal to or greater than atmospheric pressure.

Ground-water discharge: The discharge of water from the saturated zone by (1) natural processes such as groundwater seepage into stream channels and ground-water evapotranspiration and (2) discharge through wells and other manmade structures.

Ground-water divide: A hypothetical line on a water table on each side of which the water table slopes downward in a direction away from the line. In the vertical dimension, a plane across which ground water does not flow.

Ground-water evapotranspiration: Ground water discharged into the atmosphere in the gaseous state either by direct evaporation from the water table or by the transpiration of plants.

Ground-water recharge: Water that is added to the saturated zone of an aquifer.

Ground-Water Site-Inventory (GWSI) data base: A computerized file maintained by the U.S. Geological Survey that contains information about wells and springs collected throughout the United States. 
Head, static: The height of the surface of a water column above a standard datum that can be supported by the static pressure of a given point.

Hydraulic conductivity (K): A measure of the ability of a porous medium to transmit a fluid that can be expressed in unit length per unit time. A material has a hydraulic conductivity of 1 foot per day if it will transmit in 1 day, 1 cubic foot of water at the prevailing kinematic viscosity through a 1-foot square cross section of aquifer, measured at right angles to the direction of flow, under a hydraulic gradient, of 1-foot change in head over 1-foot length of flow path.

Hydraulic gradient: The change in static head per unit of distance in a given direction. If not specified, the direction is generally understood to be that of the maximum rate of decrease in head.

Hydrograph: A graph showing stage (height), flow velocity, or other property of water with respect to time.

Ice-contact deposits: Stratified drift deposited in contact with melting glacial ice. Landforms include eskers, kames, kame terraces, and grounding-line deltas.

Igneous: Descriptive term for rocks or minerals solidified from molten or partially molten material, that is, from a magma, such as basalt or granite.

Induced infiltration: The process by which water infiltrates an aquifer from an adjacent surface-water body in response to ground-water withdrawal from that aquifer.

Kame: A ridge, mound, or hummock that may be irregular and is composed of stratified sand and gravel deposited by glacial meltwater; the precise mode of formation is uncertain.

Kame terrace: A ridge consisting of stratified sand and gravel deposited in a glaciofluvial environment between a melting glacier or stagnant ice lobe and a high valley wall. The deposit has a terrace appearance after the ice has retreated.

Lacustrine: Pertaining to lake environments. As described in this report, it refers to areas associated with glacial-lake environments.

Lodgement till: A firm, compact clay-rich till deposited beneath a moving glacier, containing abraded stones oriented, in general, with their long axes parallel to the direction of ice movement.

Median: The middle value of a set of measurements that are ordered from lowest to highest, 50 percent of the measurements are lower than the median and 50 percent are higher than the median.
Metamorphic: Descriptive term for rocks such as gneiss and schist that have formed, in the solid state, from other rocks due to changes in temperature and pressure.

Micrograms per liter $(\mu \mathrm{g} / \mathrm{L})$ : A unit expressing the concentration of chemical constituents in solution as the mass (micrograms) of a constituent per unit volume (liter) of water. One thousand micrograms per liter is equivalent to one milligram per liter.

Milligrams per liter (mg/L): A unit for expressing the concentration of chemical constituents in solution as the mass (milligrams) of a constituent per unit volume (liter) of water.

Outwash: Stratified deposits chiefly of sand and gravel removed or "washed out" from a glacier by meltwater streams and deposited beyond the margin of a glacier, usually found in flat or gently sloping outwash plains.

Outwash deltas: Deltas formed beyond the margin of the glacier where glacial meltwater entered a water body.

pH: The negative logarithm of the hydrogen ion concentration. A pH of 7.0 indicates neutrality; values below 7.0 denote acidity and those above 7.0 denote alkalinity.

Phi grade scale: A logarithmic transformation of the Wentworth grade scale based on the negative logarithm to the base 2 of the particle diameter, in millimeters.

Porosity: The property of a rock or unconsolidated deposit that is a measure of the size and number of internal voids or open spaces; it may be expressed quantitatively as the ratio of the volume of its open spaces to its total volume.

Rotten Rock (grus): The fragmental products of in-situ granular disintegration of granite and granitic rocks.

Runoff: That part of the precipitation that appears in streams. It is the same as streamflow unaffected by artificial diversions, storage, or other human activities in or on the stream channels.

Saturated thickness (of stratified drift): Thickness of stratified drift extending down from the water table to the till or bedrock surface.

Saturated zone: The subsurface zone in which all open (interconnected) spaces are filled with water. Water below the water table, the upper limit of the saturated zone, is under pressure greater than atmospheric.

Schist: A metamorphic rock with subparallel orientation of the visible micaceous minerals, which dominate its composition. 
Sediment: Fragmental material that originates from weathering of rocks. It can be transported by, suspended in, or deposited by water.

Specific capacity (of a well): The rate of discharge of water divided by the corresponding drawdown of the water level in the well. Stated in this report in gallons per minute per foot.

Specific yield: The ratio of the volume of water that a rock or soil will yield, by gravity drainage, after being saturated to the total volume of the rock or soil.

Standard deviation: A measure of the amount of variability in a sample; it is the square root of the average of the squares of the deviations about the arithmetic mean of a set of data.

Storage coefficient: The volume of water an aquifer releases from or takes into storage per unit surface area of the aquifer per unit change in head. In an unconfined aquifer, the storage coefficient is virtually equal to the specific yield.

Stratified drift: Sorted and layered unconsolidated material deposited in meltwater streams flowing from glaciers or settled from suspension in quiet-water bodies fed by meltwater streams.

Superposition: A principle that states: for linear systems, the solution to individual parts of a problem can be added together to solve composite problems.

Surficial geology: The study of or distribution of unconsolidated deposits at or near the land surface.

Third quartile: For a set of measurements arranged in order of magnitude, that value where 75 percent of the measurements are lower in magnitude and 25 percent of the measurements are higher in magnitude.

Till: A predominantly nonsorted, nonstratified sediment deposited directly by a glacier and composed of boulders, gravel, sand, silt and clay mixed in various proportions.

Transmissivity: The rate at which water is transmitted through a unit width of aquifer under a unit hydraulic gradient. Equal to the average hydraulic conductivity times the saturated thickness.

Unconfined aquifer (water-table aquifer): An aquifer only partly filled with water. In such aquifers, the water or upper surface of the saturated zone is at atmospheric pressure and is free to rise and fall.

Unconsolidated deposit: A sediment in which the particles are not firmly cemented together, such as sand, in contrast to sandstone.
Unsaturated zone: The zone between the water table and the land surface in which the open spaces are not completely filled with water.

Water table: The upper surface of the saturated zone. Water at the water table is at atmospheric pressure. 


\section{APPENDIX 1. Description of selected wells, borings, and springs in the Saco and Ossipee River Basins, east-central New Hampshire}


Table 1-1. Description of selected wells, borings, and springs in the Saco and Ossipee River Basins, east-central New Hampshire.

Local site number: First two characters are U.S. Geological Survey town code. Third-character codes indicates -- A, boring done for hydrologic purposes; $\mathrm{B}$, boring done for constructional purposes; $\mathrm{S}$, spring; $\mathrm{W}$, well. The numbers are sequential numbers for each town.

Latitude, longitude: Accurate within 5 seconds.

Owner or user: NHDOT, New Hampshire Department of Transportation; USGS, U.S. Geological Survey.

Altitude: Altitudes are expressed in feet above sea level: Altitudes given in whole feet are interpolated from U.S. Geological Survey topographic maps and are accurate to plus or minus half the contour interval of the map ( ${ }^{+} /-10$ to 20 feet); altitudes in tenths of feet are determined by instrument.

Depth to bottom of casing: Depth to bottom of well casing in feet below land-surface datum.

Casing material: Material from which the casings are made: $\mathrm{C}$, concrete; $\mathrm{G}$, galvonized iron; $\mathrm{P}$, plastic; $\mathbf{R}$, rock or stone; $\mathrm{S}$, steel; or T, tile.

Type of finish: Method of finish or nature of the openings that allow water to enter the well: G, gravel-pack well with a screen; $\mathrm{O}$, open end (cased to the bottom of the well so water can only enter the well through the bottom of the hole); $\mathrm{P}$, perforated or slotted with holes punched or slots cut into the casing to admit water; S, screen (manufactured); T, sand point for driven wells; $\mathrm{X}$, open hole where the casing does not extend to the bottom of the hole (ususally a bedrock well).

Depth to bottom of open section: Depth to the bottom of the screen or open section in which water enters the well in feet below land surface.

Type of site: BrW, bedrock well; Sp, spring; TH, test hole; Wells or borings in surficial deposits: Bor, bored or augered; Cbl, cable-tool well; Dug, dug well; Dvn, driven well; Wsh, drive and wash well.

Water level: Water level, in feet below land-surface datum; mm-dd-yy is month-day-year.

Use: Use of site: $\mathrm{O}$, observation well drilled for water-level or water-quality observations; $\mathrm{T}$, test hole. Use of water: $\mathrm{C}$, commercial; H, domestic; I, irrigation; P, public; N, industrial; S, stock; T, institutional; U, unused; Z, other.

Maximum well yield: Discharge, in gallons per minute.

Drawdown: in feet, observed at a pumping well.

Specific capacity: In gallons per minute per foot of drawdown ((gal/min)/foot).

Pumping period: The length of time, in hours, that the well was pumped before the measurement of production levels.

Remarks: CA, chemical analysis summarized in appendix 5; USGS, well test hole drilled by the U.S. Geological Survey for this investigation; GS, well inventoried by Glen Stewart (past New Hampshire State Geologist); F, the well was flowing: $\mathrm{R}$, the well had been pumped recently; $\mathrm{pH}$ is the negative logarithm of the hydrogen-ion concentration; Fe, iron; ppm, parts per million; hard, hardness of water. 
Table 1-1. Descrlption of selected wells, borings, and springs in the Saco and Ossipee River Baslns, east-central $[--$, no data $]$

\begin{tabular}{|c|c|c|c|c|c|c|c|c|c|c|c|}
\hline $\begin{array}{l}\text { Local } \\
\text { site } \\
\text { number }\end{array}$ & $\begin{array}{l}\text { Lat- } \\
\text { itude }\end{array}$ & $\begin{array}{l}\text { Long- } \\
\text { itude }\end{array}$ & Owner or user & $\begin{array}{l}\text { Year } \\
\text { com- } \\
\text { pleted }\end{array}$ & $\begin{array}{l}\text { Atli- } \\
\text { tude } \\
\text { above } \\
\text { sea } \\
\text { level } \\
\text { (feet) }\end{array}$ & $\begin{array}{l}\text { Dia- } \\
\text { meter } \\
\text { of } \\
\text { well } \\
\text { casing } \\
\text { (inches) }\end{array}$ & $\begin{array}{l}\text { Depth } \\
\text { to } \\
\text { bottom } \\
\text { of } \\
\text { casing } \\
\text { (feet) }\end{array}$ & $\begin{array}{l}\text { Casing } \\
\text { material }\end{array}$ & $\begin{array}{l}\text { Type } \\
\text { of } \\
\text { finish }\end{array}$ & $\begin{array}{l}\text { Depth } \\
\text { to } \\
\text { bottom } \\
\text { of open } \\
\text { section } \\
\text { (feet) }\end{array}$ & $\begin{array}{l}\text { Type } \\
\text { of } \\
\text { site }\end{array}$ \\
\hline
\end{tabular}

$\begin{array}{llll}A D B & 2 & 435945 & 712039\end{array}$

ADB $\quad 5 \quad 435546 \quad 711316$

$\begin{array}{llll}\mathrm{ADB} & 6 & 435948 & 711937\end{array}$

ADB $7 \quad 435950 \quad 711926$

$\begin{array}{llll}\text { ADB } & 8 & 435929 & 711730\end{array}$

$\begin{array}{llll}\mathrm{ADB} & 9 & 435925 & 711757\end{array}$

ADB $\quad 10 \quad 435955 \quad 711641$

ADB $\quad 11 \quad 440058 \quad 711914$

ADB $\quad 12 \quad 440003 \quad 711408$

ADW $\quad 1 \quad 435943 \quad 71125$

ADW $\quad 2 \quad 435911 \quad 711208$

ADW $\quad 3 \quad 435914 \quad 711200$

ADW $\quad 4 \quad 435908 \quad 711207$

ADW $\quad 5 \quad 435912 \quad 711159$

ADW $\quad 6 \quad 435908 \quad 711200$

ADW $\quad 7 \quad 435607 \quad 711328$

ADW $\quad 8 \quad 435808 \quad 710836$

ADW $\quad 9 \quad 435437 \quad 712131$

ADW $\quad 10 \quad 435528 \quad 711253$

$\begin{array}{llll}\text { ADW } & 11 & 435538 & 711330\end{array}$

ADW $\quad 12 \quad 435812 \quad 710838$

ADW $\quad 13 \quad 435524 \quad 711326$

ADW $\quad 14 \quad 435948 \quad 712203$

ADW $\quad 15 \quad 435948 \quad 712203$

ADW $\quad 15 \quad 435948 \quad 712203$

ADW $\quad 16 \quad 435936 \quad 712104$

ADW $\quad 17 \quad 435754 \quad 711024$

ADW $\quad 18 \quad 435822 \quad 710940$

ADW $\quad 19 \quad 435807 \quad 710854$

$\begin{array}{llll}A D W & 20 & 435802 & 711032\end{array}$

ADW $\quad 21 \quad 435656 \quad 711222$

$\begin{array}{llll}\text { ADW } & 22 & 435620 & 711338\end{array}$

ADW $\quad 23 \quad 435903 \quad 711006$

$\begin{array}{llll}A D W & 24 & 435621 & 711337\end{array}$

ADW $\quad 25 \quad 435831 \quad 711030$

ADW $26 \quad 435806 \quad 710951$

ADW $\quad 27 \quad 440023 \quad 710929$

ADW $\quad 28 \quad 435814 \quad 710954$

ADW $29 \quad 435813 \quad 710952$

ADW $\quad 30 \quad 435837 \quad 711022$

$\begin{array}{llll}A D W & 31 & 435925 & 711021\end{array}$

ADW $\quad 32 \quad 440017 \quad 710934$

ADW $\begin{array}{llll}33 & 435613 & 711251\end{array}$

ADW $\quad 34 \quad 435918 \quad 710949$

ADW

ADW

ADW

ADW
John C1ay

Towle

Chartier

McVicar

Pero

Casarano

Smith

Croteau

Hunt

valladares

Abbott

Hutchinson

Moul ton

Hatch

Cunningham

Pinciaro

walker

carruthers

Howard

wilson

Newton

Dunham

wi 11 iams

Smith

waters

McConarty

Croteau

Andersen

Ma11et

wilson

Albany

\begin{tabular}{|c|c|c|c|c|c|c|}
\hline$\cdots$ & 1.221 .9 & $\cdots$ & $-\cdot$ & $\cdots$ & -- & $\cdots$ \\
\hline-- & 1,430 & $\cdots$ & $\cdots$ & $\cdots$ & $\cdots$ & $\cdots$ \\
\hline- & $1,189.3$ & - & - & $\cdots$ & - & $\cdots$ \\
\hline$\cdots$ & 1,220 & -- & $\cdots$ & $\cdots$ & $\cdots$ & $\cdots$ \\
\hline-- & $1,217.5$ & $\cdots$ & $-\cdot$ & $\cdots$ & $\cdots$ & $\cdots$ \\
\hline-- & $1,247.7$ & -- & $\cdots$ & $\cdots$ & -- & -- \\
\hline-- & $1,138.8$ & $\cdots$ & -- & - & $\cdots$ & $\cdots$ \\
\hline-- & 651.8 & - & - & $\cdots$ & - & $\cdots$ \\
\hline$-\cdot$ & 840 & - & - & $\cdots$ & $\cdots$ & $\cdots$ \\
\hline 1991 & 825 & 6 & 60 & $\mathrm{~s}$ & $\mathrm{x}$ & -- \\
\hline 1988 & 890 & -- & - - & $\cdots$ & $\mathbf{P}$ & $\cdots$ \\
\hline 1988 & 830 & $\cdots$ & - & -- & $P$ & $\cdots$ \\
\hline 1988 & 870 & -- & $\cdots$ & $\cdots$ & P & -- \\
\hline 1988 & 850 & - & -- & $\cdots$ & $\mathrm{P}$ & $-\cdot$ \\
\hline 1988 & 850 & - & - & $\cdots$ & $\mathrm{P}$ & -- \\
\hline 1984 & 670 & -- & 284 & $\cdots$ & $x$ & -- \\
\hline 1985 & 470 & $-\cdot$ & 99 & $\cdots$ & $s$ & -- \\
\hline 1985 & 1,140 & $\cdots$ & 9 & $\cdots$ & $\cdots$ & $-\cdot$ \\
\hline 1987 & 625 & - & 1 & -- & -- & $\cdots$ \\
\hline 1987 & 635 & - & - - & $\cdots$ & $-\cdot$ & $\cdots$ \\
\hline 1989 & 470 & $\cdots$ & 31.5 & -- & $\mathbf{S}$ & - - \\
\hline 1992 & 625 & 2 & 50.4 & P & $\mathrm{s}$ & 52.4 \\
\hline 1992 & 1,250 & 2 & 77.5 & $P$ & $\mathrm{~S}$ & 79.5 \\
\hline 1992 & 1,250 & 2 & 13.5 & P & $\mathrm{s}$ & 15.5 \\
\hline.- & 1,250 & $\cdots$ & -- & $\cdots$ & $\cdots$ & 18 \\
\hline 1992 & 1,250 & 2 & 48 & P & $\mathbf{S}$ & 50 \\
\hline 1985 & 820 & $\cdots$ & 10.5 & $\therefore$ & $\mathrm{x}$ & - \\
\hline 1985 & 800 & $\cdots$ & 262 & $\cdots$ & $\mathrm{x}$ & $\cdots$ \\
\hline 1987 & 540 & .. & 39 & - . & $\mathbf{x}$ & - \\
\hline
\end{tabular}

1987

1987

1987

1988

1988

1988

1988

1988

1988

1988

1988

1988

1989

1989

1989

1989

1990

1989

1990

1990
$830 \quad \ldots \quad 30$

$840 \quad \ldots \quad 28$

$\begin{array}{lll}750 & \ldots & 239\end{array}$

$800 \quad \ldots \quad 120$

$750 \quad-. \quad 241$

$$
960
$$

810

610

760

770

1,080

630

580

880

590

600
820

1,010

640

700
- $\quad 129$

.. 127

.. 40

-. 21

- $\quad 19$

-. 44

. 31

.. 40

- 279

-. 231

.. 40

. 232

-
- 80

$\begin{array}{rr}- & 211 \\ \ldots & 29\end{array}$ 


\begin{tabular}{|c|c|c|c|c|c|c|c|c|}
\hline $\begin{array}{l}\text { Local } \\
\text { site } \\
\text { number }\end{array}$ & $\begin{array}{l}\text { Water } \\
\text { level } \\
\text { depth } \\
\text { (feet) }\end{array}$ & $\begin{array}{l}\text { Date } \\
\mathrm{mm} / \mathrm{dd} / \mathrm{yy}\end{array}$ & Use & $\begin{array}{l}\text { Maximum } \\
\text { well } \\
\text { yield } \\
\text { (gallons } \\
\text { per } \\
\text { minute) }\end{array}$ & $\begin{array}{l}\text { Draw- } \\
\text { down } \\
\text { (feet) }\end{array}$ & $\begin{array}{c}\text { Specific } \\
\text { capacity } \\
\text { (gallons per } \\
\text { minute } \\
\text { per } \\
\text { foot) }\end{array}$ & $\begin{array}{l}\text { Pumping } \\
\text { period } \\
\text { (hours) }\end{array}$ & Remarks \\
\hline & & & & & & CARROLI & OUNTY & \\
\hline
\end{tabular}

USGS Albany Landfil1

USGS Albany Landfill

USGS Albany Landfill

USGS Albany Landfill

USGS Albany Landfill
USGS

USGS

USGS

UsGS 
Table 1-1. Description of selected wells, borings, and springs in the Saco and Ossipee River Basins, east-central

\begin{tabular}{|c|c|c|c|c|c|c|c|c|c|c|c|}
\hline $\begin{array}{l}\text { Local } \\
\text { site } \\
\text { number }\end{array}$ & $\begin{array}{l}\text { Lat- } \\
\text { itude }\end{array}$ & $\begin{array}{l}\text { Long- } \\
\text { itude }\end{array}$ & Owner or user & $\begin{array}{l}\text { Year } \\
\text { com- } \\
\text { pleted }\end{array}$ & $\begin{array}{l}\text { Alti- } \\
\text { tude } \\
\text { above } \\
\text { sea } \\
\text { level } \\
\text { (feet) }\end{array}$ & $\begin{array}{l}\text { Dia- } \\
\text { meter } \\
\text { of } \\
\text { well } \\
\text { casing } \\
\text { (inches) }\end{array}$ & $\begin{array}{l}\text { Depth } \\
\text { to } \\
\text { bottom } \\
\text { of } \\
\text { casing } \\
\text { (feet) }\end{array}$ & $\begin{array}{l}\text { Casing } \\
\text { material }\end{array}$ & $\begin{array}{l}\text { Type } \\
\text { of } \\
\text { finish }\end{array}$ & $\begin{array}{l}\text { Depth } \\
\text { to } \\
\text { bottom } \\
\text { of open } \\
\text { section } \\
\text { (feet) }\end{array}$ & $\begin{array}{c}\text { Type } \\
\text { of } \\
\text { site }\end{array}$ \\
\hline
\end{tabular}

\begin{tabular}{|c|c|c|c|c|c|c|c|c|c|c|c|c|}
\hline ADW & 40 & 435739 & 711125 & s. George & 1991 & 700 & $\cdots$ & 40 & $-\cdot$ & $\mathrm{x}$ & $\cdots$ & Brw \\
\hline ADW & 41 & 435629 & 711235 & Saxby & 1991 & 830 & $\cdots$ & 271 & - & $\mathrm{x}$ & $\cdots$ & Brw \\
\hline ADW & 42 & 440003 & 710954 & Smith & 1991 & 640 & $\cdots$ & 39 & - - & $x$ & $\cdots$ & BrW \\
\hline ADW & 43 & 435943 & 711253 & C1ay & 1991 & 820 & $\cdots$ & 60 & $\cdots$ & $\mathrm{x}$ & $\cdots$ & BrW \\
\hline ADW & 44 & 435746 & 711021 & Ferris & 1991 & 730 & $\cdots$ & 59 & - - & $x$ & $\cdots$ & BrW \\
\hline ADW & 45 & 435948 & 710945 & Diceo & 1991 & 600 & $\cdots$ & 39 & $\cdots$ & $\mathrm{x}$ & $\cdots$ & BrW \\
\hline ADW & 46 & 435919 & 711004 & Schreiber & 1991 & 650 & $\cdots$ & 189 & $\cdots$ & $\mathrm{x}$ & $\cdots$ & BrW \\
\hline ADW & 47 & 435805 & 710845 & Harrow & 1991 & 470 & $\cdots$ & 80 & $\cdots$ & $x$ & $\cdots$ & BrW \\
\hline ADW & 48 & 435724 & 711239 & Wiggin & 1992 & 730 & $\cdots$ & 92 & - & $\mathrm{x}$ & $\cdots$ & BrW \\
\hline$A D W$ & 49 & 435950 & 711023 & Hardiss & 1991 & 620 & $\cdots$ & 139 & $\cdots$ & $\mathrm{x}$ & $-\cdot$ & Brw \\
\hline $\mathrm{ADW}$ & 50 & 435947 & 711020 & Howl and & 1991 & 620 & $\cdots$ & 127 & $\cdots$ & $\mathrm{x}$ & $\cdots$ & BrW \\
\hline$A D W$ & 51 & 435936 & 710928 & Darcy & 1991 & 550 & $\cdots$ & 37 & - & $\mathrm{x}$ & $\cdots$ & BrW \\
\hline ADW & 52 & 435932 & 710924 & Hanson & 1991 & 530 & $\cdots$ & 19 & - & $\mathrm{x}$ & $\cdots$ & BrW \\
\hline$A D W$ & 53 & 435938 & 710934 & Avignone & 1991 & 560 & $\cdots$ & 119 & $\cdots$ & $x$ & $\cdots$ & BrW \\
\hline ADW & 54 & 435938 & 710936 & Sermon & 1991 & 560 & $\cdots$ & 49 & $\cdots$ & $x$ & $\cdots$ & BrW \\
\hline ADW & 55 & 435942 & 710939 & Barber & 1991 & 560 & $\cdots$ & 40 & $-\cdot$ & $\mathrm{x}$ & $\cdots$ & Brw \\
\hline$A D W$ & 56 & 435948 & 711008 & Loring & 1991 & 600 & $\cdots$ & 113 & $\cdots$ & $\mathrm{x}$ & $\cdots$ & Brw \\
\hline ADW & 57 & 435948 & 711003 & Doherty & 1991 & 600 & $-\cdot$ & 103 & -. & $x$ & $\cdots$ & BrW \\
\hline$A D W$ & 58 & 435949 & 711006 & Robbins & 1991 & 600 & $\cdots$ & 103 & $\cdots$ & $\mathrm{x}$ & $\cdots$ & BrW \\
\hline ADW & 59 & 435942 & 710942 & Chesley & 1991 & 580 & $\cdots$ & 19 & $\cdots$ & $\mathrm{x}$ & $\cdots$ & BrW \\
\hline ADW & 60 & 435946 & 710954 & & $-\cdot$ & 600 & - & 35 & $\cdots$ & $\mathrm{x}$ & $\cdots$ & Brw \\
\hline$A D W$ & 61 & 435949 & 711000 & Santolucito & 1991 & 600 & $\cdots$ & 75 & $\cdots$ & $\mathrm{x}$ & $-\cdot$ & BrW \\
\hline$A D W$ & 62 & 435948 & 711002 & Berry & 1991 & 600 & $\cdots$ & 93 & $-\cdot$ & $\mathrm{x}$ & - & Brw \\
\hline$A D W$ & 63 & 435945 & 710949 & Head & 1991 & 600 & $\cdots$ & 34 & $\cdots$ & $\mathrm{x}$ & - & BrW \\
\hline $\mathrm{ADW}$ & 64 & 435944 & 710948 & Gorman & 1991 & 600 & $\cdots$ & 19 & $\cdots$ & $\mathrm{x}$ & $\cdots$ & Brw \\
\hline$A D W$ & 65 & 435937 & 710931 & Darcy & 1991 & 550 & $\cdots$ & 59 & - & $\mathrm{x}$ & - & BrW \\
\hline $\mathrm{ADW}$ & 66 & 435925 & 711019 & Davidson & 1992 & 630 & $\cdots$ & 39 & $\cdots$ & $\mathrm{x}$ & $\cdots$ & Brw \\
\hline$A D W$ & 67 & 435941 & 711252 & Robert and Mary Diamond & 1969 & 805 & 6 & 12 & $\mathrm{P}$ & 0 & - & Dug \\
\hline ADW & 68 & 435940 & 711253 & & $-\cdot$ & 800 & 24 & 2.1 & c & 0 & $\cdots$ & Dug \\
\hline$A D W$ & 69 & 435437 & 712119 & Andrew Thompson & 1971 & 1,115 & 36 & 6 & $\mathrm{c}$ & 0 & $\cdots$ & Dug \\
\hline$A D W$ & 70 & 435438 & 712133 & Doug Mcvicar & $-\cdot$ & 1,130 & 36 & 9.6 & C & 0 & $\cdots$ & Dug \\
\hline & \multicolumn{12}{|c|}{ Bartlett } \\
\hline $\mathrm{BCB}$ & 2 & 440525 & 711219 & & $\cdots$ & 632.5 & $\cdots$ & $\cdots$ & $\cdots$ & $\cdots$ & $\cdots$ & $\mathrm{TH}$ \\
\hline $\mathrm{BCB}$ & 4 & 440216 & 711913 & & - & 960.6 & $\cdots$ & $\cdots$ & $\cdots$ & $\cdots$ & $\cdots$ & $\mathrm{TH}$ \\
\hline $\mathrm{BCB}$ & 5 & 440239 & 711637 & & $\cdots$ & 520.3 & $\cdots$ & $\cdots$ & $\cdots$ & -. & $\cdots$ & $\mathrm{TH}$ \\
\hline $\mathrm{BCB}$ & 6 & 440718 & 710751 & & $\cdots$ & 771.5 & $\cdots$ & $\cdots$ & $\cdots$ & $\cdots$ & $\cdots$ & $\mathrm{TH}$ \\
\hline $\mathrm{BCB}$ & 7 & 440501 & 711708 & & $\cdots$ & 665.2 & $\cdots$ & $\cdots$ & $\cdots$ & $\cdots$ & $\cdots$ & $\mathrm{TH}$ \\
\hline $\mathrm{BCW}$ & 7 & 440641 & 711043 & & $\cdots$ & 570 & $\cdots$ & $\cdots$ & $\cdots$ & $-\cdot$ & $\cdots$ & $\cdots$ \\
\hline $\mathrm{BCW}$ & 8 & 440607 & 711053 & & $\cdots$ & 620 & $\cdots$ & $-\cdot$ & $\cdots$ & $\cdots$ & $\cdots$ & BrW \\
\hline $\mathrm{BCW}$ & 9 & 440506 & 710956 & & $\cdots$ & 540 & $\cdots$ & $\cdots$ & $\cdots$ & $\cdots$ & $\cdots$ & BrW \\
\hline $\mathrm{BCW}$ & 10 & 440506 & 710848 & & $\cdots$ & 490 & $\cdots$ & $\cdots$ & $\cdots$ & $\cdots$ & $\cdots$ & $\cdots$ \\
\hline $\mathrm{BCW}$ & 11 & 440457 & 710950 & & $\cdot-$ & 560 & $\cdots$ & $\cdot-$ & $\cdots$ & $\cdots$ & $\cdots$ & Brw \\
\hline $\mathrm{BCW}$ & 12 & 440438 & 710951 & & $\cdots$ & 545 & $\cdots$ & $\cdots$ & $\cdots$ & $\cdots$ & $\cdots$ & $\cdots$ \\
\hline $\mathrm{BCW}$ & 13 & 440550 & 710934 & & $\cdots$ & 516 & $\cdots$ & $\cdots$ & $\cdots$ & $\cdots$ & $\cdots$ & $\cdots$ \\
\hline$B C W$ & 14 & 440549 & 710932 & & $\cdots$ & 100 & $\cdots$ & $\cdots$ & $\cdots$ & $\cdots$ & $\cdots$ & $\cdots$ \\
\hline $\mathrm{BCW}$ & 15 & 440549 & 710932 & & $\cdots$ & 100 & $\cdots$ & $\cdots$ & $\cdots$ & $\cdots$ & $\cdots$ & $\cdots$ \\
\hline $\mathrm{BCW}$ & 16 & 440439 & 710832 & & $\cdots$ & 492 & $\cdots$ & $\cdots$ & $\cdots$ & $\cdots$ & $\cdots$ & $\cdots$ \\
\hline BCW & 17 & 440511 & 710903 & & $\cdots$ & 100 & $\cdots$ & $\cdots$ & $\cdots$ & $\cdots$ & $\cdots$ & $\cdots$ \\
\hline $\mathrm{BCW}$ & 18 & 440502 & 710829 & & $\cdots$ & 502 & $\cdots$ & $\cdots$ & $\cdots$ & $\cdots$ & $\cdots$ & $\cdots$ \\
\hline $\mathrm{BCW}$ & 19 & 440712 & 711303 & Hamblet & 1984 & 1,040 & $\cdots$ & 40 & $\cdots$ & $\mathbf{x}$ & $\cdots$ & Brw \\
\hline $\mathrm{BCW}$ & 20 & 440447 & 711408 & Attitash Lift Corp & 1984 & 740 & $\cdots$ & 40 & $\cdots$ & $\mathbf{x}$ & $\cdots$ & BrW \\
\hline $\mathrm{BCW}$ & 21 & 440744 & 711207 & Tarr & 1984 & 1,080 & $\cdots$ & 19 & $\cdots$ & $\mathbf{x}$ & $\cdots$ & BrW \\
\hline
\end{tabular}




\begin{tabular}{|c|c|c|c|c|c|c|c|c|}
\hline $\begin{array}{l}\text { Local } \\
\text { site } \\
\text { number }\end{array}$ & $\begin{array}{l}\text { Water } \\
\text { level } \\
\text { depth } \\
\text { (feet) }\end{array}$ & $\begin{array}{l}\text { Date } \\
\mathrm{mm} / \mathrm{dd} / \mathrm{yy}\end{array}$ & Use & $\begin{array}{l}\text { Maximum } \\
\text { well } \\
\text { yield } \\
\text { (gallons } \\
\text { per } \\
\text { minute) }\end{array}$ & $\begin{array}{l}\text { Draw- } \\
\text { down } \\
\text { (feet) }\end{array}$ & $\begin{array}{c}\text { Specific } \\
\text { capacity } \\
\text { (gallons per } \\
\text { minute } \\
\text { per } \\
\text { foot) }\end{array}$ & $\begin{array}{l}\text { Pumping } \\
\text { period } \\
\text { (hours) }\end{array}$ & Remarks \\
\hline
\end{tabular}

Albany - - Continuned

\begin{tabular}{|c|c|c|c|c|c|c|c|c|}
\hline$A D W$ & 40 & $\cdots$ & $\cdots$ & $\mathbf{H}$ & 5 & $\cdots$ & $\cdots$ & 1 \\
\hline ADW & 41 & - & $\cdots$ & $\mathbf{H}$ & 18 & $\cdots$ & $\cdots$ & 1 \\
\hline ADW & 42 & 95 & $08-28-91$ & $\mathbf{H}$ & 1 & $\cdots$ & $\cdots$ & 1 \\
\hline ADW & 43 & 20 & $04-14-91$ & $\mathbf{H}$ & 2 & $\cdots$ & $\cdots$ & .5 \\
\hline ADW & 44 & 30 & $06-20-91$ & $\mathbf{H}$ & 0.5 & $\cdots$ & $\cdots$ & 1 \\
\hline ADW & 45 & 30 & $06-20-91$ & H & 2 & $\cdots$ & $\cdots$ & 1 \\
\hline ADW & 46 & 65 & $11-16-91$ & $\mathbf{H}$ & 2.5 & $\cdots$ & $\cdots$ & 24 \\
\hline ADW & 47 & 30 & $09-30-91$ & $\mathbf{H}$ & 0.5 & $\cdots$ & $\cdots$ & 1 \\
\hline ADW & 48 & - & $\cdots$ & H & 9 & $-\cdot$ & -- & 1 \\
\hline ADW & 49 & $\cdots$ & $\cdots$ & $\mathbf{H}$ & 6 & $\cdots$ & $\cdots$ & 2 \\
\hline ADW & 50 & $\cdots$ & $\cdots$ & H & 2.5 & $\cdots$ & $\cdots$ & 2 \\
\hline ADW & 51 & $\cdots$ & $\cdots$ & H & 9 & $\cdots$ & $\cdots$ & 2 \\
\hline ADW & 52 & $\cdots$ & $\cdots$ & $\mathbf{H}$ & 20 & $\cdots$ & $\cdots$ & .5 \\
\hline ADW & 53 & $\cdots$ & $\cdots$ & $\mathbf{H}$ & 3 & $\cdots$ & $\cdots$ & 2 \\
\hline ADW & 54 & $\cdots$ & $\cdots$ & $\mathbf{H}$ & 2 & $\cdots$ & $\cdots$ & 2 \\
\hline ADW & 55 & $\cdots$ & $\cdots$ & $\mathbf{H}$ & 8 & $-\cdot$ & $\cdots$ & $\cdots$ \\
\hline ADW & 56 & $\cdots$ & $\cdots$ & $\mathbf{K}$ & 4 & $\cdots$ & $\cdots$ & 2 \\
\hline$A D W$ & 57 & $\cdots$ & $\cdots$ & $\mathbf{H}$ & 1 & - & $\cdots$ & 2 \\
\hline ADW & 58 & $\cdots$ & $\cdots$ & $\mathbf{H}$ & 5 & $\cdots$ & $\cdots$ & 2 \\
\hline$A D W$ & 59 & $\cdots$ & $\cdots$ & H & 3 & $\cdots$ & $\cdots$ & 2 \\
\hline ADW & 60 & $\cdots$ & - & H & $\cdots$ & $\cdots$ & $\cdots$ & $\cdots$ \\
\hline ADW & 61 & $\cdots$ & $\cdots$ & $\mathrm{H}$ & 2 & $\cdots$ & $\cdots$ & 2 \\
\hline ADW & 62 & $\cdots$ & $\cdots$ & H & 1.5 & $\cdots$ & $\cdots$ & 2 \\
\hline ADW & 63 & $\cdots$ & $\cdots$ & H & 10 & $\cdots$ & $\cdots$ & 2 \\
\hline ADW & 64 & $\cdots$ & $\cdots$ & $\mathbf{H}$ & 3 & $\cdots$ & $\cdots$ & 2 \\
\hline ADW & 65 & $\cdots$ & $\cdots$ & H & 0.8 & $\cdots$ & $\cdots$ & 2 \\
\hline ADW & 66 & $\cdots$ & $\cdots$ & $\mathbf{H}$ & 100 & $\cdots$ & $\cdots$ & 1 \\
\hline ADW & 67 & 10 & $08-08-91$ & $\mathbf{H}$ & 13.6 & $\cdots$ & $\cdots$ & $\cdots$ \\
\hline ADW & 68 & 1.4 & $08-08-91$ & $\mathbf{v}$ & $\cdots$ & $\cdots$ & $\cdots$ & $\cdots$ \\
\hline ADW & 69 & 2.8 & $08-09-91$ & $\mathbf{H}$ & $\cdots$ & $\cdots$ & $\cdots$ & $\cdots$ \\
\hline ADW & 70 & 6.8 & $08-09 \cdot 91$ & $\mathbf{H}$ & $\cdots$ & $\cdots$ & $\cdots$ & $\cdots$ \\
\hline & & & & & & & $\max$ & \\
\hline $\mathrm{BCB}$ & 2 & $\cdots$ & $\cdots$ & $\mathbf{v}$ & $\cdots$ & $\cdots$ & $\cdots$ & $\cdots$ \\
\hline $\mathrm{BCB}$ & 4 & 6.8 & $12 \ldots-63$ & $\mathbf{u}$ & $\cdots$ & $\cdots$ & $\cdots$ & $\cdots$ \\
\hline $\mathrm{BCB}$ & 5 & $\cdots$ & $\cdots$ & $u$ & $\cdots$ & - & $\cdots$ & $\cdots$ \\
\hline $\mathrm{BCB}$ & 6 & $\cdots$ & $\cdots$ & $\mathbf{v}$ & $\cdots$ & $\cdots$ & $\cdots$ & $\cdots$ \\
\hline $\mathrm{BCB}$ & 7 & $\cdots$ & $\cdots$ & $\mathbf{U}$ & $\cdots$ & $\cdots$ & $\cdots$ & $\cdots$ \\
\hline $\mathrm{BCW}$ & 7 & 25 & $11-10 \cdot 66$ & $\cdots$ & $\cdots$ & $\cdots$ & $\cdots$ & $\cdots$ \\
\hline $\mathrm{BCW}$ & 8 & 100 & $08-16-81$ & $\cdots$ & $\cdots$ & $\cdots$ & $\cdots$ & $\cdots$ \\
\hline $\mathrm{BCW}$ & 9 & 18 & $07-21-70$ & $\cdots$ & $\cdots$ & $\cdots$ & $\cdots$ & $\cdots$ \\
\hline BCw & 10 & 10 & $07-21-81$ & $\cdots$ & $\cdots$ & - & $\cdots$ & $\cdots$ \\
\hline $\mathrm{BCw}$ & 11 & $\cdots$ & $\cdots$ & $\cdots$ & $\cdots$ & $\cdots$ & $\cdots$ & $\cdots$ \\
\hline Bcw & 12 & $\cdots$ & $\cdots$ & $\cdots$ & - & $\cdots$ & $\cdots$ & $\cdots$ \\
\hline BCw & 13 & $\cdots$ & $\cdots$ & 0 & $\cdots$ & $\cdots$ & $\cdots$ & $\cdots$ \\
\hline BCw & 14 & $\cdots$ & - & 0 & $\cdots$ & $\cdots$ & $\cdots$ & $\cdots$ \\
\hline BCw & 15 & $\cdots$ & $\cdots$ & 0 & $\cdots$ & - & $\cdots$ & $\cdots$ \\
\hline $\mathrm{BCW}$ & 16 & $\cdots$ & $\cdots$ & 0 & $\cdots$ & $\cdots$ & - & $-\cdot$ \\
\hline BCw & 17 & $\cdots$ & $\cdots$ & $\mathbf{P}$ & $\cdots$ & - & $\cdots$ & $\cdots$ \\
\hline BCw & 18 & $\cdots$ & $\cdots$ & 0 & $\cdots$ & $\cdots$ & $\cdots$ & $\cdots$ \\
\hline BCw & 19 & 40 & $05-10-84$ & $\mathbf{H}$ & .5 & $-\cdot$ & $\cdots$ & 1 \\
\hline Bcw & 20 & $\cdots$ & $\cdots$ & $\mathbf{P}$ & 30 & $\cdots$ & $\cdots$ & .5 \\
\hline BCw & 21 & $\cdots$ & $\cdots$ & $\mathbf{H}$ & 1 & $\cdots$ & $\cdots$ & .5 \\
\hline
\end{tabular}

Project well (Johnson and others, 1987) Project well (Johnson and others, 1987) Profect well (Johnson and others, 1987) Project well (Johnson and others, 1987) Project well (Johnson and others, 1987) 
Table 1-1. Description of selected wells, borings, and springs in the Saco and Ossipee River Basins, east-central

\begin{tabular}{|c|c|c|c|c|c|c|c|c|c|c|c|}
\hline $\begin{array}{l}\text { Local } \\
\text { site } \\
\text { number }\end{array}$ & $\begin{array}{l}\text { Lat- } \\
\text { itude }\end{array}$ & $\begin{array}{l}\text { Long- } \\
\text { itude }\end{array}$ & Owner or user & $\begin{array}{l}\text { Year } \\
\text { com- } \\
\text { pleted }\end{array}$ & $\begin{array}{c}\text { Alti- } \\
\text { tude } \\
\text { above } \\
\text { sea } \\
\text { level } \\
\text { (feet) }\end{array}$ & $\begin{array}{l}\text { Dia- } \\
\text { meter } \\
\text { of } \\
\text { well } \\
\text { casing } \\
\text { (inches) }\end{array}$ & $\begin{array}{l}\text { Depth } \\
\text { to } \\
\text { bottom } \\
\text { of } \\
\text { casing } \\
\text { (feet) }\end{array}$ & $\begin{array}{l}\text { Casing } \\
\text { material }\end{array}$ & $\begin{array}{l}\text { Type } \\
\text { of } \\
\text { finish }\end{array}$ & $\begin{array}{l}\text { Depth } \\
\text { to } \\
\text { bottom } \\
\text { of open } \\
\text { section } \\
\text { (feet) }\end{array}$ & $\begin{array}{c}\text { Type } \\
\text { of } \\
\text { site }\end{array}$ \\
\hline
\end{tabular}

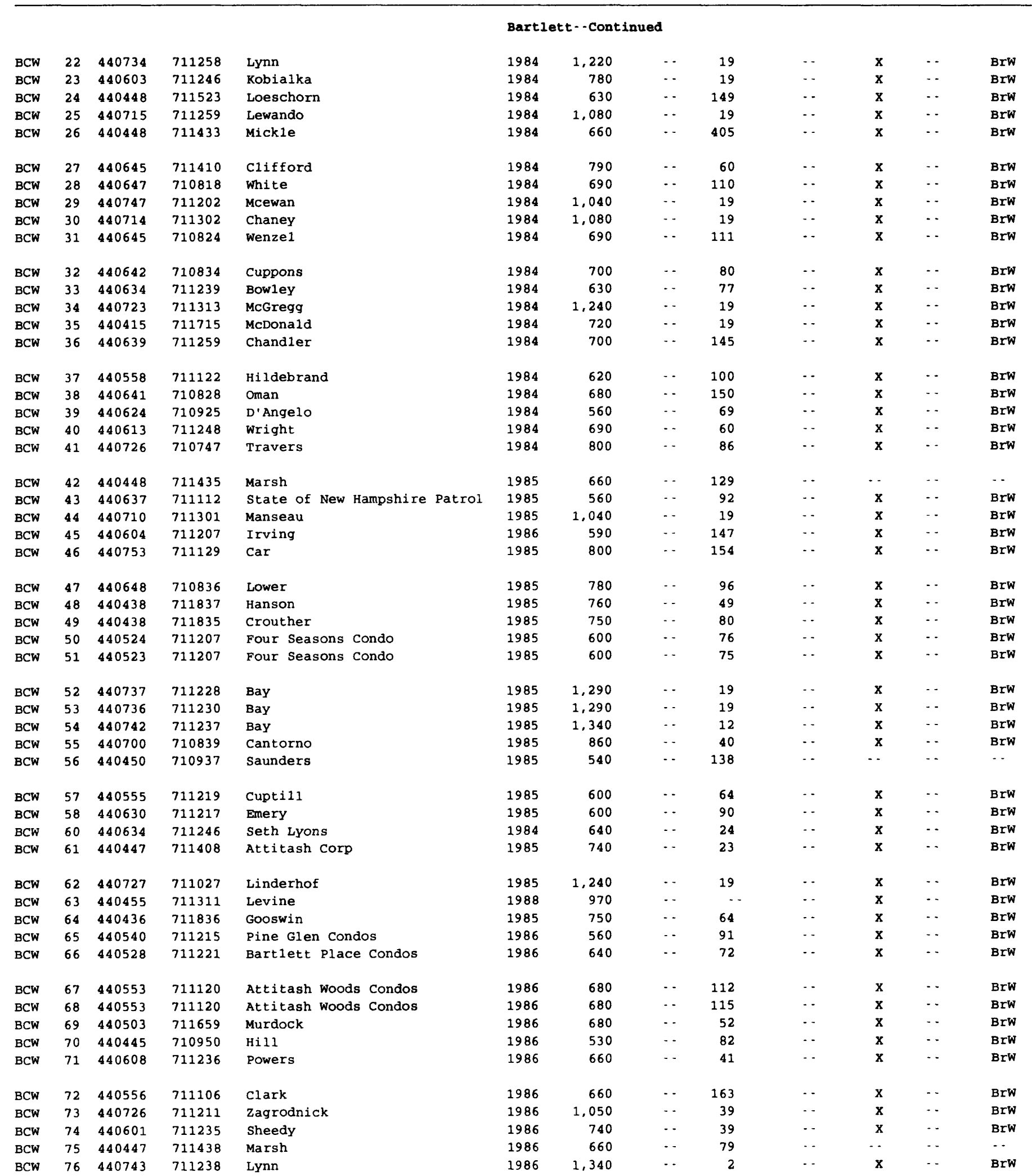




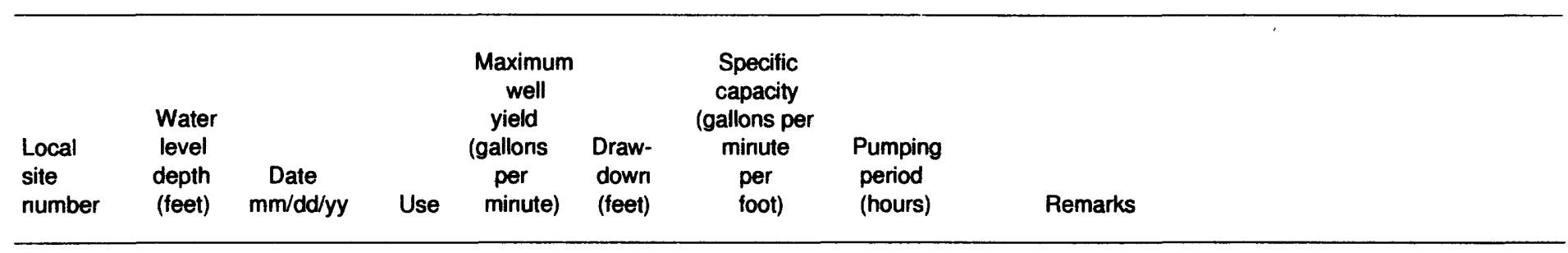

Bartlett--Continued

\begin{tabular}{|c|c|c|c|c|c|c|c|c|}
\hline $\mathrm{BCW}$ & 22 & 10 & $05-29-84$ & H & 8 & $\cdots$ & $\cdots$ & .8 \\
\hline $\mathrm{BCW}$ & 23 & $\cdots$ & - & H & 1.5 & $\cdots$ & $\cdots$ & 1 \\
\hline BCW & 24 & $\cdots$ & $\cdots$ & H & 1.5 & $\cdots$ & $\cdots$ & 1 \\
\hline $\mathrm{BCW}$ & 25 & $\cdots$ & - & H & 1 & -- & - & .8 \\
\hline BCW & 26 & 45 & $06 \cdot 16 \cdot 84$ & H & 2 & $\cdots$ & $\cdots$ & .5 \\
\hline $\mathrm{BCW}$ & 27 & 15 & $07-27-84$ & H & 1 & -. & -. & .3 \\
\hline $\mathrm{BCW}$ & 28 & $\cdots$ & - & H & 10 & $\cdots$ & $\cdots$ & $\cdots$ \\
\hline $\mathrm{BCW}$ & 29 & $\cdots$ & $\cdots$ & H & 1.5 & $\cdots$ & - & 1 \\
\hline $\mathrm{BCW}$ & 30 & $\cdots$ & $\cdots$ & H & 4.5 & $\cdots$ & -- & 1 \\
\hline $\mathrm{BCW}$ & 31 & 20 & $10-05-84$ & H & 15 & $\cdots$ & $\cdots$ & .5 \\
\hline $\mathrm{BCW}$ & 32 & $\cdots$ & $\cdots$ & H & 90 & -. & $\cdots$ & .2 \\
\hline $\mathrm{BCW}$ & 33 & 15 & $11-03-84$ & H & 3 & $\cdots$ & -. & 2 \\
\hline $\mathrm{BCW}$ & 34 & - & - & H & 2 & $\cdots$ & - & 1 \\
\hline BCW & 35 & 10 & $11-27-84$ & H & 20 & $\cdots$ & $\cdots$ & 1 \\
\hline $\mathrm{BCW}$ & 36 & - & - & H & 20 & $\cdots$ & - & 1 \\
\hline BCW & 37 & $\cdots$ & - & H & 150 & $\cdots$ & -. & 1 \\
\hline $\mathrm{BCW}$ & 38 & 2 & $11-15-84$ & H & 30 & $\cdots$ & $\cdots$ & .5 \\
\hline $\mathrm{BCW}$ & 39 & 15 & $11-21-84$ & H & 4 & -. & -. & .5 \\
\hline $\mathrm{BCW}$ & 40 & 40 & $12-18-84$ & H & 5 & $\cdots$ & - & .5 \\
\hline BCW & 41 & $\cdots$ & - & $\mathrm{H}$ & 9 & $\cdots$ & $\cdots$ & .5 \\
\hline BCW & 42 & $\cdots$ & - & H & 10 & $\cdots$ & $-\cdot$ & .5 \\
\hline BCW & 43 & 30 & $04-26-85$ & H & 10 & $\cdots$ & -. & 1 \\
\hline BCW & 44 & 20 & $05-02-85$ & $\mathrm{H}$ & 5 & $\cdots$ & $\cdots$ & 1 \\
\hline BCW & 45 & 30 & $04-03-86$ & H & 20 & $\cdots$ & $\cdots$ & .5 \\
\hline BCW & 46 & 50 & $04-09-85$ & P & 120 & $\cdots$ & $\cdots$ & 1 \\
\hline BCW & 47 & 80 & $05-15-85$ & H & 20 & $\cdots$ & $\cdots$ & .5 \\
\hline BCW & 48 & 20 & $06-04-85$ & H & 2.5 & $\cdots$ & $\cdots$ & 1 \\
\hline BCW & 49 & 35 & $06-04-85$ & H & 40 & $\cdots$ & $-\cdot$ & .5 \\
\hline BCW & 50 & 25 & $06-18-85$ & $\mathbf{P}$ & 200 & $\cdots$ & $\cdots$ & 1 \\
\hline $\mathrm{BCW}$ & 51 & 25 & $06-18-85$ & $\mathbf{P}$ & 200 & $\cdots$ & $\cdots$ & 1 \\
\hline $\mathrm{BCW}$ & 52 & -. & $\cdots$ & H & 0.5 & $\cdots$ & $\cdots$ & .5 \\
\hline $\mathrm{BCW}$ & 53 & $\cdots$ & $\cdots$ & H & 4 & $\cdots$ & $-\cdot$ & .5 \\
\hline BCW & 54 & - & $\cdots$ & H & 1.5 & -. & -. & .5 \\
\hline $\mathrm{BCW}$ & 55 & $\cdots$ & $-\cdot$ & $\mathbf{P}$ & 50 & $\cdots$ & $\cdots$ & .5 \\
\hline $\mathrm{BCW}$ & 56 & $\cdots$ & $\cdots$ & H & 15 & $\cdots$ & $\cdots$ & 12 \\
\hline $\mathrm{BCW}$ & 57 & $\cdots$ & - & H & 6.5 & $\cdots$ & $\cdots$ & 1 \\
\hline BCW & 58 & 10 & $06-24-85$ & H & 7 & $\cdots$ & .. & 1 \\
\hline $\mathrm{BCW}$ & 60 & - & - & H & 6 & $\cdots$ & $\cdots$ & .5 \\
\hline BCW & 61 & $\cdots$ & $\cdots$ & $\mathbf{P}$ & 50 & $\cdots$ & $\cdots$ & 1 \\
\hline BCW & 62 & $\cdots$ & $\cdots$ & $\mathrm{z}$ & 75 & $\cdots$ & $\cdots$ & $\cdots$ \\
\hline BCW & 63 & 100 & $01-23-88$ & H & 12 & $\cdots$ & $\cdots$ & 1 \\
\hline BCW & 64 & 45 & $04 \cdot 16 \cdot 85$ & H & 7 & $\cdots$ & . & .5 \\
\hline BCW & 65 & $\cdots$ & $\cdots$ & $\mathbf{P}$ & 75 & $\cdots$ & $\cdots$ & 1 \\
\hline $\mathrm{BCW}$ & 66 & $\cdots$ & $\cdots$ & P & 100 & $\cdots$ & $\cdots$ & 1 \\
\hline $\mathrm{BCW}$ & 67 & 5 & $05-19-86$ & P & 45 & $\cdots$ & $-\cdot$ & 1 \\
\hline $\mathrm{BCW}$ & 68 & $\cdots$ & - & P & 20 & $\cdots$ & $\cdots$ & .5 \\
\hline $\mathrm{BCW}$ & 69 & $\cdots$ & $\cdots$ & H & 2.5 & $\cdots$ & $\cdots$ & 1 \\
\hline $\mathrm{BCW}$ & 70 & $\cdots$ & $\cdots$ & H & 3.5 & $\cdots$ & $\cdots$ & 1 \\
\hline BCW & 71 & $\cdots$ & $\cdots$ & H & 5 & $\cdots$ & $\cdots$ & 1 \\
\hline $\mathrm{BCW}$ & 72 & 15 & $07-31-86$ & H & 5.5 & $\cdots$ & $\cdots$ & .5 \\
\hline $\mathrm{BCW}$ & 73 & 30 & $07-22-86$ & H & 12 & - & -. & 1 \\
\hline$B C W$ & 74 & 75 & $07-25-86$ & H & 45 & $\cdots$ & $-\cdot$ & 1 \\
\hline $\mathrm{BCW}$ & 75 & . & - & $\mathrm{H}$ & 20 & $\cdots$ & $\cdots$ & 1 \\
\hline $\mathrm{BCW}$ & 76 & $\cdots$ & $\cdots$ & $\mathrm{H}$ & 2 & $\cdots$ & $-\cdot$ & .5 \\
\hline
\end{tabular}


Table 1-1. Description of selected wells, borings, and springs in the Saco and Ossipee River Basins, east-central

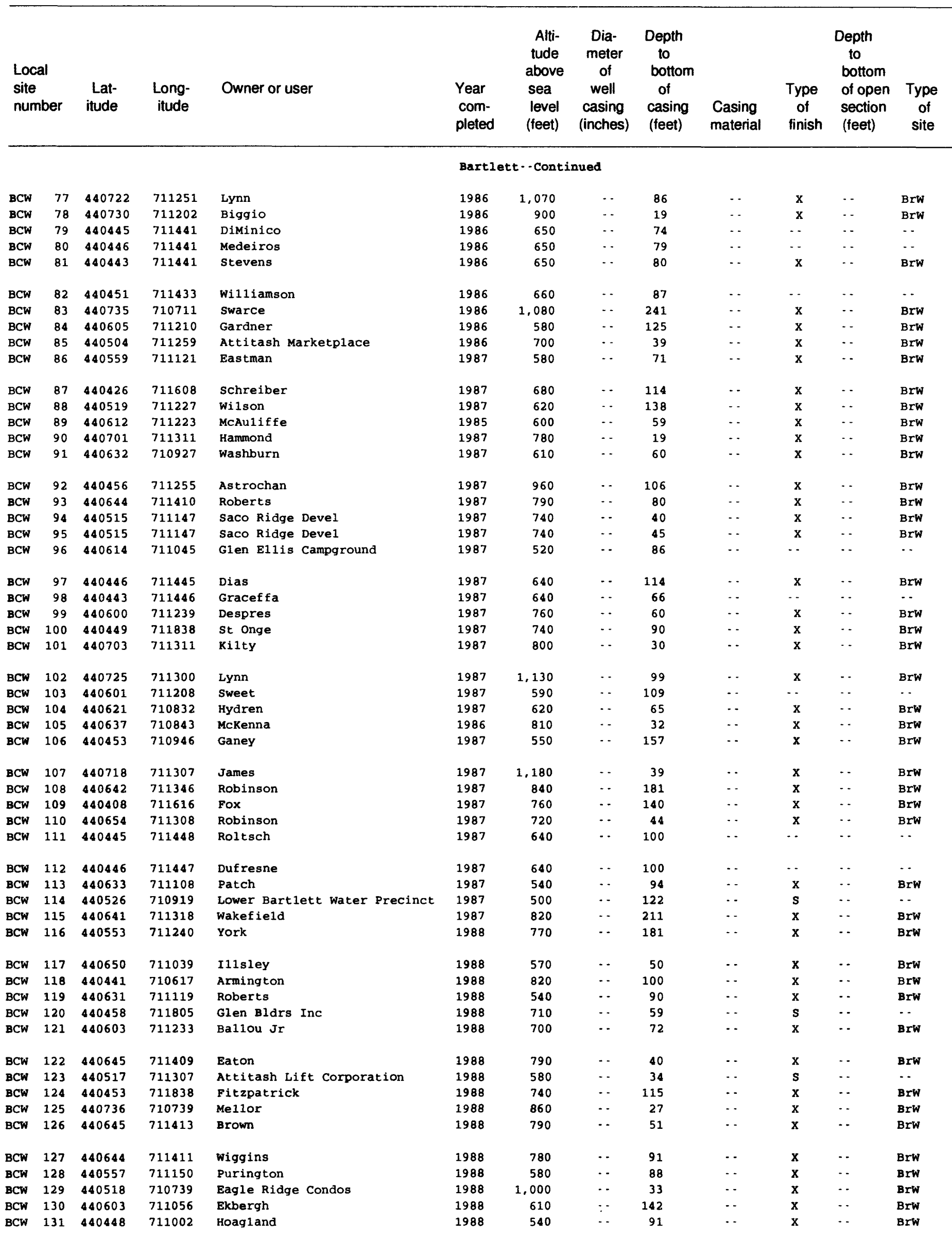




\begin{tabular}{|c|c|c|c|c|c|c|c|c|}
\hline $\begin{array}{l}\text { Local } \\
\text { site } \\
\text { number }\end{array}$ & $\begin{array}{l}\text { Water } \\
\text { level } \\
\text { depth } \\
\text { (feet) }\end{array}$ & $\begin{array}{l}\text { Date } \\
\mathrm{mm} / \mathrm{dd} / \mathrm{yy}\end{array}$ & Use & $\begin{array}{l}\text { Maximum } \\
\text { well } \\
\text { yield } \\
\text { (gallons } \\
\text { per } \\
\text { minute) }\end{array}$ & $\begin{array}{l}\text { Draw- } \\
\text { down } \\
\text { (feet) }\end{array}$ & $\begin{array}{l}\text { Specific } \\
\text { capacity } \\
\text { (gallons per } \\
\text { minute } \\
\text { per } \\
\text { foot) }\end{array}$ & $\begin{array}{l}\text { Pumping } \\
\text { period } \\
\text { (hours) }\end{array}$ & Remarks \\
\hline
\end{tabular}

\begin{tabular}{|c|c|c|c|c|c|c|c|c|}
\hline \multirow[b]{2}{*}{$\mathrm{BCW}$} & \multirow[b]{2}{*}{77} & \multirow[b]{2}{*}{-. } & \multirow[b]{2}{*}{$\cdots$} & \multirow[b]{2}{*}{ H } & \multirow[b]{2}{*}{6} & \multirow[b]{2}{*}{ - } & \multicolumn{2}{|c|}{ Bartlett--Cont } \\
\hline & & & & & & & - & .5 \\
\hline $\mathrm{BCW}$ & 78 & - & - & $\mathrm{H}$ & 20 & - & -. & .5 \\
\hline BCW & 79 & - - & - & H & 30 & $\cdots$ & .. & 1 \\
\hline BCW & 80 & 25 & $09-09-86$ & H & 25 & . & -. & 24 \\
\hline $\mathrm{BCW}$ & 81 & 40 & $09-10-86$ & H & 10 & $-\cdot$ & $\cdots$ & 1 \\
\hline BCW & 82 & 25 & $09-11-86$ & H & 25 & $\cdots$ & -. & 24 \\
\hline $\mathrm{BCW}$ & 83 & 85 & $11-01-86$ & H & 10 & - & -. & .5 \\
\hline BCW & 84 & 50 & $10-16-86$ & H & 7.5 & $\cdots$ & -. & .5 \\
\hline BCW & 85 & 60 & $10-15-86$ & $\mathrm{z}$ & 1 & - & - - & - \\
\hline BCW & 86 & - & - & H & 150 & $\cdots$ & $\cdots$ & .5 \\
\hline BCW & 87 & 9 & $05-05-87$ & H & 3 & - - & $-\cdot$ & 1 \\
\hline $\mathrm{BCW}$ & 88 & 50 & $01-28-87$ & H & 6.5 & - & - & .5 \\
\hline $\mathrm{BCW}$ & 89 & 25 & $11-23 \cdot 85$ & H & 10 & $-\cdot$ & $\cdots$ & $\cdots$ \\
\hline $\mathrm{BCW}$ & 90 & -. & . & H & 20 & $\cdots$ & . & 1 \\
\hline BCW & 91 & - & $-\cdot$ & H & 75 & $\cdots$ & $\cdots$ & 1 \\
\hline $\mathrm{BCW}$ & 92 & 6 & $04-27-87$ & P & 30 & $\cdots$ & $\cdots$ & 1 \\
\hline BCW & 93 & 15 & $06-10-87$ & H & 75 & - & -. & .5 \\
\hline $\mathrm{BCW}$ & 94 & 6 & $05-04-87$ & H & 20 & $\cdots$ & $\cdots$ & .5 \\
\hline $\mathrm{BCW}$ & 95 & 20 & $04 \cdot 30 \cdot 87$ & H & 17 & $\cdots$ & $\cdots$ & 1 \\
\hline BCW & 96 & 25 & $05-16-87$ & P & 35 & - & $\cdots$ & 48 \\
\hline BCW & 97 & $\cdots$ & $\cdots$ & H & 4 & $\cdots$ & $\cdots$ & 1 \\
\hline $\mathrm{BCW}$ & 98 & $\cdots$ & $\cdots$ & H & 15 & $\cdots$ & $\cdots$ & 48 \\
\hline $\mathrm{BCW}$ & 99 & $\cdots$ & $\cdots$ & $\mathrm{H}$ & 100 & $\cdots$ & $\cdots$ & 1 \\
\hline $\mathrm{BCW}$ & 100 & - & $\cdots$ & H & 25 & -. & $\cdots$ & 1 \\
\hline BCW & 101 & $\cdots$ & $-\cdot$ & H & 3 & $\cdots$ & $\cdots$ & 1 \\
\hline BCW & 102 & $\cdots$ & $\cdots$ & H & 3 & $\cdots$ & $\cdots$ & .5 \\
\hline $\mathrm{BCW}$ & 103 & - - & $-\cdot$ & H & 10 & $\cdots$ & - & .5 \\
\hline $\mathrm{BCW}$ & 104 & $\cdots$ & $\cdots$ & $\mathrm{P}$ & 150 & $\cdots$ & $\cdots$ & 1 \\
\hline BCW & 105 & - & - & H & 50 & $\cdots$ & $\cdots$ & .5 \\
\hline $\mathrm{BCW}$ & 106 & 75 & $11-06-87$ & H & 5 & $\cdots$ & $\cdots$ & .5 \\
\hline $\mathrm{BCW}$ & 107 & 80 & $11-07-87$ & H & -. & $\cdots$ & $\cdots$ & $\cdots$ \\
\hline $\mathrm{BCW}$ & 108 & $\cdots$ & - & $\mathrm{H}$ & 15 & $\cdots$ & $\cdots$ & 1 \\
\hline $\mathrm{BCW}$ & 109 & $\cdots$ & $-\cdot$ & H & 100 & $\cdots$ & -. & 1 \\
\hline $\mathrm{BCW}$ & 110 & - & - & H & 15 & $\cdots$ & $\cdots$ & 1 \\
\hline $\mathrm{BCW}$ & 111 & 30 & $11-05-87$ & H & 20 & $\cdots$ & $\cdots$ & 12 \\
\hline $\mathrm{BCW}$ & 112 & 20 & $11-06-87$ & н & 12 & $\cdots$ & . & 12 \\
\hline $\mathrm{BCW}$ & 113 & $\cdot \cdot$ & - & c & 10 & $\cdots$ & - & 1 \\
\hline $\mathrm{BCW}$ & 114 & 17.6 & $12-15-87$ & $\mathrm{P}$ & 750 & -. & -. & 121 \\
\hline BCW & 115 & . & . & H & 15 & $\cdots$ & -. & .5 \\
\hline BCW & 116 & 150 & $03-15-88$ & H & 2.5 & $\cdots$ & $\cdots$ & 1 \\
\hline $\mathrm{BCW}$ & 117 & $\cdots$ & $\cdots$ & P & 18 & $\cdots$ & -. & 1 \\
\hline $\mathrm{BCW}$ & 118 & $\cdots$ & . & H & 30 & -. & .. & 1 \\
\hline $\mathrm{BCW}$ & 119 & 6 & $04-20-88$ & $\mathrm{H}$ & 100 & $\cdots$ & $\cdots$ & 1 \\
\hline $\mathrm{BCW}$ & 120 & $\cdots$ & . & H & 25 & $\cdots$ & $\cdots$ & 8 \\
\hline BCW & 121 & $\cdots$ & $\cdots$ & H & 2 & $\cdots$ & -. & 1 \\
\hline $\mathrm{BCW}$ & 122 & 40 & $07-22-88$ & H & 12 & - & $\cdots$ & .5 \\
\hline $\mathrm{BCW}$ & 123 & 20 & $07-20 \cdot 88$ & c & 60 & - & .. & 1 \\
\hline BCW & 124 & 10 & $07-05-88$ & H & 3 & $\cdots$ & $\cdots$ & .5 \\
\hline BCW & 125 & 18 & $06-17-88$ & H & 75 & $\cdots$ & $\cdots$ & .5 \\
\hline $\mathrm{BCW}$ & 126 & 50 & $05-25-88$ & H & 50 & $\cdots$ & $\cdots$ & .5 \\
\hline BCW & 127 & 40 & 05.25 .88 & H & 5.5 & $\cdots$ & $\cdots$ & .5 \\
\hline BCW & 128 & 40 & $09-15-88$ & H & 20 & $\cdots$ & .. & .5 \\
\hline BCW & 129 & 20 & $10-13-88$ & P & 50 & -. & -. & 1 \\
\hline $\mathrm{BCW}$ & 130 & - & . & H & 4 & $\cdots$ & $\cdots$ & 1 \\
\hline BCW & 131 & $\cdots$ & .. & H & 10 & .. & .. & 1 \\
\hline
\end{tabular}


Table 1-1. Description of selected wells, borings, and springs in the Saco and Ossipee River Basins, east-central

\begin{tabular}{|c|c|c|c|c|c|c|c|c|c|c|c|}
\hline $\begin{array}{l}\text { Local } \\
\text { site } \\
\text { number }\end{array}$ & $\begin{array}{l}\text { Lat- } \\
\text { itude }\end{array}$ & $\begin{array}{l}\text { Long- } \\
\text { itude }\end{array}$ & Owner or user & $\begin{array}{l}\text { Year } \\
\text { com- } \\
\text { pleted }\end{array}$ & $\begin{array}{l}\text { Alti- } \\
\text { tude } \\
\text { above } \\
\text { sea } \\
\text { level } \\
\text { (feet) }\end{array}$ & $\begin{array}{l}\text { Dia- } \\
\text { meter } \\
\text { of } \\
\text { well } \\
\text { casing } \\
\text { (inches) }\end{array}$ & $\begin{array}{l}\text { Depth } \\
\text { to } \\
\text { bottom } \\
\text { of } \\
\text { casing } \\
\text { (feet) }\end{array}$ & $\begin{array}{l}\text { Casing } \\
\text { material }\end{array}$ & $\begin{array}{l}\text { Type } \\
\text { of } \\
\text { finish }\end{array}$ & $\begin{array}{l}\text { Depth } \\
\text { to } \\
\text { bottom } \\
\text { of open } \\
\text { section } \\
\text { (feet) }\end{array}$ & $\begin{array}{c}\text { Type } \\
\text { of } \\
\text { site }\end{array}$ \\
\hline
\end{tabular}

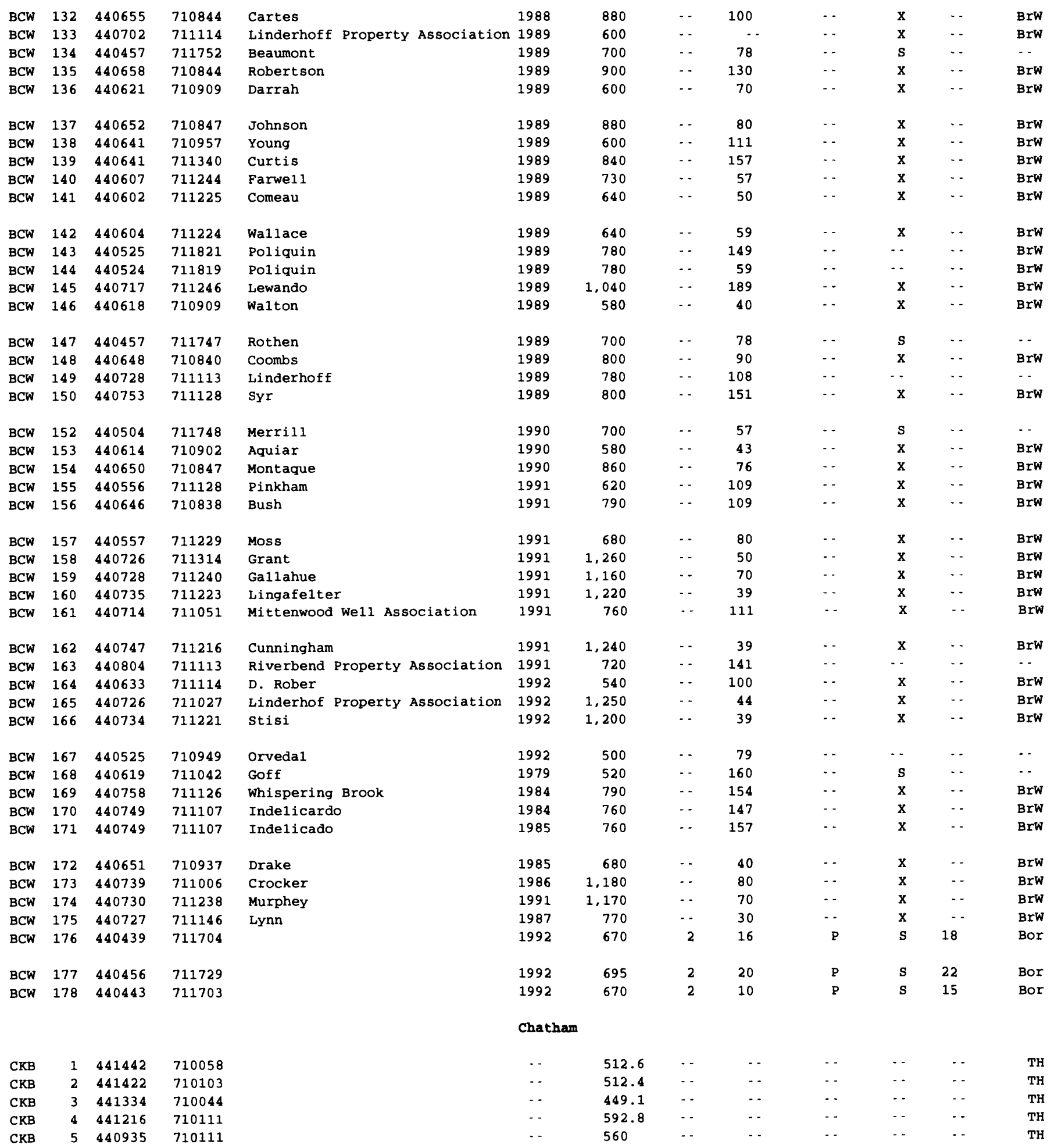




\begin{tabular}{|c|c|c|c|c|c|c|c|c|}
\hline $\begin{array}{l}\text { Local } \\
\text { site } \\
\text { number }\end{array}$ & $\begin{array}{l}\text { Water } \\
\text { level } \\
\text { depth } \\
\text { (feet) }\end{array}$ & $\begin{array}{l}\text { Date } \\
\mathrm{mm} / \mathrm{dd} / \mathrm{yy}\end{array}$ & Use & $\begin{array}{l}\text { Maximum } \\
\text { well } \\
\text { yield } \\
\text { (gallons } \\
\text { per } \\
\text { minute) }\end{array}$ & $\begin{array}{l}\text { Draw- } \\
\text { down } \\
\text { (feet) }\end{array}$ & $\begin{array}{c}\text { Specific } \\
\text { capacity } \\
\text { (gallons per } \\
\text { minute } \\
\text { per } \\
\text { foot) }\end{array}$ & $\begin{array}{l}\text { Pumping } \\
\text { period } \\
\text { (hours) }\end{array}$ & Remarks \\
\hline
\end{tabular}

Bartlett - - Continued

\begin{tabular}{|c|c|c|c|c|c|c|c|c|c|}
\hline $\mathrm{BCW}$ & 132 & 10 & $11 \cdot 17-88$ & $\mathrm{H}$ & 4 & $\cdots$ & - & .5 & \\
\hline $\mathrm{BCW}$ & 133 & - & - & $\mathrm{P}$ & 60 & -- & - & 1 & \\
\hline $\mathrm{BCW}$ & 134 & $\cdots$ & - & $\mathrm{H}$ & 25 & $\cdots$ & - & 1 & \\
\hline $\mathrm{BCW}$ & 135 & 50 & $03-01-89$ & $\mathrm{H}$ & 15 & - & $\cdots$ & .5 & \\
\hline $\mathrm{BCW}$ & 136 & 25 & $05-10-89$ & $\mathrm{H}$ & 15 & -- & $\cdots$ & 1 & \\
\hline BCW & 137 & $\cdots$ & $\cdots$ & $\mathrm{H}$ & 7 & $\cdots$ & $\cdots$ & 1 & \\
\hline $\mathrm{BCW}$ & 138 & - & - & $\mathrm{H}$ & 100 & $\cdots$ & $-\cdot$ & 1 & \\
\hline $\mathrm{BCW}$ & 139 & 80 & $06-28-89$ & H & 30 & $\cdots$ & - & 1 & \\
\hline $\mathrm{BCW}$ & 140 & 40 & $07-28-89$ & $\mathrm{H}$ & 10 & -- & $\cdots$ & 1 & \\
\hline $\mathrm{BCW}$ & 141 & - & - & $\mathrm{H}$ & 3.5 & $\cdots$ & $\cdots$ & 1 & \\
\hline BCW & 142 & 78 & $07-26 \cdot 89$ & $\mathrm{H}$ & 3 & $\cdots$ & $\cdots$ & 1 & \\
\hline BCW & 143 & - - & - & $\mathrm{P}$ & 35 & $\cdots$ & $-\cdot$ & 8 & \\
\hline $\mathrm{BCW}$ & 144 & - & $\cdots$ & $P$ & 30 & - & $\cdots$ & 8 & \\
\hline $\mathrm{BCW}$ & 145 & - & - & $\mathrm{H}$ & .0 .5 & $\cdots$ & -- & .5 & \\
\hline $\mathrm{BCW}$ & 146 & 10 & $08-25-89$ & $\mathrm{H}$ & 8 & $\cdots$ & - & .5 & \\
\hline $\mathrm{BCW}$ & 147 & - & $\cdots$ & $\mathrm{H}$ & 25 & $-\cdot$ & $\cdots$ & 36 & \\
\hline $\mathrm{BCW}$ & 148 & $\cdots$ & - & $\mathrm{H}$ & 10 & $\cdots$ & $\cdots$ & 1 & \\
\hline $\mathrm{BCW}$ & 149 & - & $\cdots$ & $\mathrm{P}$ & 75 & $\cdots$ & $\cdots$ & 24 & \\
\hline $\mathrm{BCW}$ & 150 & $\cdots$ & $\cdots$ & $\mathrm{P}$ & 75 & $\cdots$ & $-\cdot$ & 1 & \\
\hline BCW & 152 & - & - & $\mathrm{H}$ & 30 & - & - & 8 & \\
\hline $\mathrm{BCW}$ & 153 & 30 & $12-04-90$ & $\mathrm{H}$ & 5 & $-\cdot$ & -- & .5 & \\
\hline $\mathrm{BCW}$ & 154 & 5 & $09-15-90$ & H & 10 & $-\cdot$ & $\cdots$ & .5 & \\
\hline $\mathrm{BCW}$ & 155 & 60 & $07-03-91$ & H & 6 & $\cdots$ & $\cdots$ & .5 & \\
\hline $\mathrm{BCW}$ & 156 & 10 & $08-15-91$ & H & 5 & $\cdots$ & $\cdots$ & .5 & \\
\hline $\mathrm{BCW}$ & 157 & -- & $\cdots$ & H & 6 & -- & $\cdots$ & 1 & \\
\hline $\mathrm{BCW}$ & 158 & $\cdots$ & $\cdots$ & $\mathrm{H}$ & 6 & $\cdots$ & - & 1 & \\
\hline $\mathrm{BCW}$ & 159 & $\cdots$ & $\cdots$ & $\mathrm{H}$ & 20 & $\cdots$ & $\cdots$ & 1 & \\
\hline $\mathrm{BCW}$ & 160 & - & - & H & 60 & $\cdots$ & $\cdots$ & 1 & \\
\hline $\mathrm{BCW}$ & 161 & 75 & $11-01-91$ & $\mathbf{P}$ & 30 & $\cdots$ & $\cdots$ & .5 & \\
\hline $\mathrm{BCW}$ & 162 & $\cdots$ & - & H & 30 & - & - & 1 & \\
\hline BCW & 163 & $\cdots$ & $\cdots$ & $\mathrm{H}$ & 75 & - & -- & 1 & \\
\hline $\mathrm{BCW}$ & 164 & $\cdots$ & $\cdots$ & H & 45 & $\cdots$ & $\cdots$ & 1 & \\
\hline $\mathrm{BCW}$ & 165 & 620 & $06-07-92$ & $\mathbf{P}$ & 150 & $\cdots$ & $\cdots$ & 1 & \\
\hline BCW & 166 & $\cdots$ & - & $\mathrm{H}$ & 10 & $\cdots$ & - & 1 & \\
\hline $\mathrm{BCW}$ & 167 & 10 & $07-29-92$ & H & 55 & $\cdots$ & $\cdots$ & $\cdots$ & \\
\hline BCW & 168 & $\cdots$ & $\cdots$ & $\mathbf{P}$ & 30 & $\cdots$ & $\cdots$ & $\cdots$ & \\
\hline BCW & 169 & - - & $\cdots$ & $\mathrm{P}$ & 25 & $-\cdot$ & $\cdots$ & 1 & \\
\hline $\mathrm{BCW}$ & 170 & 60 & $10-05-84$ & $\mathrm{P}$ & 20 & $\cdots$ & $-\cdot$ & 7 & \\
\hline $\mathrm{BCW}$ & 171 & 75 & $06-22-85$ & $\mathrm{P}$ & 20 & -- & $-\cdot$ & 6 & \\
\hline $\mathrm{BCW}$ & 172 & 50 & $09-30-85$ & $\mathrm{H}$ & 18 & $\cdots$ & $\cdots$ & 1 & \\
\hline BCW & 173 & - & - & $\mathrm{H}$ & 45 & $\cdots$ & $\cdots$ & 1 & \\
\hline BCW & 174 & $\cdots$ & $\cdots$ & H & 6 & $-\cdot$ & $\cdots$ & 1 & \\
\hline $\mathrm{BCW}$ & 175 & - & $\cdots$ & $\mathrm{P}$ & 120 & $\cdots$ & $\cdots$ & .5 & \\
\hline BCW & 176 & $\cdots$ & $\cdots$ & 0 & - - & $\cdots$ & $\cdots$ & $\cdots$ & USGS \\
\hline BCW & 177 & $\cdots$ & $-\cdot$ & 0 & - & $-\cdot$ & $-\cdot$ & $\cdots$ & USGS \\
\hline $\mathrm{BCW}$ & 178 & $\cdots$ & $\cdots$ & 0 & $\cdots$ & $\cdots$ & $\cdots$ & $\cdots$ & USGS \\
\hline
\end{tabular}

\begin{tabular}{|c|c|c|c|c|c|c|c|}
\hline CKB & 1 & $\cdots$ & $\cdots$ & $\mathrm{U}$ & $\cdots$ & $\cdots$ & $\cdots$ \\
\hline $\mathrm{CKB}$ & 2 & $\ldots$ & $\cdots$ & $\mathrm{U}$ & $\cdots$ & $\cdots$ & $\cdots$ \\
\hline CKB & 3 & - & $\cdots$ & $\mathrm{U}$ & $\cdots$ & $\cdots$ & $\cdots$ \\
\hline CKB & 4 & $\cdots$ & $\cdots$ & $\mathrm{U}$ & $\cdots$ & $\cdots$ & $\cdots$ \\
\hline CKB & 5 & $\ldots$ & - & $\mathrm{U}$ & $\cdots$ & $\cdots$ & $\cdots$ \\
\hline
\end{tabular}




\begin{tabular}{|c|c|c|c|c|c|c|c|c|c|c|c|}
\hline $\begin{array}{l}\text { Local } \\
\text { site } \\
\text { number }\end{array}$ & $\begin{array}{l}\text { Lat- } \\
\text { itude }\end{array}$ & $\begin{array}{l}\text { Long- } \\
\text { itude }\end{array}$ & Owner or user & $\begin{array}{l}\text { Year } \\
\text { com- } \\
\text { pleted }\end{array}$ & $\begin{array}{l}\text { Alti- } \\
\text { tude } \\
\text { above } \\
\text { sea } \\
\text { level } \\
\text { (feet) }\end{array}$ & $\begin{array}{l}\text { Dia- } \\
\text { meter } \\
\text { of } \\
\text { well } \\
\text { casing } \\
\text { (inches) }\end{array}$ & $\begin{array}{l}\text { Depth } \\
\text { to } \\
\text { bottom } \\
\text { of } \\
\text { casing } \\
\text { (feet) }\end{array}$ & $\begin{array}{l}\text { Casing } \\
\text { material }\end{array}$ & $\begin{array}{l}\text { Type } \\
\text { of } \\
\text { finish }\end{array}$ & $\begin{array}{l}\text { Depth } \\
\text { to } \\
\text { bottom } \\
\text { of open } \\
\text { section } \\
\text { (feet) }\end{array}$ & $\begin{array}{c}\text { Type } \\
\text { of } \\
\text { site }\end{array}$ \\
\hline
\end{tabular}

\begin{tabular}{|c|c|c|c|}
\hline CKB & 6 & 440442 & 710145 \\
\hline $\mathrm{CKB}$ & 7 & 440553 & 710025 \\
\hline CKS & 1 & 440941 & 710111 \\
\hline CKW & 1 & 440944 & 710105 \\
\hline CKW & 2 & 441013 & 710059 \\
\hline CKW & 3 & 441037 & 710137 \\
\hline CKW & 4 & 441019 & 710038 \\
\hline CKW & 5 & 441057 & 710046 \\
\hline CKW & 6 & 441217 & 710034 \\
\hline CKW & 7 & 441217 & 710034 \\
\hline$C K W$ & 8 & 441513 & 710104 \\
\hline CKW & 9 & 441452 & 710051 \\
\hline CKW & 10 & 441357 & 710050 \\
\hline CKW & 11 & 441004 & 710040 \\
\hline CKW & 12 & 440512 & 710018 \\
\hline$C K W$ & 13 & 440555 & 710025 \\
\hline CKW & 14 & 440614 & 710018 \\
\hline CKW & 15 & 441111 & 710043 \\
\hline CKW & 16 & 441019 & 710029 \\
\hline CKW & 17 & 440649 & 710019 \\
\hline CKW & 18 & 441150 & 710028 \\
\hline CKW & 19 & 440548 & 710014 \\
\hline$C K W$ & 20 & 440629 & 710017 \\
\hline CKW & 21 & 441427 & 710112 \\
\hline CKW & 22 & 441445 & 710102 \\
\hline CKW & 23 & 441146 & 710033 \\
\hline CKW & 24 & 441303 & 710026 \\
\hline CKW & 25 & 440623 & 710019 \\
\hline CKW & 26 & 440646 & 710030 \\
\hline CKW & 27 & 440513 & 710019 \\
\hline CKW & 28 & 440515 & 710020 \\
\hline CKW & 29 & 440941 & 710039 \\
\hline CKW & 30 & 440645 & 710027 \\
\hline CKW & 31 & 440441 & 710127 \\
\hline CKW & 32 & 441503 & 710043 \\
\hline CKW & 33 & 441051 & 710152 \\
\hline CKW & 34 & 440554 & 710024 \\
\hline CKW & 35 & 441039 & 710046 \\
\hline CWA & 1 & 440407 & 710110 \\
\hline CWW & 10 & 440232 & 710830 \\
\hline CWW & 44 & 440417 & 710946 \\
\hline CWW & 45 & 440336 & 710952 \\
\hline CWW & 46 & 440307 & 710925 \\
\hline$C W W$ & 47 & 440305 & 710924 \\
\hline CWW & 48 & 440253 & 710901 \\
\hline CWW & 49 & 440246 & 710845 \\
\hline CWW & 50 & 440238 & 710855 \\
\hline CWW & 51 & 440235 & 710854 \\
\hline$C W w$ & 52 & 440223 & 710836 \\
\hline CWW & 53 & 440212 & 710827 \\
\hline CWW & 54 & 440156 & 710914 \\
\hline
\end{tabular}

Richard Theot

wiggin

Marie Limmer

Linda Miles

James Maloney

Wayne and Norma Mcallis

Richard and Gail Dembow

Engle

Mitchel1

Mckenzie

calomb

Foster

Brown

Doo

Forbes

Beaton

Mcviney

Brooks

Kubik

Roy

Bagosius

Davis

Hilliard

wilfong

stryker

Pevear

K. Saunders

Kennet t

Fernald

MacPherson
Chatham--Continued

\begin{tabular}{|c|c|c|c|c|c|c|c|}
\hline$\cdots$ & 486 & $\cdots$ & $\cdots$ & $\cdots$ & $\cdots$ & $\cdots$ & $\mathrm{TH}$ \\
\hline$\cdots$ & 421.5 & $\cdots$ & $\cdots$ & $\cdots$ & $\cdots$ & $\cdots$ & $\mathrm{TH}$ \\
\hline$\cdots$ & 575 & $\cdots$ & $-\cdot$ & $\cdots$ & $\cdots$ & $\cdots$ & sp \\
\hline 1971 & 550 & 36 & 8.9 & c & 0 & $\cdots$ & Dug \\
\hline 1966 & 510 & 36 & 8.3 & C & 0 & - & Dug \\
\hline - & 555 & 72 & 12.5 & C & 0 & $\cdots$ & Dug \\
\hline 1960 & 510 & $\cdots$ & - & $\cdots$ & $\mathrm{x}$ & $\cdots$ & BrW \\
\hline 1970 & 510 & 36 & 7.5 & C & 0 & - & Dug \\
\hline$\cdots$ & 510 & $\cdots$ & $\cdots$ & $\cdots$ & $\mathrm{x}$ & $\cdots$ & BrW \\
\hline - & 510 & $\cdots$ & $\cdots$ & $\cdots$ & 0 & $\cdots$ & Dug \\
\hline- & 525 & 36 & 10.7 & C & 0 & - & Dug \\
\hline 1992 & 535 & 2 & 27.5 & P & $\mathbf{S}$ & 29.5 & Bor \\
\hline 1992 & 510 & 2 & 57.9 & $\mathrm{P}$ & $\mathbf{s}$ & 59.9 & Bor \\
\hline 1992 & 530 & 2 & 57.7 & $\mathrm{P}$ & S & 59.7 & Bor \\
\hline 1984 & 490 & $\cdots$ & 181 & $\cdots$ & $\mathrm{x}$ & - - & $B r W$ \\
\hline$\cdots$ & 420 & $\cdots$ & 27 & $\cdots$ & $\mathrm{x}$ & $\cdots$ & BrW \\
\hline 1985 & 390 & $\cdots$ & 70 & $\cdots$ & $\mathrm{x}$ & $\cdots$ & BrW \\
\hline 1985 & 480 & $\cdots$ & 19 & $\cdots$ & $\mathrm{x}$ & $\cdots$ & BrW \\
\hline 1986 & 540 & $\cdots$ & 95 & $\cdots$ & $\mathrm{x}$ & $\cdots$ & Brw \\
\hline 1987 & 500 & $\cdots$ & 99 & $\cdots$ & $\mathrm{x}$ & $\cdots$ & BrW \\
\hline 1987 & 520 & $\cdots$ & 43 & $\cdots$ & $x$ & $\cdots$ & Brw \\
\hline 1987 & 390 & $\cdots$ & 38 & $\cdots$ & $x$ & $\cdots$ & BrW \\
\hline 1987 & 410 & $\cdots$ & 19 & $\cdots$ & $\mathrm{x}$ & $\cdots$ & BrW \\
\hline 1987 & 550 & $\cdots$ & 75 & $\cdots$ & $x$ & $\cdots$ & BrW \\
\hline 1988 & 520 & $\cdots$ & 40 & $\cdots$ & $\mathrm{x}$ & $\cdots$ & BrW \\
\hline 1988 & 520 & $\cdots$ & 52 & $\cdots$ & $x$ & $\cdots$ & $\mathrm{BrW}$ \\
\hline 1989 & 430 & $\cdots$ & 80 & $\cdots$ & $\mathrm{x}$ & $\cdots$ & BrW \\
\hline 1989 & 420 & $\cdots$ & 59 & $\cdots$ & $x$ & $\cdots$ & BrW \\
\hline 1989 & 510 & $\cdots$ & 67 & $\cdots$ & $\mathrm{x}$ & - & BrW \\
\hline 1989 & 490 & $\cdots$ & 180 & $\cdots$ & $\mathrm{x}$ & $\cdots$ & BrW \\
\hline 1990 & 500 & $\cdots$ & 171 & $\cdots$ & $\mathrm{x}$ & $\cdots$ & BrW \\
\hline 1990 & 480 & - & 39 & $\cdots$ & $\mathrm{x}$ & $\cdots$ & Brw \\
\hline 1991 & 490 & $\cdots$ & 39 & $\cdots$ & $\mathbf{x}$ & $\cdots$ & BrW \\
\hline 1991 & 480 & $\cdots$ & 89 & $\cdots$ & $\mathrm{x}$ & $\cdots$ & $\mathrm{BrW}$ \\
\hline 1991 & 540 & $\cdots$ & 64 & $\cdots$ & $\mathrm{x}$ & $\cdots$ & BrW \\
\hline 1991 & 720 & - & 39 & $\cdots$ & $\mathrm{x}$ & $\cdots$ & BrW \\
\hline 1991 & 420 & $\cdots$ & 50 & $\cdots$ & $\mathrm{x}$ & $\cdots$ & Brw \\
\hline 1991 & 522 & - & 60 & $\cdots$ & $\mathrm{x}$ & $\cdots$ & \\
\hline
\end{tabular}

Conway

\begin{tabular}{|c|c|c|c|c|c|c|c|}
\hline 1992 & 430 & - & $\cdots$ & $\cdots$ & $\cdots$ & $\cdots$ & Bor \\
\hline - & 100 & $\cdots$ & $\cdots$ & $\cdots$ & $\cdots$ & $\cdots$ & $\cdots$ \\
\hline - & 545 & $\cdots$ & $\cdots$ & $\cdots$ & $\cdots$ & $\cdots$ & $\cdots$ \\
\hline - & 520 & $\cdots$ & $\cdots$ & $\cdots$ & $\cdots$ & $\cdots$ & $\cdots$ \\
\hline . & 500 & $\cdots$ & $\cdots$ & $\cdots$ & $\cdots$ & $\cdots$ & BrW \\
\hline$\cdots$ & 500 & $\cdots$ & $\cdots$ & $\cdots$ & $\cdots$ & $\cdots$ & BrW \\
\hline$\cdots$ & 510 & $\cdots$ & $\cdots$ & $\cdots$ & $\cdots$ & $\cdots$ & Brw \\
\hline$\cdots$ & 480 & $\cdots$ & $\cdots$ & $\cdots$ & $\cdots$ & $\cdots$ & Brw \\
\hline$\cdots$ & 524 & $\cdots$ & $\cdots$ & $\cdots$ & $\cdots$ & $\cdots$ & Brw \\
\hline - & 524 & $\cdots$ & -- & $\cdots$ & $\cdots$ & $\cdots$ & BrW \\
\hline$\cdots$ & 521 & $\cdots$ & $\cdots$ & - & $\cdots$ & - & Brw \\
\hline$\cdots$ & 510 & $\cdots$ & $\cdots$ & $\cdots$ & $\cdots$ & $\cdots$ & BrW \\
\hline$\cdots$ & 510 & $\cdots$ & $\cdots$ & $\cdots$ & $\cdots$ & $\cdots$ & $\cdots$ \\
\hline
\end{tabular}




\begin{tabular}{|c|c|c|c|c|c|c|c|c|}
\hline $\begin{array}{l}\text { Local } \\
\text { site } \\
\text { number }\end{array}$ & $\begin{array}{l}\text { Water } \\
\text { level } \\
\text { depth } \\
\text { (feet) }\end{array}$ & $\begin{array}{l}\text { Date } \\
\mathrm{mm} / \mathrm{dd} / \mathrm{yy}\end{array}$ & Use & $\begin{array}{l}\text { Maximum } \\
\text { well } \\
\text { yield } \\
\text { (gallons } \\
\text { per } \\
\text { minute) }\end{array}$ & $\begin{array}{l}\text { Draw- } \\
\text { down } \\
\text { (feet) }\end{array}$ & $\begin{array}{c}\text { Specific } \\
\text { capacity } \\
\text { (gallons per } \\
\text { minute } \\
\text { per } \\
\text { foot) }\end{array}$ & $\begin{array}{l}\text { Pumping } \\
\text { period } \\
\text { (hours) }\end{array}$ & Remarks \\
\hline
\end{tabular}

Conway - - Continued

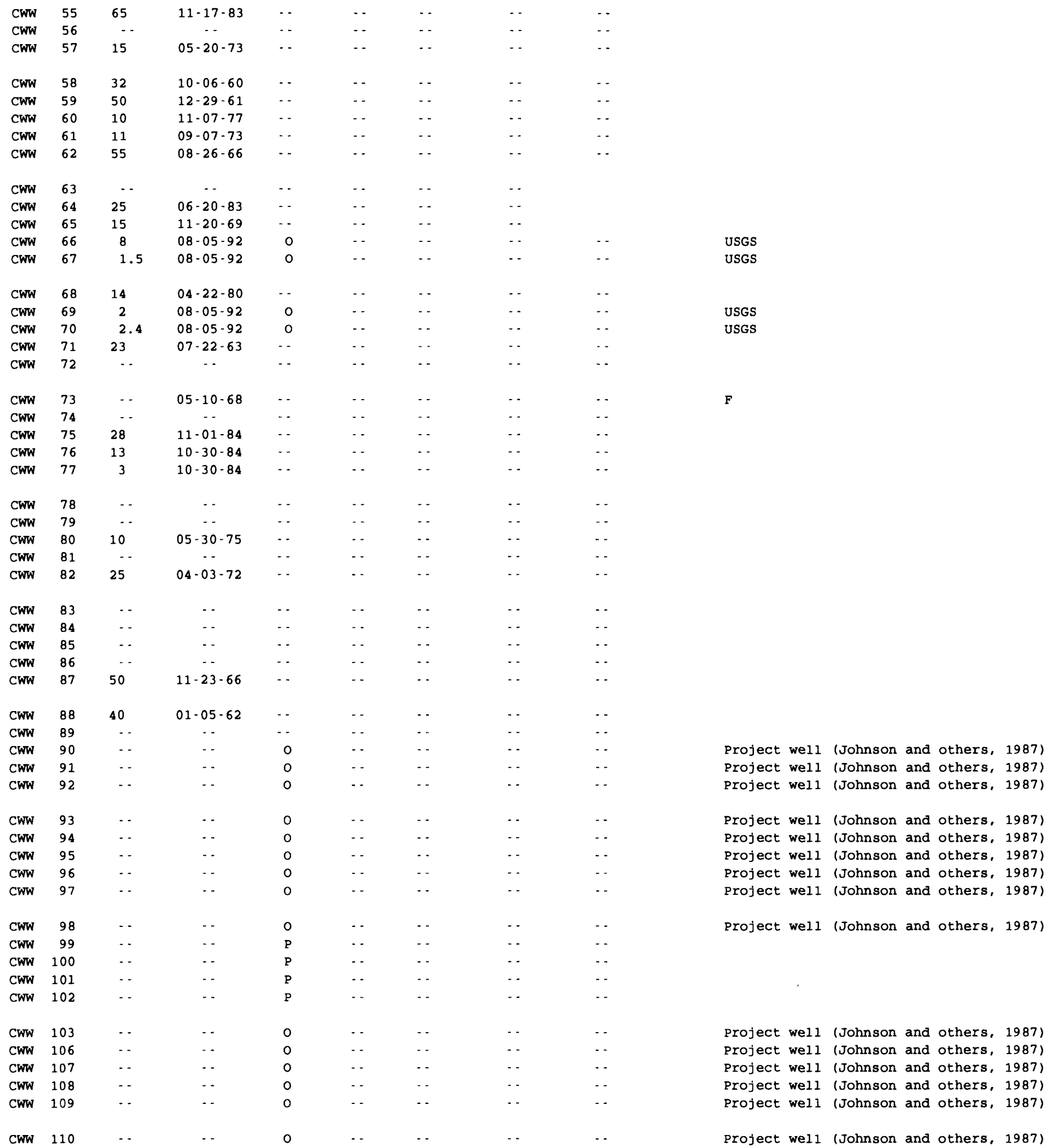


Table 1-1. Description of selected wells, borings, and springs in the Saco and Ossipee River Basins, east-central

\begin{tabular}{|c|c|c|c|c|c|c|c|c|c|c|c|}
\hline $\begin{array}{l}\text { Local } \\
\text { site } \\
\text { number }\end{array}$ & $\begin{array}{l}\text { Lat- } \\
\text { itude }\end{array}$ & $\begin{array}{l}\text { Long- } \\
\text { itude }\end{array}$ & Owner or user & $\begin{array}{l}\text { Year } \\
\text { com- } \\
\text { pleted }\end{array}$ & $\begin{array}{l}\text { Alti- } \\
\text { tude } \\
\text { above } \\
\text { sea } \\
\text { level } \\
\text { (feet) }\end{array}$ & $\begin{array}{l}\text { Dia- } \\
\text { meter } \\
\text { of } \\
\text { well } \\
\text { casing } \\
\text { (inches) }\end{array}$ & $\begin{array}{l}\text { Depth } \\
\text { to } \\
\text { bottom } \\
\text { of } \\
\text { casing } \\
\text { (feet) }\end{array}$ & $\begin{array}{l}\text { Casing } \\
\text { material }\end{array}$ & $\begin{array}{l}\text { Type } \\
\text { of } \\
\text { finish }\end{array}$ & $\begin{array}{l}\text { Depth } \\
\text { to } \\
\text { bottom } \\
\text { of open } \\
\text { section } \\
\text { (feet) }\end{array}$ & $\begin{array}{c}\text { Type } \\
\text { of } \\
\text { site }\end{array}$ \\
\hline
\end{tabular}

\begin{tabular}{|c|c|c|c|}
\hline CWW & 111 & 440143 & 710721 \\
\hline CWW & 112 & 440202 & 710729 \\
\hline CWW & 113 & 440202 & 710729 \\
\hline CWW & 114 & 440121 & 710818 \\
\hline CWW & 115 & 440125 & 710750 \\
\hline CWW & 116 & 440128 & 710717 \\
\hline CWW & 117 & 440128 & 710717 \\
\hline CWW & 118 & 440101 & 710659 \\
\hline CWW & 119 & 440025 & 710731 \\
\hline CWW & 120 & 440022 & 710701 \\
\hline CWW & 121 & 440022 & 710701 \\
\hline CWW & 122 & 435920 & 710753 \\
\hline CWW & 123 & 435916 & 710720 \\
\hline CWW & 124 & 435846 & 710800 \\
\hline CWW & 125 & 440040 & 710319 \\
\hline CWW & 126 & 440302 & 710748 \\
\hline CWW & 127 & 440300 & 710738 \\
\hline CWW & 128 & 440305 & 710729 \\
\hline CWW & 129 & 440236 & 710749 \\
\hline CWW & 130 & 440234 & 710748 \\
\hline$C W w$ & 131 & 440234 & 710748 \\
\hline CWW & 132 & 440233 & 710741 \\
\hline CWW & 133 & 440223 & 710729 \\
\hline CWW & 134 & 440122 & 710709 \\
\hline CWW & 135 & 440122 & 710709 \\
\hline CWW & 136 & 440117 & 710644 \\
\hline CWW & 137 & 440121 & 710108 \\
\hline$C W W$ & 138 & 440051 & 710014 \\
\hline CWW & 139 & 440044 & 710800 \\
\hline CWW & 140 & 435958 & 710729 \\
\hline & 141 & 435954 & 710720 \\
\hline$C W W$ & 142 & 440038 & 71075 \\
\hline
\end{tabular}

$\begin{array}{lrlll}\text { ECW } & 1 & 435456 & 710423 & \text { Waukeela Camp } \\ \text { ECW } & 2 & 435453 & 710403 & \text { Hennigan } \\ \text { ECW } & 3 & 435347 & 710405 & \text { Sundman } \\ \text { ECW } & 4 & 435350 & 710405 & \\ \text { ECW } & 5 & 435350 & 710421 & \text { Lee } \\ & & & & \\ \text { ECW } & 6 & 435417 & 710419 & \text { Heath } \\ \text { ECW } & 7 & 435343 & 710411 & \text { Gospodarek } \\ \text { ECW } & 8 & 435349 & 710355 & \text { Hil1 } \\ \text { ECW } & 9 & 435341 & 710356 & \text { Larson } \\ \text { ECW } & 10 & 435425 & 710412 & \text { Philbrick } \\ & & & & \\ \text { ECW } & 11 & 435250 & 710501 & \text { Reynolds } \\ \text { ECW } & 12 & 435522 & 710529 & \text { Bungeroth } \\ \text { ECW } & 13 & 435136 & 710441 & \text { Latour } \\ \text { ECW } & 14 & 435441 & 710342 & \text { Lance } \\ \text { ECW } & 15 & 435422 & 710534 & \text { Lesser } \\ & & & & \\ \text { ECW } & 16 & 435430 & 710239 & \text { D'Angelo } \\ \text { ECW } & 17 & 435446 & 710321 & \text { Wilson } \\ \text { ECW } & 18 & 435133 & 710420 & \text { Graf } \\ \text { ECW } & 19 & 435403 & 710420 & \text { Guerringue } \\ \text { ECW } & 20 & 435140 & 710328 & \text { Stewart }\end{array}$

Conway - - Continued

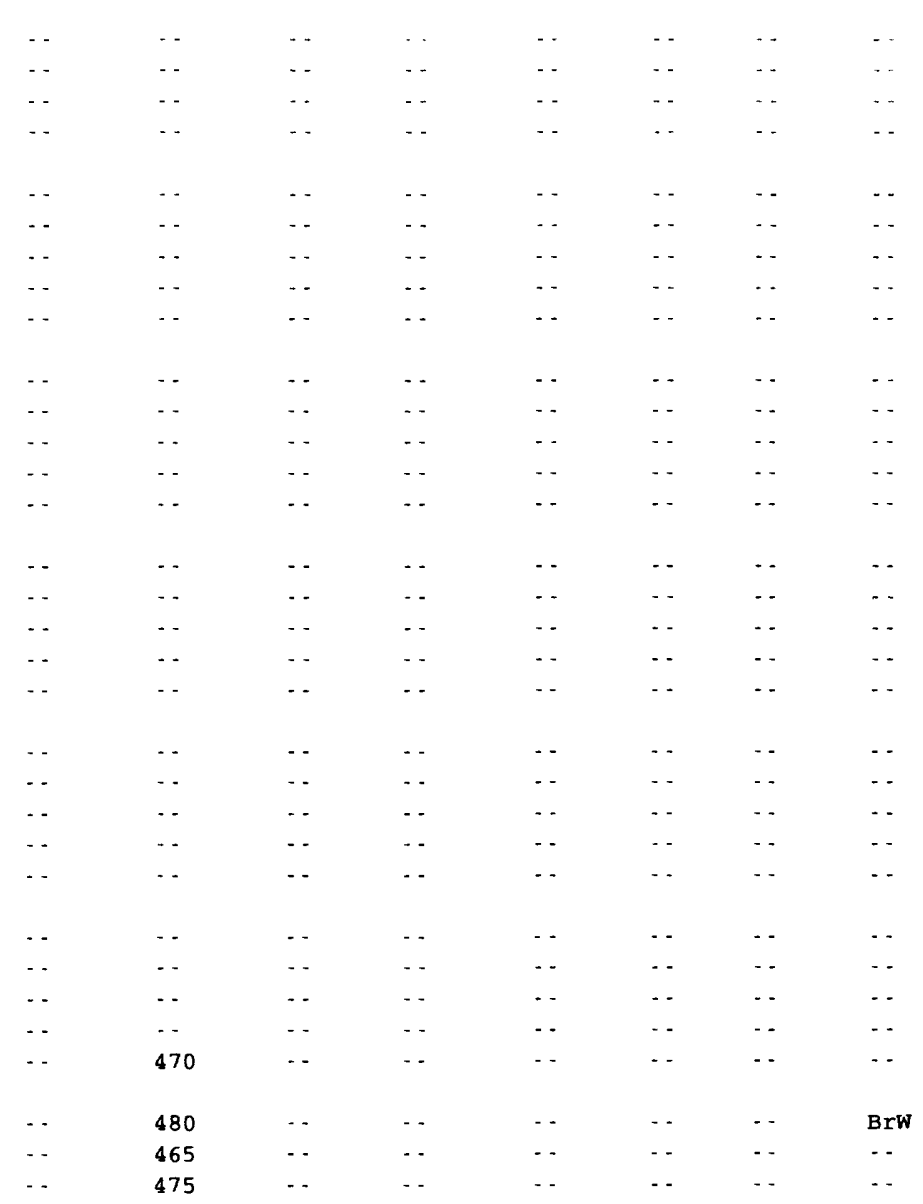

Baton

\begin{tabular}{|c|c|c|c|c|c|c|c|}
\hline$\cdots$ & 475 & 36 & 7.5 & C & 0 & $\cdots$ & Dug \\
\hline$\cdots$ & 500 & 2 & 26 & $\mathrm{~s}$ & $T$ & $\cdots$ & Dvn \\
\hline 1979 & 485 & . & - & - & $x$ & $\cdots$ & BrW \\
\hline 1978 & 480 & - - & - & - & $x$ & $\cdots$ & BrW \\
\hline 1984 & 615 & - & 20 & - & $x$ & - & BrW \\
\hline 1986 & 490 & $\cdots$ & 60 & $\cdots$ & $x$ & $\cdots$ & BrW \\
\hline 1987 & 470 & - & 64 & $\cdots$ & $\cdots$ & $\cdots$ & $\cdots$ \\
\hline 1988 & 530 & -- & 42 & $\cdots$ & $\mathrm{x}$ & $\cdots$ & BrW \\
\hline 1988 & 540 & . & 71 & $\cdots$ & $\mathrm{x}$ & - & BrW \\
\hline 1989 & 545 & $\cdots$ & 33 & $\cdots$ & $x$ & $\cdots$ & BrW \\
\hline 1985 & 470 & - & - & $\cdots$ & $\cdots$ & $\cdots$ & Dug \\
\hline 1985 & 800 & - & 49 & - & $x$ & $\cdots$ & BrW \\
\hline 1986 & 660 & $\cdots$ & 83 & - & $x$ & $\cdots$ & BrW \\
\hline 1988 & 540 & - & 39 & $\cdots$ & $x$ & $\cdots$ & Brw \\
\hline 1988 & 950 & - & 40 & - & $x$ & $\cdots$ & BrW \\
\hline 1988 & 860 & - & 40 & $\cdots$ & $x$ & - & BrW \\
\hline 1988 & 560 & - & 50 & - & $x$ & $\cdots$ & BrW \\
\hline 1988 & 770 & $\cdots$ & 201 & - & $x$ & - & Brw \\
\hline 1988 & 520 & $\cdots$ & 139 & $\cdots$ & $x$ & $\cdots$ & BrW \\
\hline 1989 & 750 & - & 24 & - & $x$ & - & BrW \\
\hline
\end{tabular}




\begin{tabular}{|c|c|c|c|c|c|c|c|c|}
\hline $\begin{array}{l}\text { Local } \\
\text { site } \\
\text { number }\end{array}$ & $\begin{array}{l}\text { Water } \\
\text { level } \\
\text { depth } \\
\text { (feet) }\end{array}$ & $\begin{array}{l}\text { Date } \\
\mathrm{mm} / \mathrm{dd} / \mathrm{yy}\end{array}$ & Use & $\begin{array}{l}\text { Maximum } \\
\text { well } \\
\text { yield } \\
\text { (gallons } \\
\text { per } \\
\text { minute) }\end{array}$ & $\begin{array}{l}\text { Draw- } \\
\text { down } \\
\text { (feet) }\end{array}$ & $\begin{array}{l}\text { Specific } \\
\text { capacity } \\
\text { (gallons per } \\
\text { minute } \\
\text { per } \\
\text { foot) }\end{array}$ & $\begin{array}{l}\text { Pumping } \\
\text { period } \\
\text { (hours) }\end{array}$ & Remarks \\
\hline
\end{tabular}

Chatham--Continued

\begin{tabular}{|c|c|c|c|c|c|}
\hline CKB & 6 & $\cdots$ & $\cdots$ & U & $\cdots$ \\
\hline CKB & 7 & $\cdots$ & $\cdots$ & $\mathrm{U}$ & $\cdots$ \\
\hline CKS & 1 & $\cdots$ & $\cdots$ & $S$ & $\cdots$ \\
\hline$C K W$ & 1 & 4 & $08-12 \cdot 91$ & H & $\cdots$ \\
\hline CKW & 2 & 3.7 & $08-12-91$ & $\mathrm{H}$ & $\cdots$ \\
\hline CKW & 3 & 9.7 & $08-12-91$ & H & $\cdots$ \\
\hline CKW & 4 & $\cdots$ & $\cdots$ & H & $\cdots$ \\
\hline CKW & 5 & 0.5 & $08-12-91$ & $\mathbf{H}$ & $\cdots$ \\
\hline
\end{tabular}

CK

CKW

$\begin{array}{ccccc}. & \ldots & \text { H S } & \ldots & \ldots \\ - & \ldots & \text { S I } & \ldots & \ldots \\ 6.4 & 08-12-91 & \text { H } & \ldots & \ldots \\ 21.9 & 08-04-92 & \text { O } & \ldots & \ldots \\ 24.7 & 08-04-92 & \text { O } & \ldots & \ldots\end{array}$

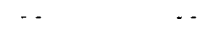

CKW 8

CKW 9

CKW 10

$24.7 \quad 08.04-92$

$$
\text { - } \quad \cdot
$$

CKW 11

$37.5 \quad 08-04-9$

-.

$\cdots$
$-07-85$

$10 \cdot 08-85$

$$
0.5
$$$$
\text { - }
$$

CKW 13

CKW 14

5

20

- $08-85$

CKW 16

$$
\begin{array}{ll}
\ldots & \ldots \\
\cdots & \ldots
\end{array}
$$$$
\text { -. }
$$$$
10
$$

-.

-

-

$$
\text { - }
$$$$
\begin{array}{ll}
- & \\
&
\end{array}
$$

$$
\cdots
$$

-

75

75

-

$11 \cdot 22 \cdot 90$

15

$01 \cdot 05 \cdot 91$

-.

35

20

-

$08-05 \cdot 91$

..

$10-11 \cdot 91$

$$
\text { -. }
$$$$
2.3
$$$$
15
$$$$
\begin{array}{cc}
2.5 & \cdots \\
150 & \cdots
\end{array}
$$$$
\begin{array}{r}
5 \\
6
\end{array}
$$$$
30
$$

$$
5.5 \quad \cdots
$$

$$
\begin{array}{r}
5 . \\
60
\end{array}
$$$$
\begin{array}{r}
60 \\
20
\end{array}
$$

60

$\begin{array}{cc}40 & \\ 2.5 & \\ 30 & \\ 10 & \\ 3 & \\ 3 & \\ 10 & \\ 3 & \\ 4 & \\ 2.5 & \cdots\end{array}$

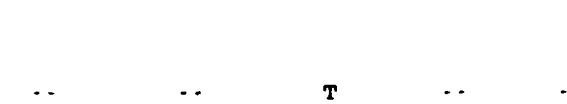

$\cdots$
$\cdots$
$\cdots$
$\cdots$

$$
\begin{aligned}
& \cdots \\
& \cdots \\
& \cdots
\end{aligned}
$$$$
\begin{array}{lll}
. . & \cdots & \text { USGS }
\end{array}
$$$$
\begin{array}{lll}
. & . & \text { USGS }
\end{array}
$$$$
\begin{array}{llll}
\ldots & \ldots & \text { USGS }
\end{array}
$$$$
\text { ck }
$$

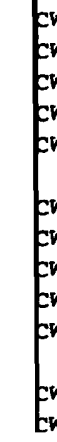

-.

$04-19-80$ $06-18-77$

$07-09 \cdot 83$

$\begin{array}{ll}\cdots & \cdots \\ \cdots & \end{array}$

$11 \cdot 19 \cdot 74$ $05-21-66$ $01-26 \cdot 83$

-

$05-20-73$$$
\begin{array}{ll}
\cdots & 1 \\
\cdots & \cdots \\
\cdots & 1 \\
\cdots & 2
\end{array}
$$

- 1$$
\cdots
$$$$
-
$$$$
\cdots
$$

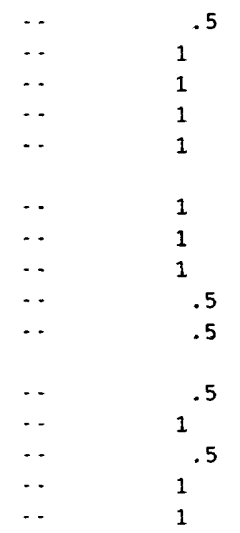

Conway

USGS

Project well (Johnson and others, 1987) 
Table 1-1. Description of selected wells, borings, and springs in the Saco and Ossipee River Basins, east-central

\begin{tabular}{|c|c|c|c|c|c|c|c|c|c|c|c|}
\hline $\begin{array}{l}\text { Local } \\
\text { site } \\
\text { number }\end{array}$ & $\begin{array}{l}\text { Lat- } \\
\text { itude }\end{array}$ & $\begin{array}{c}\text { Long- } \\
\text { itude }\end{array}$ & Owner or user & $\begin{array}{l}\text { Year } \\
\text { com- } \\
\text { pleted }\end{array}$ & $\begin{array}{l}\text { Alti- } \\
\text { tude } \\
\text { above } \\
\text { sea } \\
\text { level } \\
\text { (feet) }\end{array}$ & $\begin{array}{l}\text { Dia- } \\
\text { meter } \\
\text { of } \\
\text { well } \\
\text { casing } \\
\text { (inches) }\end{array}$ & $\begin{array}{l}\text { Depth } \\
\text { to } \\
\text { bottom } \\
\text { of } \\
\text { casing } \\
\text { (feet) }\end{array}$ & $\begin{array}{l}\text { Casing } \\
\text { material }\end{array}$ & $\begin{array}{l}\text { Type } \\
\text { of } \\
\text { finish }\end{array}$ & $\begin{array}{l}\text { Depth } \\
\text { to } \\
\text { bottom } \\
\text { of open } \\
\text { section } \\
\text { (feet) }\end{array}$ & $\begin{array}{c}\text { Type } \\
\text { of } \\
\text { site }\end{array}$ \\
\hline
\end{tabular}

\begin{tabular}{|c|c|c|c|}
\hline WW & 55 & & 710817 \\
\hline WW & 56 & 440155 & 710816 \\
\hline WW & 57 & 440156 & 710914 \\
\hline WW & 58 & 440152 & 10815 \\
\hline CWW & 59 & 440148 & 710811 \\
\hline CWW & 60 & 440144 & 10812 \\
\hline CWW & 61 & 440139 & 10800 \\
\hline CWW & 62 & 440125 & 710755 \\
\hline CWW & 63 & 440121 & 710801 \\
\hline CWW & 64 & 440107 & 710752 \\
\hline CWW & 65 & 440047 & 710759 \\
\hline CWW & 66 & 435815 & \\
\hline CWW & 67 & & \\
\hline CWW & 68 & 435958 & 710749 \\
\hline CWW & 69 & 440430 & \\
\hline CWW & 70 & & \\
\hline CWW & 71 & 435939 & 710644 \\
\hline CWW & 72 & 435900 & 710549 \\
\hline CWW & 73 & 435919 & \\
\hline CWW & 74 & 435928 & 510 \\
\hline CWW & 75 & 435 & 108 \\
\hline CWW & 76 & 440 & \\
\hline CWW & 77 & 440 & \\
\hline CWW & 78 & & \\
\hline CWW & 79 & 435 & \\
\hline CWW & 80 & 435 & 710242 \\
\hline CWW & 81 & 440014 & 710242 \\
\hline CWW & 82 & 440217 & 710829 \\
\hline CWW & 83 & & \\
\hline CWW & 84 & 435 & \\
\hline CWW & 85 & 44000 & \\
\hline CWW & 86 & 440 & \\
\hline CWW & 87 & 440120 & 10749 \\
\hline WW & 88 & & \\
\hline CWW & 89 & & \\
\hline CWW & 90 & 440 & \\
\hline CWW & 91 & 440 & 17 \\
\hline CWW & 92 & 440403 & 710917 \\
\hline VW & & & \\
\hline CWW & 94 & 440329 & 300 \\
\hline CWW & 95 & 440326 & \\
\hline CWW & 96 & & \\
\hline CWW & 97 & 440301 & 710810 \\
\hline wi & & & \\
\hline CWW & 99 & & 10825 \\
\hline $\mathrm{CWW}$ & & & \\
\hline CWW & & & \\
\hline CWW & 102 & 435854 & 710803 \\
\hline & & & \\
\hline & 106 & & \\
\hline & 107 & & \\
\hline CWW & 108 & 440225 & 710747 \\
\hline & & & \\
\hline & & & \\
\hline
\end{tabular}

Conway - Continued

$\begin{array}{llllllll}- & 510 & \ldots & \ldots & \ldots & \ldots & -. & \text { BrW }\end{array}$

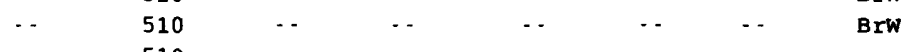

$\begin{array}{llllllll}- & 510 & \ldots & \ldots & \ldots & \ldots & \ldots & \ldots\end{array}$

$\begin{array}{llllllll}- & 515 & \ldots & \ldots & \ldots & \ldots & \ldots & \ldots\end{array}$

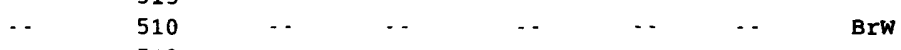

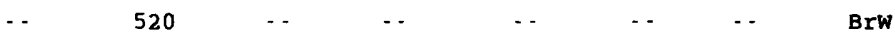

$\begin{array}{llllllll}- & 458 & \ldots & \ldots & -. & \ldots & -. & - \\ . & 499 & . & \ldots & -. & \ldots & . . & \text { Brw }\end{array}$

$\begin{array}{llllllll}. & 500 & \ldots & \ldots & \ldots & \ldots & \ldots & \text { BrW }\end{array}$

$\begin{array}{llllllll}. & 470 & . & \ldots & \ldots & \ldots & . & \text { BrW }\end{array}$

$\begin{array}{cccccccc}-\cdots & 470 & - & \cdots & - & - & - & \text { BrW } \\ 1992 & 465 & 2 & 47.5 & \text { P } & \text { S } & 49.5 & \text { Bor }\end{array}$

$\begin{array}{llllllll}1992 & 430 & 2 & 66.4 & \text { P } & \text { S } & 68.4 & \text { Bor }\end{array}$

$\begin{array}{llllllll}. . & 510 & . & \ldots & . & \ldots & \ldots & \text { Brw }\end{array}$

$\begin{array}{llllllll}1992 & 435 & 2 & 55 & \text { P } & \text { S } & 57 & \text { Bor }\end{array}$

$\begin{array}{llllllll}1992 & 435 & 2 & 16 & \text { P } & \text { S } & 18 & \text { Bor }\end{array}$

$\begin{array}{llllllll}\ldots & 480 & \ldots & \ldots & . & \ldots & \ldots & \text { Brw } \\ .- & 555 & \ldots & \ldots & \ldots & \ldots & . & \text { BrW }\end{array}$

$\begin{array}{llllllll}. & 420 & \ldots & \ldots & \ldots & \ldots & \ldots & \text { BrW }\end{array}$

$\begin{array}{llllllll}- & 450 & . & \ldots & \ldots & \ldots & \ldots & \text { BrW }\end{array}$

$\begin{array}{llllllll}- & 465 & \ldots & \ldots & \ldots & \ldots & \ldots & \text { BrW }\end{array}$

$\begin{array}{llllllll}- & 445 & . & \ldots & \ldots & \ldots & \ldots & \text { BrW }\end{array}$

$\begin{array}{llllllll}. & 455 & \ldots & \ldots & \ldots & \ldots & \ldots & \text { BrW }\end{array}$

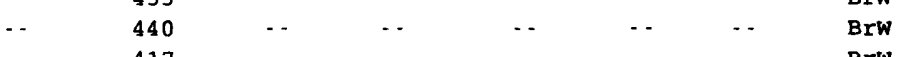

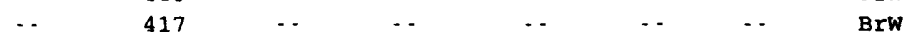

$\begin{array}{llllllll}-. & 465 & -. & . & \ldots & \ldots & \ldots & \text { BrW } \\ . . & 520 & -. & \ldots & \ldots & \ldots & \ldots & \text { BrW }\end{array}$

$\begin{array}{llllllll}. & 420 & \ldots & \ldots & \ldots & \ldots & \ldots & \text { BrW }\end{array}$

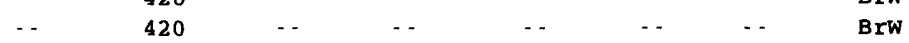

$\begin{array}{llllllll}. & 440 & . & \ldots & \ldots & \ldots & \ldots & \text { Brw } \\ . . & 415 & \ldots & \ldots & \ldots & \ldots & \ldots & \text { Brw }\end{array}$

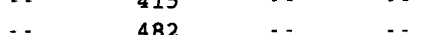

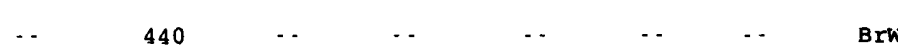

\begin{tabular}{llllllll}
\hline & 475 & $\ldots$ & $\ldots$ & $\ldots$ & $\ldots$ &. & BrW
\end{tabular}

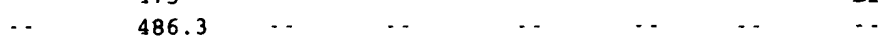

$\begin{array}{llllllll}. & 484.5 & \ldots & . & . & \ldots & . & \ldots \\ . & 484.3 & \ldots & \ldots & \ldots & \ldots & \ldots & \ldots\end{array}$

$\begin{array}{llllll} & & & & & \\ & 499.4 & \ldots & \ldots & \ldots & \ldots\end{array}$

$\begin{array}{llllllll}- & 499.4 & . & - & - & \ldots & . & \ldots \\ - & 527.9 & \ldots & \ldots & -. & \ldots & \ldots & \ldots\end{array}$

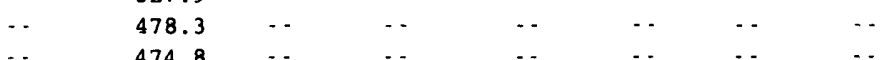

$\begin{array}{llllllll}\ldots & 474.8 & . & \ldots & \ldots & \ldots & \ldots & \ldots \\ .- & 469 & . & \ldots & \ldots & \ldots & \ldots & .\end{array}$

66 Geohydrology and Water Quality of Stratified-Drift Aquifers in the Saco and Ossipee River Basins, East-Central New Hampshire 


\begin{tabular}{|c|c|c|c|c|c|c|c|c|}
\hline $\begin{array}{l}\text { Local } \\
\text { site } \\
\text { number }\end{array}$ & $\begin{array}{l}\text { Water } \\
\text { level } \\
\text { depth } \\
\text { (feet) }\end{array}$ & $\begin{array}{l}\text { Date } \\
\mathrm{mm} / \mathrm{dd} / \mathrm{yy}\end{array}$ & Use & $\begin{array}{l}\text { Maximum } \\
\text { well } \\
\text { yield } \\
\text { (gallons } \\
\text { per } \\
\text { minute) }\end{array}$ & $\begin{array}{l}\text { Draw- } \\
\text { down } \\
\text { (feet) }\end{array}$ & $\begin{array}{c}\text { Specific } \\
\text { capacity } \\
\text { (gallons per } \\
\text { minute } \\
\text { per } \\
\text { foot) }\end{array}$ & $\begin{array}{l}\text { Pumping } \\
\text { period } \\
\text { (hours) }\end{array}$ & Remarks \\
\hline
\end{tabular}

Conway - Continued

\begin{tabular}{|c|c|c|c|}
\hline CWW & 111 & - & - \\
\hline CWW & 112 & $\cdots$ & - \\
\hline CWW & 113 & $\cdots$ & $\cdots$ \\
\hline CWW & 114 & $\cdots$ & $\cdots$ \\
\hline CWW & 115 & $\cdots$ & $\cdots$ \\
\hline CWW & 116 & $\cdots$ & $\cdots$ \\
\hline CWW & 117 & -- & $\cdots$ \\
\hline CWW & 118 & $\cdots$ & $\cdots$ \\
\hline CWW & 119 & $\cdots$ & -- \\
\hline CWW & 120 & $\cdots$ & -- \\
\hline CWW & 121 & $\cdots$ & - \\
\hline CWW & 122 & -- & -- \\
\hline CWW & 123 & $\cdots$ & $\cdots$ \\
\hline CWW & 124 & - & - \\
\hline CWW & 125 & $-\cdot$ & -- \\
\hline CWW & 126 & -- & -- \\
\hline $\mathrm{CWW}$ & 127 & $\cdots$ & $-\cdot$ \\
\hline CWW & 128 & - & - \\
\hline CWW & 129 & $\cdots$ & $\cdots$ \\
\hline CWW & 130 & -- & - \\
\hline CWW & 131 & - & $\cdots$ \\
\hline CWW & 132 & - & $-\cdot$ \\
\hline CWW & 133 & -- & - \\
\hline CWW & 134 & $\cdots$ & - \\
\hline CWW & 135 & $\cdots$ & $\cdots$ \\
\hline CWW & 136 & $\cdot-$ & -- \\
\hline CWW & 137 & -- & - \\
\hline CWW & 138 & - - & - - \\
\hline CWW & 139 & 15 & $06-01-65$ \\
\hline CWW & 140 & 30 & $04-05-82$ \\
\hline CWW & 141 & 40 & $01-05-83$ \\
\hline CWW & 142 & 15 & $06-11-76$ \\
\hline
\end{tabular}

Project well (Johnson and others, 1987) Project well (Johnson and others, 1987) Project we11 (Johnson and others, 1987) Project wel1 (Johnson and others, 1987)

Project well (Johnson and others, 1987) Project well (Johnson and others, 1987) Project well (Johnson and others, 1987) project well (Johnson and others, 1987) Project well (Johnson and others, 1987)

Project we11 (Johnson and others, 1987) Project well (Johnson and others, 1987) Project we11 (Johnson and others, 1987) Project well (Johnson and others, 1987) Project well (Johnson and others, 1987)

Project well (Johnson and others, 1987) Project well (Johnson and others, 1987) Project well (Johnson and others, 1987) Project well (Johnson and others, 1987) Project well (Johnson and others, 1987)

Project we11 (Johnson and others, 1987) Project we11 (Johnson and others, 1987) project well (Johnson and others, 1987) Project well (Johnson and others, 1987) Project well (Johnson and others, 1987)

Project well (Johnson and others, 1987) Project well (Johnson and others, 1987) Project well (Johnson and others, 1987) project well (Johnson and others, 1987)

\section{Baton}

ECW

ECW

ECW

ECW

ECW

ECW

ECW

ECW

ECW

ECW 10

ECW 11

ECW 12

ECW 13

ECW 14

ECW 15

ECW $16 \quad 75$

ECW $17 \quad 50$

ECW $18 \quad 80$

ECW 19

ECW 20
$08-05-91$

$08-05-91$

08-05-91

-.

$-$

-

-

-

-

$01-04-89$

$09-20-85$

$10-18-85$

-

$06-17-88$

$05-13-88$

$09-19-88$

$08-11-88$

-.

$07-21-89$
H T

H S

H

$\mathrm{H}$

$\mathrm{H}$
$\mathrm{H}$

$\mathrm{H}$

$\mathrm{H}$

$\mathrm{H}$

$\mathrm{H}$
$\mathrm{H}$

$\mathrm{H}$

$\mathrm{H}$

$\mathrm{H}$
$\mathrm{H}$
$\mathrm{H}$
$\mathrm{H}$
$\mathrm{H}$

\begin{tabular}{|c|c|}
\hline$\cdots$ & $\cdots$ \\
\hline - - & - - \\
\hline - & 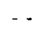 \\
\hline$\therefore$ & - \\
\hline$\ldots$ & .5 \\
\hline$\therefore$ & 1 \\
\hline - - & 1 \\
\hline- & 1 \\
\hline$\cdots$ & 1 \\
\hline$\cdots$ & 1.5 \\
\hline$\ldots$ & $\ldots$ \\
\hline$\ldots$ & .5 \\
\hline - & .8 \\
\hline- & 1 \\
\hline- & 1 \\
\hline- & .5 \\
\hline- & 1 \\
\hline-- & 1 \\
\hline$\cdots$ & 1 \\
\hline . & 1 \\
\hline
\end{tabular}

Wel1 went Dry $(8 / 4 / 91)$ 
Table 1-1. Description of selected wells, borings, and springs in the Saco and Ossipee River Basins, east-central

\begin{tabular}{|c|c|c|c|c|c|c|c|c|c|c|c|}
\hline $\begin{array}{l}\text { Local } \\
\text { site } \\
\text { number }\end{array}$ & $\begin{array}{l}\text { Lat- } \\
\text { itude }\end{array}$ & $\begin{array}{l}\text { Long- } \\
\text { itude }\end{array}$ & Owner or user & $\begin{array}{l}\text { Year } \\
\text { com- } \\
\text { pleted }\end{array}$ & $\begin{array}{c}\text { Alti- } \\
\text { tude } \\
\text { above } \\
\text { sea } \\
\text { level } \\
\text { (feet) }\end{array}$ & $\begin{array}{l}\text { Dia- } \\
\text { meter } \\
\text { of } \\
\text { well } \\
\text { casing } \\
\text { (inches) }\end{array}$ & $\begin{array}{l}\text { Depth } \\
\text { to } \\
\text { bottom } \\
\text { of } \\
\text { casing } \\
\text { (feet) }\end{array}$ & $\begin{array}{l}\text { Casing } \\
\text { material }\end{array}$ & $\begin{array}{l}\text { Type } \\
\text { of } \\
\text { finish }\end{array}$ & $\begin{array}{l}\text { Depth } \\
\text { to } \\
\text { bottom } \\
\text { of open } \\
\text { section } \\
\text { (feet) }\end{array}$ & $\begin{array}{c}\text { Type } \\
\text { of } \\
\text { site }\end{array}$ \\
\hline
\end{tabular}

$\begin{array}{lllll}\text { ECW } & 21 & \mathbf{4 3 5 4 0 1} & 710110 & \text { Whicher } \\ \text { ECW } & 22 & \mathbf{4 3 5 5 1 0} & 710255 & \text { L. Kande1 } \\ \text { ECW } & 23 & \mathbf{4 3 5 1 4 1} & 710326 & \text { Stavis } \\ \text { ECW } & 24 & \mathbf{4 3 5 3 5 4} & 710234 & \text { P1iskin } \\ \text { ECW } & 25 & 435359 & 710133 & \text { E1a }\end{array}$

$\begin{array}{lllll}\text { EFA } & 1 & 434248 & 710407 & \\ & & & & \\ \text { EFB } & 1 & 434746 & 710325 & \text { NHDO } \\ \text { EFB } & 2 & 434731 & 710157 & \text { NHDO }\end{array}$

$\begin{array}{lllll}\text { EFB } & 3 & 434437 & 710520 & \text { NHDOT }\end{array}$

EFS $\quad 1 \quad 434120 \quad 710047$

EFW $\quad 2 \quad 434734 \quad 710459$

$\begin{array}{llll}\text { EFW } & 3 & 434757 & 7102 \\ \text { EFW } & 4 & 434736 & 7104\end{array}$

$\begin{array}{llll}\text { EFW } & 5 & 434329 & 710415\end{array}$

$\begin{array}{llll}\text { EFW } & 6 & 434225 & 710136\end{array}$

$\begin{array}{lrrr}\text { EFW } & 7 & 434120 & 710049 \\ \text { EFW } & 8 & 434120 & 710048 \\ \text { EFW } & 9 & 434608 & 710640 \\ \text { EFW } & 10 & 434225 & 710136\end{array}$

EFW $11 \quad 434544 \quad 7105$

$\begin{array}{lllll}\text { EFW } & 12 & 434403 & 710545 & \text { Piekut } \\ \text { EFW } & 13 & \mathbf{4 3 4 3 5 9} & 710523 & \text { Moison } \\ \text { EFW } & 14 & 434517 & 710003 & \text { Chapman } \\ \text { EFW } & 15 & 434401 & 710545 & \text { Vivenzio }\end{array}$

EFW $\quad 16 \quad 434407 \quad 710029$ Lane

$\begin{array}{llll}\text { EFW } & 17 & 434422 & 710524\end{array}$

EFW $\quad \begin{array}{lll}18 & 434752 & 710225\end{array}$

EFW $\quad \begin{array}{llll}19 & 434522 & 710550\end{array}$

$\begin{array}{llll}\text { EFW } & 20 & \mathbf{4 3 4 5 2 2} & 705951\end{array}$

$\begin{array}{llll}\text { EFW } & 21 & 434458 & 710013\end{array}$

$\begin{array}{llll}\text { EFW } & 22 & 434358 & 710439\end{array}$

$\begin{array}{llll}\text { EFW } & 23 & 434110 & 710035\end{array}$

$\begin{array}{llll}\text { EFW } & 24 & 434430 & 710523\end{array}$

$\begin{array}{llll}\text { EFW } & 25 & 434752 & 710239\end{array}$

$\begin{array}{llll}\text { EFW } & 26 & \mathbf{4 3 4 4 3 2} & 710524\end{array}$

$\begin{array}{llll}\text { EFW } & 27 & 434204 & 705947\end{array}$

$\begin{array}{llll}\text { EFW } & 28 & 434717 & 705938\end{array}$

$\begin{array}{llll}\text { EFW } & 29 & 434749 & 710222\end{array}$

$\begin{array}{llll}\text { EFW } & 30 & 434649 & 710231\end{array}$

EFW $\quad 31 \quad 434649 \quad 710233$

$\begin{array}{llll}\text { EFW } & 32 & 434648 & 7103\end{array}$

$\begin{array}{llll}\text { EFW } & 33 & 434357 & 7104\end{array}$

EFW $34 \quad 434646 \quad 710417$

$\begin{array}{llll}\text { EFW } & 35 & 434542 & 710549\end{array}$

$\begin{array}{lll}\text { EFW } & 36 & 434235\end{array}$

$\begin{array}{llll}\text { EFW } & 37 & 434319 & 710054\end{array}$

$\begin{array}{llll}E F W & 38 & 434524 & 705918\end{array}$

$\begin{array}{llll}\text { EFW } & 39 & 434348 & 710008\end{array}$

EFW $\quad 40 \quad \mathbf{4 3 4 4 3 4} \quad 710519$

EFW $\quad 41 \quad 434239 \quad 705929$

EFW $\quad 42 \quad 434630 \quad 710529$
English

Robert Burns

Camp Marist

Fields

Philip Blay

Merrow, Harry, C

Fields

pinkston

Barrett

Ke11y

Cragin

Molloy

Miller

Wright

Woods

Thurston

Simeal Properties

Suter

Sargent

Highwatch Const

Highwatch Const

Highwatch Learning Center

Smith

Troy

Soule

Lebe 1

Edwards

Clansey

Edwards

Clark

Forbush
Edwards

Baton--Continued

$\begin{array}{rrrr}1989 & 1,060 & \cdots & 40 \\ 1989 & 800 & \cdots & 39 \\ 1990 & 740 & \cdots & 42 \\ 1990 & 940 & \cdots & 39 \\ 1990 & 1,040 & \cdots & 143\end{array}$

Bffingham

\begin{tabular}{|c|c|c|c|c|c|c|}
\hline$\cdots$ & 530 & $\cdots$ & - & $\cdots$ & - & $\cdots$ \\
\hline - - & 389 & $\ldots$ & - - & - & $\cdots$ & - . \\
\hline .. & 381 & - & . & - & $\cdots$ & - \\
\hline .. & 425 & .. & - & $\ldots$ & . & $\ldots$ \\
\hline
\end{tabular}

$\begin{array}{cc}1991 & 415 \\ -- & 39 \\ 1981 & 41 \\ -- & 450 \\ 1977 & 535 \\ 1986 & 495 \\ -\cdots & 495 \\ 1991 & 420 \\ 1985 & 530 \\ 1985 & 435 \\ 1985 & 510 \\ 1986 & 530 \\ 1986 & 465 \\ 1986 & 510 \\ 1986 & 410\end{array}$

$\begin{array}{rr}6 & 150 \\ 2 & \\ 36 & \\ 36 & \end{array}$

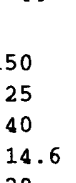

$\mathrm{sp}$

1987

1987

1988

1988

1988

1989

1989

1991

1990

1990

1990

1984

1987

1984

$1984 \quad 980$

$1984 \quad 660$

1985

1985

1985

1985

440

520

440

510

$1985 \quad 570$

1985

1985

1985

1986

1986

440

$-\cdot \quad 126$

$\begin{array}{llll}- & \cdots & \cdots & \text { Sp } \\ \text { S } & \text { X } & \cdots & \text { BrW } \\ \text { S } & \text { T } & \cdots & \text { Dvn } \\ \text { S } & \text { G } & \cdots & - \\ \text { C } & 0 & \cdots & \text { Dug } \\ \text { C } & \text { O } & \cdots & \text { Dug }\end{array}$

$\begin{array}{llllll}- & 16 & \ldots & \mathrm{X} & \ldots & \mathrm{BrW}\end{array}$

$\begin{array}{llllll}36 & 12 & C & 0 & - & \text { Dug }\end{array}$

$\begin{array}{llllll}2 & 72 & P & S & 74.5 & \text { Bor }\end{array}$

$\begin{array}{llllll}- & - & \ldots & \mathrm{x} & - & \mathrm{Brh} \\ - & -. & \ldots & \mathrm{s} & \ldots & \ldots\end{array}$

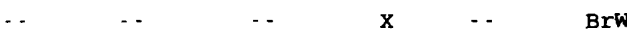

$\begin{array}{llllll}- & 213 & \ldots & x & \ldots & \text { BrW }\end{array}$

$\begin{array}{llllll}- & 65 & \ldots & \mathrm{X} & \ldots & \text { BrW }\end{array}$

$\begin{array}{llllll}- & 115 & \ldots & \mathrm{X} & \ldots & \mathrm{BrW}\end{array}$

$\begin{array}{llllll} & 30 & \ldots & \mathrm{x} & \ldots & \text { BrW }\end{array}$

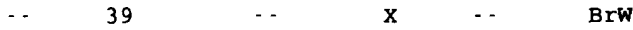

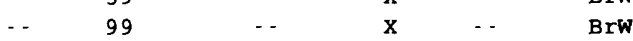

$\begin{array}{llllll}- & 36 & \ldots & \mathrm{x} & \ldots & \mathrm{BrW}\end{array}$

$\begin{array}{llllll}\ldots & 40 & \ldots & \mathrm{x} & \ldots & \mathrm{BrW}\end{array}$

$\begin{array}{llllll}- & 60 & \ldots & \mathrm{x} & - & \end{array}$

$\begin{array}{lllllll}- & 79 & \ldots & \mathrm{x} & \ldots & \mathrm{BrW}\end{array}$

$\begin{array}{llllll}79 & 69 & \ldots & \mathrm{x} & \ldots & \mathrm{BrW}\end{array}$

-. 68

- 80

-. 147

-. 53

-.

$-\quad 40$

-. 40

128

.. 60

- 159

- 26

151

․ 70

180

-. 40

$\begin{array}{rr}- & 40 \\ - & 92 \\ - & 148\end{array}$

Highwatch Learning Center 


\begin{tabular}{|c|c|c|c|c|c|c|c|c|}
\hline $\begin{array}{l}\text { Local } \\
\text { site } \\
\text { number }\end{array}$ & $\begin{array}{l}\text { Water } \\
\text { level } \\
\text { depth } \\
\text { (feet) }\end{array}$ & $\begin{array}{l}\text { Date } \\
\mathrm{mm} / \mathrm{dd} / \mathrm{yy}\end{array}$ & Use & $\begin{array}{c}\text { Maximum } \\
\text { well } \\
\text { yield } \\
\text { (gallons } \\
\text { per } \\
\text { minute) }\end{array}$ & $\begin{array}{l}\text { Draw- } \\
\text { down } \\
\text { (feet) }\end{array}$ & $\begin{array}{l}\text { Specific } \\
\text { capacity } \\
\text { (gallons per } \\
\text { minute } \\
\text { per } \\
\text { foot) }\end{array}$ & $\begin{array}{l}\text { Pumping } \\
\text { period } \\
\text { (hours) }\end{array}$ & Remarks \\
\hline
\end{tabular}

Eaton--Continued

$\begin{array}{lcccccccc}\text { ECW } & 21 & 1 & 08-02-89 & \text { H } & 0.8 & \ldots & \ldots & 1 \\ \text { ECW } & 22 & \ldots & -- & \text { H } & 5 & \ldots & \ldots & 1 \\ \text { ECW } & 23 & 16 & 08-21-90 & \text { H } & 2.5 & \ldots & \ldots & 1 \\ \text { ECW } & 24 & \cdots & -- & \text { H } & 60 & \ldots & \ldots & .5 \\ \text { ECW } & 25 & \cdots & - & \text { H } & 1.5 & \ldots & \ldots & .5\end{array}$

\section{Effingham}

$\begin{array}{ccccccccc}\text { EFA } & 1 & 65 & 00-00-91 & \text { U } & \ldots & \ldots & \ldots \\ \text { EFB } & 1 & \ldots & \ldots & \text { T } & \ldots & \ldots & \ldots \\ \text { EFB } & 2 & \ldots & \ldots & \text { T } & \ldots & \ldots & \ldots & \ldots \\ \text { EFB } & 3 & \ldots & \ldots & \text { T } & \ldots & \ldots & \ldots & \ldots \\ \text { EFS } & 1 & \ldots & \ldots & \ldots & \ldots & \ldots & \ldots & \ldots\end{array}$

$\begin{array}{lcccccccc}\text { EFW } & 2 & \ldots & \ldots & \text { H T } & 60 & \ldots & \ldots & \ldots \\ \text { EFW } & 3 & \ldots & \ldots & \text { H } & \ldots & \ldots & \ldots & \ldots \\ \text { EFW } & 4 & 18 & 07-10-91 & \text { H T } & \ldots & \ldots & \ldots & \ldots \\ \text { EFW } & 5 & 6.4 & 07-10-91 & \ldots & \ldots & \ldots & \ldots & \ldots \\ \text { EFW } & 6 & 10.6 & 07-10-91 & \text { H } & \ldots & \ldots & \ldots & \ldots \\ \text { EFW } & 7 & \ldots & \ldots 7-10-91 & \text { H T } & \ldots & \ldots & \ldots & \ldots \\ \text { EFW } & 8 & 9 & 07-30-91 & \text { T } & \ldots & \ldots & \ldots & 1 \\ \text { EFW } & 9 & 27.5 & 07-08-85 & \text { H } & 3 & \ldots & \ldots & \text { USGS } \\ \text { EFW } & 10 & 20 & 05-08 & \text { H T } & 22 & \ldots & \ldots\end{array}$

\begin{tabular}{|c|c|c|c|c|c|c|c|c|}
\hline EFW & 12 & 25 & $10-20-85$ & H & 4.5 & - & $\cdots$ & 1 \\
\hline EFW & 13 & 150 & $04-11-86$ & H & 5.5 & $\cdots$ & $\cdots$ & .5 \\
\hline EFW & 14 & - & - & H & 10 & $\cdots$ & $\cdots$ & 1 \\
\hline EFW & 15 & 22 & $06-20-86$ & H & 25 & $\cdots$ & $\cdots$ & .5 \\
\hline EFW & 16 & & - & H & 5 & $\cdots$ & $\cdots$ & 1 \\
\hline
\end{tabular}

\begin{tabular}{|c|c|c|c|c|c|c|c|c|}
\hline EFW & 16 & - & $\cdots$ & $\mathrm{H}$ & 5 & $\cdots$ & $\cdots$ & 1 \\
\hline EFW & 17 & - & - & H & 6 & $\cdots$ & $\cdots$ & 1 \\
\hline EFW & 18 & 35 & $10-27-87$ & $\mathrm{H}$ & 20 & $\cdots$ & $\cdots$ & .5 \\
\hline EFW & 19 & - & - & $\mathrm{H}$ & 6 & $\cdots$ & $\cdots$ & .2 \\
\hline EFW & 20 & 50 & $07-05-88$ & $\mathrm{H}$ & 1.3 & -. & - & 1 \\
\hline EFW & 21 & 20 & $12 \cdot 08 \cdot 88$ & $\mathrm{H}$ & 20 & - & $\cdots$ & .5 \\
\hline EFW & 22 & - & - & $\mathrm{H}$ & 2 & . & $\cdots$ & 1.3 \\
\hline EFW & 23 & - & - & $\mathrm{H}$ & 50 & $\cdots$ & $\cdots$ & .5 \\
\hline EFW & 24 & 27 & $01-06-91$ & $\mathrm{H}$ & 12 & $\cdots$ & $\cdots$ & 1 \\
\hline EFW & 25 & - & - & H & 18 & - & $\cdots$ & 12 \\
\hline EFW & 26 & 50 & $11-28-90$ & H & 0.5 & $\cdots$ & $\cdots$ & 1.5 \\
\hline
\end{tabular}

\begin{tabular}{|c|c|c|c|c|c|c|c|c|}
\hline EFW & 26 & 50 & $11-28-90$ & H & 0.5 & $\cdots$ & $\cdots$ & 1.5 \\
\hline EFW & 27 & - & - - & H & 32 & $\cdots$ & $\cdots$ & .3 \\
\hline EFW & 28 & 40 & $11-06-84$ & H & 3 & $-\cdot$ & $\cdots$ & .5 \\
\hline EFW & 29 & 10 & $09--87$ & H & - & $\cdots$ & $\cdots$ & $\cdots$ \\
\hline EFW & 30 & $\cdots$ & - - & $\mathrm{T}$ & 150 & $\cdots$ & $\cdots$ & .5 \\
\hline EFW & 31 & 10 & $06-21-84$ & $\mathrm{~T}$ & 100 & $\cdots$ & $\cdots$ & .5 \\
\hline EFW & 32 & 120 & $08-14-84$ & $\mathrm{~T}$ & 12 & $\cdots$ & $\cdots$ & .5 \\
\hline EFW & 33 & 15 & $03-07-85$ & $\mathrm{H}$ & 2 & $\cdots$ & $\cdots$ & 1 \\
\hline EFW & 34 & 50 & $11-04-85$ & $\mathrm{H}$ & 1 & $\cdots$ & $\cdots$ & $\cdots$ \\
\hline EFW & 35 & 19.5 & $06-15-85$ & $\mathrm{H}$ & 10 & $\cdots$ & - & 3 \\
\hline EFW & 36 & - & $\cdots$ & $\mathbf{H}$ & 40 & $\cdots$ & $\cdots$ & 1 \\
\hline EFW & 37 & 40 & $10-29-85$ & $\mathrm{H}$ & 30 & $\cdots$ & $\cdots$ & .5 \\
\hline EFW & 38 & 45 & $10-31-85$ & $\mathrm{H}$ & 6 & $\cdots$ & $\cdots$ & .5 \\
\hline EFW & 39 & - & $\cdots$ & $\mathrm{H}$ & 100 & $\cdots$ & $\cdots$ & .5 \\
\hline EFW & 40 & 70 & $12-15-85$ & $\mathrm{H}$ & 0.3 & $\cdots$ & $\cdots$ & 1 \\
\hline EFW & 41 & - & - & $\mathrm{H}$ & 3 & - - & - & 1 \\
\hline EFW & 42 & 10 & $04-18-86$ & $\mathrm{H}$ & 2.5 & - & $\cdots$ & .5 \\
\hline
\end{tabular}




\begin{tabular}{|c|c|c|c|c|c|c|c|c|c|c|c|}
\hline $\begin{array}{l}\text { Local } \\
\text { site } \\
\text { number }\end{array}$ & $\begin{array}{l}\text { Lat- } \\
\text { itude }\end{array}$ & $\begin{array}{l}\text { Long- } \\
\text { itude }\end{array}$ & Owner or user & $\begin{array}{l}\text { Year } \\
\text { com- } \\
\text { pleted }\end{array}$ & $\begin{array}{l}\text { Alti- } \\
\text { tude } \\
\text { above } \\
\text { sea } \\
\text { level } \\
\text { (feet) }\end{array}$ & $\begin{array}{c}\text { Dia- } \\
\text { meter } \\
\text { of } \\
\text { well } \\
\text { casing } \\
\text { (inches) }\end{array}$ & $\begin{array}{l}\text { Depth } \\
\text { to } \\
\text { bottom } \\
\text { of } \\
\text { casing } \\
\text { (feet) }\end{array}$ & $\begin{array}{l}\text { Casing } \\
\text { material }\end{array}$ & $\begin{array}{l}\text { Type } \\
\text { of } \\
\text { finish }\end{array}$ & $\begin{array}{l}\text { Depth } \\
\text { to } \\
\text { bottom } \\
\text { of open } \\
\text { section } \\
\text { (feet) }\end{array}$ & $\begin{array}{c}\text { Type } \\
\text { of } \\
\text { site }\end{array}$ \\
\hline
\end{tabular}

\begin{tabular}{|c|c|c|c|c|}
\hline EFW & 43 & 434427 & 710113 & Peterson \\
\hline EFW & 44 & 434441 & 710720 & Johnson \\
\hline EFW & 45 & 434728 & 710228 & Murray \\
\hline EFW & 46 & 434451 & 710727 & Sheehan \\
\hline EFW & 47 & 434432 & 710457 & Bowes \\
\hline EFW & 48 & 434550 & 710545 & Griffin \\
\hline EFW & 49 & 434457 & 710007 & Revin \\
\hline EFW & 50 & 434636 & 710546 & Brennick \\
\hline EFW & 51 & 434636 & 710545 & Brennick \\
\hline EFW & 52 & 434744 & 710326 & Gardner \\
\hline EFW & 53 & 434410 & 710444 & McClean \\
\hline EFW & 54 & 434405 & 710550 & Gagne \\
\hline EFW & 55 & 434344 & 710046 & Ravel1 \\
\hline EFW & 56 & 434741 & 710322 & Peckham \\
\hline EFW & 57 & 434745 & 710322 & DeFanti \\
\hline EFW & 58 & 434444 & 710057 & Applei \\
\hline EFW & 59 & 434420 & 710042 & Simmons \\
\hline EFW & 60 & 434447 & 710717 & Angelo \\
\hline EFW & 61 & 434442 & 710725 & Leona \\
\hline EFW & 62 & 434447 & 710735 & Hodge \\
\hline EFW & 63 & 434653 & 710315 & Fahy \\
\hline EFW & 64 & 434529 & 705957 & Libby \\
\hline EFW & 65 & 434242 & 705936 & Le Be1 \\
\hline EFW & 66 & 434527 & 705924 & L. Seamans \\
\hline EFW & 67 & 434449 & 710058 & Bedard \\
\hline EFW & 68 & 434401 & 710439 & Robertson \\
\hline EFW & 69 & 434521 & 710001 & Jean \\
\hline EFW & 70 & 434449 & 710722 & K. Haggerty \\
\hline EFW & 71 & 434315 & 710004 & Amara 1 \\
\hline EFW & 72 & 434446 & 710720 & Mitchell \\
\hline EFW & 73 & 434620 & 705947 & Smith \\
\hline EFW & 74 & 434443 & 710524 & K. Baribeau \\
\hline EFW & 75 & 434451 & 710742 & Tuttle \\
\hline EFW & 76 & 434301 & 710142 & Ferber \\
\hline EFW & 77 & 434738 & 710326 & Tow1e \\
\hline EFW & 78 & 434426 & 710702 & Brienzo \\
\hline EFW & 79 & 434744 & 710305 & Raynes \\
\hline EFW & 80 & 434746 & 710304 & Hartford \\
\hline EFW & 81 & 434721 & 710521 & Dig n Doze \\
\hline EFW & 82 & 434720 & 705936 & J. Lemming-Little \\
\hline EFW & 83 & 434427 & 710510 & A. Cosentino \\
\hline EFW & 84 & 434621 & 705940 & Bookholz \\
\hline EFW & 85 & 434423 & 710043 & Town of Effingham \\
\hline EFW & 86 & 434421 & 710044 & Keville \\
\hline EFW & 87 & 434620 & 705938 & Columbo \\
\hline EFW & 88 & 434745 & 710318 & Spiller \\
\hline EFW & 89 & 434200 & 710013 & Fai11 \\
\hline EFW & 90 & 434724 & 710501 & Ossipee Lake Camping \\
\hline EFW & 91 & 434447 & 710056 & Stackpole \\
\hline EFW & 92 & 434549 & 710009 & Riordan \\
\hline EFW & 93 & 434718 & 705934 & Leeming \\
\hline
\end{tabular}

Effingham - Continued

\begin{tabular}{|c|c|c|c|c|c|c|c|}
\hline 1986 & 530 & $\cdots$ & 117 & - & $x$ & . & BrW \\
\hline 1986 & 490 & - & 120 & - - & $x$ & - & Brw \\
\hline 1986 & 440 & - & 80 & - & $x$ & $\ldots$ & BrW \\
\hline 1987 & 440 & - & 39 & $\cdots$ & $\mathrm{S}$ & - & $\cdots$ \\
\hline 1987 & 480 & $\cdots$ & 25 & - & $x$ & - & BrW \\
\hline 1987 & 440 & - & 80 & -- & $x$ & - - & BrW \\
\hline 1987 & 420 & $\cdots$ & 70 & $\cdots$ & $x$ & $\cdots$ & BrW \\
\hline 1987 & 420 & $\cdots$ & 61 & - & $\cdots$ & $\cdots$ & BrW \\
\hline 1987 & 420 & $\cdots$ & 55 & - & $\mathbf{S}$ & $\ldots$ & $\cdots$ \\
\hline 1987 & 400 & -. & 54 & $\ldots$ & $x$ & - - & BrW \\
\hline 1987 & 470 & - & 39 & -- & $x$ & - - & BrW \\
\hline 1987 & 500 & $\cdots$ & 45 & - & $x$ & - - & BrW \\
\hline 1987 & 530 & $\ldots$ & 39 & - & $\mathrm{x}$ & $\cdots$ & BrW \\
\hline 1987 & 420 & - & 79 & $\cdots$ & $x$ & $\cdots$ & BrW \\
\hline 1987 & 400 & - & 80 & -- & $x$ & - & BrW \\
\hline 1988 & 530 & -- & 100 & - - & $x$ & - & BrW \\
\hline 1988 & 470 & $\cdots$ & 84 & - & $x$ & $\cdots$ & BrW \\
\hline 1988 & 460 & - & 53 & - - & $\mathbf{S}$ & - & $\cdots$ \\
\hline 1988 & 480 & - & 79 & $\cdots$ & $x$ & - & BrW \\
\hline 1988 & 480 & - & 91 & - - & $x$ & - & BrW \\
\hline 1988 & 660 & - & 103 & $\cdots$ & $x$ & - & BrW \\
\hline 1988 & 520 & - - & 47 & - - & $x$ & -- & BrW \\
\hline 1988 & 520 & - & 148 & - & $x$ & - & BrW \\
\hline 1988 & 560 & $\cdots$ & 186 & - & $x$ & $\cdots$ & BrW \\
\hline 1988 & 540 & $\cdots$ & 106 & $\ldots$ & $\mathrm{x}$ & - - & BrW \\
\hline 1988 & 480 & $\cdots$ & 30 & - & $x$ & - & BrW \\
\hline 1988 & 500 & $\cdots$ & 117 & $\cdots$ & $x$ & $\cdots$ & Brw \\
\hline 1988 & 450 & - & 47 & - & $\mathbf{S}$ & -- & $\cdots$ \\
\hline 1988 & 500 & - & 63 & - & $x$ & $\cdots$ & BrW \\
\hline 1988 & 450 & - & 47 & - - & $\mathrm{s}$ & -- & $\cdots$ \\
\hline 1988 & 500 & - & 141 & $\cdots$ & $\mathrm{x}$ & - - & BrW \\
\hline 1989 & 440 & $\cdots$ & 74 & - - & $\cdots$ & -- & $\cdots$ \\
\hline 1989 & 460 & $\cdots$ & 59 & - - & $S$ & - - & $\cdots$ \\
\hline 1989 & 520 & - & 49 & $\cdots$ & $x$ & $\cdots$ & BrW \\
\hline 1989 & 420 & -- & 60 & - & $\mathrm{x}$ & - & BrW \\
\hline 1989 & 480 & $\cdots$ & 213 & - - & $x$ & - & BrW \\
\hline 1990 & 400 & $\ldots$ & 80 & - & $x$ & - - & BrW \\
\hline 1991 & 400 & $\cdots$ & 80 & - & $x$ & - & Brw \\
\hline 1991 & 420 & -- & 201 & - & $x$ & $\cdots$ & BrW \\
\hline 1989 & 410 & $\cdots$ & 98 & $\cdots$ & $x$ & - & BrW \\
\hline 1991 & 460 & $\cdots$ & 140 & - & $x$ & - & BrW \\
\hline 1991 & 440 & - & 20 & $\cdots$ & $S$ & - & $\cdots$ \\
\hline 1991 & 480 & $\cdots$ & 120 & - & $x$ & - & BrW \\
\hline 1991 & 480 & $\cdots$ & 120 & $\cdots$ & $x$ & $\cdots$ & BrW \\
\hline 1991 & 440 & $\cdots$ & 20 & $\cdots$ & $\mathbf{S}$ & $\cdots$ & - \\
\hline 1991 & 400 & $\cdots$ & 151 & -- & $x$ & - & BrW \\
\hline 1991 & 485 & $\cdots$ & 120 & - & $x$ & - & BrW \\
\hline 1992 & 415 & $\cdots$ & 100 & - & $x$ & - & BrW \\
\hline 1992 & 540 & - & 100 & - & $x$ & - & BrW \\
\hline 1992 & 570 & $\cdots$ & 120 & . & $x$ & . & BrW \\
\hline 1992 & 410 & $\cdots$ & 150 & - & $x$ & - & BrW \\
\hline
\end{tabular}




\begin{tabular}{|c|c|c|c|c|c|c|c|c|c|}
\hline \multicolumn{2}{|c|}{$\begin{array}{l}\text { Local } \\
\text { site } \\
\text { number }\end{array}$} & \multirow[t]{2}{*}{$\begin{array}{l}\text { Water } \\
\text { level } \\
\text { depth } \\
\text { (feet) }\end{array}$} & \multirow[t]{2}{*}{$\begin{array}{c}\text { Date } \\
\mathrm{mm} / \mathrm{dd} / \mathrm{yy}\end{array}$} & \multirow[t]{2}{*}{ Use } & \multirow[t]{2}{*}{$\begin{array}{l}\text { Maximum } \\
\text { well } \\
\text { yield } \\
\text { (gallons } \\
\text { per } \\
\text { minute) }\end{array}$} & \multirow[t]{2}{*}{$\begin{array}{l}\text { Draw- } \\
\text { down } \\
\text { (feet) }\end{array}$} & \multirow{2}{*}{$\begin{array}{c}\begin{array}{c}\text { Specific } \\
\text { capacity } \\
\text { (gallons per } \\
\text { minute } \\
\text { per } \\
\text { foot) }\end{array} \\
\text { Effinghan }\end{array}$} & \multirow{2}{*}{$\begin{array}{l}\text { Pumping } \\
\begin{array}{l}\text { period } \\
\text { (hours) }\end{array} \\
\text { - Continued }\end{array}$} & \multirow[t]{2}{*}{ Remarks } \\
\hline & & & & & & & & & \\
\hline EFW & 43 & -. & - & H & 15 & - & - - & 2.5 & \\
\hline EFW & 44 & 55 & $10-03-86$ & $\mathrm{H}$ & 30 & - & $\cdots$ & .5 & \\
\hline EFW & 45 & 40 & $09-25-86$ & $\mathrm{H}$ & 3 & - & -. & .5 & \\
\hline EFW & 46 & 25.5 & $05-13-87$ & $\mathrm{H}$ & 17 & - & $-\cdot$ & 2 & \\
\hline EFW & 47 & - & . & H & 15 & -. & -. & 1 & \\
\hline EFW & 48 & 60 & $06-24-87$ & $\mathrm{H}$ & 6 & $\cdots$ & $\cdots$ & 1 & \\
\hline EFW & 49 & 50 & $06-03-87$ & H & 0.7 & - & - - & .5 & \\
\hline EFW & 50 & 7 & $05-13-87$ & $\mathbf{T}$ & 20 & -. & -. & 1 & \\
\hline EFW & 51 & 7 & $05-12-87$ & $T$ & 55 & $\cdots$ & $\cdots$ & 48 & \\
\hline EFW & 52 & 20 & $09-09-87$ & H & 2 & -- & $\cdots$ & .5 & \\
\hline EFW & 53 & 14 & $12-14-87$ & $\mathrm{H}$ & 1 & - & - & .5 & \\
\hline EFW & 54 & 65 & $12-22-87$ & $\mathrm{H}$ & 1.8 & $\cdots$ & $\cdots$ & 1 & \\
\hline EFW & 55 & 65 & $10-28-87$ & $\mathrm{H}$ & 5 & - & $\cdots$ & .5 & \\
\hline EFW & 56 & -. & - - & $\mathrm{H}$ & 15 & - & $\cdots$ & 1 & \\
\hline EFW & 57 & .. & - & H & 10 & - & - & 1 & \\
\hline EFW & 58 & 40 & $03-17-88$ & н & 50 & -. & - & .5 & \\
\hline EFW & 59 & 5 & $05-08-88$ & $\mathrm{H}$ & 18 & - & $\cdots$ & 1 & \\
\hline EFW & 60 & 39 & $04-17-88$ & $\mathrm{H}$ & 12 & -. & -. & 1 & \\
\hline EFW & 61 & - - & - & $\mathrm{H}$ & 6 & $-\cdot$ & - & .5 & \\
\hline EFW & 62 & $\cdots$ & $\cdots$ & н & 6 & - & - - & .5 & \\
\hline EFW & 63 & -. & . & H & 15 & .. & -. & 1 & \\
\hline EFW & 64 & 8 & $05-19-88$ & H & 4.5 & . & . & .5 & \\
\hline EFW & 65 & $\cdots$ & - & H & 6 & $\cdots$ & $\cdots$ & 1 & \\
\hline EFW & 66 & $\cdots$ & $-\cdot$ & $\mathrm{H}$ & 4 & $\cdots$ & $\cdots$ & 1 & \\
\hline EFW & 67 & -. & - & $\mathrm{H}$ & 8 & $\cdots$ & - & 1 & \\
\hline SFW & 68 & 20 & $09-27-88$ & H & 6 & - & $\cdots$ & 1 & \\
\hline IFW & 69 & 10 & $08-11-88$ & $\mathrm{H}$ & 12 & - & - & .5 & \\
\hline $\mathrm{BFW}$ & 70 & 31 & $10-22-88$ & $\mathrm{H}$ & 16.5 & $\cdots$ & $\cdots$ & 1 & \\
\hline SFW & 71 & -. & - & $\mathrm{H}$ & 15 & $\cdots$ & $-\cdot$ & 1 & \\
\hline SFW & 72 & 31.5 & $10-26-88$ & $\mathrm{H}$ & 16.5 & $-\cdot$ & $\cdots$ & 1.5 & \\
\hline EFW & 73 & - & - & $\mathrm{H}$ & 13 & $\cdots$ & - & 1 & \\
\hline $\mathrm{BFW}$ & 74 & $\cdots$ & $\cdots$ & $\mathrm{H}$ & 30 & -. & $\cdots$ & 8 & \\
\hline BFW & 75 & -. & . & H & 5.5 & - & $\cdots$ & 6 & \\
\hline SFW & 76 & 20 & $08 \cdot 11 \cdot 89$ & $\mathbf{H}$ & 30 & $\cdots$ & -. & 1 & \\
\hline :FW & 77 & 10 & $08-30-89$ & $\mathrm{H}$ & 3 & - & - & .5 & \\
\hline :FW & 78 & 35 & $08-30-89$ & $\mathrm{H}$ & 5 & - & - - & 1 & \\
\hline :FW & 79 & 20 & $08-22-90$ & $\mathrm{H}$ & 4 & - & $\cdots$ & 1 & \\
\hline :FW & 80 & 25 & $04-11-91$ & н & 40 & - & $\cdots$ & .5 & \\
\hline :FW & 81 & 30 & $04-02-91$ & $\mathrm{H}$ & 1.5 & -. & $\cdots$ & 1 & \\
\hline :FW & 82 & 6 & $08-16-89$ & H & 5.5 & - & - & .5 & \\
\hline :FW & 83 & & - & н & 15 & .. & .. & 1 & \\
\hline :FW & 84 & 16 & $08-04-91$ & H & 8 & -. & - & 1 & \\
\hline FW & 85 & 20 & $07-20.91$ & н & 8 & - & $\cdots$ & 1 & \\
\hline FW & 86 & 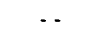 & 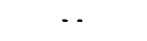 & $\mathrm{H}$ & 4.5 & -. & $\cdots$ & 1 & \\
\hline FW & 87 & 16 & $08-24-91$ & H & 6 & $\cdots$ & -. & 1 & \\
\hline FW & 88 & 3 & $08-15-91$ & H & 50 & -. & $\cdots$ & 1 & \\
\hline FW & 89 & 6 & $10-17-91$ & $\mathrm{H}$ & 14 & $\cdots$ & - & 1 & \\
\hline FW & 90 & $\cdots$ & - & $\mathrm{H}$ & 7.5 & $\cdots$ & - & 1 & \\
\hline FW & 91 & $\cdots$ & $\cdots$ & $\mathrm{H}$ & 30 & $\cdots$ & $\cdots$ & 1 & \\
\hline FW & 92 & - & . & н & 5.5 & $\cdots$ & . & 1 & \\
\hline FW & 93 & 40 & $08 \cdot 25-92$ & H & 25 & $\cdots$ & - & 1 & \\
\hline
\end{tabular}


Table 1-1. Description of selected welis, borings, and springs in the Saco and Ossipee River Basins, east-central

\begin{tabular}{|c|c|c|c|c|c|c|c|c|c|c|c|}
\hline $\begin{array}{l}\text { Local } \\
\text { site } \\
\text { number }\end{array}$ & $\begin{array}{l}\text { Lat- } \\
\text { itude }\end{array}$ & $\begin{array}{l}\text { Long- } \\
\text { itude }\end{array}$ & Owner or user & $\begin{array}{l}\text { Year } \\
\text { com- } \\
\text { pleted }\end{array}$ & $\begin{array}{l}\text { Alti- } \\
\text { tude } \\
\text { above } \\
\text { sea } \\
\text { level } \\
\text { (feet) }\end{array}$ & $\begin{array}{l}\text { Dia- } \\
\text { meter } \\
\text { of } \\
\text { well } \\
\text { casing } \\
\text { (inches) }\end{array}$ & $\begin{array}{l}\text { Depth } \\
\text { to } \\
\text { bottom } \\
\text { of } \\
\text { casing } \\
\text { (feet) }\end{array}$ & $\begin{array}{l}\text { Casing } \\
\text { material }\end{array}$ & $\begin{array}{l}\text { Type } \\
\text { of } \\
\text { finish }\end{array}$ & $\begin{array}{l}\text { Depth } \\
\text { to } \\
\text { bottom } \\
\text { of open } \\
\text { section } \\
\text { (feet) }\end{array}$ & $\begin{array}{c}\text { Type } \\
\text { of } \\
\text { site }\end{array}$ \\
\hline
\end{tabular}

\begin{tabular}{|c|c|c|c|c|c|c|c|c|c|c|c|c|}
\hline FLS & 1 & 434941 & 710607 & & $\cdots$ & 420 & - & $\cdots$ & $\cdots$ & - & $\cdots$ & sp \\
\hline FLW & 3 & 434753 & 710122 & Davis & 1990 & 400 & $\cdots$ & -. & - & $T$ & $\cdots$ & Dvn \\
\hline FLW & 4 & 434744 & 710138 & Schluter & 1988 & 405 & 2 & 15 & $\mathrm{~s}$ & $\mathrm{~T}$ & - & Dvn \\
\hline FLW & 5 & 434745 & 710138 & schluter & - & 395 & 2 & 18 & $\mathrm{~s}$ & $T$ & $\cdots$ & Dvn \\
\hline FLW & 6 & 434844 & 710455 & & 1972 & 422 & 6 & 20 & $\mathrm{~s}$ & $\mathrm{x}$ & $\cdots$ & Brw \\
\hline FLW & 7 & 434845 & 710454 & & 1988 & 425 & $\cdots$ & - & $\cdots$ & $T$ & $\cdots$ & Dvn \\
\hline FLW & 8 & 434843 & 710451 & & - & 425 & - & $\cdots$ & -. & $T$ & $\cdots$ & Dvn \\
\hline FLW & 9 & 434839 & 710605 & & 1987 & 409 & $\cdots$ & -. & $\cdots$ & $T$ & $\cdots$ & Dvn \\
\hline FLW & 10 & 434833 & 710549 & & 1970 & 415 & 2 & 15 & - & $T$ & $\cdots$ & Dvn \\
\hline FLW & 11 & 434828 & 710540 & & 1950 & 420 & 3 & 50 & $\mathrm{~s}$ & $T$ & - & Dvn \\
\hline FLW & 12 & 434812 & 710513 & & 1986 & 420 & - & - & - & $\mathbf{x}$ & $\cdots$ & BrW \\
\hline FLW & 13 & 434918 & 710626 & Mitchell & $\cdots$ & 455 & 2 & 61 & $\mathrm{~s}$ & $T$ & $\cdots$ & Dvn \\
\hline FLW & 14 & 434919 & 710619 & & 1986 & 410 & -. & $\cdots$ & -. & $T$ & $\cdots$ & Dvn \\
\hline FLW & 15 & 434859 & 710622 & & -. & 410 & - & 13 & $s$ & $T$ & - & Dvn \\
\hline FLW & 16 & 434900 & 710633 & John Gallant & $\cdots$ & 425 & $\cdots$ & $\cdots$ & $\cdots$ & $\cdots$ & $\cdots$ & $\cdots$ \\
\hline FLW & 17 & 434842 & 710625 & Camp Huckins & -. & 408 & 48 & 8.3 & c & 0 & $\cdots$ & Dug \\
\hline FLW & 18 & 434822 & 710627 & Hormel1 & 1971 & 415 & 1 & 22 & s & $T$ & $\cdots$ & Dvn \\
\hline FLW & 19 & 434904 & 710916 & Camp Calumet & 1976 & 418 & - & $-\cdot$ & -. & $\mathrm{x}$ & $\cdots$ & Brw \\
\hline FLW & 20 & 434912 & 710926 & Camp Calumet & 1985 & 428 & - & $\cdots$ & - & $\mathrm{x}$ & - & BrW \\
\hline FLW & 21 & 435049 & 710540 & & 1989 & 410 & 3 & 4.9 & C & 0 & $\cdots$ & Dug \\
\hline FLW & 22 & 434941 & 710606 & & 1969 & 410 & $\cdots$ & 27 & $\mathrm{~s}$ & $T$ & $\cdots$ & Dvn \\
\hline FLW & 23 & 434944 & 711030 & North Country Lumber & 1986 & 455 & 2 & 35 & G & $T$ & $\cdots$ & Dvn \\
\hline FLW & 24 & 434846 & 710644 & YMCA Camp Huckins & 1991 & 415 & 2 & 58.7 & P & $\mathrm{s}$ & 58.7 & Bor \\
\hline FLW & 25 & 434856 & 710940 & Hobbs, Richard, L & 1991 & 415 & 2 & 37.5 & $\mathrm{P}$ & $\mathbf{s}$ & 39.5 & Bor \\
\hline FLW & 26 & 434730 & 710123 & USGS & 1991 & 390 & 2 & 18 & P & $s$ & 20 & Bor \\
\hline FLW & 27 & 434734 & 705926 & Lorraine Gaguine & - & 405 & 6 & -. & $\mathrm{s}$ & $\mathrm{x}$ & -. & BrW \\
\hline FLW & 28 & 434750 & 710101 & Edward Reed & 1968 & 395 & 3 & 25 & $s$ & $T$ & $\cdots$ & Dvn \\
\hline FLW & 29 & 434857 & 710455 & Buck & 1984 & 450 & - & $\cdots$ & - & $\mathrm{x}$ & $-\cdot$ & Brw \\
\hline FLW & 30 & 434821 & 710503 & Mcvey & 1984 & 430 & - - & $\cdots$ & - & $\mathrm{x}$ & $\cdots$ & BrW \\
\hline FLW & 31 & 434924 & 710613 & silver & 1984 & 430 & $\cdots$ & $\cdots$ & $\cdots$ & $\mathrm{s}$ & $\cdots$ & $\cdots$ \\
\hline FLW & 32 & 434917 & 710609 & Fielding & 1984 & 420 & - & $\cdots$ & $\cdots$ & $s$ & $\cdots$ & $\cdots$ \\
\hline FLW & 33 & 434736 & 710633 & Hardy & 1984 & 450 & $\cdots$ & $\cdots$ & $\cdots$ & $\mathrm{s}$ & $\cdots$ & $\cdots$ \\
\hline FLW & 34 & 434854 & 710442 & Cullinane & 1985 & 460 & -. & $\cdots$ & $\cdots$ & $\mathrm{x}$ & $\cdots$ & Brw \\
\hline FLW & 35 & 434850 & 710451 & Hill & 1985 & 450 & -. & -. & $\cdots$ & $\mathrm{x}$ & -. & BrW \\
\hline FLW & 36 & 434743 & 710154 & Dorian & 1985 & 410 & - - & 25 & $\cdots$ & $s$ & $\cdots$ & $\cdots$ \\
\hline FLW & 37 & 434731 & 710653 & Moore & 1986 & 440 & $\cdots$ & 99 & $\cdots$ & $\mathrm{x}$ & - & BrW \\
\hline FLW & 38 & 434907 & 710608 & Falanga & 1986 & 445 & $\cdots$ & 119 & $\cdots$ & $\mathrm{x}$ & $\cdots$ & Brw \\
\hline FLW & 39 & 434827 & 710508 & widmer & 1986 & 430 & $\cdots$ & 160 & $\cdots$ & $\mathrm{x}$ & - & BrW \\
\hline FLW & 40 & 434828 & 710501 & Adderley & 1986 & 430 & $\cdots$ & 30.5 & $\cdots$ & $\mathrm{s}$ & $\cdots$ & $\cdots$ \\
\hline FLW & 41 & 434807 & 710441 & MacDonald & 1986 & 430 & $\cdots$ & 112 & $\cdots$ & $\mathrm{x}$ & $\cdots$ & BrW \\
\hline FLW & 42 & 434855 & 710445 & Cullinane & 1985 & 455 & $\cdots$ & 19 & $\cdots$ & $\mathrm{x}$ & $-\cdot$ & BrW \\
\hline FLW & 43 & 434819 & 710508 & Rotandi & 1986 & 430 & $\cdots$ & 41 & $\cdots$ & $s$ & $\cdots$ & $\cdots$ \\
\hline FLW & 44 & 434825 & 710455 & Milotte Association control & 1986 & 430 & $\cdots$ & 30.5 & $\cdots$ & $\mathrm{s}$ & $\cdots$ & $\cdots$ \\
\hline FLW & 45 & 434819 & 710450 & Storey & 1987 & 430 & - & 29 & - & $\mathbf{s}$ & - & $\cdots$ \\
\hline FLW & 46 & 434820 & 710458 & Fitzpastrick & 1987 & 430 & $\cdots$ & 34 & $\cdots$ & $\mathrm{s}$ & $\cdots$ & $\cdots$ \\
\hline FLW & 47 & 434734 & 710631 & Codner & 1987 & 430 & -. & 62 & - & $\mathrm{s}$ & - & - \\
\hline FLW & 48 & 434921 & 710628 & Anson & 1987 & 465 & $\cdots$ & 62 & $\cdots$ & $\mathrm{s}$ & $\cdots$ & $\cdots$ \\
\hline FLW & 49 & 434747 & 710148 & Rainone & 1987 & 405 & - & 27 & - & $\mathrm{s}$ & $\cdots$ & $\cdots$ \\
\hline FLW & 50 & 434756 & 710454 & Blake & 1987 & 420 & $\cdots$ & 140 & $\cdots$ & $\mathrm{x}$ & $\cdots$ & BrW \\
\hline FLW & 51 & 434818 & 710508 & zampante & 1987 & 430 & - & 44 & $\cdots$ & $s$ & - & $\cdots$ \\
\hline FLW & 52 & 434729 & 710650 & Lawnicki & 1987 & 460 & $\cdots$ & 113 & $\cdots$ & $\mathrm{x}$ & $\cdots$ & BrW \\
\hline FLW & 53 & 434802 & 710449 & Boyd & 1988 & 430 & $\cdots$ & 100 & $\cdots$ & $x$ & $\cdots$ & BrW \\
\hline FLW & 54 & 434910 & 710607 & We1ch & 1988 & 450 & $\cdots$ & 71 & $\cdots$ & $\mathrm{x}$ & $\cdots$ & BrW \\
\hline
\end{tabular}




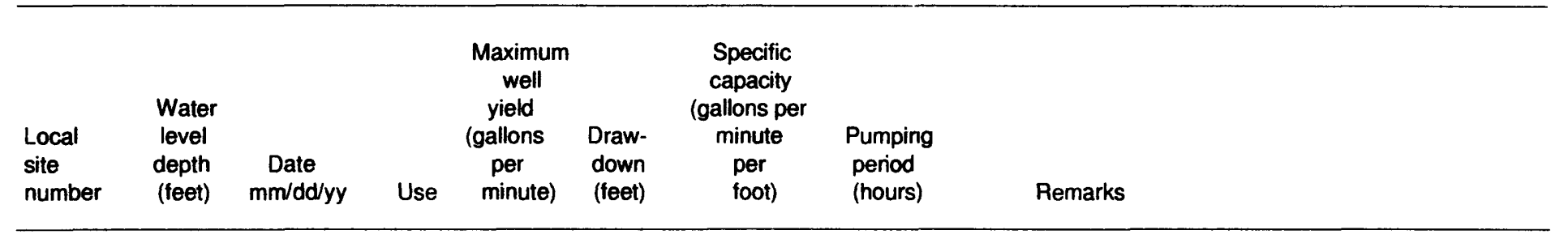

\begin{tabular}{|c|c|c|c|c|c|c|c|c|c|}
\hline \multirow[b]{2}{*}{ FLS } & \multirow[b]{2}{*}{1} & \multirow[b]{2}{*}{$\cdots$} & \multirow[b]{2}{*}{--} & \multirow[b]{2}{*}{$\cdots$} & \multirow[b]{2}{*}{$-\cdot$} & \multirow[b]{2}{*}{-} & \multicolumn{2}{|c|}{ Freedom } & \\
\hline & & & & & & & - - & $\cdots$ & \\
\hline FLW & 3 & $\cdots$ & -. & H & $\cdots$ & -. & -. & $\cdots$ & \\
\hline FLW & 4 & -- & $\cdots$ & H & $\cdots$ & - & - - & -. & \\
\hline FLW & 5 & -. & $\cdots$ & $\mathrm{U}$ & $\cdots$ & - & .. & $\cdots$ & Rust stains \\
\hline FLW & 6 & $\cdots$ & $\cdots$ & н & - & -. & -. & - & \\
\hline FKW & 8 & - & $\cdots$ & H & $\cdots$ & - & -. & - & \\
\hline FKW & 9 & $\cdots$ & $\cdots$ & H & $\cdots$ & -. & -. & $\cdots$ & point still draws up sand \\
\hline FLW & 9 & - & $\cdots$ & H & $\cdots$ & $\cdots$ & $\cdots$ & $\cdots$ & \\
\hline FLW & 10 & $\cdots$ & -. & н & $\cdots$ & -. & $\cdots$ & $\cdots$ & \\
\hline FLW & 11 & - & $\cdots$ & $T$ & $\cdots$ & $\cdots$ & -. & $\cdots$ & \\
\hline FLW & 12 & -. & - & н & 5.5 & $\cdots$ & -. & -. & \\
\hline FLW & 13 & 36 & $\cdots-83$ & H & $\cdots$ & - & -. & $\cdots$ & \\
\hline FLW & 14 & - & $\cdots$ & H & -. & -. & - - & - & \\
\hline FLW & 15 & - & - & H & $\cdots$ & -. & -. & -. & \\
\hline FLW & 16 & $\cdots$ & - & $\mathrm{H}$ & $\cdots$ & $\cdots$ & $\cdots$ & $\cdots$ & \\
\hline FLW & 17 & 2.6 & $06-05-91$ & $T$ & $\cdots$ & -. & - & $\cdots$ & $\mathbf{R}$ \\
\hline FLW & 18 & $\cdots$ & - & H & $\cdots$ & $\cdots$ & $\cdots$ & $\cdots$ & \\
\hline FLW & 19 & $\cdots$ & $\cdots$ & T H & $\cdots$ & $\cdots$ & $\cdots$ & $\cdots$ & \\
\hline FLW & 20 & -. & - - & T H & $\cdots$ & $\cdots$ & - & $-\cdot$ & \\
\hline FLW & 21 & 3.6 & $06-06-91$ & H & $\cdots$ & $\cdots$ & .. & - & Well will go dry \\
\hline FLW & 22 & - & - & H & -. & -. & -. & -. & \\
\hline FLW & 23 & 32 & $\cdots 86$ & T H & $\cdots$ & $\cdots$ & $\cdots$ & $\cdots$ & \\
\hline FLW & 24 & 7.5 & $07-30-91$ & 0 & $\cdots$ & $\cdots$ & -. & - & USGS \\
\hline FLW & 25 & 15.3 & $07-31-91$ & 0 & $\cdots$ & $\cdots$ & $\cdots$ & $\cdots$ & USGS \\
\hline FLW & 26 & 10 & $10-09-91$ & 0 & $\ldots$ & .. & -. & -. & USGS \\
\hline FLW & 27 & - & $\cdots$ & H & -- & - & $\cdots$ & - & \\
\hline FLW & 28 & $\cdots$ & $\cdots$ & H & $\cdots$ & - & $\cdots$ & - & \\
\hline FLW & 29 & 48 & $12-24-84$ & H & 2 & . & .. & .5 & \\
\hline FLW & 30 & .. & - & $\mathrm{H}$ & 20 & $\cdots$ & -. & $\cdots$ & \\
\hline FLW & 31 & 47.5 & $10-11-84$ & $\mathrm{H}$ & 7.5 & - & - & 3 & \\
\hline FLW & 32 & 16.5 & $12-05-84$ & H & 25 & - & -. & 2 & \\
\hline FLW & 33 & 45.5 & $08-29-84$ & H & 10 & $\cdots$ & - & -. & \\
\hline FLW & 34 & 40 & $11-18 \cdot 85$ & H & 1 & - & -. & .. & \\
\hline FLW & 35 & -. & - & $\mathrm{H}$ & 1.5 & $\cdots$ & -. & 1 & \\
\hline FLW & 36 & 18 & $10-25-85$ & - & 25 & - & -. & 2 & \\
\hline FLW & 37 & 50 & $07-03-86$ & $\mathrm{H}$ & 0.8 & $\cdots$ & $\cdots$ & 1 & \\
\hline FLW & 38 & 45 & $06-27-86$ & H & 2.5 & $\cdots$ & $\cdots$ & .5 & \\
\hline FLW & 39 & 85 & $09-16-86$ & H & 12 & -. & -. & 1 & \\
\hline FLW & 40 & 22 & $10-11-86$ & H & 5 & .. & .. & 2 & \\
\hline FLW & 41 & 25 & $09-20-86$ & H & 40 & - & .. & .5 & \\
\hline FLW & 42 & 40 & $11-18-85$ & H & 1 & $\cdots$ & -. & $\cdots$ & \\
\hline FLW & 43 & 27.5 & $11-18-86$ & H & 16.5 & $\cdots$ & $\cdots$ & 2 & \\
\hline FLW & 44 & 22 & $11-22-86$ & H & 12 & -. & - & 2 & \\
\hline FLW & 45 & 19 & $04-24-87$ & H & 7 & $\cdots$ & - & 2 & \\
\hline FLW & 46 & 23 & $05-07-87$ & H & 6.5 & -. & .. & 2 & \\
\hline FLW & 47 & - & . & H & 10 & $\cdots$ & .. & 2 & \\
\hline FLW & 48 & 49 & $06-11-87$ & H & 10 & -. & .. & 3 & \\
\hline FLW & 49 & 20 & $06-06-87$ & $\mathrm{H}$ & 30 & .. & .. & 2 & \\
\hline FLW & 50 & 10 & $08-18-87$ & $\mathrm{H}$ & 1.5 & - & $\cdots$ & 1 & \\
\hline FLW & 51 & 28 & $10-10-87$ & $\mathrm{H}$ & 15 & $\cdots$ & $\cdots$ & 1 & \\
\hline FLW & 52 & 40 & $10-14-87$ & $\mathrm{H}$ & 25 & -. & - & .5 & \\
\hline FLW & 53 & 35 & $01-08-88$ & H & 4 & $\cdots$ & $\cdots$ & .5 & \\
\hline FLW & 54 & .. & $\cdots$ & H & 25 & - & -. & 1 & \\
\hline
\end{tabular}


Table 1-1. Description of selected wells, borings, and springs in the Saco and Ossipee River Basins, east-central

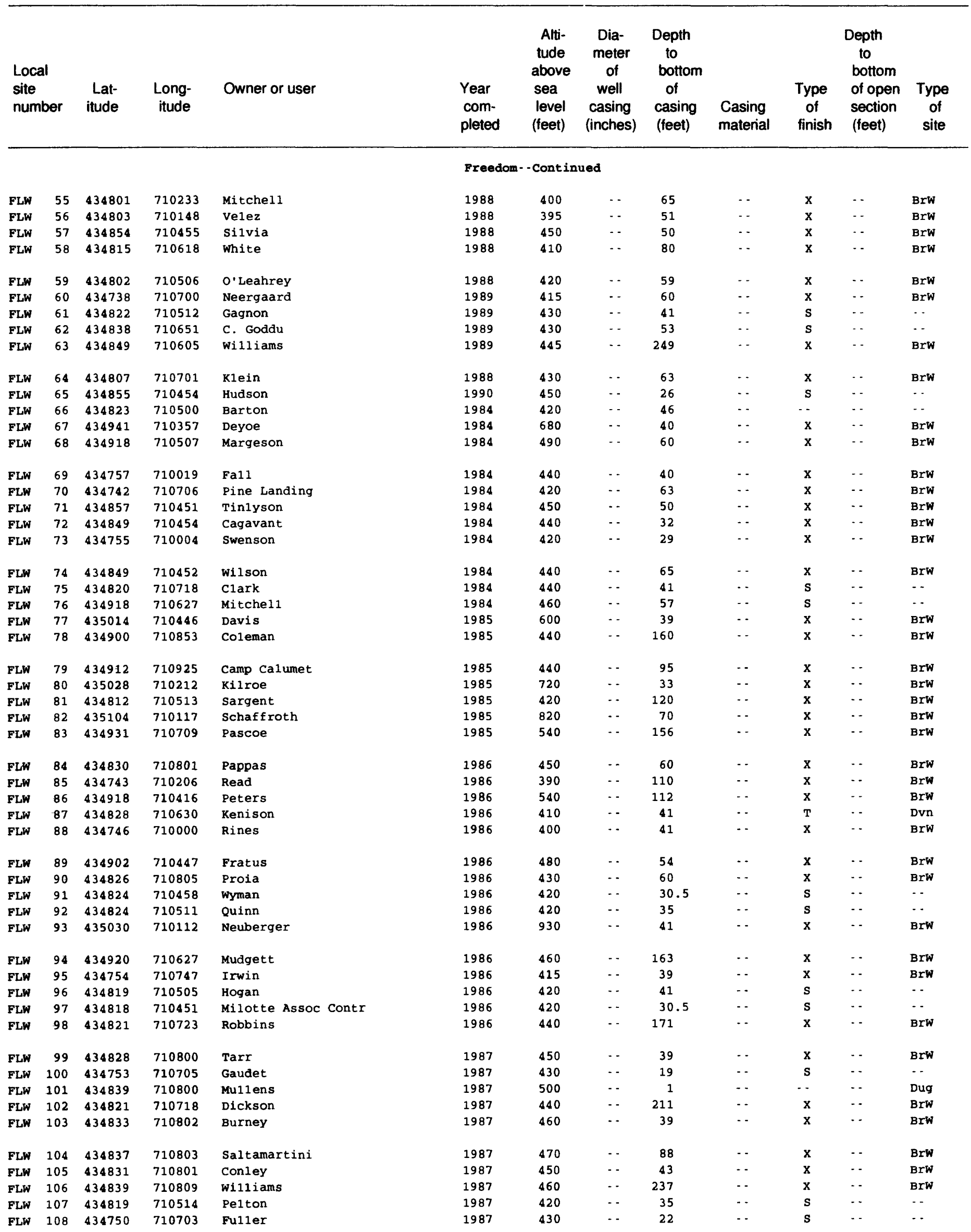




\begin{tabular}{|c|c|c|c|c|c|c|c|c|}
\hline $\begin{array}{l}\text { Local } \\
\text { site } \\
\text { number }\end{array}$ & $\begin{array}{l}\text { Water } \\
\text { level } \\
\text { depth } \\
\text { (feet) }\end{array}$ & $\begin{array}{l}\text { Date } \\
\mathrm{mm} / \mathrm{dd} / \mathrm{yy}\end{array}$ & Use & $\begin{array}{l}\text { Maximum } \\
\text { well } \\
\text { yield } \\
\text { (gallons } \\
\text { per } \\
\text { minute) }\end{array}$ & $\begin{array}{l}\text { Draw- } \\
\text { down } \\
\text { (feet) }\end{array}$ & $\begin{array}{l}\text { Specific } \\
\text { capacity } \\
\text { (gallons per } \\
\text { minute } \\
\text { per } \\
\text { foot) }\end{array}$ & $\begin{array}{l}\text { Pumping } \\
\text { period } \\
\text { (hours) }\end{array}$ & Remarks \\
\hline
\end{tabular}

Freedom - - Continued

\begin{tabular}{|c|c|c|c|c|c|c|c|c|}
\hline FLW & 55 & 15 & $05-13-88$ & $\mathrm{H}$ & 3 & $\cdots$ & $\cdots$ & .5 \\
\hline FLW & 56 & 40 & $07-05-88$ & $\mathrm{H}$ & 1 & $\cdots$ & $\cdots$ & .5 \\
\hline FLW & 57 & 20 & $07-15-88$ & $\mathrm{H}$ & 7 & $\cdots$ & - & .5 \\
\hline FLW & 58 & 40 & $07-16-88$ & H & 3.5 & $\cdots$ & $\cdots$ & .5 \\
\hline FLW & 59 & 15 & $10-27-88$ & H & 2 & $\cdots$ & $\cdots$ & .5 \\
\hline FLW & 60 & $\cdots$ & $-\cdot$ & $\mathrm{H}$ & 15 & $-\cdot$ & $\cdots$ & .5 \\
\hline FLW & 61 & 25 & $06-03-89$ & $\mathrm{H}$ & 12 & - & $\cdots$ & 1 \\
\hline FLW & 62 & 33 & $07-29-89$ & $\mathrm{H}$ & 7 & $\cdots$ & $\cdots$ & 2 \\
\hline FLW & 63 & 50 & $07-13-89$ & $\mathrm{H}$ & 5 & - & $\cdots$ & .5 \\
\hline FLW & 64 & - & - - & $\mathrm{H}$ & 12 & - & - & 1 \\
\hline FLW & 65 & 21 & $11-04-90$ & $\mathrm{H}$ & 3.5 & - & - & 2 \\
\hline FLW & 66 & 22.5 & $04-25-84$ & $\mathrm{H}$ & 12 & $\cdots$ & 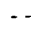 & 2 \\
\hline FLW & 67 & 30 & $06 \cdot 02 \cdot 84$ & $\mathrm{H}$ & 8 & $\cdots$ & - & .5 \\
\hline FLW & 68 & 40 & $06-29-84$ & $\mathrm{H}$ & 0.8 & $\cdots$ & $\cdots$ & 1 \\
\hline FLW & 69 & 25 & $06-29-84$ & H & 2 & $\cdots$ & - & .5 \\
\hline FLW & 70 & - & - - & $P$ & 6 & -- & $\cdots$ & 48 \\
\hline FLW & 71 & $\cdots$ & - & H & 4 & $\cdots$ & - - & 1 \\
\hline FLW & 72 & 20 & $08 \cdot 07 \cdot 84$ & $\mathrm{H}$ & 3 & $\cdots$ & $\cdots$ & .5 \\
\hline FLW & 73 & 40 & $09-17-84$ & $\mathrm{H}$ & 25 & - & $\cdots$ & .5 \\
\hline FLW & 74 & 15 & $10-13-84$ & $\mathrm{H}$ & 4 & -- & $\cdots$ & .5 \\
\hline FLW & 75 & 21 & $10-27-84$ & $\mathrm{H}$ & 6 & - & - & 5 \\
\hline FLW & 76 & 46.5 & $10-26-84$ & $\mathrm{H}$ & 10 & - & - & 3 \\
\hline FLW & 77 & 45 & $05 \cdot 25 \cdot 85$ & H & 0.5 & - & - & 2 \\
\hline FLW & 78 & 35 & $07-06-85$ & $\mathrm{H}$ & 1.3 & - & $\cdots$ & 1 \\
\hline FLW & 79 & 10 & $05-21-85$ & $\mathrm{H}$ & 12 & $\cdots$ & $\cdots$ & .5 \\
\hline FLW & 80 & $\cdots$ & - & $\mathrm{H}$ & 5 & -- & $\cdots$ & .8 \\
\hline FLW & 81 & 50 & $12-04-85$ & H & 3 & - & -- & .5 \\
\hline FLW & 82 & 20 & $12-15-85$ & $\mathrm{H}$ & 6.5 & - - & $\cdots$ & .5 \\
\hline FLW & 83 & -- & $\cdots$ & $\mathrm{H}$ & 9 & - & - & 1 \\
\hline FLW & 84 & 20 & $01-28-86$ & $\mathrm{H}$ & 60 & - & $\cdots$ & .5 \\
\hline FLW & 85 & 15 & $04-22-86$ & $\mathrm{H}$ & 2 & -- & - & .5 \\
\hline FLW & 86 & 25 & $05-15-86$ & $\mathrm{H}$ & 1.5 & $\cdots$ & $-\cdot$ & .5 \\
\hline FLW & 87 & 5 & $05-23-86$ & $\mathrm{H}$ & 10 & $\cdots$ & - & 10 \\
\hline FLW & 88 & $\cdots$ & -- & $\mathrm{H}$ & 150 & $\cdots$ & - & 1 \\
\hline FLW & 89 & 80 & $09-17-86$ & H & 4 & $\cdots$ & $\cdots$ & .5 \\
\hline FLW & 90 & 4 & $09-17-86$ & $\mathrm{H}$ & 15 & -- & $\cdots$ & .5 \\
\hline FLW & 91 & 22 & $10-03-86$ & $\mathrm{H}$ & 15 & - & $\cdots$ & 1 \\
\hline FLW & 92 & 28 & $10-11-86$ & $\mathrm{H}$ & 15 & $\cdots$ & $\cdots$ & 1 \\
\hline FLW & 93 & 70 & $10-17-86$ & H & 3.5 & $\cdots$ & - - & .5 \\
\hline FLW & 94 & 45 & $10-21-86$ & $\mathrm{H}$ & 15 & $\cdots$ & $\cdots$ & .5 \\
\hline FLW & 95 & 15 & $11-15-86$ & $\mathrm{H}$ & 0.3 & $\cdots$ & $\cdots$ & 1 \\
\hline FLW & 96 & 25.5 & $11-29-86$ & $\mathrm{H}$ & 16.5 & $\cdots$ & $\cdots$ & 1 \\
\hline FLW & 97 & 21 & $11-28-86$ & $\mathrm{H}$ & 16 & $\cdots$ & $\cdots$ & 2 \\
\hline FLW & 98 & 40 & $12-11-86$ & $\mathrm{H}$ & 12 & $\cdots$ & - & .5 \\
\hline FLW & 99 & 20 & $03-10-87$ & H & 10 & .. & - & .5 \\
\hline FLW & 100 & 11 & $04-13-87$ & $\mathrm{H}$ & 20 & $\cdots$ & $\cdots$ & 2 \\
\hline FLW & 101 & 8 & $03-03-87$ & $\mathrm{H}$ & 28 & $\cdots$ & $\cdots$ & $\cdots$ \\
\hline FLW & 102 & - & - & $\mathrm{H}$ & 3 & $\cdots$ & $\cdots$ & 1 \\
\hline FLW & 103 & 28 & $05-28-87$ & $\mathrm{H}$ & 150 & $\cdots$ & - & .5 \\
\hline FLW & 104 & 20 & $05-28-87$ & $\mathrm{H}$ & 50 & $\cdots$ & $\cdots$ & .5 \\
\hline FLW & 105 & 25 & $05-29-87$ & $\mathrm{H}$ & 100 & $\cdots$ & $\cdots$ & .5 \\
\hline FLW & 106 & 20 & $06-01-87$ & $\mathrm{H}$ & 100 & $\cdots$ & $\cdots$ & .5 \\
\hline FLW & 107 & 25 & $07-04-87$ & $\mathrm{H}$ & 16 & - & - & 1 \\
\hline FLW & 108 & 11 & $06 \cdot 07 \cdot 87$ & $\mathrm{H}$ & 10 & -- & $\cdots$ & 3 \\
\hline
\end{tabular}


Table 1-1. Description of selected wells, borings, and springs in the Saco and Ossipee Rlver Basins, east-central

\begin{tabular}{|c|c|c|c|c|c|c|c|c|c|c|c|}
\hline $\begin{array}{l}\text { Local } \\
\text { site } \\
\text { number }\end{array}$ & $\begin{array}{l}\text { Lat- } \\
\text { itude }\end{array}$ & $\begin{array}{l}\text { Long- } \\
\text { itude }\end{array}$ & Owner or user & $\begin{array}{l}\text { Year } \\
\text { com- } \\
\text { pleted }\end{array}$ & $\begin{array}{l}\text { Alti- } \\
\text { tude } \\
\text { above } \\
\text { sea } \\
\text { level } \\
\text { (feet) }\end{array}$ & $\begin{array}{c}\text { Dia- } \\
\text { meter } \\
\text { of } \\
\text { well } \\
\text { casing } \\
\text { (inches) }\end{array}$ & $\begin{array}{l}\text { Depth } \\
\text { to } \\
\text { bottom } \\
\text { of } \\
\text { casing } \\
\text { (feet) }\end{array}$ & $\begin{array}{l}\text { Casing } \\
\text { material }\end{array}$ & $\begin{array}{l}\text { Type } \\
\text { of } \\
\text { finish }\end{array}$ & $\begin{array}{l}\text { Depth } \\
\text { to } \\
\text { bottom } \\
\text { of open } \\
\text { section } \\
\text { (feet) }\end{array}$ & $\begin{array}{c}\text { Type } \\
\text { of } \\
\text { site }\end{array}$ \\
\hline
\end{tabular}

\begin{tabular}{|c|c|c|c|c|c|c|c|c|c|c|c|c|}
\hline FLW & 109 & 434844 & 710453 & Grunst & 1987 & 430 & -- & 31 & -- & $\mathbf{s}$ & -- & $\cdots$ \\
\hline FLW & 110 & 434801 & 710218 & Atherton & 1987 & 420 & - & 19 & -- & $\mathrm{x}$ & - & BrW \\
\hline FLW & 111 & 434832 & 710805 & Mckay & 1987 & 440 & -- & 30 & $\cdots$ & $\mathrm{x}$ & -- & BrW \\
\hline FLW & 112 & 434817 & 710806 & Becht & 1987 & 410 & - & 105 & -- & $x$ & $\cdots$ & BrW \\
\hline FLW & 113 & 435126 & 710325 & Hampton & 1987 & 740 & -- & 60 & $\cdots$ & $\mathrm{x}$ & - & BrW \\
\hline FLW & 114 & 434826 & 710807 & Phillips & 1987 & 430 & - & 73 & -- & $\mathbf{x}$ & - & BrW \\
\hline FLW & 115 & 434842 & 710803 & Foley & 1987 & 490 & $\cdots$ & 192 & - & $\mathrm{x}$ & - & BrW \\
\hline FLW & 116 & 434844 & 710805 & Coles & 1987 & 480 & $-\cdot$ & 191 & -- & $\mathrm{x}$ & $\cdots$ & BrW \\
\hline FLW & 117 & 434817 & 710717 & Mccarthy & 1987 & 440 & $\cdots$ & 35 & $\cdots$ & $\mathbf{S}$ & $\cdots$ & $\cdots$ \\
\hline FLW & 118 & 434927 & 710626 & McCowan & 1987 & 460 & $\cdots$ & 71 & $\cdots$ & $\mathbf{s}$ & $\cdots$ & $\cdots$ \\
\hline FLW & 119 & 434758 & 710707 & Poisson Const & 1987 & 430 & $\cdots$ & 60 & $\cdots$ & $\mathbf{x}$ & $-\cdot$ & BrW \\
\hline FLW & 120 & 434934 & 710640 & Quinn & 1987 & 440 & $\cdots$ & 53 & - & $\mathbf{s}$ & $\cdots$ & $\cdots$ \\
\hline FLW & 121 & 434821 & 710807 & Perella & 1987 & 415 & - & 151 & -- & $\mathrm{x}$ & - & BrW \\
\hline FLW & 122 & 435041 & 710350 & Gafner & 1987 & 1,060 & $\cdots$ & 39 & -- & $\mathbf{x}$ & $\cdots$ & BrW \\
\hline FLW & 123 & 434832 & 710805 & McKay & 1987 & 440 & $\cdots$ & 32 & -- & $\mathbf{x}$ & $\cdots$ & BrW \\
\hline FLW & 124 & 434830 & 710806 & Gilbride & 1987 & 430 & $\cdots$ & 30 & $\cdots$ & $x$ & $\cdots$ & BrW \\
\hline FLW & 125 & 434755 & 710209 & cloutier & 1987 & 410 & $\cdots$ & 29 & $\cdots$ & $x$ & $\cdots$ & BrW \\
\hline FLW & 126 & 434747 & 710732 & Tersolo & 1987 & 420 & -- & 39 & $\cdots$ & $\mathrm{x}$ & -- & BrW \\
\hline FLW & 127 & 434745 & 710400 & Freedom Village Condo D & 1988 & 410 & $\cdots$ & 47 & $\cdots$ & $\mathbf{x}$ & - & BrW \\
\hline FLW & 128 & 434745 & 710400 & Freedom Village Condo D & 1988 & 410 & $\cdots$ & 40 & $\cdots$ & $\mathbf{x}$ & -- & BrW \\
\hline FLW & 129 & 434804 & 710332 & Haynes & 1988 & 420 & -- & 60 & $-\cdot$ & $\mathbf{x}$ & $\cdots$ & BrW \\
\hline FLW & 130 & 434837 & 710802 & Chevalier & 1988 & 490 & $\cdots$ & 74 & - & $\mathrm{x}$ & $\cdots$ & BrW \\
\hline FLW & 131 & 434830 & 710504 & Dig \& Doze Const & 1988 & 420 & $\cdots$ & 31 & $\cdots$ & $\mathbf{s}$ & -- & $\cdots$ \\
\hline FLW & 132 & 434915 & 710652 & Davidson Jr & 1988 & 440 & $\cdots$ & 51 & $\cdots$ & $\mathbf{S}$ & $\cdots$ & $-\cdot$ \\
\hline FLW & 133 & 434913 & 710628 & Tabor & 1988 & 440 & $-\cdots$ & 57 & $\cdots$ & $\mathbf{S}$ & $\cdots$ & $\cdots$ \\
\hline FLW & 134 & 434924 & 710627 & Be1ville & 1988 & 460 & $\cdots$ & 70 & $\cdots$ & $\mathbf{s}$ & $\cdots$ & $\cdots$ \\
\hline FLW & 135 & 434749 & 710700 & Mailman & 1988 & 430 & -- & 40 & $-\cdot$ & $\mathrm{x}$ & $-\cdot$ & BrW \\
\hline FLW & 136 & 434857 & 710613 & Sands & 1988 & 420 & $-\cdot$ & 27 & $\cdots$ & $\mathbf{s}$ & $\cdots$ & $\cdots$ \\
\hline FLW & 137 & 434851 & 710452 & a11en & 1988 & 440 & $\cdots$ & 35 & $\cdots$ & $\mathbf{S}$ & $\cdots$ & $\cdots$ \\
\hline FLW & 138 & 435032 & 710116 & Reks & 1988 & 900 & $\cdots$ & 60 & $\cdots$ & $\mathbf{x}$ & $-\cdot$ & BrW \\
\hline FLW & 139 & 434807 & 710716 & C. Campgrounds Inc & 1988 & 440 & - & 73 & -- & $\mathbf{s}$ & $\cdots$ & $\cdots$ \\
\hline FLW & 140 & 434837 & 710809 & Leonard & 1988 & 450 & $\cdots$ & 210 & $\cdots$ & $\mathbf{x}$ & $-\cdot$ & BrW \\
\hline FLW & 141 & 434846 & 710808 & Grimes & 1988 & 480 & $\cdots$ & 265 & $\cdots$ & $\mathrm{x}$ & $\cdots$ & BrW \\
\hline FLW & 142 & 434902 & 710858 & Neergard & 1988 & 430 & $\cdots$ & 60 & $\cdots$ & $\mathrm{x}$ & $\cdots$ & BrW \\
\hline FLW & 143 & 434924 & 710421 & Paris & 1988 & 540 & $-\cdot$ & 87 & $-\cdot$ & $\mathrm{x}$ & $\cdots$ & BrW \\
\hline FLW & 144 & 434826 & 710758 & Staton & 1988 & 440 & $\cdots$ & 40 & $\cdots$ & $x$ & $\cdots$ & BrW \\
\hline FLW & 145 & 434751 & 710658 & Nelson & 1988 & 430 & $-\cdot$ & 33 & $\cdots$ & $\mathrm{x}$ & $\cdots$ & $\mathrm{BrW}$ \\
\hline FLW & 146 & 434820 & 710248 & Battles & 1988 & 460 & $\cdots$ & 80 & $-\cdot$ & $\mathrm{x}$ & $\cdots$ & BrW \\
\hline FLW & 147 & 434742 & 710706 & Pine Landing Devel & 1988 & 420 & $\cdots$ & 41 & $-\cdot$ & $\mathbf{s}$ & $\cdots$ & $-\cdot$ \\
\hline FLW & 148 & 434833 & 710802 & Cardin & 1987 & 460 & $\cdots$ & 40 & $\cdots$ & $\mathbf{x}$ & $\cdots$ & BrW \\
\hline FLW & 149 & 434837 & 710806 & Soep & 1987 & 460 & $\cdots$ & 100 & $\cdots$ & $\mathbf{x}$ & - & BrW \\
\hline FLW & 150 & 435103 & 710153 & Perry & 1988 & 1,040 & $\cdots$ & 40 & $-\cdot$ & $\mathrm{x}$ & $\cdots$ & BrW \\
\hline FLW & 151 & 434830 & 710212 & Nason & 1988 & 440 & -- & 69 & $\cdots$ & $\mathrm{x}$ & $\cdots$ & BrW \\
\hline FLW & 152 & 434801 & 710152 & Donahue & 1988 & 400 & $\cdots$ & 41 & $\cdots$ & $\mathrm{x}$ & $\cdots$ & BrW \\
\hline FLW & 153 & 434851 & 710439 & Morin & 1988 & 460 & $\cdots$ & 40 & $\cdots$ & $x$ & $\cdots$ & BrW \\
\hline FLW & 154 & 435108 & 710411 & Roberts & 1988 & 700 & $\cdots$ & 261 & $\cdots$ & $\mathbf{x}$ & $\cdots$ & BrW \\
\hline FLW & 155 & 434813 & 710312 & Niblett & 1988 & 400 & -- & 79 & $\cdots$ & $\mathrm{x}$ & - & BrW \\
\hline FLW & 156 & 434833 & 710425 & Goode & 1988 & 490 & $\cdots$ & 40 & $\cdots$ & $\mathbf{x}$ & $\cdots$ & BrW \\
\hline FLW & 157 & 434827 & 710805 & Casey & 1988 & 430 & $\cdots$ & 66 & -- & $\mathbf{x}$ & $-\cdot$ & BrW \\
\hline FLW & 158 & 434815 & 710257 & Dig \& Doze & 1989 & 420 & $\cdots$ & 40 & - & $\mathbf{x}$ & $\cdots$ & BrW \\
\hline FLW & 159 & 434834 & 710811 & Tauro & 1989 & 440 & $\cdots$ & 125 & $\cdots$ & $x$ & $\cdots$ & BrW \\
\hline FLW & 160 & 434843 & 710759 & Nigro & 1989 & 500 & - & 101 & $\cdots$ & $\mathrm{x}$ & $-\cdot$ & BrW \\
\hline FLW & 161 & 434846 & 710805 & Dix & 1989 & 490 & - & 254 & $\cdots$ & $\mathrm{x}$ & $-\cdot$ & BrW \\
\hline FLW & 162 & 434849 & 710801 & Long & 1989 & 480 & - & 179 & -- & $\mathrm{x}$ & -- & BrW \\
\hline FLW & 163 & 434913 & 710657 & Palmer & 1989 & 440 & - & 44 & $\cdots$ & $\mathbf{s}$ & $-\cdot$ & $-\cdots$ \\
\hline
\end{tabular}




\begin{tabular}{|c|c|c|c|c|c|c|c|c|}
\hline $\begin{array}{l}\text { Local } \\
\text { site } \\
\text { number }\end{array}$ & $\begin{array}{l}\text { Water } \\
\text { level } \\
\text { depth } \\
\text { (feet) }\end{array}$ & $\begin{array}{l}\text { Date } \\
\mathrm{mm} / \mathrm{dd} / \mathrm{yy}\end{array}$ & Use & $\begin{array}{c}\text { Maximum } \\
\text { well } \\
\text { yield } \\
\text { (gallons } \\
\text { per } \\
\text { minute) }\end{array}$ & $\begin{array}{l}\text { Draw- } \\
\text { down } \\
\text { (feet) }\end{array}$ & $\begin{array}{c}\text { Specific } \\
\text { capacity } \\
\text { (gallons per } \\
\text { minute } \\
\text { per } \\
\text { foot) }\end{array}$ & $\begin{array}{l}\text { Pumping } \\
\text { period } \\
\text { (hours) }\end{array}$ & Remarks \\
\hline
\end{tabular}

Freedom - - Continued

\begin{tabular}{|c|c|c|c|c|c|c|c|c|}
\hline FLW & 109 & 15 & $07-11-87$ & $\mathrm{H}$ & 25 & - & - & 3.5 \\
\hline FLW & 110 & 25 & $08-27-87$ & H & 15 & $\cdots$ & $\cdots$ & .5 \\
\hline FLW & 111 & 25 & $09-11-87$ & $\mathrm{H}$ & 9 & $\cdots$ & $\cdots$ & 1 \\
\hline FLW & 112 & - & $\cdots$ & $\mathrm{H}$ & 8 & $\cdots$ & $\cdots$ & .5 \\
\hline FLW & 113 & 20 & $08-13-87$ & H & 1 & $\cdots$ & $\cdots$ & 1 \\
\hline FLW & 114 & $\cdots$ & - - & $\mathrm{H}$ & 15 & $\cdots$ & -- & 1 \\
\hline FLW & 115 & 43 & $08-13-87$ & H & 2.5 & $\cdots$ & -- & 1 \\
\hline FLW & 116 & 54 & $08-11-87$ & $\mathrm{H}$ & 3 & $\cdots$ & -- & 1 \\
\hline FLW & 117 & 17 & $09-22-87$ & $\mathbf{H}$ & 7 & $\cdots$ & $\cdots$ & 2 \\
\hline FLW & 118 & 52 & $10-10-87$ & $\mathrm{H}$ & 8 & $\cdots$ & $\cdots$ & 1 \\
\hline FLW & 119 & 30 & $08-27-87$ & $\mathrm{H}$ & 4 & $\cdots$ & - & 1 \\
\hline FLW & 120 & 38 & $10-10-87$ & $\mathrm{H}$ & 15 & $\cdots$ & - & 2 \\
\hline FLW & 121 & $\cdots$ & - & H & 2 & $\cdots$ & - & 1 \\
\hline FLW & 122 & 100 & $11-04-87$ & $\mathrm{H}$ & 5 & - & $\cdots$ & 2 \\
\hline FLW & 123 & 35 & $11-23-87$ & H & 1 & $\cdots$ & $\cdots$ & 1 \\
\hline FLW & 124 & 18 & $11-24-87$ & $\mathrm{H}$ & 5 & $\cdots$ & $\cdots$ & .5 \\
\hline FLW & 125 & 25 & $10-27-87$ & $\mathrm{H}$ & 2.5 & $\cdots$ & $\cdots$ & .5 \\
\hline FLW & 126 & 20 & $10-14-87$ & $P$ & 60 & $\cdots$ & $\cdots$ & 1 \\
\hline FLW & 127 & 10 & $03-29-88$ & P & 40 & $\cdots$ & $\cdots$ & .5 \\
\hline FLW & 128 & 10 & $03-30-88$ & $P$ & 40 & $\cdots$ & $\cdots$ & .5 \\
\hline FLW & 129 & 10 & $04-11-88$ & $\mathrm{H}$ & 3 & $\cdots$ & $\cdots$ & 1 \\
\hline FLW & 130 & 35 & $04-06-88$ & $\mathrm{H}$ & 4 & - & - & .5 \\
\hline FLW & 131 & 23.5 & $04-30-88$ & H & 7 & $\cdots$ & $\cdots$ & 1.5 \\
\hline FLW & 132 & 31 & $05-07-88$ & $\mathrm{z}$ & 10 & - & $\cdots$ & 1 \\
\hline FLW & 133 & 41 & $06-13-88$ & $\mathrm{H}$ & 15 & $\cdots$ & $\cdots$ & 1 \\
\hline FLW & 134 & 51 & $06-11-88$ & $\mathrm{H}$ & 12 & $\cdots$ & $\cdots$ & 2 \\
\hline FLW & 135 & $\cdots$ & $\cdots$ & $\mathrm{H}$ & 100 & $\cdots$ & -- & 1 \\
\hline FLW & 136 & 12 & $06-18-88$ & $\cdots$ & 40 & $\cdots$ & - & 1 \\
\hline FLW & 137 & 21 & $07-30-88$ & $\mathrm{H}$ & 12 & -- & -- & 2 \\
\hline FLW & 138 & 25 & $08-01-88$ & $\mathrm{H}$ & 0.3 & $\cdots$ & $\cdots$ & 1 \\
\hline FLW & 139 & 30 & $05-03-88$ & C & 200 & $\cdots$ & $\cdots$ & 4 \\
\hline FLW & 140 & 60 & $05-25-88$ & $\mathrm{H}$ & 150 & $\cdots$ & -- & .5 \\
\hline FLW & 141 & 20 & $05-31-88$ & $\mathrm{H}$ & 60 & $\cdots$ & $\cdots$ & .5 \\
\hline FLW & 142 & 25 & $05-31-88$ & $\mathrm{H}$ & 5 & $\cdots$ & -- & 1 \\
\hline FLW & 143 & 45 & $06-24-88$ & $\mathrm{H}$ & 5 & $\cdots$ & - & .5 \\
\hline FLW & 144 & 30 & $07-19-88$ & H & 20 & $\cdots$ & - & .5 \\
\hline FLW & 145 & 25 & $07-19-88$ & $\mathrm{H}$ & 7.5 & $-\cdot$ & - & .5 \\
\hline FLW & 146 & 25 & $07-16-88$ & H & 3 & -- & - & .5 \\
\hline FLW & 147 & 22 & $07-04-88$ & $\mathrm{P}$ & 13 & $\cdots$ & $\cdots$ & 1.5 \\
\hline FLW & 148 & 15 & $05-05-87$ & H & 6 & $\cdots$ & $\cdots$ & .5 \\
\hline FLW & 149 & - & $\cdots$ & $\mathrm{H}$ & 5 & - & $\cdots$ & .5 \\
\hline FLW & 150 & 60 & $11-09-88$ & $\mathrm{H}$ & 10 & - & - & .5 \\
\hline FLW & 151 & 10 & $10-27-88$ & H & 100 & - & - & .5 \\
\hline FLW & 152 & 10 & $10-28-88$ & $\mathrm{H}$ & 150 & $\cdots$ & $\cdots$ & .5 \\
\hline FLW & 153 & 60 & $11-04-88$ & $\mathrm{H}$ & 0.5 & $\cdots$ & $-\cdot$ & 1 \\
\hline FLW & 154 & $\cdots$ & $\cdots$ & H & 0.8 & $\cdots$ & $\cdots$ & .5 \\
\hline FLW & 155 & - & $\cdots$ & H & 10 & $\cdots$ & $\cdots$ & 1 \\
\hline FLW & 156 & 45 & $12-01-88$ & $\mathbf{H}$ & 1 & - & $\cdots$ & 1 \\
\hline FLW & 157 & $\cdots$ & $\cdots$ & H & 9 & $\cdots$ & $-\cdot$ & 1 \\
\hline FLW & 158 & 18 & $04-20-89$ & H & 2.5 & $\cdots$ & -- & .5 \\
\hline FLW & 159 & 30 & $04-20-89$ & H & 15 & - & $\cdots$ & 1 \\
\hline FLW & 160 & - - & $\cdots$ & H & 1 & $\cdots$ & -- & 1 \\
\hline FLW & 161 & -- & -- & H & 12 & - & $\cdots$ & 1 \\
\hline FLW & 162 & $\cdots$ & $\cdots$ & $\mathrm{H}$ & 10 & $\cdots$ & $-\cdots$ & 1 \\
\hline FLW & 163 & 18 & $04-29-89$ & H & 15 & $\cdots$ & $\cdots$ & 2 \\
\hline
\end{tabular}


Table 1-1. Description of selected wells, borings, and springs In the Saco and Ossipee River Basins, east-centrai

\begin{tabular}{|c|c|c|c|c|c|c|c|c|c|c|c|}
\hline $\begin{array}{l}\text { Local } \\
\text { site } \\
\text { number }\end{array}$ & $\begin{array}{l}\text { Lat- } \\
\text { itude }\end{array}$ & $\begin{array}{l}\text { Long- } \\
\text { itude }\end{array}$ & Owner or user & $\begin{array}{l}\text { Year } \\
\text { com- } \\
\text { pleted }\end{array}$ & $\begin{array}{l}\text { Alti- } \\
\text { tude } \\
\text { above } \\
\text { sea } \\
\text { level } \\
\text { (feet) }\end{array}$ & $\begin{array}{l}\text { Dia- } \\
\text { meter } \\
\text { of } \\
\text { well } \\
\text { casing } \\
\text { (inches) }\end{array}$ & $\begin{array}{l}\text { Depth } \\
\text { to } \\
\text { bottom } \\
\text { of } \\
\text { casing } \\
\text { (feet) }\end{array}$ & $\begin{array}{l}\text { Casing } \\
\text { material }\end{array}$ & $\begin{array}{l}\text { Type } \\
\text { of } \\
\text { finish }\end{array}$ & $\begin{array}{l}\text { Depth } \\
\text { to } \\
\text { bottom } \\
\text { of open } \\
\text { section } \\
\text { (feet) }\end{array}$ & $\begin{array}{c}\text { Type } \\
\text { of } \\
\text { site }\end{array}$ \\
\hline
\end{tabular}

Freedom - - Continued

\begin{tabular}{|c|c|c|c|c|}
\hline FLW & 164 & 435059 & 710153 & Perry \\
\hline FLW & 165 & 434848 & 710447 & Owens \\
\hline FLW & 166 & 434845 & 710759 & Corcoran \\
\hline FLW & 167 & 434843 & 710803 & Amico \\
\hline FLW & 168 & 434816 & 710720 & Kondrat \\
\hline FLW & 169 & 434902 & 710901 & McKechnie \\
\hline FLW & 170 & 434736 & 710631 & Abode \\
\hline FLW & 171 & 435051 & 710200 & Bittner \\
\hline FLW & 172 & 434841 & 710800 & Stone \\
\hline FLW & 173 & 434900 & 710448 & Johnson \\
\hline FLW & 174 & 434818 & 710324 & Eldridge \\
\hline FLW & 175 & 434922 & 710337 & Wheeler \\
\hline FLW & 176 & 434747 & 710329 & Lawler \\
\hline FLW & 177 & 434845 & 710805 & Mcfaun \\
\hline FLW & 178 & 434843 & 710705 & Neal \\
\hline FLW & 179 & 434831 & 710751 & Bolduc \\
\hline FLW & 180 & 434830 & 710749 & Bolduc \\
\hline FLW & 181 & 434821 & 710756 & Hu1slander \\
\hline FLW & 182 & 434820 & 710756 & Coughlin \\
\hline FLW & 183 & 434819 & 710754 & Shel thammer \\
\hline FLW & 184 & 434810 & 710439 & Rollins \\
\hline FLW & 185 & 434748 & 710619 & Keenan \\
\hline FLW & 186 & 434837 & 710645 & Hunt \\
\hline FLW & 187 & 434901 & 710620 & Deluca \\
\hline FLW & 188 & 434846 & 710711 & Collins \\
\hline FLW & 189 & 435021 & 710447 & Wa1z \\
\hline FLW & 190 & 434830 & 710442 & Sanders \\
\hline FLW & 191 & 434746 & 710141 & Farnella \\
\hline FLW & 192 & 434933 & 710631 & Ogren \\
\hline FLW & 193 & 434756 & 710218 & Cummings \\
\hline FLW & 194 & 434815 & 710803 & Mazzeo \\
\hline FLW & 195 & 434952 & 710031 & worth \\
\hline FLW & 196 & 434746 & 710735 & Boynton \\
\hline FLW & 197 & 434921 & 710422 & Brooks \\
\hline FLW & 198 & 434800 & 710236 & Grossman \\
\hline FLW & 199 & 435041 & 710221 & Fauver \\
\hline FLW & 200 & 434758 & 710219 & Farine1la \\
\hline
\end{tabular}

\begin{tabular}{|c|c|c|c|c|c|c|c|}
\hline 1989 & 1,020 & $\cdots$ & 60 & $\cdots$ & $x$ & $\cdots$ & Brw \\
\hline 1989 & 440 & $\cdots$ & 50 & $\cdots$ & $\mathbf{x}$ & . & Brw \\
\hline 1989 & 500 & $\cdots$ & 141 & $\cdots$ & $\mathrm{x}$ & $\cdots$ & Brw \\
\hline 1989 & 500 & $\cdots$ & 153 & $\cdots$ & $x$ & $\cdots$ & BrW \\
\hline 1989 & 440 & $\cdots$ & 195 & - & $x$ & $\cdots$ & BrW \\
\hline 1989 & 420 & $\cdots$ & 71 & $\cdots$ & $x$ & $\cdots$ & BrW \\
\hline 1989 & 460 & $\cdots$ & 172 & - & $\mathrm{x}$ & $\cdots$ & Brw \\
\hline 1990 & 1,040 & $\cdots$ & 39 & - & $x$ & $\cdots$ & BrW \\
\hline 1989 & 500 & $\cdots$ & 91 & $\cdots$ & $\mathrm{x}$ & $\cdots$ & BrW \\
\hline 1990 & 480 & $\cdots$ & 60 & $\cdots$ & $x$ & $\cdots$ & Brw \\
\hline 1990 & 420 & $\cdots$ & 60 & $\cdots$ & $x$ & $\cdots$ & BrW \\
\hline 1990 & 620 & $\cdots$ & 34 & $\cdots$ & $x$ & $\cdots$ & BrW \\
\hline 1990 & 420 & - & 53 & - & $\mathrm{x}$ & $\cdots$ & BrW \\
\hline 1990 & 490 & $\cdots$ & 196 & $\cdots$ & $x$ & $\cdots$ & BrW \\
\hline 1991 & 440 & $\cdots$ & 26 & $\cdots$ & $\mathbf{S}$ & $\cdots$ & $\cdots$ \\
\hline 1989 & 540 & $\cdots$ & 43 & $\cdots$ & $\mathbf{x}$ & $\cdots$ & $\mathrm{BrW}$ \\
\hline 1989 & 520 & - & 43 & $\cdots$ & $x$ & $\cdots$ & BrW \\
\hline 1989 & 430 & - & 41 & $\cdots$ & $x$ & $\cdots$ & BrW \\
\hline 1989 & 430 & $\ldots$ & 86 & $\cdots$ & $\mathrm{x}$ & $\cdots$ & BrW \\
\hline 1989 & 430 & - & 86 & - & $x$ & $\cdots$ & BrW \\
\hline 1991 & 420 & $\cdots$ & 11 & $\cdots$ & $\cdots$ & $\cdots$ & - \\
\hline 1991 & 420 & - & 35 & $\cdots$ & $\mathbf{S}$ & - & - \\
\hline 1991 & 440 & $\ldots$ & 53 & - & $\mathrm{S}$ & - & $\cdots$ \\
\hline 1991 & 420 & - & 58 & - & $\cdots$ & $\cdots$ & - \\
\hline 1991 & 450 & - & 29 & - & $\mathbf{S}$ & $\cdots$ & $\cdots$ \\
\hline 1991 & 590 & . & 49 & - & $x$ & - & BrW \\
\hline 1991 & 440 & $\cdots$ & 49 & - & $x$ & $\cdots$ & BrW \\
\hline 1992 & 400 & - & 33.5 & - & $\mathbf{S}$ & - & $\cdots$ \\
\hline 1992 & 420 & - & 35 & - & $\mathbf{S}$ & $\cdots$ & $\cdots$ \\
\hline 1992 & 400 & $\cdots$ & 49 & - & $x$ & $\cdots$ & BrW \\
\hline 1992 & 410 & $\cdots$ & 74 & - - & $x$ & $\cdots$ & BrW \\
\hline 1992 & 830 & $\cdots$ & 36 & $\cdots$ & $x$ & - & BrW \\
\hline 1992 & 420 & - & 21 & $\cdots$ & $\mathbf{x}$ & - & BrW \\
\hline 1992 & 540 & $\cdots$ & 108 & - & $x$ & $\cdots$ & BrW \\
\hline 1992 & 400 & - & 80 & $\cdots$ & $x$ & $\cdots$ & BrW \\
\hline 1992 & 890 & $\cdots$ & 119 & $\cdots$ & $\mathrm{x}$ & $\cdots$ & $\mathrm{BrW}$ \\
\hline 1993 & 400 & . & 54 & . & $x$ & $\cdots$ & BrW \\
\hline
\end{tabular}

HJW $\quad 1 \quad \mathbf{4 4 0 4 4 4} \quad \mathbf{7 1 1 9 1 9}$ Badger Rea1ty

HJW $2 \quad 440442 \quad 711913 \quad$ Stucchi

$\begin{array}{llll}\text { HJB } & 1 & 441200 & 712425\end{array}$

HJB $\quad 2 \quad \mathbf{4 4 1 1 5 2} \quad \mathbf{7 1 2 4 2 8}$

HJB $3440911 \quad 712154$

$\begin{array}{llll}\text { HJB } & 4 & \mathbf{4 4 0 6 4 7} & \mathbf{7 1 2 1 1 5}\end{array}$

HJB $5 \quad \mathbf{4 4 0 6 4 5} 712115$

HJB $\quad 6 \quad 441013 \quad 712306$
Hart's Location

$\begin{array}{cccccccc}1985 & 760 & \ldots & 119 & \ldots & \text { X } & \ldots & \text { BrW } \\ 1990 & 760 & \ldots & 79 & \ldots & \text { S } & \ldots & \ldots \\ \ldots & 1,487.5 & \ldots & \ldots & \ldots & \ldots & \ldots & \text { TH } \\ \ldots & 1,459.8 & \ldots & \ldots & \ldots & \ldots & \ldots & \text { TH } \\ \ldots & 1,153.7 & \ldots & \ldots & \ldots & \ldots & \ldots & \text { TH } \\ \ldots & 1,020.1 & \ldots & \ldots & \ldots & \ldots & \ldots & \text { TH } \\ \ldots & 1,002.6 & \ldots & \ldots & \ldots & \ldots & \ldots & \text { TH } \\ & 1,287 & \ldots & \ldots & \ldots & \ldots & \ldots & \text { TH }\end{array}$

Jackson

$\begin{array}{cccccccc}\ldots & 1,583.3 & \ldots & \ldots & \ldots & \ldots & \ldots & \text { TH } \\ \ldots & 1,152.6 & \ldots & \ldots & \ldots & \ldots & \ldots & \text { TH } \\ \ldots & 992 & \ldots & \ldots & \ldots & \ldots & \ldots & \text { TH }\end{array}$

152.6
992

$-$

$-\cdot$

TH $\begin{array}{llll}\text { JAB } & 1 & 441331 & 711512 \\ \text { JAB } & 2 & 441207 & 711418\end{array}$

$\begin{array}{llll}J A B & 3 & 440843 & 711122\end{array}$ 
New Hampshire--Continued

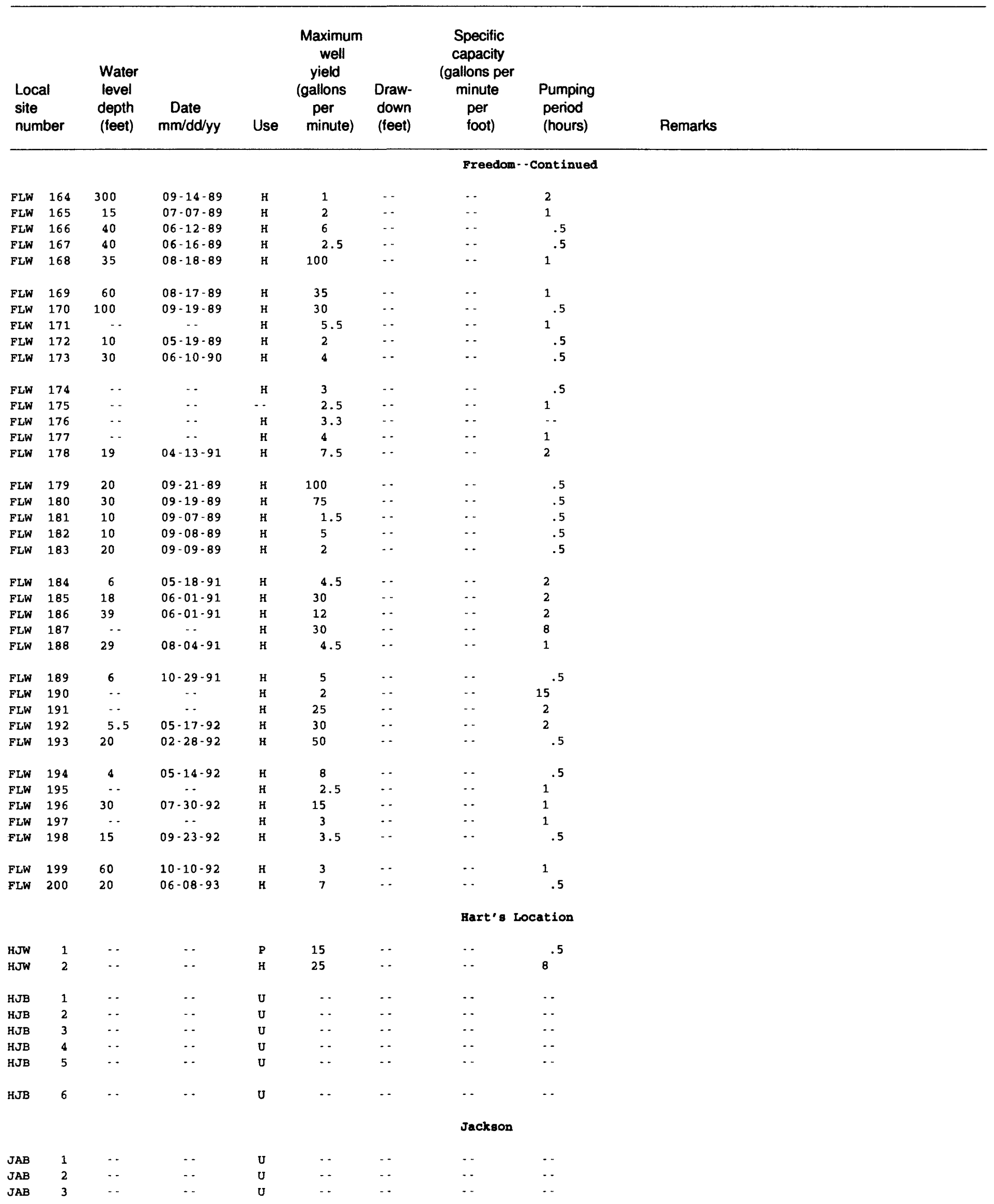

Appendlx 181 
Table 1-1. Description of selected wells, borings, and springs in the Saco and Ossipee River Basins, east-centrai

\begin{tabular}{|c|c|c|c|c|c|c|c|c|c|c|c|}
\hline $\begin{array}{l}\text { Local } \\
\text { site } \\
\text { number }\end{array}$ & $\begin{array}{l}\text { Lat- } \\
\text { itude }\end{array}$ & $\begin{array}{l}\text { Long- } \\
\text { itude }\end{array}$ & Owner or user & $\begin{array}{l}\text { Year } \\
\text { com- } \\
\text { pleted }\end{array}$ & $\begin{array}{l}\text { Alti- } \\
\text { tude } \\
\text { above } \\
\text { sea } \\
\text { level } \\
\text { (feet) }\end{array}$ & $\begin{array}{l}\text { Dia- } \\
\text { meter } \\
\text { of } \\
\text { well } \\
\text { casing } \\
\text { (inches) }\end{array}$ & $\begin{array}{l}\text { Depth } \\
\text { to } \\
\text { bottom } \\
\text { of } \\
\text { casing } \\
\text { (feet) }\end{array}$ & $\begin{array}{l}\text { Casing } \\
\text { material }\end{array}$ & $\begin{array}{l}\text { Type } \\
\text { of } \\
\text { finish }\end{array}$ & $\begin{array}{l}\text { Depth } \\
\text { to } \\
\text { bottom } \\
\text { of open } \\
\text { section } \\
\text { (feet) }\end{array}$ & $\begin{array}{c}\text { Type } \\
\text { of } \\
\text { site }\end{array}$ \\
\hline
\end{tabular}

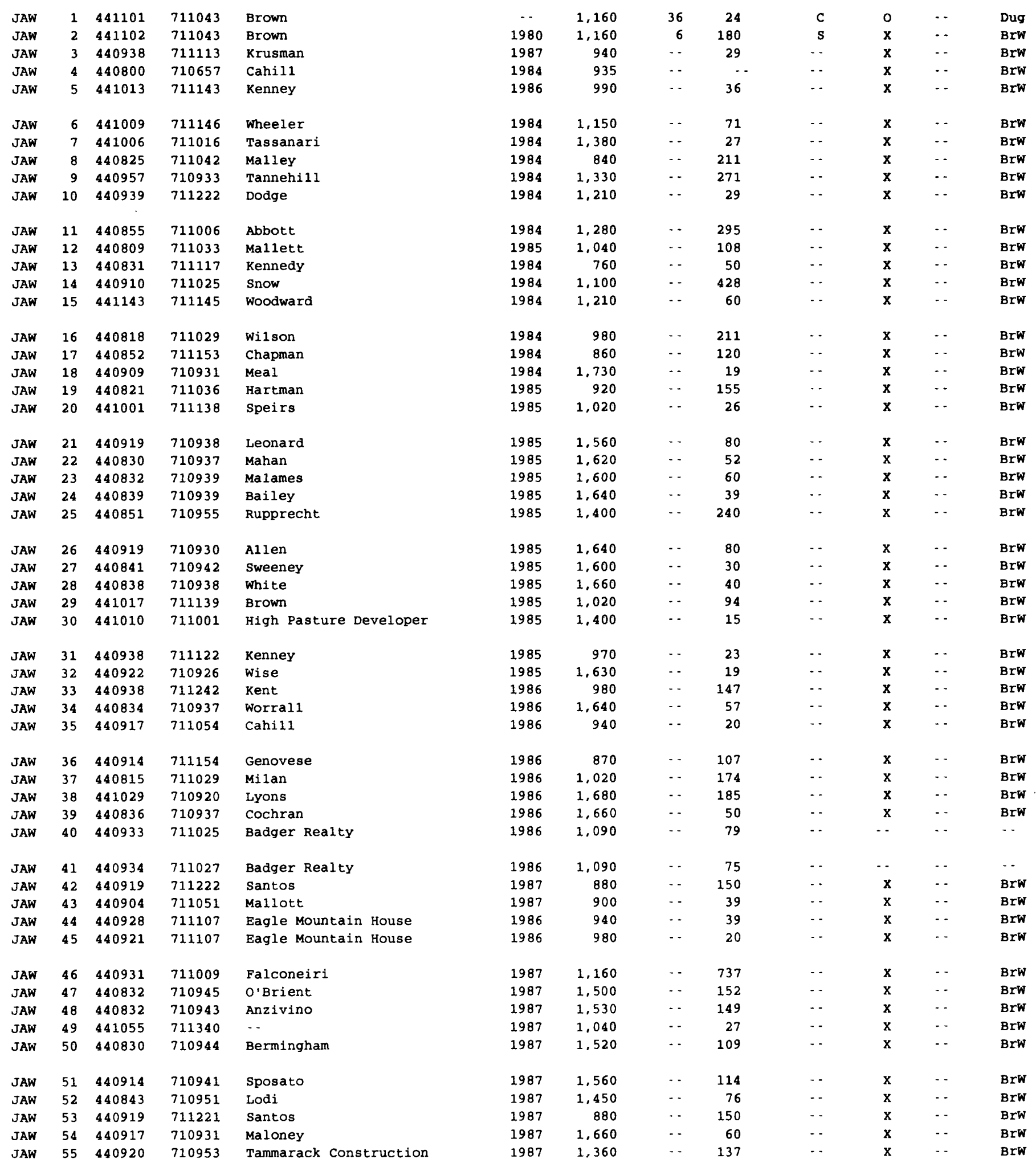




\begin{tabular}{|c|c|c|c|c|c|c|c|c|}
\hline $\begin{array}{l}\text { Local } \\
\text { site } \\
\text { number }\end{array}$ & $\begin{array}{l}\text { Water } \\
\text { level } \\
\text { depth } \\
\text { (feet) }\end{array}$ & $\begin{array}{l}\text { Date } \\
\mathrm{mm} / \mathrm{dd} / \mathrm{yy}\end{array}$ & Use & $\begin{array}{l}\text { Maximum } \\
\text { well } \\
\text { yield } \\
\text { (gallons } \\
\text { per } \\
\text { minute) }\end{array}$ & $\begin{array}{l}\text { Draw- } \\
\text { down } \\
\text { (feet) }\end{array}$ & $\begin{array}{c}\text { Specific } \\
\text { capacity } \\
\text { (gallons per } \\
\text { minute } \\
\text { per } \\
\text { foot) }\end{array}$ & $\begin{array}{l}\text { Pumping } \\
\text { period } \\
\text { (hours) }\end{array}$ & Remarks \\
\hline
\end{tabular}

\begin{tabular}{|c|c|c|c|c|c|c|c|c|c|}
\hline JAW & 1 & 18 & 08-07-91 & I & -. & - - & $\cdots$ & $\cdots$ & contains iron \\
\hline JAW & 2 & -. & - & H & 10 & - & - & $\cdots$ & \\
\hline JAW & 3 & - & - & H & 12 & -. & -. & .5 & \\
\hline JAW & 4 & 40 & $06-06-84$ & H & 50 & - & $\cdots$ & 1 & \\
\hline JAW & 5 & .. & .. & H & 55 & .. & -. & .5 & \\
\hline
\end{tabular}

$\begin{array}{lllllllll}\text { JAW } & 5 & \cdots & \cdots & \text { H } & 55 & \cdots & \cdots & .5\end{array}$

\begin{tabular}{lllllllll} 
JAW & 6 & $\ldots$ & $\ldots$ & H & 10 & $\ldots$ & $\ldots$ & 1 \\
\hline
\end{tabular}

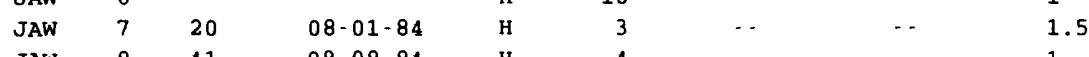

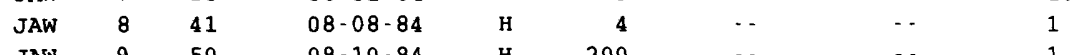

$\begin{array}{lrcccrccc}\text { JAW } & 9 & 50 & 08-10-84 & \text { H } & 200 & \ldots & \ldots & 1 \\ \text { JAW } & 10 & \ldots & \cdots & \text { H } & 5 & \ldots & \ldots & 1\end{array}$

$\begin{array}{lllllllll}\text { JAW } & 11 & \ldots & \ldots & \mathrm{H} & 2.8 & \ldots & \ldots & 1\end{array}$

$\begin{array}{lllllllll}\text { JAW } & 12 & 45 & 09-23-85 & \text { H } & 2.5 & \cdots & \cdots & \text {. } \\ \text { JAW } & 13 & 15 & 10-03-84 & \text { H } & 2 & \cdots & \cdots & 1 \\ \text { JAW } & 14 & 90 & 09-07-84 & \text { H } & 3.5 & \cdots & \cdots & \text {. }\end{array}$

$\begin{array}{lllllllll}\text { JAW } & 14 & 90 & 09-07-84 & \text { H } & 3.5 & . & . & .5 \\ \text { JAW } & 15 & 15 & 08-27-84 & \text { H } & 9 & -. & . . & .5\end{array}$

$\begin{array}{lllllllll}\text { JAW } & 16 & \ldots & \ldots & \text { H } & 2 & \ldots & \ldots & 1\end{array}$

$\begin{array}{lcccccccc}\text { JAW } & 17 & \cdots & \cdots & \text { H } & 4 & . & \ldots & 1 \\ \text { JAW } & 18 & 80 & 11-24-84 & \text { H } & 1 & \ldots & \ldots & 1\end{array}$

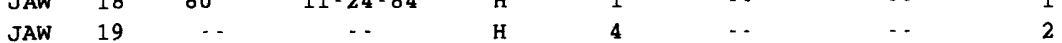

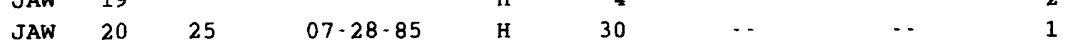

$\begin{array}{lllllllll}\text { JAW } & 21 & \ldots & \ldots & \text { H } & 6 & \ldots & \ldots & 1\end{array}$

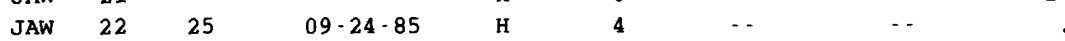

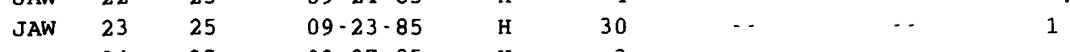

$\begin{array}{lcccccccc}\text { JAW } & 24 & 35 & 09-27-85 & \text { H } & 3 & . & . . & .5 \\ \text { JAW } & 25 & . & -. & \text { H } & 0.3 & . & . & \end{array}$

$\begin{array}{llccccccc}\text { JAW } & 26 & \ldots & \ldots & \text { H } & 8 & \ldots & \ldots & \ldots \\ & 27 & 36 & 10-30-85 & \text { H } & 2 & -. & \ldots & 2\end{array}$

$\begin{array}{lcccccccc}\text { JAW } & 27 & 36 & 10-30-85 & \text { H } & 2 & -- & - & 2 \\ \text { JAW } & 28 & - & -- & \text { H } & 1.5 & -- & - & 1\end{array}$

$\begin{array}{lllllllll}\text { JAW } & 29 & -. & \ldots & \text { H } & 10 & \ldots & \ldots & 1 \\ \text { JAW } & 30 & -. & \ldots & \text { H } & 55 & \ldots & \ldots & .5\end{array}$

$\begin{array}{lllllllll}\text { JAW } & 31 & \ldots & \ldots & \text { H } & 40 & \ldots & \ldots & .5\end{array}$

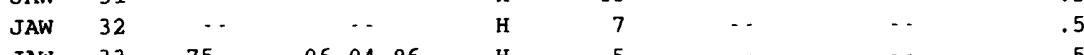

$\begin{array}{lccccrccc}\text { JAW } & 33 & 75 & 06-04-86 & \text { H } & 5 & -. & -. & .5 \\ \text { JAW } & 34 & -. & -.- & \text { H } & 10 & -. & -. & 1\end{array}$

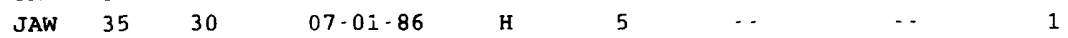

$\begin{array}{llllllll}\text { JAW } & 36 & 15 & 09-19-86 & \text { H } & 20 & \ldots & \ldots\end{array}$

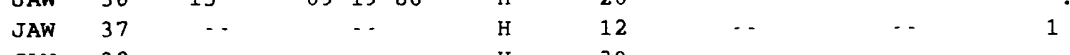

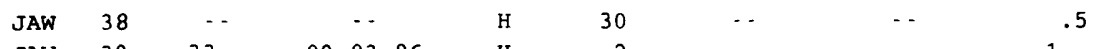

$\begin{array}{rccccrccc}\text { JAW } & 39 & 33 & 09-03-86 & \text { H } & 2 & -. & \ldots & 1 \\ \text { JAW } & 40 & -. & -. & \text { H } & 100 & \ldots & . & .5\end{array}$

$\begin{array}{lllllllll}\text { JAW } & 41 & \ldots & \ldots & \text { H } & 100 & \ldots & \ldots & .5\end{array}$

$\begin{array}{llllll}-. & \text { H } & 50 & \cdots & \text {. } & 1\end{array}$

$\begin{array}{lllllllll}\text { JAW } & 43 & 145 & 07-20-87 & \text { P } & 50 & \cdots & \cdots & 1\end{array}$

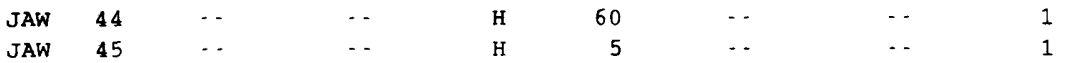

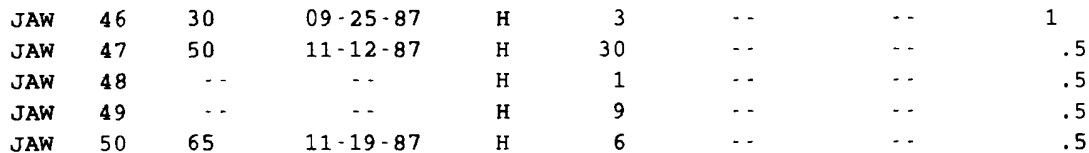

$\begin{array}{lllllllll}\text { JAW } & 51 & 150 & 11-11-87 & \text { H } & 30 & \ldots & \ldots & 1\end{array}$

$\begin{array}{ccccccccc}\text { JAW } & 52 & 80 & 11-16-87 & \text { H } & 3.5 & - & \ldots & 1 \\ \text { JAW } & 53 & -. & -. & \text { H } & 35 & - & \ldots & 1 \\ \text { JAW } & 54 & -. & -. & \text { H } & 12 & -. & \ldots & 1\end{array}$

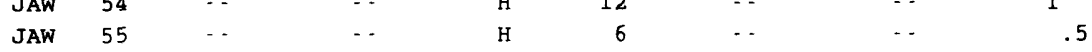


Table 1-1. Description of selected wells, borings, and springs in the Saco and Ossipee River Basins, east-central

\begin{tabular}{|c|c|c|c|c|c|c|c|c|c|c|c|c|}
\hline \multicolumn{2}{|c|}{$\begin{array}{l}\text { Local } \\
\text { site } \\
\text { number }\end{array}$} & $\begin{array}{l}\text { Lat- } \\
\text { itude }\end{array}$ & $\begin{array}{l}\text { Long- } \\
\text { itude }\end{array}$ & Owner or user & $\begin{array}{l}\text { Year } \\
\text { com- } \\
\text { pleted }\end{array}$ & 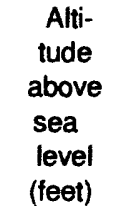 & $\begin{array}{l}\text { Dia- } \\
\text { meter } \\
\text { of } \\
\text { well } \\
\text { casing } \\
\text { (inches) }\end{array}$ & $\begin{array}{l}\text { Depth } \\
\text { to } \\
\text { bottom } \\
\text { of } \\
\text { casing } \\
\text { (feet) }\end{array}$ & $\begin{array}{l}\text { Casing } \\
\text { material }\end{array}$ & $\begin{array}{l}\text { Type } \\
\text { of } \\
\text { finish }\end{array}$ & $\begin{array}{l}\text { Depth } \\
\text { to } \\
\text { bottom } \\
\text { of open } \\
\text { section } \\
\text { (feet) }\end{array}$ & $\begin{array}{c}\text { Type } \\
\text { of } \\
\text { site }\end{array}$ \\
\hline \multicolumn{13}{|c|}{ Jackson--Continued } \\
\hline JAW & 56 & 440905 & 711036 & Chase & 1987 & 960 & $\cdots$ & 137 & -. & $\mathrm{x}$ & .. & $\mathrm{BrW}$ \\
\hline JAW & 57 & 441147 & 711354 & Poirier & 1987 & 1,200 & - & 19 & $\cdots$ & $\mathbf{x}$ & -. & BrW \\
\hline JAW & 58 & 440957 & 711317 & Helbich & 1988 & 940 & -. & 101 & -. & $x$ & - & BrW \\
\hline JAW & 59 & 440859 & 711003 & Leonard & 1988 & 1,330 & $\cdots$ & 358 & $-\cdot$ & $x$ & $\cdots$ & BrW \\
\hline JAW & 60 & 440818 & 711043 & Kramp & 1988 & 920 & $\cdots$ & 153 & $-\cdot$ & $\mathbf{x}$ & $\cdots$ & Brw \\
\hline JAW & 61 & 440947 & 711129 & Heistand & 1988 & 980 & - & 40 & $\cdots$ & $\mathbf{x}$ & -. & Brw \\
\hline JAW & 62 & 441138 & 711136 & Chipman & 1988 & 1,220 & .. & 100 & $\cdots$ & $\mathrm{x}$ & .. & BrW \\
\hline JAW & 63 & 441021 & 710911 & Peterson & 1988 & 1,610 & -. & 232 & $\cdots$ & $x$ & .. & BrW \\
\hline JAW & 64 & 440855 & 711220 & Graves & 1989 & 1,000 & $\cdots$ & 135 & $\cdots$ & $\mathrm{x}$ & $\cdots$ & Brw \\
\hline JAW & 65 & 440836 & 710946 & Lafond & 1989 & 1,480 & $\cdots$ & 130 & $-\cdot$ & $x$ & $\cdots$ & BrW \\
\hline JAW & 66 & 441007 & 711133 & Jenkins & 1989 & 980 & $\cdots$ & 45 & $\cdots$ & $\mathrm{x}$ & -. & Brw \\
\hline JAW & 67 & 440927 & 711213 & Salerno & 1989 & 1,000 & $\cdots$ & 160 & $\cdots$ & $x$ & $\cdots$ & Brw \\
\hline JAW & 68 & 441130 & 711345 & Maynard & 1989 & 1,060 & -. & 60 & $\cdots$ & $x$ & $\cdots$ & Brw \\
\hline JAW & 69 & 440903 & 711243 & Lovering & 1990 & 1,030 & $\cdots$ & 147 & -- & $\mathrm{x}$ & .. & BrW \\
\hline JAW & 70 & 440946 & 711230 & Tesone & 1990 & 1,120 & $\cdots$ & 70 & -. & $x$ & $\cdots$ & BrW \\
\hline JAW & 71 & 440935 & 711108 & Williams & 1991 & 940 & $\cdots$ & 40 & $\cdots$ & $x$ & $\cdots$ & BrW \\
\hline JAW & 72 & 440954 & 711125 & Bordash & 1991 & 980 & $\cdots$ & 60 & $\cdots$ & $\mathrm{x}$ & .. & BrW \\
\hline JAW & 73 & 440957 & 711134 & Kehoe & 1991 & 1,000 & $\cdots$ & 50 & $\cdots$ & $x$ & $\cdots$ & BrW \\
\hline JAW & 74 & 441001 & 711048 & Jenkins & 1991 & 1,100 & - & 122 & $\cdots$ & $x$ & .. & Brw \\
\hline JAW & 75 & 440912 & 711024 & Stone & 1992 & 1,110 & $\cdots$ & 432 & $\cdots$ & $x$ & $\cdots$ & BrW \\
\hline JAW & 76 & 440912 & 710948 & Russo & 1992 & 1,460 & - & 220 & $\cdots$ & $\mathrm{x}$ & - & BrW \\
\hline JAW & 77 & 440737 & 710735 & Tinker & 1988 & 900 & $\cdots$ & 42 & $\cdots$ & $\mathbf{x}$ & $\cdots$ & BrW \\
\hline JAW & 78 & 440744 & 710658 & Holmes & 1988 & 1,160 & $\cdots$ & 121 & $\cdots$ & $\mathrm{x}$ & $\cdots$ & BrW \\
\hline \multicolumn{13}{|c|}{ Madison } \\
\hline MBA & 1 & 435618 & 710917 & & 1992 & 500 & $\cdots$ & $\cdots$ & - & $\cdots$ & $\cdots$ & Bor \\
\hline MBB & 1 & 435053 & 711019 & NHDOT & $\cdots$ & 464.5 & $\cdots$ & $\cdots$ & $\cdots$ & .. & -. & $\mathbf{T H}$ \\
\hline MBB & 2 & 435315 & 711012 & NHDOT & $\cdots$ & 469 & $\cdots$ & $\cdots$ & $\cdots$ & - & $\cdots$ & TH \\
\hline MBB & 3 & 435547 & 710931 & NHDOT & $\cdots$ & 492 & $\cdots$ & $\cdots$ & $\cdots$ & $\cdots$ & -. & $\mathbf{T H}$ \\
\hline MBB & 4 & 435333 & 710935 & NHDOT & $\cdots$ & 480 & $\cdots$ & $\cdots$ & $\cdots$ & $\cdots$ & $\cdots$ & $\mathrm{TH}$ \\
\hline MBS & 1 & 435314 & 711048 & JOHN F. CHICK & $\cdots$ & 485 & $\cdots$ & $\cdots$ & $\cdots$ & $\cdots$ & $\cdots$ & sp \\
\hline MBW & 1 & 435010 & 711017 & New Eng Lumber & 1956 & 456 & 6 & - & -. & $\mathbf{s}$ & $\cdots$ & $\cdots$ \\
\hline MBW & 2 & 435002 & 711111 & Madison Lumber Mill & 1983 & 450 & $\cdots$ & $-\cdot$ & $\cdots$ & $\mathrm{x}$ & $\cdots$ & $\cdots$ \\
\hline MBW & 3 & 435102 & 710954 & Garland & 1974 & 495 & 6 & -. & $\mathbf{s}$ & $\mathrm{x}$ & .. & Brw \\
\hline MBW & 4 & 435102 & 710954 & Garland & 1969 & 495 & 36 & 15.5 & c & 0 & $\cdots$ & Dug \\
\hline MBW & 5 & 435059 & 710957 & Josepth Vonhandorf & 1981 & 485 & 8 & 87 & $\mathbf{s}$ & G & $\cdots$ & $\mathrm{Cb} 1$ \\
\hline MBW & 6 & 435104 & 711046 & Kevin and Kate walker & 1980 & 485 & 2 & 55 & $\mathbf{G}$ & $\mathbf{T}$ & $-\cdot$ & Dvn \\
\hline MBW & 7 & 435317 & 711021 & Henry Forrest & 1981 & 475 & 36 & 10.3 & $\mathrm{c}$ & 0 & $\cdots$ & Dug \\
\hline MBW & 9 & 435529 & 710956 & Kevin VOLLMAR & & 505 & 2 & & $\mathbf{s}$ & $\mathbf{T}$ & -. & Dvn \\
\hline MBW & 10 & 435054 & 711132 & USGS & 1991 & 480 & 2 & 59 & $P$ & $\mathbf{s}$ & 59 & Bor \\
\hline MBW & 11 & 435052 & 711027 & Kurinskas & 1984 & 470 & $\cdots$ & $\cdots$ & $\cdots$ & $\cdots$ & $\cdots$ & $\cdots$ \\
\hline MBW & 12 & 435142 & 711102 & MCNa11 & 1984 & 470 & $\cdots$ & - & $\cdots$ & $\mathbf{x}$ & -. & Brw \\
\hline MBW & 13 & 435545 & 710853 & Daigel & 1984 & 550 & $-\cdot$ & -- & $\cdots$ & $\mathrm{x}$ & $\cdots$ & Brw \\
\hline MBW & 14 & 435122 & 711121 & D'Arezzo & 1985 & 490 & $\cdots$ & - & $\cdots$ & $\therefore$ & $\cdots$ & $\cdots$ \\
\hline MBW & 15 & 435155 & 711111 & Cormack & 1984 & 510 & $\cdots$ & 23 & $\cdots$ & $\mathbf{x}$ & $\cdots$ & Brw \\
\hline MBW & 16 & 435059 & 711108 & Shank & 1985 & 485 & $\cdots$ & 51.5 & -. & $\mathbf{s}$ & $\cdots$ & $\cdots$ \\
\hline MBW & 17 & 435259 & 711044 & Nichols & 1986 & 480 & $\cdots$ & 61 & $-\cdot$ & $\mathrm{x}$ & . & BrW \\
\hline MBW & 18 & 435054 & 711038 & VonHandorf & 1986 & 475 & $\cdots$ & 39 & - & $\mathbf{s}$ & $\cdots$ & $\cdots$ \\
\hline MBW & 19 & 435141 & 711117 & Wasson & 1986 & 510 & $\cdots$ & 30 & $\cdots$ & $x$ & $\cdots$ & BrW \\
\hline MBW & 20 & 435145 & 711116 & Ham & 1986 & 510 & - & 50 & $\cdots$ & $x$ & -. & BrW \\
\hline MBW & 21 & 435130 & 711120 & Fern Const & 1986 & 490 & $\cdots$ & 41 & - & $x$ & $\cdots$ & Brw \\
\hline MBW & 22 & 435054 & 711043 & Cummings & 1986 & 485 & $\cdots$ & 29 & -. & $\mathrm{s}$ & - & $\cdots$ \\
\hline MBW & 23 & 435054 & 711042 & McLough1 in & 1986 & 485 & $\cdots$ & 49 & $\cdots$ & $\mathbf{s}$ & $\cdots$ & - \\
\hline
\end{tabular}




\begin{tabular}{|c|c|c|c|c|c|c|c|c|}
\hline $\begin{array}{l}\text { Local } \\
\text { site } \\
\text { number }\end{array}$ & $\begin{array}{l}\text { Water } \\
\text { level } \\
\text { depth } \\
\text { (feet) }\end{array}$ & $\begin{array}{l}\text { Date } \\
\mathrm{mm} / \mathrm{dd} / \mathrm{yy}\end{array}$ & Use & $\begin{array}{l}\text { Maximum } \\
\text { well } \\
\text { yield } \\
\text { (gallons } \\
\text { per } \\
\text { minute) }\end{array}$ & $\begin{array}{l}\text { Draw- } \\
\text { down } \\
\text { (feet) }\end{array}$ & $\begin{array}{c}\text { Specific } \\
\text { capacity } \\
\text { (gallons per } \\
\text { minute } \\
\text { per } \\
\text { foot) }\end{array}$ & $\begin{array}{l}\text { Pumping } \\
\text { period } \\
\text { (hours) }\end{array}$ & Remarks \\
\hline
\end{tabular}

Jackson - - Continued

\begin{tabular}{|c|c|c|c|c|c|c|c|c|}
\hline JAW & 56 & $\cdots$ & $\cdots$ & $\mathrm{H}$ & 2 & $\cdots$ & - & .5 \\
\hline JAW & 57 & $\cdots$ & $\cdots$ & $\mathrm{H}$ & 5 & $\cdots$ & - & .3 \\
\hline JAW & 58 & - & - & $\mathrm{H}$ & 8 & $\cdots$ & - & 1 \\
\hline JAW & 59 & 180 & $07-13-88$ & $\mathrm{H}$ & 3 & $\cdots$ & $\cdots$ & 1 \\
\hline JAW & 60 & - & - & $\mathrm{H}$ & 25 & $-\cdot$ & $\cdots$ & 1 \\
\hline JAW & 61 & 25 & $07-29-88$ & $\mathrm{H}$ & 12 & -- & $\cdots$ & 1 \\
\hline JAW & 62 & 5 & $09-20-88$ & $\mathrm{H}$ & 6.5 & $\cdots$ & $\cdots$ & .5 \\
\hline JAW & 63 & - & - & $\mathrm{H}$ & 125 & $\cdots$ & -- & 1 \\
\hline JAW & 64 & $\cdots$ & $\cdots$ & $\mathrm{H}$ & 15 & $\cdots$ & $\cdots$ & 1 \\
\hline JAW & 65 & $\cdots$ & $\cdots$ & $\mathrm{H}$ & 6 & $\cdots$ & $\cdots$ & 1 \\
\hline JAW & 66 & $\cdots$ & $\cdots$ & $\mathrm{H}$ & 75 & $\cdots$ & $-\cdot$ & 1 \\
\hline JAW & 67 & - & - & $\mathrm{H}$ & 6.5 & $\cdots$ & $\cdots$ & 1 \\
\hline JAW & 68 & 6 & $06-16-89$ & $\mathrm{H}$ & 15 & $\cdots$ & $\cdots$ & .5 \\
\hline JAW & 69 & $\cdots$ & - & $\mathrm{H}$ & 20 & $\cdots$ & $\cdots$ & 1 \\
\hline JAW & 70 & $\cdots$ & $\cdots$ & $\mathrm{H}$ & 5 & $\cdots$ & $\cdots$ & 1 \\
\hline JAW & 71 & 10 & $05-03-91$ & $\mathrm{H}$ & 45 & $\cdots$ & $\cdots$ & 1 \\
\hline JAW & 72 & - & - & $\mathrm{H}$ & 150 & $\cdots$ & $\cdots$ & 1 \\
\hline JAW & 73 & $\cdots$ & $\cdots$ & $\mathrm{H}$ & 7 & -- & - & 1 \\
\hline JAW & 74 & - & $\cdots$ & $\mathrm{H}$ & 5.5 & $\cdots$ & $\cdots$ & 1 \\
\hline JAW & 75 & $\cdots$ & $\cdots$ & H & 12 & $\cdots$ & $\cdots$ & 1 \\
\hline JAW & 76 & $\cdots$ & $\cdots$ & H & 12 & $\cdots$ & $\cdots$ & 1 \\
\hline JAW & 77 & $\cdots$ & $\cdots$ & $\mathrm{H}$ & 150 & - & $\cdots$ & 1 \\
\hline JAW & 78 & $\cdots$ & $\cdots$ & $\mathrm{H}$ & 6 & $\cdots$ & $\cdots$ & 1 \\
\hline
\end{tabular}

\begin{tabular}{|c|c|c|c|c|c|c|c|c|c|}
\hline MBA & 1 & $\cdots$ & $\cdots$ & $T$ & $\cdots$ & $\cdots$ & $\cdots$ & $\cdots$ & USGS \\
\hline MBB & 1 & $\cdots$ & $\cdots$ & $T$ & $\cdots$ & $\cdots$ & $\cdots$ & $\cdots$ & \\
\hline MBB & 2 & $\cdots$ & - & $\mathrm{T}$ & - & $\cdots$ & - & - & \\
\hline MBB & 3 & $\cdots$ & $\cdots$ & $T$ & $\cdots$ & $\cdots$ & $\cdots$ & $\cdots$ & \\
\hline MBB & 4 & $\cdots$ & $\cdots$ & $\mathrm{T}$ & $\cdots$ & $\cdots$ & $\cdots$ & $\cdots$ & \\
\hline MBS & 1 & $\cdots$ & $\cdots$ & $\cdots$ & $\cdots$ & $\cdots$ & $\cdots$ & $\cdots$ & Spring is water supply for fi \\
\hline MBW & 1 & 9 & $06-01-66$ & $\mathrm{U}$ & $\cdots$ & $\cdots$ & $\cdots$ & $\cdots$ & \\
\hline MBW & 2 & $\cdots$ & $\cdots$ & $T$ & $-\cdot$ & $\cdots$ & $\cdots$ & $\cdots$ & \\
\hline MBW & 3 & $\cdots$ & $\cdots$ & $\mathrm{H}$ & 6 & $\cdots$ & $\cdots$ & -- & \\
\hline MBW & 4 & 9.9 & $07-16-91$ & $\mathrm{U}$ & $\cdots$ & $\cdots$ & $\cdots$ & $\cdots$ & \\
\hline MBW & 5 & $\cdots$ & $\cdots$ & H & $\cdots$ & $-\cdot$ & $\cdots$ & $\cdots$ & slightiy hard \\
\hline MBW & 6 & $\cdots$ & $\cdots$ & $\mathrm{H}$ & $\cdots$ & $\cdots$ & $\cdots$ & $\cdots$ & Iron stains in tub and toilet \\
\hline MBW & 7 & 6.2 & $07-16-91$ & H S & $\cdots$ & $\cdots$ & $\cdots$ & $\cdots$ & $\mathbf{R}$ \\
\hline MBW & 9 & $\cdots$ & $\cdots$ & $\mathrm{H}$ & $\cdots$ & $\cdots$ & $\cdots$ & $\cdots$ & High in iron \\
\hline MBW & 10 & 35 & $08-01-91$ & 0 & $\cdots$ & $\cdots$ & $\cdots$ & $\cdots$ & USGS \\
\hline MBW & 11 & 20.5 & $05-19-84$ & H & 30 & $\cdots$ & $\cdots$ & 1 & \\
\hline MBW & 12 & 70 & $10-25-84$ & $\mathrm{H}$ & 20 & $\cdots$ & $\cdots$ & .3 & \\
\hline MBW & 13 & 30 & $11-14-84$ & $\mathrm{H}$ & 4.5 & $\cdots$ & $\cdots$ & .5 & \\
\hline MBW & 14 & 42 & $07-06-85$ & $\mathrm{H}$ & 10 & $\cdots$ & $\cdots$ & 3 & \\
\hline MBW & 15 & $\cdots$ & - & $\mathrm{H}$ & 1.5 & $\cdots$ & $\cdots$ & .5 & \\
\hline MBW & 16 & 37 & $10-27-85$ & $\mathrm{H}$ & 15 & $\cdots$ & $\cdots$ & 2 & \\
\hline MBW & 17 & 30 & $02-15-86$ & $\mathrm{H}$ & 0.7 & $\cdots$ & $\cdots$ & 1 & \\
\hline MBW & 18 & 23 & $04-26-86$ & $\mathrm{H}$ & 15 & $\cdots$ & $\cdots$ & 2 & \\
\hline MBW & 19 & $-\cdot$ & $\cdots$ & $\mathrm{H}$ & 2 & $\cdots$ & $\cdots$ & 1 & \\
\hline MBW & 20 & $\cdots$ & $\cdots$ & $\mathrm{H}$ & 4.5 & $\cdots$ & $\cdots$ & 2 & \\
\hline MBW & 21 & 11 & $06-21-86$ & $\mathrm{H}$ & 60 & $\cdots$ & $\cdots$ & 2 & \\
\hline MBW & 22 & 22 & $09-13-86$ & $\mathrm{H}$ & 15 & $\cdots$ & $\cdots$ & 1 & \\
\hline MBW & $23^{\circ}$ & 29 & $06-08-86$ & $\mathrm{H}$ & 12 & $\cdots$ & $\cdots$ & 2 & \\
\hline
\end{tabular}


Table 1-1. Description of selected wells, borings, and springs in the Saco and Ossipee River Basins, east-central

\begin{tabular}{|c|c|c|c|c|c|c|c|c|c|c|c|c|}
\hline \multicolumn{2}{|c|}{$\begin{array}{l}\text { Local } \\
\text { site } \\
\text { number }\end{array}$} & $\begin{array}{l}\text { Lat- } \\
\text { itude }\end{array}$ & $\begin{array}{l}\text { Long- } \\
\text { itude }\end{array}$ & Owner or user & $\begin{array}{l}\text { Year } \\
\text { com- } \\
\text { pleted }\end{array}$ & $\begin{array}{l}\text { Alti- } \\
\text { tude } \\
\text { above } \\
\text { sea } \\
\text { level } \\
\text { (feet) }\end{array}$ & $\begin{array}{l}\text { Dia- } \\
\text { meter } \\
\text { of } \\
\text { well } \\
\text { casing } \\
\text { (inches) }\end{array}$ & $\begin{array}{l}\text { Depth } \\
\text { to } \\
\text { bottom } \\
\text { of } \\
\text { casing } \\
\text { (feet) }\end{array}$ & $\begin{array}{l}\text { Casing } \\
\text { material }\end{array}$ & $\begin{array}{l}\text { Type } \\
\text { of } \\
\text { finish }\end{array}$ & $\begin{array}{l}\text { Depth } \\
\text { to } \\
\text { bottom } \\
\text { of open } \\
\text { section } \\
\text { (feet) }\end{array}$ & $\begin{array}{c}\text { Type } \\
\text { of } \\
\text { site }\end{array}$ \\
\hline & & \multicolumn{11}{|c|}{ Madison--Continued } \\
\hline MBW & 24 & 435054 & 711040 & Mosche1la & 1986 & 480 & $\cdots$ & 49 & $\cdots$ & $\mathrm{s}$ & $\cdots$ & $\cdots$ \\
\hline MBW & 25 & 435124 & 711117 & Cummings & 1986 & 490 & - & 29 & - & $\mathrm{s}$ & - & - \\
\hline MBW & 26 & 435128 & 711120 & Simkins & 1986 & 490 & - & 70 & $\cdots$ & $\mathrm{x}$ & $\cdots$ & Brw \\
\hline MBW & 27 & 435552 & 710948 & Hill & 1986 & 500 & - & 120 & $\cdots$ & $\mathrm{x}$ & $\cdots$ & BrW \\
\hline MBW & 28 & 435142 & 711108 & Cunio & 1986 & 490 & $\cdots$ & 67 & - & $\mathrm{x}$ & $\cdots$ & BrW \\
\hline MBW & 29 & 435055 & 711045 & Cummings & 1987 & 485 & $\cdots$ & 49 & -. & $\mathrm{s}$ & - & -. \\
\hline MBW & 30 & 435057 & 711111 & Nickerson & 1987 & 490 & $\cdots$ & 55 & $\cdots$ & $\mathrm{s}$ & $\cdots$ & $\cdots$ \\
\hline MBW & 31 & 435049 & 711111 & wills & 1987 & 470 & $\cdots$ & 110 & $\cdots$ & $\cdots$ & $\cdots$ & $\cdots$ \\
\hline MBW & 32 & 435132 & 711105 & Bourque & 1987 & 480 & $\cdots$ & 100 & -. & $\mathrm{x}$ & $\cdots$ & $\mathrm{BrW}$ \\
\hline MBW & 33 & 435053 & 711114 & Hamel & 1987 & 470 & $\cdots$ & 53 & $-\cdot$ & $\mathrm{s}$ & $\cdots$ & - \\
\hline MBW & 34 & 435146 & 711116 & Ham & 1987 & 510 & $\cdots$ & 30 & $\cdots$ & $\mathrm{x}$ & $\cdots$ & $\mathrm{BrW}$ \\
\hline MBW & 35 & 435049 & 711111 & wills & 1987 & 470 & - & 58 & - & $x$ & -. & BrW \\
\hline MBW & 36 & 435311 & 710958 & Dudley & 1987 & 500 & $-\cdot$ & 60 & $\cdots$ & $\mathrm{x}$ & $\cdots$ & Brw \\
\hline MBW & 37 & 435148 & 711111 & Herzog & 1988 & 510 & $\cdots$ & 57 & $\cdots$ & $\mathrm{s}$ & $\cdots$ & $\cdots$ \\
\hline MBW & 38 & 435032 & 711113 & Ross & 1988 & 470 & - & 44 & $\cdots$ & $s$ & $\cdots$ & - \\
\hline MBW & 39 & 435620 & 710900 & Durkee & 1988 & 570 & $\cdots$ & 60 & $-\cdot$ & $\mathrm{x}$ & - & BrW \\
\hline MBW & 40 & 435053 & 711035 & Vonhandorf & 1988 & 470 & $\cdots$ & 32 & $\cdots$ & $s$ & $\cdots$ & $\cdots$ \\
\hline MBW & 41 & 435124 & 711113 & James & 1988 & 490 & $\cdots$ & 59 & - & $s$ & - & $\cdots$ \\
\hline MBW & 42 & 435129 & 711117 & Dalis & 1988 & 490 & $\cdots$ & 29 & - & $\mathrm{s}$ & - & $\cdots$ \\
\hline MBW & 43 & 435323 & 711014 & Sherwood & 1988 & 470 & -. & 17 & - & - & $\cdots$ & Dug \\
\hline MBW & 44 & 435538 & 710922 & Husted & 1988 & 505 & $\cdots$ & 113 & $\cdots$ & $\mathrm{x}$ & $\cdots$ & BrW \\
\hline MBW & 45 & 435059 & 711116 & Luca & 1989 & 470 & $\cdots$ & 59 & -. & $\mathrm{s}$ & - & $\cdots$ \\
\hline MBW & 46 & 435548 & 710930 & Knott & 1989 & 490 & $-\cdot$ & 90 & -- & $x$ & - & Brw \\
\hline MBW & 47 & 435048 & 711122 & Aglwin & 1989 & 475 & $\cdots$ & 53 & $\cdots$ & $\mathbf{s}$ & - & $\cdots$ \\
\hline MBW & 48 & 435142 & 711105 & Rand & 1989 & 475 & - & 52 & - & $\mathrm{x}$ & - & BrW \\
\hline MBW & 49 & 435324 & 711017 & Sherwood & 1989 & 470 & - & 60 & $\cdots$ & $\mathrm{x}$ & $\cdots$ & BrW \\
\hline MBW & 50 & 435050 & 711116 & Eldridge & 1990 & 470 & - & 58 & - & $s$ & - & $\cdots$ \\
\hline MBW & 51 & 435238 & 711003 & Beck & 1990 & 510 & $\cdots$ & 54 & $\cdots$ & s & - - & $\cdots$ \\
\hline MBW & 52 & 435540 & 710957 & Te110 & 1990 & 495 & -. & 50 & $\cdots$ & $x$ & -. & BrW \\
\hline MBW & 53 & 435057 & 711116 & Chase & 1990 & 470 & - - & 174 & $\cdots$ & - & -. & $\cdots$ \\
\hline MBW & 54 & 435549 & 710946 & Scoppettuolo & 1988 & 500 & - & 109 & -. & $\cdots$ & - & $\cdots$ \\
\hline MBW & 55 & 435642 & 710914 & & 1992 & 475 & 2 & 47 & $\mathrm{P}$ & $\mathrm{s}$ & 49 & Bor \\
\hline MBW & 56 & 435646 & 710932 & USGS & 1992 & 550 & 2 & 66 & P & $s$ & 69.1 & Bor \\
\hline MBW & 57 & 435715 & 710913 & & 1992 & 470 & 2 & 47 & $\mathrm{P}$ & $s$ & 49 & Bor \\
\hline MBW & 58 & 435606 & 710832 & Village District of Eidelweiss & $\cdots$ & 580 & - & - & -. & $\cdots$ & - & $\cdots$ \\
\hline MBW & 59 & 435240 & 711002 & Dreston & 1984 & 670 & - & 60 & $\cdots$ & $\mathrm{x}$ & $\cdots$ & Brw \\
\hline MBW & 60 & 435244 & 710956 & Rame11 & 1985 & 540 & - & 20 & $\cdots$ & $\mathrm{x}$ & - & Brw \\
\hline MBW & 61 & 435638 & 710716 & Packard & 1984 & 650 & -. & 106 & $\cdots$ & $\mathrm{x}$ & - & BrW \\
\hline MBW & 62 & 435455 & 710857 & Chaplick & 1984 & 720 & $\cdots$ & 93 & $\cdots$ & $\mathrm{x}$ & $\cdots$ & $\mathrm{BrW}$ \\
\hline MBW & 63 & 435530 & 710959 & Shipu1ski & 1984 & 540 & - & 110 & . & $\mathrm{x}$ & -. & BrW \\
\hline MBW & 64 & 435347 & 711106 & High Street Association & 1984 & 640 & $\cdots$ & 35 & $\cdots$ & $\mathrm{x}$ & $\cdots$ & Brw \\
\hline MBW & 65 & 435356 & 710847 & Fleischmann & 1984 & 580 & -. & 61 & - & $\mathrm{x}$ & $\cdots$ & BrW \\
\hline MBW & 66 & 435610 & 710654 & Tangur & 1984 & 660 & - & 99 & - & $\mathrm{x}$ & $\cdots$ & BrW \\
\hline MBW & 67 & 435003 & 711111 & Madison Saw Mill & 1984 & 440 & - & 59 & $\cdots$ & - & $\cdots$ & -. \\
\hline MBW & 68 & 435003 & 711111 & Madison Saw Mill & 1984 & 440 & $-\cdot$ & 60 & - & $-\cdot$ & $\cdots$ & $\cdots$ \\
\hline MBW & 69 & 435300 & 711130 & Bartlett & 1984 & 720 & -. & 19 & $\cdots$ & $\mathrm{x}$ & $\cdots$ & BrW \\
\hline MBW & 70 & 435402 & 710940 & Arnold & 1985 & 610 & $-\cdot$ & 17 & - & - & - & Dug \\
\hline MBW & 71 & 435339 & 710924 & Town of Madison & 1985 & 510 & $\cdots$ & 19 & - & $\cdots$ & $\cdots$ & Dug \\
\hline MBW & 72 & 435557 & 710834 & Village Dist of Eidlewe & 1985 & 560 & - & 36 & - & $\cdots$ & - & - \\
\hline MBW & 73 & 435616 & 710706 & Macleon & 1985 & 655 & - & 35 & $\cdots$ & $\mathrm{x}$ & $\cdots$ & BrW \\
\hline MBW & 74 & 435459 & 710856 & McNau1 to & 1985 & 700 & $\cdots$ & 86 & $\cdots$ & $x$ & $\cdots$ & $\mathrm{BrW}$ \\
\hline MBW & 75 & 435223 & 710622 & Pitts & 1985 & 760 & - & 59 & $\cdots$ & $\mathrm{x}$ & - & $\mathrm{BrW}$ \\
\hline MBW & 76 & 435610 & 710712 & Potvin & 1985 & 655 & - & - - & $\cdots$ & - & - & Dug \\
\hline
\end{tabular}




\begin{tabular}{|c|c|c|c|c|c|c|c|c|}
\hline $\begin{array}{l}\text { Local } \\
\text { site } \\
\text { number }\end{array}$ & $\begin{array}{l}\text { Water } \\
\text { level } \\
\text { depth } \\
\text { (feet) }\end{array}$ & $\begin{array}{l}\text { Date } \\
\mathrm{mm} / \mathrm{dd} / \mathrm{yy}\end{array}$ & Use & $\begin{array}{c}\text { Maximum } \\
\text { well } \\
\text { yield } \\
\text { (gallons } \\
\text { per } \\
\text { minute) }\end{array}$ & $\begin{array}{l}\text { Draw- } \\
\text { down } \\
\text { (feet) }\end{array}$ & $\begin{array}{c}\text { Specific } \\
\text { capacity } \\
\text { (gallons per } \\
\text { minute } \\
\text { per } \\
\text { foot) }\end{array}$ & $\begin{array}{l}\text { Pumping } \\
\text { period } \\
\text { (hours) }\end{array}$ & Remarks \\
\hline
\end{tabular}

\begin{tabular}{|c|c|c|c|c|c|c|c|c|c|}
\hline MBW & 24 & 27 & $06-29-86$ & $\mathrm{H}$ & 15 & -- & - & 2 & \\
\hline MBW & 25 & 22 & $07-05-86$ & $\mathrm{H}$ & 15 & -- & $\cdots$ & 1 & \\
\hline MBW & 26 & - - & - - & $\mathrm{H}$ & 20 & - & $\cdots$ & 1 & \\
\hline MBW & 27 & - & . & H & 15 & - & - & 1 & \\
\hline MBW & 28 & 40 & $09-30-86$ & $\mathrm{H}$ & 2 & - & $\cdots$ & 1 & \\
\hline MBW & 29 & 31 & $04-13-87$ & $\mathrm{H}$ & 17 & $-\cdot$ & - & 2 & \\
\hline MBW & 30 & 33 & $05-16-87$ & $\mathrm{H}$ & 15 & $\cdots$ & $\cdots$ & 1.5 & \\
\hline MBW & 31 & 40 & $06-25-87$ & $\mathrm{H}$ & 30 & $\cdots$ & $-\cdot$ & 48 & \\
\hline MBW & 32 & 40 & $08-01-87$ & $\mathrm{H}$ & 5.5 & $\cdots$ & $\cdots$ & 1 & \\
\hline MBW & 33 & 34 & $07 \cdot 29-87$ & $\mathrm{H}$ & 12.5 & $\cdots$ & - & 2 & \\
\hline MMBW & 34 & 30 & $08-09-87$ & $\mathrm{H}$ & 7 & - & $\cdots$ & 1 & \\
\hline MBW & 35 & 30 & $12-28-87$ & $\mathrm{H}$ & 30 & -- & - & 12 & \\
\hline MBW & 36 & 60 & $11-13-87$ & $\mathrm{H}$ & 1.3 & - & -- & .5 & \\
\hline MBW & 37 & 39 & $04-02-88$ & $\mathrm{H}$ & 10 & - & - & 2 & \\
\hline MBW & 38 & 27 & $05-21 \cdot 88$ & $\mathrm{H}$ & 16.5 & - & - & 2 & \\
\hline MBW & 39 & - - & - - & $\mathrm{H}$ & 2 & $\cdots$ & - & 1 & \\
\hline MBW & 40 & 10 & $08-21-88$ & $\mathrm{H}$ & 30 & $\cdots$ & - & 1 & \\
\hline MBW & 41 & 44 & $07-11-88$ & $\mathrm{H}$ & 9 & $-\cdot$ & $-\cdot$ & 1 & \\
\hline MBW & 42 & 23.5 & $07-06-88$ & $\mathrm{H}$ & 15 & $\cdots$ & - & 1 & \\
\hline MBW & 43 & 9 & $07-29-88$ & $\mathrm{H}$ & - & - & - & - & \\
\hline MBW & 44 & 50 & $12-20-88$ & $\mathrm{H}$ & 2 & - & - & 3 & \\
\hline MBW & 45 & 30 & $01-17-89$ & $\mathrm{H}$ & 25 & - & $\cdots$ & 12 & \\
\hline MBW & 46 & - & - & $\mathrm{H}$ & 20 & - & - - & 1 & \\
\hline MBW & 47 & 36 & $06-24-89$ & $\mathrm{H}$ & 13 & - & - & 2 & \\
\hline MBW & 48 & 20 & $06-06-89$ & $\mathrm{H}$ & 6 & $\cdots$ & - & 1 & \\
\hline MBW & 49 & 28 & $10-25-89$ & $\mathrm{H}$ & 1 & - & - & .5 & \\
\hline MBW & 50 & 34 & $01-21 \cdot 90$ & $\mathrm{H}$ & 15 & - & - & 1 & \\
\hline MBW & 51 & 42.5 & $08-19-90$ & $\mathrm{H}$ & 15 & - & $\cdots$ & 2 & \\
\hline MBW & 52 & 30 & $11-01-90$ & $\mathrm{H}$ & 100 & -- & $\cdots$ & .5 & \\
\hline MBW & 53 & 40 & $11 \cdot 05 \cdot 90$ & $\mathrm{H}$ & 30 & - & - & .5 & \\
\hline MBW & 54 & 15 & $09-13-88$ & $\mathrm{H}$ & 20 & - & - & .5 & \\
\hline MBW & 55 & 6.4 & $08-07-92$ & 0 & - & - & $\cdots$ & $\cdots$ & USGS \\
\hline MBW & 56 & 66 & $08-07-92$ & 0 & - & - & - & - & UsGS \\
\hline MBW & 57 & 10 & $08-06-92$ & 0 & - & $\cdots$ & $-\cdot$ & - & USGS \\
\hline MBW & 58 & - & - & $P$ & $\cdots$ & - & $-\cdot$ & $-\cdot$ & \\
\hline MBW & 59 & 40 & $05-15-84$ & $\mathrm{H}$ & 3.5 & $\cdots$ & - & .5 & \\
\hline MBW & 60 & 40 & $05-16-85$ & $\mathrm{H}$ & 0.5 & - & $\cdots$ & 1 & \\
\hline MBW & 61 & - & - & $\mathrm{H}$ & 5 & - & - & .5 & \\
\hline MBW & 62 & 35 & $06-08 \cdot 84$ & $\mathrm{H}$ & 4 & - & - & .5 & \\
\hline MBW & 63 & 50 & $06-10-84$ & $\mathrm{H}$ & 3 & . & $\cdots$ & .5 & \\
\hline MBW & 64 & 60 & $07-04-84$ & $\mathrm{H}$ & 8 & - & - & .5 & \\
\hline MBW & 65 & 20 & $08-06-84$ & $\mathrm{H}$ & 4 & - & $\cdots$ & - & \\
\hline MBW & 66 & 20 & $10-01-84$ & $\mathrm{H}$ & 40 & $\cdots$ & $\cdots$ & .5 & \\
\hline MBW & 67 & 20 & $11-14-84$ & $\mathrm{~N}$ & 35 & . & - & 3 & \\
\hline MBW & 68 & 20 & $11-14-84$ & $\mathrm{~N}$ & 35 & - & $\cdots$ & 4 & \\
\hline MBW & 69 & - & - & H & 0.3 & $\cdots$ & $\cdots$ & .5 & \\
\hline MBW & 70 & 8 & $05-22-85$ & $\mathrm{H}$ & - & - & $\cdots$ & $\cdots$ & \\
\hline MBW & 71 & 7 & $06-13-85$ & $\mathrm{c}$ & - & - & - & .. & \\
\hline MBW & 72 & 16 & $06-14-85$ & $P$ & 35 & - & $\cdots$ & 1 & \\
\hline MBW & 73 & 6 & $03-19 \cdot 85$ & $\mathrm{H}$ & 25 & - & $\cdots$ & .5 & \\
\hline MBW & 74 & 120 & $04 \cdot 05 \cdot 85$ & $\mathrm{H}$ & 30 & $-\cdot$ & $\cdots$ & .5 & \\
\hline MBW & 75 & 2 & $06-09-85$ & $\mathrm{H}$ & 0.8 & $\cdots$ & $\cdots$ & 1 & \\
\hline MBW & 76 & 6 & $04-15-85$ & $\mathrm{H}$ & 20 & . & - & 2 & \\
\hline
\end{tabular}


Table 1-1. Description of selected wells, borings, and springs in the Saco and Ossipee River Basins, east-central

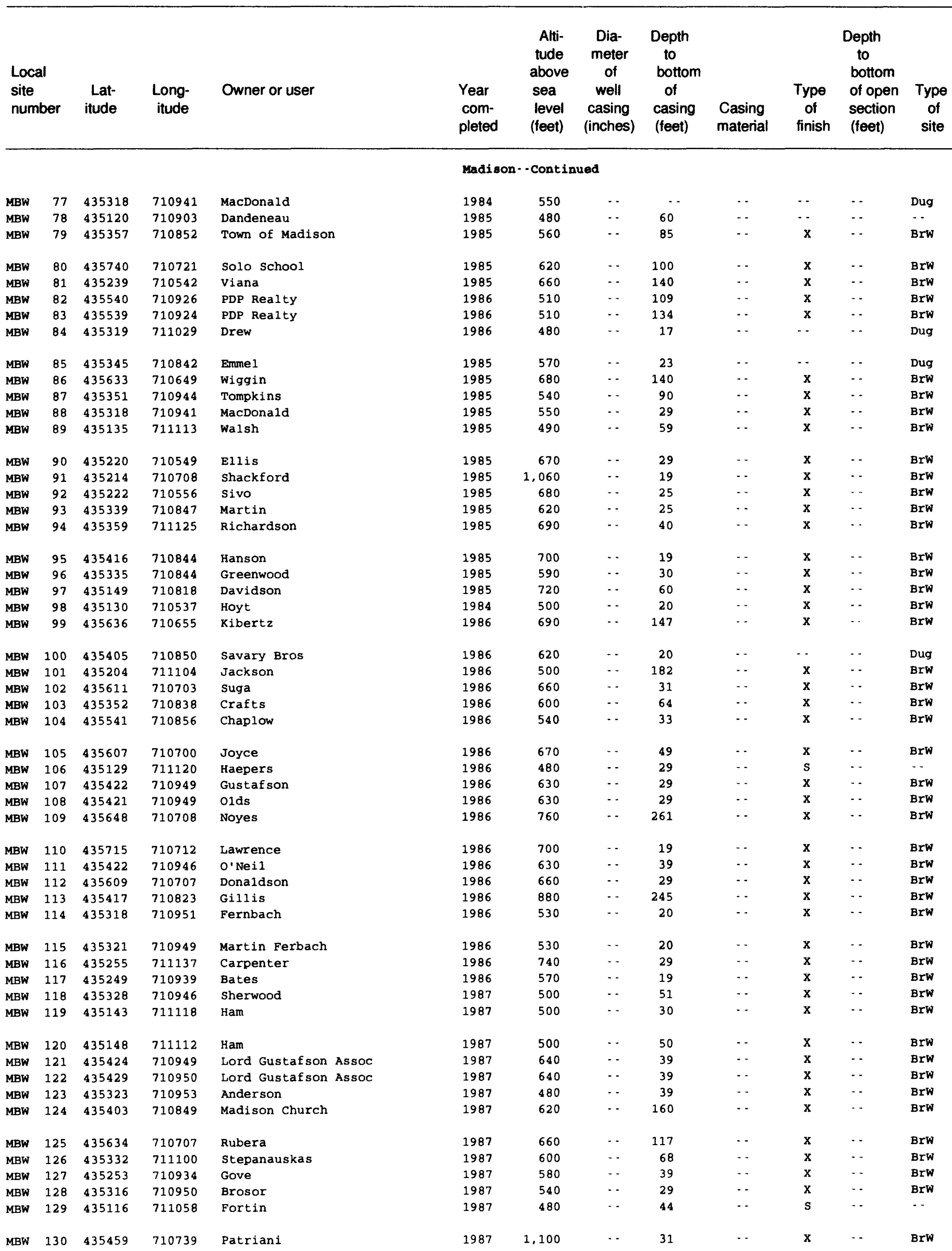




\begin{tabular}{|c|c|c|c|c|c|c|c|c|}
\hline $\begin{array}{l}\text { Local } \\
\text { site } \\
\text { number }\end{array}$ & $\begin{array}{l}\text { Water } \\
\text { level } \\
\text { depth } \\
\text { (feet) }\end{array}$ & $\begin{array}{l}\text { Date } \\
\mathrm{mm} / \mathrm{dd} / \mathrm{yy}\end{array}$ & Use & $\begin{array}{l}\text { Maximum } \\
\text { well } \\
\text { yield } \\
\text { (gallons } \\
\text { per } \\
\text { minute) }\end{array}$ & $\begin{array}{l}\text { Draw- } \\
\text { down } \\
\text { (feet) }\end{array}$ & $\begin{array}{c}\text { Specific } \\
\text { capacity } \\
\text { (gallons per } \\
\text { minute } \\
\text { per } \\
\text { foot) }\end{array}$ & $\begin{array}{l}\text { Pumping } \\
\text { period } \\
\text { (hours) }\end{array}$ & Remarks \\
\hline
\end{tabular}

Madison--Continued

\begin{tabular}{|c|c|c|c|c|c|c|c|c|}
\hline MBW & 77 & 12 & $10-20-84$ & H & 10 & $\cdots$ & $\cdots$ & 2 \\
\hline MBW & 78 & 28 & $07-02-85$ & $\mathrm{H}$ & 50 & $\cdots$ & $-\cdot$ & 2 \\
\hline MBW & 79 & 90 & $07-22-85$ & H & 35 & $\cdots$ & - & .5 \\
\hline MBW & 80 & 40 & $08-25-85$ & $T$ & 60 & $\cdots$ & - & .5 \\
\hline MBW & 81 & 25 & $09-28-85$ & H & 3 & $\cdots$ & $\cdots$ & .5 \\
\hline MBW & 82 & 30 & $05-19-86$ & $\mathbf{H}$ & 10 & $\cdots$ & $\cdots$ & .5 \\
\hline MBW & 83 & 30 & $05-20-86$ & H & 8 & $\cdots$ & $\cdots$ & .5 \\
\hline MBW & 84 & - & - & H & $\cdots$ & $\cdots$ & $\cdots$ & $-\cdot$ \\
\hline MBW & 85 & 20 & $08-27-85$ & $\mathrm{H}$ & $\cdots$ & $\cdots$ & $\cdots$ & $\cdots$ \\
\hline MMBW & 86 & - & $\cdots$ & H & 9 & $\cdots$ & $\cdots$ & 1 \\
\hline MBW & 87 & 20 & $08-15-85$ & $\mathrm{H}$ & 5 & $\cdots$ & $\cdots$ & 1 \\
\hline MBW & 88 & $\cdots$ & $\cdots$ & $\mathrm{H}$ & 20 & $\cdots$ & - & 1 \\
\hline MBW & 89 & 30 & $09-11-85$ & H & 3 & $\cdots$ & $\cdots$ & .3 \\
\hline MBW & 90 & 45 & $10-18-85$ & H & 1.8 & $\cdots$ & $-\cdot$ & 1 \\
\hline MBW & 91 & 15 & $12-18-85$ & H & 1.5 & $\cdots$ & $\cdots$ & 2 \\
\hline MBW & 92 & 40 & $12-19-85$ & H & 75 & $\cdots$ & $\cdots$ & .5 \\
\hline MBW & 93 & $\cdots$ & $\cdots$ & $\mathrm{H}$ & 22 & $\cdots$ & $\cdots$ & 1 \\
\hline MBW & 94 & - & $\cdots$ & $\mathrm{H}$ & 4 & $\cdots$ & $\cdots$ & 1 \\
\hline MBW & 95 & $\cdots$ & $\cdots$ & $\mathrm{H}$ & 50 & $\cdots$ & $\cdots$ & 1 \\
\hline MBW & 96 & $\cdots$ & $\cdots$ & $\mathrm{H}$ & 40 & $\cdots$ & - & 1 \\
\hline MBW & 97 & $\cdots$ & $\cdots$ & H & 5.5 & $\cdots$ & $\cdots$ & 1 \\
\hline MBW & 98 & - & - & $\mathrm{H}$ & 2.5 & $\cdots$ & $\cdots$ & .5 \\
\hline MBW & 99 & 70 & $05-14-86$ & $\mathbf{H}$ & 7 & $\cdots$ & $\cdots$ & 1 \\
\hline MBW & 100 & 7 & $07-09-86$ & H & $\cdots$ & -- & $\cdots$ & $\cdots$ \\
\hline MBW & 101 & 45 & $06-02-86$ & $\mathrm{H}$ & 0.5 & $\cdots$ & $\cdots$ & 2 \\
\hline MBW & 102 & 12 & $06-22-86$ & H & 50 & $\cdots$ & $\cdots$ & 2 \\
\hline MBW & 103 & 12 & $08-20-86$ & $\mathrm{H}$ & 7 & $-\cdot$ & $\cdots$ & .5 \\
\hline MBW & 104 & 40 & $07-22-86$ & н & 2.5 & $\cdots$ & $\cdots$ & 2 \\
\hline MBW & 205 & - & - & $\mathbf{H}$ & 5.5 & $-\cdot$ & $\cdots$ & 1 \\
\hline MBW & 106 & 21.5 & $08-19-86$ & $\mathrm{H}$ & 15 & $\cdots$ & $\cdots$ & 2 \\
\hline MBW & 107 & 67 & $08-15-86$ & $\mathrm{H}$ & 20 & $\cdots$ & $\cdots$ & 1 \\
\hline MBW & 108 & 30 & $08-14-86$ & $\mathrm{H}$ & 150 & $\cdots$ & $\cdots$ & 1 \\
\hline MBW & 109 & 65 & $08-21-86$ & H & 4 & $\cdots$ & $\cdots$ & 1 \\
\hline VBW & 110 & $\cdots$ & $\cdots$ & H & 3 & $\cdots$ & $\cdots$ & 1 \\
\hline VBW & 111 & $\cdots$ & $\cdots$ & H & 150 & $\cdots$ & $\cdots$ & 1 \\
\hline VBW & 112 & 5 & $20-17-86$ & $\mathbf{H}$ & 150 & $\cdots$ & $\cdots$ & 2 \\
\hline VBW & 113 & 70 & $10-24-86$ & $\mathbf{H}$ & 30 & $\cdots$ & $\cdots$ & 1 \\
\hline VBW & 114 & $\cdots$ & $\cdots$ & H & 1.5 & $\cdots$ & $\cdots$ & 1 \\
\hline GBW & 115 & $\cdots$ & $\cdots$ & H & 5 & $\cdots$ & $\cdots$ & 2 \\
\hline IBW & 116 & $\cdots$ & $\cdots$ & K & 4 & $\cdots$ & $\cdots$ & 1 \\
\hline IBW & 117 & $\cdots$ & $\cdots$ & H & 12 & $\cdots$ & $\cdots$ & 1 \\
\hline BWW & 118 & 20 & $04-14-87$ & H & 12 & $\cdots$ & $\cdots$ & .5 \\
\hline ABW & 119 & $\cdots$ & $\cdots$ & H & 4 & $\cdots$ & $\cdots$ & 1 \\
\hline LBW & 220 & $\cdots$ & - & $\mathbf{H}$ & 3 & - & $\cdots$ & 1 \\
\hline IBW & 121 & $\cdots$ & $\cdots$ & H & 100 & $\cdots$ & $\cdots$ & 1 \\
\hline IBW & 122 & $\cdots$ & $\cdots$ & $\mathrm{H}$ & 9 & $\cdots$ & . & 1 \\
\hline EW & 123 & 25 & $02 \cdot 28 \cdot 87$ & H & 4 & $\cdots$ & - & 1 \\
\hline IBW & 124 & 25 & $04-17-87$ & H & 3.5 & $\cdots$ & $\cdots$ & .5 \\
\hline IBW & 125 & 5 & $06-03-87$ & H & 4 & $\cdots$ & 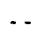 & .5 \\
\hline EBW & 126 & 20 & $05-11-87$ & H & 25 & $\cdots$ & $\cdots$ & .5 \\
\hline IBW & 127 & 20 & $06-30-87$ & H & 2 & $\cdots$ & $\cdots$ & .5 \\
\hline LBW & 128 & $\cdots$ & $\cdots$ & H & 3 & $\cdots$ & $\cdots$ & 2 \\
\hline LBW & 129 & 34 & $07-16-87$ & H & 12 & $\cdots$ & $\cdots$ & 2 \\
\hline $\mathbb{E B W}$ & 130 & 60 & $08-18-87$ & H & 50 & $\cdots$ & $\cdots$ & .5 \\
\hline
\end{tabular}




\begin{tabular}{|c|c|c|c|c|c|c|c|c|c|c|c|}
\hline $\begin{array}{l}\text { Local } \\
\text { site } \\
\text { number }\end{array}$ & $\begin{array}{l}\text { Lat- } \\
\text { itude }\end{array}$ & $\begin{array}{l}\text { Long- } \\
\text { itude }\end{array}$ & Owner or user & $\begin{array}{l}\text { Year } \\
\text { com- } \\
\text { pleted }\end{array}$ & $\begin{array}{l}\text { Alti- } \\
\text { tude } \\
\text { above } \\
\text { sea } \\
\text { level } \\
\text { (feet) }\end{array}$ & $\begin{array}{l}\text { Dia- } \\
\text { meter } \\
\text { of } \\
\text { well } \\
\text { casing } \\
\text { (inches) }\end{array}$ & $\begin{array}{l}\text { Depth } \\
\text { to } \\
\text { bottom } \\
\text { of } \\
\text { casing } \\
\text { (feet) }\end{array}$ & $\begin{array}{l}\text { Casing } \\
\text { material }\end{array}$ & $\begin{array}{l}\text { Type } \\
\text { of } \\
\text { finish }\end{array}$ & $\begin{array}{l}\text { Depth } \\
\text { to } \\
\text { bottom } \\
\text { of open } \\
\text { section } \\
\text { (feet) }\end{array}$ & $\begin{array}{c}\text { Type } \\
\text { of } \\
\text { site }\end{array}$ \\
\hline
\end{tabular}

\begin{tabular}{|c|c|c|c|c|c|c|c|c|c|c|c|c|}
\hline MBW & 131 & 435316 & 710941 & Chute & 1987 & 560 & $\cdots$ & 39 & $\cdots$ & $\mathbf{x}$ & $\cdots$ & BrW \\
\hline MBW & 132 & 435654 & 710712 & Pettingell & 1987 & 820 & - & 209 & $\cdots$ & $x$ & - & $\mathrm{BrW}$ \\
\hline MBW & 133 & 435221 & 710651 & watson & 1987 & 900 & $\cdots$ & 20 & $\cdots$ & $\cdots$ & $\cdots$ & Dug \\
\hline MBW & 134 & 435049 & 711113 & Gillis & 1987 & 480 & $\cdots$ & 59 & $\cdots$ & $\mathbf{S}$ & $\cdots$ & $\cdots$ \\
\hline MBW & 135 & 435257 & 711129 & Medeiros & 1987 & 740 & $\cdots$ & 39 & $\cdots$ & $x$ & $\cdots$ & $\mathrm{BrW}$ \\
\hline MBW & 136 & 435215 & 710633 & Elworthy & 1987 & 860 & $\cdots$ & 51 & $\cdots$ & $x$ & - & BrW \\
\hline MBW & 137 & 435057 & 711114 & O'Neil & 1987 & 480 & $\cdots$ & 58 & - & $\mathrm{s}$ & $\cdots$ & $\cdots$ \\
\hline MBW & 138 & 435417 & 710723 & McKnight & 1987 & 920 & $\cdots$ & 17 & - & - & $\cdots$ & Dug \\
\hline MBW & 139 & 435203 & 710524 & Purity Spring Resort & 1987 & 460 & $\cdots$ & 49 & $\cdots$ & $x$ & $\cdots$ & BrW \\
\hline MBW & 140 & 435418 & 710842 & Fuller & 1987 & 730 & - & 38 & $\cdots$ & $x$ & $\cdots$ & Brw \\
\hline MBW & 141 & 435431 & 710949 & Pequawket Assoc & 1987 & 630 & $\cdots$ & 29 & - & $x$ & $\cdots$ & Brw \\
\hline MBW & 142 & 435431 & 710946 & Thompson & 1987 & 620 & $\cdots$ & 39 & $\cdots$ & $x$ & $\cdots$ & Brw \\
\hline MBW & 143 & 435352 & 710842 & Goss & 1987 & 580 & $\cdots$ & 39 & $\cdots$ & $\mathbf{x}$ & - & Brw \\
\hline MBW & 144 & 435112 & 711113 & Con1ey & 1987 & 480 & $\cdots$ & 57 & $-\cdot$ & $\mathbf{S}$ & $\cdots$ & $\cdots$ \\
\hline MBW & 145 & 435610 & 710706 & Village District of Eidelweiss & 1987 & 660 & $\cdots$ & 80 & -- & $\mathbf{x}$ & $-\cdot$ & Brw \\
\hline MBW & 146 & 435241 & 710758 & McNulty & 1987 & 680 & - & 60 & - & $\mathbf{x}$ & - & Brw \\
\hline MBW & 147 & 435259 & 710558 & Kemensky & 1987 & 840 & $\cdots$ & 54 & - & $\mathrm{x}$ & $\cdots$ & $\mathrm{BrW}$ \\
\hline MBW & 148 & 435528 & 711143 & Bryant & 1986 & 760 & - & 30 & - & $\mathbf{x}$ & - & $\mathrm{BrW}$ \\
\hline MBW & 149 & 435230 & 710600 & Sherman & 1987 & 740 & $-\cdot$ & 49 & $\cdots$ & $\mathrm{x}$ & $\cdots$ & Brw \\
\hline MBW & 150 & 435316 & 710952 & Newsom & 1987 & 530 & -- & 30 & -- & $\mathrm{x}$ & $\cdots$ & Brw \\
\hline MBW & 151 & 435046 & 711116 & Savary Bros & 1987 & 480 & $\cdots$ & 51.5 & $\cdots$ & $\mathrm{S}$ & $\cdots$ & -- \\
\hline MBW & 152 & 435305 & 710940 & Tromb1ey & 1987 & 560 & $\cdots$ & 149 & $\cdots$ & $\mathrm{x}$ & -- & Brw \\
\hline MBW & 153 & 435325 & 710944 & Hurd & 1987 & 520 & $\cdots$ & 37 & - & $x$ & - & Brw \\
\hline MBW & 154 & 435733 & 710651 & Smith & 1987 & 520 & $\cdots$ & 80 & $\cdots$ & $x$ & $-\cdot$ & BrW \\
\hline MBW & 155 & 435538 & 710922 & PDP Realty & 1988 & 510 & $\cdots$ & 134 & $\cdots$ & $x$ & $-\cdot$ & Brw \\
\hline MBW & 156 & 435319 & 710940 & Brown & 1988 & 540 & - - & 60 & $\cdots$ & $x$ & - & BrW \\
\hline MBW & 157 & 435228 & 710543 & Lancashing & 1988 & 750 & - & 34 & - & $x$ & $\cdots$ & Brw \\
\hline MBW & 158 & 435258 & 711133 & waterhouse & 1988 & 720 & - & 30 & $\cdots$ & $x$ & - & Brw \\
\hline MBW & 159 & 435359 & 710940 & Forgues & 1988 & 600 & - & 50 & $\cdots$ & $x$ & $\cdots$ & BrW \\
\hline MBW & 160 & 435148 & 711114 & walker & 1988 & 500 & $\cdots$ & 27 & - & $\mathrm{x}$ & . & BrW \\
\hline MBW & 161 & 435555 & 710836 & Eidelweiss & 1988 & 560 & $\cdots$ & 29 & - & $x$ & $\cdots$ & Brw \\
\hline MBW & 162 & 435727 & 710732 & Harrow & 1988 & 820 & - & 80 & $\cdots$ & $x$ & - & Brw \\
\hline MBW & 163 & 435618 & 710649 & Denoncourt & 1988 & 660 & - & 100 & $\cdots$ & $x$ & $\cdots$ & Brw \\
\hline MBW & 164 & 435457 & 710857 & Tessier & 1988 & 700 & - & 90 & $\cdots$ & $x$ & - & $\mathrm{BrW}$ \\
\hline MBW & 165 & 435623 & 710648 & Bennett & 1988 & 660 & $\cdots$ & 80 & $\cdots$ & $x$ & $\cdots$ & Brw \\
\hline MBW & 166 & 435512 & 711158 & Baker & 1988 & 720 & $\cdots$ & 80 & $-\cdot$ & $x$ & - & Brw \\
\hline MBW & 167 & 435611 & 710652 & Previte Jr & 1988 & 670 & $\cdots$ & 84 & $\cdots$ & $\mathbf{x}$ & $-\cdot$ & BrW \\
\hline MBW & 168 & 435358 & 711118 & Shackford & 1988 & 670 & - & 41 & $\cdots$ & $\mathbf{x}$ & - & Brw \\
\hline MBW & 169 & 435734 & 710712 & Hill & 1988 & 590 & $\cdots$ & 40 & $\cdots$ & $x$ & - & BrW \\
\hline MBW & 170 & 435416 & 710817 & Curtis & 1988 & 870 & - & 221 & $\cdots$ & $\mathbf{x}$ & $\cdots$ & BrW \\
\hline MBW & 171 & 435337 & 710933 & Plummer and R. Smokla & 1988 & 500 & $\cdots$ & 80 & $\cdots$ & $x$ & $\cdots$ & Brw \\
\hline MBW & 172 & 435405 & 711152 & McKinney & 1988 & 580 & $\cdots$ & 114 & $\cdots$ & $\mathrm{x}$ & $\cdots$ & Brw \\
\hline MBW & 173 & 435313 & 711033 & Saury & 1988 & 480 & $\cdots$ & 44 & $\cdots$ & $\mathbf{S}$ & $\cdots$ & $\cdots$ \\
\hline MBW & 174 & 435432 & 710906 & Risma & 1988 & 660 & $\cdots$ & 42 & - & $\mathrm{x}$ & $\cdots$ & BrW \\
\hline MBW & 175 & 435432 & 710910 & Gwyther & 1988 & 630 & $\cdots$ & 44 & - & $x$ & $\cdots$ & BrW \\
\hline MBW & 176 & 435434 & 710901 & Mcwalter & 1988 & 700 & $\cdots$ & 39 & - & $\mathrm{x}$ & - & Brw \\
\hline MBW & 177 & 435230 & 710543 & williams & 1988 & 720 & $\cdots$ & 71 & $\cdots$ & $\mathrm{x}$ & $\cdots$ & BrW \\
\hline MBW & 178 & 435101 & 711126 & Nelson & 1988 & 480 & $\cdots$ & 56 & - & $\mathrm{s}$ & $\cdots$ & $\cdots$ \\
\hline MBW & 179 & 435446 & 710724 & Thompson-Tucker & 1988 & 1.080 & $\cdots$ & 74 & -- & $\mathrm{x}$ & $\cdots$ & Brw \\
\hline MBW & 180 & 435732 & 711014 & Savary & 1988 & 660 & $\cdots$ & 170 & $\cdots$ & $x$ & $\cdots$ & Brw \\
\hline MBW & 181 & 435314 & 710943 & Brady & 1988 & 560 & $\cdots$ & 40 & $\cdots$ & $x$ & $\cdots$ & BrW \\
\hline MBW & 182 & 435320 & 710947 & Soroka & 1988 & 560 & $\cdots$ & 39 & - & $x$ & $\cdots$ & Brw \\
\hline MBW & 183 & 435338 & 710748 & Keylock Homes & 1988 & 840 & $\cdots$ & 40 & $\cdots$ & $\mathrm{x}$ & $\cdots$ & BrW \\
\hline MBW & 184 & 435740 & 710845 & Talbot & 1989 & 480 & $\cdots$ & 20 & $\cdots$ & $\cdots$ & $\cdots$ & Dug \\
\hline
\end{tabular}




\begin{tabular}{|c|c|c|c|c|c|c|c|c|}
\hline $\begin{array}{l}\text { Local } \\
\text { site } \\
\text { number }\end{array}$ & $\begin{array}{l}\text { Water } \\
\text { level } \\
\text { depth } \\
\text { (feet) }\end{array}$ & $\begin{array}{l}\text { Date } \\
\mathrm{mm} / \mathrm{dd} / \mathrm{yy}\end{array}$ & Use & $\begin{array}{l}\text { Maximum } \\
\text { well } \\
\text { yield } \\
\text { (gallons } \\
\text { per } \\
\text { minute) }\end{array}$ & $\begin{array}{l}\text { Draw- } \\
\text { down } \\
\text { (feet) }\end{array}$ & $\begin{array}{l}\text { Specific } \\
\text { capacity } \\
\text { (gallons per } \\
\text { minute } \\
\text { per } \\
\text { foot) }\end{array}$ & $\begin{array}{l}\text { Pumping } \\
\text { period } \\
\text { (hours) }\end{array}$ & Remarks \\
\hline
\end{tabular}

\begin{tabular}{|c|c|c|c|c|c|c|c|c|}
\hline MBW & 131 & 40 & $08-29-87$ & $\mathrm{H}$ & 5 & $\cdots$ & $\cdots$ & 1 \\
\hline MBW & 132 & 60 & $09-02-87$ & $\mathrm{H}$ & 20 & $\cdots$ & - & .5 \\
\hline MBW & 133 & 10 & $08-19-87$ & $\mathrm{H}$ & - & $\cdots$ & - & - \\
\hline MBW & 134 & 36 & $08-10-87$ & $\mathrm{H}$ & 30 & $\cdots$ & - & 48 \\
\hline MBW & 135 & - & - & $\mathrm{H}$ & 3 & $\cdots$ & - & 1 \\
\hline MBW & 136 & 20 & $08-08-87$ & $\mathrm{H}$ & 4 & $\cdots$ & - & 1 \\
\hline MBW & 137 & 35 & $07-22-87$ & $\mathrm{H}$ & 30 & $\cdots$ & -- & 48 \\
\hline MBW & 138 & 8 & $01-08-87$ & $\mathrm{H}$ & - - & - & - & - \\
\hline MBW & 139 & $\cdots$ & - - & $\mathbf{P}$ & 30 & -- & - & 1.5 \\
\hline MMBW & 140 & 40 & $07 \cdot 30 \cdot 87$ & $\mathrm{H}$ & 75 & - & - & .5 \\
\hline MBW & 141 & - & $\ldots$ & $\mathrm{H}$ & 4 & - & $\cdots$ & 1 \\
\hline MBW & 142 & 47 & $09-15-87$ & $\mathrm{H}$ & 15 & $\cdots$ & -- & 1 \\
\hline MBW & 143 & 21 & $09-11-87$ & $\mathrm{H}$ & 4 & - & - & 1 \\
\hline MBW & 144 & 41 & $10 \cdot 25 \cdot 87$ & $\mathrm{H}$ & 15 & $\cdots$ & - & 1.5 \\
\hline MBW & 145 & 25 & $11-02 \cdot 87$ & $P$ & 125 & $\cdots$ & - & 12 \\
\hline MBW & 146 & $\cdots$ & - & $\mathrm{H}$ & 100 & - & - & 1 \\
\hline MBW & 147 & 25 & $10-26-87$ & $\mathrm{H}$ & 6 & - & - & 1 \\
\hline MBW & 148 & - - & - & $\mathrm{H}$ & 9 & - & - & .5 \\
\hline MBW & 149 & $\cdots$ & - & $\mathrm{H}$ & 4 & - & - - & 1 \\
\hline MBW & 150 & - & - & $\mathrm{H}$ & 100 & - & - & 1 \\
\hline MBW & 151 & 35 & $12-05-87$ & $\mathrm{H}$ & 15 & - & - & 2 \\
\hline MBW & 152 & 25 & $12-16-87$ & $\mathrm{H}$ & 5 & - & - & .5 \\
\hline MBW & 153 & 25 & $12 \cdot 02-87$ & $\mathrm{H}$ & 15 & -- & $\cdots$ & .5 \\
\hline MBW & 154 & 20 & $11 \cdot 19 \cdot 87$ & $\mathrm{H}$ & 7.5 & - & $\cdots$ & .5 \\
\hline MBW & 155 & 60 & $04-15-88$ & $\mathrm{H}$ & 10 & - & -- & .5 \\
\hline MBW & 156 & 15 & $01-26-88$ & $\mathrm{H}$ & 6 & -. & - - & .5 \\
\hline MBW & 157 & 90 & $01-22-88$ & $\mathrm{H}$ & 0.7 & - - & - & 1 \\
\hline MBW & 158 & - - & - & $\mathrm{H}$ & 3 & - & -- & 1 \\
\hline MBW & 159 & 15 & $04-19-88$ & $\mathrm{H}$ & 9 & $\cdots$ & $\cdots$ & 1 \\
\hline MBW & 160 & 30 & $01-29-88$ & $\mathrm{H}$ & 3 & - & - - & 1 \\
\hline MBW & 161 & 15 & $01-21-88$ & $\cdots$ & 20 & - & -- & 1 \\
\hline MBW & 162 & 130 & $04-26-88$ & $\mathrm{H}$ & 6 & -- & $\cdots$ & 1 \\
\hline MBW & 163 & - & - - & $\mathrm{H}$ & 100 & - & - - & 1 \\
\hline MBW & 164 & 146 & $05-21-88$ & $\mathrm{H}$ & 10 & $\cdots$ & $\cdots$ & 1 \\
\hline MBW & 165 & $\cdots$ & -- & $\mathrm{H}$ & 20 & $\cdots$ & $\cdots$ & 1 \\
\hline MBW & 166 & - & $\cdots$ & $\mathrm{H}$ & 4 & $\cdots$ & - & 1 \\
\hline MBW & 167 & - - & - & $\mathrm{H}$ & 75 & $\ldots$ &.- & 1 \\
\hline MBW & 168 & 90 & $07-18-88$ & $\mathrm{H}$ & 3 & - & $\cdots$ & 1 \\
\hline MBW & 169 & 40 & $06-11-88$ & $\mathrm{H}$ & 1.5 & $-\cdot$ & $\cdots$ & .5 \\
\hline MBW & 170 & 60 & $07-05-88$ & $\mathrm{H}$ & 3 & - & - & 1 \\
\hline MBW & 171 & 10 & $07-12-88$ & $\mathrm{H}$ & 12 & - & - - & .5 \\
\hline MBW & 172 & 30 & $07-12-88$ & $\mathrm{H}$ & 1 & $\cdots$ & -- & 1 \\
\hline MBW & 173 & 28 & $08-16-88$ & $\mathrm{H}$ & 15 & - & -. & 2 \\
\hline MBW & 174 & - - & - & $\mathrm{H}$ & 3 & $\cdots$ & $\cdots$ & 1 \\
\hline MBW & 175 & 40 & $07-20-88$ & $\mathrm{H}$ & 6 & - & $\cdots$ & 1 \\
\hline MBW & 176 & - & - & $\mathrm{H}$ & 3 & - & $\cdots$ & 1 \\
\hline MBW & 177 & $\cdots$ & - & $\mathrm{H}$ & - & $-\cdots$ & - & 1 \\
\hline MBW & 178 & 42 & $11-19-88$ & $\mathrm{H}$ & 15 & - & $-\cdot$ & 1 \\
\hline MBW & 179 & - & - - & $\mathrm{H}$ & 5.5 & - & $\cdots$ & 1 \\
\hline MBW & 180 & - & $\cdots$ & $\mathrm{H}$ & 3 & $\cdots$ & . & 1 \\
\hline MBW & 181 & 21 & $08-30-88$ & $\mathrm{H}$ & 5 & $\cdots$ & 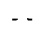 & 1 \\
\hline MBW & 182 & - - & - & $\mathrm{H}$ & 25 & - - & $\cdots$ & 1 \\
\hline MBW & 183 & 80 & $12-02-88$ & $\mathrm{H}$ & 0.8 & $\cdots$ & $-\cdot$ & 1 \\
\hline MBW & 184 & 15 & $01-11-89$ & $\mathrm{H}$ & $\ldots$ & $-\cdot$ & - & - \\
\hline
\end{tabular}


Table 1-1. Description of selected wells, borings, and springs in the Saco and Ossipee River Basins, east-central

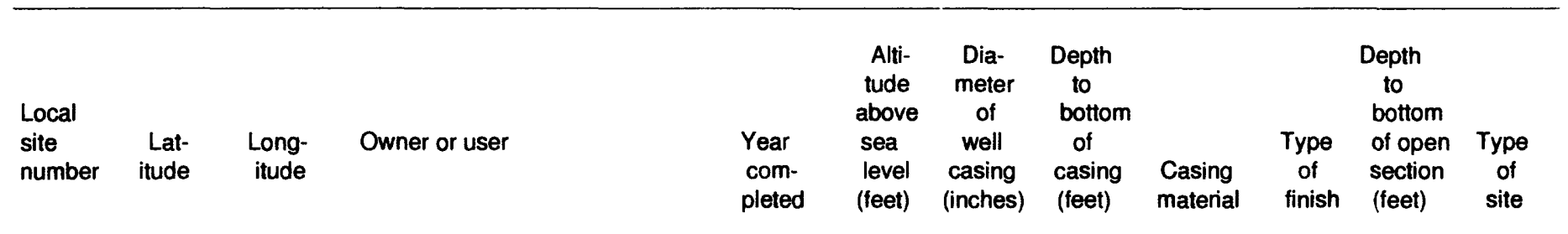

\begin{tabular}{|c|c|c|c|c|c|c|c|c|c|c|c|c|}
\hline MBW & 185 & 435353 & 710727 & Rodgers & 1989 & 1,080 & $\cdots$ & 40 & $\cdots$ & $\mathrm{x}$ & $\cdots$ & Brw \\
\hline MBW & 186 & 435219 & 710617 & Cyr & 1989 & 760 & -- & 46 & $\cdots$ & $\mathrm{x}$ & $\cdots$ & Brw \\
\hline MBW & 187 & 435525 & 711148 & McInnis & 1989 & 750 & $\cdots$ & 54 & $\cdots$ & $x$ & $\cdots$ & BrW \\
\hline MBW & 188 & 435238 & 710548 & Van Raden & 1989 & 660 & $\cdots$ & 93 & $\cdots$ & $x$ & $\cdots$ & Brw \\
\hline MBW & 189 & 435421 & 710643 & Hatten & 1989 & 1,080 & $\cdots$ & 80 & $\cdots$ & $x$ & $\cdots$ & Brw \\
\hline MBW & 190 & 435353 & 710914 & Madison School Board & 1989 & 520 & $\cdots$ & 94 & $\cdots$ & $\mathrm{x}$ & $\cdots$ & BrW \\
\hline MBW & 191 & 435436 & 710903 & Flanders & 1989 & 680 & $\cdots$ & 105 & $\cdots$ & $\mathrm{x}$ & - & Brw \\
\hline MBW & 192 & 435607 & 710827 & Vatcher & 1989 & 640 & $\cdots$ & 120 & $-\cdot$ & $\mathrm{x}$ & $\cdots$ & Brw \\
\hline MBW & 193 & 435633 & 710658 & Howland & 1989 & 660 & $\cdots$ & 110 & - & $\mathrm{x}$ & $\cdots$ & BrW \\
\hline MBW & 194 & 435529 & 710958 & street Jr & 1989 & 530 & $\cdots$ & 91 & $\cdots$ & $\mathrm{x}$ & $\cdots$ & BrW \\
\hline MBW & 195 & 435336 & 710840 & Litchfield & 1988 & 580 & $\cdots$ & 62 & -- & $x$ & $\cdots$ & BrW \\
\hline MBW & 196 & 435337 & 710824 & Collord & 1989 & 630 & - & 17 & - & - & - & Dug \\
\hline MBW & 197 & 435343 & 710933 & Rioux & 1989 & 520 & $\cdots$ & 16 & $\cdots$ & - & $\cdots$ & Dug \\
\hline MBW & 198 & 435419 & 710942 & Wonsor & 1989 & 620 & $\cdots$ & 45 & $\cdots$ & $\mathbf{x}$ & $\cdots$ & BrW \\
\hline MBW & 199 & 435349 & 710932 & Ferry & 1989 & 560 & $\cdots$ & 80 & - & $\mathrm{x}$ & $\cdots$ & Brw \\
\hline MBW & 200 & 435417 & 710810 & Van Wickler & 1989 & 880 & $\cdots$ & 189 & $\cdots$ & $\mathrm{x}$ & $\cdots$ & Brw \\
\hline MBW & 201 & 435416 & 710939 & Littlefield & 1989 & 640 & - & 60 & $\cdots$ & $\mathrm{x}$ & $\cdots$ & Brw \\
\hline MBW & 202 & 435353 & 710914 & Madison Elementary School & 1989 & 520 & $\cdots$ & 39 & $\cdots$ & $\mathbf{S}$ & $\cdots$ & \\
\hline MBW. & 203 & 435120 & 711051 & Fortin & 1990 & 480 & $\cdots$ & 44 & $-\cdot$ & $\mathbf{S}$ & $\cdots$ & \\
\hline MBW & 204 & 435248 & 710721 & D. Wood & 1990 & 920 & $\cdots$ & 39 & $\cdots$ & $\mathrm{x}$ & $\cdots$ & BrW \\
\hline MBW & 205 & 435532 & 711002 & Silver Lake Association & 1990 & 550 & $\cdots$ & 79 & $-\cdot$ & $\mathrm{x}$ & $\cdots$ & BrW \\
\hline MBW & 206 & 435413 & 710846 & Toscano & 1990 & 680 & -- & 70 & $\cdots$ & $\mathrm{x}$ & $\cdots$ & BrW \\
\hline MBW & 207 & 435221 & 710613 & Case & 1990 & 700 & $\cdots$ & 60 & $\cdots$ & $\mathrm{x}$ & $\cdots$ & Brw \\
\hline MBW & 208 & 435322 & 710949 & Mckinley & 1990 & 530 & $\cdots$ & 40 & $\cdots$ & $\mathrm{x}$ & $\cdots$ & Brw \\
\hline MBW & 209 & 435219 & 710623 & พa11 & 1990 & 800 & $\cdots$ & 40 & $-\cdot$ & $\mathrm{x}$ & $\cdots$ & BrW \\
\hline MBW & 210 & 435331 & 710935 & Dolly Madison's Country & 1991 & 480 & $\cdots$ & 17 & $\cdots$ & $\mathbf{S}$ & $\cdots$ & \\
\hline MBW & 211 & 435127 & 710514 & Gillard & 1991 & 480 & $\cdots$ & 20 & $\cdots$ & $\cdots$ & $\cdots$ & Dug \\
\hline MBW & 212 & 435415 & 711059 & Deaderick & 1991 & 680 & $\cdots$ & 20 & $\cdots$ & $\cdots$ & $\cdots$ & Dug \\
\hline MBW & 213 & 435019 & 711130 & Audette Jr & 1991 & 460 & - & 35 & $-\cdot$ & $\mathrm{s}$ & $\cdots$ & \\
\hline MBW & 214 & 435117 & 711141 & Chagnon & 1991 & 480 & $\cdots$ & 68 & -- & $s$ & $\cdots$ & \\
\hline MBW & 215 & 435518 & 710637 & Cody & 1991 & 1,140 & $\cdots$ & 39 & $\cdots$ & $\mathrm{x}$ & $\cdots$ & BrW \\
\hline MBW & 216 & 435258 & 711136 & Leavitt & 1991 & 720 & $-\cdot$ & 45 & $\cdots$ & $\mathrm{x}$ & $\cdots$ & Brw \\
\hline MBW & 217 & 435735 & 710909 & Doe & 1991 & 520 & $\cdots$ & 159 & $\cdots$ & $\mathrm{s}$ & $\cdots$ & \\
\hline MBW & 218 & 435311 & 710957 & Hutchinson & 1991 & 500 & - & 60 & $\cdots$ & $x$ & $-\cdot$ & BrW \\
\hline MBW & 219 & 435140 & 711128 & Callahan & 1991 & 500 & $\cdots$ & 34.8 & $\cdots$ & $\mathbf{s}$ & $\cdots$ & \\
\hline MBW & 220 & 435250 & 710735 & Morri11 & 1991 & 890 & $\cdots$ & 60 & $\cdots$ & $\mathrm{x}$ & $\cdots$ & Brw \\
\hline MBW & 221 & 435155 & 711109 & Thompson & 1991 & 500 & - & 70 & - & $\cdots$ & $\cdots$ & \\
\hline MBW & 222 & 435640 & 710700 & Belisle & 1991 & 720 & $\cdots$ & 191 & - & $\mathrm{x}$ & $\cdots$ & Brw \\
\hline MBW & 223 & 435131 & 711137 & Savory Bros & 1991 & 460 & $\cdots$ & 26 & - & $\mathbf{S}$ & $\cdots$ & \\
\hline MBW & 224 & 435316 & 711024 & Batchelder & 1991 & 477 & $\cdots$ & 100 & $\cdots$ & $\mathrm{x}$ & $\cdots$ & BrW \\
\hline MBW & 225 & 435340 & 710936 & Fanciullo & 1992 & 500 & $\cdots$ & 70 & $\cdots$ & $\mathrm{x}$ & $\cdots$ & BrW \\
\hline MBW & 226 & 435108 & 711138 & starkey & 1992 & 480 & $\cdots$ & 71 & -- & $\mathbf{s}$ & $\cdots$ & \\
\hline MBW & 227 & 435026 & 711116 & Govoni & 1992 & 470 & $\cdots$ & 44 & $\cdots$ & 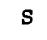 & $\cdots$ & \\
\hline MBW & 228 & 435118 & 711041 & Rosse & 1992 & 480 & $\cdots$ & 35 & $\cdots$ & $\mathbf{s}$ & $\cdots$ & \\
\hline MBW & 229 & 435116 & 711141 & Terrasi & 1992 & 480 & $\cdots$ & 68 & $\cdots$ & $\mathbf{s}$ & $\cdots$ & \\
\hline MBW & 230 & 435211 & 711058 & Arnold & 1992 & 480 & $\cdots$ & 157 & $\cdots$ & $\mathrm{x}$ & $\cdots$ & BrW \\
\hline MBW & 231 & 435536 & 711002 & Perkins & 1992 & 520 & $\cdots$ & 61 & $\cdots$ & $\mathrm{x}$ & $\cdots$ & BrW \\
\hline MBW & 232 & 435425 & 711111 & Shackford Jr & 1992 & 640 & $\cdots$ & 23 & - & $\cdots$ & $\cdots$ & Dug \\
\hline MBW & 233 & 435326 & 711057 & Palica & 1992 & 560 & $\cdots$ & 113 & $\cdots$ & $\mathrm{x}$ & $\cdots$ & Brw \\
\hline MBW & 234 & 435057 & 711127 & Destremps & 1992 & 480 & $\cdots$ & 58 & $\cdots$ & $\mathrm{s}$ & $\cdots$ & \\
\hline MBW & 235 & 435735 & 711020 & VanDyne & 1987 & 680 & $\cdots$ & 219 & $\cdots$ & $\mathrm{x}$ & - & BrW \\
\hline MBW & 236 & 435120 & 711139 & A. Bradshaw & 1992 & 480 & $\cdots$ & 69 & $\cdots$ & -- & $\cdots$ & \\
\hline MBW & 237 & 435113 & 711118 & Murdock & 1992 & 480 & $\cdots$ & 59 & $\cdots$ & $\cdots$ & $\cdots$ & \\
\hline MBW & 238 & 435733 & 710922 & Rowell & 1992 & 570 & $\cdots$ & 221 & $\cdots$ & $\mathrm{x}$ & $\cdots$ & BrW \\
\hline MBW & 239 & 435257 & 711129 & Medeiros & 1992 & 740 & $\cdots$ & 39 & $\cdots$ & $\mathrm{x}$ & $\cdots$ & Brw \\
\hline
\end{tabular}




\begin{tabular}{|c|c|c|c|c|c|c|c|c|}
\hline $\begin{array}{l}\text { Local } \\
\text { site } \\
\text { number }\end{array}$ & $\begin{array}{l}\text { Water } \\
\text { level } \\
\text { depth } \\
\text { (feet) }\end{array}$ & $\begin{array}{l}\text { Date } \\
\mathrm{mm} / \mathrm{dd} / \mathrm{yy}\end{array}$ & Use & $\begin{array}{l}\text { Maximum } \\
\text { well } \\
\text { yield } \\
\text { (gallons } \\
\text { per } \\
\text { minute) }\end{array}$ & $\begin{array}{l}\text { Draw- } \\
\text { down } \\
\text { (feet) }\end{array}$ & $\begin{array}{l}\text { Specific } \\
\text { capacity } \\
\text { (gallons per } \\
\text { minute } \\
\text { per } \\
\text { foot) }\end{array}$ & $\begin{array}{l}\text { Pumping } \\
\text { period } \\
\text { (hours) }\end{array}$ & Remarks \\
\hline
\end{tabular}

\begin{tabular}{|c|c|c|c|c|c|c|c|c|}
\hline MBW & 185 & - & $\cdots$ & $\mathrm{H}$ & 5 & $\cdots$ & $\cdots$ & 1 \\
\hline MBW & 186 & 28 & $05-01 \cdot 89$ & $\mathrm{H}$ & 1 & $\cdots$ & $\cdots$ & 1 \\
\hline MBW & 187 & 5 & $03-27-89$ & $\mathrm{H}$ & 3.5 & - & - & 1 \\
\hline MBW & 188 & 40 & $03-29-89$ & $\mathrm{H}$ & 0.7 & - & -. & 2 \\
\hline MBW & 189 & 95 & $06-23-89$ & $\mathrm{H}$ & 50 & - & - - & 1 \\
\hline MBW & 190 & $\cdots$ & $\cdots$ & $\mathbf{z}$ & 7 & $\cdots$ & $\cdots$ & 1 \\
\hline MBW & 191 & $\cdots$ & $\cdots$ & $\mathrm{H}$ & 30 & - - & $\cdots$ & 1 \\
\hline MBW & 192 & $\cdots$ & $\cdots$ & $\mathrm{H}$ & 15 & - & $\cdots$ & 1 \\
\hline MBW & 193 & - & $\cdots$ & $\mathrm{H}$ & 12 & $\cdots$ & $\cdots$ & 1 \\
\hline MBW & 194 & - & $-\cdot$ & $\mathrm{H}$ & 8 & - & $\cdots$ & 1 \\
\hline MMBW & 195 & - & $\cdots$ & H & 10 & - & - & .8 \\
\hline MBW & 196 & 4 & $08 \cdot 27 \cdot 89$ & $\mathrm{H}$ & - & $\cdots$ & $\cdots$ & $\cdots$ \\
\hline MBW & 197 & 9 & $10-31 \cdot 89$ & $\mathrm{H}$ & 60 & - & $\cdots$ & .5 \\
\hline MBW & 198 & 50 & $08-11-89$ & $\mathrm{H}$ & 13 & - - & $\cdots$ & 1 \\
\hline MBW & 199 & - & - - & $\mathrm{H}$ & 7.5 & $\cdots$ & $\cdots$ & 1 \\
\hline MBW & 200 & 45 & $12-03-89$ & $\mathrm{H}$ & 3 & - & - & .5 \\
\hline MBW & 201 & 30 & $12-08-89$ & $\mathrm{H}$ & 100 & - & $\cdots$ & 1 \\
\hline MBW & 202 & - & - & $T$ & 24 & - & $\ldots$ & 48 \\
\hline MBW & 203 & 30 & $04-29-90$ & $\mathrm{H}$ & 20 & $\cdots$ & $\cdots$ & 1 \\
\hline MBW & 204 & 25 & $05-26-90$ & $\mathrm{H}$ & 4 & $\cdots$ & - & 1 \\
\hline MBW & 205 & - & - & H & 25 & $\cdots$ & $\cdots$ & .5 \\
\hline MBW & 206 & 25 & $08-29-90$ & $\mathrm{H}$ & 15 & $\cdots$ & $\cdots$ & 1 \\
\hline MBW & 207 & - & - & $\mathrm{H}$ & 20 & - & $-\cdot$ & 1 \\
\hline MBW & 208 & - & - & $\mathrm{H}$ & 11 & - & $\cdots$ & 1 \\
\hline MBW & 209 & 30 & $05-10-90$ & $\mathrm{H}$ & 2.5 & $\cdots$ & $\cdots$ & .5 \\
\hline MBW & 210 & 5 & $04-27-91$ & C & 20 & $\cdots$ & $\cdots$ & 1 \\
\hline MBW & 211 & 10 & $05-12-91$ & $\mathrm{H}$ & - & $\cdots$ & $\cdots$ & $\cdots$ \\
\hline MBW & 212 & 3 & $05-05-91$ & $\mathrm{H}$ & $\cdots$ & $\cdots$ & - & - \\
\hline MBW & 213 & 26 & $06-18-91$ & H & 15 & - & $\cdots$ & 1 \\
\hline MBW & 214 & 54 & $06-30-91$ & $\mathrm{H}$ & 10 & $\cdots$ & $\cdots$ & 2 \\
\hline MBW & 215 & 5 & $07-25-91$ & $\mathrm{H}$ & 3.5 & - & - & .5 \\
\hline MBW & 216 & $\cdots$ & - & $\mathrm{H}$ & 2 & - & - & 1 \\
\hline MBW & 217 & $\cdots$ & $\cdots$ & $\mathrm{H}$ & 25 & - & $\cdots$ & 12 \\
\hline MBW & 218 & - & - & $\mathrm{H}$ & 7 & $-\cdot$ & - & 1 \\
\hline MBW & 219 & 31 & $09-20-91$ & H & 15 & $\cdots$ & $\cdots$ & 1 \\
\hline MBW & 220 & 15 & $10-31-91$ & $\mathbf{H}$ & 12 & - & $\cdots$ & 1 \\
\hline MBW & 221 & - & $\cdots$ & $\mathrm{P}$ & 20 & $\cdots$ & $\cdots$ & 48 \\
\hline MBW & 222 & 70 & $11-12-91$ & $\mathrm{H}$ & 15 & - & - & .5 \\
\hline MBW & 223 & 24 & $10-28-91$ & $\mathrm{H}$ & 5 & $\cdots$ & $\cdots$ & 2 \\
\hline MBW & 224 & - & $\cdots$ & H & 10 & $\cdots$ & $\cdots$ & 1 \\
\hline MBW & 225 & 17 & $01-16-92$ & H & 2 & $\cdots$ & - & 1 \\
\hline MBW & 226 & 48 & $06-20-92$ & $\mathrm{H}$ & 11 & - & - & 2 \\
\hline MBW & 227 & 29 & $05-02-92$ & $\mathrm{H}$ & 17 & $\cdots$ & $\cdots$ & 2 \\
\hline MBW & 228 & 24 & $05-17-92$ & $\mathrm{H}$ & 17 & $\cdots$ & $\cdots$ & 1 \\
\hline MBW & 229 & 52 & $05-09-92$ & H & 10 & $\cdots$ & $\cdots$ & 2 \\
\hline MBW & 230 & - - & $\cdots$ & $\mathrm{H}$ & 6 & $\cdots$ & $\cdots$ & 1 \\
\hline MBW & 231 & 40 & $06-11-92$ & $\mathrm{H}$ & 7 & $\cdots$ & $\cdots$ & .5 \\
\hline MBW & 232 & 9 & $06-27-92$ & $\mathrm{H}$ & 20 & - & $\cdots$ & 16 \\
\hline MBW & 233 & 60 & $08-11-92$ & $\mathrm{H}$ & 12 & -- & - & 1 \\
\hline MBW & 234 & 40 & $07-28-92$ & $\mathrm{H}$ & 35 & - & - - & 5 \\
\hline MBW & 235 & 40 & $10-16 \cdot 87$ & $\mathrm{H}$ & 4 & - & $\cdots$ & .5 \\
\hline MBW & 236 & 54 & $08-15-92$ & $\mathrm{H}$ & 11 & $\cdots$ & $\cdots$ & 2 \\
\hline MBW & 237 & 43 & $09-07-92$ & $\mathrm{H}$ & 11 & $\cdots$ & $\cdots$ & 1.5 \\
\hline MBW & 238 & 80 & $10-29-92$ & $\mathrm{H}$ & 0.8 & - & $\cdots$ & 1 \\
\hline MBW & $239^{\circ}$ & - & - & $\mathrm{H}$ & 2 & $-\cdot$ & - & 1 \\
\hline
\end{tabular}


Table 1-1. Description of selected wells, borings, and springs in the Saco and Ossipee River Basins, east-central

\begin{tabular}{|c|c|c|c|c|c|c|c|c|c|c|c|}
\hline $\begin{array}{l}\text { Local } \\
\text { site } \\
\text { number }\end{array}$ & $\begin{array}{l}\text { Lat- } \\
\text { itude }\end{array}$ & $\begin{array}{l}\text { Long- } \\
\text { itude }\end{array}$ & Owner or user & $\begin{array}{l}\text { Year } \\
\text { com- } \\
\text { pleted }\end{array}$ & $\begin{array}{l}\text { Alti- } \\
\text { tude } \\
\text { above } \\
\text { sea } \\
\text { level } \\
\text { (feet) }\end{array}$ & $\begin{array}{c}\text { Dia- } \\
\text { meter } \\
\text { of } \\
\text { well } \\
\text { casing } \\
\text { (inches) }\end{array}$ & $\begin{array}{l}\text { Depth } \\
\text { to } \\
\text { bottom } \\
\text { of } \\
\text { casing } \\
\text { (feet) }\end{array}$ & $\begin{array}{l}\text { Casing } \\
\text { material }\end{array}$ & $\begin{array}{l}\text { Type } \\
\text { of } \\
\text { finish }\end{array}$ & $\begin{array}{l}\text { Depth } \\
\text { to } \\
\text { bottom } \\
\text { of open } \\
\text { section } \\
\text { (feet) }\end{array}$ & $\begin{array}{c}\text { Type } \\
\text { of } \\
\text { site }\end{array}$ \\
\hline
\end{tabular}

\begin{tabular}{|c|c|c|c|c|c|c|c|c|c|c|c|c|}
\hline MBW & 240 & 435742 & 710900 & Kitchen Jr & 1992 & 470 & - & 145 & $-\cdot$ & $-\cdot$ & $\cdots$ & \\
\hline MBW & 241 & 435113 & 711138 & Spence & 1992 & 480 & $\cdots$ & 69 & $\cdots$ & $\mathbf{s}$ & $\cdots$ & \\
\hline MBW & 242 & 435057 & 711129 & Mermet & 1992 & 480 & $\cdots$ & 59 & $\cdots$ & $\mathbf{s}$ & $\cdots$ & \\
\hline MBW & 243 & 435209 & 710547 & Pritchard & 1992 & 540 & $\cdots$ & 40 & $\cdots$ & $\mathrm{x}$ & $\cdots$ & BrW \\
\hline MBW & 244 & 435224 & 710604 & Laliberte & 1992 & 720 & $\cdots$ & 20 & $\cdots$ & $\mathrm{x}$ & $\cdots$ & BrW \\
\hline MBW & 245 & 435139 & 710521 & Purity Spring Resort & 1992 & 460 & $\cdots$ & 120 & $\cdots$ & $\mathbf{x}$ & $\cdots$ & BrW \\
\hline MBW & 246 & 435133 & 711142 & Goc & 1992 & 460 & $\cdots$ & 68 & $\cdots$ & $\mathbf{x}$ & $\cdots$ & BrW \\
\hline MBW & 247 & 435336 & 710926 & Jones & 1993 & 510 & - & 93 & -- & $\mathrm{x}$ & $\cdots$ & BrW \\
\hline MBW & 248 & 435204 & 711058 & Ballentyne & 1993 & 475 & - & 171 & $-\cdot$ & $\mathrm{x}$ & $\cdots$ & BrW \\
\hline MBW & 249 & 435638 & 710901 & Village District of Eidelweiss & 1993 & 520 & - & 65 & $\mathbf{s}$ & $\mathrm{x}$ & $\cdots$ & BrW \\
\hline MBW & 250 & 435030 & 711114 & Jones & 1993 & 460 & -- & 49 & - & $\mathbf{S}$ & $\cdots$ & \\
\hline MBW & 251 & 435027 & 711125 & Lowry & 1993 & 460 & $\cdots$ & 54.5 & $\cdots$ & $\mathbf{s}$ & - & \\
\hline MBW & 252 & 435002 & 711109 & Madison Lumber Mill & $\cdots$ & 450 & $\cdots$ & - & $\cdots$ & $\cdots$ & $\cdots$ & \\
\hline MBW & 253 & 435610 & 710702 & Village District of Eidelweiss & - & 0 & $\cdots$ & $\cdots$ & $\cdots$ & $\cdots$ & $\cdots$ & $-\cdot$ \\
\hline
\end{tabular}

Madison--Continued

\begin{tabular}{|c|c|c|c|c|}
\hline OXA & 1 & 434828 & 711004 & Leconte, Paul \\
\hline OXA & 2 & 434558 & 710837 & \\
\hline OXA & 3 & 434829 & 711003 & \\
\hline $\mathrm{OXB}$ & 1 & 434909 & 711224 & NHDOT \\
\hline OXB & 2 & 434750 & 711055 & NHDOT \\
\hline OXB & 3 & 434756 & 711052 & NHDOT \\
\hline OXB & 4 & 434547 & 710908 & NHDOT \\
\hline OXB & 5 & 434522 & 710828 & NHDOT \\
\hline OXB & 6 & 434511 & 710814 & NHDOT \\
\hline OXB & 7 & 434543 & 710807 & NHDOT \\
\hline OXB & 8 & 434129 & 710707 & NHDOT \\
\hline OXB & 9 & 434642 & 710958 & NHDOT \\
\hline OXB & 10 & 434516 & 711018 & NHDOT \\
\hline OXB & 11 & 434514 & 711033 & NHDOT \\
\hline OXB & 12 & 434105 & 710519 & NHDOT \\
\hline OXB & 13 & 434010 & 710426 & NHDOT \\
\hline OXB & 14 & 434030 & 710449 & NHDOT \\
\hline OXB & 15 & 434051 & 710649 & NHDOT \\
\hline OXB & 16 & 434900 & 711146 & NHDOT \\
\hline oxs & 1 & 434709 & 711139 & $\cdots$ \\
\hline oxs & 2 & 434335 & 710937 & $\cdots$ \\
\hline oxs & 3 & 434053 & 710539 & - \\
\hline oxs & 4 & 434056 & 710529 & - \\
\hline oxs & 5 & 433909 & 710350 & $\cdots$ \\
\hline $\mathrm{OXW}$ & 4 & 434923 & 711115 & $\cdots$ \\
\hline oxw & 5 & 434920 & 711116 & - \\
\hline oxw & 6 & 434927 & 711231 & La11 \\
\hline oxw & 7 & 434926 & 711238 & Roland Lord \\
\hline OXW & 8 & 434920 & 711220 & U.S. Post office, Ossipee \\
\hline oxw & 9 & 434916 & 711250 & Nudd \\
\hline oxw & 10 & 434903 & 711200 & Virgil Abbott \\
\hline oxw & 11 & 434905 & 711157 & Virgil abbott \\
\hline oxw & 12 & 434856 & 711133 & Doe \\
\hline oxw & 13 & 434849 & 711126 & Walter Penny \\
\hline oxw & 14 & 434826 & 710949 & Stanley ward \\
\hline oxw & 15 & 434811 & 711039 & Galente \\
\hline oxw & 16 & 434806 & 711048 & Kim Ross \\
\hline oxw & 17 & 434339 & 710926 & Peter Cook \\
\hline
\end{tabular}

Ossipee

\begin{tabular}{|c|c|c|c|c|c|c|c|}
\hline-- & 415 & $\cdots$ & $\cdots$ & $\cdots$ & $\cdots$ & $\cdots$ & TH \\
\hline$\cdots$ & 410 & $\cdots$ & - & - & $\cdots$ & $\cdots$ & \\
\hline 1991 & 415 & $\cdots$ & $\cdots$ & $\cdots$ & $\cdots$ & $\cdots$ & Bor \\
\hline$\cdots$ & 414 & $\cdots$ & $\cdots$ & $\cdots$ & $\cdots$ & $\cdots$ & $\mathrm{TH}$ \\
\hline$\cdots$ & 413 & $\cdots$ & $\cdots$ & $\cdots$ & - & $\cdots$ & $\mathbf{T H}$ \\
\hline$\cdots$ & 415 & $\cdots$ & $\cdots$ & $\cdots$ & $\cdots$ & $\cdots$ & $\mathrm{TH}$ \\
\hline$\cdots$ & 424 & $\cdots$ & $\cdots$ & $\cdots$ & $\cdots$ & $\cdots$ & $\mathrm{TH}$ \\
\hline$\cdots$ & 434.5 & $\cdots$ & $\cdots$ & $\cdots$ & $\cdots$ & $\cdots$ & $\mathrm{TH}$ \\
\hline$\cdots$ & 415 & $\cdots$ & $\cdots$ & $\cdots$ & $\cdots$ & $\cdots$ & TH \\
\hline$\cdots$ & 412 & $\cdots$ & $\cdots$ & $\cdots$ & $\cdots$ & $\cdots$ & $\mathrm{TH}$ \\
\hline - & 649.5 & $\cdots$ & - & $\cdots$ & $\cdots$ & $\cdots$ & $\mathrm{TH}$ \\
\hline$\cdots$ & 440 & - & $\cdots$ & $\cdots$ & $\cdots$ & $\cdots$ & $\mathrm{TH}$ \\
\hline$\cdots$ & 556 & $\cdots$ & $\cdots$ & $\cdots$ & $-\cdot$ & $\cdots$ & TH \\
\hline- & 569 & $\cdots$ & $\cdots$ & $\cdots$ & $\cdots$ & $\cdots$ & $\mathrm{TH}$ \\
\hline$\cdots$ & 515 & $\cdots$ & $\ldots$ & $\cdots$ & $\cdots$ & $\cdots$ & $\mathrm{TH}$ \\
\hline$\cdots$ & 508 & $\cdots$ & $\cdots$ & - & $\ldots$ & $\cdots$ & $\mathrm{TH}$ \\
\hline$\cdots$ & 490 & $\cdots$ & $\cdots$ & $\cdots$ & $\cdots$ & $\cdots$ & $\mathrm{TH}$ \\
\hline -. & 635.5 & $\cdots$ & $\cdots$ & - & $\cdots$ & $\cdots$ & $\mathbf{T H}$ \\
\hline$\cdots$ & 422 & $\cdots$ & $\cdots$ & $\cdots$ & $\cdots$ & $\cdots$ & $\mathrm{TH}$ \\
\hline$\cdots$ & 590 & $\cdots$ & $\cdots$ & $\cdots$ & $\cdots$ & $\cdots$ & $\mathrm{sp}$ \\
\hline$\cdots$ & 530 & $\cdots$ & $\cdots$ & $\cdots$ & $\cdots$ & $\cdots$ & sp \\
\hline$\cdots$ & 565 & $\cdots$ & $\cdots$ & $\cdots$ & $\cdots$ & $\cdots$ & $\mathrm{sp}$ \\
\hline$\cdots$ & 525 & $\cdots$ & $\cdots$ & $\cdots$ & $\cdots$ & $\cdots$ & $\mathrm{Sp}$ \\
\hline$\cdots$ & 575 & $\cdots$ & $\cdots$ & $\cdots$ & $\cdots$ & $\cdots$ & Sp \\
\hline 1979 & 430 & $\cdots$ & - & $\cdots$ & $\mathbf{T}$ & $\cdots$ & Dvn \\
\hline 1987 & 430 & 2 & 12 & G & $\mathrm{T}$ & $\cdots$ & Dvn \\
\hline 1987 & 425 & 6 & 100 & $\mathrm{~s}$ & $\mathbf{F}$ & $\cdots$ & Wsh \\
\hline$\cdots$ & 425 & 1 & 25 & $\mathbf{S}$ & $\mathrm{T}$ & $\cdots$ & Dvn \\
\hline 1985 & 420 & $\cdots$ & - & $\cdots$ & $\mathrm{T}$ & $\cdots$ & Dvn \\
\hline 1989 & 435 & 2 & 48 & $\mathrm{~s}$ & $\mathrm{~T}$ & $\cdots$ & Dvn \\
\hline 1973 & 425 & 6 & 250 & $\mathbf{S}$ & G & $\cdots$ & Brw \\
\hline - & 425 & 2 & 13 & G & $\mathrm{T}$ & $\cdots$ & Dvn \\
\hline 1989 & 430 & 36 & 12 & C & C & $\cdots$ & Dug \\
\hline$\cdots$ & 435 & 36 & 9.8 & $\mathrm{~T}$ & 0 & $\cdots$ & Dug \\
\hline 1951 & 410 & $\cdots$ & - & - & $\mathrm{T}$ & $\cdots$ & Dvn \\
\hline 1964 & 415 & 2 & 18 & G & $\mathrm{T}$ & $\cdots$ & Dvn \\
\hline 1986 & 425 & 2 & 17.5 & $\mathbf{s}$ & $\mathrm{T}$ & $\cdots$ & Dvn \\
\hline - . & 545 & $\cdots$ & - & $\cdots$ & $\mathrm{T}$ & $\cdots$ & Dvm \\
\hline
\end{tabular}




\begin{tabular}{|c|c|c|c|c|c|c|c|c|}
\hline $\begin{array}{l}\text { Local } \\
\text { site } \\
\text { number }\end{array}$ & $\begin{array}{l}\text { Water } \\
\text { level } \\
\text { depth } \\
\text { (feet) }\end{array}$ & $\begin{array}{l}\text { Date } \\
\mathrm{mm} / \mathrm{dd} / \mathrm{yy}\end{array}$ & Use & $\begin{array}{l}\text { Maximum } \\
\text { well } \\
\text { yield } \\
\text { (gallons } \\
\text { per } \\
\text { minute) }\end{array}$ & $\begin{array}{l}\text { Draw- } \\
\text { down } \\
\text { (feet) }\end{array}$ & $\begin{array}{c}\text { Specific } \\
\text { capacity } \\
\text { (gallons per } \\
\text { minute } \\
\text { per } \\
\text { foot) }\end{array}$ & $\begin{array}{l}\text { Pumping } \\
\text { period } \\
\text { (hours) }\end{array}$ & Remarks \\
\hline
\end{tabular}

$\begin{array}{cccc}\text { MBW } & 240 & 10 & 11-07-92 \\ \text { MBW } & 241 & 51 & 10-24-92 \\ \text { MBW } & 242 & 43 & 12-12-92 \\ \text { MBW } & 243 & 25 & 06-28-92 \\ \text { MBW } & 244 & 20 & 06-26-92 \\ & & & \\ \text { MBW } & 245 & 2 & 06-24-92 \\ \text { MBW } & 246 & 2 & 05-27-92 \\ \text { MBW } & 247 & 50 & 05-03-93 \\ \text { MBW } & 248 & 12 & 05-20-93 \\ \text { MBW } & 249 & 40 & 07-\cdots-93 \\ & & & \\ \text { MBW } & 250 & 28 & 05-22-93 \\ \text { MBW } & 251 & 29 & 08-28-93 \\ \text { MBW } & 252 & \cdots & \cdots \\ \text { MBW } & 253 & 60 & 08-18-87\end{array}$

Madison- - Continued

\begin{tabular}{|c|c|c|c|c|c|c|c|c|c|}
\hline \multicolumn{10}{|c|}{ Ossipee } \\
\hline OXA & 1 & 6 & $07-29-91$ & $\mathrm{~T}$ & $\cdots$ & $\cdots$ & $\cdots$ & $\cdots$ & \\
\hline OXA & 2 & $-\cdot$ & $\cdots$ & $\mathrm{U}$ & $\cdots$ & $\cdots$ & $\cdots$ & $\cdots$ & \\
\hline OXA & 3 & 6 & $07-29-91$ & 0 & $\cdots$ & $\cdots$ & $\cdots$ & $\cdots$ & \\
\hline $\mathrm{OXB}$ & 1 & $\cdots$ & $\cdots$ & $\mathrm{T}$ & $\cdots$ & $\cdots$ & $\cdots$ & $\cdots$ & \\
\hline OXB & 2 & $-\cdot$ & $\cdots$ & $\mathrm{T}$ & - & - & $\cdots$ & - & \\
\hline OXB & 3 & $\cdots$ & $\cdots$ & $\mathrm{T}$ & $\cdots$ & $\cdots$ & $\cdots$ & $\cdots$ & \\
\hline $\mathrm{OXB}$ & 4 & $\cdots$ & $\cdots$ & $T$ & $\cdots$ & $\cdots$ & $-\cdot$ & $\cdots$ & \\
\hline OXB & 5 & $\cdots$ & $\cdots$ & $\mathrm{T}$ & $\cdots$ & $\cdots$ & $\cdots$ & $\cdots$ & \\
\hline $\mathrm{OXB}$ & 6 & $\cdots$ & $\cdots$ & $T$ & $\cdots$ & -- & $\cdots$ & $\cdots$ & \\
\hline $\mathrm{OXB}$ & 7 & $\cdots$ & $\cdots$ & $\mathbf{T}$ & $\cdots$ & $\cdots$ & $\cdots$ & $\cdots$ & \\
\hline $\mathrm{OXB}$ & 8 & - & $\cdots$ & $\mathrm{T}$ & $\cdots$ & - & $\cdots$ & $\cdots$ & \\
\hline OXB & 9 & - & $\cdots$ & $T$ & $\cdots$ & $\cdots$ & $\cdots$ & $\cdots$ & \\
\hline $\mathrm{OXB}$ & 10 & $\cdots$ & $-\cdot$ & $T$ & $\cdots$ & $\cdots$ & $\cdots$ & $\cdots$ & \\
\hline OXB & 11 & -- & $\cdots$ & $T$ & $\cdots$ & $\cdots$ & - & -- & \\
\hline $\mathrm{OXB}$ & 12 & $\cdots$ & $\cdots$ & $\mathrm{T}$ & $-\cdot$ & $\cdots$ & $\cdots$ & $\cdots$ & \\
\hline $\mathrm{OXB}$ & 13 & $\cdots$ & $\cdots$ & $T$ & $\cdots$ & $\cdots$ & $\cdots$ & $\cdots$ & \\
\hline $\mathrm{OXB}$ & 14 & $\cdots$ & $\cdots$ & $\mathrm{T}$ & $-\cdot$ & $\cdots$ & $\cdots$ & $\cdots$ & \\
\hline$O X B$ & 15 & $\cdots$ & $\cdots$ & $T$ & $\cdots$ & -- & $\cdots$ & $\cdots$ & \\
\hline $\mathrm{OXB}$ & 16 & $\cdots$ & $\cdots$ & $\mathrm{T}$ & $\cdots$ & $\cdots$ & $\cdots$ & $\cdots$ & \\
\hline oxs & 1 & $\cdots$ & $\cdots$ & $\cdots$ & 1.2 & $\cdots$ & $\cdots$ & $\cdots$ & \\
\hline oxs & 2 & $\cdots$ & $\cdots$ & $\cdots$ & $\cdots$ & $\cdots$ & $\cdots$ & $\cdots$ & \\
\hline oxs & 3 & $\cdots$ & $\cdots$ & $\cdots$ & $\cdots$ & $\cdots$ & $\cdots$ & $\cdots$ & \\
\hline oxs & 4 & $\cdots$ & $\cdots$ & $\cdots$ & $\cdots$ & $\cdots$ & $\cdots$ & $\cdots$ & \\
\hline oxs & 5 & $\cdots$ & $\cdots$ & $\cdots$ & $\cdots$ & $\cdots$ & $\cdots$ & $\cdots$ & More than one spring in the area \\
\hline oxw & 4 & $\cdots$ & - & $\mathrm{H}$ & $\cdots$ & $-\cdot$ & $\cdots$ & $\cdots$ & \\
\hline OXW & 5 & 4 & $\ldots-87$ & $T$ & - & $\cdots$ & $\cdots$ & $-\cdot$ & High in iron and manganese \\
\hline $\mathrm{oxw}$ & 6 & 20 & $06-13-87$ & $\mathrm{~T}$ & 25 & $\cdots$ & $\cdots$ & $\cdots$ & $\mathrm{pH}=5.8, F e=1.1 \mathrm{ppm}$, hard $=20$ \\
\hline oxw & 7 & 20 & $06-11-91$ & H & - & $\cdots$ & - & - & \\
\hline oxw & 8 & - & $\cdots$ & $\mathrm{H}$ & $\cdots$ & $\cdots$ & $\cdots$ & $\cdots$ & High iron, they do not drink water \\
\hline oxw & 9 & 20 & $\ldots 89$ & H & $\cdots$ & $\cdots$ & $-\cdot$ & $\cdots$ & Contains salts and iron \\
\hline oxw & 10 & 7.5 & $06-11-91$ & H & -- & $\cdots$ & -- & $\cdots$ & $\mathrm{R}$ Treat water with lime, iron stains, sulfur $\mathrm{S}$ \\
\hline OXW & 11 & 5 & $06-11-91$ & $\mathbf{I}$ & $\cdots$ & $\cdots$ & $\cdots$ & $\cdots$ & \\
\hline OXW & 12 & 7.1 & $06-11-91$ & H & $\cdots$ & $\cdots$ & $\cdots$ & $\cdots$ & $\mathbf{R}$ \\
\hline oxw & 13 & 3.8 & $06-11-91$ & $\mathrm{H}$ & $\cdots$ & $\cdots$ & $\cdots$ & $-\cdot$ & \\
\hline OXW & 14 & $\cdots$ & $\cdots$ & $\mathrm{H}$ & $\cdots$ & $\cdots$ & $\cdots$ & $\cdots$ & \\
\hline OXW & 15 & $\cdots$ & $\cdots$ & $\mathrm{H}$ & $\cdots$ & $\cdots$ & $\cdots$ & - & \\
\hline OXW & 16 & $-\cdot$ & $\cdots$ & $\mathrm{H}$ & $\cdots$ & - & $\cdots$ & $\cdots$ & Contains iron \\
\hline OXW & 17 & $\cdots$ & $-\cdot$ & $\mathrm{H}$ & $\cdots$ & $\cdots$ & - & $\cdots$ & \\
\hline
\end{tabular}


Table 1-1. Description of selected wells, borings, and springs in the Saco and Ossipee River Basins, east-central

\begin{tabular}{|c|c|c|c|c|c|c|c|c|c|c|c|}
\hline $\begin{array}{l}\text { Local } \\
\text { site } \\
\text { number }\end{array}$ & $\begin{array}{l}\text { Lat- } \\
\text { itude }\end{array}$ & $\begin{array}{l}\text { Long- } \\
\text { itude }\end{array}$ & Owner or user & $\begin{array}{l}\text { Year } \\
\text { com- } \\
\text { pleted }\end{array}$ & $\begin{array}{l}\text { Alti- } \\
\text { tude } \\
\text { above } \\
\text { sea } \\
\text { level } \\
\text { (feet) }\end{array}$ & $\begin{array}{c}\text { Dia- } \\
\text { meter } \\
\text { of } \\
\text { well } \\
\text { casing } \\
\text { (inches) }\end{array}$ & $\begin{array}{l}\text { Depth } \\
\text { to } \\
\text { bottom } \\
\text { of } \\
\text { casing } \\
\text { (feet) }\end{array}$ & $\begin{array}{l}\text { Casing } \\
\text { material }\end{array}$ & $\begin{array}{l}\text { Type } \\
\text { of } \\
\text { finish }\end{array}$ & $\begin{array}{l}\text { Depth } \\
\text { to } \\
\text { bottom } \\
\text { of open } \\
\text { section } \\
\text { (feet) }\end{array}$ & $\begin{array}{c}\text { Type } \\
\text { of } \\
\text { site }\end{array}$ \\
\hline
\end{tabular}

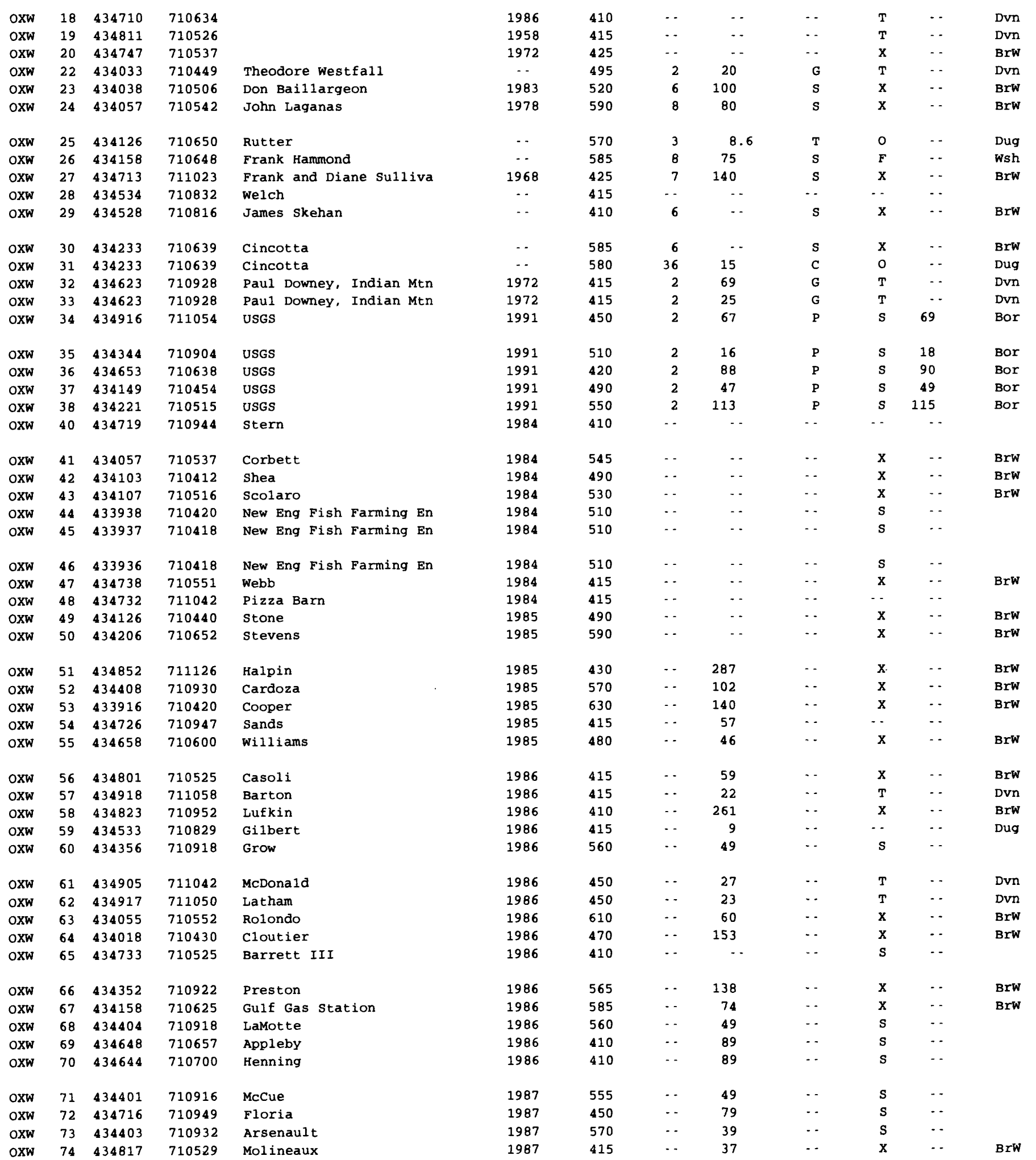




\begin{tabular}{|c|c|c|c|c|c|c|c|c|c|}
\hline \multicolumn{2}{|c|}{$\begin{array}{l}\text { Local } \\
\text { site } \\
\text { number }\end{array}$} & \multirow[t]{2}{*}{$\begin{array}{l}\text { Water } \\
\text { level } \\
\text { depth } \\
\text { (feet) }\end{array}$} & \multirow[t]{2}{*}{$\begin{array}{l}\text { Date } \\
\mathrm{mm} / \mathrm{dd} / \mathrm{yy}\end{array}$} & \multirow[t]{2}{*}{ Use } & \multirow[t]{2}{*}{$\begin{array}{l}\text { Maximum } \\
\text { well } \\
\text { yield } \\
\text { (gallons } \\
\text { per } \\
\text { minute) }\end{array}$} & \multirow[t]{2}{*}{$\begin{array}{l}\text { Draw- } \\
\text { down } \\
\text { (feet) }\end{array}$} & \multirow[t]{2}{*}{$\begin{array}{c}\text { Specific } \\
\text { capacity } \\
\text { (gallons per } \\
\text { minute } \\
\text { per } \\
\text { foot) }\end{array}$} & \multirow{2}{*}{$\begin{array}{l}\begin{array}{l}\text { Pumping } \\
\text { period } \\
\text { (hours) }\end{array} \\
\text { Continued }\end{array}$} & \multirow[t]{2}{*}{ Remarks } \\
\hline & & & & & & & & & \\
\hline oxw & 18 & 12 & $\cdots-86$ & $\mathrm{H}$ & $-\cdot$ & $\cdots$ & - & $\cdots$ & \\
\hline oxw & 19 & 15 & $-\ldots 58$ & $\mathrm{H}$ & $\cdots$ & $-\cdot$ & $-\cdot$ & - & \\
\hline oxw & 20 & -. & -. & $\mathrm{H}$ & $\cdots$ & $\cdots$ & - & -. & Treat water \\
\hline oxw & 22 & 12 & $\cdots-88$ & H & $-\cdot$ & $\cdots$ & -- & - & Some iron \\
\hline oxw & 23 & - & $\cdots$ & $T$ & - & $\cdots$ & -. & $\cdots$ & \\
\hline oxw & 24 & $\cdots$ & $\cdots$ & H & 75 & $-\cdot$ & $-\cdot$ & $\cdots$ & Contains sediments, odor \\
\hline oxw & 25 & 4.6 & $06-14-91$ & $\mathrm{H}$ & $\cdots$ & - & - & $\cdots$ & R Salt contamination from state shed across \\
\hline $\mathrm{oxw}$ & 26 & 20 & -..91 & $\mathrm{H}$ & -. & -. & -. & - & Lots of salt \\
\hline $\mathrm{oxw}$ & 27 & - - & - & $\mathbf{T}$ & 15 & $-\cdot$ & - & -- & \\
\hline oxw & 28 & - & - & $\mathrm{H}$ & $\cdots$ & $-\cdot$ & $-\cdot$ & $-\cdot$ & Traces of iron \\
\hline oxw & 29 & $\cdot \cdot$ & $-\cdot$ & H & 2 & $\cdots$ & $\cdot-$ & $\cdots$ & \\
\hline oxw & 30 & $\cdots$ & $\cdots$ & $\mathrm{H}$ & -. & - - & -. & - & \\
\hline oxw & 31 & 7 & $06-18-91$ & $\mathrm{U}$ & $\cdot \cdot$ & $-\cdot$ & $\cdot-$ & $-\cdot$ & \\
\hline oxw & 32 & $-\cdot$ & -- & $\mathrm{TH}$ & 33 & $\cdots$ & $-\cdot$ & - & Water supply for a subdivision \\
\hline oxw & 33 & -- & - & T H & 36 & $\cdots$ & $\cdots$ & -- & Water supply for a subdivision \\
\hline oxw & 34 & 19 & $10-15-91$ & o & 2.5 & $\cdots$ & $\cdots$ & $\cdots$ & USGS \\
\hline oxw & 35 & $-\cdot$ & - & 0 & $-\cdot$ & $\cdots$ & - & - & USGS \\
\hline oxw & 36 & 7 & $10-10-91$ & 0 & -. & - - & - - & - & USGS \\
\hline oxw & 37 & 27 & $08-02-91$ & 0 & - & $\cdots$ & -. & $\cdots$ & USGS \\
\hline oxw & 38 & 37 & $09-23-91$ & 0 & $\cdots$ & $\cdots$ & $-\cdot$ & -. & USGS \\
\hline oxw & 40 & 11 & $07-09-84$ & H & 50 & $\cdot-$ & $\cdots$ & 2 & \\
\hline oxw & 41 & 10 & $09-14-84$ & H & 2 & - & - & 1.5 & \\
\hline $\mathrm{oxw}$ & 42 & -. & $\cdots$ & H & 100 & - & -- & - & \\
\hline oxw & 43 & 35 & $11-30-84$ & $\mathrm{H}$ & 0.8 & $\cdots$ & $-\cdot$ & 1 & \\
\hline oxw & 44 & 2.7 & $11-13-84$ & $\mathbf{z}$ & 281 & $\cdots$ & $-\cdot$ & 6 & \\
\hline oxw & 45 & 2.8 & $11-06-84$ & $\mathrm{z}$ & 330 & $\cdots$ & $\cdots$ & 6 & \\
\hline oxw & 46 & 1.6 & $11 \cdot 15-84$ & $\mathbf{z}$ & 290 & $\cdots$ & $\cdots$ & 6 & \\
\hline oxw & 47 & - & - & $\mathrm{H}$ & 40 & $\cdots$ & $\cdots$ & $\cdots$ & \\
\hline oxw & 48 & 12 & $09-29-84$ & C & 10.5 & $\cdots$ & $\cdots$ & 2 & \\
\hline oxw & 49 & 40 & $07-10-85$ & $\mathrm{H}$ & 80 & $\cdots$ & -- & .5 & \\
\hline oxw & 50 & 28 & $09-13-85$ & $\mathrm{H}$ & 7.5 & $\cdots$ & $\cdots$ & .5 & \\
\hline oxw & 51 & 30 & $11-13 \cdot 85$ & -. & 2.5 & $\cdots$ & $\cdots$ & 1 & \\
\hline oxw & 52 & 30 & $11-13-85$ & $\mathrm{H}$ & 2.5 & $\cdots$ & -. & 1 & \\
\hline oxw & 53 & $\cdots$ & - & $\mathrm{H}$ & 0.2 & $\cdots$ & $\cdots$ & 2 & \\
\hline oxw & 54 & 6 & $10-04-85$ & P & 60 & $-\cdot$ & -. & 3 & \\
\hline oxw & 55 & $\cdots$ & - & H & 5.5 & $\cdots$ & $\cdots$ & .5 & \\
\hline oxw & 56 & 10 & $04-23-86$ & $\mathrm{H}$ & 10 & $\cdots$ & $\cdots$ & .5 & \\
\hline oxw & 57 & 16 & $04-19-86$ & $\mathrm{H}$ & 5 & - & $-\cdot$ & .5 & \\
\hline oxw & 58 & -. & . & H & 5.5 & $\cdots$ & $\cdots$ & 1 & \\
\hline oxw & 59 & 5 & $05-20-86$ & H & 30 & $\cdots$ & - & 3 & \\
\hline oxw & 60 & 36 & $04-20-86$ & $\mathrm{H}$ & 12 & $\cdot-$ & $\cdot \cdot$ & 2 & \\
\hline oxw & 61 & 26 & $07-03-86$ & H & 10 & $\cdots$ & $\cdots$ & 2 & \\
\hline $\mathrm{oxw}$ & 62 & 28 & $07-03-86$ & H & 20 & - & - & 2 & \\
\hline oxw & 63 & - & - & H & 40 & $\cdots$ & $\cdots$ & .5 & \\
\hline oxw & 64 & 20 & $07-01.86$ & $\mathrm{H}$ & 20 & $-\cdot$ & - & .5 & \\
\hline oxw & 65 & 70 & $06-26-86$ & H & 20 & $\cdots$ & $\cdots$ & 12 & \\
\hline oxw & 66 & 35 & $08-17-86$ & H & 1.5 & $\cdots$ & $\cdots$ & 1 & \\
\hline oxw & 67 & 20 & $08-15-86$ & $\mathbf{N}$ & 60 & $-\cdot$ & $\cdots$ & 1 & \\
\hline oxw & 68 & 35 & $06-28-86$ & $\mathrm{H}$ & 15 & $-\cdot$ & $\cdots$ & 1 & \\
\hline oxw & 69 & 2 & $06 \cdot 01-86$ & $\mathrm{H}$ & 35 & $\cdots$ & $\cdots$ & $-\cdot$ & \\
\hline oxw & 70 & 18 & $06-01-86$ & $\mathrm{H}$ & 30 & $\cdots$ & $\cdots$ & 1 & \\
\hline oxw & 71 & $\cdots$ & $\cdots$ & $\mathrm{H}$ & 10 & $\cdots$ & $-\cdot$ & 2 & \\
\hline oxw & 72 & 56 & $04-14-87$ & $\mathrm{H}$ & 10 & $\cdots$ & $\cdots$ & 1.5 & \\
\hline oxw & 73 & 34 & $04-12-87$ & $\mathrm{H}$ & 10 & $\cdots$ & $\cdots$ & 2 & \\
\hline oxw & 74 & 10 & $05-05-87$ & $\mathrm{H}$ & 6 & $\cdots$ & $\cdots$ & .5 & \\
\hline
\end{tabular}


Table 1-1. Description of selected wells, borings, and springs in the Saco and Ossipee River Basins, east-central

\begin{tabular}{|c|c|c|c|c|c|c|c|c|c|c|c|}
\hline $\begin{array}{l}\text { Local } \\
\text { site } \\
\text { number }\end{array}$ & $\begin{array}{l}\text { Lat- } \\
\text { itude }\end{array}$ & $\begin{array}{l}\text { Long- } \\
\text { itude }\end{array}$ & Owner or user & $\begin{array}{l}\text { Year } \\
\text { com- } \\
\text { pleted }\end{array}$ & $\begin{array}{l}\text { Alti- } \\
\text { tude } \\
\text { above } \\
\text { sea } \\
\text { level } \\
\text { (feet) }\end{array}$ & $\begin{array}{l}\text { Dia- } \\
\text { meter } \\
\text { of } \\
\text { well } \\
\text { casing } \\
\text { (inches) }\end{array}$ & $\begin{array}{l}\text { Depth } \\
\text { to } \\
\text { bottom } \\
\text { of } \\
\text { casing } \\
\text { (feet) }\end{array}$ & $\begin{array}{l}\text { Casing } \\
\text { material }\end{array}$ & $\begin{array}{l}\text { Type } \\
\text { of } \\
\text { finish }\end{array}$ & $\begin{array}{l}\text { Depth } \\
\text { to } \\
\text { bottom } \\
\text { of open } \\
\text { section } \\
\text { (feet) }\end{array}$ & $\begin{array}{c}\text { Type } \\
\text { of } \\
\text { site }\end{array}$ \\
\hline
\end{tabular}

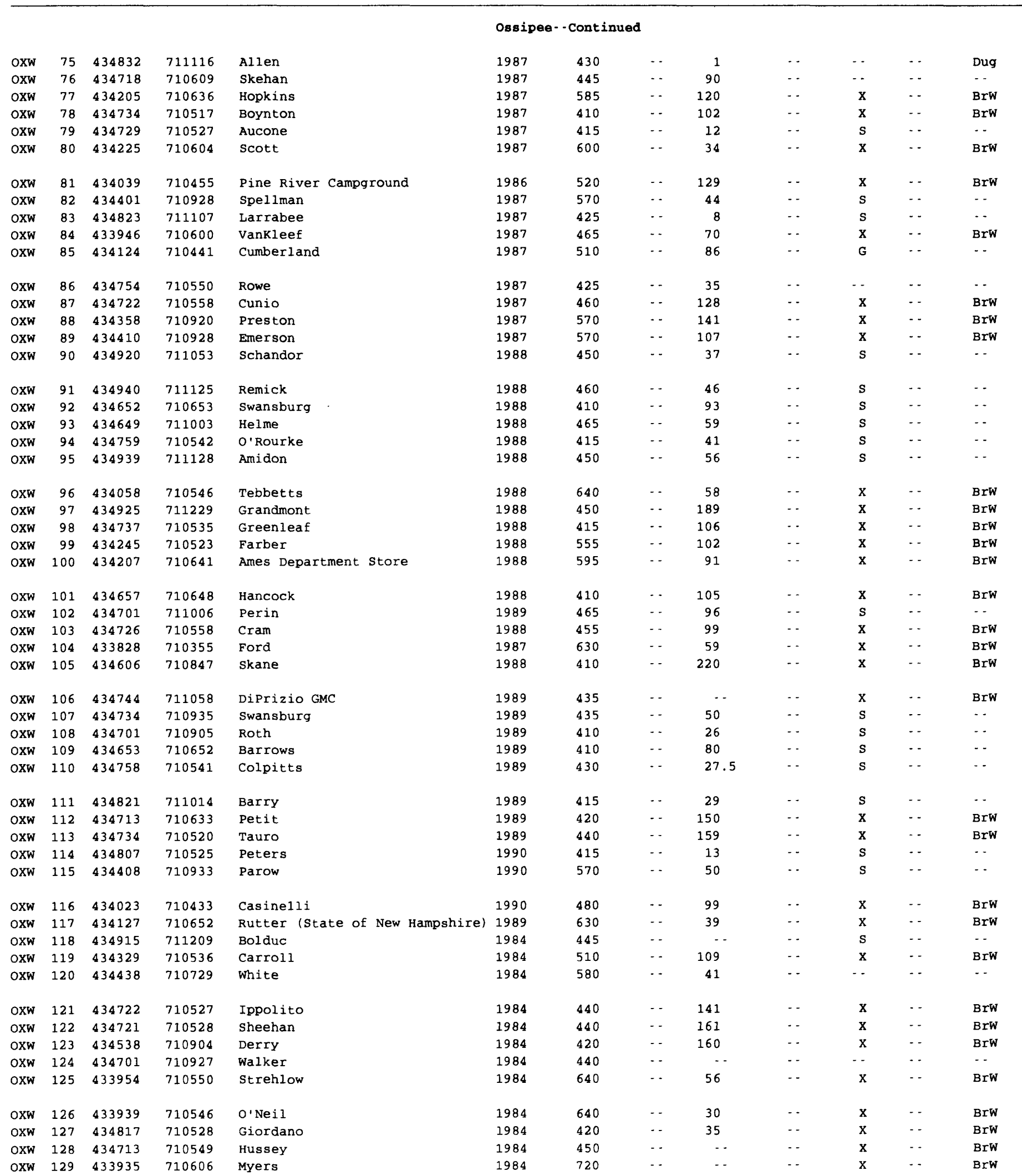




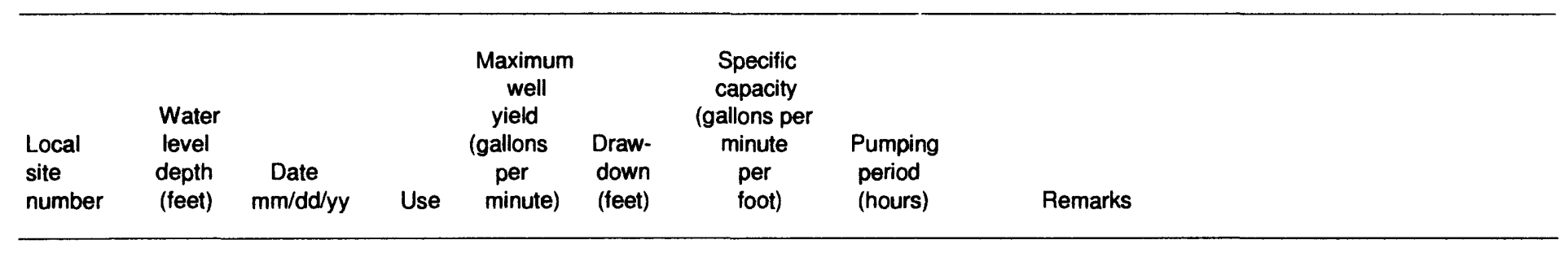

\begin{tabular}{|c|c|c|c|c|c|c|c|c|}
\hline \multirow[b]{2}{*}{ oxw } & \multirow[b]{2}{*}{75} & \multirow[b]{2}{*}{8} & \multirow[b]{2}{*}{$03-12-87$} & \multirow[b]{2}{*}{ H } & \multirow[b]{2}{*}{20} & \multirow[b]{2}{*}{ - } & \multicolumn{2}{|c|}{ Ossipee--Continu } \\
\hline & & & & & & & -. & - \\
\hline oxw & 76 & 50 & $06-12-87$ & $\mathrm{H}$ & 25 & .. & .. & 48 \\
\hline OXW & 77 & -. & - & $\mathrm{H}$ & 100 & - & - - & 1 \\
\hline oxw & 78 & 10 & 07.01 .87 & $\mathrm{H}$ & 15 & - - & -. & 1 \\
\hline oxw & 79 & 6 & $07-06-87$ & $\mathrm{H}$ & 20 & - - & .. & 2 \\
\hline oxw & 80 & 18 & $07-28-87$ & $\mathrm{H}$ & 2.5 & - & - & .5 \\
\hline oxw & 81 & 50 & $11-01-86$ & $\mathrm{H}$ & 10 & - & .. & 1 \\
\hline OXW & 82 & 36 & $08-16-87$ & H & 10 & .. & .. & 3 \\
\hline oxw & 83 & 6 & $08-15-87$ & $\mathrm{H}$ & 25.5 & .. & -. & 2 \\
\hline OXW & 84 & 20 & $08 \cdot 28 \cdot 87$ & H & 40 & -. & -. & 1 \\
\hline oxw & 85 & -. & - & H & 25 & $\cdots$ & - & 12 \\
\hline oxw & 86 & 24.5 & $09-22-87$ & H & 15 & - & - & 1 \\
\hline OXW & 87 & - & - & H & 3 & $\cdots$ & - - & 1 \\
\hline oxw & 88 & - & . & H & 6 & - & -. & 1 \\
\hline OXW & 89 & 35 & $11-05-87$ & H & 50 & -. & - & .5 \\
\hline oxw & 90 & 25 & $04-16-88$ & H & 18 & - - & - & 2 \\
\hline oxw & 91 & .. & - & $\mathrm{H}$ & 15 & -. & -. & 1 \\
\hline oxw & 92 & 5 & $06-23-88$ & H & 27.5 & .. & .. & 1 \\
\hline oxw & 93 & 49 & $08-30-88$ & $\mathrm{H}$ & 10 & -. & .. & 1 \\
\hline OXW & 94 & .. & . & H & 20 & -. & -. & 1 \\
\hline OXW & 95 & 33.5 & $09-30-88$ & H & 15 & $\cdots$ & -. & 2 \\
\hline$O X W$ & 96 & 10 & $07-06-88$ & H & 80 & $\cdots$ & - - & .5 \\
\hline oxw & 97 & 20 & 07.07 .88 & H & 8.5 & -. & - & .5 \\
\hline oxw & 98 & 35 & $06-11-88$ & $\mathrm{H}$ & 5 & - - & -. & .5 \\
\hline oxw & 99 & 30 & $05-20-88$ & $\mathrm{H}$ & 10 & -. & .. & .5 \\
\hline oxw & 100 & 30 & $05-23-88$ & H & 15 & - & - & 1 \\
\hline oxw & 101 & 3 & $08-10-88$ & H & 0.8 & - - & .. & 1 \\
\hline OXW & 102 & - & .. & $\mathrm{H}$ & 25 & .. & -. & 2.5 \\
\hline OXW & 103 & .. & .. & $\mathrm{H}$ & 100 & $\cdots$ & .. & .5 \\
\hline oxw & 104 & - & - & H & 5 & .. & -. & -. \\
\hline oxw & 105 & 5 & $11-11-88$ & H & 6 & - & $\cdots$ & .5 \\
\hline oxw & 106 & 10 & $03-11-89$ & H & 12 & - & .. & 1 \\
\hline oxw & 107 & 2 & $04-30-89$ & H & 60 & - & - & 2 \\
\hline oxw & 108 & 2 & $06-30-89$ & H & 35 & . & -. & 1 \\
\hline oxw & 109 & 5.3 & $08-05-89$ & H & 30 & .. & .. & 1 \\
\hline oxw & 110 & 12 & $09-25-89$ & $\mathrm{H}$ & 30 & $\cdots$ & -. & 2 \\
\hline oxw & 111 & 4 & $11-19-89$ & $\mathrm{H}$ & 40 & .. & -. & 2 \\
\hline oxw & 112 & 30 & $11-30-89$ & $\mathrm{H}$ & 12 & .. & .. & .5 \\
\hline oxw & 113 & - & . & H & 4 & - & -. & 1 \\
\hline oXw & 114 & 8 & $06-16-90$ & $\mathrm{H}$ & 35 & - & - - & 2 \\
\hline OXW & 115 & 43 & $08-20-90$ & H & 4.5 & - & $\cdots$ & 1 \\
\hline oxw & 116 & - - & .. & H & 30 & .. & .. & .3 \\
\hline oxw & 117 & 144 & $11-28-89$ & H & 0.5 & - & - & 1 \\
\hline OXW & 118 & 9 & $08.06-84$ & C & 30 & - & -. & 3 \\
\hline oxw & 119 & - & - . & $\mathrm{H}$ & 1 & - & -. & .5 \\
\hline oxw & 120 & 38 & $05-13-84$ & $\mathrm{H}$ & 5 & - - & $\cdots$ & 2 \\
\hline oxw & 121 & 30 & $05-31-84$ & H & 100 & - & -. & .5 \\
\hline oxw & 122 & 30 & $05-31-84$ & H & 100 & $\cdots$ & -. & .5 \\
\hline oxw & 123 & 1 & $07-18-84$ & H & 50 & .. & -. & .5 \\
\hline oxw & 124 & 13 & $07-12-84$ & H & 40 & - & -. & 2 \\
\hline oxw & 125 & 15 & $09-29-84$ & H & 6 & - & $\cdots$ & .3 \\
\hline oxw & 126 & - & -. & $\mathrm{H}$ & 4 & $\cdots$ & - & .3 \\
\hline oxw & 127 & 28 & $08-15-84$ & $\mathrm{H}$ & 3.5 & - - & .. & .5 \\
\hline oxw & 128 & 40 & $10-12-84$ & $\cdots$ & 1.5 & - & - & .5 \\
\hline oxw & $129^{\circ}$ & - - & - & $\mathrm{H}$ & 4 & - & - & 1 \\
\hline
\end{tabular}


Table 1-1. Description of selected wells, borings, and springs in the Saco and Ossipee River Basins, east-central

\begin{tabular}{|c|c|c|c|c|c|c|c|c|c|c|c|}
\hline $\begin{array}{l}\text { Local } \\
\text { site } \\
\text { number }\end{array}$ & $\begin{array}{l}\text { Lat- } \\
\text { itude }\end{array}$ & $\begin{array}{l}\text { Long- } \\
\text { itude }\end{array}$ & Owner or user & $\begin{array}{l}\text { Year } \\
\text { com- } \\
\text { pleted }\end{array}$ & $\begin{array}{c}\text { Alti- } \\
\text { tude } \\
\text { above } \\
\text { sea } \\
\text { level } \\
\text { (feet) }\end{array}$ & $\begin{array}{l}\text { Dia- } \\
\text { meter } \\
\text { of } \\
\text { well } \\
\text { casing } \\
\text { (inches) }\end{array}$ & $\begin{array}{l}\text { Depth } \\
\text { to } \\
\text { bottom } \\
\text { of } \\
\text { casing } \\
\text { (feet) }\end{array}$ & $\begin{array}{l}\text { Casing } \\
\text { material }\end{array}$ & $\begin{array}{l}\text { Type } \\
\text { of } \\
\text { finish }\end{array}$ & $\begin{array}{l}\text { Depth } \\
\text { to } \\
\text { bottom } \\
\text { of open } \\
\text { section } \\
\text { (feet) }\end{array}$ & $\begin{array}{c}\text { Type } \\
\text { of } \\
\text { site }\end{array}$ \\
\hline
\end{tabular}

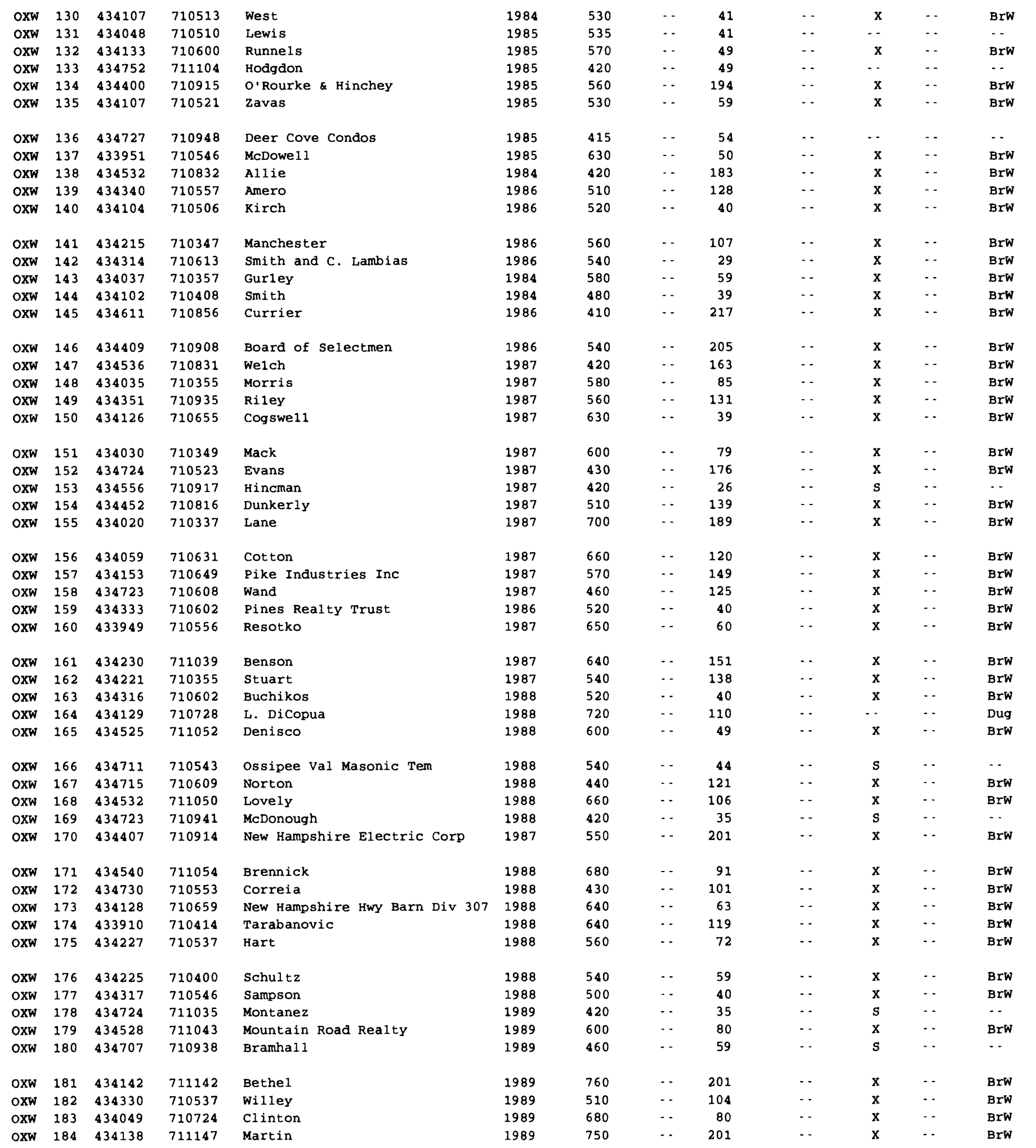




\begin{tabular}{|c|c|c|c|c|c|c|c|c|}
\hline $\begin{array}{l}\text { Local } \\
\text { site } \\
\text { number }\end{array}$ & $\begin{array}{l}\text { Water } \\
\text { level } \\
\text { depth } \\
\text { (feet) }\end{array}$ & $\begin{array}{l}\text { Date } \\
\mathrm{mm} / \mathrm{dd} / \mathrm{yy}\end{array}$ & Use & $\begin{array}{l}\text { Maximum } \\
\text { well } \\
\text { yield } \\
\text { (gallons } \\
\text { per } \\
\text { minute) }\end{array}$ & $\begin{array}{l}\text { Draw- } \\
\text { down } \\
\text { (feet) }\end{array}$ & $\begin{array}{c}\text { Specific } \\
\text { capacity } \\
\text { (gallons per } \\
\text { minute } \\
\text { per } \\
\text { foot) }\end{array}$ & $\begin{array}{l}\text { Pumping } \\
\text { period } \\
\text { (hours) }\end{array}$ & Remarks \\
\hline
\end{tabular}

\begin{tabular}{|c|c|c|c|c|c|c|c|c|}
\hline OXW & 130 & - & - & $\mathrm{H}$ & 2 & $-\cdot$ & $\ldots$ & .5 \\
\hline OXW & 131 & 36 & $06 \cdot 29 \cdot 85$ & $\mathrm{H}$ & 10 & $\cdots$ & - & 3 \\
\hline OXW & 132 & 25 & $04-27-85$ & $\mathrm{H}$ & 60 & $\cdots$ & $\cdots$ & 2 \\
\hline OXW & 133 & 150 & $03-13-85$ & $\mathrm{H}$ & 1.5 & -- & -- & 1 \\
\hline OXW & 134 & 22 & $06-20-85$ & $\mathrm{H}$ & 3 & $\cdots$ & $\cdots$ & .5 \\
\hline oxw & 135 & 30 & $10-26-85$ & $\mathrm{H}$ & 11 & $-\cdot$ & $\cdots$ & .5 \\
\hline oxw & 136 & 7.5 & $10-05-85$ & $\mathbf{P}$ & 60 & -- & - & 2 \\
\hline oxw & 137 & 15 & $08-28-85$ & $\mathrm{H}$ & 3 & - & -- & 1 \\
\hline oxw & 138 & - & - - & $\mathrm{H}$ & 2.5 & -- & $\cdots$ & .5 \\
\hline oxw & 139 & - - & - & $\mathrm{H}$ & 2 & - & $\cdots$ & .5 \\
\hline OXW & 140 & 40 & $06-02-86$ & $\mathrm{H}$ & 25 & -- & $\cdots$ & .5 \\
\hline oxw & 141 & 25 & $06-06-86$ & $\mathrm{H}$ & 3.5 & $\cdots$ & $\cdots$ & .5 \\
\hline oXw & 142 & 11 & $08-25-86$ & $\mathrm{H}$ & 30 & $\cdots$ & - & 1 \\
\hline oxw & 143 & 55 & $04-27-84$ & $\mathrm{H}$ & 15 & - & - & 2 \\
\hline $\mathrm{OXW}$ & 144 & $\cdots$ & -- & $\mathrm{H}$ & 5 & - & - & 1 \\
\hline $\mathrm{OXW}$ & 145 & 5 & $12-04-86$ & $\mathrm{H}$ & 1.8 & - & $\cdots$ & 1 \\
\hline OXW & 146 & 20 & $12-04-86$ & $\mathrm{~N}$ & 25 & - & -. & 1 \\
\hline oxw & 147 & 20 & $05-06-87$ & $\mathrm{H}$ & 4 & -- & -- & .5 \\
\hline oxw & 148 & 25 & $05-14-87$ & $\mathrm{H}$ & 30 & $\cdots$ & - & .5 \\
\hline OXW & 149 & 20 & $05-28-87$ & $\mathrm{H}$ & 25 & $\cdots$ & $\cdots$ & .5 \\
\hline oxw & 150 & 20 & $05-18-87$ & $\mathrm{H}$ & 0.8 & - & $\ldots$ & 1 \\
\hline oxw & 151 & - - & - & $\mathrm{H}$ & 4 &.- & - & 1 \\
\hline oxw & 152 & 50 & $04-16-87$ & $\mathrm{H}$ & 15 & - & -. & .5 \\
\hline oxw & 153 & 8 & $07-30-87$ & $\mathrm{H}$ & 30 & -- & -- & 2 \\
\hline oxw & 154 & -- & -- & $\mathrm{H}$ & 35 & - & - & 2 \\
\hline oxw & 155 & $\cdots$ & - & $\mathrm{H}$ & 20 & $\cdots$ & $\cdots$ & 1.5 \\
\hline oxw & 156 & 50 & $08-27-87$ & $\mathrm{H}$ & 2 & -- & - & 1 \\
\hline oxw & 157 & 60 & $03-13-87$ & $\mathrm{C}$ & 1 & $\cdots$ & -- & 1 \\
\hline oxw & 158 & - - & - & $\mathrm{H}$ & 35 & - & - & $\cdots$ \\
\hline oxw & 159 & -- & - & $\mathrm{H}$ & 30 & $\cdots$ & - & .5 \\
\hline oxw & 160 & 20 & $12-12-87$ & $\mathrm{H}$ & 1.5 & $\cdots$ & $\cdots$ & .5 \\
\hline oxw & 161 & 50 & $09-22-87$ & $\mathrm{H}$ & 2.5 & $\cdots$ & - & 1 \\
\hline OXW & 162 & - & - & $\mathrm{H}$ & 4 & -- & -- & 1 \\
\hline OXW & 163 & 45 & $04-15-88$ & $\mathrm{H}$ & 1 & $\cdots$ & $\cdots$ & 1 \\
\hline oxw & 164 & 60 & $02 \cdot 25 \cdot 88$ & $\mathrm{H}$ & 15 & - & - & 72 \\
\hline OXW & 165 & 20 & $05-20-88$ & $\mathrm{H}$ & 4 & $\cdots$ & $\cdots$ & 1 \\
\hline oxw & 166 & 36.5 & $07-09-88$ & $\mathrm{H}$ & 11 & $\cdots$ & - & 2 \\
\hline oxw & 167 & 30 & $05-04-88$ & $\mathrm{H}$ & 50 & $\cdots$ & - & .5 \\
\hline OXW & 168 & 40 & $05-04 \cdot 88$ & $\mathrm{H}$ & 1 & -- & - & 1 \\
\hline oxw & 169 & 7.5 & $09-26-88$ & $\mathrm{H}$ & 35 & $\cdots$ & -- & 1 \\
\hline oxw & 170 & $\cdots$ & - & $\mathrm{N}$ & 8.5 & - & $\cdots$ & .5 \\
\hline oXw & 171 & $\cdots$ & $\cdots$ & $\mathrm{H}$ & 30 & $\cdots$ & - & 1 \\
\hline $\mathrm{OXW}$ & 172 & -- & $\cdots$ & $\mathrm{H}$ & 10 & $\cdots$ & - & .8 \\
\hline OXW & 173 & $-\cdot$ & - & -- & 1.8 & $\cdots$ & - & 1 \\
\hline OXW & 174 & $\cdots$ & - & $\mathrm{H}$ & - & $\cdots$ & $\ldots$ & - \\
\hline oxw & 175 & $\cdots$ & $\cdots$ & $\mathrm{H}$ & $\cdots$ & $\cdots$ & - & 1 \\
\hline oxw & 176 & - & - & $\mathrm{H}$ & 6 & -- & $\cdots$ & 1 \\
\hline oxw & 177 & 25 & $11-11-88$ & $\mathrm{H}$ & 1.8 & - & - & 1 \\
\hline$O X W$ & 178 & 7 & $06-03-89$ & $\mathrm{H}$ & 20 & - & - & 1 \\
\hline oxw & 179 & 20 & $05-15 \cdot 89$ & $\mathrm{H}$ & 1 & $\cdots$ & - & .5 \\
\hline oxw & 180 & 42 & $04-30-89$ & $\mathrm{H}$ & 13.5 & $\cdots$ & -- & 1 \\
\hline OXW & 181 & 45 & $07-08-89$ & $\mathrm{H}$ & 15 & $\cdots$ & - & 1 \\
\hline oxw & 182 & 25 & $09-06-89$ & $\mathrm{H}$ & 30 & - & - & .5 \\
\hline oxw & 183 & 25 & $12 \cdot 20 \cdot 89$ & $\mathrm{H}$ & 4 & - & $\cdots$ & .5 \\
\hline OXW & 184 & 10 & $11-15-89$ & H & 5 & - & $\cdots$ & .5 \\
\hline
\end{tabular}


Table 1-1. Description of selected wells, borings, and springs in the Saco and Ossipee River Basins, east-central

\begin{tabular}{|c|c|c|c|c|c|c|c|c|c|c|c|}
\hline $\begin{array}{l}\text { Local } \\
\text { site } \\
\text { number }\end{array}$ & $\begin{array}{l}\text { Lat- } \\
\text { itude }\end{array}$ & $\begin{array}{l}\text { Long- } \\
\text { itude }\end{array}$ & Owner or user & $\begin{array}{l}\text { Year } \\
\text { com- } \\
\text { pleted }\end{array}$ & 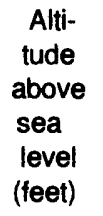 & $\begin{array}{c}\text { Dia- } \\
\text { meter } \\
\text { of } \\
\text { well } \\
\text { casing } \\
\text { (inches) }\end{array}$ & $\begin{array}{l}\text { Depth } \\
\text { to } \\
\text { bottom } \\
\text { of } \\
\text { casing } \\
\text { (feet) }\end{array}$ & $\begin{array}{l}\text { Casing } \\
\text { material }\end{array}$ & $\begin{array}{l}\text { Type } \\
\text { of } \\
\text { finish }\end{array}$ & $\begin{array}{l}\text { Depth } \\
\text { to } \\
\text { bottom } \\
\text { of open } \\
\text { section } \\
\text { (feet) }\end{array}$ & $\begin{array}{c}\text { Type } \\
\text { of } \\
\text { site }\end{array}$ \\
\hline
\end{tabular}

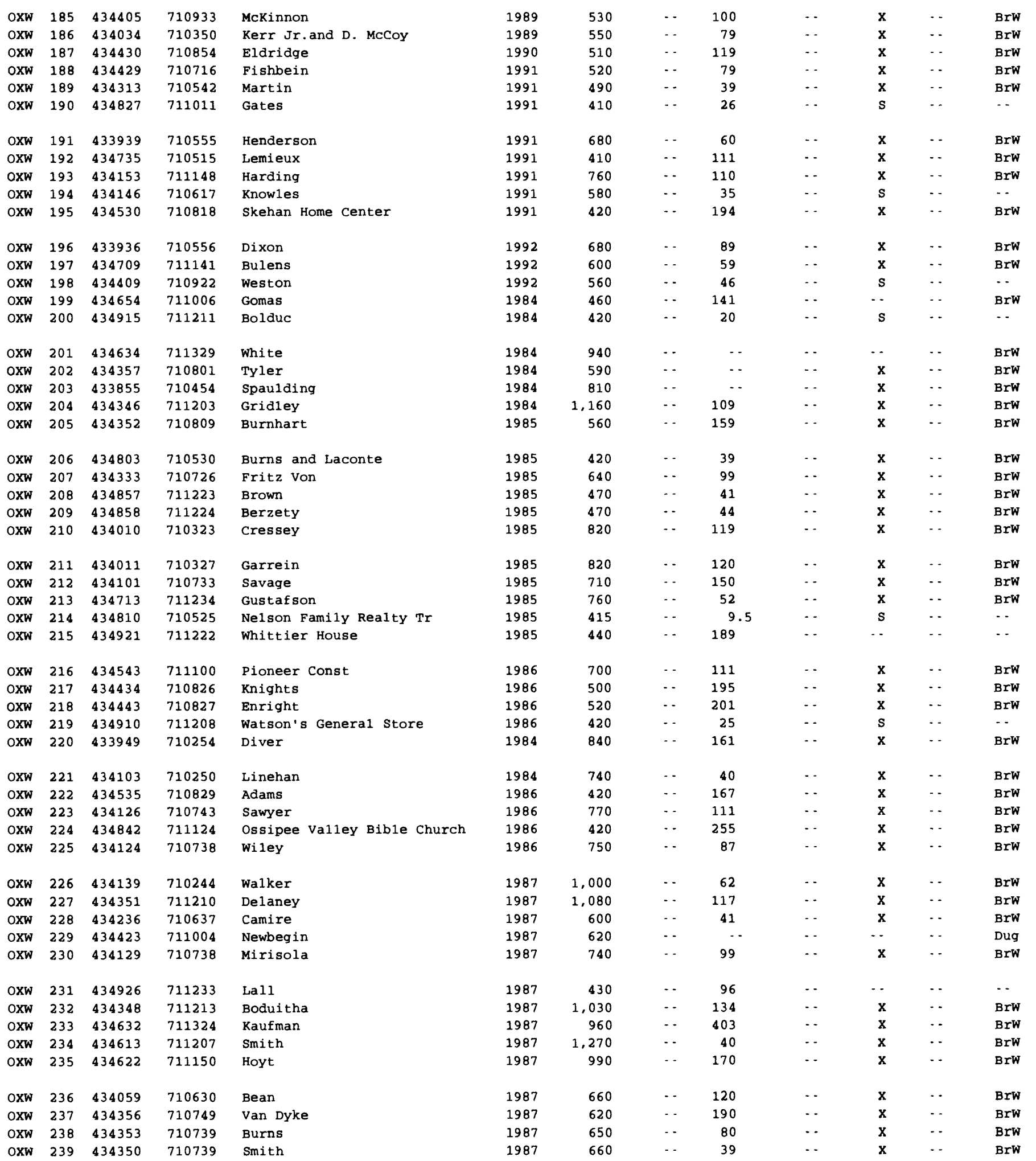




\section{New Hampshire--Continued}

\begin{tabular}{|c|c|c|c|c|c|c|c|c|}
\hline $\begin{array}{l}\text { Local } \\
\text { site } \\
\text { number }\end{array}$ & $\begin{array}{l}\text { Water } \\
\text { level } \\
\text { depth } \\
\text { (feet) }\end{array}$ & $\begin{array}{l}\text { Date } \\
\mathrm{mm} / \mathrm{dd} / \mathrm{yy}\end{array}$ & Use & $\begin{array}{c}\text { Maximum } \\
\text { well } \\
\text { yield } \\
\text { (gallons } \\
\text { per } \\
\text { minute) }\end{array}$ & $\begin{array}{l}\text { Draw- } \\
\text { down } \\
\text { (feet) }\end{array}$ & $\begin{array}{c}\text { Specific } \\
\text { capacity } \\
\text { (gallons per } \\
\text { minute } \\
\text { per } \\
\text { foot) }\end{array}$ & $\begin{array}{l}\text { Pumping } \\
\text { period } \\
\text { (hours) }\end{array}$ & Remarks \\
\hline
\end{tabular}

\begin{tabular}{|c|c|c|c|c|c|c|c|c|}
\hline oxw & 185 & 48 & $11-03-89$ & $\mathbf{H}$ & 0.7 & - & $\cdots$ & 1 \\
\hline oxw & 186 & - & $\cdots$ & $\mathbf{H}$ & 7 & - & -- & .5 \\
\hline oxw & 187 & $\cdots$ & $\cdots$ & H & 1.5 & - & -- & .8 \\
\hline OXW & 188 & 25 & $01-04-91$ & H & 30 & - & - - & 1.5 \\
\hline oxw & 189 & 15 & $02-05-91$ & H & 40 & $\cdots$ & $\cdots$ & .5 \\
\hline OXW & 190 & 4 & $05-18-91$ & H & 30 & $\cdots$ & - & 2 \\
\hline OXW & 191 & 25 & $08-15-91$ & $\mathrm{H}$ & 4 & $\cdots$ & $\cdots$ & 1 \\
\hline OXW & 192 & 3.5 & $09-10-91$ & $\mathrm{H}$ & 50 & $\cdots$ & $\cdots$ & .5 \\
\hline OXW & 193 & - - & $\cdots$ & $\mathbf{H}$ & 10 & $\cdots$ & - & 1 \\
\hline oxw & 194 & 26 & $08-17-91$ & H & 15 & -- & - & 1 \\
\hline oxw & 195 & 20 & $04-04-91$ & C & 2.5 & $\cdots$ & $\cdots$ & .5 \\
\hline oxw & 196 & 40 & $07-03-92$ & $\mathrm{H}$ & 1.5 & $\cdots$ & - & .5 \\
\hline OXW & 197 & 45 & $06-17-92$ & $\mathrm{H}$ & 2.3 & $\cdots$ & $\cdots$ & 1 \\
\hline oxw & 198 & 36 & $07-11-92$ & $\mathrm{H}$ & 10 & $\cdots$ & $\cdots$ & 3 \\
\hline oxw & 199 & 20 & $08-23-84$ & H & 15 & $\cdots$ & $\cdots$ & .5 \\
\hline oxw & 200 & 9 & $06 \cdot 08 \cdot 84$ & C & 30 & $-\cdot$ & $\cdots$ & 3 \\
\hline OXW & 201 & 50 & $07-25-84$ & H & 6 & $\cdots$ & $\cdots$ & .5 \\
\hline OXW & 202 & 30 & $10-11-84$ & $\mathrm{H}$ & 3 & $\cdots$ & $\cdots$ & .5 \\
\hline oxw & 203 & 28 & $10-06-84$ & H & 4 & $\cdots$ & $\cdots$ & .5 \\
\hline oxw & 204 & 15 & $07-02 \cdot 84$ & $\mathrm{H}$ & 30 & $\cdots$ & - & 1 \\
\hline oxw & 205 & 45 & $04-24-85$ & $\mathrm{H}$ & 2.5 & $\cdots$ & $\cdots$ & 1 \\
\hline OXW & 206 & 30 & $05-31-85$ & $\mathrm{H}$ & 6 & $\cdots$ & $\cdots$ & .5 \\
\hline OXW & 207 & 150 & $07-09-85$ & H & 1.5 & $\cdots$ & $\cdots$ & 1 \\
\hline OXW & 208 & 50 & $07-16 \cdot 85$ & H & 8.5 & - & $\cdots$ & .5 \\
\hline OXW & 209 & 21 & $08-22-85$ & $\mathrm{H}$ & 6 & $\cdots$ & $\cdots$ & .5 \\
\hline OXW & 210 & 100 & $08-22-85$ & H & 15 & -- & $\cdots$ & .5 \\
\hline OXW & 211 & 100 & $08-23-85$ & H & 15 & - & $\cdots$ & .5 \\
\hline OXW & 212 & 20 & $10-24-85$ & H & 7.5 & $\cdots$ & $\cdots$ & 1 \\
\hline OXW & 213 & - & $\cdots$ & $\mathbf{H}$ & 25 & $\cdots$ & $\cdots$ & .5 \\
\hline OXW & 214 & 5.5 & $10-19-85$ & $\mathrm{H}$ & 40 & $\cdots$ & $\cdots$ & 2 \\
\hline OXW & 215 & 15 & $10-25-85$ & C & 150 & $\cdots$ & - & 2 \\
\hline OXW & 216 & 55 & $06-17-86$ & $\mathrm{H}$ & 6 & $\cdots$ & $\cdots$ & .5 \\
\hline oxw & 217 & 55 & $06-23-86$ & H & 2 & $\cdots$ & $\cdots$ & .5 \\
\hline OXW & 218 & 55 & $06-12-86$ & $\mathrm{H}$ & 50 & $\cdots$ & $\cdots$ & .5 \\
\hline OXW & 219 & 15.5 & $10-03-86$ & C & 30 & $\cdots$ & $\cdots$ & 2 \\
\hline OXW & 220 & 35 & $08-19-84$ & $\mathrm{H}$ & 5 & $\cdots$ & $\cdots$ & .5 \\
\hline oxw & 221 & $\cdots$ & $\cdots$ & H & 1.5 & $\cdots$ & $\cdots$ & .5 \\
\hline OXW & 222 & 60 & $09-20-86$ & $\mathrm{H}$ & 15 & $\cdots$ & - & .5 \\
\hline OXW & 223 & 20 & $11-25-86$ & $\mathbf{H}$ & 25 & $\cdots$ & $\cdots$ & .5 \\
\hline OXW & 224 & 25 & $12-31-86$ & H & 35 & $\cdots$ & $\cdots$ & .5 \\
\hline OXW & 225 & 25 & $12-24-86$ & H & 4 & $\cdots$ & $\cdots$ & .5 \\
\hline OXW & 226 & $\cdots$ & $\cdots$ & H & 5 & $\cdots$ & $\cdots$ & 1 \\
\hline OXW & 227 & $\cdots$ & $\cdots$ & $\mathrm{H}$ & 0.8 & -- & $\cdots$ & 1 \\
\hline OXW & 228 & $\cdots$ & $\cdots$ & H & 7 & $\cdots$ & $\cdots$ & - \\
\hline OXW & 229 & 4 & $05 \cdot 28 \cdot 87$ & $\mathrm{H}$ & $\cdots$ & $\cdots$ & $\cdots$ & $\cdots$ \\
\hline oxw & 230 & $\cdots$ & - & H & 100 & $-\cdot$ & $-\cdot$ & 1 \\
\hline OXW & 231 & 20 & $06-10-87$ & $\mathrm{C}$ & 25 & $\cdots$ & $\cdots$ & 48 \\
\hline oxw & 232 & $\cdots$ & $\cdots$ & $\mathrm{H}$ & 10 & $\cdots$ & $\cdots$ & 1 \\
\hline OXW & 233 & 40 & $07-22-87$ & H & 15 & $\cdots$ & $\cdots$ & .5 \\
\hline oxw & 234 & 250 & $08-14-87$ & $\mathrm{H}$ & 50 & - & - & .5 \\
\hline oxw & 235 & 20 & $08-17-87$ & $\mathrm{H}$ & 3.5 & $\cdots$ & $\cdots$ & .5 \\
\hline oxw & 236 & 62 & $08-24-87$ & $\mathrm{H}$ & 1.5 & -- & $\cdots$ & 1 \\
\hline oxw & 237 & 49 & $10-16-87$ & $\mathrm{H}$ & 3 & - & - & 1 \\
\hline OXW & 238 & 45 & $10-15-87$ & H & 6 & -- & -- & 1 \\
\hline OXW & 239 & 29 & $10-13 \cdot 87$ & $\mathrm{H}$ & 4 & $\cdots$ & $\cdots$ & 1 \\
\hline
\end{tabular}


Table 1-1. Description of selected wells, borings, and springs in the Saco and Ossipee River Basins, east-central

\begin{tabular}{|c|c|c|c|c|c|c|c|c|c|c|c|c|}
\hline \multicolumn{2}{|c|}{$\begin{array}{l}\text { Local } \\
\text { site } \\
\text { number }\end{array}$} & \multirow[t]{2}{*}{$\begin{array}{l}\text { Lat- } \\
\text { itude }\end{array}$} & \multirow[t]{2}{*}{$\begin{array}{l}\text { Long- } \\
\text { itude }\end{array}$} & \multirow[t]{2}{*}{ Owner or user } & \multirow[t]{2}{*}{$\begin{array}{l}\text { Year } \\
\text { com- } \\
\text { pleted }\end{array}$} & \multirow[t]{2}{*}{$\begin{array}{c}\text { Alti- } \\
\text { tude } \\
\text { above } \\
\text { sea } \\
\text { level } \\
\text { (feet) }\end{array}$} & \multirow[t]{2}{*}{$\begin{array}{l}\text { Dia- } \\
\text { meter } \\
\text { of } \\
\text { well } \\
\text { casing } \\
\text { (inches) }\end{array}$} & \multirow[t]{2}{*}{$\begin{array}{l}\text { Depth } \\
\text { to } \\
\text { bottom } \\
\text { of } \\
\text { casing } \\
\text { (feet) }\end{array}$} & \multirow[t]{2}{*}{$\begin{array}{l}\text { Casing } \\
\text { material }\end{array}$} & \multirow[t]{2}{*}{$\begin{array}{l}\text { Type } \\
\text { of } \\
\text { finish }\end{array}$} & \multirow[t]{2}{*}{$\begin{array}{l}\text { Depth } \\
\text { to } \\
\text { bottom } \\
\text { of open } \\
\text { section } \\
\text { (feet) }\end{array}$} & \multirow[t]{2}{*}{$\begin{array}{c}\text { Type } \\
\text { of } \\
\text { site }\end{array}$} \\
\hline & & & & & & & & & & & & \\
\hline oxw & 240 & 434354 & 710942 & Eldridge & 1987 & 570 & $\cdots$ & 109 & $\cdots$ & $\mathrm{x}$ & -- & Brw \\
\hline oxw & 241 & 433851 & 710446 & Verge & 1987 & 810 & - & 25 & $\cdots$ & $\mathrm{x}$ & - & Brw \\
\hline oxw & 242 & 434842 & 711204 & Perkins & 1986 & 490 & $\cdots$ & 60 & $\cdots$ & $\mathrm{x}$ & $\cdots$ & Brw \\
\hline oxw & 243 & 434841 & 711202 & Perkins & 1986 & 490 & - & 49 & $\cdots$ & $\mathrm{x}$ & - & BrW \\
\hline oxw & 244 & 434028 & 710736 & Mountainside Business Center & 1987 & 760 & - & 47 & -. & $\mathrm{x}$ & $\cdots$ & Brw \\
\hline oxw & 245 & 434618 & 711205 & MacIssac & 1987 & 1,140 & $\cdots$ & 37 & $\cdots$ & $\mathrm{x}$ & $\cdots$ & Brw \\
\hline oxw & 246 & 434522 & 711100 & valeri & 1987 & 700 & $\cdots$ & 40 & - & $\mathrm{x}$ & $\cdots$ & Brw \\
\hline oxw & 247 & 434544 & 711102 & Fillipon & 1987 & 710 & $\cdots$ & 151 & $\cdots$ & $\mathrm{x}$ & $\cdots$ & Brw \\
\hline oxw & 248 & 434027 & 710110 & Capone & 1987 & 730 & -- & 79 & $\cdots$ & $x$ & $\cdots$ & BrW \\
\hline oxw & 249 & 434446 & 710814 & wentworth & 1988 & 520 & $\cdots$ & 159 & $\cdots$ & $\mathrm{x}$ & $\cdots$ & Brw \\
\hline oxw & 250 & 434613 & 711300 & Button & 1988 & 1,120 & $\cdots$ & 229 & $\cdots$ & $\mathrm{x}$ & $\cdots$ & Brw \\
\hline oxw & 251 & 434052 & 710931 & Demers & 1988 & 1,190 & $\cdots$ & 19 & $\cdots$ & $\mathrm{x}$ & $-\cdot$ & Brw \\
\hline oxw & 252 & 434447 & 710815 & wentworth & 1988 & 520 & -. & 166 & -. & $x$ & -. & Brw \\
\hline oxw & 253 & 434649 & 710629 & Town of Ossipee & 1988 & 440 & $\cdots$ & 99 & $\cdots$ & $\mathrm{x}$ & - & BrW \\
\hline oxw & 254 & 434946 & 711136 & Morse & 1988 & 440 & $\cdots$ & 53 & $\cdots$ & $\mathrm{s}$ & $\cdots$ & $\cdots$ \\
\hline oxw & 255 & 433932 & 710226 & Alves & 1988 & 780 & $\cdots$ & 29 & $\cdots$ & $x$ & $-\cdot$ & Brw \\
\hline oxw & 256 & 434653 & 711009 & Harrington & 1988 & 470 & - & 59 & $\cdots$ & $\mathrm{s}$ & $\cdots$ & $-\cdot$ \\
\hline oxw & 257 & 434936 & 711124 & Mottola & 1988 & 460 & $\cdots$ & 50 & $\cdots$ & $\mathrm{s}$ & $\cdots$ & $\cdots$ \\
\hline oxw & 258 & 434647 & 711414 & Thurston & 1988 & 920 & $\cdots$ & 80 & $\cdots$ & $\mathrm{x}$ & $\cdots$ & BrW \\
\hline oxw & 259 & 434131 & 710735 & P. Tilton & 1988 & 720 & $\cdots$ & 119 & $\cdots$ & $\mathrm{x}$ & $\cdots$ & Brw \\
\hline oxw & 260 & 434108 & 710811 & Freely & 1988 & 840 & $-\cdot$ & 41 & $\cdots$ & $x$ & $\cdots$ & BrW \\
\hline oxw & 261 & 434138 & 710331 & Watson & 1988 & 740 & $\cdots$ & 99 & $\cdots$ & $\mathrm{x}$ & $\cdots$ & Brw \\
\hline oxw & 262 & 434406 & 711304 & wright & 1989 & 1,040 & $\cdots$ & 111 & $\cdots$ & $x$ & -- & Brw \\
\hline oXw & 263 & 434901 & 711228 & Chase & 1988 & 470 & $\cdots$ & 77 & $\cdots$ & $\mathrm{x}$ & $\cdots$ & Brw \\
\hline oxw & 264 & 434131 & 710334 & Ventola & 1988 & 720 & $\cdots$ & 101 & $\cdots$ & $x$ & $\cdots$ & Brw \\
\hline oxw & 265 & 434357 & 710809 & Powers Jr & 1989 & 580 & $\cdots$ & 140 & $\cdots$ & $x$ & $\cdots$ & Brw \\
\hline oxw & 266 & 434000 & 710635 & Babson Jr & 1989 & 770 & - - & 40 & - & $x$ & - & BrW \\
\hline oxw & 267 & 434649 & 711210 & Roberts & 1989 & 780 & $\cdots$ & 82.5 & $\cdots$ & $\mathrm{x}$ & $\cdots$ & BrW \\
\hline oxw & 268 & 434346 & 710806 & Porter & 1989 & 540 & -- & 100 & $\cdots$ & $\mathrm{x}$ & $\cdots$ & BrW \\
\hline oxw & 269 & 434053 & 710903 & Dixey & 1989 & 1,000 & $\cdots$ & 40 & $\cdots$ & $\mathrm{x}$ & $\cdots$ & BrW \\
\hline oxw & 270 & 434356 & 710755 & Leonard & 1989 & 600 & $\cdots$ & 193 & $\cdots$ & $\mathrm{x}$ & $\cdots$ & BrW \\
\hline oxw & 271 & 434315 & 710606 & Lanci & 1989 & 540 & 6 & 17.5 & $\mathrm{~s}$ & $\mathrm{x}$ & - & Brw \\
\hline oxw & 272 & 434756 & 710537 & Smarao & 1989 & 420 & $-\cdot$ & 11 & $\cdots$ & $\mathrm{s}$ & - & -. \\
\hline oxw & 273 & 434620 & 711201 & Howard SI & 1989 & 1,080 & $\cdots$ & 139 & $\cdots$ & $\mathrm{x}$ & $\cdots$ & BrW \\
\hline oxw & 274 & 434510 & 711230 & Sullivan and S. Martin & 1989 & 960 & $\cdots$ & 39 & $\cdots$ & $\mathrm{s}$ & $\cdots$ & $\cdots$ \\
\hline oxw & 275 & 434127 & 710735 & Lavigne & 1990 & 740 & $\cdots$ & 111 & $\cdots$ & $\mathrm{x}$ & $\cdots$ & BrW \\
\hline oxw & 276 & 434808 & 710953 & Westward Shores Camping & 1990 & 410 & - & 25 & - & $\mathrm{s}$ & - & $-\cdot$ \\
\hline oxw & 277 & 434643 & 711420 & c. Googins & 1990 & 920 & $\cdots$ & 175 & $\cdots$ & $\mathrm{x}$ & $\cdots$ & BrW \\
\hline oxw & 278 & 434614 & 711206 & Smith & 1990 & 1.160 & $\cdots$ & 49 & $\cdots$ & $\mathrm{x}$ & $\cdots$ & BrW \\
\hline oxw & 279 & 434052 & 711053 & Whiting Jr(New Hampshire) & 1988 & 840 & $-\cdot$ & 139 & - & $\mathrm{x}$ & - & BrW \\
\hline oxw & 280 & 434030 & 710733 & Ambrust & 1991 & 740 & $\cdots$ & 24 & $\cdots$ & $\mathrm{x}$ & $\cdots$ & Brw \\
\hline oxw & 281 & 434800 & 710533 & Tobin & 1991 & 415 & $\cdots$ & 39 & $\cdots$ & $\mathrm{x}$ & $\cdots$ & BrW \\
\hline oxw & 282 & 434802 & 710519 & Fogg & 1991 & 420 & $\cdots$ & 31 & $\cdots$ & $\mathrm{s}$ & $\cdots$ & $\cdots$ \\
\hline oxw & 283 & 434756 & 710537 & M. Sparaco & 1991 & 420 & -- & 20 & $\cdots$ & $\mathrm{s}$ & $-\cdot$ & $\cdots$ \\
\hline oxw & 284 & 434405 & 710930 & Brooks & 1991 & 570 & -. & 38 & -. & $\mathrm{s}$ & $-\cdot$ & $\cdots$ \\
\hline oxw & 285 & 434940 & 711124 & Remick & 1991 & 460 & $\cdots$ & 84 & $\cdots$ & $\mathrm{s}$ & $\cdots$ & $-\cdot$ \\
\hline oxw & 286 & .434931 & 711224 & webster & 1991 & 440 & - & 44 & $\cdots$ & $\mathrm{s}$ & $\cdots$ & $\cdots$ \\
\hline oxw & 287 & 434943 & 711128 & cole & 1991 & 460 & $\cdots$ & 77 & - & $\mathrm{s}$ & $\cdots$ & $\cdots$ \\
\hline oxw & 288 & 434348 & 711213 & Bodurtha & 1991 & 1.030 & $\cdots$ & 60 & $\cdots$ & $\mathrm{x}$ & $\cdots$ & Brw \\
\hline oxw & 289 & 434837 & 711152 & Turner & 1991 & 450 & $\cdots$ & 70 & $\cdots$ & $\mathrm{x}$ & $\cdots$ & BrW \\
\hline oxw & 290 & 434140 & 710251 & walker & 1991 & 960 & $\cdots$ & 39 & $\cdots$ & $x$ & $\cdots$ & BrW \\
\hline oxw & 291 & 434558 & 711114 & Potter & 1991 & 780 & $\cdots$ & 99 & $\cdots$ & $\mathrm{x}$ & $\cdots$ & BrW \\
\hline oxw & 292 & 434358 & 710745 & Burns & 1992 & 620 & $\cdots$ & 249 & -- & $\mathrm{x}$ & $\cdots$ & BrW \\
\hline oxw & 293 & 434128 & 710753 & Kellacher & 1991 & 800 & $\cdots$ & 54 & $\cdots$ & $\mathrm{x}$ & $\cdots$ & Brw \\
\hline oxw & 294 & 434102 & 710737 & Savage & 1992 & 730 & $-\cdot$ & 153 & $\cdots$ & $\mathrm{x}$ & $\cdots$ & Brw \\
\hline
\end{tabular}




\begin{tabular}{|c|c|c|c|c|c|c|c|c|}
\hline $\begin{array}{l}\text { Local } \\
\text { site } \\
\text { number }\end{array}$ & $\begin{array}{l}\text { Water } \\
\text { level } \\
\text { depth } \\
\text { (feet) }\end{array}$ & $\begin{array}{l}\text { Date } \\
\mathrm{mm} / \mathrm{dd} / \mathrm{yy}\end{array}$ & Use & $\begin{array}{l}\text { Maximum } \\
\text { well } \\
\text { yield } \\
\text { (gallons } \\
\text { per } \\
\text { minute) }\end{array}$ & $\begin{array}{l}\text { Draw- } \\
\text { down } \\
\text { (feet) }\end{array}$ & $\begin{array}{c}\text { Specific } \\
\text { capacity } \\
\text { (gallons per } \\
\text { minute } \\
\text { per } \\
\text { foot) }\end{array}$ & $\begin{array}{l}\text { Pumping } \\
\text { period } \\
\text { (hours) }\end{array}$ & Remarks \\
\hline
\end{tabular}

\begin{tabular}{|c|c|c|c|c|c|c|c|c|}
\hline \multirow[b]{2}{*}{ oxw } & \multirow[b]{2}{*}{240} & \multirow[b]{2}{*}{$\cdots$} & \multirow[b]{2}{*}{$\cdots$} & \multirow[b]{2}{*}{ H } & \multirow[b]{2}{*}{1} & \multirow[b]{2}{*}{.. } & \multicolumn{2}{|c|}{ Ossipee-Contin } \\
\hline & & & & & & & $\cdots$ & 1 \\
\hline oxw & 241 & $\cdots$ & $-\cdot$ & H & 1 & $\cdots$ & $\cdots$ & 1 \\
\hline oxw & 242 & $\cdots$ & . & $\mathrm{H}$ & 0.8 & -. & -. & .5 \\
\hline oxw & 243 & - & $\cdots$ & H & 20 & $\cdots$ & $\cdots$ & .5 \\
\hline oxw & 244 & $\cdots$ & - & $\mathrm{H}$ & 10 & $\cdots$ & $\cdots$ & .5 \\
\hline oxw & 245 & 65 & $11-04-87$ & H & 3.5 & $\cdots$ & $\cdots$ & 1 \\
\hline oxw & 246 & 150 & $10-15-87$ & н & 0.7 & $\cdots$ & $\cdots$ & 1 \\
\hline oxw & 247 & 28 & $10-13-87$ & $\mathrm{H}$ & 3.5 & $\cdots$ & - & .5 \\
\hline oxw & 248 & -. & - & $\mathrm{H}$ & 3.5 & $\cdots$ & $\cdots$ & 1 \\
\hline oxw & 249 & $\cdots$ & $\cdots$ & $\mathrm{H}$ & 2 & $\cdots$ & $\cdots$ & 1 \\
\hline oxw & 250 & $\cdots$ & $\cdots$ & $\mathrm{H}$ & 4 & -. & $\cdots$ & 1 \\
\hline oxw & 251 & $\cdots$ & $\cdots$ & $\mathrm{H}$ & 1 & $\cdots$ & . & 1.5 \\
\hline oxw & 252 & - & - & $\mathrm{H}$ & 0.4 & $\cdots$ & - & 1.5 \\
\hline oxw & 253 & $\cdots$ & - & $\mathrm{H}$ & 3 & $\cdots$ & $\cdots$ & 1.8 \\
\hline oxw & 254 & 32.5 & $09-10-88$ & H & 12 & $\cdots$ & - & 2 \\
\hline oxw & 255 & 15 & $07-12-88$ & $\mathrm{H}$ & 20 & $\cdots$ & $\cdots$ & .5 \\
\hline oxw & 256 & - & - & H & 10 & $\cdots$ & - & 1 \\
\hline OXW & 257 & 28.5 & $10-02-88$ & $\mathrm{H}$ & 13 & $\cdots$ & $-\cdot$ & 1 \\
\hline oxw & 258 & -. & - & H & 50 & $\cdots$ & $\cdots$ & .5 \\
\hline oxw & 259 & $\cdots$ & $\cdots$ & $\mathrm{H}$ & 5 & $\cdots$ & $\cdots$ & .5 \\
\hline $\mathrm{OXW}$ & 260 & $\cdots$ & $\cdots$ & H & 35 & $\cdots$ & $\cdots$ & .5 \\
\hline oxw & 261 & -. & - & H & 2.5 & $\cdots$ & $\cdots$ & 1.5 \\
\hline oxw & 262 & 60 & $01-11-89$ & H & 6 & $\cdots$ & $\cdots$ & 1 \\
\hline oxw & 263 & 10 & $05-05-88$ & $\mathrm{H}$ & 15 & $\cdots$ & $\cdots$ & .5 \\
\hline oxw & 264 & 10 & $11-23-88$ & H & 20 & $\cdots$ & $\cdots$ & .5 \\
\hline oxw & 265 & - & - & H & 6 & $\cdots$ & $\cdots$ & 1 \\
\hline oxw & 266 & $\cdots$ & - & H & 150 & -. & $\cdots$ & 1 \\
\hline $\mathrm{oxw}$ & 267 & -. & - & H & 8 & $\cdots$ & $\cdots$ & 1 \\
\hline oXw & 268 & 5 & $09-12-89$ & H & 50 & $\cdots$ & $\cdots$ & .5 \\
\hline oxw & 269 & $\cdots$ & - & $\mathbf{H}$ & 4 & $\cdots$ & $\cdots$ & .5 \\
\hline oxw & 270 & 50 & $07-25-89$ & H & 12 & $\cdots$ & $\cdots$ & .5 \\
\hline oxw & 271 & $\cdots$ & - & H & 3 & $\cdots$ & $\cdots$ & $\cdots$ \\
\hline JXW & 272 & 4 & $09-11-89$ & H & 20 & $\cdots$ & $\cdots$ & 2 \\
\hline JXW & 273 & - & - & $\mathrm{H}$ & 2 & $\cdots$ & $\cdots$ & 1.3 \\
\hline$\partial X W$ & 274 & 18 & $11-29-89$ & H & 12 & $\cdots$ & $\cdots$ & .5 \\
\hline$J x w$ & 275 & - & - & $\mathrm{H}$ & 12 & $\cdots$ & $\cdots$ & 1 \\
\hline JXW & 276 & 4 & $03-03-90$ & H & 60 & $\cdots$ & $\cdots$ & 2 \\
\hline JXW & 277 & -. & - & H & 2 & $\cdots$ & $\cdots$ & -. \\
\hline$J X w$ & 278 & 50 & $09-20-90$ & H & 6 & $\cdots$ & $\cdots$ & .5 \\
\hline Jxw & 279 & 20 & $11-01-88$ & H & 6 & $\cdots$ & $\cdots$ & 1 \\
\hline bxw & 280 & - & $\cdots$ & н & 15 & $\cdots$ & $\cdots$ & 1 \\
\hline DXW & 281 & -. & - & H & 6 & $\cdots$ & -. & .5 \\
\hline oxw & 282 & 15 & $04-06-91$ & H & 25 & $\cdots$ & $\cdots$ & 1 \\
\hline bxw & 283 & $\cdots$ & - & H & 30 & $\cdots$ & $\cdots$ & 2 \\
\hline pXw & 284 & 33 & $07-03-91$ & H & 10 & $\cdots$ & $\cdots$ & 1 \\
\hline $\mathrm{pxw}$ & 285 & 28 & $06-22-91$ & H & 15 & $\cdots$ & $\cdots$ & 2 \\
\hline bxw & 286 & 20 & $08-10-91$ & H & 30 & $\cdots$ & $\cdots$ & 1.5 \\
\hline bxw & 287 & 28 & $07-20-91$ & H & 12 & $\cdots$ & $\cdots$ & 1 \\
\hline DXW & 288 & 30 & $06-25-91$ & H & 50 & $\cdots$ & $\cdots$ & .5 \\
\hline DXw & 289 & 35 & $07-25-91$ & H & 2.5 & $\cdots$ & $\cdots$ & .5 \\
\hline JXw & 290 & - & - - & H & 5 & $\cdots$ & $\cdots$ & 66 \\
\hline JXW & 291 & 40 & $11-23-91$ & H & 3.5 & $\cdots$ & $\cdots$ & 1 \\
\hline JXW & 292 & 80 & $12-09-92$ & $z$ & 2 & $\cdots$ & $\cdots$ & 2 \\
\hline JXW & 293 & 20 & $07-06-91$ & H & 3.5 & $\cdots$ & $\cdots$ & .5 \\
\hline$J X W$ & 294 & 10 & $02-28-92$ & H & 15 & -. & $\cdots$ & .5 \\
\hline
\end{tabular}


Table 1-1. Description of selected wells, borings, and springs in the Saco and Ossipee River Basins, east-central

\begin{tabular}{|c|c|c|c|c|c|c|c|c|c|c|c|}
\hline $\begin{array}{l}\text { Local } \\
\text { site } \\
\text { number }\end{array}$ & $\begin{array}{l}\text { Lat- } \\
\text { itude }\end{array}$ & $\begin{array}{l}\text { Long- } \\
\text { itude }\end{array}$ & Owner or user & $\begin{array}{l}\text { Year } \\
\text { com- } \\
\text { pleted }\end{array}$ & $\begin{array}{c}\text { Alti- } \\
\text { tude } \\
\text { above } \\
\text { sea } \\
\text { level } \\
\text { (feet) }\end{array}$ & $\begin{array}{l}\text { Dia- } \\
\text { meter } \\
\text { of } \\
\text { well } \\
\text { casing } \\
\text { (inches) }\end{array}$ & $\begin{array}{l}\text { Depth } \\
\text { to } \\
\text { bottom } \\
\text { of } \\
\text { casing } \\
\text { (feet) }\end{array}$ & $\begin{array}{l}\text { Casing } \\
\text { material }\end{array}$ & $\begin{array}{l}\text { Type } \\
\text { of } \\
\text { finish }\end{array}$ & $\begin{array}{l}\text { Depth } \\
\text { to } \\
\text { bottom } \\
\text { of open } \\
\text { section } \\
\text { (feet) }\end{array}$ & $\begin{array}{c}\text { Type } \\
\text { of } \\
\text { site }\end{array}$ \\
\hline
\end{tabular}

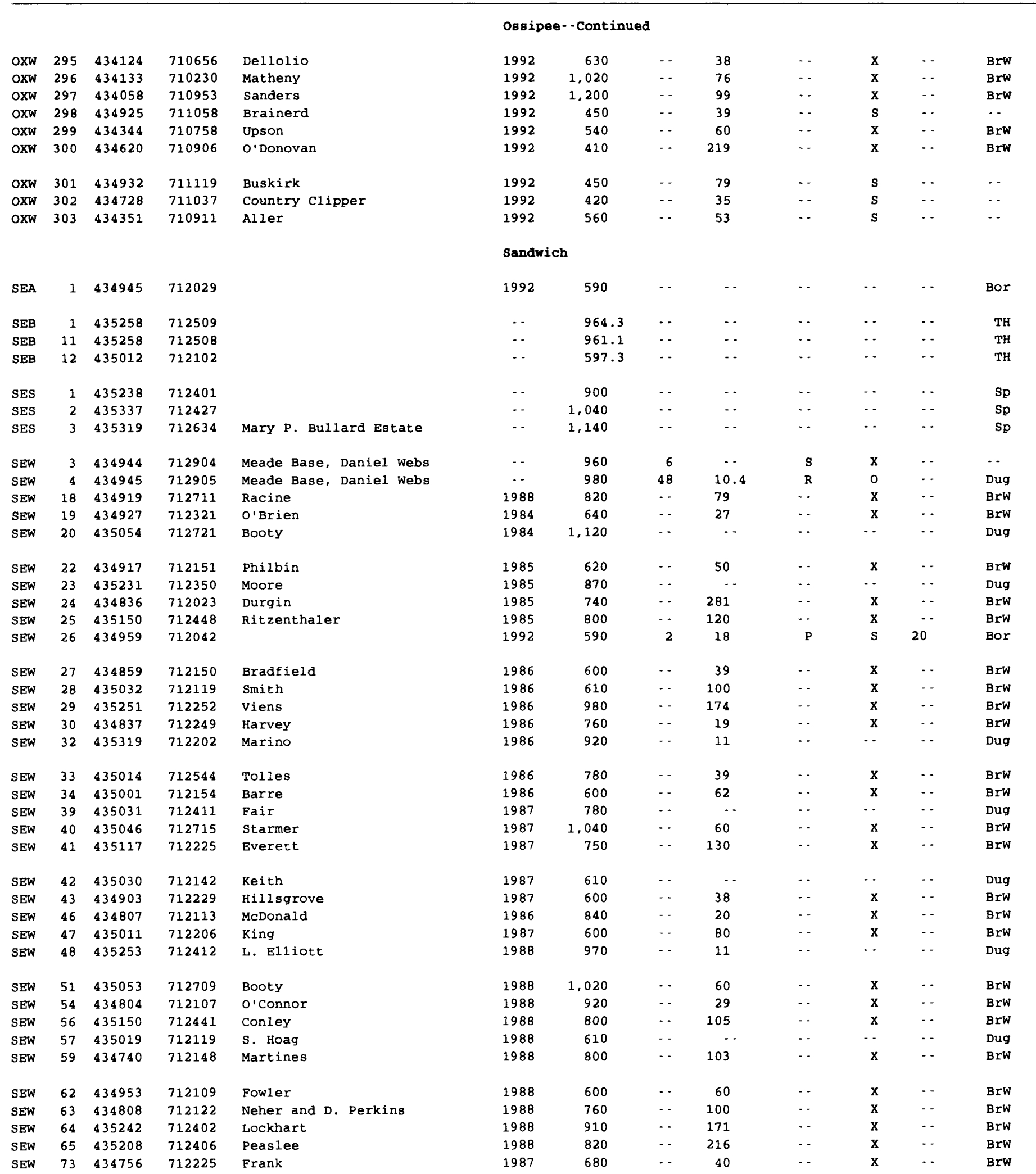




\begin{tabular}{|c|c|c|c|c|c|c|c|c|}
\hline $\begin{array}{l}\text { Local } \\
\text { site } \\
\text { number }\end{array}$ & $\begin{array}{l}\text { Water } \\
\text { level } \\
\text { depth } \\
\text { (feet) }\end{array}$ & $\begin{array}{l}\text { Date } \\
\mathrm{mm} / \mathrm{dd} / \mathrm{yy}\end{array}$ & Use & $\begin{array}{l}\text { Maximum } \\
\text { well } \\
\text { yield } \\
\text { (gallons } \\
\text { per } \\
\text { minute) }\end{array}$ & $\begin{array}{l}\text { Draw- } \\
\text { down } \\
\text { (feet) }\end{array}$ & $\begin{array}{c}\text { Specific } \\
\text { capacity } \\
\text { (gallons per } \\
\text { minute } \\
\text { per } \\
\text { foot) }\end{array}$ & $\begin{array}{l}\text { Pumping } \\
\text { period } \\
\text { (hours) }\end{array}$ & Remarks \\
\hline
\end{tabular}

\begin{tabular}{|c|c|c|c|c|c|c|c|c|}
\hline & & & & & & & oss & ontin \\
\hline oxw & 295 & 600 & $05-06-92$ & $\mathrm{H}$ & - - & - & - & - - \\
\hline oxw & 296 & 30 & $05-23-92$ & $\mathrm{H}$ & 1.5 & - & .. & 1 \\
\hline oxw & 297 & 60 & $07-08-92$ & H & 4 & - & - & .5 \\
\hline OXW & 298 & 28 & $09-19-92$ & H & 17 & - & -. & 2 \\
\hline oxw & 299 & 40 & $11-01-92$ & H & 3.5 & - & -. & 1 \\
\hline oxw & 300 & 3 & $08-27-92$ & H & 30 & - & - & .5 \\
\hline oxw & 301 & 35 & $10-31-92$ & $\mathrm{H}$ & 13 & - & $\cdots$ & - - \\
\hline oxw & 302 & 9 & $11-29-92$ & H & 5.3 & - - & - & 1 \\
\hline oxw & 303 & 43 & $12-13-92$ & H & 9 & - & . & 2 \\
\hline
\end{tabular}

\begin{tabular}{|c|c|c|c|c|c|c|c|c|c|}
\hline SEA & 1 & - & -. & $\mathrm{T}$ & $\cdots$ & $\cdots$ & $-\cdot$ & $\cdots$ & USGS \\
\hline SEB & 1 & - & $\cdots$ & $\mathrm{T}$ & - & $\cdots$ & -. & .. & \\
\hline SEB & 11 & $\cdots$ & -. & $\mathrm{U}$ & -. & - & $\cdots$ & $\cdots$ & \\
\hline SEB & 12 & . & $\cdots$ & $\mathrm{U}$ & - - & $-\cdot$ & - & - & \\
\hline SES & 1 & $\cdots$ & -. & -. & 3.5 & -. & -. & -. & \\
\hline SES & 2 & $\cdots$ & $\cdots$ & $\cdots$ & 10 & $\cdots$ & -. & $\cdots$ & \\
\hline SES & 3 & - & - & $-\cdot$ & 4 & $\cdots$ & $\cdots$ & - & \\
\hline SEW & 3 & -. & - - & H T & -. & - & - & - & \\
\hline SEW & 4 & 10.4 & $07-29-91$ & $\mathrm{U}$ & -. & - & - & $\cdots$ & \\
\hline SEW & 18 & 15 & $03-03-88$ & $\mathrm{H}$ & 3 & .. & -. & .8 & \\
\hline SEW & 19 & 30 & $07-17-84$ & H & 10 & . & .. & 1 & \\
\hline SEW & 20 & 3 & $09-24-84$ & $\mathrm{H}$ & & - & - & - & \\
\hline SEW & 22 & 15 & $06-11-85$ & $\mathrm{H}$ & 4 & - & $\cdots$ & 1 & \\
\hline SEW & 23 & 3 & $08-09-85$ & H & & -. & $\cdots$ & $-\cdot$ & \\
\hline SEW & 24 & 60 & $07-31-85$ & $\mathrm{H}$ & 30 & - - & $-\cdot$ & .5 & \\
\hline SEW & 25 & 80 & $11-20-85$ & H & 1.5 & -. & - & .5 & \\
\hline SEW & 26 & 6 & $08-27-92$ & 0 & - & - & - & - & USGS \\
\hline SEW & 27 & 10 & $06-01-86$ & $\mathrm{H}$ & 2 & -. & $-\cdot$ & .5 & \\
\hline SEW & 28 & 25 & $08-21-86$ & $\mathrm{H}$ & 12 & - & -. & .5 & \\
\hline SEW & 29 & 55 & $09-10.86$ & H & 1.8 & - & $\cdots$ & .5 & \\
\hline SEW & 30 & 4 & $08-16-86$ & H & 1.3 & - & $\cdots$ & 1 & \\
\hline SEW & 32 & 6 & $09-08-86$ & $\mathrm{H}$ & $-\cdot$ & - & -. & $\cdots$ & \\
\hline SEW & 33 & 35 & $10-07-86$ & H & 30 & - & -. & .5 & \\
\hline SEW & 34 & 40 & $10-10-86$ & H & 2.5 & - & - & .5 & \\
\hline SEW & 39 & 1 & $06-18-87$ & H & 3 & - & -. & $-\cdot$ & \\
\hline SEW & 40 & 30 & $06-15-87$ & H & 30 & -. & - & 1 & \\
\hline SEW & 41 & 85 & $05-30-87$ & H & 3 & - & -. & 1 & \\
\hline SEW & 42 & 10 & $06-25-87$ & H & 5 & - & - & 1.3 & \\
\hline SEW & 43 & 30 & $06-15-87$ & H & 7 & - & - & 2 & \\
\hline SEW & 46 & - & - & H & 2 & - & $\cdots$ & .5 & \\
\hline SEW & 47 & 10 & $09-16-87$ & H & 3.5 & - & -. & .5 & \\
\hline SEW & 48 & 3 & $01-13-88$ & H & $-\cdot$ & $\cdots$ & $\cdots$ & $\cdots$ & \\
\hline SEW & 51 & - & - - & $\mathrm{H}$ & 30 & - & -. & 1 & \\
\hline SEW & 54 & $\cdots$ & $\cdots$ & H & 1.5 & -. & - & 1 & \\
\hline SEW & 56 & - & - - & H & 3 & -. & -. & 1 & \\
\hline SEW & 57 & 8 & $07-30-88$ & H & 30 & $\cdots$ & -. & 3 & \\
\hline SEW & 59 & 40 & $08-06-88$ & $\mathrm{H}$ & 20 & - - & $\cdots$ & .5 & \\
\hline SEW & 62 & 20 & $05-18-88$ & H & 2 & $\cdots$ & - & .5 & \\
\hline SEW & 63 & 50 & $04-22-88$ & $\mathrm{H}$ & 3 & .. & - & .5 & \\
\hline SEW & 64 & - - & - & $\mathrm{H}$ & 7 & - & $\cdots$ & 1 & \\
\hline SEW & 65 & 80 & $10-06-88$ & $\mathrm{H}$ & 2.5 & - & - & .5 & \\
\hline SEW & 73 & 10 & $08-19-87$ & $\mathrm{H}$ & 15 & - & -. & .5 & \\
\hline
\end{tabular}


Table 1-1. Description of selected wells, borings, and springs in the Saco and Ossipee River Basins, east-central

\begin{tabular}{|c|c|c|c|c|c|c|c|c|c|c|c|}
\hline $\begin{array}{l}\text { Local } \\
\text { site } \\
\text { number }\end{array}$ & $\begin{array}{l}\text { Lat- } \\
\text { itude }\end{array}$ & $\begin{array}{l}\text { Long- } \\
\text { itude }\end{array}$ & Owner or user & $\begin{array}{l}\text { Year } \\
\text { com- } \\
\text { pleted }\end{array}$ & $\begin{array}{l}\text { Alti- } \\
\text { tude } \\
\text { above } \\
\text { sea } \\
\text { level } \\
\text { (feet) }\end{array}$ & $\begin{array}{l}\text { Dia- } \\
\text { meter } \\
\text { of } \\
\text { well } \\
\text { casing } \\
\text { (inches) }\end{array}$ & $\begin{array}{l}\text { Depth } \\
\text { to } \\
\text { bottom } \\
\text { of } \\
\text { casing } \\
\text { (feet) }\end{array}$ & $\begin{array}{l}\text { Casing } \\
\text { material }\end{array}$ & $\begin{array}{l}\text { Type } \\
\text { of } \\
\text { finish }\end{array}$ & $\begin{array}{l}\text { Depth } \\
\text { to } \\
\text { bottom } \\
\text { of open } \\
\text { section } \\
\text { (feet) }\end{array}$ & $\begin{array}{c}\text { Type } \\
\text { of } \\
\text { site }\end{array}$ \\
\hline
\end{tabular}

\begin{tabular}{|c|c|c|c|c|}
\hline SEW & 74 & 434759 & 712204 & Trombley \\
\hline SEW & 75 & 435018 & 712123 & Ingari and $\mathrm{R}$. Demars \\
\hline SEW & 76 & 434805 & 712109 & Hunt \\
\hline SEW & 77 & 434925 & 712834 & Milbury \\
\hline SEW & 81 & 435023 & 712415 & Litzel1 \\
\hline SEW & 82 & 435040 & 712118 & Gove \\
\hline SEW & 84 & 435324 & 712643 & Berg \\
\hline SEW & 85 & 435253 & 712359 & Twaddle \\
\hline SEW & 86 & 435122 & 712202 & Azerrad \\
\hline SEW & 87 & 435306 & 712231 & Father \& Son Construction \\
\hline SEW & 88 & 434923 & 712207 & Hof fman \\
\hline SEW & 89 & 435014 & 712710 & Nee \\
\hline SEW & 92 & 435144 & 712224 & Lombardi \\
\hline SEW & 96 & 435115 & 712428 & Caradona \\
\hline SEW & 97 & 434732 & 712206 & Everett \\
\hline SEW & 98 & 435118 & 712445 & Danielovich \\
\hline SEW & 99 & 434835 & 712202 & Bauderob \\
\hline SEW & 101 & 435012 & 712358 & Town of sandwich \\
\hline SEW & 102 & 435233 & 712403 & El1iott \\
\hline SEW & 103 & 435319 & 712156 & Daniels \\
\hline SEW & 104 & 435319 & 712620 & winship \\
\hline SEW & 111 & 435331 & 712424 & \\
\hline SEW & 152 & 434856 & 712244 & Mumford \\
\hline SEW & 153 & 434929 & 712901 & Wilber Cook \\
\hline SEW & 154 & 434931 & 712849 & wilber Cook \\
\hline SEW & 155 & 434942 & 712721 & E.H. Harding \\
\hline SEW & 156 & 434929 & 712629 & Legault \\
\hline SEW & 157 & 435327 & 712413 & 011y Moulton \\
\hline SEW & 158 & 435259 & 712447 & Ed Wondoloski \\
\hline
\end{tabular}

Sandwich - - Continued

\begin{tabular}{|c|c|c|c|c|c|c|c|}
\hline 1988 & 700 & $\cdots$ & 131 & $\cdots$ & $x$ & $\cdots$ & Brw \\
\hline 1989 & 610 & $\cdots$ & 17 & -- & $\cdots$ & $\cdots$ & Dug \\
\hline 1988 & 900 & -- & 40 & $\cdots$ & $x$ & $\cdots$ & Brw \\
\hline 1988 & 920 & $\cdots$ & 99 & $\cdots$ & $x$ & $\cdots$ & BrW \\
\hline 1988 & 780 & $\cdots$ & 85 & $\cdots$ & $x$ & $\cdots$ & Brw \\
\hline 1988 & 620 & $\cdots$ & 224 & -- & $x$ & $\cdots$ & Brw \\
\hline 1989 & 1,220 & $\cdots$ & 43 & $-\cdot$ & $x$ & $\cdots$ & BrW \\
\hline 1989 & 960 & -- & 266 & $\cdots$ & $x$ & $\cdots$ & BrW \\
\hline 1989 & 640 & $\cdots$ & 111 & $\cdots$ & $\mathbf{x}$ & $\cdots$ & BrW \\
\hline 1989 & 1,000 & $\cdots$ & 56 & $-\cdot$ & $x$ & $\cdots$ & Brw \\
\hline 1989 & 600 & $\cdots$ & 99 & -- & $x$ & $\cdots$ & BrW \\
\hline 1989 & 880 & $\cdots$ & 45 & $\cdots$ & $\mathrm{x}$ & -- & Brw \\
\hline 1989 & 800 & $\cdots$ & 14 & $\cdots$ & $\cdots$ & $\cdots$ & Dug \\
\hline 1988 & 900 & $\cdots$ & 19 & $\cdots$ & $\mathrm{x}$ & $\cdots$ & BrW \\
\hline 1988 & 760 & $\cdots$ & 69 & $\cdots$ & $\mathbf{x}$ & $\cdots$ & BrW \\
\hline 1989 & 980 & $\cdots$ & - & $\cdots$ & $-\cdot$ & - & Dug \\
\hline 1989 & 700 & $\cdots$ & 57 & $\cdots$ & $x$ & -- & BrW \\
\hline 1989 & 670 & $-\cdot$ & 53 & $\cdots$ & $x$ & $\cdots$ & BrW \\
\hline 1989 & 870 & $\cdots$ & 251 & $\cdots$ & $\mathrm{x}$ & $\cdots$ & BrW \\
\hline 1990 & 910 & $\cdots$ & 14 & $\cdots$ & $\cdots$ & $\cdots$ & Dug \\
\hline 1990 & 1,160 & $\cdots$ & 19 & $\cdots$ & $x$ & $\cdots$ & BrW \\
\hline - - & 1,020 & $\cdots$ & - & $\cdots$ & 0 & $\cdots$ & Dug \\
\hline 1986 & 600 & $\cdots$ & 50 & $\cdots$ & $x$ & $\cdots$ & Brw \\
\hline 1971 & 890 & 36 & 12 & C & 0 & $\cdots$ & Dug \\
\hline - - & 890 & 36 & 4 & $\mathbf{R}$ & 0 & - & Dug \\
\hline 1960 & 785 & -- & - & - & $\mathrm{x}$ & $\cdots$ & $\cdots$ \\
\hline 1979 & 975 & 6 & 40 & $\mathbf{s}$ & $x$ & $\cdots$ & BrW \\
\hline 1975 & 990 & $\cdots$ & - & -- & $x$ & $\cdots$ & $\cdots$ \\
\hline - - & 985 & 36 & 10.6 & C & 0 & -- & Dug \\
\hline
\end{tabular}

Tamworth

\begin{tabular}{|c|c|c|c|c|c|c|c|c|c|c|c|c|}
\hline TAB & 1 & 435135 & 711555 & NHDOT & $\cdots$ & 503 & -- & $\cdots$ & -- & $\cdots$ & $\cdots$ & $\mathrm{TH}$ \\
\hline TAB & 2 & 435430 & 712034 & NHDOT & -- & 1,085 & $-\cdot$ & $\cdots$ & - & $\cdots$ & $\cdots$ & $\mathrm{TH}$ \\
\hline $\mathrm{TAB}$ & 3 & 435332 & 711755 & NHDOT & $\cdots$ & 732 & $-\cdot$ & - & - & -- & $\cdots$ & $\mathrm{TH}$ \\
\hline TAB & 4 & 434955 & 711602 & NHDOT & -- & 465 & -- & $\cdots$ & - & $\cdots$ & -- & $\mathrm{TH}$ \\
\hline TAB & 5 & 435022 & 711554 & NHDOT & -- & 449 & -- & $\cdots$ & $\cdots$ & $\cdots$ & - & $\mathrm{TH}$ \\
\hline TAB & 6 & 435049 & 711557 & NHDOT & $\cdots$ & 480 & $\cdots$ & - & $\cdots$ & - - & $\cdots$ & $\mathrm{TH}$ \\
\hline TAB & 7 & 434936 & 711414 & NHDOT & $\cdots$ & 444.5 & $\cdots$ & - & $\cdots$ & - & $\cdots$ & $\mathrm{TH}$ \\
\hline TAB & 8 & 434951 & 711631 & NHDOT & $\cdots$ & 485 & $\cdots$ & $\cdots$ & $\cdots$ & $\cdots$ & $\cdots$ & $\mathrm{TH}$ \\
\hline TAB & 9 & 435428 & 712105 & & $\cdots$ & $1,102.7$ & -- & $\cdots$ & $\cdots$ & - & $\cdots$ & $\mathrm{TH}$ \\
\hline TAS & 1 & 435038 & 711528 & & - & 450 & - & $\cdots$ & - & $\cdots$ & $\cdots$ & sp \\
\hline TAS & 2 & 435132 & 711906 & & - & 550 & $\cdots$ & - & $\cdots$ & $\cdots$ & $-\cdot$ & Sp \\
\hline TAW & 1 & 434936 & 711947 & Kar1 Bickford & - & 590 & $\cdots$ & $\cdots$ & $\cdots$ & $\cdots$ & - & $\cdots$ \\
\hline TAW & 2 & 435012 & 712016 & crory & 1985 & 605 & $\cdots$ & $\cdots$ & - & $\mathrm{x}$ & $\cdots$ & $\cdots$ \\
\hline TAW & 3 & 435000 & 711948 & & - & 595 & $\cdots$ & - & $\cdots$ & $-\cdot$ & -- & BrW \\
\hline TAW & 4 & 435008 & 711931 & Valentine & 1984 & 595 & 2 & 32 & G & $\mathbf{T}$ & $\cdots$ & Dvn \\
\hline TAW & 5 & 434941 & 711943 & Kar1 Bickford & - - & 595 & - & - & $\cdots$ & $\mathrm{x}$ & $\cdots$ & Brw \\
\hline TAW & 6 & 434933 & 711857 & Andy Norcorss & $\cdots$ & 585 & 36 & 13.2 & $\mathbf{R}$ & 0 & $\cdots$ & Dug \\
\hline TAW & 7 & 434934 & 711859 & Irene Norcorss & - - & 590 & -- & $\cdots$ & $\cdots$ & $\mathrm{x}$ & $\cdots$ & $-\cdot$ \\
\hline TAW & 8 & 434934 & 711911 & John Cook & 1981 & 595 & 6 & 60 & $\mathbf{s}$ & $\mathrm{x}$ & $\cdots$ & BrW \\
\hline TAW & 9 & 434940 & 711919 & David Whiting & 1989 & 598 & -- & $\cdots$ & $-\cdot$ & G & -- & BrW \\
\hline TAW & 10 & 435035 & 711557 & Woodward & 1939 & 490 & 1 & 46 & $\mathbf{s}$ & $T$ & $\cdots$ & Dvn \\
\hline TAW & 11 & 435052 & 711549 & Shaun Holladay & $\cdots$ & 480 & $\cdots$ & $-\cdots$ & $\cdots$ & $\cdots$ & $\cdots$ & $\cdots$ \\
\hline TAW & 12 & 435040 & 711526 & Dana and Laurie Bonica & 1960 & 465 & 2 & 30 & $\mathbf{s}$ & $\mathrm{T}$ & $\cdots$ & Dvn \\
\hline
\end{tabular}




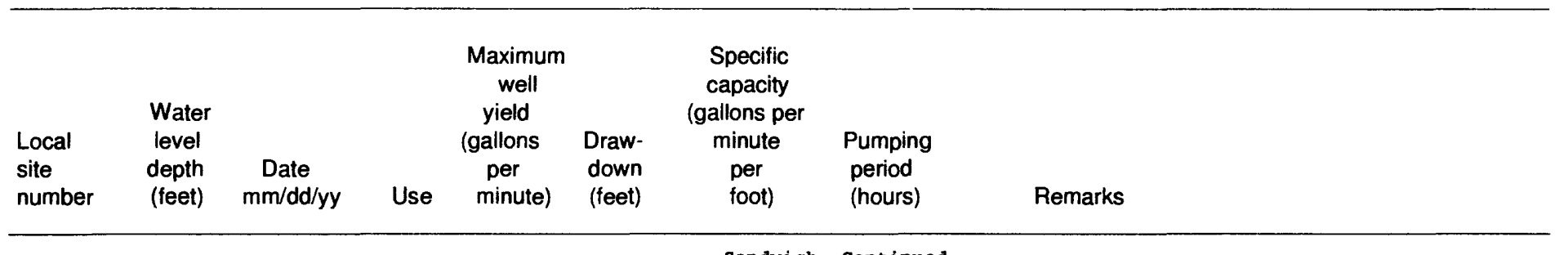

Sandwich - - Continued

\begin{tabular}{|c|c|c|c|c|c|c|c|c|c|}
\hline SEW & 74 & - & $\cdots$ & $\mathrm{H}$ & 3 & $\cdots$ & $\cdots$ & 1 & \\
\hline SEW & 75 & 11.5 & $01-06-89$ & $\mathrm{H}$ & 30 & $\cdots$ & $\cdots$ & $\cdots$ & \\
\hline SEW & 76 & 60 & $11-20-88$ & $\mathrm{H}$ & 1.5 & $\cdots$ & $\cdots$ & 1 & \\
\hline SEW & 77 & - & $\cdots$ & $\mathrm{H}$ & 8 & $\cdots$ & $\cdots$ & 1 & \\
\hline SEW & 81 & 40 & $06-01-88$ & $\mathrm{H}$ & 4 & $\cdots$ & $\cdots$ & .5 & \\
\hline SEW & 82 & 50 & $11-04-88$ & $\mathrm{H}$ & 3 & $\cdots$ & $\cdots$ & .5 & \\
\hline SEW & 84 & $\cdots$ & $-\cdot$ & $\mathrm{H}$ & 25 & $\cdots$ & $-\cdot$ & 1 & \\
\hline SEW & 85 & 35 & $03-29-89$ & $\mathrm{H}$ & 10 & $\cdots$ & $-\cdot$ & .5 & \\
\hline SEW & 86 & $\cdots$ & $\cdots$ & $\mathrm{H}$ & 8 & $\cdots$ & $\cdots$ & 1 & \\
\hline SEW & 87 & $\cdots$ & $\cdots$ & $\mathrm{H}$ & 3 & $\cdots$ & $\cdots$ & $\cdots$ & \\
\hline SEW & 88 & $\cdots$ & $\cdots$ & $\mathrm{H}$ & 20 & $\cdots$ & $\cdots$ & 48 & \\
\hline SEW & 89 & $\cdots$ & $\cdots$ & $\mathrm{H}$ & 2 & $\cdots$ & $\cdots$ & 1 & \\
\hline SEW & 92 & 3 & $10-24-89$ & $\mathrm{H}$ & 4.5 & $\cdots$ & $\cdots$ & .5 & \\
\hline SEW & 96 & $\cdots$ & $\cdots$ & $\mathrm{H}$ & 30 & $\cdots$ & $\cdots$ & 1 & \\
\hline SEW & 97 & $\cdots$ & $\cdots$ & $\mathrm{H}$ & 2 & $\cdots$ & $\cdots$ & 1 & \\
\hline SEW & 98 & 8 & $09-29-89$ & $\mathrm{H}$ & 5 & $-\cdot$ & $\cdots$ & 3.5 & \\
\hline SEW & 99 & $\cdots$ & $\cdots$ & $\mathrm{H}$ & 3 & $-\cdot$ & $-\cdot$ & .5 & \\
\hline SEW & 101 & $\cdots$ & $\cdots$ & $\mathrm{H}$ & 2 & $\cdots$ & $\cdots$ & 1 & \\
\hline SEW & 102 & $\cdots$ & - & $\mathrm{H}$ & 1 & $-\cdot$ & $\cdots$ & 1 & \\
\hline SEW & 103 & 6 & $02-28-90$ & $\mathrm{H}$ & 40 & $\cdots$ & $-\cdot$ & 6 & \\
\hline SEW & 104 & - - & $-\cdot$ & $\mathrm{H}$ & 25 & -- & $\cdots$ & .3 & \\
\hline SEW & 111 & 11.5 & $07-29-91$ & -- & - & $\cdots$ & $-\cdot$ & $\cdots$ & \\
\hline SEW & 152 & 15 & $05-01-86$ & $\mathrm{H}$ & 3.5 & -- & $\cdots$ & .5 & \\
\hline SEW & 153 & - & $\cdots$ & $\mathrm{H} \mathrm{S}$ & $\cdots$ & $\cdots$ & $\cdots$ & -- & We11 was dry at the time of the interview \\
\hline SEW & 154 & 3.9 & $07-29-91$ & $\mathrm{U}$ & $-\cdot$ & $-\cdot$ & $\cdots$ & $\cdots$ & \\
\hline SEW & 155 & $-\cdot$ & $-\cdot$ & $\mathrm{H}$ & $\cdots$ & $\cdots$ & $\cdots$ & $\cdots$ & \\
\hline SEW & 156 & $-\cdot$ & $\cdots$ & $\mathrm{H}$ & 0.8 & $\cdots$ & $\cdots$ & -- & \\
\hline SEW & 157 & $\cdots$ & $-\cdot$ & $\mathrm{H}$ & 11 & $\cdots$ & -- & $-\cdot$ & \\
\hline SEW & 158 & 7.7 & $07-30-91$ & $\mathrm{H}$ & $\cdots$ & $\cdots$ & $\cdots$ & $\cdots$ & \\
\hline
\end{tabular}

Tamworth

\begin{tabular}{|c|c|c|c|}
\hline$T A B$ & 1 & $\cdots$ & - \\
\hline$T A B$ & 2 & $\cdots$ & - \\
\hline TAB & 3 & -- & $\cdots$ \\
\hline TAB & 4 & - & $\cdots$ \\
\hline TAB & 5 & -- & $\cdots$ \\
\hline TAB & 6 & -- & $\cdots$ \\
\hline TAB & 7 & $\cdots$ & $\cdots$ \\
\hline TAB & 8 & $\cdots$ & $\cdots$ \\
\hline TAB & 9 & -- & $\cdots$ \\
\hline TAS & 1 & $\cdots$ & - - \\
\hline TAS & 2 & $\cdots$ & $\cdots$ \\
\hline TAW & 1 & - & $\cdots$ \\
\hline TAW & 2 & - & $\cdots$ \\
\hline TAW & 3 & -- & - \\
\hline TAW & 4 & 18 & $06-19-91$ \\
\hline TAW & 5 & $\cdots$ & $-\cdot$ \\
\hline TAW & 6 & 9.5 & $06-19-91$ \\
\hline TAW & 7 & $\cdots$ & $\cdots$ \\
\hline TAW & 8 & -- & $\cdots$ \\
\hline TAW & 9 & $\cdots$ & $\cdots$ \\
\hline TAW & 10 & 18 & $06-19-91$ \\
\hline TAW & 11 & $\cdots$ & $\cdots$ \\
\hline TAW & 12 & $\cdots$ & $\cdots$ \\
\hline
\end{tabular}

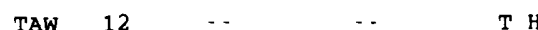


Table 1-1. Description of selected wells, borings, and springs in the Saco and Ossipee River Basins, east-central

\begin{tabular}{|c|c|c|c|c|c|c|c|c|c|c|c|}
\hline $\begin{array}{l}\text { Local } \\
\text { site } \\
\text { number }\end{array}$ & $\begin{array}{l}\text { Lat- } \\
\text { itude }\end{array}$ & $\begin{array}{l}\text { Long- } \\
\text { itude }\end{array}$ & Owner or user & $\begin{array}{l}\text { Year } \\
\text { com- } \\
\text { pleted }\end{array}$ & $\begin{array}{c}\text { Alti- } \\
\text { tude } \\
\text { above } \\
\text { sea } \\
\text { level } \\
\text { (feet) }\end{array}$ & $\begin{array}{l}\text { Dia- } \\
\text { meter } \\
\text { of } \\
\text { well } \\
\text { casing } \\
\text { (inches) }\end{array}$ & $\begin{array}{l}\text { Depth } \\
\text { to } \\
\text { bottom } \\
\text { of } \\
\text { casing } \\
\text { (feet) }\end{array}$ & $\begin{array}{l}\text { Casing } \\
\text { material }\end{array}$ & $\begin{array}{l}\text { Type } \\
\text { of } \\
\text { finish }\end{array}$ & $\begin{array}{l}\text { Depth } \\
\text { to } \\
\text { bottom } \\
\text { of open } \\
\text { section } \\
\text { (feet) }\end{array}$ & $\begin{array}{c}\text { Type } \\
\text { of } \\
\text { site }\end{array}$ \\
\hline
\end{tabular}

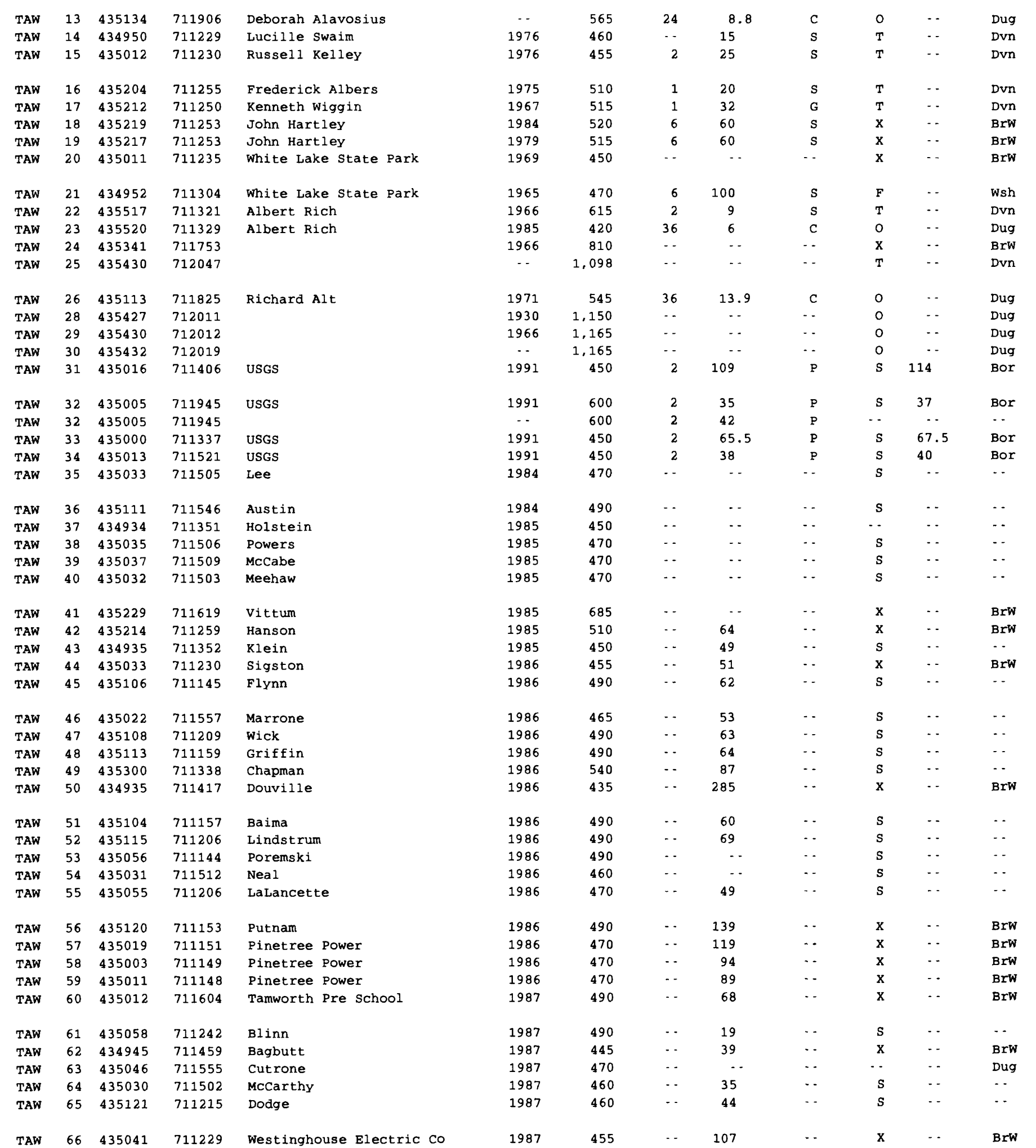




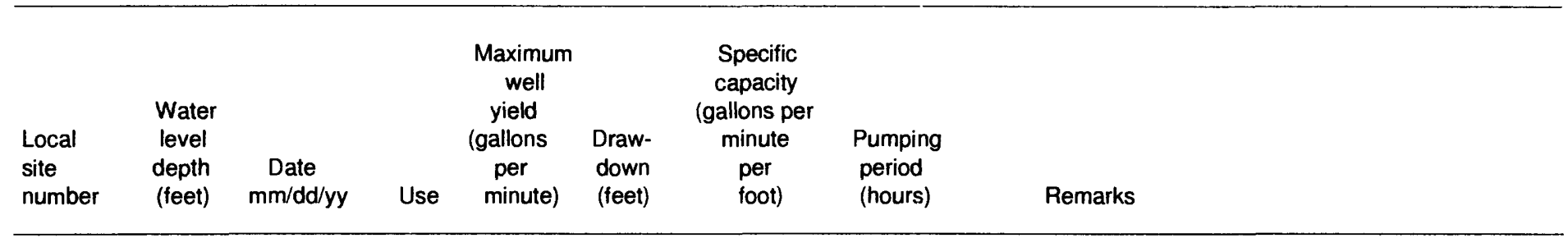

Tamworth--Continued

\begin{tabular}{|c|c|c|c|c|c|c|c|c|c|}
\hline TAW & 13 & 6.4 & $06-19-91$ & $\mathrm{H}$ & - & $\cdots$ & -- & $-\cdot$ & $\mathbf{R}$ \\
\hline TAW & 14 & - & $\cdots$ & $\mathrm{H}$ & $\cdots$ & $\cdots$ & $-\cdot$ & $-\cdot$ & Lots of iron and magnesium \\
\hline TAW & 15 & 14 & $\cdots 76$ & $\mathrm{H}$ & $\cdots$ & $\cdots$ & $-\cdot$ & $-\cdot$ & Contains iron \\
\hline TAW & 16 & - - & - & $\mathrm{H}$ & 6 & $-\cdot$ & -- & - & \\
\hline TAW & 17 & 17 & $\cdots-67$ & $\mathrm{H}$ & $-\cdot$ & $\cdots$ & $\cdots$ & $\cdots$ & A little hard \\
\hline TAW & 18 & $\cdots$ & - & $\mathrm{H}$ & 1.5 & $-\cdot$ & $\cdots$ & $-\cdot$ & \\
\hline TAW & 19 & $-\cdot$ & $-\cdot$ & $\mathrm{H}$ & 3.5 & -- & $\cdots$ & - & \\
\hline TAW & 20 & -- & $\cdots$ & $\mathrm{TH}$ & 18 & $-\cdot$ & $\cdots$ & $\cdots$ & \\
\hline TAW & 21 & $\cdots$ & + & $\mathrm{TH}$ & 200 & - & - & -- & \\
\hline TAW & 22 & 3 & $06-21-91$ & $\mathrm{H}$ & - & - & $-\cdot$ & $\cdots$ & \\
\hline TAW & 23 & 4 & $06-21 \cdot 91$ & $\mathrm{H}$ & $\cdots$ & -- & -- & - & \\
\hline TAW & 24 & 30 & $-\cdots 66$ & $\mathrm{H}$ & 3 & $\cdots$ & $\cdots$ & - & Hard water \\
\hline TAW & 25 & $-\cdot$ & - & $\mathrm{H}$ & -- & -- & $-\cdot$ & $\cdots$ & \\
\hline TAW & 26 & 4.8 & $06-21-91$ & I H & $\cdots$ & $\cdots$ & - & $\cdots$ & $\mathbf{R}$ \\
\hline TAW & 28 & 5.2 & $06-21-91$ & $\mathrm{H}$ & $-\cdot$ & $\cdots$ & $\cdots$ & $\cdots$ & High iron \\
\hline TAW & 29 & 10 & $06-21-91$ & $\mathrm{H}$ & $-\cdot$ & -- & $\cdots$ & $\cdots$ & High iron, will go dry if water garden too long \\
\hline TAW & 30 & 2 & $06-21-91$ & $\mathrm{H}$ & $\cdots$ & - & -- & - & \\
\hline TAW & 31 & 18 & $09-27-91$ & 0 & - & - & $-\cdot$ & $-\cdot$ & USGS \\
\hline TAW & 32 & -- & $\cdots$ & 0 & $-\cdot$ & - & -- & - & USGS \\
\hline TAW & 32 & - - & $\cdots$ & 0 & $\cdots$ & - & $\cdots$ & -- & \\
\hline TAW & 33 & 27 & $10-17-91$ & 0 & - - & - & - & - & USGS \\
\hline TAW & 34 & - - & - & 0 & 12 & - - & - & -- & USGS \\
\hline TAW & 35 & $-\cdot$ & - & $\mathrm{H}$ & 13 & $-\cdot$ & $\cdots$ & 2 & \\
\hline TAW & 36 & 18 & $08 \cdot 30 \cdot 84$ & $\mathrm{H}$ & 7.5 & -- & -- & 2 & \\
\hline TAW & 37 & 12 & $04-16-85$ & H & 16.5 & -- & - & 2 & \\
\hline TAW & 38 & 21 & $04-14 \cdot 85$ & $\mathrm{H}$ & 12 & -- & -- & 3 & \\
\hline TAW & 39 & 21 & $07-07-85$ & $\mathrm{H}$ & 30 & - & $-\cdot$ & 1 & \\
\hline TAW & 40 & 27 & $06-29-85$ & H & 16.5 & $\cdots$ & $\cdots$ & 2 & \\
\hline TAW & 41 & 20 & $09-06-85$ & H & 2 & - & - & .5 & \\
\hline TAW & 42 & 30 & $12-06-85$ & $\mathrm{H}$ & 10 & - & $-\cdot$ & .5 & \\
\hline TAW & 43 & 12 & $09-22-85$ & $\mathrm{H}$ & 50 & - & $\cdots$ & 2 & \\
\hline TAW & 44 & $\cdots$ & - & $\mathbf{P}$ & 40 & - & $\cdots$ & 1 & \\
\hline TAW & 45 & 50 & $01-18-86$ & $\mathrm{H}$ & 7.5 & $\cdots$ & $\cdots$ & 3 & \\
\hline TAW & 46 & 41 & $03-09-86$ & H & 6 & $\cdots$ & -- & 2 & \\
\hline TAW & 47 & 52 & $05-18-86$ & H & 8 & - & $\cdots$ & 2 & \\
\hline TAW & 48 & 47 & $05 \cdot 18 \cdot 86$ & H & 5 & -- & - & 2 & \\
\hline TAW & 49 & 30 & $07-08-86$ & H & 20 & $\cdots$ & -- & 12 & \\
\hline TAW & 50 & 15 & $08-13-86$ & H & 15 & $\cdots$ & $\cdots$ & .5 & \\
\hline TAW & 51 & 46 & $10 \cdot 25 \cdot 86$ & $\mathrm{H}$ & 10 & $\cdots$ & $-\cdot$ & 1 & \\
\hline TAW & 52 & 54 & $09-07-86$ & $\mathrm{H}$ & 6 & $\cdots$ & $\cdots$ & 2 & \\
\hline TAW & 53 & 44 & $08-23-86$ & $\mathrm{H}$ & 10 & $\cdots$ & - & 2 & \\
\hline TAW & 54 & 12 & $08-09-86$ & $\mathrm{H}$ & 40 & $\cdots$ & - & 1 & \\
\hline TAW & 55 & 33 & $07-19-86$ & $\mathrm{H}$ & 15 & $-\cdot$ & $\cdots$ & 2 & \\
\hline TAW & 56 & 50 & $10-07-86$ & H & 1.5 & $\cdots$ & -- & 1 & \\
\hline TAW & 57 & 30 & $06-25-86$ & $\cdots$ & 5 & $\cdots$ & -- & 1 & \\
\hline TAW & 58 & 30 & $06-25-86$ & $\cdots$ & 50 & $\cdots$ & $\cdots$ & 2 & \\
\hline TAW & 59 & 30 & $06-27-86$ & $\mathrm{C}$ & 150 & $\cdots$ & -- & .5 & \\
\hline TAW & 60 & 30 & $01-24-87$ & $\mathrm{H}$ & 1.3 & - & -- & 1 & \\
\hline TAW & 61 & 15 & $05-07-87$ & $\mathrm{H}$ & 35 & - & - & 1 & \\
\hline TAW & 62 & 30 & $06-29-87$ & H & 3.5 & $\cdots$ & $-\cdot$ & .5 & \\
\hline TAW & 63 & 9 & $06-24-87$ & $\mathrm{H}$ & - & -- & $\cdots$ & -- & \\
\hline TAW & 64 & 22 & $07-08-87$ & $\mathrm{H}$ & 15 & -- & $\cdots$ & 2 & \\
\hline TAW & 65 & 30.5 & $06-09-87$ & H & 8.5 & $\cdots$ & - & 2 & \\
\hline TAW & 66 & - - & - & - - & 2 & - - & - - & - & \\
\hline
\end{tabular}


Table 1-1. Description of selected wells, borings, and springs in the Saco and Ossipee River Basins, east-central

\begin{tabular}{|c|c|c|c|c|c|c|c|c|c|c|c|}
\hline $\begin{array}{l}\text { Local } \\
\text { site } \\
\text { number }\end{array}$ & $\begin{array}{l}\text { Lat- } \\
\text { itude }\end{array}$ & $\begin{array}{l}\text { Long- } \\
\text { itude }\end{array}$ & Owner or user & $\begin{array}{l}\text { Year } \\
\text { com- } \\
\text { pleted }\end{array}$ & $\begin{array}{l}\text { Alti- } \\
\text { tude } \\
\text { above } \\
\text { sea } \\
\text { level } \\
\text { (feet) }\end{array}$ & $\begin{array}{l}\text { Dia- } \\
\text { meter } \\
\text { of } \\
\text { well } \\
\text { casing } \\
\text { (inches) }\end{array}$ & $\begin{array}{l}\text { Depth } \\
\text { to } \\
\text { bottom } \\
\text { of } \\
\text { casing } \\
\text { (feet) }\end{array}$ & $\begin{array}{l}\text { Casing } \\
\text { material }\end{array}$ & $\begin{array}{l}\text { Type } \\
\text { of } \\
\text { finish }\end{array}$ & $\begin{array}{l}\text { Depth } \\
\text { to } \\
\text { bottom } \\
\text { of open } \\
\text { section } \\
\text { (feet) }\end{array}$ & $\begin{array}{c}\text { Type } \\
\text { of } \\
\text { site }\end{array}$ \\
\hline
\end{tabular}

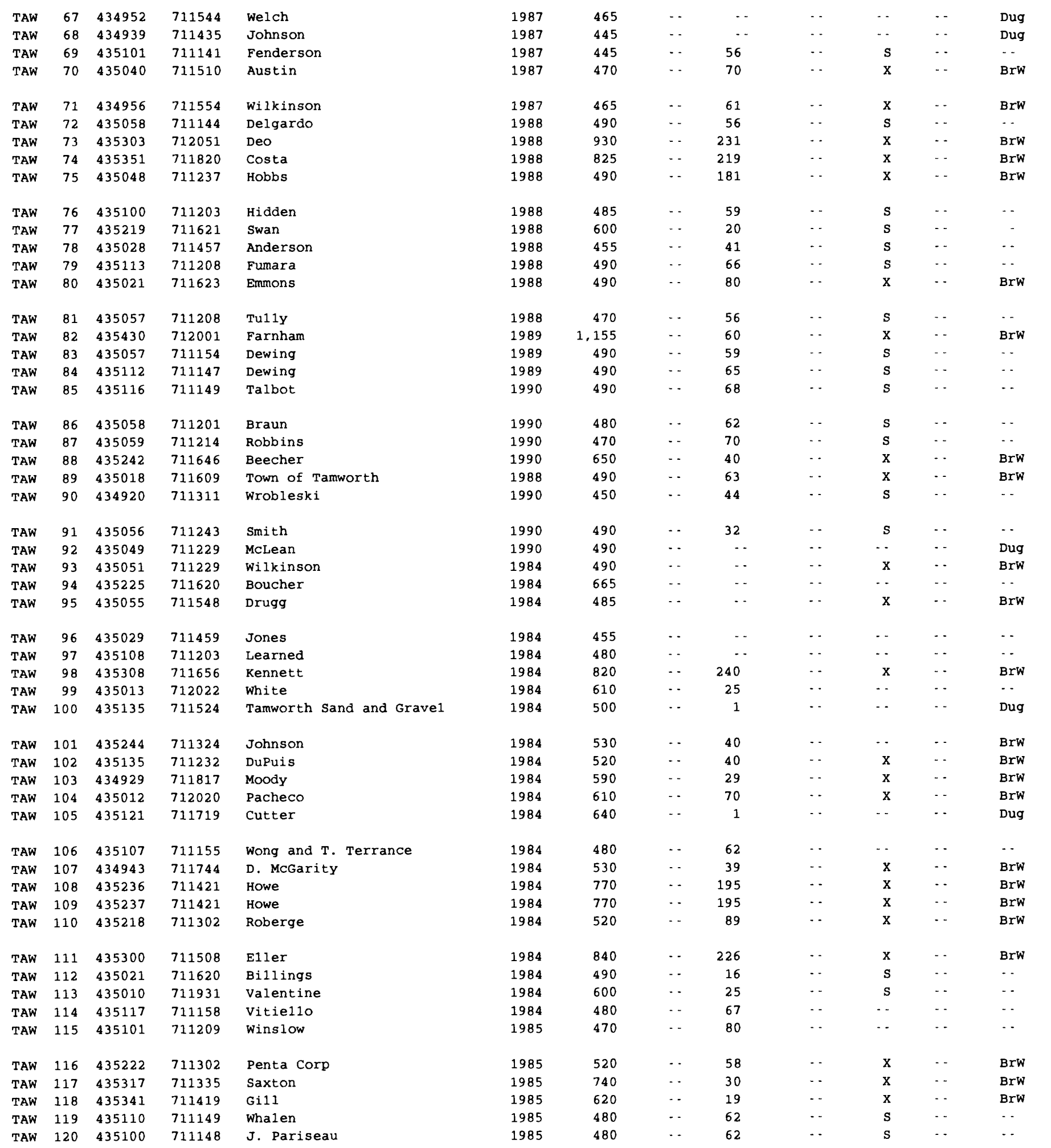




\begin{tabular}{|c|c|c|c|c|c|c|c|c|}
\hline $\begin{array}{l}\text { Local } \\
\text { site } \\
\text { number }\end{array}$ & $\begin{array}{l}\text { Water } \\
\text { level } \\
\text { depth } \\
\text { (feet) }\end{array}$ & $\begin{array}{l}\text { Date } \\
\mathrm{mm} / \mathrm{dd} / \mathrm{yy}\end{array}$ & Use & $\begin{array}{l}\text { Maximum } \\
\text { well } \\
\text { yield } \\
\text { (gallons } \\
\text { per } \\
\text { minute) }\end{array}$ & $\begin{array}{l}\text { Draw- } \\
\text { down } \\
\text { (feet) }\end{array}$ & $\begin{array}{c}\text { Specific } \\
\text { capacity } \\
\text { (gallons per } \\
\text { minute } \\
\text { per } \\
\text { foot) }\end{array}$ & $\begin{array}{l}\text { Pumping } \\
\text { period } \\
\text { (hours) }\end{array}$ & Remarks \\
\hline
\end{tabular}

Tamworth - Continued

\begin{tabular}{|c|c|c|c|c|c|c|c|c|}
\hline TAW & 67 & $\cdots$ & $\cdots$ & $\mathrm{H}$ & $\cdots$ & -- & $\cdots$ & $\therefore$ \\
\hline TAW & 68 & - - & - & $\mathrm{H}$ & - & $-\cdot$ & - & -- \\
\hline TAW & 69 & 42 & $11-14-87$ & H & 15 & - & $\cdots$ & 2 \\
\hline TAW & 70 & 40 & $09-24-87$ & H & 6 & $-\cdot$ & $\cdots$ & .5 \\
\hline TAW & 71 & 10 & $11-30-87$ & H & 0.5 & $\cdots$ & $\cdots$ & 1 \\
\hline TAW & 72 & 42 & $05-28-88$ & $\mathrm{H}$ & 10 & $\cdots$ & -- & 2 \\
\hline TAW & 73 & $\cdots$ & $\cdots$ & $\mathbf{H}$ & 35 & - & $\cdots$ & 1 \\
\hline TAW & 74 & $\cdots$ & $\cdots$ & $\cdots$ & 3 & $\cdots$ & $\cdots$ & 1 \\
\hline TAW & 75 & $\cdots$ & -- & H & 20 & $\cdots$ & $\cdots$ & .5 \\
\hline TAW & 76 & 44 & $10-02-88$ & $\mathrm{H}$ & 12 & - & $\cdots$ & 1 \\
\hline TAW & 77 & 17 & $10-31-88$ & $\mathrm{H}$ & 30 & $\cdots$ & $\cdots$ & 1 \\
\hline TAW & 78 & 18 & $11-19 \cdot 88$ & $\mathrm{H}$ & 30 & $\cdots$ & $\cdots$ & 2 \\
\hline TAW & 79 & 54 & $10-29-88$ & $\mathrm{H}$ & 15 & - & $\cdots$ & 1 \\
\hline TAW & 80 & 28 & $10-25-88$ & $\mathrm{H}$ & 1 & $\cdots$ & $\cdots$ & 1 \\
\hline TAW & 81 & 43 & $12 \cdot 10-88$ & $\mathrm{H}$ & 15 & - & $\cdots$ & 2 \\
\hline TAW & 82 & 35 & $03-27-89$ & H & 50 & - & $\cdots$ & .5 \\
\hline TAW & 83 & 44.5 & $05-07-89$ & $\mathrm{H}$ & 12 & $\cdots$ & $\cdots$ & 2 \\
\hline TAW & 84 & 50 & $09-10-89$ & $\mathrm{H}$ & 9.5 & $\cdots$ & - & 2 \\
\hline TAW & 85 & 50 & $02-24-90$ & $\mathrm{H}$ & 10 & $\cdots$ & $\cdots$ & 1 \\
\hline TAW & 86 & 47 & $01-03-90$ & $\mathrm{H}$ & 15 & $\cdots$ & $\cdots$ & 1 \\
\hline TAW & 87 & 55 & $01-28-90$ & $\mathrm{H}$ & 9 & $\cdots$ & $\cdots$ & 1 \\
\hline TAW & 88 & $\cdots$ & - & $\mathrm{H}$ & 40 & $\cdots$ & $\cdots$ & 1 \\
\hline TAW & 89 & $\cdots$ & - & $\cdots$ & 15 & $\cdots$ & $\cdots$ & 1 \\
\hline TAW & 90 & 7 & $11 \cdot 04 \cdot 90$ & $\mathrm{H}$ & 30 & $\cdots$ & $\cdots$ & 1.5 \\
\hline TAW & 91 & 29 & $12-01-90$ & $\mathrm{H}$ & 15 & $\cdots$ & $\cdots$ & 1 \\
\hline TAW & 92 & 7 & $11-27-90$ & $\mathrm{H}$ & 10 & - & - & 1.5 \\
\hline TAW & 93 & 30 & $06-19-84$ & $\mathrm{H}$ & 1 & $-\cdot$ & $\cdots$ & 1 \\
\hline TAW & 94 & 54 & $06 \cdot 05 \cdot 84$ & $\mathrm{H}$ & 6 & $\cdots$ & $\cdots$ & 3 \\
\hline TAW & 95 & 15 & $10-31-84$ & $\mathrm{H}$ & 20 & $\cdots$ & $\cdots$ & .3 \\
\hline TAW & 96 & 25 & $09-29-84$ & $\mathrm{H}$ & 15 & $\cdots$ & $\cdots$ & 2 \\
\hline TAW & 97 & 55 & $02-16-84$ & $\mathrm{H}$ & 20 & - & $\cdots$ & 18 \\
\hline TAW & 98 & 90 & $03-12-84$ & $\mathrm{H}$ & 2 & $\cdots$ & $\cdots$ & .8 \\
\hline TAW & 99 & 13.5 & $06-13-84$ & $\mathrm{H}$ & 25 & -- & -- & 2 \\
\hline TAW & 100 & 2 & $07-02-84$ & $\mathbf{z}$ & - & $\cdots$ & - & $\cdots$ \\
\hline TAW & 101 & 20 & $07-05-84$ & $\mathrm{H}$ & 12 & $\cdots$ & - & .5 \\
\hline TAW & 102 & 20 & $07-12 \cdot 84$ & $\mathrm{H}$ & 4.5 & $\cdots$ & - & .5 \\
\hline TAW & 103 & $\cdots$ & - & H & 3 & $-\cdot$ & -- & 1 \\
\hline TAW & 104 & $-\cdot$ & $\cdots$ & $\mathrm{H}$ & 5 & $\cdots$ & $\cdots$ & 1 \\
\hline TAW & 105 & $\cdots$ & $\cdots$ & $\mathrm{H}$ & $\cdots$ & $\cdots$ & $\cdots$ & - \\
\hline TAW & 106 & 46 & $05-22-84$ & $\mathrm{H}$ & 10 & - & $\cdots$ & 2 \\
\hline TAW & 107 & 10 & $10 \cdot 20 \cdot 84$ & $\mathrm{H}$ & 12 & $\cdots$ & $\cdots$ & .5 \\
\hline TAW & 108 & 90 & $12-18-84$ & $\mathrm{H}$ & 3 & $\cdots$ & $\cdots$ & .5 \\
\hline TAW & 109 & $\cdots$ & - & $\mathrm{H}$ & - & - & - & .5 \\
\hline TAW & 110 & 25 & $12-27-84$ & $\mathrm{H}$ & 75 & $\cdots$ & $\cdots$ & .5 \\
\hline TAW & 111 & $\cdots$ & - & H & 5 & - & $\cdots$ & .5 \\
\hline TAW & 112 & $\cdots$ & - & $\mathrm{H}$ & 20 & $\cdots$ & $\cdots$ & 2 \\
\hline TAW & 113 & 12 & $08-15-84$ & $\mathbf{H}$ & 40 & - & -- & 2 \\
\hline TAW & 114 & 53 & $11-15-84$ & $\mathrm{H}$ & 8 & - & $\cdots$ & 2 \\
\hline TAW & 115 & 45 & $05-17-85$ & $\mathrm{H}$ & 30 & $\cdots$ & $-\cdot$ & 12 \\
\hline TAW & 116 & 60 & $03 \cdot 06 \cdot 85$ & $\mathrm{H}$ & 25 & $\cdots$ & $\cdots$ & .5 \\
\hline TAW & 117 & 55 & $05-04-85$ & $\mathrm{H}$ & 6 & $\cdots$ & $\cdots$ & .5 \\
\hline TAW & 118 & 30 & $06-06-85$ & $\mathrm{H}$ & 5 & $\cdots$ & $\cdots$ & .5 \\
\hline TAW & 119 & 49 & $07-20-85$ & $\mathrm{H}$ & 9 & $-\cdot$ & $-\cdot$ & 3 \\
\hline TAW & 120 & - & $\cdots$ & $\mathrm{H}$ & 10 & $\cdots$ & $\cdots$ & 2 \\
\hline
\end{tabular}


Table 1-1. Description of selected wells, borings, and springs in the Saco and Ossipee River Basins, east-central

\begin{tabular}{|c|c|c|c|c|c|c|c|c|c|c|c|}
\hline $\begin{array}{l}\text { Local } \\
\text { site } \\
\text { number }\end{array}$ & $\begin{array}{l}\text { Lat- } \\
\text { itude }\end{array}$ & $\begin{array}{l}\text { Long- } \\
\text { itude }\end{array}$ & Owner or user & $\begin{array}{l}\text { Year } \\
\text { com- } \\
\text { pleted }\end{array}$ & $\begin{array}{l}\text { Alti- } \\
\text { tude } \\
\text { above } \\
\text { sea } \\
\text { level } \\
\text { (feet) }\end{array}$ & $\begin{array}{l}\text { Dia- } \\
\text { meter } \\
\text { of } \\
\text { well } \\
\text { casing } \\
\text { (inches) }\end{array}$ & $\begin{array}{l}\text { Depth } \\
\text { to } \\
\text { bottom } \\
\text { of } \\
\text { casing } \\
\text { (feet) }\end{array}$ & $\begin{array}{l}\text { Casing } \\
\text { material }\end{array}$ & $\begin{array}{l}\text { Type } \\
\text { of } \\
\text { finish }\end{array}$ & $\begin{array}{l}\text { Depth } \\
\text { to } \\
\text { bottom } \\
\text { of open } \\
\text { section } \\
\text { (feet) }\end{array}$ & $\begin{array}{c}\text { Type } \\
\text { of } \\
\text { site }\end{array}$ \\
\hline
\end{tabular}

\begin{tabular}{|c|c|c|c|c|}
\hline TAW & 121 & 435117 & 711205 & Deme110 \\
\hline TAW & 122 & 435301 & 711624 & Mahler \\
\hline TAW & 123 & 435251 & 711348 & Brothers \\
\hline TAW & 124 & 435037 & 711855 & Freeto \\
\hline TAW & 125 & 435339 & 711242 & Mille \\
\hline TAW & 126 & 435240 & 711324 & Robinson \\
\hline TAW & 127 & 434947 & 711730 & Whillemois \\
\hline TAW & 128 & 435136 & 711416 & McPeck \\
\hline TAW & 129 & 435058 & 711213 & Lavoie, Jr \\
\hline TAW & 130 & 435234 & 711321 & Pugh \\
\hline TAW & 131 & 435347 & 711232 & Brown \\
\hline TAW & 132 & 435129 & 711831 & Webster \\
\hline TAW & 133 & 435055 & 711555 & Woodside \\
\hline TAW & 134 & 435249 & 711410 & Gagnon \\
\hline TAW & 135 & 435110 & 711157 & Fusco \\
\hline TAW & 136 & 435101 & 711144 & wogman \\
\hline TAW & 137 & 435106 & 711146 & clayton \\
\hline TAW & 138 & 435113 & 711204 & zilrat \\
\hline TAW & 139 & 435118 & 711205 & Stanley \\
\hline TAW & 140 & 435105 & 711157 & N. Lisano \\
\hline TAW & 141 & 435056 & 711236 & Avellani \\
\hline TAW & 142 & 435144 & 711648 & Palmer \\
\hline TAW & 143 & 434929 & 711811 & Tappen \\
\hline TAW & 144 & 435103 & 711205 & Powers \\
\hline TAW & 145 & 435058 & 711150 & Pieroni \\
\hline TAW & 146 & 435059 & 711145 & F. Pizzut \\
\hline TAW & 147 & 435117 & 711148 & Randozza \\
\hline TAW & 148 & 435105 & 711152 & Foley \\
\hline TAW & 149 & 435107 & 711158 & Boisvert \\
\hline TAW & 150 & 435203 & 711649 & Reigate Realty Trust \\
\hline TAW & 151 & 434804 & 711826 & Perry \\
\hline TAW & 152 & 435236 & 711325 & Roberts \\
\hline TAW & 153 & 435257 & 711456 & Marston \\
\hline TAW & 154 & 435018 & 711233 & Robiller \\
\hline TAW & 155 & 435016 & 711233 & Robiller \\
\hline TAW & 156 & 435115 & 711202 & S. Leong \\
\hline TAW & 157 & 435250 & 711240 & Chocorua Meadows Association \\
\hline TAW & 158 & 435000 & 711214 & Lundberg \\
\hline TAW & 159 & 435249 & 711419 & Yannalfo \\
\hline TAW & 160 & 435459 & 711333 & Creps \\
\hline TAW & 161 & 435238 & 711320 & Dyrenforth \\
\hline TAW & 162 & 435248 & 711423 & Kaufhold \\
\hline TAW & 163 & 435112 & 711147 & M. Pepin \\
\hline TAW & 164 & 435107 & 711200 & M. Merrill \\
\hline TAW & 165 & 435123 & 711213 & T. Greco \\
\hline TAW & 166 & 435125 & 711212 & M. Knapp \\
\hline TAW & 167 & 435135 & 711228 & Lloyd \\
\hline TAW & 168 & 435251 & 711452 & E11iott \\
\hline TAW & 169 & 435105 & 711200 & Kohut \\
\hline TAW & 170 & 435247 & 711328 & Whitting's Auto Body \\
\hline TAW & 1.71 & 435226 & 711623 & Norcross \\
\hline TAW & 172 & 435003 & 711148 & Pinetree Power \\
\hline TAW & 173 & 435059 & 711153 & J. Deslauriers \\
\hline TAW & 174 & 435118 & 711207 & H. Booska \\
\hline TAW & 175 & 435425 & 711734 & Lenauer \\
\hline
\end{tabular}

Tamworth - Continued

\begin{tabular}{|c|c|c|c|c|c|c|c|}
\hline 1985 & 480 & $\cdots$ & 62 & $\cdots$ & $\mathbf{S}$ & - & - \\
\hline 1985 & 820 & $\cdots$ & 60 & $\cdots$ & $x$ & - & BrW \\
\hline 1985 & 550 & $\cdots$ & 151 & - & $x$ & $\cdots$ & BrW \\
\hline 1985 & 720 & $\cdots$ & 209 & - & $x$ & $\cdots$ & BrW \\
\hline 1985 & 770 & - & 153 & - & $\mathrm{x}$ & -- & BrW \\
\hline 1985 & 560 & - & 25 & . & $x$ & - & BrW \\
\hline 1985 & 510 & $\cdots$ & 35 & - - & $x$ & $\cdots$ & BrW \\
\hline 1985 & 700 & - & 200 & $\cdots$ & $x$ & $\cdots$ & BrW \\
\hline 1984 & 470 & $\cdots$ & 62 & - & $\mathbf{s}$ & $\cdots$ & $\cdots$ \\
\hline 1985 & 560 & $\cdots$ & 36 & - & $x$ & $\cdots$ & BrW \\
\hline 1985 & 820 & $\cdots$ & 59 & $\cdots$ & $x$ & $\cdots$ & BrW \\
\hline 1986 & 560 & $\cdots$ & 40 & $\cdots$ & $x$ & $\cdots$ & Brw \\
\hline 1985 & 480 & $\cdots$ & 36 & - & $x$ & - & BrW \\
\hline 1985 & 640 & $\cdots$ & 49 & - & $\mathrm{x}$ & $\cdots$ & BrW \\
\hline 1985 & 480 & - & 62 & - & $\mathbf{S}$ & $\cdots$ & $\cdots$ \\
\hline 1985 & 480 & - - & 62 & $\cdots$ & $\mathrm{S}$ & $\cdots$ & $\cdots$ \\
\hline 1985 & 480 & $\cdots$ & 63 & - & $\mathbf{S}$ & - & $\cdots$ \\
\hline 1985 & 480 & $\cdots$ & 62 & - & $\mathrm{S}$ & $\cdots$ & $\cdots$ \\
\hline 1985 & 480 & - & 62 & $\cdots$ & $\mathbf{S}$ & - & - \\
\hline 1985 & 480 & $\cdots$ & 62 & $\cdots$ & $\mathbf{S}$ & $\cdots$ & $\cdots$ \\
\hline 1985 & 480 & $\cdots$ & 41 & $\cdots$ & $\mathbf{S}$ & $\cdots$ & 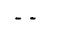 \\
\hline 1986 & 670 & - & 174 & $\cdots$ & $x$ & $\cdots$ & BrW \\
\hline 1986 & 600 & $\cdots$ & 39 & $\cdots$ & $x$ & $\cdots$ & BrW \\
\hline 1986 & 480 & $\cdots$ & 50 & - & $\mathrm{s}$ & $\cdots$ & $\cdots$ \\
\hline 1986 & 480 & $\cdots$ & 62 & - & $\mathbf{s}$ & $\cdots$ & $\cdots$ \\
\hline 1986 & 480 & - & 59 & - - & $\mathrm{s}$ & - & $\ldots$ \\
\hline 1986 & 480 & $\cdots$ & 67 & $\cdots$ & $\mathrm{S}$ & -- & $\cdots$ \\
\hline 1986 & 480 & $\cdots$ & 59 & - & $\mathrm{s}$ & $\cdots$ & $\cdots$ \\
\hline 1986 & 480 & $\cdots$ & 59 & - & $\mathrm{s}$ & - & $\cdots$ \\
\hline 1986 & 640 & $\cdots$ & 120 & $\cdots$ & $x$ & $\cdots$ & BrW \\
\hline 1986 & 1.080 & $\cdots$ & 199 & $\cdots$ & $x$ & $\cdots$ & BrW \\
\hline 1986 & 580 & $\cdots$ & 21 & $\cdots$ & $x$ & $\cdots$ & BrW \\
\hline 1986 & 840 & $\cdots$ & 131 & $\cdots$ & $x$ & $\cdots$ & $\mathrm{BrW}$ \\
\hline 1987 & 450 & $\cdots$ & 28 & - & $\mathrm{S}$ & $\cdots$ & - \\
\hline 1987 & 450 & $\cdots$ & 28 & $-\cdot$ & $\mathbf{S}$ & $\cdots$ & $\cdots$ \\
\hline 1987 & 480 & $\cdots$ & 63 & $\cdots$ & $\mathbf{S}$ & $\cdots$ & $-\cdots$ \\
\hline 1987 & 590 & $\cdots$ & 173 & $\cdots$ & $x$ & $\cdots$ & BrW \\
\hline 1987 & 430 & $\cdots$ & 44 & $\cdots$ & $\cdots$ & $\cdots$ & $\cdots$ \\
\hline 1987 & 680 & - & - & $\cdots$ & $\mathrm{x}$ & $\cdots$ & BrW \\
\hline 1987 & 680 & $\cdots$ & 19 & $\cdots$ & $x$ & $\cdots$ & BrW \\
\hline 1987 & 540 & $\cdots$ & 39 & -- & $x$ & $\cdots$ & BrW \\
\hline 1987 & 730 & $\cdots$ & 37 & $\cdots$ & $x$ & $\cdots$ & BrW \\
\hline 1987 & 480 & $-\cdot$ & 62 & $\cdots$ & $\mathbf{S}$ & $\cdots$ & $\cdots$ \\
\hline 1987 & 480 & $\cdots$ & 62 & $\cdots$ & $\mathrm{S}$ & $\cdots$ & $\cdots$ \\
\hline 1987 & 450 & $\cdots$ & 26 & $\cdots$ & $\mathrm{s}$ & $\cdots$ & $\cdots$ \\
\hline 1987 & 450 & $\cdots$ & 21 & $-\cdot$ & $\mathrm{S}$ & $\cdots$ & $\cdots$ \\
\hline 1987 & 500 & - & 129 & $-\cdot$ & $x$ & - & BrW \\
\hline 1987 & 840 & - & 60 & -- & $x$ & $\cdots$ & BrW \\
\hline 1.987 & 480 & - & 62 & $\cdots$ & $\mathbf{S}$ & $\cdots$ & $\cdots$ \\
\hline 1987 & 530 & $\cdots$ & 20 & $\cdots$ & $\mathbf{S}$ & $\cdots$ & $\cdots$ \\
\hline 1987 & 660 & $\cdots$ & 120 & $\cdots$ & $x$ & $\cdots$ & BrW \\
\hline 1987 & 470 & $\cdots$ & 135 & - & $x$ & $\cdots$ & Brw \\
\hline 1987 & 480 & $\cdots$ & 62 & $\cdots$ & $\mathrm{S}$ & $\cdots$ & - \\
\hline 1987 & 480 & $\cdots$ & 71 & $\cdots$ & $s$ & $\cdots$ & $\cdots$ \\
\hline 1987 & 840 & - & 253 & -. & $x$ & - & $\mathrm{BrW}$ \\
\hline
\end{tabular}




\begin{tabular}{|c|c|c|c|c|c|c|c|c|}
\hline $\begin{array}{l}\text { Local } \\
\text { site } \\
\text { number }\end{array}$ & $\begin{array}{l}\text { Water } \\
\text { level } \\
\text { depth } \\
\text { (feet) }\end{array}$ & $\begin{array}{l}\text { Date } \\
\mathrm{mm} / \mathrm{dd} / \mathrm{yy}\end{array}$ & Use & $\begin{array}{l}\text { Maximum } \\
\text { well } \\
\text { yield } \\
\text { (gallons } \\
\text { per } \\
\text { minute) }\end{array}$ & $\begin{array}{l}\text { Draw- } \\
\text { down } \\
\text { (feet) }\end{array}$ & $\begin{array}{c}\text { Specific } \\
\text { capacity } \\
\text { (gallons per } \\
\text { minute } \\
\text { per } \\
\text { foot) }\end{array}$ & $\begin{array}{l}\text { Pumping } \\
\text { period } \\
\text { (hours) }\end{array}$ & Remarks \\
\hline
\end{tabular}

\begin{tabular}{|c|c|c|c|c|c|c|c|c|}
\hline \multirow[b]{2}{*}{ TAW } & \multirow[b]{2}{*}{121} & \multirow[b]{2}{*}{52.5} & \multirow[b]{2}{*}{$07-12-85$} & \multirow[b]{2}{*}{ H } & \multirow[b]{2}{*}{7.5} & \multirow[b]{2}{*}{ - } & \multicolumn{2}{|c|}{ Tamworth--Cont $i$} \\
\hline & & & & & & & -. & 3 \\
\hline TAW & 122 & 75 & $07-17-85$ & $\mathrm{H}$ & 8.5 & - & -. & .5 \\
\hline TAW & 123 & 60 & $08-02-85$ & H & 30 & . & $\cdots$ & .5 \\
\hline TAW & 124 & 100 & $08-14-85$ & H & 1.5 & $\ldots$ & .. & .5 \\
\hline TAW & 125 & - & - & $\mathrm{H}$ & 0.1 & - & -. & 1 \\
\hline TAW & 126 & 15 & $08-20-85$ & H & 15 & $\cdots$ & - & .5 \\
\hline TAW & 127 & 12 & $08-27-85$ & $\mathrm{H}$ & 50 & $\cdots$ & - & .5 \\
\hline TAW & 128 & & - & H & 75 & $\cdots$ & $\cdots$ & .5 \\
\hline TAW & 129 & 48 & $11-02-84$ & H & 10 & .. & - & 3 \\
\hline TAW & 130 & 45 & $10-12 \cdot 85$ & H & 0.8 & - & $\cdots$ & 1 \\
\hline TAW & 131 & 60 & $10-15-85$ & $\mathrm{H}$ & 25 & - & -. & .5 \\
\hline TAW & 132 & 35 & $10-18-86$ & H & 1.8 & $\cdots$ & .. & 1 \\
\hline TAW & 133 & 30 & $11-08-85$ & H & 1.8 & $\cdots$ & -. & .5 \\
\hline TAW & 134 & 90 & $12-23 \cdot 85$ & H & 20 & - & - & .5 \\
\hline TAW & 135 & 49 & $09-13-85$ & $\mathrm{H}$ & 10 & - & - & 2 \\
\hline TAW & 136 & 48 & $09-28-85$ & H & 10 & $\cdots$ & $\cdots$ & 2 \\
\hline TAW & 137 & 49.5 & $09-29-85$ & H & 7.5 & -. & -. & 2 \\
\hline TAW & 138 & 54 & $08-10-85$ & H & 8.5 & $\cdots$ & $\cdots$ & 2 \\
\hline TAW & 139 & 53 & $08-17-85$ & H & 8 & $\ldots$ & -. & 2 \\
\hline TAW & 140 & 47.5 & $08-01-85$ & $\mathrm{H}$ & 9 & $\cdots$ & $\cdots$ & 3 \\
\hline TAW & 141 & 33 & $08-03-85$ & $\mathrm{H}$ & 6.5 & $\cdots$ & - & 5 \\
\hline TAW & 142 & - & - & H & 1.3 & - & - & .5 \\
\hline TAW & 143 & 25 & $09-18-86$ & H & 50 & $\cdots$ & $\cdots$ & .5 \\
\hline TAW & 144 & 35 & $10-12-86$ & H & 10 & - & -. & 2 \\
\hline TAW & 145 & 45 & $10-02-86$ & H & 6 & - & - & 1 \\
\hline TAW & 146 & 45 & $06-10-86$ & H & 12 & $\cdots$ & - & 1.5 \\
\hline TAW & 147 & 49 & $08-30-86$ & н & 10 & - & -. & 1 \\
\hline TAW & 148 & 42 & $08-30-86$ & H & 12 & $\cdots$ & $\cdots$ & 1.5 \\
\hline TAW & 149 & 46 & $08-30-86$ & н & 10.5 & - & - & 1 \\
\hline TAW & 150 & 100 & $11-06-86$ & H & 30 & - & $\cdots$ & .5 \\
\hline TAW & 151 & 13 & $10-24-86$ & $\mathrm{H}$ & 30 & $\cdots$ & - & 2 \\
\hline TAW & 152 & 40 & $12-04-86$ & H & 6 & - & - & .5 \\
\hline TAW & 153 & 40 & $12-30-86$ & H & 1.5 & - & -. & .5 \\
\hline TAW & 154 & 19 & $05-17-87$ & H & 20 & -. & $\cdots$ & 1 \\
\hline TAW & 155 & 19 & $05-17-87$ & H & 20 & $\cdots$ & - & 1 \\
\hline TAW & 156 & 48 & $05-16-87$ & H & 12 & $\cdots$ & $\cdots$ & 2 \\
\hline TAW & 157 & - & . & P & 25 & $\cdots$ & $\cdots$ & 1 \\
\hline TAW & 158 & 15 & $01-05-87$ & P & 35 & $\cdots$ & $\cdots$ & 6 \\
\hline TAW & 159 & 110 & $06.03 \cdot 87$ & $\mathrm{H}$ & 6.5 & - & - & .5 \\
\hline TAW & 160 & 30 & $06-18-87$ & H & 0.3 & - & - & 1 \\
\hline TAW & 161 & - & - & H & 1.3 & $\cdots$ & $\cdots$ & 1 \\
\hline TAW & 162 & 10 & $04-23-87$ & H & 15 & - & -. & .5 \\
\hline TAW & 163 & 48 & $07-19-87$ & $\mathrm{H}$ & 10 & $\cdots$ & -. & 3 \\
\hline TAW & 164 & 48 & $08 \cdot 07 \cdot 87$ & H & 11 & $\cdots$ & -. & 3 \\
\hline TAW & 165 & 11 & $08-08-87$ & H & 40 & $\cdots$ & $\cdots$ & 2 \\
\hline TAW & 166 & 6 & $08-08-87$ & H & 50 & $\cdots$ & $\cdots$ & 2 \\
\hline TAW & 167 & 150 & $08-27-87$ & $\mathrm{H}$ & 2.5 & $\cdots$ & $\cdots$ & .5 \\
\hline TAW & 168 & 60 & $07-10-87$ & $\mathrm{H}$ & 12 & $\cdots$ & $\cdots$ & .5 \\
\hline TAW & 169 & 46.5 & $08-15-87$ & H & 11 & $\cdots$ & $\cdots$ & 2 \\
\hline TAW & 170 & 2 & $09-05-87$ & H & 30 & $\cdots$ & $\cdots$ & 2 \\
\hline TAW & 171 & $\cdots$ & $\cdots$ & H & 2 & $\cdots$ & - & 1 \\
\hline TAW & 172 & - & - & - & 10 & $\cdots$ & $\cdots$ & - \\
\hline TAW & 173 & 44 & $09-19-87$ & $\mathrm{H}$ & 10 & $\cdots$ & $\cdots$ & 2 \\
\hline TAW & 174 & 52 & $10-03-87$ & H & 10 & - & -. & 2 \\
\hline TAW & 175 & - & - & н & 1.5 & - & - & 1 \\
\hline
\end{tabular}




\begin{tabular}{|c|c|c|c|c|c|c|c|c|c|c|c|}
\hline $\begin{array}{l}\text { Local } \\
\text { site } \\
\text { number }\end{array}$ & $\begin{array}{l}\text { Lat- } \\
\text { itude }\end{array}$ & $\begin{array}{l}\text { Long- } \\
\text { itude }\end{array}$ & Owner or user & $\begin{array}{l}\text { Year } \\
\text { com- } \\
\text { pleted }\end{array}$ & $\begin{array}{c}\text { Alti- } \\
\text { tude } \\
\text { above } \\
\text { sea } \\
\text { level } \\
\text { (feet) }\end{array}$ & $\begin{array}{l}\text { Dia- } \\
\text { meter } \\
\text { of } \\
\text { well } \\
\text { casing } \\
\text { (inches) }\end{array}$ & $\begin{array}{l}\text { Depth } \\
\text { to } \\
\text { bottom } \\
\text { of } \\
\text { casing } \\
\text { (feet) }\end{array}$ & $\begin{array}{l}\text { Casing } \\
\text { material }\end{array}$ & $\begin{array}{c}\text { Type } \\
\text { of } \\
\text { finish }\end{array}$ & $\begin{array}{l}\text { Depth } \\
\text { to } \\
\text { bottom } \\
\text { of open } \\
\text { section } \\
\text { (feet) }\end{array}$ & $\begin{array}{c}\text { Type } \\
\text { of } \\
\text { site }\end{array}$ \\
\hline
\end{tabular}

\begin{tabular}{|c|c|c|c|c|}
\hline TAW & 176 & 435106 & 711202 & R. Boudrot \\
\hline TAW & 177 & 435101 & 711201 & Butler \\
\hline TAW & 178 & 435112 & 711204 & P. Coscia \\
\hline TAW & 179 & 435006 & 711222 & Kirkpatrick \\
\hline TAW & 180 & 435008 & 711222 & Miracle \\
\hline TAW & 181 & 435055 & 711620 & Webster \\
\hline TAW & 182 & 435201 & 711618 & Lord \\
\hline TAW & 183 & 435206 & 711612 & Dulski \\
\hline TAW & 184 & 435010 & 711130 & Cook Family Trust \\
\hline TAW & 185 & 435106 & 711207 & Fiumara \\
\hline TAW & 186 & 435103 & 711152 & K. Finlestein \\
\hline TAW & 187 & 435114 & 711146 & G. Learned \\
\hline TAW & 188 & 435104 & 711145 & Ruo \\
\hline TAW & 189 & 435313 & 711347 & Woodward \\
\hline TAW & 190 & 435107 & 711148 & Katsos \\
\hline TAW & 191 & 435108 & 711206 & Riccardi \\
\hline TAW & 192 & 435122 & 711217 & Hervieux \\
\hline TAW & 193 & 435341 & 711820 & O'Brien \\
\hline TAW & 194 & 435140 & 711641 & Burton \\
\hline TAW & 195 & 435252 & 711449 & B. Shipley \\
\hline TAW & 196 & 435455 & 711331 & New Dartmouth Bank \\
\hline TAW & 197 & 435100 & 711151 & Huddleston \\
\hline TAW & 198 & 435103 & 711145 & J. Ashe \\
\hline TAW & 199 & 435140 & 711640 & Burton \\
\hline TAW & 200 & 434958 & 711229 & Capalbo \\
\hline TAW & 201 & 435135 & 711421 & Staples \\
\hline TAW & 202 & 435142 & 711240 & Lonigro \\
\hline TAW & 203 & 435102 & 711142 & Campbe11-Smith \\
\hline TAW & 204 & 435249 & 711343 & Cavalieri \\
\hline TAW & 205 & 435212 & 711238 & O'Hanian \\
\hline TAW & 206 & 435103 & 711201 & Fusco \\
\hline TAW & 207 & 435103 & 711209 & Birth \\
\hline TAW & 208 & 435114 & 711207 & Austin \\
\hline TAW & 209 & 435003 & 711601 & Martin Carrier Corp \\
\hline TAW & 210 & 435005 & 711601 & Martin Carrier Corp \\
\hline TAW & 211 & 434944 & 711222 & Chute \\
\hline TAW & 212 & 434929 & 711818 & N. Nielson \\
\hline TAW & 213 & 435017 & 711232 & Robiller \\
\hline TAW & 214 & 435311 & 711357 & T. Leonard \\
\hline TAW & 215 & 435150 & 711233 & Sampson \\
\hline TAW & 216 & 435059 & 711200 & C. Rebello \\
\hline TAW & 217 & 435111 & 711204 & L. Perry \\
\hline TAW & 218 & 435238 & 711608 & Elliott \\
\hline TAW & 219 & 435044 & 711227 & Ross \\
\hline TAW & 220 & 435110 & 711154 & c. Tierney \\
\hline TAW & 221 & 435109 & 711151 & D. Kenney \\
\hline TAW & 222 & 435243 & 711312 & Fortier \\
\hline TAW & 223 & 435240 & 711310 & L. Brett \\
\hline TAW & 224 & 435242 & 711310 & Free Baptist Society \\
\hline TAW & 225 & 435158 & 711707 & Hidden \\
\hline TAW & 226 & 435122 & 711829 & Bunker Hill Trust \\
\hline TAW & 227 & 435105 & 711155 & Ayers \\
\hline TAW & 228 & 435429 & 711859 & S. Cargil1 \\
\hline TAW & 229 & 435102 & 711151 & Bradshaw \\
\hline TAW & 230 & 435004 & 711606 & woodman \\
\hline
\end{tabular}

\section{Tamworth - - Continued}

\begin{tabular}{|c|c|c|c|c|c|c|c|}
\hline 1987 & 480 & $\cdots$ & 62 & $\cdots$ & $\mathbf{S}$ & $\cdots$ & $\cdots$ \\
\hline 1987 & 480 & - & 62 & -- & $\mathrm{s}$ & $\cdots$ & $\cdots$ \\
\hline 1987 & 480 & $\cdots$ & 63.5 & $\cdots$ & $\mathrm{s}$ & - & $\cdots$ \\
\hline 1987 & 440 & -- & 69 & $\cdots$ & $x$ & - & BrW \\
\hline 1988 & 440 & - & 101 & $\cdots$ & $x$ & -- & Brw \\
\hline 1987 & 520 & -- & 202 & $\cdots$ & $x$ & $\cdots$ & BrW \\
\hline $1988^{\circ}$ & 560 & . & 90 & - - & $x$ & $\cdots$ & Brw \\
\hline 1988 & 580 & -- & 132 & - & $\mathrm{x}$ & $\cdots$ & BrW \\
\hline 1988 & 460 & -- & 39 & $\cdots$ & $\mathrm{s}$ & - & - \\
\hline 1988 & 480 & $\cdots$ & 50 & $\cdots$ & $\mathrm{S}$ & $\cdots$ & - \\
\hline 1988 & 480 & $\cdots$ & 62 & $\cdots$ & $\mathbf{S}$ & $\cdots$ & .. \\
\hline 1988 & 480 & $\cdots$ & 68 & - & $\mathbf{s}$ & $\cdots$ & -- \\
\hline 1988 & 480 & $\ldots$ & 60 & - & $\mathrm{s}$ & - & $\cdots$ \\
\hline 1988 & 640 & $\cdots$ & 80 & -- & $x$ & -- & BrW \\
\hline 1988 & 480 & - & 59 & $\cdots$ & $\mathrm{s}$ & $\cdots$ & $\cdots$ \\
\hline 1988 & 480 & $\cdots$ & 64 & - & $\mathrm{s}$ & - & $\cdots$ \\
\hline 1988 & 450 & - & 120 & - & $x$ & $\cdots$ & BrW \\
\hline 1988 & 840 & 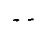 & 241 & $\cdots$ & $\mathrm{x}$ & -- & Brw \\
\hline 1988 & 640 & $\cdots$ & 2 & -- & $\cdots$ & $\cdots$ & Dug \\
\hline 1988 & 840 & $\cdots$ & 59 & $\cdots$ & $x$ & $\cdots$ & BrW \\
\hline 1988 & 700 & $\ldots$ & 19 & - & $\mathrm{x}$ & $\cdots$ & Brw \\
\hline 1988 & 480 & $\cdots$ & 60 & $\cdots$ & $\mathbf{s}$ & $\cdots$ & -- \\
\hline 1988 & 480 & - & 62 & $\cdots$ & $\mathrm{S}$ & - & $\cdots$ \\
\hline 1988 & 640 & $\cdots$ & 214 & - & $x$ & - - & BrW \\
\hline 1988 & 450 & $\cdots$ & 26 & - & $s$ & $\cdots$ & $\cdots$ \\
\hline 1988 & 740 & $\cdots$ & 249 & - & $x$ & $\cdots$ & BrW \\
\hline 1988 & 510 & $\cdots$ & 29 & - & $x$ & - & BrW \\
\hline 1988 & 480 & - & 64 & - & $\mathrm{s}$ & - & $\cdots$ \\
\hline 1988 & 540 & - & 20 & - & $\mathrm{S}$ & $\cdots$ & $\cdots$ \\
\hline 1988 & 500 & $\cdots$ & 105 & $\cdots$ & $x$ & $\cdots$ & BrW \\
\hline 1988 & 480 & - & 63 & $\cdots$ & $\mathbf{s}$ & $\cdots$ & $\cdots$ \\
\hline 1988 & 470 & - - & 84 & - & $\mathrm{s}$ & - & $\ldots$ \\
\hline 1988 & 480 & - - & 79 & $\cdots$ & $\mathrm{s}$ & $\cdots$ & $\cdots$ \\
\hline 1988 & 480 & $\cdots$ & 90 & -- & $x$ & $\cdots$ & BrW \\
\hline 1988 & 480 & $\cdots$ & 85 & - - & $x$ & $\cdots$ & BrW \\
\hline 1988 & 440 & - - & 74 & $\cdots$ & $x$ & - & BrW \\
\hline 1988 & 590 & $\cdots$ & 39 & - & $x$ & $\cdots$ & $\mathrm{BrW}$ \\
\hline 1989 & 450 & - & 65 & . & $x$ & - & BrW \\
\hline 1989 & 580 & - & 146 & $\cdots$ & $x$ & $\cdots$ & Brw \\
\hline 1989 & 480 & $\cdots$ & 62 & - & $x$ & $\cdots$ & BrW \\
\hline 1989 & 480 & - & 65 & - & $\mathrm{s}$ & - & - \\
\hline 1989 & 480 & $\cdots$ & 68 & - - & $\mathrm{s}$ & . & $\cdots$ \\
\hline 1989 & 740 & $\cdots$ & 204 & - - & $x$ & $\cdots$ & BrW \\
\hline 1989 & 480 & -. & 76 & - & $\mathrm{s}$ & - - & -- \\
\hline 1989 & 480 & $\cdots$ & 70.5 & - & $\mathrm{s}$ & - & $\therefore$ \\
\hline 1989 & 480 & $\cdots$ & 62 & $\cdots$ & $\mathbf{s}$ & - - & -- \\
\hline 1989 & 520 & $\cdots$ & 138 & $\cdots$ & $x$ & - & BrW \\
\hline 1989 & 520 & - & 118 & - - & $x$ & $\cdots$ & BrW \\
\hline 1989 & 520 & $\cdots$ & 111 & - & $x$ & $\cdots$ & Brw \\
\hline 1989 & 750 & - - & 108 & - & $x$ & - & BrW \\
\hline 1990 & 560 & - & 80 & - & $x$ & - & BrW \\
\hline 1990 & 480 & - & 62 & - & G & $\cdots$ & -. \\
\hline 1990 & 960 & -. & 14 & - & $\cdots$ & $\cdots$ & Dug \\
\hline 1990 & 480 & - & 62 & - & $\mathbf{s}$ & - & - \\
\hline 1990 & 480 & -. & 75 & $\cdots$ & $\mathrm{x}$ & - & BrW \\
\hline
\end{tabular}




\begin{tabular}{|c|c|c|c|c|c|c|c|c|}
\hline $\begin{array}{l}\text { Local } \\
\text { site } \\
\text { number }\end{array}$ & $\begin{array}{l}\text { Water } \\
\text { level } \\
\text { depth } \\
\text { (feet) }\end{array}$ & $\begin{array}{l}\text { Date } \\
\mathrm{mm} / \mathrm{dd} / \mathrm{yy}\end{array}$ & Use & $\begin{array}{l}\text { Maximum } \\
\text { well } \\
\text { yield } \\
\text { (gallons } \\
\text { per } \\
\text { minute) }\end{array}$ & $\begin{array}{l}\text { Draw- } \\
\text { down } \\
\text { (feet) }\end{array}$ & $\begin{array}{l}\text { Specific } \\
\text { capacity } \\
\text { (gallons per } \\
\text { minute } \\
\text { per } \\
\text { foot) }\end{array}$ & $\begin{array}{l}\text { Pumping } \\
\text { period } \\
\text { (hours) }\end{array}$ & Remarks \\
\hline
\end{tabular}

\begin{tabular}{|c|c|c|c|c|c|c|c|c|}
\hline TAW & 176 & 48.5 & $12-05-87$ & $\mathrm{H}$ & 12 & $\ldots$ & $\cdots$ & 2 \\
\hline TAW & 177 & 47 & $12 \cdot 05-87$ & $\mathrm{H}$ & 13 & $\cdots$ & $\cdots$ & 2 \\
\hline TAW & 178 & 50 & $11-14-87$ & $\mathrm{H}$ & 10 & $\cdots$ & $\cdots$ & 2 \\
\hline TAW & 179 & - & - & $\mathrm{H}$ & 25 & $\cdots$ & - & 2 \\
\hline TAW & 180 & $\cdots$ & $\cdots$ & $\mathrm{H}$ & 3 & $\cdots$ & $\cdots$ & 1.5 \\
\hline TAW & 181 & 27 & $12 \cdot 02 \cdot 87$ & $\mathrm{H}$ & 4 & $\cdots$ & $\cdots$ & 1 \\
\hline TAW & 182 & 30 & $03-09-88$ & $\mathrm{H}$ & 100 & -- & - & .5 \\
\hline TAW & 183 & 60 & $03-10-88$ & $\mathrm{H}$ & 60 & $\ldots$ & $-\cdot$ & .5 \\
\hline TAW & 184 & 27 & $04-17-88$ & $\mathrm{H}$ & 10 & - & $\cdots$ & 2 \\
\hline TAW & 185 & 32 & $04-16-88$ & $H$ & 15 & $\cdots$ & -- & 2 \\
\hline TAW & 186 & 44 & $04-30-88$ & $\mathrm{H}$ & 12 & $\cdots$ & $\cdots$ & 1.5 \\
\hline TAW & 187 & 53 & $05-09-88$ & $\mathrm{H}$ & 10 & $\cdots$ & $\cdots$ & 1 \\
\hline TAW & 188 & 48 & $05-28-88$ & $\mathrm{H}$ & 10 & - & - & 2 \\
\hline TAW & 189 & - & - & $\mathrm{H}$ & 8 & $\cdots$ & $\cdots$ & 1 \\
\hline TAW & 190 & 48 & $07-24-88$ & $\mathrm{H}$ & 11 & $\cdots$ & $\cdots$ & 2 \\
\hline TAW & 191 & 48.5 & $07-24-88$ & $\mathrm{H}$ & 10 & -- & $-\cdot$ & 1 \\
\hline TAW & 192 & 30 & $06-03-88$ & $\mathrm{H}$ & 4 & - & $\cdots$ & .5 \\
\hline TAW & 193 & 60 & $07-20-88$ & $\mathrm{H}$ & 40 & $\cdots$ & $\cdots$ & .5 \\
\hline TAW & 194 & - - & - & $\mathrm{H}$ & - - & $\cdots$ & -- & -- \\
\hline TAW & 195 & 50 & $06-30-88$ & $\mathrm{H}$ & 1 & - & $\cdots$ & .5 \\
\hline TAW & 196 & 30 & $07-14-88$ & $\mathrm{H}$ & 10 & $\cdots$ & $\cdots$ & 1 \\
\hline TAW & 197 & 47 & $10-30-88$ & $\mathrm{H}$ & 13 & $\cdots$ & - & 1 \\
\hline TAW & 198 & 50 & $11 \cdot 20 \cdot 88$ & $\mathrm{H}$ & 12 & $\cdots$ & $-\cdot$ & 1 \\
\hline TAW & 199 & - - & - & $\mathrm{H}$ & 7 & $\cdots$ & - & 1 \\
\hline TAW & 200 & 19 & $10-22-88$ & $\mathrm{H}$ & 12 & $-\cdot$ & $\cdots$ & 1 \\
\hline TAW & 201 & $\cdots$ & - & $\mathrm{H}$ & 30 & $\cdots$ & $\cdots$ & 1 \\
\hline TAW & 202 & 20 & $09-10-88$ & $\mathrm{H}$ & 11 & $\cdots$ & $-\cdot$ & .5 \\
\hline TAW & 203 & 50 & $12 \cdot 11 \cdot 88$ & $\mathrm{H}$ & 10 & $\cdots$ & $\cdots$ & 1.5 \\
\hline TAW & 204 & 5 & $12-10-88$ & $\mathrm{H}$ & 30 & - & - & 2 \\
\hline TAW & 205 & $\cdots$ & $\cdots$ & $\mathrm{H}$ & 60 & $\cdots$ & $\cdots$ & 1 \\
\hline TAW & 206 & 47.5 & $12-11-88$ & $\mathrm{H}$ & 14 & . & $\cdots$ & 3 \\
\hline TAW & 207 & - & - & $\mathrm{H}$ & 25 & $\cdots$ & $\cdots$ & 12 \\
\hline TAW & 208 & 60 & $12 \cdot 14 \cdot 88$ & $\mathrm{H}$ & 25 & $\cdots$ & $\cdots$ & 1 \\
\hline TAW & 209 & 20 & $12-20-88$ & $\mathrm{H}$ & 50 & - & . & .5 \\
\hline TAW & 210 & 20 & $12-20-88$ & $\mathrm{H}$ & 50 & $\cdots$ & $\cdots$ & .5 \\
\hline TAW & 211 & 28 & $11-30-88$ & $\mathrm{H}$ & 4.5 & $\cdots$ & $\cdots$ & 1 \\
\hline TAW & 212 & 15 & $06-07-88$ & $\mathrm{H}$ & 0.5 & - & - & 1 \\
\hline TAW & 213 & 60 & $06-01-89$ & $\mathrm{H}$ & 10 & $\cdots$ & - & 1 \\
\hline TAW & 214 & 10 & $03-27-89$ & $\mathrm{H}$ & 5 & $\cdots$ & $\cdots$ & .5 \\
\hline TAW & 215 & - & $\cdots$ & $\mathrm{H}$ & 25 & $\cdots$ & $\cdots$ & .8 \\
\hline TAW & 216 & 47.5 & $04-02-89$ & $\mathrm{H}$ & 13 & - & - & 2 \\
\hline TAW & 217 & 52.5 & $07-04-89$ & $\mathrm{H}$ & 10 & $\cdots$ & - & 1 \\
\hline TAW & 218 & 70 & $06-15-89$ & $\mathrm{H}$ & 3 & $\cdots$ & $\cdots$ & 2 \\
\hline TAW & 219 & 47 & $08-27-89$ & $\mathrm{H}$ & 8.5 & $\cdots$ & $\cdots$ & 2 \\
\hline TAW & 220 & 52 & $10-18-89$ & H & 9 & $\cdots$ & $\cdots$ & .5 \\
\hline TAW & 221 & 49 & $09-24-89$ & $\mathrm{H}$ & 10 & $\cdots$ & - & 2 \\
\hline TAW & 222 & $\cdots$ & - & $\mathrm{H}$ & 4 & -- & - & 1 \\
\hline TAW & 223 & - - & $\cdots$ & $\mathrm{H}$ & 3 & - & $\cdots$ & 1 \\
\hline TAW & 224 & 20 & $09-20-89$ & $\mathrm{H}$ & 4 & - & - & 1 \\
\hline TAW & 225 & 10 & $09-22 \cdot 89$ & $\cdots$ & 2 & $\cdots$ & $\cdots$ & .5 \\
\hline TAW & 226 & 14 & $01-20-90$ & $\mathrm{H}$ & 9 & $\cdots$ & $\cdots$ & .8 \\
\hline TAW & 227 & 43 & $05-05-90$ & $\mathrm{H}$ & 16 & $-\cdot$ & $\cdots$ & 2 \\
\hline TAW & 228 & 6 & $09-07-90$ & $\mathrm{H}$ & 1 & $\cdots$ & - & - \\
\hline TAW & 229 & 47 & $09-29-90$ & $\mathrm{H}$ & 12 & $\cdots$ & $\cdots$ & 1 \\
\hline TAW & 230 & 20 & $06-11-90$ & $\mathrm{H}$ & 80 & $\cdots$ & $\cdots$ & .5 \\
\hline
\end{tabular}


Table 1-1. Description of selected wells, borings, and springs in the Saco and Ossipee River Basins, east-central

\begin{tabular}{|c|c|c|c|c|c|c|c|c|c|c|c|}
\hline $\begin{array}{l}\text { Local } \\
\text { site } \\
\text { number }\end{array}$ & $\begin{array}{l}\text { Lat- } \\
\text { itude }\end{array}$ & $\begin{array}{l}\text { Long- } \\
\text { itude }\end{array}$ & Owner or user & $\begin{array}{l}\text { Year } \\
\text { com- } \\
\text { pleted }\end{array}$ & $\begin{array}{l}\text { Alti- } \\
\text { tude } \\
\text { above } \\
\text { sea } \\
\text { level } \\
\text { (feet) }\end{array}$ & $\begin{array}{l}\text { Dia- } \\
\text { meter } \\
\text { of } \\
\text { well } \\
\text { casing } \\
\text { (inches) }\end{array}$ & $\begin{array}{l}\text { Depth } \\
\text { to } \\
\text { bottom } \\
\text { of } \\
\text { casing } \\
\text { (feet) }\end{array}$ & $\begin{array}{l}\text { Casing } \\
\text { material }\end{array}$ & $\begin{array}{l}\text { Type } \\
\text { of } \\
\text { finish }\end{array}$ & $\begin{array}{l}\text { Depth } \\
\text { to } \\
\text { bottom } \\
\text { of open } \\
\text { section } \\
\text { (feet) }\end{array}$ & $\begin{array}{c}\text { Type } \\
\text { of } \\
\text { site }\end{array}$ \\
\hline
\end{tabular}

\begin{tabular}{lllll} 
TAW & 231 & 435414 & 711842 & Pray \\
TAW & 232 & 434952 & 711627 & King \\
TAW & 233 & 434945 & 711230 & Keough \\
TAW & 234 & 435100 & 711146 & De1p \\
TAW & 235 & 434935 & 711354 & Geary \\
& & & & \\
TAW & 236 & 435032 & 711704 & Ryder \\
TAW & 237 & 435055 & 711159 & Burt \\
TAW & 238 & 435304 & 711624 & Stevens \\
TAW & 239 & 435312 & 711313 & Diphilippo \\
TAW & 240 & 435240 & 711312 & Scolaro \\
& & & & \\
TAW & 241 & 435059 & 711210 & Twoney \\
TAW & 242 & 435105 & 711148 & Azibert \\
TAW & 243 & 435103 & 711147 & L. Cox \\
TAW & 244 & 435029 & 711557 & Remick \\
TAW & 245 & 434950 & 711228 & Swain \\
& & & & \\
TAW & 246 & 435200 & 712003 & Bleakney \\
TAW & 247 & 435056 & 711149 & Eaton \\
TAW & 248 & 434938 & 711356 & LaForge \\
TAW & 249 & 434941 & 711351 & Spotholtz Family Trust \\
TAW & 250 & 435126 & 711613 & B. Brinsack \\
& & & & \\
TAW & 251 & 435309 & 711328 & Wright \\
TAW & 252 & 435328 & 711751 & Baker \\
TAW & 253 & 434939 & 711550 & M.and s. Temkin \\
TAW & 254 & 435304 & 711315 & Lessard \\
TAW & 255 & 435109 & 711553 & Forst \\
& & & & \\
TAW & 256 & 435012 & 711247 & Mason \\
TAW & 257 & 435017 & 711230 & Rosie's Resturant \\
\hline
\end{tabular}

Tamworth--Continued

TAW $257 \quad 435017 \quad 711230$ Rosie's Resturant

\begin{tabular}{|c|c|c|c|c|c|c|c|}
\hline 1990 & 815 & $\cdots$ & 164 & $\cdots$ & $x$ & $\cdots$ & $\mathrm{BrW}$ \\
\hline 1990 & 480 & $\cdots$ & 40 & - & $x$ & $\cdots$ & $\mathrm{BrW}$ \\
\hline 1990 & 440 & $\cdots$ & 122 & -- & $\mathrm{x}$ & - & BrW \\
\hline 1990 & 480 & $\cdots$ & 62 & - - & $S$ & - & $\cdots$ \\
\hline 1990 & 450 & $\cdots$ & 35 & - & $\mathrm{S}$ & $\cdots$ & $\cdots$ \\
\hline 1990 & 510 & $\cdots$ & $\cdots$ & $\cdots$ & $\cdots$ & $\cdots$ & Dug \\
\hline 1990 & 480 & $\cdots$ & 59 & $\cdots$ & $\mathrm{s}$ & - & - \\
\hline 1990 & 880 & - & 40 & $\cdots$ & $x$ & - & BrW \\
\hline 1990 & 690 & $\cdots$ & 151 & $\cdots$ & $x$ & $\cdots$ & BrW \\
\hline 1990 & 450 & $\cdots$ & 120 & $\cdots$ & $x$ & - & BrW \\
\hline 1991 & 470 & $\cdots$ & 100 & $\cdots$ & $\cdots$ & $\cdots$ & $\cdots$ \\
\hline 1991 & 480 & $\cdots$ & 58 & $\cdots$ & $\mathrm{s}$ & - & $\cdots$ \\
\hline 1991 & 480 & $\cdots$ & 59 & - & $\mathrm{S}$ & - & - \\
\hline 1991 & 480 & $\cdots$ & 60 & $\cdots$ & $x$ & $\cdots$ & BrW \\
\hline 1991 & 440 & $\cdots$ & 111 & - & $x$ & $\cdots$ & $\mathrm{BrW}$ \\
\hline 1991 & 770 & - & 128 & $\cdots$ & $x$ & - & BrW \\
\hline 1992 & 480 & $\cdots$ & 59 & $\cdots$ & $\mathrm{s}$ & $\cdots$ & $\cdots$ \\
\hline 1992 & 450 & $\cdots$ & 23 & $\cdots$ & $S$ & - & $\cdots$ \\
\hline 1992 & 450 & $\cdots$ & 14 & $\cdots$ & $s$ & - & - \\
\hline 1992 & 510 & $\cdots$ & 221 & $\cdots$ & $x$ & $\cdots$ & BrW \\
\hline 1992 & 700 & $\cdots$ & 141 & $\cdots$ & $x$ & - & BrW \\
\hline 1992 & 760 & $\cdots$ & 281 & - & $x$ & - & BrW \\
\hline 1992 & 490 & - & 100 & $\cdots$ & $x$ & $\cdots$ & $\mathrm{BrW}$ \\
\hline 1992 & 680 & $\cdots$ & 130 & $\cdots$ & $x$ & $\cdots$ & BrW \\
\hline 1992 & 480 & - & 70 & $\cdots$ & $x$ & $\cdots$ & $\mathrm{BrW}$ \\
\hline 1992 & 450 & $\cdots$ & 49 & $\cdots$ & $\cdots$ & $\cdots$ & $\cdots$ \\
\hline 1991 & 450 & $\cdots$ & 32 & $\cdots$ & $S$ & $\cdots$ & $\cdots$ \\
\hline
\end{tabular}

$\begin{array}{rrrrl}\text { TZW } & 37 & \mathbf{4 3 4 2 0 4} & 711239 & \text { McClure } \\ \text { TZW } & 73 & 434108 & 711147 & \text { Workman } \\ \text { TZW } & 96 & \mathbf{4 3 4 1 1 0} & 711135 & \text { Libby } \\ \text { TZW } & 134 & \mathbf{4 3 3 9 4 8} & 711223 & \text { Ringer } \\ \text { TZW } & 159 & 434144 & 711207 & \text { Mott } \\ & & & & \\ \text { TZW } & 160 & 433949 & 711214 & \text { Banks } \\ \text { TZW } & 165 & 434333 & 711415 & \text { Camp Merrow Vista }\end{array}$

Tuf tonboro

\begin{tabular}{|c|c|c|c|c|c|c|c|}
\hline 1985 & 1,060 & $\cdots$ & 20 & $\cdots$ & $x$ & $\cdots$ & BrW \\
\hline 1987 & 860 & $\cdots$ & - & $\cdots$ & $\cdots$ & $\cdots$ & Dug \\
\hline 1987 & 880 & $\cdots$ & 59 & $\cdots$ & $x$ & $\cdots$ & BrW \\
\hline 1989 & 980 & $\cdots$ & 54 & $\cdots$ & $x$ & $\cdots$ & BrW \\
\hline 1990 & 830 & $\cdots$ & 209 & $\cdots$ & $x$ & $\cdots$ & BrW \\
\hline 1990 & 980 & $\cdots$ & 69 & $-\cdot$ & $x$ & $\cdots$ & $\mathrm{BrW}$ \\
\hline 1990 & 960 & $\cdots$ & 50 & $\cdots$ & $x$ & $\cdots$ & BrW \\
\hline
\end{tabular}

Wakefield

\begin{tabular}{|c|c|c|c|c|}
\hline WAA & 3 & 433830 & 710306 & \\
\hline WAB & 1 & 433807 & 710311 & 1955 \\
\hline WAB & 2 & 433741 & 710251 & NHDOT \\
\hline WAW & 3 & 433722 & 710258 & Lupien \\
\hline WAW & 5 & 433914 & 710005 & Vrettos \\
\hline WAW & 6 & 433714 & 705913 & slack \\
\hline WAW & 7 & 433707 & 705919 & McHugh \\
\hline WAW & 8 & 434049 & 705937 & McNur \\
\hline WAW & 9 & 433808 & 710307 & McCormick \\
\hline WAW & 10 & 433811 & 710308 & McCormick \\
\hline WAW & 12 & 433714 & 705901 & Galabrun \\
\hline WAW & 13 & 433718 & 705901 & Galabrun \\
\hline WAW & 15 & 433752 & 710122 & Gray \\
\hline WAW & 16 & 433723 & 705853 & Donne11y \\
\hline WAW & 17 & 433717 & 705921 & Dwyer \\
\hline
\end{tabular}

\begin{tabular}{|c|c|c|c|c|c|c|c|}
\hline - & 610 & - - & $\cdots$ & $\cdots$ & $\cdots$ & - & $\cdots$ \\
\hline . & 585 & - & $\cdots$ & - & $\cdots$ & $-\cdot$ & $\mathrm{TH}$ \\
\hline$\cdots$ & 689 & $\cdots$ & $\cdots$ & $\cdots$ & - & $\cdots$ & $\mathrm{TH}$ \\
\hline 1984 & 780 & $\cdots$ & 40 & $\cdots$ & $x$ & - & BrW \\
\hline 1984 & 600 & $\cdots$ & 9.5 & $\cdots$ & - & - & $\cdots$ \\
\hline 1984 & 580 & - & 181 & $\cdots$ & $x$ & $\cdots$ & $\mathrm{BrW}$ \\
\hline 1984 & 580 & $\cdots$ & 62 & $\cdots$ & $\cdots$ & $\cdots$ & $\cdots$ \\
\hline 1984 & 500 & $\cdots$ & 60 & - & $x$ & - & BrW \\
\hline 1984 & 580 & $\cdots$ & 60 & - & $\cdots$ & $\cdots$ & BrW \\
\hline 1984 & 580 & $\cdots$ & 60 & $\cdots$ & $\cdots$ & $\cdots$ & BrW \\
\hline 1984 & 560 & . & 123 & $\cdots$ & $\mathrm{X}$ & $\cdots$ & BrW \\
\hline 1984 & 580 & - & 107 & $\cdots$ & $\cdots$ & $\cdots$ & $\mathrm{BrW}$ \\
\hline 1984 & 620 & - & 104 & $\cdots$ & -- & $\cdots$ & $B r W$ \\
\hline 1985 & 560 & $\cdots$ & 16 & $\cdots$ & $\mathbf{S}$ & $\cdots$ & $\cdots$ \\
\hline 1984 & 620 & - & 48 & $\cdots$ & $\mathbf{S}$ & - & - \\
\hline
\end{tabular}




\begin{tabular}{|c|c|c|c|c|c|c|c|c|}
\hline $\begin{array}{l}\text { Local } \\
\text { site } \\
\text { number }\end{array}$ & $\begin{array}{l}\text { Water } \\
\text { level } \\
\text { depth } \\
\text { (feet) }\end{array}$ & $\begin{array}{l}\text { Date } \\
\mathrm{mm} / \mathrm{dd} / \mathrm{yy}\end{array}$ & Use & $\begin{array}{l}\text { Maximum } \\
\text { well } \\
\text { yield } \\
\text { (gallons } \\
\text { per } \\
\text { minute) }\end{array}$ & $\begin{array}{l}\text { Draw- } \\
\text { down } \\
\text { (feet) }\end{array}$ & $\begin{array}{c}\text { Specific } \\
\text { capacity } \\
\text { (galions per } \\
\text { minute } \\
\text { per } \\
\text { foot) }\end{array}$ & $\begin{array}{l}\text { Pumping } \\
\text { period } \\
\text { (hours) }\end{array}$ & Remarks \\
\hline
\end{tabular}

Tamworth - - Continued

\begin{tabular}{|c|c|c|c|c|c|c|c|c|}
\hline TAW & 231 & - - & $\cdots$ & $\mathrm{H}$ & 8 & $\cdots$ & $-\cdot$ & .5 \\
\hline TAW & 232 & 10 & $07-18-90$ & $\mathrm{H}$ & 6.5 & $\cdots$ & $\cdots$ & .5 \\
\hline TAW & 233 & 40 & $07-18-90$ & $\mathrm{H}$ & 2.5 & - & $\cdots$ & 1 \\
\hline TAW & 234 & $\cdots$ & $\cdots$ & $\mathrm{H}$ & 13 & $\cdots$ & $\cdots$ & 1.5 \\
\hline TAW & 235 & 20 & $08-20-90$ & $\mathrm{H}$ & 30 & $\cdots$ & - & 2 \\
\hline TAW & 236 & 10 & $05-10-90$ & $\mathrm{H}$ & 6 & $-\cdot$ & - & 4 \\
\hline TAW & 237 & 42 & $12-01-90$ & $\mathrm{H}$ & 15 & $-\cdot$ & $-\cdot$ & 2 \\
\hline TAW & 238 & 10 & $08-18-90$ & $\mathrm{H}$ & 15 & $\cdots$ & $\cdots$ & .5 \\
\hline TAW & 239 & 40 & $12-01-90$ & $\mathrm{H}$ & 2.5 & $\cdots$ & - & 1 \\
\hline TAW & 240 & 30 & $10-06-90$ & $\mathrm{H}$ & 1.3 & $\cdots$ & $-\cdot$ & 1 \\
\hline TAW & 241 & 55 & $04-05-91$ & $\mathrm{H}$ & 30 & $\cdots$ & $\cdots$ & 1 \\
\hline TAW & 242 & 47 & $07-27-91$ & $\mathrm{H}$ & 13 & -- & $\cdots$ & .5 \\
\hline TAW & 243 & 46.5 & $08-10-91$ & $\mathrm{H}$ & 10 & -- & - - & 2 \\
\hline TAW & 244 & 60 & $08 \cdot 29-91$ & $\mathrm{H}$ & 1 & $\cdots$ & $-\cdot$ & 1 \\
\hline TAW & 245 & 30 & $08 \cdot 15-91$ & $\mathrm{H}$ & 7 & $\cdots$ & $\cdots$ & 1 \\
\hline TAW & 246 & 30 & $07-17-91$ & $\mathrm{H}$ & 1.5 & $-\cdot$ & $\cdots$ & 1 \\
\hline TAW & 247 & 44.5 & $04-16-92$ & $\mathrm{H}$ & 15 & - & - & 2 \\
\hline TAW & 248 & 12 & $06-08-92$ & $\mathrm{H}$ & 35 & $\cdots$ & - & 2 \\
\hline TAW & 249 & 11.5 & $06-20-92$ & $\mathrm{H}$ & 25 & - & $\cdots$ & 4 \\
\hline IAW & 250 & 15 & $07-09-92$ & $\mathrm{H}$ & 30 & $-\cdot$ & $\cdots$ & .3 \\
\hline TAW & 251 & 100 & $08-15-92$ & $\mathrm{H}$ & 1.5 & $\cdots$ & $\cdots$ & 1 \\
\hline TAW & 252 & 85 & $07-28-92$ & $\mathrm{H}$ & 10 & $\cdots$ & $\cdots$ & .5 \\
\hline TAW & 253 & 50 & $07-22-92$ & $\mathrm{H}$ & 50 & - - & - & 1 \\
\hline TAW & 254 & $\cdots$ & - & $\mathrm{H}$ & 6 & - & $\cdots$ & 1 \\
\hline IAW & 255 & 15 & $10-31-92$ & $\mathrm{H}$ & 6 & $\cdots$ & $\cdots$ & 1 \\
\hline TAW & 256 & 10 & $10-25-92$ & $\mathbf{P}$ & 70 & - & $\cdots$ & 18 \\
\hline \multirow[t]{2}{*}{ TAW } & 257 & 24 & $04-20-91$ & $\mathrm{C}$ & 12 & - & $\cdots$ & 2 \\
\hline & & & & & & & \multicolumn{2}{|c|}{ Tuf tonboro } \\
\hline TZW & 37 & $\cdots$ & - & $\mathrm{H}$ & 2.5 & - & $\cdots$ & .5 \\
\hline TZW & 73 & 8 & $08-26-87$ & $\mathrm{H}$ & $\cdots$ & $\cdots$ & $\cdots$ & $\cdots$ \\
\hline TZW & 96 & - & - & $\mathrm{H}$ & 9 & - & $-\cdot$ & 1.5 \\
\hline TZW & 134 & $\cdots$ & $\cdots$ & $\mathrm{H}$ & 10 & $\cdots$ & - & .5 \\
\hline TZW & 159 & $\cdots$ & $\cdots$ & $\mathrm{H}$ & 0.8 & $\cdots$ & $\cdots$ & .7 \\
\hline TZW & 160 & 25 & $09-04-90$ & $\mathrm{H}$ & 4 & - & $\cdots$ & 1 \\
\hline \multirow[t]{2}{*}{ TZW } & 165 & 40 & $10-27-90$ & $\mathrm{H}$ & 40 & - & $\cdots$ & .5 \\
\hline & & & & & & & \multicolumn{2}{|c|}{ Wakefield } \\
\hline WAA & 3 & $\cdots$ & $\cdots$ & $\mathrm{U}$ & $\cdots$ & - & $\cdots$ & $-\cdot$ \\
\hline WAB & 1 & $\cdots$ & $\cdots$ & $\mathrm{T}$ & $\cdots$ & $\cdots$ & - & $\cdots$ \\
\hline WAB & 2 & $\cdots$ & $\cdots$ & $\mathrm{T}$ & $-\cdot$ & $\cdots$ & $-\cdot$ & $\cdots$ \\
\hline WAW & 3 & $\cdots$ & - & $\mathrm{H}$ & 5 & $\cdots$ & $\cdots$ & .5 \\
\hline WAW & 5 & 2 & $.06-14-84$ & $\mathrm{H}$ & 30 & $-\cdot$ & $-\cdot$ & 2 \\
\hline WAW & 6 & $\cdots$ & $\cdots$ & $\mathrm{H}$ & 45 & $\cdots$ & $\cdots$ & .5 \\
\hline WAW & 7 & 46.5 & $07-20-84$ & $\mathrm{H}$ & 9 & $\cdots$ & $\cdots$ & 2 \\
\hline WAW & 8 & 20 & $09-11-84$ & $\mathrm{H}$ & 6 & $\cdots$ & $\cdots$ & .5 \\
\hline WAW & 9 & 30 & $08-23-84$ & $\mathrm{H}$ & 4 & - & $\cdots$ & .5 \\
\hline WAW & 10 & 30 & $08-20-84$ & $\mathrm{H}$ & 0.2 & $\cdots$ & $\cdots$ & 1 \\
\hline WAW & 12 & 35 & $10-26 \cdot 84$ & $\mathrm{H}$ & 20 & - & - & .5 \\
\hline WAW & 13 & - & $\cdots$ & $\mathrm{H}$ & $\cdots$ & $\cdots$ & $\cdots$ & .5 \\
\hline WAW & 15 & $\cdots$ & $\cdots$ & $\mathrm{H}$ & $\cdots$ & $\cdots$ & $\cdots$ & $\cdots$ \\
\hline WAW & 16 & 7 & $05-19-85$ & $\mathrm{H}$ & 15 & $\cdots$ & $\cdots$ & 2 \\
\hline WAW & 17 & 30 & $11-06-84$ & $\mathrm{H}$ & 16 & $\cdots$ & -- & 1 \\
\hline
\end{tabular}


rable 1-1. Descriptlon of selected wells, borlngs, and springs In the Saco and Ossipee River Basins, east-central

\begin{tabular}{|c|c|c|c|c|c|c|c|c|c|c|c|}
\hline $\begin{array}{l}\text { Local } \\
\text { site } \\
\text { number }\end{array}$ & $\begin{array}{l}\text { Lat- } \\
\text { itude }\end{array}$ & $\begin{array}{l}\text { Long- } \\
\text { itude }\end{array}$ & Owner or user & $\begin{array}{l}\text { Year } \\
\text { com- } \\
\text { pleted }\end{array}$ & $\begin{array}{c}\text { Alti- } \\
\text { tude } \\
\text { above } \\
\text { sea } \\
\text { level } \\
\text { (feet) }\end{array}$ & $\begin{array}{c}\text { Dia- } \\
\text { meter } \\
\text { of } \\
\text { well } \\
\text { casing } \\
\text { (inches) }\end{array}$ & $\begin{array}{l}\text { Depth } \\
\text { to } \\
\text { bottom } \\
\text { of } \\
\text { casing } \\
\text { (feet) }\end{array}$ & $\begin{array}{l}\text { Casing } \\
\text { material }\end{array}$ & $\begin{array}{l}\text { Type } \\
\text { of } \\
\text { finish }\end{array}$ & $\begin{array}{l}\text { Depth } \\
\text { to } \\
\text { bottom } \\
\text { of open } \\
\text { section } \\
\text { (feet) }\end{array}$ & $\begin{array}{c}\text { Type } \\
\text { of } \\
\text { site }\end{array}$ \\
\hline
\end{tabular}

\begin{tabular}{|c|c|c|c|c|}
\hline WAW & 18 & 433716 & 710000 & Aaronian \\
\hline WAW & 19 & 433738 & 705907 & Patten \\
\hline WAW & 21 & 433804 & 710257 & Donnel1y \\
\hline WAW & 22 & 433726 & 710029 & st. Cyr \\
\hline WAW & 23 & 433828 & 705932 & Harrison \\
\hline WAW & 25 & 433753 & 705853 & Chick \\
\hline WAW & 26 & 433839 & 705938 & Anderson \\
\hline WAW & 27 & 433716 & 710000 & Sapp \\
\hline WAW & 28 & 433721 & 710000 & Seftan \\
\hline WAW & 29 & 433719 & 705947 & Creighton \\
\hline WAW & 33 & 433816 & 710300 & Pike \\
\hline WAW & 34 & 433833 & 710247 & Lake Land Deve \\
\hline WAW & 35 & 433841 & 705905 & Harleb \\
\hline WAW & 36 & 433812 & 710313 & Ford \\
\hline WAW & 37 & 433826 & 710305 & Blackadar \\
\hline WAW & 41 & 433853 & 705921 & Toriano \\
\hline WAW & 43 & 433749 & 705843 & Downing \\
\hline WAW & 45 & 433724 & 710013 & Armbrust \\
\hline WAW & 46 & 433725 & 710024 & St Cyr \\
\hline WAW & 47 & 433728 & 710036 & Doherty \\
\hline WAW & 48 & 433726 & 705946 & Burgess \\
\hline WAW & 49 & 433818 & 710303 & Donnel1y \\
\hline WAW & 50 & 433722 & 710106 & Frechet te \\
\hline WAW & 51 & 433905 & 705930 & Hagen \\
\hline WAW & 52 & 433940 & 705936 & Minnon \\
\hline WAW & 54 & 433743 & 710206 & Colella \\
\hline WAW & 97 & 433944 & 705942 & Minnon \\
\hline WAW & 99 & 433652 & 705913 & Carpentier \\
\hline WAW & 100 & 433740 & 710212 & Jones \\
\hline WAW & 101 & 433706 & 705911 & Gear \\
\hline WAW & 102 & 433914 & 705926 & McGrath \\
\hline WAW & 103 & 433915 & 705926 & Yeo \\
\hline WAW & 104 & 434052 & 705958 & Santos \\
\hline WAW & 105 & 434032 & 705926 & Hewitt \\
\hline WAW & 106 & 433712 & 710100 & walsh \\
\hline WAW & 107 & 433838 & 705858 & Nichinielio \\
\hline WAW & 108 & 433943 & 705936 & Trask \\
\hline WAW & 109 & 433944 & 705947 & Michaud \\
\hline WAW & 110 & 434101 & 705940 & Fiore \\
\hline WAW & 111 & 433828 & 705850 & Tobin \\
\hline WAW & 112 & 433747 & 710232 & walking \\
\hline WAW & 113 & 433653 & 705917 & Tandus $\mathrm{Jr}$ \\
\hline WAW & 114 & 434107 & 705950 & Chick \\
\hline WAW & 115 & 433845 & 705902 & Noviello \\
\hline WAW & 116 & 433727 & 710131 & Carney \\
\hline WAW & 117 & 433755 & 710139 & Fox \\
\hline WAW & 118 & 433902 & 705922 & Jaeger \\
\hline WAW & 119 & 433841 & 705912 & Perry \\
\hline WAW & 120 & 433735 & 710156 & Marston \\
\hline WAW & 121 & 433757 & 710229 & David \\
\hline WAW & 122 & 433842 & 705928 & Buxton \\
\hline WAW & 123 & 433747 & 710226 & B1ack \\
\hline WAW & 124 & 433925 & 705934 & Tryder \\
\hline WAW & 125 & 433751 & 710234 & O'Leary \\
\hline
\end{tabular}

Wakefield--Continued

\begin{tabular}{|c|c|c|c|c|c|c|c|}
\hline 1985 & 600 & $\cdots$ & 112 & - & $\cdots$ & $\cdots$ & BrW \\
\hline 1985 & 560 & $\cdots$ & 161 & $\cdots$ & $\cdots$ & - & BrW \\
\hline 1985 & 600 & $\cdots$ & 80 & $\cdots$ & $\cdots$ & $\cdots$ & Brw \\
\hline 1985 & 590 & $\cdots$ & 80 & $\cdots$ & $\cdots$ & $\cdots$ & BrW \\
\hline 1985 & 600 & - & 43 & $\cdots$ & $\cdots$ & - & BrW \\
\hline 1985 & 560 & $\cdots$ & 28 & $\cdots$ & $s$ & $\cdots$ & $\cdots$ \\
\hline 1985 & 640 & $\cdots$ & 119 & $\cdots$ & $\cdots$ & $\cdots$ & BrW \\
\hline 1985 & 600 & $\cdots$ & 120 & $\cdots$ & $\cdots$ & $\cdots$ & BrW \\
\hline 1985 & 620 & $\cdots$ & 109 & $\cdots$ & $\cdots$ & $\cdots$ & $\cdots$ \\
\hline 1985 & 580 & $\cdots$ & 120 & $\cdots$ & - & - & BrW \\
\hline 1985 & 640 & $\cdots$ & 124 & $\cdots$ & $x$ & $\cdots$ & BrW \\
\hline 1985 & 600 & $\cdots$ & 59 & $\cdots$ & $\cdots$ & $\cdots$ & $\cdots$ \\
\hline 1985 & 620 & $\cdots$ & 74 & $\cdots$ & $x$ & $\cdots$ & BrW \\
\hline 1985 & 620 & $\cdots$ & 89 & - & - & $\cdots$ & BrW \\
\hline 1985 & 640 & $\cdots$ & 112 & - & $x$ & - & BrW \\
\hline 1985 & 640 & $\cdots$ & 100 & $\cdots$ & $x$ & $\cdots$ & BrW \\
\hline 1986 & 580 & $\cdots$ & 45 & $\cdots$ & $\cdots$ & $\cdots$ & BrW \\
\hline 1986 & 590 & $\cdots$ & 60 & $\cdots$ & $x$ & $\cdots$ & BrW \\
\hline 1986 & 590 & $\cdots$ & 52 & $\cdots$ & $x$ & $\cdots$ & BrW \\
\hline 1986 & 590 & $\cdots$ & 60 & $\cdots$ & $x$ & $\cdots$ & BrW \\
\hline 1986 & 590 & $\cdots$ & 120 & $\cdots$ & $x$ & $\cdots$ & BrW \\
\hline 1986 & 640 & $\cdots$ & 161 & $\cdots$ & $x$ & - . & Brw \\
\hline 1986 & 600 & $\cdots$ & 48 & $\cdots$ & $\mathrm{x}$ & $\cdots$ & BrW \\
\hline 1986 & 600 & $\cdots$ & 95 & $\cdots$ & $x$ & - & BrW \\
\hline 1986 & 640 & - & 38 & $\cdots$ & $\mathbf{x}$ & $\cdots$ & BrW \\
\hline 1986 & 640 & $\cdots$ & 91 & $\cdots$ & - & $\cdots$ & $\cdots$ \\
\hline 1986 & 590 & $\cdots$ & 38 & $\cdots$ & $x$ & $\cdots$ & BrW \\
\hline 1986 & 570 & $\cdots$ & 19 & $\cdots$ & $x$ & $\cdots$ & Brw \\
\hline 1986 & 615 & $\cdots$ & 92 & $\cdots$ & $\mathbf{x}$ & - & BrW \\
\hline 1986 & 570 & $\cdots$ & 170 & $\cdots$ & $\mathrm{x}$ & - & BrW \\
\hline 1986 & 620 & $\cdots$ & 76 & - & $x$ & $\cdots$ & BrW \\
\hline 1986 & 620 & $\cdots$ & 71 & $\cdots$ & $x$ & $\cdots$ & BrW \\
\hline 1986 & 495 & $\cdots$ & 39 & $\cdots$ & $x$ & $\cdots$ & BrW \\
\hline 1986 & 525 & $\cdots$ & 40 & $\cdots$ & $\mathrm{x}$ & $\cdots$ & BrW \\
\hline 1987 & 605 & $\cdots$ & 79 & $\cdots$ & $\mathrm{x}$ & $\cdots$ & BrW \\
\hline 1987 & 630 & $\cdots$ & 75 & - & $\mathrm{x}$ & $\cdots$ & BrW \\
\hline 1987 & 615 & $\cdots$ & 49 & $\cdots$ & $x$ & - & BrW \\
\hline 1987 & 600 & $\cdots$ & 40 & $\cdots$ & $x$ & $\cdots$ & BrW \\
\hline 1987 & 500 & $\cdots$ & 20 & $\cdots$ & $x$ & $\cdots$ & BrW \\
\hline 1987 & 595 & $\cdots$ & 60 & - & $x$ & $\cdots$ & BrW \\
\hline 1987 & 620 & $\cdots$ & 67 & $\cdots$ & $\mathrm{x}$ & $\cdots$ & BrW \\
\hline 1987 & 570 & $\cdots$ & 30 & - & $x$ & $\cdots$ & BrW \\
\hline 1987 & 490 & $\cdots$ & 27 & $\cdots$ & $x$ & - & BrW \\
\hline 1987 & 630 & $\cdots$ & 33 & $\cdots$ & $\mathrm{x}$ & $\cdots$ & BrW \\
\hline 1987 & 595 & $\cdots$ & 44 & - & $x$ & - & BrW \\
\hline 1987 & 600 & $\cdots$ & 125 & $\cdots$ & $x$ & $\cdots$ & BrW \\
\hline 1987 & 625 & - & 59 & $\cdots$ & $x$ & - & BrW \\
\hline 1987 & 610 & $\cdots$ & 100 & $\cdots$ & $x$ & . & BrW \\
\hline 1987 & 590 & $\cdots$ & 40 & $\cdots$ & $\mathrm{x}$ & $\cdots$ & BrW \\
\hline 1987 & 595 & $\cdots$ & 19 & $\cdots$ & $\mathrm{x}$ & $\cdots$ & Brw \\
\hline 1987 & 590 & $\cdots$ & 74 & $\cdots$ & $\mathrm{x}$ & $\cdots$ & BrW \\
\hline 1987 & 595 & $\cdots$ & 72 & $\cdots$ & $x$ & $\cdots$ & Brw \\
\hline 1988 & 590 & $\cdots$ & 59 & $\cdots$ & $\mathrm{x}$ & $\cdots$ & BrW \\
\hline
\end{tabular}




\begin{tabular}{|c|c|c|c|c|c|c|c|c|}
\hline $\begin{array}{l}\text { Local } \\
\text { site } \\
\text { number }\end{array}$ & $\begin{array}{l}\text { Water } \\
\text { level } \\
\text { depth } \\
\text { (feet) }\end{array}$ & $\begin{array}{l}\text { Date } \\
\mathrm{mm} / \mathrm{dd} / \mathrm{yy}\end{array}$ & Use & $\begin{array}{l}\text { Maximum } \\
\text { well } \\
\text { yield } \\
\text { (gallons } \\
\text { per } \\
\text { minute) }\end{array}$ & $\begin{array}{l}\text { Draw- } \\
\text { down } \\
\text { (feet) }\end{array}$ & $\begin{array}{c}\text { Specific } \\
\text { capacity } \\
\text { (gallons per } \\
\text { minute } \\
\text { per } \\
\text { foot) }\end{array}$ & $\begin{array}{l}\text { Pumping } \\
\text { period } \\
\text { (hours) }\end{array}$ & Remarks \\
\hline
\end{tabular}

Wakefield - Continued

\begin{tabular}{|c|c|c|c|c|c|c|c|c|}
\hline WAW & 18 & 35 & $04-17-85$ & $\mathrm{H}$ & 3.5 & - & - & 1 \\
\hline WAW & 19 & 50 & $04-19-85$ & $\mathrm{H}$ & 5 & - & - & 1 \\
\hline WAW & 21 & 50 & $05-23-85$ & $\mathbf{H}$ & 1.8 & $\cdots$ & $-\cdot$ & 1 \\
\hline WAW & 22 & 20 & $05-29-85$ & H & 9 & $\cdots$ & $-\cdot$ & .5 \\
\hline WAW & 23 & 10 & $05-29-85$ & $\mathrm{H}$ & 8 & $\cdots$ & $\cdots$ & .5 \\
\hline WAW & 25 & 19.5 & $05-01-85$ & H & 35 & -- & $\cdots$ & 2 \\
\hline WAW & 26 & 30 & $06-27-85$ & $\mathrm{H}$ & 1.5 & -- & $\cdots$ & .5 \\
\hline WAW & 27 & 35 & $07-18-85$ & $\mathrm{H}$ & 8 & - & - - & .5 \\
\hline WAW & 28 & 40 & $07-18-85$ & H & 9 & - & - & 4 \\
\hline WAW & 29 & 40 & $08-08-85$ & H & 40 & $\cdots$ & $\cdots$ & .5 \\
\hline WAW & 33 & - & - & $\mathrm{H}$ & 25 & $\cdots$ & - & 1 \\
\hline WAW & 34 & 20 & $08-03-85$ & $\mathrm{P}$ & 80 & - & $-\cdots$ & 1 \\
\hline WAW & 35 & $\cdots$ & $\cdots$ & H & 4 & $-\cdot$ & $\cdots$ & 1 \\
\hline WAW & 36 & $\cdots$ & $\cdots$ & H & 7 & - & -- & 2 \\
\hline WAW & 37 & - & - & $\mathrm{H}$ & 5 & - & -- & 1 \\
\hline WAW & 41 & 60 & $12-09-85$ & $\mathbf{H}$ & 30 & $\cdots$ & $\cdots$ & .5 \\
\hline WAW & 43 & $\cdots$ & - & H & 4 & $\cdots$ & $\cdots$ & 2 \\
\hline WAW & 45 & 10 & $04-01-86$ & H & 12 & $\cdots$ & $\cdots$ & .5 \\
\hline WAW & 46 & 10 & $04-02-86$ & H & 5 & - & -- & .5 \\
\hline WAW & 47 & 10 & $04-03-86$ & H & 20 & - & $-\cdot$ & .5 \\
\hline WAW & 48 & 50 & $04-04-86$ & $\mathbf{H}$ & 5 & -- & - & .5 \\
\hline WAW & 49 & 55 & $04-10-86$ & $\mathbf{H}$ & 1.5 & $\cdots$ & $\cdots$ & .5 \\
\hline WAW & 50 & 10 & $04-14-86$ & H & 60 & $\cdots$ & $-\cdot$ & .5 \\
\hline WAW & 51 & 4 & $04-29-86$ & $\mathrm{H}$ & 15 & - & -- & .5 \\
\hline WAW & 52 & 10 & $05-09-86$ & H & 10 & -- & $\cdots$ & 1.5 \\
\hline WAW & 54 & 50 & $05-08-86$ & $\mathrm{H}$ & 50 & $\cdots$ & $\cdots$ & 1 \\
\hline WAW & 97 & 10 & $05-09-86$ & $\mathrm{H}$ & 10 & -- & $-\cdot$ & 1.5 \\
\hline WAW & 99 & $\cdots$ & - - & $\mathrm{H}$ & 4 & - & -- & $\cdots$ \\
\hline WAW & 100 & - & $\cdots$ & $\mathrm{H}$ & 1 & $\cdots$ & $\cdots$ & $I$ \\
\hline WAW & 101 & 20 & $08-18 \cdot 86$ & $\mathbf{H}$ & 25 & $-\cdot$ & $\cdots$ & .5 \\
\hline WAW & 102 & 60 & $07-14-86$ & $\mathrm{H}$ & 30 & $\cdots$ & $\cdots$ & .5 \\
\hline WAW & 103 & 50 & $07-17-86$ & $\mathrm{H}$ & 10 & $\cdots$ & $\cdots$ & .5 \\
\hline WAW & 104 & 50 & $11-29-86$ & H & 2 & $\cdots$ & $\cdots$ & .5 \\
\hline WAW & 105 & $\cdots$ & $\cdots$ & $\mathbf{H}$ & 12 & $\cdots$ & $\cdots$ & 1 \\
\hline WAW & 106 & - & $\cdots$ & $\mathrm{H}$ & 15 & $\cdots$ & $\cdots$ & 1 \\
\hline WAW & 107 & 40 & $08-07-87$ & H & 1 & $\cdots$ & $\cdots$ & .5 \\
\hline WAW & 108 & 25 & $08-11-87$ & $\mathrm{H}$ & 5 & $\cdots$ & - & .5 \\
\hline WAW & 109 & 10 & $08-28-87$ & $\mathrm{H}$ & 5 & $\cdots$ & -- & .5 \\
\hline NAW & 110 & - & $\cdots$ & $\mathrm{H}$ & 3 & $\cdots$ & - & 1 \\
\hline NAW & 111 & - & - & $\mathrm{H}$ & 5 & $\cdots$ & -- & .8 \\
\hline NAW & 112 & -- & -- & $\mathrm{H}$ & 50 & $\cdots$ & -- & 1 \\
\hline NAW & 113 & $\cdots$ & $\cdots$ & $\mathrm{H}$ & 100 & - & - & - \\
\hline NAW & 114 & $\cdots$ & $\cdots$ & $\mathrm{H}$ & 10 & $\cdots$ & $\cdots$ & $\cdots$ \\
\hline NAW & 115 & -- & $\cdots$ & $\mathbf{H}$ & 4 & $\cdots$ & $\ldots$ & 1 \\
\hline NAW & 116 & $\cdots$ & $\cdots$ & $\mathrm{H}$ & 3 & -- & $\cdots$ & 1 \\
\hline NAW & 117 & $\cdots$ & $\cdots$ & $\mathrm{H}$ & 2 & - - & - & 1 \\
\hline NAW & 118 & - & - & $\mathrm{H}$ & 1 & $\cdots$ & $\cdots$ & 1.5 \\
\hline NAW & 119 & 45 & $08-09-87$ & $\mathrm{H}$ & 35 & $\cdots$ & $-\cdot$ & .5 \\
\hline NAW & 120 & $\cdots$ & $\cdots$ & H & 25 & - & - & .3 \\
\hline NAW & 121 & $\cdots$ & - & $\mathbf{H}$ & 0.3 & . & - & 1 \\
\hline NAW & 122 & 20 & $05-07-87$ & $\mathrm{H}$ & 7.5 & $\cdots$ & $\cdots$ & .5 \\
\hline VAW & 123 & - & $\cdots$ & H & 100 & - & - & 1 \\
\hline VAW & 124 & - & $\cdots$ & H & 5 & $-\cdot$ & $\cdots$ & 1 \\
\hline NAW & 125 & $\cdots$ & $\cdots$ & $\mathrm{H}$ & 0.2 & $\cdots$ & -- & 4.5 \\
\hline
\end{tabular}


Table 1-1. Description of selected wells, borings, and springs in the Saco and Ossipee River Basins, east-central

\begin{tabular}{|c|c|c|c|c|c|c|c|c|c|c|c|c|}
\hline \multicolumn{2}{|c|}{$\begin{array}{l}\text { Local } \\
\text { site } \\
\text { number }\end{array}$} & $\begin{array}{l}\text { Lat- } \\
\text { itude }\end{array}$ & $\begin{array}{l}\text { Long- } \\
\text { itude }\end{array}$ & Owner or user & $\begin{array}{l}\text { Year } \\
\text { com- } \\
\text { pleted }\end{array}$ & $\begin{array}{c}\text { Alti- } \\
\text { tude } \\
\text { above } \\
\text { sea } \\
\text { level } \\
\text { (feet) }\end{array}$ & $\begin{array}{l}\text { Dia- } \\
\text { meter } \\
\text { of } \\
\text { well } \\
\text { casing } \\
\text { (inches) }\end{array}$ & $\begin{array}{l}\text { Depth } \\
\text { to } \\
\text { bottom } \\
\text { of } \\
\text { casing } \\
\text { (feet) }\end{array}$ & $\begin{array}{l}\text { Casing } \\
\text { material }\end{array}$ & $\begin{array}{l}\text { Type } \\
\text { of } \\
\text { finish }\end{array}$ & $\begin{array}{l}\text { Depth } \\
\text { to } \\
\text { bottom } \\
\text { of open } \\
\text { section } \\
\text { (feet) }\end{array}$ & $\begin{array}{c}\text { Type } \\
\text { of } \\
\text { site }\end{array}$ \\
\hline \multicolumn{13}{|c|}{ Wakefield.-Continued } \\
\hline WAW & 126 & 433640 & 705844 & S. Larson & 1988 & 600 & -. & 74 & .. & $\mathrm{x}$ & $\cdots$ & BrW \\
\hline WAW & 127 & 434042 & 705852 & Flaherty & 1988 & 540 & $\cdots$ & 93 & $-\cdot$ & $x$ & $\cdots$ & BrW \\
\hline WAW & 128 & 433830 & 705927 & Fisher & 1988 & 595 & - & 40 & -. & $\mathrm{x}$ & . & BrW \\
\hline WAW & 129 & 433931 & 705928 & Kench & 1988 & 620 & $\cdots$ & 41 & $-\cdot$ & $x$ & - & $B r W$ \\
\hline WAW & 130 & 434100 & 710020 & Dolaher & 1988 & 520 & - & 59 & - & $\mathrm{x}$ & - - & Brw \\
\hline WAW & 131 & 434040 & 705931 & Gillespie & 1988 & 500 & - - & 50 & - & $\mathrm{x}$ & -. & BrW \\
\hline WAW & 132 & 433745 & 710127 & Brown & 1988 & 610 & - & 131 & $\cdots$ & $\mathrm{x}$ & $\cdots$ & $\mathrm{BrW}$ \\
\hline WAW & 133 & 433736 & 710200 & E11is & 1988 & 595 & -. & 84 & - . & $\mathrm{x}$ & - - & $\mathrm{BrW}$ \\
\hline WAW & 134 & 433754 & 705911 & R. Stevens & 1988 & 560 & - & 129 & - - & $\mathrm{x}$ & - & BrW \\
\hline WAW & 135 & 433753 & 710233 & Clark & 1988 & 640 & $\cdots$ & 130 & $\cdots$ & $\mathrm{x}$ & -- & BrW \\
\hline WAW & 136 & 433700 & 710002 & Spinale & 1989 & 665 & - & 106 & . & $\mathrm{x}$ & $\cdots$ & BrW \\
\hline WAW & 137 & 433924 & 705936 & Perrin MD & 1989 & 590 & - & 80 & - & $\mathrm{x}$ & - & BrW \\
\hline WAW & 138 & 433751 & 710214 & Irving & 1988 & 600 & - & - & . & $\mathrm{s}$ & . & BrW \\
\hline WAW & 139 & 433904 & 705920 & Irving & 1989 & 635 & $-\cdot$ & $\cdots$ & $\cdots$ & $\mathrm{s}$ & $\cdots$ & BrW \\
\hline WAW & 140 & 433658 & 710000 & st.cyr & 1989 & 670 & - & 107 & -. & $\mathrm{x}$ & - & BrW \\
\hline WAW & 141 & 433844 & 705900 & Hackett & 1989 & 630 & - - & 79 & -. & $\mathrm{x}$ & - & BrW \\
\hline WAW & 142 & 433730 & 710150 & Tomasello & 1989 & 590 & .. & 59 & - & $\mathrm{x}$ & - & BrW \\
\hline WAW & 143 & 433904 & 705949 & Gautreau & 1989 & 660 & - & 129 & -. & $x$ & - & BrW \\
\hline WAW & 144 & 433903 & 705947 & O'Connor & 1989 & 660 & - & 113 & - & $x$ & $-\cdot$ & BrW \\
\hline WAW & 145 & 434056 & 705951 & Liani & 1989 & 530 & $\cdots$ & 35 & - & $\mathrm{x}$ & - . & BrW \\
\hline WAW & 146 & 433745 & 710221 & Dodge & 1990 & 650 & .. & 100 & .. & $x$ & .. & $\mathrm{BrW}$ \\
\hline WAW & 147 & 433722 & 705945 & Mccarthy & 1990 & 615 & - & - &.- & -. & $\cdots$ & BrW \\
\hline WAW & 148 & 433815 & 710215 & Plouffe & $\cdots$ & 590 & - & -. & .. & $\mathrm{T}$ & - & Dvn \\
\hline WAW & 149 & 433814 & 710204 & Ayles & $\cdots$ & 595 & 36 & 10.7 & $\mathrm{C}$ & 0 & $\cdots$ & Dug \\
\hline WAW & 150 & 434037 & 705919 & Harold Mallett & 1980 & 510 & 72 & 14.2 & c & 0 & $-\cdot$ & Dug \\
\hline WAW & 151 & 434036 & 705919 & Artist Remick & 1971 & 530 & 36 & 15.7 & $\mathrm{c}$ & 0 & -. & Dug \\
\hline WAW & 152 & 434042 & 705919 & Asher & 1950 & 490 & 72 & 9.5 & $\mathrm{R}$ & 0 & - & Dug \\
\hline WAW & 153 & 434046 & 705928 & Scott Carpenter & 1976 & 490 & $\cdots$ & 8 & G & $\mathrm{T}$ & - & Dvn \\
\hline WAW & 154 & 433815 & 705850 & Gobbi & 1976 & 600 & -. & 22 & $\mathrm{~s}$ & $\mathrm{~T}$ & $-\cdot$ & Dvn \\
\hline WAW & 155 & 433640 & 705910 & Gary Baker & 1988 & 570 & 8 & - & $\mathrm{s}$ & G & -. & - \\
\hline WAW & 156 & 433641 & 705910 & & . & 570 & 36 & 11.3 & $\mathrm{c}$ & 0 & - & Dug \\
\hline \multirow[t]{2}{*}{ WAW } & 157 & 433820 & 710319 & USGS & 1991 & 620 & 2 & 47 & $P$ & $\mathbf{s}$ & 49 & Bor \\
\hline & \multicolumn{12}{|c|}{ Wolfeboro } \\
\hline WRW & 28 & 433834 & 710707 & Brown & 1985 & 1,090 & -. & 95 & .. & $\mathrm{x}$ & -. & Brw \\
\hline WRW & 38 & 434006 & 711136 & Lavare110 & 1986 & 1,160 & - & 38 & - & $\mathrm{x}$ & - & Brw \\
\hline WRW & 50 & 433915 & 710559 & Aversa & 1986 & 720 & - & 38 & - & $x$ & - & $\mathrm{BrW}$ \\
\hline WRW & 63 & 433836 & 711144 & Olimpio & 1987 & 1,220 & .. & 39 & .. & $\mathrm{x}$ & - & BrW \\
\hline WRW & 65 & 433855 & 710504 & wright & 1987 & 840 & - & 80 & -. & $\mathrm{x}$ & - - & BrW \\
\hline WRW & 98 & 433848 & 710453 & Deyab and P. Martin & 1988 & 840 & -. & 40 & - & $\mathrm{x}$ & - & BrW \\
\hline WRW & 135 & 433912 & 710544 & Lush & 1989 & 770 & -. & 85 & .. & $\mathrm{x}$ & . & $\mathrm{BrW}$ \\
\hline WRW & 140 & 433855 & 710511 & Gray & 1990 & 840 & .. & 53 & -. & $\mathrm{x}$ & . & $\mathrm{BrW}$ \\
\hline WRW & 144 & 433836 & 710708 & Kelly & 1990 & 1,100 & .. & 60 & .. & $\mathrm{x}$ & .. & $\mathrm{BrW}$ \\
\hline WRW & 156 & 433913 & 710550 & Nepveu & 1991 & 760 & - & 79 & -. & $\mathrm{x}$ & - & BrW \\
\hline
\end{tabular}




\begin{tabular}{|c|c|c|c|c|c|c|c|c|}
\hline $\begin{array}{l}\text { Local } \\
\text { site } \\
\text { number }\end{array}$ & $\begin{array}{l}\text { Water } \\
\text { level } \\
\text { depth } \\
\text { (feet) }\end{array}$ & $\begin{array}{l}\text { Date } \\
\mathrm{mm} / \mathrm{dd} / \mathrm{yy}\end{array}$ & Use & $\begin{array}{l}\text { Maximum } \\
\text { well } \\
\text { yield } \\
\text { (gallons } \\
\text { per } \\
\text { minute) }\end{array}$ & $\begin{array}{l}\text { Draw- } \\
\text { down } \\
\text { (feet) }\end{array}$ & $\begin{array}{c}\text { Specific } \\
\text { capacity } \\
\text { (gallons per } \\
\text { minute } \\
\text { per } \\
\text { foot) }\end{array}$ & $\begin{array}{l}\text { Pumping } \\
\text { period } \\
\text { (hours) }\end{array}$ & Remarks \\
\hline
\end{tabular}

wakefield--Continued

\begin{tabular}{|c|c|c|c|c|c|c|c|c|c|}
\hline WAW & 126 & - & - & $\mathrm{H}$ & 8 & $\cdots$ & - & 1 & \\
\hline WAW & 127 & 12 & $06-18-88$ & H & 8 & $\cdots$ & . & .5 & \\
\hline WAW & 128 & 15 & $07-05-88$ & H & 30 & $\cdots$ & $\cdots$ & .5 & \\
\hline WAW & 129 & -. & - & H & 1 & $\cdots$ & $\cdots$ & 1.3 & \\
\hline WAW & 130 & $\cdots$ & $\cdots$ & H & 100 & $\cdots$ & $\cdots$ & .5 & \\
\hline WAW & 131 & $\cdots$ & - & H & 12 & $\cdots$ & $\cdots$ & 1 & \\
\hline WAW & 132 & $\cdots$ & $\cdots$ & H & 15 & $\cdots$ & $\cdots$ & .8 & \\
\hline WAW & 133 & $\cdots$ & $\cdots$ & H & 50 & $\cdots$ & $\cdots$ & .8 & \\
\hline WAW & 134 & $\cdots$ & $\cdots$ & H & 5 & $\cdots$ & $\cdots$ & 1 & \\
\hline WAW & 135 & 30 & $12-08-88$ & H & 5 & - & - & .5 & \\
\hline WAW & 136 & - & - & H & 2 & $\cdots$ & $\cdots$ & 1.5 & \\
\hline WAW & 137 & $\cdots$ & - & H & 20 & $\cdots$ & $\cdots$ & 1 & \\
\hline WAW & 138 & 3 & $06-23-88$ & H & 20 & $\cdots$ & -. & 5 & \\
\hline WAW & 139 & 25 & $09-11-89$ & H & 15 & - & $\cdots$ & 5 & \\
\hline WAW & 140 & 20 & $07-28-89$ & H & 20 & $\cdots$ & $\cdots$ & .5 & \\
\hline WAW & 141 & 75 & $09-06.89$ & H & 100 & $\cdots$ & - & 1 & \\
\hline WAW & 142 & $-\cdot$ & - & H & 12 & $\cdots$ & $\cdots$ & 1 & \\
\hline WAW & 143 & $\cdots$ & $\cdots$ & H & 30 & . & $\cdots$ & 1 & \\
\hline WAW & 144 & 30 & $05-09-89$ & н & 50 & $\cdots$ & $\cdots$ & 1 & \\
\hline WAW & 145 & $\cdots$ & - & H & 15 & $-\cdot$ & $\cdots$ & 1 & \\
\hline WAW & 146 & 40 & $08-02-90$ & $\mathrm{H}$ & 10 & $\cdots$ & - & 1 & \\
\hline WAW & 147 & 15 & $05-19-90$ & $\mathrm{H}$ & 15 & $\cdots$ & $\cdots$ & 8 & \\
\hline WAW & 148 & 3.2 & $07-16-91$ & H & $-\cdot$ & $\cdots$ & $\cdots$ & $\cdots$ & \\
\hline WAW & 149 & 3 & $07-16-91$ & $\mathrm{H}$ & - & $\cdots$ & $\cdots$ & $\cdots$ & \\
\hline WAW & 150 & 13.1 & $07-16-91$ & $\mathrm{H}$ & $\cdots$ & $\cdots$ & $\cdots$ & $\cdots$ & $\mathrm{R}$ \\
\hline WAW & 151 & 5.7 & $07-16-91$ & H & $\cdots$ & $\cdots$ & $\cdots$ & $\cdots$ & \\
\hline WAW & 152 & 4.9 & $07-17-91$ & H & $-\cdot$ & $\cdots$ & $\cdots$ & $\cdots$ & $\mathrm{R}$ \\
\hline WAW & 153 & 4 & $07-17-91$ & T H & $\cdots$ & $\cdots$ & $\cdots$ & $\cdots$ & $R$ \\
\hline WAW & 154 & 12 & $07-17-91$ & H & $\cdots$ & $\cdots$ & $\cdots$ & $-\cdot$ & Contains iron \\
\hline WAW & 155 & -. & - & H & - & - & - & - & \\
\hline WAW & 156 & 5 & $07-17-91$ & H & $\cdots$ & $\cdots$ & $\cdots$ & $\cdots$ & \\
\hline WAW & 157 & 17 & $10-11-91$ & 0 & $\cdots$ & $\cdots$ & $\cdots$ & $\cdots$ & USGS \\
\hline & & & & \multicolumn{6}{|c|}{ Wolfeboro } \\
\hline WRW & 28 & 20 & $12-18-85$ & H & 2.5 & $\cdots$ & $\cdots$ & 1 & \\
\hline WRW & 38 & 12 & $06-19 \cdot 86$ & H & 28 & $\cdots$ & $\cdots$ & 72 & \\
\hline WRW & 50 & - & - & H & 1 & $\cdots$ & $\cdots$ & 1 & \\
\hline WRW & 63 & - & - & $\mathrm{H}$ & 40 & $\cdots$ & $\cdots$ & 1 & \\
\hline WRW & 65 & 30 & $08-13-87$ & H & 12 & $\cdots$ & $\cdots$ & .5 & \\
\hline WRW & 98 & 15 & $07-05-88$ & H & 6 & - & $\cdots$ & .5 & \\
\hline WRW & 135 & $\cdots$ & . & H & 35 & $\cdots$ & $\cdots$ & .5 & \\
\hline WRW & 140 & 20 & $09-18-90$ & H & 20 & $\cdots$ & $\cdots$ & 1 & \\
\hline WRW & 144 & 60 & $05-15-90$ & H & 1 & - & $\cdots$ & 1 & \\
\hline WRW & 156 & - & - & H & 7.5 & - & $\cdots$ & .4 & \\
\hline
\end{tabular}


APPENDIX 2. Stratigraphic logs of selected wells and borings in the Saco and Ossipee River Basins, east-central, New Hampshire 
Table 2-1. Stratigraphic logs of selected wells and borings in the Saco and Ossipee River Basins, east-central New Hampshire.

Local Site number: First two characters are U.S. Geological Survey town and code. Third character indicates -- A, borings done for hydrologic purposes; $\mathrm{B}$, borings done primarily for constructional purposes; S, springs; W, wells. The numbers are sequential numbers for each town.

Depth drilled: Depth drilled in feet below land-surface datum.

Depth of well: Depth of well in feet below land-surface datum.

Depth to refusal: Depth to bedrock or refusal in feet below land-surface datum.

Depth to top: Depth to top of lithologic unit, in feet below land-surface datum.

Depth to bottom: Depth to bottom of lithologic unit, in feet below land-surface datum.

\section{Aquifer code:}

Codes for the following geologic ages and aquifer materials are listed below.

$\begin{array}{llll}\text { 110SDMN } & \text { Quaternary sediment, undifferentiated } & \text { 110SOIL } & \text { Quaternary soil } \\ \text { 111SDMN } & \text { Holocene sediments, undifferentiated } & 111 \text { FILL } & \text { Holocene fill } \\ \text { 111SWMP } & \text { Holocene swamp deposits } & 112 \text { SRFD } & \text { Pleistocene stratified drift } \\ \text { 112GLCL } & \text { Pleistocene glacial deposits, undifferentiated } & 112 \text { TILL } & \text { Pleistocene till } \\ 112 \mathrm{CSR} & \text { Pleistocene lacustrine deposits } & \text { BEDROCK } & \text { bedrock }\end{array}$

\section{Lithology Code:}

The following lithologic codes are used to describe aquifer units.

$\begin{array}{llll}\text { ALVM } & \text { alluvium } & \text { BLDR } & \text { boulders } \\ \text { GLCL } & \text { glacial (undifferentiated) } & \text { GRNT } & \text { granite } \\ \text { GRSC } & \text { gravel, silt, and clay } & \text { GRVL } & \text { gravel } \\ \text { MUCK } & \text { muck } & \text { OTHR } & \text { other } \\ \text { SAND } & \text { sand } & \text { SDCL } & \text { sand and clay } \\ \text { SDST } & \text { sand and silt } & \text { SGVC } & \text { sand, gravel, and clay } \\ \text { CLAY } & \text { clay } & \text { GRDS } & \text { gravel, sand, and silt } \\ \text { LOAM } & \text { loam } & \text { PEAT } & \text { peat } \\ \text { SDGL } & \text { sand and gravel } & \text { SILT } & \text { silt } \\ \text { STCL } & \text { silt and clay } & \text { TILL } & \text { till }\end{array}$

The following abbreviations are used to modify the lithologic descriptions:

$\begin{array}{clrl}\text { VF } & \text { Very fine } & \text { C } & \text { Coarse } \\ \text { F } & \text { Fine } & \text { VC } & \text { Very Coarse } \\ \text { M } & \text { Medium } & \text { S } & \text { Sand }\end{array}$


Table 2-1. Stratigraphic logs of wells and borings in the Saco and Ossipee River Basins, eastcentral New Hampshire

[--, A dash indicates no data available]

\begin{tabular}{|c|c|c|c|c|c|c|c|}
\hline $\begin{array}{l}\text { Local } \\
\text { site } \\
\text { no. }\end{array}$ & $\begin{array}{l}\text { Depth } \\
\text { drilled } \\
\text { (feet) }\end{array}$ & $\begin{array}{l}\text { Depth } \\
\text { of } \\
\text { well } \\
\text { (feet) }\end{array}$ & $\begin{array}{l}\text { Depth } \\
\text { to } \\
\text { refusal } \\
\text { (feet) }\end{array}$ & $\begin{array}{l}\text { Depth } \\
\text { to } \\
\text { top } \\
\text { (feet) }\end{array}$ & $\begin{array}{l}\text { Depth } \\
\text { to } \\
\text { bottom } \\
\text { (feet) }\end{array}$ & $\begin{array}{l}\text { Aquifer } \\
\text { code }\end{array}$ & Lithology \\
\hline
\end{tabular}

CARROLL COUNTY

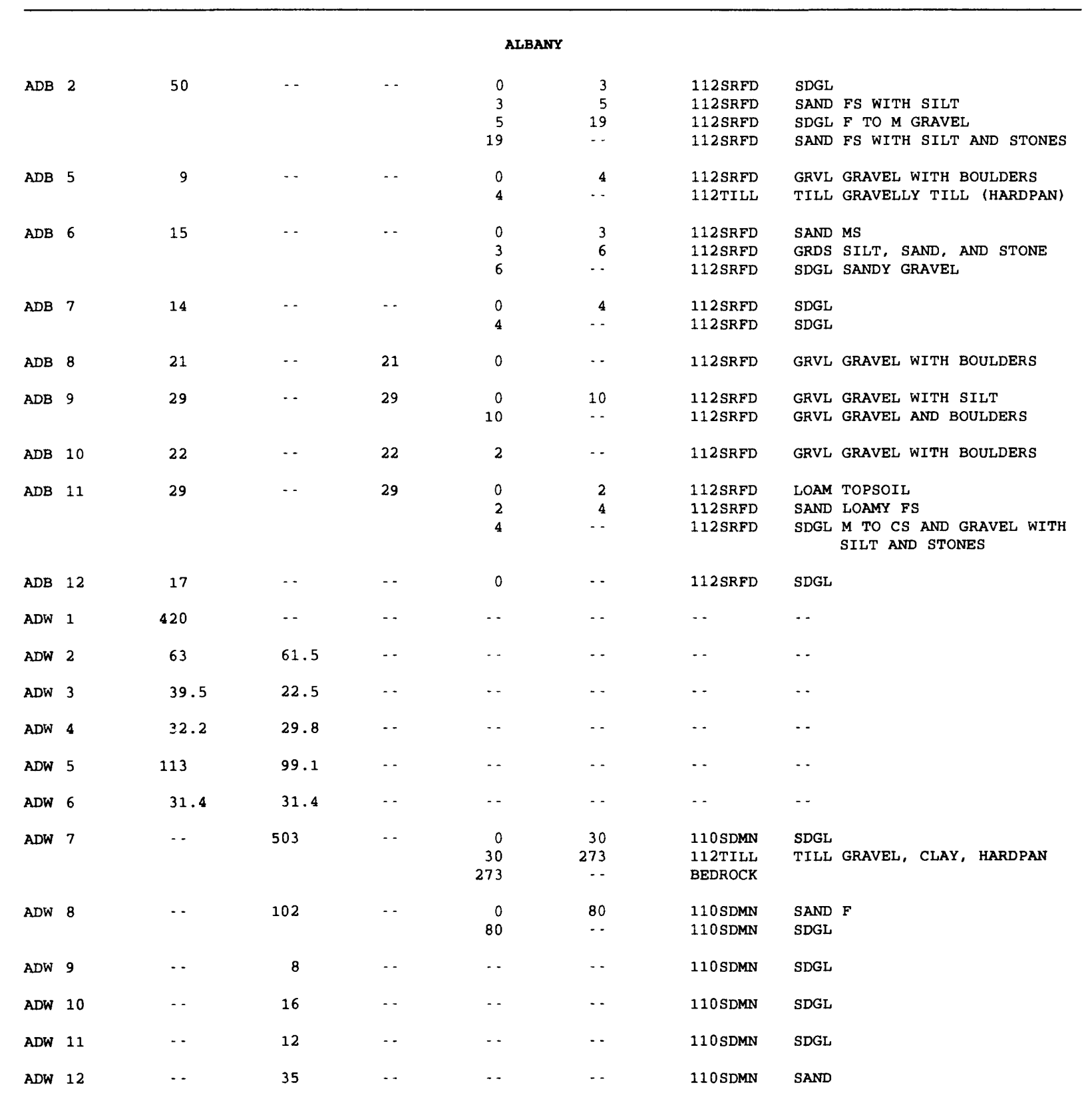


Table 2-1. Stratigraphic logs of wells and borings in the Saco and Ossipee River Basins, eastcentral New Hampshire--Continued

\begin{tabular}{|c|c|c|c|c|c|c|c|}
\hline $\begin{array}{l}\text { Local } \\
\text { site } \\
\text { No. }\end{array}$ & $\begin{array}{l}\text { Depth } \\
\text { drilled } \\
\text { (feet) }\end{array}$ & $\begin{array}{l}\text { Depth } \\
\text { of } \\
\text { well } \\
\text { (feet) }\end{array}$ & $\begin{array}{l}\text { Depth } \\
\text { to } \\
\text { refusal } \\
\text { (feet) }\end{array}$ & $\begin{array}{l}\text { Depth } \\
\text { to } \\
\text { top } \\
\text { (feet) }\end{array}$ & $\begin{array}{l}\text { Depth } \\
\text { to } \\
\text { bottom } \\
\text { (feet) }\end{array}$ & $\begin{array}{l}\text { Aquifer } \\
\text { code }\end{array}$ & Lithology \\
\hline
\end{tabular}

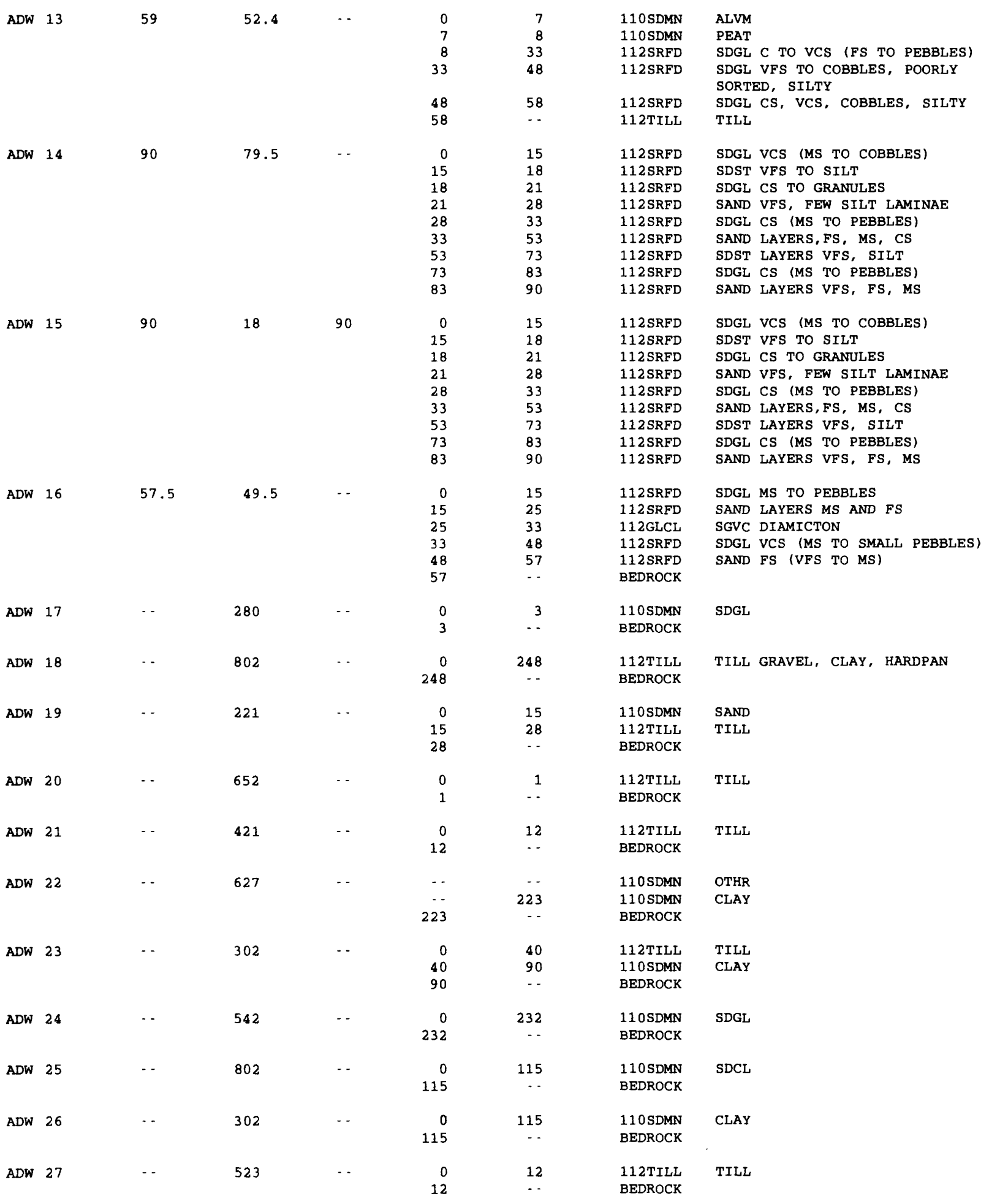


Table 2-1. Stratigraphic logs of wells and borings in the Saco and Ossipee River Basins, eastcentral New Hampshire--Continued

\begin{tabular}{|c|c|c|c|c|c|c|c|c|}
\hline \multicolumn{2}{|c|}{$\begin{array}{l}\text { Local } \\
\text { site } \\
\text { no. }\end{array}$} & $\begin{array}{l}\text { Depth } \\
\text { drilled } \\
\text { (feet) }\end{array}$ & $\begin{array}{l}\text { Depth } \\
\text { of } \\
\text { well } \\
\text { (feet) }\end{array}$ & $\begin{array}{l}\text { Depth } \\
\text { to } \\
\text { refusal } \\
\text { (feet) }\end{array}$ & $\begin{array}{l}\text { Depth } \\
\text { to } \\
\text { top } \\
\text { (feet) }\end{array}$ & $\begin{array}{l}\text { Depth } \\
\text { to } \\
\text { bottom } \\
\text { (feet) }\end{array}$ & $\begin{array}{c}\text { Aquifer } \\
\text { code }\end{array}$ & Lithology \\
\hline \multicolumn{9}{|c|}{ ALBANY - - Continued } \\
\hline ADW & 28 & $-\cdot$ & 20 & $\cdots$ & $-\cdot$ & -- & 112TILL & TILL \\
\hline ADW & 29 & $-\cdot$ & 19 & $\cdots$ & $\begin{array}{r}0 \\
8 \\
10 \\
12\end{array}$ & $\begin{array}{r}8 \\
10 \\
12 \\
--\end{array}$ & $\begin{array}{l}112 \mathrm{TILL} \\
110 \mathrm{SDMN} \\
112 \mathrm{TILL} \\
110 \mathrm{SDMN}\end{array}$ & $\begin{array}{l}\text { TILL } \\
\text { SDGL } \\
\text { TILL } \\
\text { SDGL }\end{array}$ \\
\hline ADW & 30 & $\cdots$ & 421 & $\cdots$ & $\begin{array}{l}0 \\
2\end{array}$ & 2 & $\begin{array}{l}\text { 112TILL } \\
\text { BEDROCK }\end{array}$ & TILL \\
\hline ADW & 31 & $\cdots$ & 242 & $\cdots$ & $\begin{array}{r}0 \\
17\end{array}$ & $\begin{array}{l}17 \\
--\end{array}$ & $\begin{array}{l}\text { 112TILL } \\
\text { BEDROCK }\end{array}$ & TILL \\
\hline ADW & 32 & $\cdots$ & 202 & $\cdots$ & $\begin{array}{r}0 \\
18\end{array}$ & $\begin{array}{l}18 \\
\cdots\end{array}$ & $\begin{array}{l}\text { 112TILL } \\
\text { BEDROCK }\end{array}$ & TILL \\
\hline$A D W$ & 33 & - & 282 & $\cdots$ & $\begin{array}{r}0 \\
120 \\
270\end{array}$ & $\begin{array}{r}120 \\
270 \\
--\end{array}$ & $\begin{array}{l}\text { 112TILL } \\
\text { BEDROCK } \\
\text { BEDROCK }\end{array}$ & TILL \\
\hline ADW & 34 & $\cdots$ & 521 & $\cdots$ & $\begin{array}{r}0 \\
50 \\
220\end{array}$ & $\begin{array}{r}50 \\
220 \\
-.\end{array}$ & $\begin{array}{l}110 \text { SDMN } \\
112 \text { TILL } \\
\text { BEDROCK }\end{array}$ & $\begin{array}{l}\text { SDGL } \\
\text { TILL }\end{array}$ \\
\hline ADW & 35 & $\cdots$ & 1110 & $\cdots$ & $\begin{array}{r}0 \\
22\end{array}$ & 22 & $\begin{array}{l}112 \mathrm{TILL} \\
\text { BEDROCK }\end{array}$ & TILL \\
\hline ADW & 36 & $\cdots$ & 280 & $\cdots$ & $\begin{array}{r}0 \\
205\end{array}$ & 205 & $\begin{array}{l}\text { 112TILL } \\
\text { BEDROCK }\end{array}$ & TILL \\
\hline ADW & 37 & -- & 422 & $\cdots$ & $\begin{array}{r}0 \\
60\end{array}$ & $\begin{array}{l}60 \\
\cdots\end{array}$ & $\begin{array}{l}\text { 112TILL } \\
\text { BEDROCK }\end{array}$ & TILL \\
\hline ADW & 38 & - & 424 & $-\cdot$ & $\begin{array}{r}0 \\
180 \\
200\end{array}$ & $\begin{array}{l}180 \\
200\end{array}$ & $\begin{array}{l}\text { 112TILL } \\
\text { BEDROCK } \\
\text { BEDROCK }\end{array}$ & TILL \\
\hline $\mathrm{ADW}$ & 39 & $\cdots$ & 102 & $\cdots$ & $\begin{array}{r}0 \\
15\end{array}$ & $\begin{array}{l}15 \\
-\end{array}$ & $\begin{array}{l}\text { 112TILL } \\
\text { BEDROCK }\end{array}$ & TILL SAND, GRAVEL， HARDPAN \\
\hline $\mathrm{ADW}$ & 40 & $\cdots$ & 345 & $-\cdot$ & $\begin{array}{r}0 \\
15\end{array}$ & $\begin{array}{l}15 \\
\cdots\end{array}$ & $\begin{array}{l}110 \text { SDMN } \\
\text { BEDROCK }\end{array}$ & SAND \\
\hline ADW & 41 & - & 343 & $\cdots$ & $\begin{array}{r}0 \\
40 \\
240 \\
250\end{array}$ & $\begin{array}{r}40 \\
240 \\
250 \\
--\end{array}$ & $\begin{array}{l}110 \text { SDMN } \\
110 \text { SDMN } \\
\text { BEDROCK } \\
\text { BEDROCK }\end{array}$ & $\begin{array}{l}\text { SDGL } \\
\text { SDCL }\end{array}$ \\
\hline $\mathrm{ADW}$ & 42 & $\cdots$ & 922 & $\cdots$ & 11 & $\cdots$ & BEDROCK & \\
\hline $\mathrm{ADW}$ & 43 & - & 422 & $\cdots$ & $\begin{array}{r}0 \\
45\end{array}$ & $\begin{array}{l}45 \\
--\end{array}$ & $\begin{array}{l}\text { 112TILL } \\
\text { BEDROCK }\end{array}$ & TILL \\
\hline $\mathrm{ADW}$ & 44 & $\cdots$ & 322 & $\cdots$ & $\begin{array}{r}0 \\
25\end{array}$ & $\begin{array}{l}25 \\
\cdots\end{array}$ & $\begin{array}{l}\text { 112TILL } \\
\text { BEDROCK }\end{array}$ & TILL \\
\hline $\mathrm{ADW}$ & 45 & - & 422 & - & $\begin{array}{r}0 \\
15\end{array}$ & $\begin{array}{l}15 \\
\cdots\end{array}$ & $\begin{array}{l}\text { 112TILL } \\
\text { BEDROCK }\end{array}$ & TILL \\
\hline $\mathrm{ADW}$ & 46 & $\cdots$ & 523 & -. & $\begin{array}{r}0 \\
180\end{array}$ & $\begin{array}{r}180 \\
\cdots\end{array}$ & $\begin{array}{l}\text { 112TILL } \\
\text { BEDROCK }\end{array}$ & TILL \\
\hline $\mathrm{ADW}$ & 47 & $\cdots$ & 727 & $\cdots$ & $\begin{array}{r}0 \\
40 \\
50\end{array}$ & $\begin{array}{l}40 \\
50 \\
\cdots\end{array}$ & $\begin{array}{l}110 \text { SDMN } \\
110 \text { SDMN } \\
\text { BEDROCK }\end{array}$ & $\begin{array}{l}\text { SDCL } \\
\text { SDGL }\end{array}$ \\
\hline $\mathrm{ADW}$ & 48 & - & 325 & $\cdots$ & $\begin{array}{r}0 \\
60\end{array}$ & $\begin{array}{l}60 \\
\cdots\end{array}$ & $\begin{array}{l}\text { 112TILL } \\
\text { BEDROCK }\end{array}$ & TILL \\
\hline
\end{tabular}


Table 2-1. Stratigraphic logs of wells and borings in the Saco and Ossipee River Basins, eastcentral New Hampshire--Continued

\begin{tabular}{|c|c|c|c|c|c|c|c|c|}
\hline $\begin{array}{l}\text { Loca } \\
\text { site } \\
\text { No. }\end{array}$ & & $\begin{array}{l}\text { Depth } \\
\text { drilled } \\
\text { (feet) }\end{array}$ & $\begin{array}{l}\text { Depth } \\
\text { of } \\
\text { well } \\
\text { (feet) }\end{array}$ & $\begin{array}{l}\text { Depth } \\
\text { to } \\
\text { refusal } \\
\text { (feet) }\end{array}$ & $\begin{array}{l}\text { Depth } \\
\text { to } \\
\text { top } \\
\text { (feet) }\end{array}$ & $\begin{array}{l}\text { Depth } \\
\text { to } \\
\text { bottom } \\
\text { (feet) }\end{array}$ & $\begin{array}{l}\text { Aquifer } \\
\text { code }\end{array}$ & Lithology \\
\hline \multicolumn{9}{|c|}{ ALBANY - - Continued } \\
\hline ADW & 49 & $\cdots$ & $\begin{array}{l}385 \\
425\end{array}$ & $\cdots$ & $\begin{array}{r}0 \\
134 \\
0 \\
115\end{array}$ & $\begin{array}{r}134 \\
\cdots \\
115 \\
\cdots\end{array}$ & $\begin{array}{l}110 \text { SDMN } \\
\text { BEDROCK } \\
110 \text { SDMN } \\
\text { BEDROCK }\end{array}$ & SDGL \\
\hline ADW & 51 & - & 145 & $-\cdot$ & $\begin{array}{r}0 \\
26\end{array}$ & $\begin{array}{l}26 \\
\cdots\end{array}$ & $\begin{array}{l}110 \text { SDMN } \\
\text { BEDROCK }\end{array}$ & SDGL \\
\hline ADW & 52 & $\cdots$ & 145 & $\cdots$ & $\begin{array}{r}0 \\
11\end{array}$ & $\begin{array}{l}11 \\
\cdots\end{array}$ & $\begin{array}{l}110 \text { SDMN } \\
\text { BEDROCK }\end{array}$ & SDGL \\
\hline ADW & 53 & $\cdots$ & 225 & $\cdots$ & $\begin{array}{r}0 \\
108\end{array}$ & $\begin{array}{c}108 \\
\ldots\end{array}$ & $\begin{array}{l}110 \text { SDMN } \\
\text { BEDROCK }\end{array}$ & SDGL \\
\hline $\mathrm{ADW}$ & 54 & $\cdots$ & 225 & $\cdots$ & $\begin{array}{r}0 \\
43\end{array}$ & $\begin{array}{l}43 \\
--\end{array}$ & $\begin{array}{l}110 \text { SDMN } \\
\text { BEDROCK }\end{array}$ & SDGL \\
\hline ADW & 55 & $-\cdot$ & 165 & $\cdots$ & $\begin{array}{r}0 \\
30\end{array}$ & $\begin{array}{l}30 \\
\cdots\end{array}$ & $\begin{array}{l}110 \text { SDMN } \\
\text { BEDROCK }\end{array}$ & SDGL \\
\hline ADW & 56 & $\cdots$ & 445 & - - & $\begin{array}{r}0 \\
108\end{array}$ & $\begin{array}{c}108 \\
\ldots\end{array}$ & $\begin{array}{l}110 \text { SDMN } \\
\text { BEDROCK }\end{array}$ & SDGL \\
\hline ADW & 57 & - & 405 & $\cdots$ & $\begin{array}{r}0 \\
87\end{array}$ & $\begin{array}{l}87 \\
--\end{array}$ & $\begin{array}{l}110 \text { SDMN } \\
\text { BEDROCK }\end{array}$ & SDGL \\
\hline $\mathrm{ADW}$ & 58 & $\cdots$ & 445 & $\cdots$ & $\begin{array}{r}0 \\
94\end{array}$ & $\begin{array}{l}94 \\
\cdots\end{array}$ & $\begin{array}{l}110 \mathrm{SDMN} \\
\text { BEDROCK }\end{array}$ & SDGL \\
\hline ADW & 59 & $\cdots$ & 325 & $\cdots$ & $\begin{array}{r}0 \\
12\end{array}$ & $\begin{array}{l}12 \\
\cdots\end{array}$ & $\begin{array}{l}110 \mathrm{SDMN} \\
\text { BEDROCK }\end{array}$ & SDGL \\
\hline $\mathrm{ADW}$ & 60 & $\cdots$ & 165 & $\cdots$ & $\begin{array}{r}0 \\
22\end{array}$ & $\begin{array}{l}22 \\
\cdots\end{array}$ & $\begin{array}{l}110 \text { SDMN } \\
\text { BEDROCK }\end{array}$ & SDGL \\
\hline $\mathrm{ADW}$ & 61 & - & 285 & $\cdots$ & $\begin{array}{r}0 \\
68\end{array}$ & $\begin{array}{l}68 \\
-.\end{array}$ & $\begin{array}{l}110 \mathrm{SDMN} \\
\text { BEDROCK }\end{array}$ & SDGL \\
\hline ADW & 62 & $\cdots$ & 425 & $\cdots$ & $\begin{array}{r}0 \\
89\end{array}$ & $\begin{array}{l}89 \\
-\cdot\end{array}$ & $\begin{array}{l}110 \text { SDMN } \\
\text { BEDROCK }\end{array}$ & SDGL \\
\hline ADW & 63 & $\cdots$ & 345 & $-\cdot$ & $\begin{array}{r}0 \\
18\end{array}$ & $\begin{array}{l}18 \\
\cdots\end{array}$ & $\begin{array}{l}110 \text { SDMN } \\
\text { BEDROCK }\end{array}$ & SDGL \\
\hline ADW & 64 & - & 305 & $\cdots$ & $\begin{array}{r}0 \\
10\end{array}$ & $\begin{array}{l}10 \\
\cdots\end{array}$ & $\begin{array}{l}110 \text { SDMN } \\
\text { BEDROCK }\end{array}$ & SDGL \\
\hline $\mathrm{ADW}$ & 65 & $\cdots$ & 445 & $\cdots$ & $\begin{array}{r}0 \\
50\end{array}$ & $\begin{array}{l}50 \\
\cdots\end{array}$ & $\begin{array}{l}110 \mathrm{SDMN} \\
\text { BEDROCK }\end{array}$ & SDGL \\
\hline ADW & 66 & - & 183 & $\cdots$ & $\begin{array}{r}0 \\
10\end{array}$ & $\begin{array}{l}10 \\
\cdots\end{array}$ & $\begin{array}{l}\text { 112TILL } \\
\text { BEDROCK }\end{array}$ & TILL \\
\hline $\mathrm{ADW}$ & 67 & 12 & 12 & $\cdots$ & $\cdots$ & $\cdots$ & $\cdots$ & \\
\hline ADW & 68 & 2.1 & 2.1 & $\cdots$ & $\cdots$ & $\cdots$ & $\cdots$ & \\
\hline$A D W$ & 69 & 6 & 6 & $\cdots$ & - & $-\cdot$ & $\cdots$ & \\
\hline $\mathrm{ADW}$ & 70 & 9.6 & 9.6 & $\cdots$ & $\cdots$ & $\cdots$ & $\cdots$ & \\
\hline \multicolumn{9}{|c|}{ BARTLETT } \\
\hline$B C B$ & 2 & 11.3 & $\cdots$ & $\cdots$ & $\begin{array}{l}0 \\
2.5\end{array}$ & 2.5 & $\begin{array}{l}112 \text { SRFD } \\
112 \text { SRFD }\end{array}$ & $\begin{array}{l}\text { SAND LOAMY SAND } \\
\text { SDGL SAND AND GRAVEL WITH } \\
\text { BOULDERS }\end{array}$ \\
\hline
\end{tabular}


Table 2-1. Stratigraphic logs of wells and borings in the Saco and Ossipee River Basins, eastcentral New Hampshire--Continued

\begin{tabular}{|c|c|c|c|c|c|c|c|c|}
\hline $\begin{array}{l}\text { Loca } \\
\text { site } \\
\text { no. }\end{array}$ & & $\begin{array}{l}\text { Depth } \\
\text { drilled } \\
\text { (feet) }\end{array}$ & $\begin{array}{l}\text { Depth } \\
\text { of } \\
\text { well } \\
\text { (feet) }\end{array}$ & $\begin{array}{l}\text { Depth } \\
\text { to } \\
\text { refusal } \\
\text { (feet) }\end{array}$ & $\begin{array}{l}\text { Depth } \\
\text { to } \\
\text { top } \\
\text { (feet) }\end{array}$ & $\begin{array}{l}\text { Depth } \\
\text { to } \\
\text { bottom } \\
\text { (feet) }\end{array}$ & $\begin{array}{l}\text { Aquifer } \\
\text { code }\end{array}$ & Lithology \\
\hline & & \multicolumn{7}{|c|}{ BARTLETT - - Continued } \\
\hline BCB & 4 & 17 & $-\cdot$ & $\cdots$ & $\begin{array}{l}0 \\
5 \\
9\end{array}$ & $\begin{array}{r}5 \\
9 \\
--\end{array}$ & $\begin{array}{l}112 \text { SRFD } \\
112 \text { SRFD } \\
112 \text { SRFD }\end{array}$ & $\begin{array}{l}\text { SDGL F TO CS AND GRAVEL } \\
\text { PEAT PEAT AND WOOD } \\
\text { SDGL F TO CS, COBBLES, } \\
\text { BOULDERS, AND TRACES OF } \\
\text { SILT }\end{array}$ \\
\hline $\mathrm{BCB}$ & 5 & 7 & $\cdots$ & 7 & $\begin{array}{l}0 \\
3 \\
5\end{array}$ & $\begin{array}{r}3 \\
5 \\
-\end{array}$ & $\begin{array}{l}\text { 112SRFD } \\
112 \text { SRFD } \\
112 S R F D\end{array}$ & $\begin{array}{l}\text { GRSC GRAVEL, SAND, AND SILT } \\
\text { ROCK } \\
\text { SILT SILT WITH WEATHERED ROCK }\end{array}$ \\
\hline $\mathrm{BCB}$ & 6 & 29 & $-\cdot$ & $\cdots$ & $\begin{array}{r}0 \\
8 \\
12 \\
14\end{array}$ & $\begin{array}{r}8 \\
12 \\
14 \\
--\end{array}$ & $\begin{array}{l}112 \text { SRFD } \\
112 \text { SRFD } \\
112 \text { SRFD } \\
112 \text { SRFD }\end{array}$ & $\begin{array}{l}\text { GRVL GRAVEL WITH BOULDERS } \\
\text { BLDR CORED BOULDERS } \\
\text { GRVL } \\
\text { BLDR }\end{array}$ \\
\hline $\mathrm{BCB}$ & 7 & 27.5 & $\cdots$ & $\cdots$ & $\begin{array}{r}0 \\
8 \\
18\end{array}$ & $\begin{array}{r}8 \\
18 \\
-\end{array}$ & $\begin{array}{l}\text { 112SRFD } \\
112 \text { SRFD } \\
\text { BEDROCK }\end{array}$ & $\begin{array}{l}\text { SDGL TRACE OF SILT AND COBBLES } \\
\text { SAND SILTY SAND } \\
\text { GRNT PINK CONWAY GRANITE }\end{array}$ \\
\hline $\mathrm{BCW}$ & 7 & - & 93 & $-\cdot$ & $-\cdot$ & -- & $112 \mathrm{SRFD}$ & \\
\hline $\mathrm{BCW}$ & 8 & $\cdots$ & 305 & $\cdot-$ & 120 & $\cdots$ & BEDROCK & \\
\hline $\mathrm{BCW}$ & 9 & - & 1010 & $-\cdot$ & 100 & - & BEDROCK & \\
\hline $\mathrm{BCW}$ & 10 & $-\cdot$ & 61 & -- & $\cdots$ & - & $112 \mathrm{SRFD}$ & \\
\hline $\mathrm{BCW}$ & 11 & $\cdots$ & 289 & -- & 111 & -- & BEDROCK & \\
\hline $\mathrm{BCW}$ & 12 & $\cdots$ & 80 & $\cdots$ & $-\cdot$ & - & 112SRFD & \\
\hline $\mathrm{BCW}$ & 19 & - & 800 & $-\cdot$ & $\overline{10}$ & $\begin{array}{l}10 \\
-\cdot\end{array}$ & $\begin{array}{l}\text { 112TILL } \\
\text { BEDROCK }\end{array}$ & TILL \\
\hline $\mathrm{BCW}$ & 20 & $-\cdot$ & 753 & $-\cdot$ & $\begin{array}{r}0 \\
25\end{array}$ & $\begin{array}{l}25 \\
\cdots\end{array}$ & $\begin{array}{l}110 \text { SDMN } \\
\text { BEDROCK }\end{array}$ & SDGL \\
\hline $\mathrm{BCW}$ & 21 & $-\cdot$ & 385 & $-\cdot$ & 5 & $\cdots$ & BEDROCK & \\
\hline $\mathrm{BCW}$ & 22 & $\cdots$ & 505 & $\cdots$ & 8 & $\cdots$ & BEDROCK & \\
\hline $\mathrm{BCW}$ & 23 & - & 515 & $\cdots$ & $\begin{array}{l}0 \\
6\end{array}$ & $\begin{array}{l}6 \\
--\end{array}$ & $\begin{array}{l}\text { 112TILL } \\
\text { BEDROCK }\end{array}$ & TILL \\
\hline $\mathrm{BCW}$ & 24 & $\cdots$ & 545 & $-\cdot$ & $\begin{array}{r}0 \\
135\end{array}$ & 135 & $\begin{array}{l}110 \text { SDMN } \\
\text { BEDROCK }\end{array}$ & SAND \\
\hline $\mathrm{BCW}$ & 25 & $-\cdot$ & 245 & - & $\begin{array}{r}0 \\
18\end{array}$ & 18 & $\begin{array}{l}\text { 112TILL } \\
\text { BEDROCK }\end{array}$ & TILL \\
\hline $\mathrm{BCW}$ & 26 & -- & 527 & - & $\begin{array}{r}0 \\
70 \\
150\end{array}$ & $\begin{array}{r}70 \\
150 \\
\cdots\end{array}$ & $\begin{array}{l}112 T I L L \\
112 T I L L \\
\text { BEDROCK }\end{array}$ & $\begin{array}{l}\text { TILL GRAVEL, CLAY, HARDPAN } \\
\text { TILL CLAY, HARDPAN }\end{array}$ \\
\hline $\mathrm{BCW}$ & 27 & $\cdots$ & 265 & $-\cdot$ & $\begin{array}{r}0 \\
30\end{array}$ & $\begin{array}{l}30 \\
--\end{array}$ & $\begin{array}{l}112 \text { TILL } \\
\text { BEDROCK }\end{array}$ & TILL CLAY, HARDPAN \\
\hline $\mathrm{BCW}$ & 28 & $\cdots$ & 242 & $\cdots$ & $\begin{array}{r}0 \\
5 \\
50 \\
90\end{array}$ & $\begin{array}{r}5 \\
50 \\
90 \\
\cdots\end{array}$ & $\begin{array}{l}110 S D M N \\
110 \text { SDMN } \\
112 \text { TILL } \\
\text { BEDROCK }\end{array}$ & $\begin{array}{l}\text { SDGL } \\
\text { SAND } \\
\text { TILL }\end{array}$ \\
\hline $\mathrm{BCW}$ & 29 & $\cdots$ & 298 & $\cdots$ & $\begin{array}{l}0 \\
3\end{array}$ & $\begin{array}{c}3 \\
-\cdot\end{array}$ & $\begin{array}{l}110 S D M N \\
\text { BEDROCK }\end{array}$ & SDGL \\
\hline $\mathrm{BCW}$ & 30 & $-\cdot$ & 298 & $-\cdot$ & $\begin{array}{l}0 \\
4\end{array}$ & $\begin{array}{c}4 \\
-\end{array}$ & $\begin{array}{l}110 \text { SDMN } \\
\text { BEDROCK }\end{array}$ & OTHR \\
\hline $\mathrm{BCW}$ & 31 & $-\cdot$ & 227 & $\cdots$ & $\begin{array}{r}0 \\
50 \\
95\end{array}$ & $\begin{array}{l}50 \\
95 \\
--\end{array}$ & $\begin{array}{l}112 \text { TILL } \\
112 \text { TILL } \\
\text { BEDROCK }\end{array}$ & $\begin{array}{l}\text { TILL } \\
\text { TILL GRAVEL, HARDPAN }\end{array}$ \\
\hline
\end{tabular}


Table 2-1. Stratigraphic logs of wells and borings in the Saco and Ossipee River Basins, eastcentral New Hampshire--Continued

\begin{tabular}{|c|c|c|c|c|c|c|c|c|}
\hline \multicolumn{2}{|c|}{$\begin{array}{l}\text { Local } \\
\text { site } \\
\text { No. }\end{array}$} & $\begin{array}{l}\text { Depth } \\
\text { drilled } \\
\text { (feet) }\end{array}$ & $\begin{array}{l}\text { Depth } \\
\text { of } \\
\text { well } \\
\text { (feet) }\end{array}$ & $\begin{array}{l}\text { Depth } \\
\text { to } \\
\text { refusal } \\
\text { (feet) }\end{array}$ & $\begin{array}{l}\text { Depth } \\
\text { to } \\
\text { top } \\
\text { (feet) }\end{array}$ & $\begin{array}{l}\text { Depth } \\
\text { to } \\
\text { bottom } \\
\text { (feet) }\end{array}$ & $\begin{array}{l}\text { Aquifer } \\
\text { code }\end{array}$ & Lithology \\
\hline & & \multicolumn{7}{|c|}{ BARTLETT - - Continued } \\
\hline $\mathrm{BCW}$ & 32 & - & 277 & - & $\begin{array}{r}0 \\
60\end{array}$ & 60 & $\begin{array}{l}112 \text { TILL } \\
\text { BEDROCK }\end{array}$ & TILL \\
\hline $\mathrm{BCW}$ & 33 & $\cdots$ & 381 & $\cdots$ & $\begin{array}{r}0 \\
60\end{array}$ & $\begin{array}{l}60 \\
\cdots\end{array}$ & $\begin{array}{l}110 \text { SDMN } \\
\text { BEDROCK }\end{array}$ & SDGL \\
\hline $\mathrm{BCW}$ & 34 & $\cdots$ & 575 & $\cdots$ & $\begin{array}{l}0 \\
4\end{array}$ & $\begin{array}{r}4 \\
\therefore\end{array}$ & $\begin{array}{l}\text { 112TILL } \\
\text { BEDROCK }\end{array}$ & TILL \\
\hline BCW & 35 & $\cdots$ & 203 & $\cdots$ & $\begin{array}{l}0 \\
4\end{array}$ & $\begin{array}{c}4 \\
--\end{array}$ & $\begin{array}{l}\text { 112TILL } \\
\text { BEDROCK }\end{array}$ & TILL \\
\hline $\mathrm{BCW}$ & 36 & $\cdots$ & 321 & $\cdots$ & $\begin{array}{r}0 \\
125\end{array}$ & 125 & $\begin{array}{l}112 \text { TILL } \\
\text { BEDROCK }\end{array}$ & TILL \\
\hline $\mathrm{BCW}$ & 37 & $\cdots$ & 282 & $\cdots$ & $\begin{array}{r}0 \\
50 \\
85\end{array}$ & $\begin{array}{l}50 \\
85 \\
-.\end{array}$ & $\begin{array}{l}110 \text { SDMN } \\
110 \text { SDMN } \\
\text { BEDROCK }\end{array}$ & $\begin{array}{l}\text { SDGL } \\
\text { SAND }\end{array}$ \\
\hline $\mathrm{BCW}$ & 38 & $\cdots$ & 228 & $\cdots$ & $\begin{array}{r}0 \\
137\end{array}$ & $\begin{aligned} 137 \\
-\end{aligned}$ & $\begin{array}{l}\text { 110SDMN } \\
\text { BEDROCK }\end{array}$ & SDCL \\
\hline $\mathrm{BCW}$ & 39 & $\cdots$ & 302 & $\cdots$ & $\begin{array}{r}0 \\
61\end{array}$ & $\begin{array}{l}61 \\
-.\end{array}$ & $\begin{array}{l}\text { 112TILL } \\
\text { BEDROCK }\end{array}$ & TILL \\
\hline BCW & 40 & $\cdots$ & 687 & $\cdots$ & $\begin{array}{r}0 \\
50\end{array}$ & $\begin{array}{l}50 \\
\cdots\end{array}$ & $\begin{array}{l}\text { 110SDMN } \\
\text { BEDROCK }\end{array}$ & OTHR \\
\hline $\mathrm{BCW}$ & 41 & $\cdots$ & 477 & $\cdots$ & $\begin{array}{r}0 \\
76\end{array}$ & $\begin{array}{l}76 \\
-\cdot\end{array}$ & $\begin{array}{l}110 \text { SDMN } \\
\text { BEDROCK }\end{array}$ & OTHR \\
\hline $\mathrm{BCW}$ & 42 & $\cdots$ & 130 & - & 0 & $-\cdot$ & $110 \mathrm{SDMN}$ & SDGL \\
\hline $\mathrm{BCW}$ & 43 & - & 265 & $\cdots$ & $\begin{array}{r}0 \\
78\end{array}$ & $\begin{array}{l}78 \\
--\end{array}$ & $\begin{array}{l}110 \text { SDMN } \\
\text { BEDROCK }\end{array}$ & SDGL \\
\hline $\mathrm{BCW}$ & 44 & $\cdots$ & 298 & $\cdots$ & $\begin{array}{l}0 \\
6\end{array}$ & $\begin{array}{c}6 \\
--\end{array}$ & $\begin{array}{l}110 \text { SDMN } \\
\text { BEDROCK }\end{array}$ & SDGL \\
\hline $\mathrm{BCW}$ & 45 & $\cdots$ & 277 & $\cdots$ & $\begin{array}{r}0 \\
133\end{array}$ & 133 & $\begin{array}{l}112 \text { TILL } \\
\text { BEDROCK }\end{array}$ & TILL \\
\hline $\mathrm{BCW}$ & 46 & $-\cdot$ & 377 & $\cdots$ & $\begin{array}{r}0 \\
80 \\
141\end{array}$ & $\begin{array}{r}80 \\
141 \\
-.\end{array}$ & $\begin{array}{l}110 \text { SDMN } \\
112 \text { TILL } \\
\text { BEDROCK }\end{array}$ & $\begin{array}{l}\text { CLAY } \\
\text { TILL }\end{array}$ \\
\hline $\mathrm{BCW}$ & 47 & $\cdots$ & 452 & - & $\begin{array}{r}0 \\
80\end{array}$ & $\begin{array}{l}80 \\
\cdots\end{array}$ & $\begin{array}{l}\text { 112TILL } \\
\text { BEDROCK }\end{array}$ & TILL GRAVEL, CLAY, HARDPAN \\
\hline $\mathrm{BCW}$ & 48 & $-\cdot$ & 502 & $\cdots$ & $\begin{array}{r}0 \\
30\end{array}$ & $\begin{array}{l}30 \\
\cdots\end{array}$ & $\begin{array}{l}\text { 112TILL } \\
\text { BEDROCK }\end{array}$ & TILL GRAVEL, HARDPAN \\
\hline $\mathrm{BCW}$ & 49 & $\cdots$ & 327 & - & $\begin{array}{r}0 \\
45\end{array}$ & $\begin{array}{l}45 \\
\cdots\end{array}$ & $\begin{array}{l}\text { 112TILL } \\
\text { BEDROCK }\end{array}$ & TILL \\
\hline $\mathrm{BCW}$ & 50 & $\cdots$ & 222 & $-\cdot$ & $\begin{array}{r}0 \\
70\end{array}$ & $\begin{array}{l}70 \\
\cdots\end{array}$ & $\begin{array}{l}110 \text { SDMN } \\
\text { BEDROCK }\end{array}$ & SDGL \\
\hline $\mathrm{BCW}$ & 51 & $\cdots$ & 222 & $\cdots$ & $\begin{array}{r}0 \\
70\end{array}$ & $\begin{array}{l}70 \\
\cdots\end{array}$ & $\begin{array}{l}110 \text { SDMN } \\
\text { BEDROCK }\end{array}$ & SDGL \\
\hline $\mathrm{BCW}$ & 52 & $\cdots$ & 865 & $\cdots$ & $\begin{array}{l}0 \\
9\end{array}$ & $\begin{array}{l}9 \\
-\end{array}$ & $\begin{array}{l}110 \text { SDMN } \\
\text { BEDROCK }\end{array}$ & SDGL \\
\hline $\mathrm{BCW}$ & 53 & $\cdots$ & 985 & $\cdots$ & $\begin{array}{l}0 \\
3\end{array}$ & $\begin{array}{l}3 \\
\cdots\end{array}$ & $\begin{array}{l}110 \text { SDMN } \\
\text { BEDROCK }\end{array}$ & SDGL \\
\hline $\mathrm{BCW}$ & 54 & $\cdots$ & 645 & $\cdots$ & 0 & $\cdots$ & BEDROCK & \\
\hline $\mathrm{BCW}$ & 55 & $\cdots$ & 150 & - & $\begin{array}{r}0 \\
20\end{array}$ & 20 & $\begin{array}{l}110 \text { SDMN } \\
\text { BEDROCK }\end{array}$ & SDGL \\
\hline
\end{tabular}


Table 2-1. Stratigraphic logs of wells and borings in the Saco and Ossipee River Basins, eastcentral New Hampshire--Continued

\begin{tabular}{|c|c|c|c|c|c|c|c|c|}
\hline $\begin{array}{l}\text { Loce } \\
\text { site } \\
\text { no. }\end{array}$ & & $\begin{array}{l}\text { Depth } \\
\text { drilled } \\
\text { (feet) }\end{array}$ & $\begin{array}{l}\text { Depth } \\
\text { of } \\
\text { well } \\
\text { (feet) }\end{array}$ & $\begin{array}{l}\text { Depth } \\
\text { to } \\
\text { refusal } \\
\text { (feet) }\end{array}$ & $\begin{array}{l}\text { Depth } \\
\text { to } \\
\text { top } \\
\text { (feet) }\end{array}$ & $\begin{array}{l}\text { Depth } \\
\text { to } \\
\text { bottom } \\
\text { (feet) }\end{array}$ & $\begin{array}{l}\text { Aquifer } \\
\text { code }\end{array}$ & Lithology \\
\hline & & \multicolumn{7}{|c|}{ BARTLETT - - Continued } \\
\hline BCW & 56 & $\cdots$ & 150 & - & $\begin{array}{r}0 \\
130\end{array}$ & $\begin{array}{r}130 \\
\cdots\end{array}$ & $\begin{array}{l}110 \text { SDMN } \\
\text { BEDROCK }\end{array}$ & SDGL \\
\hline BCW & 57 & $\cdots$ & 280 & $\cdots$ & $\begin{array}{r}0 \\
58\end{array}$ & 58 & $\begin{array}{l}\text { 110SDMN } \\
\text { BEDROCK }\end{array}$ & SDGL \\
\hline $\mathrm{BCW}$ & 58 & - & 401 & $\cdots$ & $\begin{array}{r}0 \\
78\end{array}$ & $\begin{array}{l}78 \\
-\cdot\end{array}$ & $\begin{array}{l}110 \text { SDMN } \\
\text { BEDROCK }\end{array}$ & SDGL \\
\hline $\mathrm{BCW}$ & 60 & -- & 265 & $\cdots$ & $\begin{array}{r}0 \\
25\end{array}$ & 25 & $\begin{array}{l}110 \text { SDMN } \\
\text { BEDROCK }\end{array}$ & SAND \\
\hline $\mathrm{BCW}$ & 61 & - & 689 & $\cdots$ & $\begin{array}{r}0 \\
10\end{array}$ & $\begin{array}{l}10 \\
-\cdot\end{array}$ & $\begin{array}{l}\text { 112TILL } \\
\text { BEDROCK }\end{array}$ & TILL \\
\hline $\mathrm{BCW}$ & 62 & - & 990 & $-\cdot$ & $\begin{array}{l}0 \\
3\end{array}$ & $\begin{array}{l}3 \\
-\end{array}$ & $\begin{array}{l}110 \text { SDMN } \\
\text { BEDROCK }\end{array}$ & SDGL \\
\hline $\mathrm{BCW}$ & 63 & $\cdots$ & 502 & $\cdots$ & 0 & $\cdots$ & $110 \mathrm{SDMN}$ & SDGL \\
\hline $\mathrm{BCW}$ & 64 & $-\cdot$ & 177 & $\cdots$ & $\begin{array}{r}0 \\
37\end{array}$ & $\begin{array}{l}37 \\
--\end{array}$ & $\begin{array}{l}\text { 112TILL } \\
\text { BEDROCK }\end{array}$ & TILL GRAVEL, HARDPAN \\
\hline $\mathrm{BCW}$ & 65 & $\cdots$ & 242 & $\cdots$ & $\begin{array}{r}0 \\
80\end{array}$ & $\begin{array}{l}80 \\
\cdots\end{array}$ & $\begin{array}{l}\text { 110SDMN } \\
\text { BEDROCK }\end{array}$ & SDGL \\
\hline $\mathrm{BCW}$ & 66 & - & 363 & $-\cdot$ & $\begin{array}{r}0 \\
10 \\
30\end{array}$ & $\begin{array}{l}10 \\
30 \\
\ldots\end{array}$ & $\begin{array}{l}110 \mathrm{SDMN} \\
110 \mathrm{SDMN} \\
\text { BEDROCK }\end{array}$ & $\begin{array}{l}\text { SAND } \\
\text { SDGL }\end{array}$ \\
\hline $\mathrm{BCW}$ & 67 & -- & 200 & - & $\begin{array}{r}0 \\
95\end{array}$ & $\begin{array}{l}95 \\
\cdots\end{array}$ & $\begin{array}{l}\text { 112TILL } \\
\text { BEDROCK }\end{array}$ & TILL \\
\hline $\mathrm{BCW}$ & 68 & $\cdots$ & 225 & $\cdots$ & $\begin{array}{r}0 \\
97\end{array}$ & $\begin{array}{l}97 \\
\cdots\end{array}$ & $\begin{array}{l}\text { 112TILL } \\
\text { BEDROCK }\end{array}$ & TILL \\
\hline BCW & 69 & $\cdots$ & 513 & - & $\begin{array}{r}0 \\
35\end{array}$ & $\begin{array}{l}35 \\
--\end{array}$ & $\begin{array}{l}\text { 112TILL } \\
\text { BEDROCK }\end{array}$ & TILL \\
\hline $\mathrm{BCW}$ & 70 & $\cdots$ & 363 & $\cdots$ & $\begin{array}{r}0 \\
75\end{array}$ & $\begin{array}{l}75 \\
-\end{array}$ & $\begin{array}{l}\text { 112TILL } \\
\text { BEDROCK }\end{array}$ & TILL SAND, GRAVEL, HARDPAN \\
\hline $\mathrm{BCW}$ & 71 & $\cdots$ & 322 & $\cdots$ & $\begin{array}{r}0 \\
25\end{array}$ & $\begin{array}{l}25 \\
\cdots\end{array}$ & $\begin{array}{l}\text { 112TILL } \\
\text { BEDROCK }\end{array}$ & TILL \\
\hline $\mathrm{BCW}$ & 72 & - & 402 & - & $\begin{array}{r}0 \\
90 \\
143\end{array}$ & $\begin{array}{r}90 \\
143 \\
\ldots\end{array}$ & $\begin{array}{l}110 \text { SDMN } \\
112 \mathrm{TILL} \\
\text { BEDROCK }\end{array}$ & $\begin{array}{l}\text { SDGL } \\
\text { TILL GRAVEL, CLAY, HARDPAN }\end{array}$ \\
\hline BCW & 73 & $\cdots$ & 444 & $\cdots$ & $\begin{array}{r}0 \\
15\end{array}$ & $\begin{array}{l}15 \\
-\end{array}$ & $\begin{array}{l}110 \text { SDMN } \\
\text { BEDROCK }\end{array}$ & SDGL \\
\hline $\mathrm{BCW}$ & 74 & - & 383 & $\cdots$ & $\begin{array}{r}0 \\
25\end{array}$ & $\begin{array}{l}25 \\
\cdots\end{array}$ & $\begin{array}{l}110 \text { SDMN } \\
\text { BEDROCK }\end{array}$ & SDGL \\
\hline $\mathrm{BCW}$ & 75 & $\cdots$ & 80 & $\cdots$ & - & - & $110 \mathrm{SDMN}$ & SDGL \\
\hline BCW & 76 & $\cdots$ & 425 & - & $\begin{array}{l}0 \\
3\end{array}$ & $\begin{array}{r}3 \\
\therefore\end{array}$ & $\begin{array}{l}110 \mathrm{SDMN} \\
\text { BEDROCK }\end{array}$ & SDGL \\
\hline $\mathrm{BCW}$ & 77 & $\cdots$ & 305 & $\cdots$ & $\begin{array}{r}0 \\
60\end{array}$ & $\begin{array}{l}60 \\
\cdots\end{array}$ & $\begin{array}{l}110 \text { SDMN } \\
\text { BEDROCK }\end{array}$ & CLAY \\
\hline $\mathrm{BCW}$ & 78 & $\cdots$ & 185 & $\cdots$ & $\begin{array}{l}0 \\
7\end{array}$ & $\begin{array}{r}7 \\
-.\end{array}$ & $\begin{array}{l}110 \text { SDMN } \\
\text { BEDROCK }\end{array}$ & SDGL \\
\hline $\mathrm{BCW}$ & 79 & - & 75 & $\cdots$ & $-\cdot$ & $-\cdot$ & $110 \mathrm{SDMN}$ & SDGL \\
\hline $\mathrm{BCW}$ & 80 & $\cdots$ & 81 & $-\cdot$ & $\begin{array}{r}0 \\
89\end{array}$ & $\begin{array}{l}89 \\
\cdots\end{array}$ & $\begin{array}{l}110 \text { SDMN } \\
\text { BEDROCK }\end{array}$ & SDGL \\
\hline
\end{tabular}


Table 2-1. Stratigraphic iogs of welis and borings in the Saco and Ossipee River Basins, eastcentral New Hampshire--Continued

\begin{tabular}{|c|c|c|c|c|c|c|c|c|}
\hline \multicolumn{2}{|c|}{$\begin{array}{l}\text { Local } \\
\text { site } \\
\text { No. }\end{array}$} & $\begin{array}{l}\text { Depth } \\
\text { drilled } \\
\text { (feet) }\end{array}$ & $\begin{array}{l}\text { Depth } \\
\text { of } \\
\text { well } \\
\text { (feet) }\end{array}$ & $\begin{array}{l}\text { Depth } \\
\text { to } \\
\text { refusal } \\
\text { (feet) }\end{array}$ & $\begin{array}{l}\text { Depth } \\
\text { to } \\
\text { top } \\
\text { (feet) }\end{array}$ & $\begin{array}{l}\text { Depth } \\
\text { to } \\
\text { bottom } \\
\text { (feet) }\end{array}$ & $\begin{array}{l}\text { Aquifer } \\
\text { code }\end{array}$ & Lithology \\
\hline & & \multicolumn{7}{|c|}{ BARTLETT - - Continued } \\
\hline BCW & 81 & $\cdots$ & 302 & $-\cdot$ & $\begin{array}{r}0 \\
45\end{array}$ & $\begin{array}{l}45 \\
--\end{array}$ & $\begin{array}{l}\text { 112TILL } \\
\text { BEDROCK }\end{array}$ & TILL GRAVEL, HARDPAN \\
\hline BCW & 82 & $\cdots$ & 92 & $\cdots$ & $\begin{array}{r}0 \\
130\end{array}$ & $\begin{array}{r}130 \\
\cdots\end{array}$ & $\begin{array}{l}110 \text { SDMN } \\
\text { BEDROCK }\end{array}$ & SDGL \\
\hline BCW & 83 & $\cdots$ & 452 & $\cdots$ & $\begin{array}{r}0 \\
228\end{array}$ & $\begin{array}{r}228 \\
\cdots\end{array}$ & $\begin{array}{l}\text { 112TILL } \\
\text { BEDROCK }\end{array}$ & TILL GRAVEL, CLAY, HARDPAN \\
\hline BCW & 84 & $\cdots$ & 202 & $\cdots$ & $\begin{array}{r}0 \\
108\end{array}$ & $\begin{array}{c}108 \\
\cdots\end{array}$ & $\begin{array}{l}110 \text { SDMN } \\
\text { BEDROCK }\end{array}$ & OTHR \\
\hline BCW & 85 & $\cdots$ & 1100 & $\cdots$ & $\begin{array}{r}0 \\
17\end{array}$ & $\begin{array}{l}17 \\
\cdots\end{array}$ & $\begin{array}{l}\text { 112TILL } \\
\text { BEDROCK }\end{array}$ & TILL \\
\hline BCW & 86 & $\cdots$ & 200 & $\cdots$ & $\begin{array}{r}0 \\
60\end{array}$ & $\begin{array}{l}60 \\
\cdots\end{array}$ & $\begin{array}{l}110 \text { SDMN } \\
\text { BEDROCK }\end{array}$ & SDGL \\
\hline BCW & 87 & $\cdots$ & 421 & $\cdots$ & $\begin{array}{r}0 \\
40 \\
100\end{array}$ & $\begin{array}{r}40 \\
100 \\
\cdots\end{array}$ & $\begin{array}{l}110 \text { SDMN } \\
112 \text { TILL } \\
\text { BEDROCK }\end{array}$ & $\begin{array}{l}\text { SDGL } \\
\text { TILL }\end{array}$ \\
\hline BCW & 88 & $\cdots$ & 402 & $\cdots$ & $\begin{array}{r}0 \\
15 \\
115 \\
125\end{array}$ & $\begin{array}{r}15 \\
115 \\
125 \\
-.\end{array}$ & $\begin{array}{l}110 \text { SDMN } \\
112 \text { TILL } \\
\text { BEDROCK } \\
\text { BEDROCK }\end{array}$ & $\begin{array}{l}\text { SDGL } \\
\text { TILL CLAY, HARDPAN }\end{array}$ \\
\hline $\mathrm{BCW}$ & 89 & $\cdots$ & 305 & $\cdots$ & $\begin{array}{r}0 \\
4 \\
30 \\
40\end{array}$ & $\begin{array}{r}4 \\
30 \\
40 \\
-\end{array}$ & $\begin{array}{l}110 \text { SDMN } \\
110 \text { SDMN } \\
110 \text { SDMN } \\
\text { BEDROCK }\end{array}$ & $\begin{array}{l}\text { SAND } \\
\text { SDGL } \\
\text { CLAYY }\end{array}$ \\
\hline $\mathrm{BCW}$ & 90 & $\cdots$ & 141 & $\cdots$ & $\begin{array}{r}0 \\
10\end{array}$ & $\begin{array}{l}10 \\
\cdots\end{array}$ & $\begin{array}{l}\text { 112TILL } \\
\text { BEDROCK }\end{array}$ & TILL \\
\hline BCW & 91 & $-\cdot$ & 221 & $\cdots$ & $\begin{array}{r}0 \\
45\end{array}$ & $\begin{array}{l}45 \\
\cdots\end{array}$ & $\begin{array}{l}\text { 112TILL } \\
\text { BEDROCK }\end{array}$ & TILL \\
\hline $\mathrm{BCW}$ & 92 & $\cdots$ & 402 & $-\cdot$ & $\begin{array}{r}0 \\
88\end{array}$ & $\begin{array}{l}88 \\
-\end{array}$ & $\begin{array}{l}\text { 112TILL } \\
\text { BEDROCK }\end{array}$ & TILL \\
\hline $\mathrm{BCW}$ & 93 & $\cdots$ & 262 & $\cdots$ & $\begin{array}{r}0 \\
25\end{array}$ & $\begin{array}{l}25 \\
\cdots\end{array}$ & $\begin{array}{l}\text { 112TILL } \\
\text { BEDROCK }\end{array}$ & TILL GRAVEL, HARDPAN \\
\hline $\mathrm{BCW}$ & 94 & $\cdots$ & 602 & $\cdots$ & $\begin{array}{r}0 \\
13\end{array}$ & $\begin{array}{l}13 \\
\cdots\end{array}$ & $\begin{array}{l}110 \text { SDMN } \\
\text { BEDROCK }\end{array}$ & SDGL \\
\hline $\mathrm{BCW}$ & 95 & $\cdots$ & 802 & - & $\begin{array}{r}0 \\
24\end{array}$ & $\begin{array}{l}24 \\
--\end{array}$ & $\begin{array}{l}110 \text { SDMN } \\
\text { BEDROCK }\end{array}$ & SDGL \\
\hline $\mathrm{BCW}$ & 96 & -. & 90 & $\cdots$ & $\begin{array}{r}0 \\
218\end{array}$ & $\begin{array}{r}218 \\
\cdots\end{array}$ & $\begin{array}{l}110 \text { SDMN } \\
\text { BEDROCK }\end{array}$ & SDGL \\
\hline BCW & 97 & $\cdots$ & 423 & $\cdots$ & $\begin{array}{r}0 \\
100\end{array}$ & 100 & $\begin{array}{l}110 \text { SDMN } \\
\text { BEDROCK }\end{array}$ & SDGL \\
\hline $\mathrm{BCW}$ & 98 & $-\cdot$ & 70 & $\cdots$ & $\begin{array}{r}0 \\
76\end{array}$ & $\begin{array}{l}76 \\
\ldots\end{array}$ & $\begin{array}{l}110 \mathrm{SDMN} \\
\text { BEDROCK }\end{array}$ & SDGL \\
\hline $\mathrm{BCW}$ & 99 & - & 322 & $\cdots$ & $\begin{array}{r}0 \\
45\end{array}$ & $\begin{array}{l}45 \\
--\end{array}$ & $\begin{array}{l}\text { 112TILL } \\
\text { BEDROCK }\end{array}$ & TILL \\
\hline $\mathrm{BCW}$ & 100 & $\cdots$ & 244 & $\cdots$ & $\begin{array}{r}0 \\
60\end{array}$ & $\begin{array}{l}60 \\
-\end{array}$ & $\begin{array}{l}110 \text { SDMN } \\
\text { BEDROCK }\end{array}$ & SDGL \\
\hline $\mathrm{BCW}$ & 101 & $\cdots$ & 622 & $\cdots$ & $\begin{array}{r}0 \\
15\end{array}$ & $\begin{array}{l}15 \\
--\end{array}$ & $\begin{array}{l}\text { 112TILL } \\
\text { BEDROCK }\end{array}$ & TILL \\
\hline BCW & 102 & $\cdots$ & 425 & $\cdots$ & $\begin{array}{r}0 \\
85\end{array}$ & $\begin{array}{l}85 \\
-\cdot\end{array}$ & $\begin{array}{l}110 \text { SDMN } \\
\text { BEDROCK }\end{array}$ & SGVC \\
\hline
\end{tabular}


Table 2-1. Stratigraphic logs of wells and borings in the Saco and Ossipee River Basins, eastcentral New Hampshire--Continued

\begin{tabular}{|c|c|c|c|c|c|c|c|c|}
\hline \multicolumn{2}{|c|}{$\begin{array}{l}\text { Local } \\
\text { site } \\
\text { no. }\end{array}$} & $\begin{array}{l}\text { Depth } \\
\text { drilled } \\
\text { (feet) }\end{array}$ & $\begin{array}{l}\text { Depth } \\
\text { of } \\
\text { well } \\
\text { (feet) }\end{array}$ & $\begin{array}{l}\text { Depth } \\
\text { to } \\
\text { refusal } \\
\text { (feet) }\end{array}$ & $\begin{array}{l}\text { Depth } \\
\text { to } \\
\text { top } \\
\text { (feet) }\end{array}$ & $\begin{array}{l}\text { Depth } \\
\text { to } \\
\text { bottom } \\
\text { (feet) }\end{array}$ & $\begin{array}{l}\text { Aquifer } \\
\text { code }\end{array}$ & Lithology \\
\hline & & \multicolumn{7}{|c|}{ BARTLETT - - Continued } \\
\hline $\mathrm{BCW}$ & 103 & $\cdots$ & 110 & - & -- & - & $110 \mathrm{SDMN}$ & SDGL \\
\hline $\mathrm{BCW}$ & 104 & $\cdots$ & 280 & -- & $\begin{array}{r}0 \\
40 \\
50\end{array}$ & $\begin{array}{l}40 \\
50 \\
\cdots\end{array}$ & $\begin{array}{l}110 \text { SDMN } \\
110 \text { SDMN } \\
\text { BEDROCK }\end{array}$ & $\begin{array}{l}\text { SDGL } \\
\text { CLAY }\end{array}$ \\
\hline $\mathrm{BCW}$ & 105 & $-\cdot$ & 385 & $-\cdot$ & $\begin{array}{r}0 \\
24\end{array}$ & $\begin{array}{l}24 \\
\cdots\end{array}$ & $\begin{array}{l}110 \text { SDMN } \\
\text { BEDROCK }\end{array}$ & SDGL \\
\hline $\mathrm{BCW}$ & 106 & $\cdots$ & 377 & $\cdots$ & $\begin{array}{r}0 \\
139\end{array}$ & $\begin{array}{r}139 \\
\ldots\end{array}$ & $\begin{array}{l}110 \text { SDMN } \\
\text { BEDROCK }\end{array}$ & SDCL $F$ \\
\hline $\mathrm{BCW}$ & 107 & $-\cdot$ & 402 & $-\cdot$ & $\begin{array}{r}0 \\
10\end{array}$ & $\begin{array}{l}10 \\
-\cdot\end{array}$ & $\begin{array}{l}\text { 112TILL } \\
\text { BEDROCK }\end{array}$ & TILL \\
\hline BCW & 108 & $\cdots$ & 361 & $\cdots$ & $\begin{array}{r}0 \\
150\end{array}$ & $\begin{array}{r}150 \\
\cdots\end{array}$ & $\begin{array}{l}112 \mathrm{TILL} \\
\text { BEDROCK }\end{array}$ & TILL \\
\hline $\mathrm{BCW}$ & 109 & - & 420 & - & $\begin{array}{r}0 \\
80 \\
120\end{array}$ & $\begin{array}{r}80 \\
120 \\
--\end{array}$ & $\begin{array}{l}\text { 112TILL } \\
110 \text { SDMN } \\
\text { BEDROCK }\end{array}$ & $\begin{array}{l}\text { TILL } \\
\text { CLAY }\end{array}$ \\
\hline $\mathrm{BCW}$ & 110 & $\cdots$ & 141 & -- & $\begin{array}{r}0 \\
25\end{array}$ & $\begin{array}{l}25 \\
--\end{array}$ & $\begin{array}{l}\text { 112TILL } \\
\text { BEDROCK }\end{array}$ & TILL \\
\hline $\mathrm{BCW}$ & 111 & $\cdots$ & 103 & $\cdots$ & $\begin{array}{r}0 \\
115\end{array}$ & $\begin{array}{r}115 \\
\ldots\end{array}$ & $\begin{array}{l}110 \text { SDMN } \\
\text { BEDROCK }\end{array}$ & SDGL \\
\hline $\mathrm{BCW}$ & 112 & $\cdots$ & 103 & $\cdots$ & $\begin{array}{r}0 \\
115\end{array}$ & 115 & $\begin{array}{l}110 \text { SDMN } \\
\text { BEDROCK }\end{array}$ & SDGL \\
\hline BCW & 113 & - & 301 & $\cdots$ & $\begin{array}{r}0 \\
75\end{array}$ & $\begin{array}{l}75 \\
\cdots\end{array}$ & $\begin{array}{l}110 \mathrm{SDMN} \\
\text { BEDROCK }\end{array}$ & SDGL \\
\hline $\mathrm{BCW}$ & 114 & $-\cdot$ & 123 & -- & $\begin{array}{r}0 \\
5 \\
23\end{array}$ & $\begin{array}{r}5 \\
23 \\
--\end{array}$ & $\begin{array}{l}110 \mathrm{SDMN} \\
110 \mathrm{SDMN} \\
110 \mathrm{SDMN}\end{array}$ & $\begin{array}{l}\text { SAND F } \\
\text { SDGL C } \\
\text { SAND }\end{array}$ \\
\hline $\mathrm{BCW}$ & 115 & $\cdots$ & 445 & - & $\begin{array}{r}0 \\
188\end{array}$ & $\begin{array}{r}188 \\
-\end{array}$ & $\begin{array}{l}110 \text { SDMN } \\
\text { BEDROCK }\end{array}$ & SGVC \\
\hline $\mathrm{BCW}$ & 116 & $\cdots$ & 752 & -. & $\begin{array}{r}0 \\
75 \\
161\end{array}$ & $\begin{array}{r}75 \\
161 \\
\cdots\end{array}$ & $\begin{array}{l}112 \text { TILL } \\
110 \text { SDMN } \\
\text { BEDROCK }\end{array}$ & $\begin{array}{l}\text { TILL } \\
\text { SGVC }\end{array}$ \\
\hline $\mathrm{BCW}$ & 117 & $\cdots$ & 323 & -. & $\begin{array}{r}0 \\
35\end{array}$ & $\begin{array}{l}35 \\
\cdots\end{array}$ & $\begin{array}{l}\text { 112TILL } \\
\text { BEDROCK }\end{array}$ & TILL CLAY, HARDPAN \\
\hline $\mathrm{BCW}$ & 118 & $\cdots$ & 303 & $\cdots$ & $\begin{array}{r}0 \\
80\end{array}$ & 80 & $\begin{array}{l}\text { BEDROCK } \\
\text { BEDROCK }\end{array}$ & \\
\hline BCW & 119 & $-\cdot$ & 403 & - & $\begin{array}{r}0 \\
60 \\
70\end{array}$ & $\begin{array}{l}60 \\
70 \\
-.\end{array}$ & $\begin{array}{l}110 \text { SDMN } \\
110 \text { SDMN } \\
\text { BEDROCK }\end{array}$ & $\begin{array}{l}\text { SDGL } \\
\text { OTHR }\end{array}$ \\
\hline $\mathrm{BCW}$ & 120 & $\cdots$ & 60 & $\cdots$ & 0 & $\cdots$ & $110 \mathrm{SDMN}$ & SDGL \\
\hline $\mathrm{BCW}$ & 121 & $\cdots$ & 643 & - & $\begin{array}{r}0 \\
55\end{array}$ & $\begin{array}{l}55 \\
\cdots\end{array}$ & $\begin{array}{l}\text { 112TILL } \\
\text { BEDROCK }\end{array}$ & TILL \\
\hline BCW & 122 & - & 322 & $\cdots$ & $\begin{array}{r}0 \\
18\end{array}$ & $\begin{array}{l}18 \\
--\end{array}$ & $\begin{array}{l}\text { 112TILL } \\
\text { BEDROCK }\end{array}$ & TILL \\
\hline $\mathrm{BCW}$ & 123 & $\cdots$ & 38 & $-\cdot$ & $\begin{array}{r}0 \\
40 \\
60\end{array}$ & $\begin{array}{l}40 \\
60 \\
-\end{array}$ & $\begin{array}{l}110 \text { SDMN } \\
110 \text { SDMN } \\
\text { BEDROCK }\end{array}$ & $\begin{array}{l}\text { SDGL } \\
\text { SDST }\end{array}$ \\
\hline $\mathrm{BCW}$ & 124 & $\cdots$ & 352 & $\cdots$ & $\begin{array}{r}0 \\
100\end{array}$ & 100 & $\begin{array}{l}110 \text { SDMN } \\
\text { BEDROCK }\end{array}$ & SGVC \\
\hline $\mathrm{BCW}$ & 125 & $\cdots$ & 302 & $\cdots$ & $\begin{array}{l}0 \\
8\end{array}$ & $\begin{array}{c}8 \\
-\end{array}$ & $\begin{array}{l}\text { 112TILL } \\
\text { BEDROCK }\end{array}$ & TILL \\
\hline
\end{tabular}


Table 2-1. Stratigraphic logs of wells and borings in the Saco and Ossipee River Basins, eastcentral New Hampshire--Continued

\begin{tabular}{|c|c|c|c|c|c|c|c|c|}
\hline \multicolumn{2}{|c|}{$\begin{array}{l}\text { Local } \\
\text { site } \\
\text { No. }\end{array}$} & $\begin{array}{l}\text { Depth } \\
\text { drilled } \\
\text { (feet) }\end{array}$ & $\begin{array}{l}\text { Depth } \\
\text { of } \\
\text { well } \\
\text { (feet) }\end{array}$ & $\begin{array}{l}\text { Depth } \\
\text { to } \\
\text { refusal } \\
\text { (feet) }\end{array}$ & $\begin{array}{l}\text { Depth } \\
\text { to } \\
\text { top } \\
\text { (feet) }\end{array}$ & $\begin{array}{l}\text { Depth } \\
\text { to } \\
\text { bottom } \\
\text { (feet) }\end{array}$ & $\begin{array}{l}\text { Aquifer } \\
\text { code }\end{array}$ & Lithology \\
\hline & & \multicolumn{7}{|c|}{ BARTLETT - - Continued } \\
\hline $\mathrm{BCW}$ & 126 & $\cdots$ & 282 & -- & $\begin{array}{r}0 \\
38\end{array}$ & $\begin{array}{l}38 \\
\cdots\end{array}$ & $\begin{array}{l}\text { 112TILL } \\
\text { BEDROCK }\end{array}$ & TILL GRAVEL, HARDPAN \\
\hline $\mathrm{BCW}$ & 127 & $\cdots$ & 202 & $\cdots$ & $\begin{array}{r}0 \\
60 \\
75\end{array}$ & $\begin{array}{l}60 \\
75 \\
--\end{array}$ & $\begin{array}{l}\text { 112TILL } \\
\text { BEDROCK } \\
\text { BEDROCK }\end{array}$ & TILL GRAVEL， HARDPAN \\
\hline BCW & 128 & -- & 202 & $\cdots$ & $\begin{array}{r}0 \\
70\end{array}$ & 70 & $\begin{array}{l}110 \text { SDMN } \\
\text { BEDROCK }\end{array}$ & SDCL \\
\hline BCW & 129 & $\cdots$ & 402 & $\cdots$ & $\begin{array}{r}0 \\
18\end{array}$ & $\begin{array}{l}18 \\
-\cdot\end{array}$ & $\begin{array}{l}\text { 112TILL } \\
\text { BEDROCK }\end{array}$ & TILL \\
\hline BCW & 130 & $\cdots$ & 383 & $\cdots$ & $\begin{array}{r}0 \\
120\end{array}$ & 120 & $\begin{array}{l}\text { 112TILL } \\
\text { BEDROCK }\end{array}$ & TILL \\
\hline BCW & 131 & $\cdots$ & 624 & $\cdots$ & $\begin{array}{r}0 \\
80\end{array}$ & $\begin{array}{l}80 \\
\ldots\end{array}$ & $\begin{array}{l}\text { 110SDMN } \\
\text { BEDROCK }\end{array}$ & SAND \\
\hline $\mathrm{BCW}$ & 132 & $\cdots$ & 322 & $\cdots$ & $\begin{array}{r}0 \\
35 \\
50 \\
88\end{array}$ & $\begin{array}{l}35 \\
50 \\
88 \\
\cdots\end{array}$ & $\begin{array}{l}110 \text { SDMN } \\
110 \text { SDMN } \\
112 \mathrm{TILL} \\
\text { BEDROCK }\end{array}$ & $\begin{array}{l}\text { SDCL } \\
\text { CLAY } \\
\text { TILL }\end{array}$ \\
\hline $\mathrm{BCW}$ & 133 & $\cdots$ & 505 & $\cdots$ & 205 & - & BEDROCK & \\
\hline $\mathrm{BCW}$ & 134 & $\cdots$ & 81 & $\cdots$ & $\cdots$ & $\cdots$ & 110 SDMN & SDGL \\
\hline BCW & 135 & - & 152 & $\cdots$ & $\begin{array}{r}0 \\
120\end{array}$ & $\begin{array}{r}120 \\
\ldots\end{array}$ & $\begin{array}{l}\text { 112TILL } \\
\text { BEDROCK }\end{array}$ & TILL GRAVEL, CLAY, HARDPAN \\
\hline $\mathrm{BCW}$ & 136 & $\cdots$ & 303 & $\cdots$ & $\begin{array}{r}0 \\
10 \\
60\end{array}$ & $\begin{array}{l}10 \\
60 \\
\cdots\end{array}$ & $\begin{array}{l}\text { 112TILL } \\
\text { BEDROCK } \\
\text { BEDROCK }\end{array}$ & TILL \\
\hline BCW & 137 & - & 424 & -- & $\begin{array}{r}0 \\
25 \\
60\end{array}$ & $\begin{array}{l}25 \\
60 \\
\cdots\end{array}$ & $\begin{array}{l}\text { 112TILL } \\
\text { BEDROCK } \\
\text { BEDROCK }\end{array}$ & TILL \\
\hline BCW & 138 & $\cdots$ & 182 & $\cdots$ & $\begin{array}{r}0 \\
100\end{array}$ & $\begin{array}{c}100 \\
\ldots\end{array}$ & $\begin{array}{l}\text { 112TILL } \\
\text { BEDROCK }\end{array}$ & TILL \\
\hline $\mathrm{BCW}$ & 139 & - & 283 & -- & $\begin{array}{r}0 \\
100 \\
140\end{array}$ & $\begin{array}{r}100 \\
140 \\
\ldots\end{array}$ & $\begin{array}{l}\text { 112TILL } \\
\text { BEDROCK } \\
\text { BEDROCK }\end{array}$ & TILL \\
\hline $\mathrm{BCW}$ & 140 & $\cdots$ & 322 & - & $\begin{array}{r}0 \\
20 \\
58\end{array}$ & $\begin{array}{l}20 \\
58 \\
-.\end{array}$ & $\begin{array}{l}110 \text { SDMN } \\
\text { BEDROCK } \\
\text { BEDROCK }\end{array}$ & OTHR \\
\hline BCW & 141 & $\cdots$ & 424 & $\cdots$ & $\begin{array}{r}0 \\
15\end{array}$ & $\begin{array}{l}15 \\
\cdots\end{array}$ & $\begin{array}{l}\text { BEDROCK } \\
\text { BEDROCK }\end{array}$ & \\
\hline $\mathrm{BCW}$ & 142 & $\cdots$ & 505 & -- & $\begin{array}{r}0 \\
20\end{array}$ & 20 & $\begin{array}{l}\text { BEDROCK } \\
\text { BEDROCK }\end{array}$ & \\
\hline $\mathrm{BCW}$ & 143 & $\cdots$ & 150 & $\cdots$ & $\begin{array}{r}0 \\
40 \\
150\end{array}$ & $\begin{array}{r}40 \\
150 \\
\ldots\end{array}$ & $\begin{array}{l}110 \text { SDMN } \\
\text { BEDROCK } \\
\text { BEDROCK }\end{array}$ & SDGL \\
\hline $\mathrm{BCW}$ & 144 & $\cdots$ & 100 & - & $\begin{array}{r}0 \\
40\end{array}$ & 40 & $\begin{array}{l}\text { 110SDMN } \\
\text { BEDROCK }\end{array}$ & SDGL \\
\hline $\mathrm{BCW}$ & 145 & $\cdots$ & 805 & - & $\begin{array}{r}0 \\
60\end{array}$ & $\begin{array}{l}60 \\
-.\end{array}$ & $\begin{array}{l}110 \text { SDMN } \\
\text { BEDROCK }\end{array}$ & SGVC \\
\hline $\mathrm{BCW}$ & 146 & - & 142 & $\cdots$ & $\begin{array}{r}0 \\
16 \\
18\end{array}$ & $\begin{array}{l}16 \\
18 \\
\cdots\end{array}$ & $\begin{array}{l}110 \text { SDMN } \\
110 \text { SDMN } \\
\text { BEDROCK }\end{array}$ & $\begin{array}{l}\text { SAND } \\
\text { SDGL }\end{array}$ \\
\hline $\mathrm{BCW}$ & 147 & - & 81 & - & 79 & $\cdots$ & BEDROCK & \\
\hline
\end{tabular}


Table 2-1. Stratigraphic logs of wells and borings in the Saco and Ossipee River Basins, eastcentral New Hampshire--Continued

\begin{tabular}{|c|c|c|c|c|c|c|c|c|}
\hline $\begin{array}{l}\text { Local } \\
\text { site } \\
\text { no. }\end{array}$ & & $\begin{array}{l}\text { Depth } \\
\text { drilled } \\
\text { (feet) }\end{array}$ & $\begin{array}{l}\text { Depth } \\
\text { of } \\
\text { well } \\
\text { (feet) }\end{array}$ & $\begin{array}{l}\text { Depth } \\
\text { to } \\
\text { refusal } \\
\text { (feet) }\end{array}$ & $\begin{array}{l}\text { Depth } \\
\text { to } \\
\text { top } \\
\text { (feet) }\end{array}$ & $\begin{array}{l}\text { Depth } \\
\text { to } \\
\text { bottom } \\
\text { (feet) }\end{array}$ & $\begin{array}{l}\text { Aquifer } \\
\text { code }\end{array}$ & Lithology \\
\hline & & \multicolumn{7}{|c|}{ BARTLETT - - Continued } \\
\hline $\mathrm{BCW}$ & 148 & $\cdots$ & 360 & $-\cdot$ & $\begin{array}{r}0 \\
75\end{array}$ & $\begin{array}{l}75 \\
--\end{array}$ & $\begin{array}{l}112 \text { TILL } \\
\text { BEDROCK }\end{array}$ & TILL \\
\hline BCW & 149 & $-\cdot$ & 109 & $\cdots$ & $\begin{array}{r}0 \\
109\end{array}$ & $\begin{array}{r}109 \\
\ldots\end{array}$ & $\begin{array}{l}110 \text { SDMN } \\
\text { BEDROCK }\end{array}$ & SDGL \\
\hline BCW & 150 & $\cdots$ & 342 & -. & $\begin{array}{r}0 \\
90 \\
135\end{array}$ & $\begin{array}{r}90 \\
135 \\
\cdots\end{array}$ & $\begin{array}{l}\text { 112TILL } \\
\text { BEDROCK } \\
\text { BEDROCK }\end{array}$ & TILL \\
\hline BCW & 152 & - & 6 & $\cdots$ & $\begin{array}{r}0 \\
58\end{array}$ & $\begin{array}{l}58 \\
\cdots\end{array}$ & $\begin{array}{l}110 \text { SDMN } \\
\text { BEDROCK }\end{array}$ & SDGL \\
\hline $\mathrm{BCW}$ & 153 & $-\cdot$ & 222 & $\cdots$ & $\begin{array}{r}0 \\
25\end{array}$ & $\begin{array}{l}25 \\
\cdots\end{array}$ & $\begin{array}{l}110 \text { SDMN } \\
\text { BEDROCK }\end{array}$ & SGVC \\
\hline BCW & 154 & $-\cdot$ & 152 & $-\cdot$ & $\begin{array}{r}0 \\
60\end{array}$ & $\begin{array}{l}60 \\
\cdots\end{array}$ & $\begin{array}{l}\text { 112TILL } \\
\text { BEDROCK }\end{array}$ & TILL CLAY, HARDPAN \\
\hline BCW & 155 & $-\cdot$ & 252 & -- & $\begin{array}{r}0 \\
80\end{array}$ & $\begin{array}{l}80 \\
\cdots\end{array}$ & $\begin{array}{l}\text { 112TILL } \\
\text { BEDROCK }\end{array}$ & TILL GRAVEL, CLAY, HARDPAN \\
\hline BCW & 156 & - & 300 & $-\cdot$ & $\begin{array}{r}0 \\
83\end{array}$ & $\begin{array}{l}83 \\
\cdots\end{array}$ & $\begin{array}{l}\text { 112TILL } \\
\text { BEDROCK }\end{array}$ & TILL GRAVEL, CLAY, HARDPAN \\
\hline $\mathrm{BCW}$ & 157 & $\cdots$ & 525 & $-\cdot$ & $\begin{array}{r}0 \\
25 \\
60\end{array}$ & $\begin{array}{l}25 \\
60 \\
--\end{array}$ & $\begin{array}{l}\text { 112TILL } \\
110 \text { SDMN } \\
\text { BEDROCK }\end{array}$ & $\begin{array}{l}\text { TILL } \\
\text { OTHR }\end{array}$ \\
\hline BCW & 158 & $\cdots$ & 524 & $\cdots$ & $\begin{array}{r}0 \\
10\end{array}$ & $\begin{array}{l}10 \\
\cdots\end{array}$ & $\begin{array}{l}112 \text { TILL } \\
\text { BEDROCK }\end{array}$ & TILL \\
\hline $\mathrm{BCW}$ & 159 & $\cdots$ & 522 & $-\cdot$ & $\begin{array}{r}0 \\
50\end{array}$ & $\begin{array}{l}50 \\
--\end{array}$ & $\begin{array}{l}112 T I L L \\
\text { BEDROCK }\end{array}$ & TILL \\
\hline BCW & .160 & $\cdots$ & 564 & $\cdots$ & $\begin{array}{l}0 \\
3\end{array}$ & 3 & $\begin{array}{l}\text { 112TILL } \\
\text { BEDROCK }\end{array}$ & TILL \\
\hline BCW & 161 & $\cdots$ & 662 & $\cdots$ & $\begin{array}{r}0 \\
90\end{array}$ & $\begin{array}{l}90 \\
--\end{array}$ & $\begin{array}{l}\text { 112TILL } \\
\text { BEDROCK }\end{array}$ & TILL CLAY, HARDPAN \\
\hline BCW & 162 & $-\cdot$ & 424 & - & $\begin{array}{l}0 \\
2\end{array}$ & $\begin{array}{r}2 \\
--\end{array}$ & $\begin{array}{l}\text { 112TILL } \\
\text { BEDROCK }\end{array}$ & TILL \\
\hline $\mathrm{BCW}$ & 163 & $-\cdot$ & 600 & $\cdots$ & $\begin{array}{r}0 \\
118\end{array}$ & $\begin{array}{r}118 \\
--\end{array}$ & $\begin{array}{l}110 \text { SDMN } \\
\text { BEDROCK }\end{array}$ & SGVC \\
\hline $\mathrm{BCW}$ & 164 & $\cdots$ & 425 & $-\cdot$ & $\begin{array}{r}0 \\
20 \\
80\end{array}$ & $\begin{array}{l}20 \\
80 \\
\ldots\end{array}$ & $\begin{array}{l}110 \text { SDMN } \\
\text { BEDROCK } \\
\text { BEDROCK }\end{array}$ & OTHR \\
\hline BCW & 165 & $-\cdot$ & 1020 & - & $\begin{array}{l}0 \\
5\end{array}$ & $\begin{array}{r}5 \\
-\cdot\end{array}$ & $\begin{array}{l}\text { 112TILL } \\
\text { BEDROCK }\end{array}$ & TILL \\
\hline BCW & 166 & $-\cdot$ & 503 & $-\cdot$ & $\begin{array}{l}0 \\
2\end{array}$ & $\begin{array}{l}2 \\
\therefore\end{array}$ & $\begin{array}{l}112 \text { TILL } \\
\text { BEDROCK }\end{array}$ & TILL \\
\hline $\mathrm{BCW}$ & 167 & - & 83.2 & $-\cdot$ & $\begin{array}{r}0 \\
10 \\
70\end{array}$ & $\begin{array}{l}10 \\
70 \\
-.\end{array}$ & $\begin{array}{l}\text { 112SRFD } \\
112 \mathrm{SRFD} \\
112 \mathrm{SRFD}\end{array}$ & $\begin{array}{l}\text { SDGL } \\
\text { SAND } \\
\text { SDGL }\end{array}$ \\
\hline BCW & 168 & $\cdots$ & 168 & $-\cdot$ & $\cdots$ & - & $\cdots$ & \\
\hline BCW & 169 & - & 500 & $\cdots$ & $\begin{array}{r}0 \\
130\end{array}$ & $\begin{array}{r}130 \\
-.\end{array}$ & $\begin{array}{l}\text { 112TILL } \\
\text { BEDROCK }\end{array}$ & TILL \\
\hline BCW & 170 & $-\cdot$ & 440 & $\cdots$ & $\begin{array}{r}0 \\
30 \\
138\end{array}$ & $\begin{array}{r}30 \\
138 \\
\ldots\end{array}$ & $\begin{array}{l}112 \mathrm{TILL} \\
110 \text { SDMN } \\
\text { BEDROCK }\end{array}$ & $\begin{array}{l}\text { TILL } \\
\text { SDGL }\end{array}$ \\
\hline
\end{tabular}


Table 2-1. Stratigraphic logs of wells and borings in the Saco and Ossipee River Basins, eastcentral New Hampshire--Continued

\begin{tabular}{|c|c|c|c|c|c|c|c|c|}
\hline \multicolumn{2}{|c|}{$\begin{array}{l}\text { Local } \\
\text { site } \\
\text { No. }\end{array}$} & $\begin{array}{l}\text { Depth } \\
\text { drilled } \\
\text { (feet) }\end{array}$ & $\begin{array}{l}\text { Depth } \\
\text { of } \\
\text { well } \\
\text { (feet) }\end{array}$ & $\begin{array}{l}\text { Depth } \\
\text { to } \\
\text { refusal } \\
\text { (feet) }\end{array}$ & $\begin{array}{l}\text { Depth } \\
\text { to } \\
\text { top } \\
\text { (feet) }\end{array}$ & $\begin{array}{l}\text { Depth } \\
\text { to } \\
\text { bottom } \\
\text { (feet) }\end{array}$ & $\begin{array}{l}\text { Aquifer } \\
\text { code }\end{array}$ & Lithology \\
\hline & & \multicolumn{7}{|c|}{ BARTLBTT - - Continued } \\
\hline $\mathrm{BCW}$ & 171 & - & 500 & $\cdots$ & $\begin{array}{r}0 \\
80 \\
150\end{array}$ & $\begin{array}{r}80 \\
150\end{array}$ & $\begin{array}{l}\text { 112TILL } \\
\text { BEDROCK } \\
\text { BEDROCK }\end{array}$ & TILL \\
\hline $\mathrm{BCW}$ & 172 & $-\cdot$ & 422 & $-\cdot$ & $\begin{array}{r}0 \\
30\end{array}$ & $\begin{array}{l}30 \\
\cdots\end{array}$ & $\begin{array}{l}\text { 112TILL } \\
\text { BEDROCK }\end{array}$ & TILL \\
\hline BCW & 173 & $\cdots$ & 421 & $-\cdot$ & $\begin{array}{r}0 \\
60\end{array}$ & $\begin{array}{l}60 \\
\ldots\end{array}$ & $\begin{array}{l}\text { 112TILL } \\
\text { BEDROCK }\end{array}$ & TILL \\
\hline $\mathrm{BCW}$ & 174 & $-\cdot$ & 523 & $-\cdot$ & $\begin{array}{r}0 \\
20\end{array}$ & 20 & $\begin{array}{l}\text { 112TILL } \\
\text { BEDROCK }\end{array}$ & TILL \\
\hline $\mathrm{BCW}$ & 175 & $\cdots$ & 150 & - & $\begin{array}{r}0 \\
25\end{array}$ & $\begin{array}{l}25 \\
\cdots\end{array}$ & $\begin{array}{l}110 \text { SDMN } \\
\text { BEDROCK }\end{array}$ & CLAY \\
\hline $\mathrm{BCW}$ & 176 & 23 & 18 & - & $\begin{array}{r}0 \\
15 \\
18\end{array}$ & $\begin{array}{l}15 \\
18 \\
--\end{array}$ & $\begin{array}{l}110 \text { SDMN } \\
112 \text { SRFD } \\
112 \text { TILL }\end{array}$ & $\begin{array}{l}\text { SDGL COBBLE GRAVEL } \\
\text { SDGL M TO VCS AND PEBBLES } \\
\text { TILL }\end{array}$ \\
\hline $\mathrm{BCW}$ & 177 & 23.5 & 22 & 23.5 & $\begin{array}{r}0 \\
17 \\
18 \\
23\end{array}$ & $\begin{array}{l}17 \\
18 \\
23 \\
23.5\end{array}$ & $\begin{array}{l}110 \mathrm{SDMN} \\
112 \mathrm{GLCL} \\
112 \mathrm{SRFD} \\
112 \mathrm{TILL}\end{array}$ & $\begin{array}{l}\text { SDGL COBBLE GRAVEL } \\
\text { GLCL DIAMICTON } \\
\text { SDGL PEBBLE GRAVEL (F TO VCS/M } \\
\text { TO VCS MATRIX) } \\
\text { TILL }\end{array}$ \\
\hline $\mathrm{BCW}$ & 178 & 18.5 & 15 & 18.5 & $\begin{array}{r}0 \\
2 \\
7 \\
15\end{array}$ & $\begin{array}{l}2 \\
7 \\
15 \\
18.5\end{array}$ & $\begin{array}{l}110 \text { SDMN } \\
112 \text { SRFD } \\
112 \text { SRFD } \\
112 \text { TILL }\end{array}$ & $\begin{array}{l}\text { LOAM } \\
\text { SDGL COBBLE GRAVEL } \\
\text { SDGL BOULDER GRAVEL } \\
\text { TILL }\end{array}$ \\
\hline \multicolumn{9}{|c|}{ CHATHAM } \\
\hline $\mathrm{CKB}$ & 1 & 25 & $\cdots$ & $\cdots$ & $-\cdot$ & $\cdots$ & 112 SRFD & SAND M TO C SAND \\
\hline CKB & 2 & 10 & - & - & - - & - & 112SRFD & GRVL \\
\hline $\mathrm{CKB}$ & 3 & 10 & - & - & $\begin{array}{l}0 \\
5\end{array}$ & $\begin{array}{c}5 \\
\cdots\end{array}$ & $\begin{array}{l}112 \text { SRFD } \\
112 \mathrm{SRFD}\end{array}$ & $\begin{array}{l}\text { GRVL } \\
\text { SAND MS }\end{array}$ \\
\hline $\mathrm{CKB}$ & 4 & 7 & - & 7 & - & - & $112 \mathrm{SRFD}$ & GRVL \\
\hline $\mathrm{CKB}$ & 5 & 2.5 & - & $\cdots$ & . & - & 112 SRFD & SAND FS \\
\hline $\mathrm{CKB}$ & 6 & 9 & $\cdots$ & 9 & $\begin{array}{l}0 \\
4\end{array}$ & $\begin{array}{l}4 \\
--\end{array}$ & $\begin{array}{l}112 \mathrm{SRFD} \\
112 \mathrm{SRFD}\end{array}$ & $\begin{array}{l}\text { SDGL } \\
\text { TILL SANDY TILL }\end{array}$ \\
\hline $\mathrm{CKB}$ & 7 & 8 & - & 8 & $\begin{array}{l}0 \\
1\end{array}$ & $\begin{array}{l}1 \\
--\end{array}$ & $\begin{array}{l}112 \text { SRFD } \\
112 \mathrm{SRFD}\end{array}$ & $\begin{array}{l}\text { GRVL } \\
\text { GRVL SILTY GRAVEL }\end{array}$ \\
\hline CKS & 1 & -. & $\cdots$ & -. & $-\cdot$ & - & $-\cdot$ & \\
\hline CKW & 1 & 8.9 & 8.9 & -. & - & .. & -. & \\
\hline $\mathrm{CKW}$ & 2 & 8.3 & 8.3 & $\cdots$ & - & .. & $\cdots$ & \\
\hline $\mathrm{CKW}$ & 3 & 12.5 & 12.5 & $-\cdot$ & - & $\cdots$ & $\cdots$ & \\
\hline $\mathrm{CKW}$ & 4 & 240 & $-\cdot$ & $\cdots$ & - & - & $\cdots$ & \\
\hline $\mathrm{CKW}$ & 5 & 7.5 & 7.5 & - & . & - & $\cdots$ & \\
\hline $\mathrm{CKW}$ & 6 & 300 & $\cdots$ & -. & . & $\cdots$ & - & \\
\hline CKW & 7 & 8.9 & 8.9 & $\cdots$ & - & $-\cdot$ & $\cdots$ & \\
\hline $\mathrm{CKW}$ & 8 & 10.7 & 10.7 & .. & $\ldots$ & -. & $\cdots$ & \\
\hline
\end{tabular}


Table 2-1. Stratigraphic logs of wells and borings in the Saco and Ossipee Rlver Basins, eastcentral New Hampshire--Continued

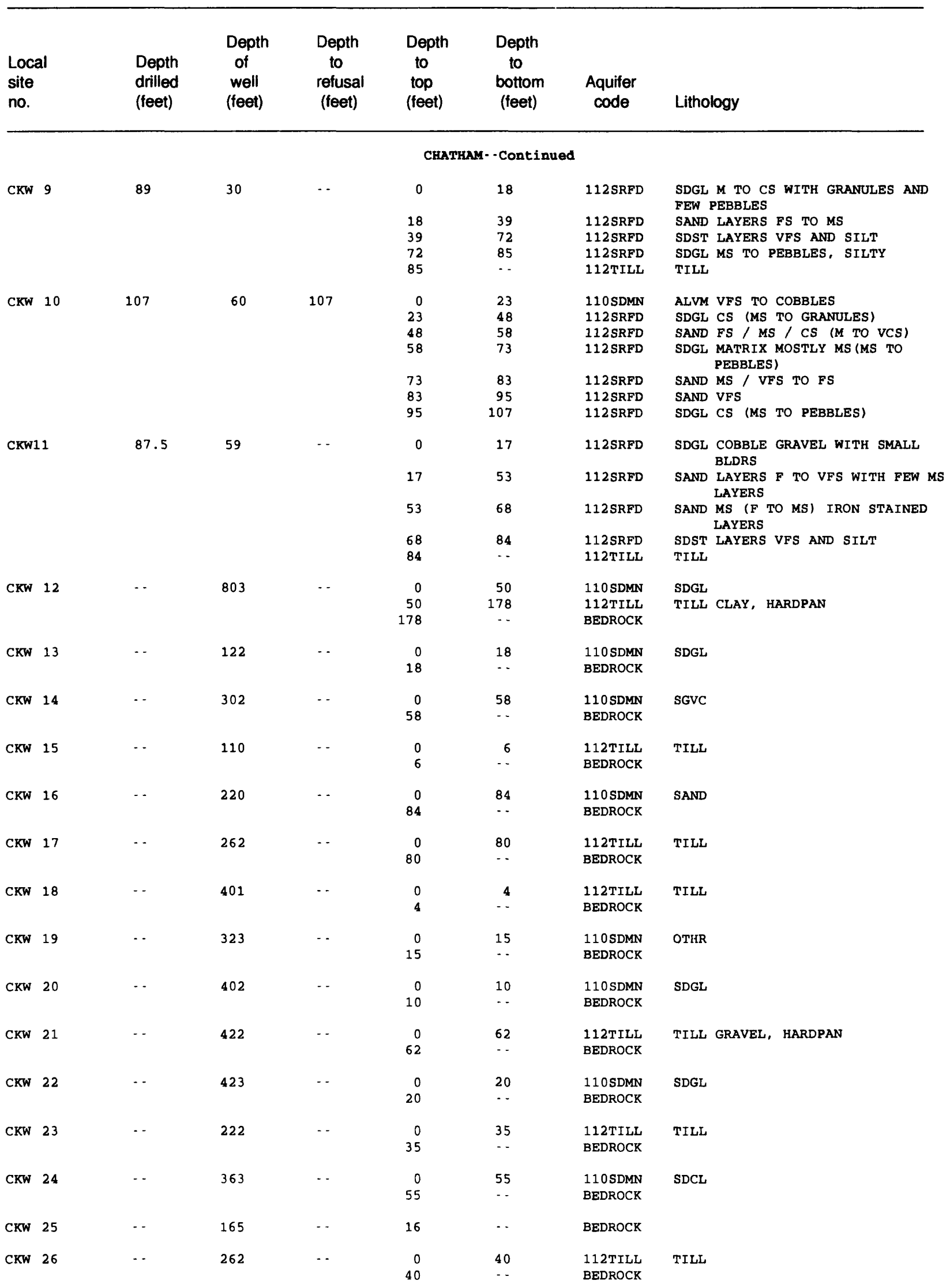


Table 2-1. Stratigraphic logs of wells and borings in the Saco and Ossipee River Basins, eastcentral New Hampshire--Continued

\begin{tabular}{|c|c|c|c|c|c|c|c|c|}
\hline \multicolumn{2}{|c|}{$\begin{array}{l}\text { Local } \\
\text { site } \\
\text { No. }\end{array}$} & $\begin{array}{l}\text { Depth } \\
\text { drilled } \\
\text { (feet) }\end{array}$ & $\begin{array}{l}\text { Depth } \\
\text { of } \\
\text { well } \\
\text { (feet) }\end{array}$ & $\begin{array}{l}\text { Depth } \\
\text { to } \\
\text { refusal } \\
\text { (feet) }\end{array}$ & $\begin{array}{l}\text { Depth } \\
\text { to } \\
\text { top } \\
\text { (feet) }\end{array}$ & $\begin{array}{l}\text { Depth } \\
\text { to } \\
\text { bottom } \\
\text { (feet) }\end{array}$ & $\begin{array}{l}\text { Aquifer } \\
\text { code }\end{array}$ & Lithology \\
\hline & & \multicolumn{7}{|c|}{ CHATHAY - Continued } \\
\hline CKW & 27 & $\cdots$ & 682 & $\cdots$ & $\begin{array}{r}0 \\
60 \\
165\end{array}$ & $\begin{array}{r}60 \\
165 \\
\cdots\end{array}$ & $\begin{array}{l}\text { 112TILL } \\
\text { BEDROCK } \\
\text { BEDROCK }\end{array}$ & TILL \\
\hline CKW & 28 & -- & 362 & $\cdots$ & $\begin{array}{r}0 \\
135 \\
145\end{array}$ & $\begin{array}{r}135 \\
145 \\
-.\end{array}$ & $\begin{array}{l}\text { 112TILL } \\
\text { BEDROCK } \\
\text { BEDROCK }\end{array}$ & TILL \\
\hline CKW & 29 & $\cdots$ & 200 & $\cdots$ & $\begin{array}{r}0 \\
32\end{array}$ & 32 & $\begin{array}{l}\text { 112TILL } \\
\text { BEDROCK }\end{array}$ & TILL \\
\hline CKW & 30 & $\cdots$ & 225 & $\cdots$ & $\begin{array}{r}0 \\
25\end{array}$ & $\begin{array}{l}25 \\
\cdots\end{array}$ & $\begin{array}{l}\text { 112TILL } \\
\text { BEDROCK }\end{array}$ & TILL \\
\hline CKW & 31 & $\cdots$ & 220 & $\cdots$ & $\begin{array}{r}0 \\
55 \\
83\end{array}$ & $\begin{array}{l}55 \\
83 \\
\cdots\end{array}$ & $\begin{array}{l}110 \text { SDMN } \\
112 \text { TILL } \\
\text { BEDROCK }\end{array}$ & $\begin{array}{l}\text { SDGL } \\
\text { TILL }\end{array}$ \\
\hline CKW & 32 & $\cdots$ & 420 & $\cdots$ & $\begin{array}{r}0 \\
15 \\
57\end{array}$ & $\begin{array}{l}15 \\
57 \\
\cdots\end{array}$ & $\begin{array}{l}110 \text { SDMN } \\
112 T I L L \\
\text { BEDROCK }\end{array}$ & $\begin{array}{l}\text { SDGL } \\
\text { TILL }\end{array}$ \\
\hline CKW & 33 & $\cdots$ & 382 & $\cdots$ & $\begin{array}{r}0 \\
20\end{array}$ & $\begin{array}{l}20 \\
\cdots\end{array}$ & $\begin{array}{l}\text { 112TILL } \\
\text { BEDROCK }\end{array}$ & TILL \\
\hline CKW & 34 & $\cdots$ & 325 & $\cdots$ & $\begin{array}{r}0 \\
12\end{array}$ & $\begin{array}{l}12 \\
-\cdot\end{array}$ & $\begin{array}{l}110 \text { SDMN } \\
\text { BEDROCK }\end{array}$ & SDGL \\
\hline CKW & 35 & $\cdots$ & 422 & $\cdots$ & $\begin{array}{r}0 \\
20 \\
45\end{array}$ & $\begin{array}{l}20 \\
45 \\
\cdots\end{array}$ & $\begin{array}{l}112 \text { TILL } \\
110 \text { SDMN } \\
\text { BEDROCK }\end{array}$ & $\begin{array}{l}\text { TILL GRAVEL, HARDPAN } \\
\text { CLAY }\end{array}$ \\
\hline & & & & & CONW & & & \\
\hline CWA & 1 & 49 & $\cdots$ & $-\cdot$ & $\begin{array}{r}0 \\
7 \\
8 \\
23 \\
33 \\
41\end{array}$ & $\begin{array}{r}7 \\
8 \\
23 \\
33 \\
41 \\
-\cdots\end{array}$ & $\begin{array}{l}110 \text { SDMN } \\
110 \text { SDMN } \\
110 \text { SDMN } \\
112 \text { SRFD } \\
112 \text { SRFD } \\
112 \text { TILL }\end{array}$ & $\begin{array}{l}\text { LOAM VFS TO SILT } \\
\text { SAND CS (MS TO VCS) } \\
\text { SDST VFS TO SILT } \\
\text { SAND VFS GRAY } \\
\text { STCL MASSIVE, GRAY } \\
\text { TILL GRAY, COMPACT }\end{array}$ \\
\hline CWW & 10 & $\cdots$ & $\cdots$ & -- & $\cdots$ & $\cdots$ & $\cdots$ & \\
\hline CWW & 44 & $\cdots$ & 110 & $\cdots$ & $\cdots$ & $-\cdot$ & $112 \mathrm{SRFD}$ & \\
\hline CWw & 45 & $\cdots$ & 185 & $\cdots$ & $\cdots$ & $\cdot-$ & 112SRFD & \\
\hline CWW & 46 & $\cdots$ & 428 & $\cdots$ & 75 & $\cdots$ & BEDROCK & \\
\hline CWw & 47 & $\cdots$ & 305 & $\cdots$ & 70 & $\cdots$ & BEDROCK & \\
\hline CWW & 48 & $\cdots$ & 530 & $\cdots$ & 80 & $\cdots$ & BEDROCK & \\
\hline CWW & 49 & $\cdots$ & 140 & $-\cdot$ & 122 & $\cdots$ & BEDROCK & \\
\hline CWW & 50 & -- & 385 & $\cdots$ & 65 & $\cdots$ & BEDROCK & \\
\hline CWW & 51 & $\cdots$ & 118 & $\cdots$ & 60 & - & BEDROCK & \\
\hline CWW & 52 & $\cdots$ & 405 & $\cdots$ & 40 & $\cdots$ & BEDROCK & \\
\hline CWW & 53 & $\cdots$ & 225 & $\cdots$ & 475 & $\cdots$ & BEDROCK & \\
\hline CWW & 54 & $\cdots$ & 100 & $\cdots$ & $\cdots$ & $\cdots$ & $112 \mathrm{SRFD}$ & \\
\hline CWw & 55 & $\cdots$ & 470 & $\cdots$ & 36 & $\cdots$ & BEDROCK & \\
\hline CWW & 56 & $\cdots$ & 500 & $\cdots$ & $\cdots$ & $\cdot \cdot$ & $\cdots$ & \\
\hline CWW & 57 & $-\cdot$ & 180 & $\cdots$ & $\cdots$ & - & 112SRFD & \\
\hline
\end{tabular}


Table 2-1. Stratigraphic logs of wells and borings in the Saco and Ossipee River Basins, eastcentral New Hampshire--Continued

\begin{tabular}{|c|c|c|c|c|c|c|c|c|}
\hline \multicolumn{2}{|c|}{$\begin{array}{l}\text { Local } \\
\text { site } \\
\text { no. }\end{array}$} & $\begin{array}{l}\text { Depth } \\
\text { drilled } \\
\text { (feet) }\end{array}$ & $\begin{array}{l}\text { Depth } \\
\text { of } \\
\text { well } \\
\text { (feet) }\end{array}$ & $\begin{array}{l}\text { Depth } \\
\text { to } \\
\text { refusal } \\
\text { (feet) }\end{array}$ & $\begin{array}{l}\text { Depth } \\
\text { to } \\
\text { top } \\
\text { (feet) }\end{array}$ & $\begin{array}{l}\text { Depth } \\
\text { to } \\
\text { bottom } \\
\text { (feet) }\end{array}$ & $\begin{array}{c}\text { Aquifer } \\
\text { code }\end{array}$ & Lithology \\
\hline \multicolumn{9}{|c|}{ CONWAY - - Continued } \\
\hline CWW & 58 & $\cdots$ & 52 & $-\cdot$ & $\cdots$ & - & 112SRFD & \\
\hline CWW & 59 & $\cdots$ & 550 & $\cdots$ & 50 & $\cdots$ & BEDROCK & \\
\hline CWW & 60 & - & 205 & $\cdots$ & 22 & $\cdots$ & BEDROCK & \\
\hline CWW & 61 & $\cdots$ & 45 & $-\cdot$ & $\cdots$ & $\cdots$ & 112SRFD & \\
\hline CWW & 62 & $-\cdot$ & 156 & $\cdots$ & 145 & $\cdots$ & BEDROCK & \\
\hline CWW & 63 & $\cdots$ & 225 & $\cdots$ & 130 & $\cdots$ & BEDROCK & \\
\hline CWW & 64 & $-\cdot$ & 515 & - & 148 & - & BEDROCK & \\
\hline CWW & 65 & $\cdots$ & 258 & $\cdots$ & 102 & $\cdots$ & BEDROCK & \\
\hline \multirow[t]{5}{*}{ CWw } & 66 & 119 & 50 & - & 0 & 23 & 112 SRFD & SDGL CS (MS TO COBBLES) \\
\hline & & & & & 23 & 43 & 112SRFD & $\begin{array}{l}\text { SDGL CS (MS TO GRANULES) FEW } \\
\text { PEBBLES ( } 1 \text { F TO MS LYR })\end{array}$ \\
\hline & & & & & 43 & 68 & 112SRFD & $\begin{array}{l}\text { SAND LAYERS MS TO CS, SOME VCS, } \\
1 \text { VFS AND SILT LAMINA }\end{array}$ \\
\hline & & & & & 68 & 78 & 112SRFD & $\begin{array}{l}\text { SDGL CS - VCS (MS TO PEBBLES, } \\
\text { SILTY) }\end{array}$ \\
\hline & & & & & 78 & $\cdots$ & 112TILL & TILL \\
\hline \multirow[t]{5}{*}{ CWW } & 67 & 75 & 69 & 75 & $\begin{array}{r}0 \\
18\end{array}$ & $\begin{array}{l}18 \\
33\end{array}$ & 112SRFD & $\begin{array}{l}\text { SDGL VCS (CS TO PEBBLES) } \\
\text { SAND VFS TO FS SOME MS }\end{array}$ \\
\hline & & & & & 33 & 43 & 112SRFD & SDST VFS AND SILT WITH CLAY \\
\hline & & & & & & & & LAMANAE \\
\hline & & & & & 43 & 65 & 112SRFD & STCL GRAY \\
\hline & & & & & 65 & 75 & 112 SRFD & SDGL MS TO VCS MATRIX, SILTY \\
\hline CWW & 68 & - & 440 & $\cdots$ & 21 & - & BEDROCK & \\
\hline \multirow[t]{7}{*}{$\mathrm{CWW}$} & 69 & 60 & 58 & - & 0 & 7 & 110 SDMN & LOAM \\
\hline & & & & & 7 & 15 & 110 SDMN & PEAT \\
\hline & & & & & 15 & 23 & $112 \mathrm{SRFD}$ & SDGL LAYERS VCS (MS TO PEBBLES) \\
\hline & & & & & 23 & 33 & 112SRFD & $\begin{array}{l}\text { SDGL LAYERS MATRIX FS, MS, TO } \\
\text { VCS }\end{array}$ \\
\hline & & & & & 33 & 53 & 112SRFD & SDST \\
\hline & & & & & 53 & 60 & 112SRFD & SDGL CD (MS TO GRAN) \\
\hline & & & & & 60 & 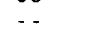 & BEDROCK & \\
\hline \multirow[t]{7}{*}{ CWW } & 70 & 60 & 19 & - & 0 & 7 & $110 \mathrm{SDMN}$ & LOAM \\
\hline & & & & & 7 & 15 & $110 \mathrm{SDMN}$ & PEAT \\
\hline & & & & & 15 & 23 & 112SRFD & SDGL LAYERS VCS (MS TO PEBBLES) \\
\hline & & & & & 23 & 33 & 112 SRFD & SDGL LAYERS MATRIX FS, MS TO VC \\
\hline & & & & & 33 & 53 & 112 SRFD & SDST \\
\hline & & & & & 53 & 60 & 112 SRFD & SDGL CS (MS TO GRAN) \\
\hline & & & & & 60 & -- & BEDROCK & \\
\hline CWW & 71 & - & 153 & $\cdots$ & 20 & $-\cdot$ & BEDROCK & \\
\hline CWW & 72 & $-\cdot$ & 325 & $\cdots$ & 50 & $\cdots$ & BEDROCK & \\
\hline CWW & 73 & $\cdots$ & 260 & - & 20 & - & BEDROCK & \\
\hline CWW & 74 & $\cdots$ & 175 & $\cdots$ & 20 & $\cdots$ & BEDROCK & \\
\hline CWW & 75 & $\cdots$ & 227 & $\cdots$ & 30 & $\cdots$ & BEDROCK & \\
\hline CWW & 76 & $\cdots$ & 101 & $\cdots$ & $-\cdot$ & $\cdots$ & 112SRFD & \\
\hline CWW & 77 & -. & 75 & - & 69 & $\cdots$ & BEDROCK & \\
\hline CWW & 78 & $\cdots$ & 158 & $\cdots$ & 33 & $\cdots$ & BEDROCK & \\
\hline$C W W$ & 79 & - & 260 & $\cdots$ & 100 & $\cdots$ & BEDROCK & \\
\hline$C W W$ & 80 & - & 480 & - & 110 & - & BEDROCK & \\
\hline
\end{tabular}


Table 2-1. Stratigraphic logs of wells and borings in the Saco and Ossipee River Basins, eastcentral New Hampshire--Continued

\begin{tabular}{|c|c|c|c|c|c|c|c|c|}
\hline \multicolumn{2}{|c|}{$\begin{array}{l}\text { Local } \\
\text { site } \\
\text { No. }\end{array}$} & $\begin{array}{l}\text { Depth } \\
\text { drilled } \\
\text { (feet) }\end{array}$ & $\begin{array}{l}\text { Depth } \\
\text { of } \\
\text { well } \\
\text { (feet) }\end{array}$ & $\begin{array}{l}\text { Depth } \\
\text { to } \\
\text { refusal } \\
\text { (feet) }\end{array}$ & $\begin{array}{l}\text { Depth } \\
\text { to } \\
\text { top } \\
\text { (feet) }\end{array}$ & $\begin{array}{l}\text { Depth } \\
\text { to } \\
\text { bottom } \\
\text { (feet) }\end{array}$ & $\begin{array}{l}\text { Aquifer } \\
\text { code }\end{array}$ & Lithology \\
\hline \multicolumn{9}{|c|}{ CONWAY - - Continued } \\
\hline CWW & 81 & -- & 117 & $\cdots$ & 105 & $\cdots$ & BEDROCK & \\
\hline CWW & 82 & $\cdots$ & 280 & $\cdots$ & 50 & $\cdots$ & BEDROCK & \\
\hline CWW & 83 & $\cdots$ & $\cdots$ & $\cdots$ & 89 & $\cdots$ & BEDROCK & \\
\hline CWW & 84 & - & 485 & $\cdots$ & 70 & $\cdots$ & BEDROCK & \\
\hline CWW & 85 & $\cdots$ & 266 & $\cdots$ & 70 & $\cdots$ & BEDROCK & \\
\hline CWW & 86 & $\cdots$ & - & $\cdots$ & 118 & $\cdots$ & BEDROCK & \\
\hline CWW & 87 & - & 79 & $\cdots$ & $\cdots$ & $-\cdot$ & $112 \mathrm{SRFD}$ & \\
\hline CWW & 88 & $\cdots$ & 413 & $\cdots$ & 90 & $\cdots$ & BEDROCK & \\
\hline CWW & 89 & $-\cdot$ & 178 & -- & 90 & -. & BEDROCK & \\
\hline CWW & 107 & -- & 15.8 & $-\cdot$ & -. & $-\cdot$ & $-\cdot$ & \\
\hline CWW & 108 & - & 36.7 & $\cdots$ & .. & $\cdots$ & $\cdots$ & \\
\hline CWW & 109 & $\cdots$ & 79.6 & $-\cdot$ & $-\cdot$ & $-\cdot$ & $-\cdot$ & \\
\hline CWW & 110 & $\cdots$ & 60.1 & $-\cdot$ & $-\cdot$ & - & $\cdots$ & \\
\hline CWW & 139 & $-\cdot$ & 120 & $\cdots$ & $\cdots$ & $\cdots$ & 112SRFD & \\
\hline $\mathrm{CWW}$ & 140 & $\cdots$ & 485 & $\cdots$ & 135 & $\cdots$ & BEDROCK & \\
\hline CWw & 141 & $\cdots$ & 140 & -. & $-\cdot$ & $\cdots$ & 112SRFD & \\
\hline CWW & 142 & $\cdots$ & 150 & $-\cdot$ & - & $\cdots$ & $112 \mathrm{SRFD}$ & \\
\hline \multicolumn{9}{|c|}{ EATON } \\
\hline $\mathrm{ECW}$ & 1 & 7.5 & 7.5 & $\cdots$ & $\cdots$ & $-\cdot$ & -- & \\
\hline ECW & 2 & 26 & 23 & $\cdots$ & $\cdots$ & $\cdots$ & $\cdots$ & \\
\hline ECW & 3 & 350 & 350 & -- & $-\cdot$ & $\cdots$ & $\cdots$ & \\
\hline ECW & 4 & 365 & $-\cdot$ & $-\cdot$ & $-\cdot$ & $-\cdot$ & . & \\
\hline ECW & 5 & - & 213 & $\cdots$ & $\begin{array}{l}0 \\
8\end{array}$ & $\begin{array}{l}8 \\
--\end{array}$ & $\begin{array}{l}\text { 110SDMN } \\
\text { BEDROCK }\end{array}$ & SDGL \\
\hline ECW & 6 & $-\cdot$ & 261 & $\cdots$ & $\begin{array}{r}0 \\
10 \\
45\end{array}$ & $\begin{array}{l}10 \\
45 \\
-.\end{array}$ & $\begin{array}{l}110 \text { SDMN } \\
110 \text { SDMN } \\
\text { BEDROCK }\end{array}$ & $\begin{array}{l}\text { SAND } \\
\text { SDGL }\end{array}$ \\
\hline ECW & 7 & $\cdots$ & 65 & -- & $\begin{array}{r}0 \\
65\end{array}$ & $\begin{array}{l}65 \\
-\cdot\end{array}$ & $\begin{array}{l}\text { 110SDMN } \\
\text { BEDROCK }\end{array}$ & SDGL \\
\hline ECW & 8 & $\cdots$ & 503 & $\cdots$ & $\begin{array}{r}0 \\
15\end{array}$ & $\begin{array}{l}15 \\
--\end{array}$ & $\begin{array}{l}\text { 110SDMN } \\
\text { BEDROCK }\end{array}$ & SDGL \\
\hline ECW & 9 & $\cdots$ & 423 & - & $\begin{array}{r}0 \\
60\end{array}$ & $\begin{array}{l}60 \\
\cdots\end{array}$ & $\begin{array}{l}110 \text { SDMN } \\
\text { BEDROCK }\end{array}$ & SDGL \\
\hline ECW & 10 & $\cdots$ & 350 & $-\cdot$ & $\begin{array}{r}0 \\
25\end{array}$ & $\begin{array}{l}25 \\
--\end{array}$ & $\begin{array}{l}\text { 110SDMN } \\
\text { BEDROCK }\end{array}$ & SDGL \\
\hline ECW & 11 & $-\cdot$ & 10 & $\cdots$ & $\cdots$ & $\begin{array}{l}- \\
\cdots\end{array}$ & $\begin{array}{l}\text { 112TILL } \\
\text { 110SDMN }\end{array}$ & $\begin{array}{l}\text { TILL } \\
\text { SDGL }\end{array}$ \\
\hline$E C W$ & 12 & $\cdots$ & 177 & -. & $\begin{array}{r}0 \\
17\end{array}$ & 17 & $\begin{array}{l}\text { 112TILL } \\
\text { BEDROCK }\end{array}$ & TILL \\
\hline
\end{tabular}


Table 2-1. Stratigraphic logs of wells and borings in the Saco and Ossipee River Basins, eastcentral New Hampshire--Continued

\begin{tabular}{|c|c|c|c|c|c|c|c|c|}
\hline \multicolumn{2}{|c|}{$\begin{array}{l}\text { Local } \\
\text { site } \\
\text { no. }\end{array}$} & $\begin{array}{l}\text { Depth } \\
\text { drilled } \\
\text { (feet) }\end{array}$ & $\begin{array}{l}\text { Depth } \\
\text { of } \\
\text { well } \\
\text { (feet) }\end{array}$ & $\begin{array}{l}\text { Depth } \\
\text { to } \\
\text { refusal } \\
\text { (feet) }\end{array}$ & $\begin{array}{l}\text { Depth } \\
\text { to } \\
\text { top } \\
\text { (feet) }\end{array}$ & $\begin{array}{l}\text { Depth } \\
\text { to } \\
\text { bottom } \\
\text { (feet) }\end{array}$ & $\begin{array}{l}\text { Aquifer } \\
\text { code }\end{array}$ & Lithology \\
\hline \multicolumn{9}{|c|}{ EATON- - Continued } \\
\hline ECW & 13 & $\cdots$ & 402 & $\cdots$ & $\begin{array}{r}0 \\
75\end{array}$ & $\begin{array}{l}75 \\
\cdots\end{array}$ & $\begin{array}{l}110 \text { SDMN } \\
\text { BEDROCK }\end{array}$ & SDGL \\
\hline ECW & 14 & $\cdots$ & 364 & $\cdots$ & $\begin{array}{r}0 \\
28\end{array}$ & $\begin{array}{l}28 \\
\cdots\end{array}$ & $\begin{array}{l}110 \text { SDMN } \\
\text { BEDROCK }\end{array}$ & SDGL \\
\hline ECW & 15 & $\cdots$ & 1200 & $\cdots$ & $\begin{array}{r}0 \\
12\end{array}$ & $\begin{array}{l}12 \\
\cdots\end{array}$ & $\begin{array}{l}\text { 112TILL } \\
\text { BEDROCK }\end{array}$ & TILL \\
\hline ECW & 16 & $-\cdot$ & 602 & $\cdots$ & $\begin{array}{r}0 \\
17\end{array}$ & $\begin{array}{l}17 \\
--\end{array}$ & $\begin{array}{l}\text { 112TILL } \\
\text { BEDROCK }\end{array}$ & TILL CLAY, HARDPAN \\
\hline ECW & 17 & $\cdots$ & 281 & $-\cdot$ & $\begin{array}{r}0 \\
20\end{array}$ & 20 & $\begin{array}{l}\text { 112TILL } \\
\text { BEDROCK }\end{array}$ & TILL \\
\hline ECW & 18 & $-\cdot$ & 482 & $\cdots$ & $\begin{array}{r}0 \\
186\end{array}$ & $\begin{array}{r}186 \\
--\end{array}$ & $\begin{array}{l}\text { 112TILL } \\
\text { BEDROCK }\end{array}$ & TILL CLAY, HARDPAN \\
\hline ECW & 19 & -- & 422 & $\cdots$ & $\begin{array}{r}0 \\
120\end{array}$ & 120 & $\begin{array}{l}\text { 110SDMN } \\
\text { BEDROCK }\end{array}$ & SDGL \\
\hline ECW & 20 & $\cdots$ & 600 & $\cdots$ & $\begin{array}{r}0 \\
12\end{array}$ & 12 & $\begin{array}{l}\text { 112TILL } \\
\text { BEDROCK }\end{array}$ & TILL \\
\hline ECW & 21 & -- & 802 & $\cdots$ & $\begin{array}{r}0 \\
25\end{array}$ & 25 & $\begin{array}{l}\text { 110SDMN } \\
\text { BEDROCK }\end{array}$ & CLAY \\
\hline ECW & 22 & $\cdots$ & 524 & $\cdots$ & $\begin{array}{r}0 \\
15\end{array}$ & $\begin{array}{l}15 \\
--\end{array}$ & $\begin{array}{l}\text { 112TILL } \\
\text { BEDROCK }\end{array}$ & TILL \\
\hline ECW & 23 & $\cdots$ & 525 & $-\cdot$ & $\begin{array}{l}0 \\
1\end{array}$ & $\begin{array}{r}1 \\
13\end{array}$ & $\begin{array}{l}112 \text { TILL } \\
\text { BEDROCK }\end{array}$ & TILL \\
\hline ECW & 24 & $\cdots$ & 406 & $\cdots$ & 13 & $-\cdot$ & BEDROCK & \\
\hline ECW & 25 & $\cdots$ & 325 & $\cdots$ & $\begin{array}{r}0 \\
130\end{array}$ & $\begin{array}{r}130 \\
-.\end{array}$ & $\begin{array}{l}110 \text { SDMN } \\
\text { BEDROCK }\end{array}$ & CLAY \\
\hline \multicolumn{9}{|c|}{ EPFINGHAM } \\
\hline EFA & 1 & 67 & - & $\cdots$ & $\begin{array}{ll}-\cdot \\
63\end{array}$ & $\begin{array}{l}\cdots \\
\cdots\end{array}$ & $\begin{array}{l}112 \mathrm{SRFD} \\
112 \mathrm{GLCL}\end{array}$ & $\begin{array}{l}\text { SAND LAYERS OF F TO VCS } \\
\text { GRDS DIAMICTON }\end{array}$ \\
\hline EFB & 1 & 14 & $\cdots$ & $\cdots$ & $\begin{array}{r}0 \\
3 \\
8 \\
14\end{array}$ & $\begin{array}{r}3 \\
8 \\
14 \\
\cdots\end{array}$ & $\begin{array}{l}111 \text { FILL } \\
112 \text { SRFD } \\
112 \text { TILL } \\
112 \text { TILL }\end{array}$ & $\begin{array}{l}\text { OTHR FILL } \\
\text { SAND SAND, SOME COBBLES } \\
\text { TILL TILL, SANDY } \\
\text { TILL REFUSAL }\end{array}$ \\
\hline EFB & 2 & 54 & $\cdots$ & $\cdots$ & $\begin{array}{r}0 \\
2 \\
45 \\
54\end{array}$ & $\begin{array}{r}2 \\
45 \\
54 \\
-\cdot\end{array}$ & $\begin{array}{l}112 \text { SRFD } \\
112 \mathrm{LCSR} \\
112 \text { SRFD } \\
\text { BEDROCK }\end{array}$ & $\begin{array}{l}\text { SAND SAND, COARSE } \\
\text { SAND SAND, FINE } \\
\text { SDGL SAND, COARSE AND GRAVEL } \\
\text { REFUSAL ON BOULDER OR BEDROCK }\end{array}$ \\
\hline EFB & 3 & 60 & - & $\cdots$ & $\begin{array}{r}0 \\
10 \\
25 \\
42 \\
47 \\
52 \\
60\end{array}$ & $\begin{array}{l}10 \\
25 \\
42 \\
47 \\
52 \\
60 \\
-.\end{array}$ & $\begin{array}{l}\text { 112SRFD } \\
112 \mathrm{SRFD} \\
112 \mathrm{LCSR} \\
112 \mathrm{SRFD} \\
112 \mathrm{LCSR} \\
112 \mathrm{TILL} \\
112 \mathrm{TILL}\end{array}$ & $\begin{array}{l}\text { SAND SAND, FINE TO MEDIUM } \\
\text { SAND SAND, MEDIUM TO COARSE } \\
\text { SAND SAND, FINE, SILTY } \\
\text { SAND SAND, MEDIUM } \\
\text { SILT SILT, FINE SANDY } \\
\text { TILL TILL, GRAVELLY } \\
\text { TILL END OF HOLE AT } 60 \text { FEET } \\
\text { (OTHER LOGS HIT REFUSAL } 52 \\
\text { FEET-62 FEET) }\end{array}$ \\
\hline EFW & 2 & 600 & $\cdots$ & $\cdots$ & $\cdots$ & $\cdots$ & $-\cdot$ & \\
\hline EFW & 3 & 25 & $\cdots$ & $\cdot \cdot$ & $\cdots$ & $\cdots$ & $\cdot \cdot$ & \\
\hline EFW & 4 & 40 & 40 & $\cdots$ & - & - & $\cdots$ & \\
\hline
\end{tabular}


Table 2-1. Stratigraphic logs of wells and borings in the Saco and Ossipee River Basins, eastcentral New Hampshire--Continued

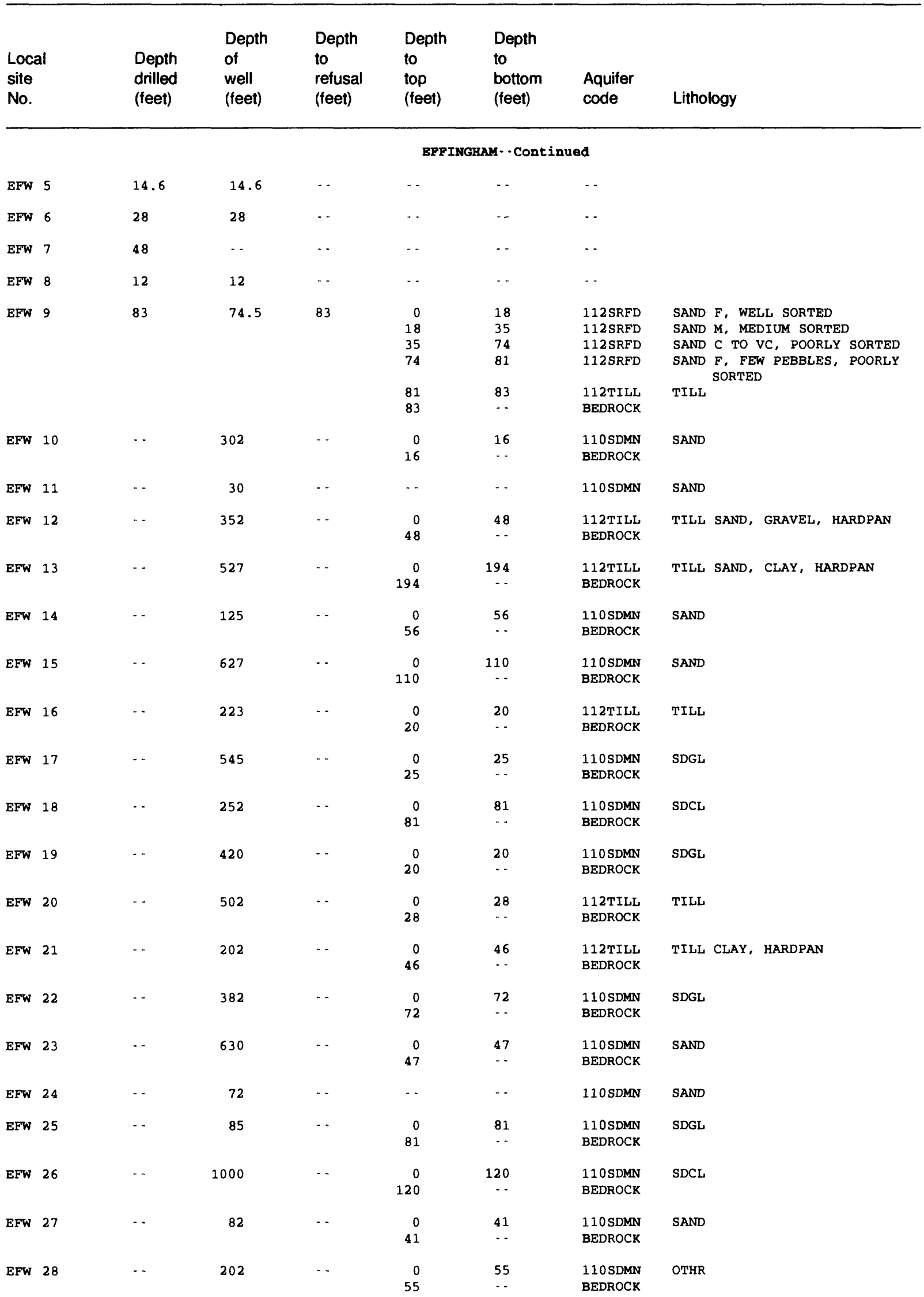


Table 2-1. Stratigraphic logs of wells and borings in the Saco and Ossipee River Basins, eastcentral New Hampshire--Continued

\begin{tabular}{|c|c|c|c|c|c|c|c|}
\hline $\begin{array}{l}\text { Local } \\
\text { site } \\
\text { no. }\end{array}$ & $\begin{array}{l}\text { Depth } \\
\text { drilled } \\
\text { (feet) }\end{array}$ & $\begin{array}{l}\text { Depth } \\
\text { of } \\
\text { well } \\
\text { (feet) }\end{array}$ & $\begin{array}{l}\text { Depth } \\
\text { to } \\
\text { refusal } \\
\text { (feet) }\end{array}$ & $\begin{array}{l}\text { Depth } \\
\text { to } \\
\text { top } \\
\text { (feet) }\end{array}$ & $\begin{array}{l}\text { Depth } \\
\text { to } \\
\text { bottom } \\
\text { (feet) }\end{array}$ & $\begin{array}{l}\text { Aquifer } \\
\text { code }\end{array}$ & Lithology \\
\hline
\end{tabular}

EPFINGHAK - - Continued

\begin{tabular}{|c|c|c|c|c|c|c|c|c|}
\hline EFW & 29 & 25 & 25 & $\cdots$ & $\cdots$ & $\cdots$ & & \\
\hline EFW & 30 & $\cdots$ & 670 & $\cdots$ & $\begin{array}{r}0 \\
10\end{array}$ & $\begin{array}{l}10 \\
\cdots\end{array}$ & $\begin{array}{l}\text { 112TILL } \\
\text { BEDROCK }\end{array}$ & TILL \\
\hline EFW & 31 & $\cdots$ & 638 & $\cdots$ & $\begin{array}{l}0 \\
8\end{array}$ & $\begin{array}{c}8 \\
\cdots\end{array}$ & $\begin{array}{l}\text { 112TILL } \\
\text { BEDROCK }\end{array}$ & TILL \\
\hline EFW & 32 & $\cdots$ & 503 & $\cdots$ & $\begin{array}{r}\because \cdot \\
0 \\
99\end{array}$ & $\begin{array}{l}\cdots \\
99 \\
\cdots\end{array}$ & $\begin{array}{l}\text { 112TILL } \\
110 \text { SDMN } \\
\text { BEDROCK }\end{array}$ & $\begin{array}{l}\text { TILL CLAY, HARDPAN } \\
\text { SGVC }\end{array}$ \\
\hline EFW & 33 & $\cdots$ & 323 & $\cdots$ & $\begin{array}{r}0 \\
30 \\
40\end{array}$ & $\begin{array}{r}30 \\
40 \\
152\end{array}$ & $\begin{array}{l}110 \text { SDMN } \\
112 T I L L \\
\text { BEDROCK }\end{array}$ & $\begin{array}{l}\text { SAND } \\
\text { TILL }\end{array}$ \\
\hline EFW & 34 & $\cdots$ & 300 & $\cdots$ & 152 & $\cdots$ & BEDROCK & \\
\hline EFW & 35 & $\cdots$ & 30 & $\cdots$ & 0 & 140 & 110SDMN & SAND \\
\hline EFW & 36 & $\cdots$ & 223 & $\cdots$ & 140 & $\cdots$ & BEDROCK & \\
\hline EFW & 37 & $\cdots$ & 127 & $\cdots$ & $\begin{array}{r}0 \\
40\end{array}$ & $\begin{array}{l}40 \\
\cdots\end{array}$ & $\begin{array}{l}\text { 112TILL } \\
\text { BEDROCK }\end{array}$ & TILL \\
\hline EFW & 38 & $\cdots$ & 303 & $\cdots$ & $\begin{array}{r}0 \\
160\end{array}$ & $\begin{array}{r}160 \\
\ldots\end{array}$ & $\begin{array}{l}\text { 112TILL } \\
\text { BEDROCK }\end{array}$ & TILL SAND, CLAY, HARDPAN \\
\hline EFW & 39 & $\cdots$ & 350 & $\cdots$ & $\begin{array}{r}0 \\
12\end{array}$ & $\begin{array}{l}12 \\
\cdots\end{array}$ & $\begin{array}{l}112 \text { TILL } \\
\text { BEDROCK }\end{array}$ & TILL \\
\hline EFW & 40 & $\cdots$ & 800 & $\cdots$ & $\begin{array}{r}0 \\
70 \\
80\end{array}$ & $\begin{array}{l}70 \\
80 \\
--\end{array}$ & $\begin{array}{l}110 \text { SDMN } \\
112 \text { TILL } \\
\text { BEDROCK }\end{array}$ & $\begin{array}{l}\text { SAND } \\
\text { TILL }\end{array}$ \\
\hline EFW & 41 & $\cdots$ & 362 & $\cdots$ & $\begin{array}{r}0 \\
50 \\
135\end{array}$ & $\begin{array}{r}50 \\
135 \\
\cdots\end{array}$ & $\begin{array}{l}110 \text { SDMN } \\
110 \text { SDMN } \\
\text { BEDROCK }\end{array}$ & $\begin{array}{l}\text { SAND } \\
\text { CLAY }\end{array}$ \\
\hline EFW & 42 & $\cdots$ & 427 & $\cdots$ & $\begin{array}{r}0 \\
107\end{array}$ & $\begin{array}{r}107 \\
-\end{array}$ & $\begin{array}{l}\text { 110SDMN } \\
\text { BEDROCK }\end{array}$ & SDCL \\
\hline EFW & 43 & $\cdots$ & 282 & $\cdots$ & $\begin{array}{r}0 \\
100\end{array}$ & 100 & $\begin{array}{l}110 \text { SDMN } \\
\text { BEDROCK }\end{array}$ & SDGL \\
\hline EFW & 44 & $\cdots$ & 377 & $\cdots$ & $\begin{array}{r}0 \\
100\end{array}$ & $\begin{array}{r}100 \\
\cdots\end{array}$ & $\begin{array}{l}110 \text { SDMN } \\
\text { BEDROCK }\end{array}$ & SAND \\
\hline EFW & 45 & $\cdots$ & 377 & $\cdots$ & $\begin{array}{r}0 \\
70\end{array}$ & $\begin{array}{l}70 \\
\cdots\end{array}$ & $\begin{array}{l}\text { 110SDMN } \\
\text { BEDROCK }\end{array}$ & SDGL \\
\hline EFW & 46 & $\cdots$ & 43 & $\cdots$ & $\begin{array}{l}\cdots \\
\therefore\end{array}$ & $\begin{array}{l}\cdots \\
\cdots\end{array}$ & $\begin{array}{l}110 \text { SDMN } \\
110 \text { SDMN }\end{array}$ & $\begin{array}{l}\text { SAND } \\
\text { SDGL }\end{array}$ \\
\hline EFW & 47 & $\cdots$ & 241 & $\cdots$ & $\begin{array}{r}0 \\
10\end{array}$ & $\begin{array}{l}10 \\
\cdots\end{array}$ & $\begin{array}{l}\text { 112TILL } \\
\text { BEDROCK }\end{array}$ & TILL \\
\hline EFW & 48 & $\cdots$ & 352 & $\cdots$ & $\begin{array}{r}0 \\
64\end{array}$ & $\begin{array}{l}64 \\
\cdots\end{array}$ & $\begin{array}{l}110 \text { SDMN } \\
\text { BEDROCK }\end{array}$ & SDCL \\
\hline EFW & 49 & $\cdots$ & 602 & $\cdots$ & $\begin{array}{r}0 \\
20 \\
35\end{array}$ & $\begin{array}{l}20 \\
35 \\
\cdots\end{array}$ & $\begin{array}{l}110 \text { SDMN } \\
110 \text { SDMN } \\
\text { BEDROCK }\end{array}$ & $\begin{array}{l}\text { SAND } \\
\text { SDGL }\end{array}$ \\
\hline EFW & 50 & $\cdots$ & 120 & $\cdots$ & $\begin{array}{r}0 \\
45 \\
62\end{array}$ & $\begin{array}{l}45 \\
62 \\
\cdots\end{array}$ & $\begin{array}{l}110 \text { SDMN } \\
110 \text { SDMN } \\
\text { BEDROCK }\end{array}$ & $\begin{array}{l}\text { SDCL } \\
\text { SDGL }\end{array}$ \\
\hline EFW & 51 & $\cdots$ & 59 & $\cdots$ & $\begin{array}{r}0 \\
46 \\
61\end{array}$ & $\begin{array}{l}46 \\
61 \\
\ldots\end{array}$ & $\begin{array}{l}110 \text { SDMN } \\
110 \text { SDMN } \\
\text { BEDROCK }\end{array}$ & $\begin{array}{l}\text { SDCL } \\
\text { SDGL }\end{array}$ \\
\hline
\end{tabular}


Table 2-1. Stratigraphic logs of wells and borlngs in the Saco and Ossipee River Basins, eastcentral New Hampshire--Continued

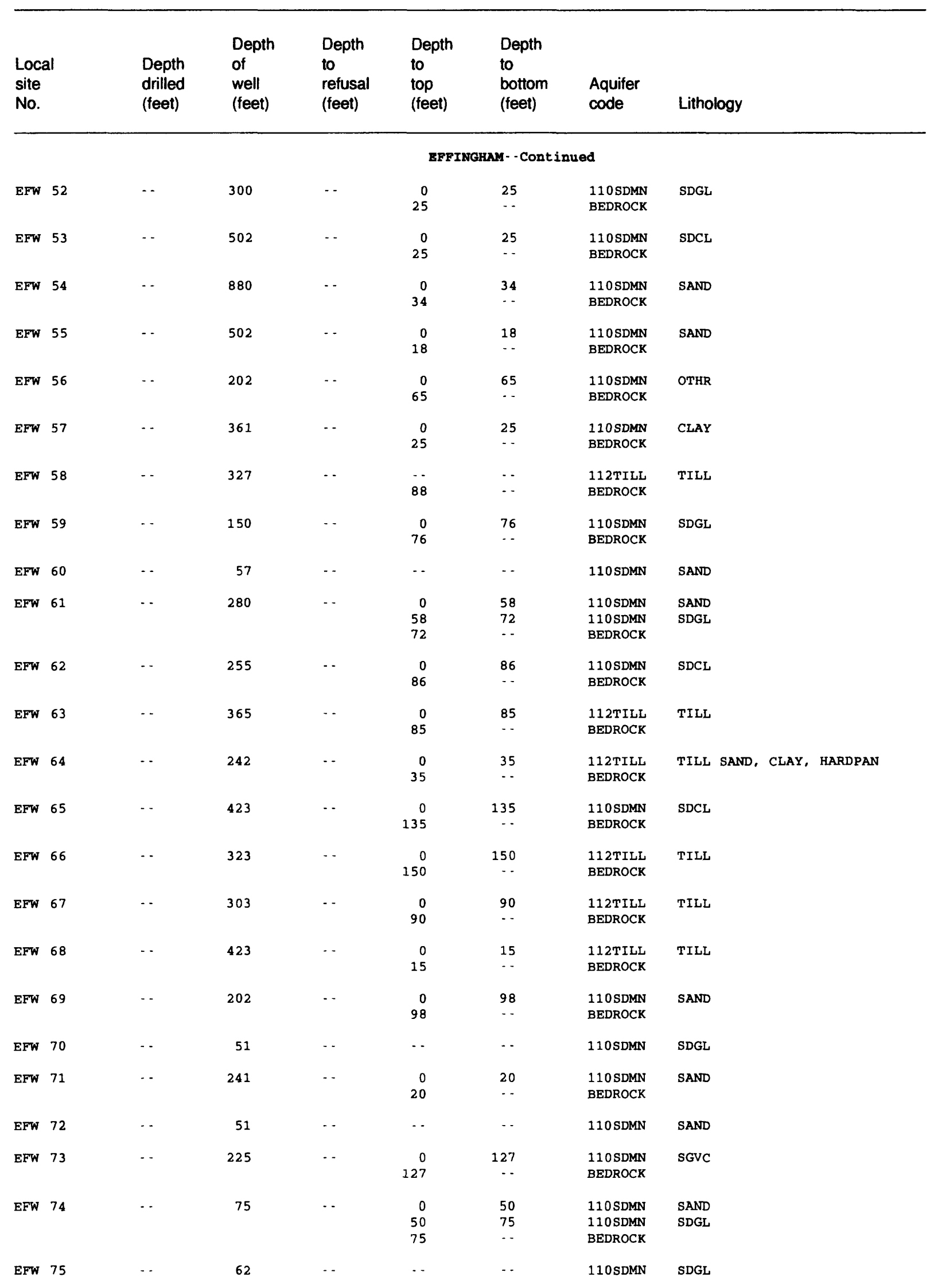


Table 2-1. Stratigraphic logs of wells and borings in the Saco and Ossipee River Basins, eastcentral New Hampshire--Continued

\begin{tabular}{llcccccc}
\hline & & Depth & Depth & Depth & Depth & & \\
Local & Depth & of & to & to & to & & \\
site & drilled & well & refusal & top & bottom & Aquifer & \\
no. & (feet) & (feet) & (feet) & (feet) & (feet) & code & Lithology
\end{tabular}

\begin{tabular}{|c|c|c|c|c|c|c|c|c|}
\hline \multirow[b]{2}{*}{ EFW } & \multirow[b]{2}{*}{76} & \multicolumn{7}{|c|}{ EFFINGHAM- - Continued } \\
\hline & & $\cdots$ & 93 & $\cdots$ & $\begin{array}{r}0 \\
30\end{array}$ & $\begin{array}{l}30 \\
\cdots\end{array}$ & $\begin{array}{l}\text { 112TILL } \\
\text { BEDROCK }\end{array}$ & TILL GRAVEL, CLAY, HARDPAN \\
\hline EFW & 77 & $\cdots$ & 322 & $\cdots$ & $\begin{array}{r}0 \\
45\end{array}$ & $\begin{array}{l}45 \\
--\end{array}$ & $\begin{array}{l}\text { 112TILL } \\
\text { BEDROCK }\end{array}$ & TILL SAND, CLAY, HARDPAN \\
\hline EFW & 78 & $\cdots$ & 422 & $\cdots$ & $\begin{array}{r}0 \\
100 \\
203\end{array}$ & $\begin{array}{l}100 \\
203\end{array}$ & $\begin{array}{l}110 \text { SDMN } \\
112 \text { TILL } \\
\text { BEDROCK }\end{array}$ & $\begin{array}{l}\text { SAND } \\
\text { TILL SAND, CLAY, HARDPAN }\end{array}$ \\
\hline EFW & 79 & $\cdots$ & 502 & $\cdots$ & $\begin{array}{r}0 \\
55\end{array}$ & $\begin{array}{l}55 \\
--\end{array}$ & $\begin{array}{l}\text { 112TILL } \\
\text { BEDROCK }\end{array}$ & TILL SAND， GRAVEL， HARDPAN \\
\hline EFW & 80 & $\cdots$ & 482 & $\cdots$ & $\begin{array}{r}0 \\
50\end{array}$ & 50 & $\begin{array}{l}110 \text { SDMN } \\
\text { BEDROCK }\end{array}$ & SDGL \\
\hline EFW & 81 & $-\cdot$ & 422 & $\cdots$ & $\begin{array}{r}0 \\
75 \\
165\end{array}$ & $\begin{array}{r}75 \\
165 \\
-.\end{array}$ & $\begin{array}{l}110 \text { SDMN } \\
112 \text { TILL } \\
\text { BEDROCK }\end{array}$ & $\begin{array}{l}\text { SAND } \\
\text { TILL GRAVEL, CLAY, HARDPAN }\end{array}$ \\
\hline EFW & 82 & $\cdots$ & 255 & $\cdots$ & $\begin{array}{r}0 \\
75\end{array}$ & $\begin{array}{l}75 \\
--\end{array}$ & $\begin{array}{l}110 \text { SDMN } \\
\text { BEDROCK }\end{array}$ & SAND \\
\hline EFW & 83 & - & 324 & $\cdots$ & $\begin{array}{r}0 \\
100 \\
120\end{array}$ & $\begin{array}{r}100 \\
120 \\
\cdots\end{array}$ & $\begin{array}{l}110 \text { SDMN } \\
110 \text { SDMN } \\
\text { BEDROCK }\end{array}$ & $\begin{array}{l}\text { SAND } \\
\text { OTHR }\end{array}$ \\
\hline EFW & 84 & $\cdots$ & 24 & $\cdots$ & $\begin{array}{r}0 \\
25\end{array}$ & 25 & $\begin{array}{l}110 \mathrm{SDMN} \\
112 \mathrm{TILL}\end{array}$ & $\begin{array}{l}\text { SAND } \\
\text { TILL }\end{array}$ \\
\hline EFW & 85 & $\cdots$ & 425 & $\cdots$ & $\begin{array}{r}0 \\
90\end{array}$ & $\begin{array}{l}90 \\
\cdots\end{array}$ & $\begin{array}{l}110 \text { SDMN } \\
\text { BEDROCK }\end{array}$ & OTHR \\
\hline EFW & 86 & $\cdots$ & 325 & $\cdots$ & $\begin{array}{r}0 \\
90\end{array}$ & 90 & $\begin{array}{l}110 \text { SDMN } \\
\text { BEDROCK }\end{array}$ & OTHR \\
\hline EFW & 87 & $\cdots$ & 24 & $\cdots$ & $\begin{array}{c}0 \\
24.5\end{array}$ & $\begin{array}{l}24.5 \\
\cdots\end{array}$ & $\begin{array}{l}110 \text { SDMN } \\
112 \text { TILL }\end{array}$ & $\begin{array}{l}\text { SDGL } \\
\text { TILL }\end{array}$ \\
\hline EFW & 88 & $\cdots$ & 324 & $\cdots$ & $\begin{array}{r}0 \\
20 \\
140\end{array}$ & $\begin{array}{r}20 \\
140 \\
-.\end{array}$ & $\begin{array}{l}110 \text { SDMN } \\
110 \text { SDMN } \\
\text { BEDROCK }\end{array}$ & $\begin{array}{l}\text { SDGL } \\
\text { OTHR ROTTEN ROCK }\end{array}$ \\
\hline EFW & 89 & $\cdots$ & 523 & $\cdots$ & $\begin{array}{r}0 \\
90\end{array}$ & $\begin{array}{l}90 \\
\cdots\end{array}$ & $\begin{array}{l}110 \text { SDMN } \\
\text { BEDROCK }\end{array}$ & SAND \\
\hline EFW & 90 & $\cdots$ & 665 & $\cdots$ & $\begin{array}{r}0 \\
90\end{array}$ & $\begin{array}{l}90 \\
\cdots\end{array}$ & $\begin{array}{l}\text { 112TILL } \\
\text { BEDROCK }\end{array}$ & TILL SAND, HARDPAN \\
\hline EFW & 91 & $\cdots$ & 503 & $\cdots$ & $\begin{array}{r}0 \\
85\end{array}$ & $\begin{array}{l}85 \\
-\end{array}$ & $\begin{array}{l}\text { 112TILL } \\
\text { BEDROCK }\end{array}$ & TILL \\
\hline EFW & 92 & $\cdots$ & 423 & $\cdots$ & $\begin{array}{r}0 \\
80 \\
100\end{array}$ & $\begin{array}{r}80 \\
100\end{array}$ & $\begin{array}{l}\text { 112TILL } \\
110 \text { SDMN } \\
\text { BEDROCK }\end{array}$ & $\begin{array}{l}\text { TILL } \\
\text { OTHR RO'TTEN ROCK }\end{array}$ \\
\hline \multicolumn{9}{|c|}{ FREEDOM } \\
\hline EFW & 93 & $\cdots$ & 303 & $\cdots$ & $\begin{array}{r}0 \\
140\end{array}$ & $\begin{array}{r}140 \\
-.\end{array}$ & $\begin{array}{l}\text { 112TILL } \\
\text { BEDROCK }\end{array}$ & TILL \\
\hline FLS & 1 & $\cdots$ & $\cdots$ & $\cdots$ & $\cdots$ & $\cdots$ & $\cdots$ & \\
\hline FLW & 3 & $\cdots$ & $\cdots$ & $\cdots$ & $\cdots$ & $\cdots$ & $\cdots$ & \\
\hline FLW & 4 & 15 & 15 & $\cdots$ & $\cdots$ & $\cdots$ & $\cdots$ & \\
\hline FLW & 5 & 18 & 18 & $\cdots$ & $\cdots$ & $\cdots$ & $\cdots$ & \\
\hline FLW & 6 & 87 & 20 & - & $\cdots$ & $\cdots$ & $\cdots$ & \\
\hline
\end{tabular}


Table 2-1. Stratigraphic logs of wells and borings in the Saco and Ossipee River Basins, eastcentral New Hampshire--Continued

\begin{tabular}{|c|c|c|c|c|c|c|c|c|}
\hline \multicolumn{2}{|c|}{$\begin{array}{l}\text { Local } \\
\text { site } \\
\text { No. }\end{array}$} & $\begin{array}{l}\text { Depth } \\
\text { drilled } \\
\text { (feet) }\end{array}$ & $\begin{array}{l}\text { Depth } \\
\text { of } \\
\text { well } \\
\text { (feet) }\end{array}$ & $\begin{array}{l}\text { Depth } \\
\text { to } \\
\text { refusal } \\
\text { (feet) }\end{array}$ & $\begin{array}{l}\text { Depth } \\
\text { to } \\
\text { top } \\
\text { (feet) }\end{array}$ & $\begin{array}{l}\text { Depth } \\
\text { to } \\
\text { bottom } \\
\text { (feet) }\end{array}$ & $\begin{array}{l}\text { Aquifer } \\
\text { code }\end{array}$ & Lithology \\
\hline & & \multicolumn{7}{|c|}{ PREBDOM- - Continued } \\
\hline FLW & 7 & 28 & 28 & $\cdots$ & -. & -. & $\cdots$ & \\
\hline FLW & 8 & 32 & 32 & - & $\cdots$ & $\cdots$ & $\cdots$ & \\
\hline FLW & 9 & 12 & 12 & $\cdots$ & $\cdots$ & $\cdots$ & $\cdots$ & \\
\hline FLW & 10 & 15 & 15 & $\cdots$ & $\cdots$ & $\cdots$ & $\cdots$ & \\
\hline FLW & 11 & 50 & 50 & $\cdots$ & $\cdots$ & $\cdots$ & -. & \\
\hline FLW & 12 & 377 & 60 & $\cdots$ & $\cdots$ & -. & $\cdots$ & \\
\hline FLW & 13 & 61 & 61 & $\cdots$ & $\cdots$ & -. & $\cdots$ & \\
\hline FLW & 14 & 18 & 18 & - & $\cdots$ & - & $\cdots$ & \\
\hline FLW & 15 & 16 & 16 & $\cdots$ & - & $\cdots$ & $\cdots$ & \\
\hline FLW & 16 & - & $-\cdot$ & - & $\cdots$ & - & $\cdots$ & \\
\hline FLW & 17 & 8.3 & 8.3 & $\cdots$ & $\cdots$ & $\cdots$ & $\cdots$ & \\
\hline FLW & 18 & 22 & 22 & $\cdots$ & - & $\cdots$ & $\cdots$ & \\
\hline FLW & 19 & $\cdots$ & $\cdots$ & $\cdots$ & $\cdots$ & $\cdots$ & $\cdots$ & \\
\hline FLW & 20 & - & $\cdots$ & - & $\cdots$ & $\cdots$ & - & \\
\hline FLW & 21 & 4.9 & 4.9 & - & $\cdots$ & - & $\cdots$ & \\
\hline FLW & 22 & 30 & 30 & $\cdots$ & $\cdots$ & $\cdots$ & - & \\
\hline FLW & 23 & 35 & 35 & $\cdots$ & $\cdots$ & $\cdots$ & - & \\
\hline FLW & 24 & 73.5 & 58.7 & 73.5 & \begin{tabular}{l}
\multicolumn{1}{c}{0} \\
10 \\
13.5 \\
16 \\
18 \\
37.5 \\
48 \\
63 \\
73.5
\end{tabular} & $\begin{array}{l}10 \\
13.5 \\
15 \\
18 \\
37.5 \\
48 \\
63 \\
73.5 \\
-.\end{array}$ & $\begin{array}{l}112 \mathrm{SRFD} \\
112 \mathrm{SRFD} \\
112 \mathrm{SRFD} \\
112 \mathrm{SRFD} \\
112 \mathrm{SRFD} \\
112 \mathrm{SRFD} \\
112 \mathrm{SRFD} \\
112 \mathrm{TILL} \\
112 \mathrm{TILL}\end{array}$ & $\begin{array}{l}\text { SAND M, POORLY SORTED } \\
\text { SAND C, POORLY SORTED } \\
\text { SAND F } \\
\text { SAND VC } \\
\text { SAND C, MEDIUM SORTED } \\
\text { SAND M, MEDIUM SORTED } \\
\text { SAND C, VERY POORLY SORTED } \\
\text { TILL } \\
\text { TILL REFUSAL }\end{array}$ \\
\hline FLW & 25 & 109 & 39.5 & 109 & $\begin{array}{l}0 \\
15 \\
23 \\
33 \\
40 \\
68 \\
73 \\
83 \\
97.5 \\
106 \\
109\end{array}$ & $\begin{array}{l}15 \\
23 \\
33 \\
40 \\
68 \\
73 \\
83 \\
97.5 \\
106 \\
109 \\
\cdots\end{array}$ & $\begin{array}{l}112 \text { SRFD } \\
112 \text { SRFD } \\
112 \text { SRFD } \\
112 \text { SRFD } \\
112 \text { SRFD } \\
112 L C S R \\
112 L C S R \\
112 L C S R \\
112 L C S R \\
112 T I L L \\
112 T I L L\end{array}$ & $\begin{array}{l}\text { SAND } M \text {, WELL SORTED } \\
\text { SAND C TO VC, MEDIUM SORTED } \\
\text { SAND } M \text {, POORLY SORTED } \\
\text { SAND VC, POORLY SORTED } \\
\text { SAND F TO M, BANDED } \\
\text { SDST VF, GRAY } \\
\text { SAND F, GRAY, MEDIUM SORTED } \\
\text { STCL GRAY } \\
\text { CLAY BROWN, VARVED } \\
\text { TILL } \\
\text { TILL REFUSAL }\end{array}$ \\
\hline FLW & 26 & 113 & 20 & 113 & $\begin{array}{r}-. \\
33 \\
63 \\
73 \\
108\end{array}$ & $\begin{array}{r}33 \\
63 \\
73 \\
108 \\
-.\end{array}$ & $\begin{array}{l}112 \text { SRFD } \\
112 \text { SRFD } \\
112 \text { SRFD } \\
112 \text { SRFD } \\
112 \text { TILL }\end{array}$ & $\begin{array}{l}\text { SAND VF TO FS } \\
\text { SDCL CLAY AND VFS LAYERS } \\
\text { STCL SILT AND CLAY LAYERS } \\
\text { SDST SILT AND F TO VFS LAYERS } \\
\text { TILL }\end{array}$ \\
\hline FLW & 27 & 200 & - & $\cdots$ & $\cdots$ & - & - & \\
\hline FLW & 28 & 25 & 25 & - & $\cdots$ & $\cdots$ & $\cdots$ & \\
\hline FLW & 29 & $\cdots$ & 453 & $\cdots$ & $\begin{array}{r}0 \\
43\end{array}$ & $\begin{array}{l}43 \\
--\end{array}$ & $\begin{array}{l}110 \text { SDMN } \\
\text { BEDROCX }\end{array}$ & SAND \\
\hline
\end{tabular}


Table 2-1. Stratigraphic logs of wells and borings in the Saco and Ossipee River Basins, eastcentral New Hampshire--Continued

\begin{tabular}{|c|c|c|c|c|c|c|c|c|}
\hline $\begin{array}{l}\text { Loca } \\
\text { site } \\
\text { no. }\end{array}$ & & $\begin{array}{l}\text { Depth } \\
\text { drilled } \\
\text { (feet) }\end{array}$ & $\begin{array}{l}\text { Depth } \\
\text { of } \\
\text { well } \\
\text { (feet) }\end{array}$ & $\begin{array}{l}\text { Depth } \\
\text { to } \\
\text { refusal } \\
\text { (feet) }\end{array}$ & $\begin{array}{l}\text { Depth } \\
\text { to } \\
\text { top } \\
\text { (feet) }\end{array}$ & $\begin{array}{l}\text { Depth } \\
\text { to } \\
\text { bottom } \\
\text { (feet) }\end{array}$ & $\begin{array}{l}\text { Aquifer } \\
\text { code }\end{array}$ & Lithology \\
\hline & & \multicolumn{7}{|c|}{ FREBDOK- - Continued } \\
\hline FLW & 30 & $\cdots$ & 242 & $\cdots$ & $\begin{array}{r}- \\
0 \\
140\end{array}$ & $\begin{array}{r}-. \\
140 \\
\ldots\end{array}$ & $\begin{array}{l}\text { 110SDMN } \\
112 \text { TILL } \\
\text { BEDROCK }\end{array}$ & $\begin{array}{l}\text { SAND } \\
\text { TILL CLAY, HARDPAN }\end{array}$ \\
\hline FLW & 31 & $\cdots$ & 66 & $\cdots$ & $\begin{array}{r}0 \\
30 \\
58\end{array}$ & $\begin{array}{l}30 \\
58 \\
--\end{array}$ & $\begin{array}{l}110 \mathrm{SDMN} \\
110 \mathrm{SDMN} \\
110 \mathrm{SDMN}\end{array}$ & $\begin{array}{l}\text { SDGL } \\
\text { SGVC } \\
\text { SDGL }\end{array}$ \\
\hline FLW & 32 & $\cdots$ & 25 & $\cdots$ & $\begin{array}{r}0 \\
12 \\
25\end{array}$ & $\begin{array}{l}12 \\
25 \\
-.\end{array}$ & $\begin{array}{l}110 \text { SDMN } \\
110 \text { SDMN } \\
110 \text { SDMN }\end{array}$ & $\begin{array}{l}\text { SGVC } \\
\text { SDGL } \\
\text { OTHR }\end{array}$ \\
\hline FLW & 33 & - & 66 & $\cdots$ & $\cdots$ & $\cdots$ & $\cdots$ & \\
\hline FLW & 34 & $\cdots$ & 530 & $\cdots$ & $\begin{array}{l}0 \\
8\end{array}$ & $\begin{array}{l}8 \\
\cdots\end{array}$ & $\begin{array}{l}\text { 112TILL } \\
\text { BEDROCK }\end{array}$ & TILL \\
\hline FLW & 35 & $-\cdot$ & 506 & $\cdots$ & $\begin{array}{r}0 \\
80\end{array}$ & $\begin{array}{l}80 \\
-\cdots\end{array}$ & $\begin{array}{l}110 \text { SDMN } \\
\text { BEDROCK }\end{array}$ & SAND \\
\hline FLW & 36 & $\cdots$ & 28 & $\cdots$ & $\begin{array}{r}0 \\
28\end{array}$ & $\begin{array}{l}28 \\
\cdots\end{array}$ & $\begin{array}{l}110 \text { SDMN } \\
112 \text { TILL }\end{array}$ & $\begin{array}{l}\text { SDGL } \\
\text { TILL }\end{array}$ \\
\hline FLW & 37 & $\cdots$ & 475 & $\cdots$ & $\begin{array}{r}0 \\
82\end{array}$ & $\begin{array}{l}82 \\
-\cdot\end{array}$ & $\begin{array}{l}110 \text { SDMN } \\
\text { BEDROCK }\end{array}$ & SAND \\
\hline FLW & 38 & $\cdots$ & 425 & $\cdot-$ & $\begin{array}{r}0 \\
105\end{array}$ & $\begin{array}{c}105 \\
-.\end{array}$ & $\begin{array}{l}110 S D M N \\
\text { BEDROCK }\end{array}$ & SDCL \\
\hline FLW & 39 & $\cdots$ & 277 & $\cdots$ & $\begin{array}{r}0 \\
70 \\
146\end{array}$ & $\begin{array}{r}70 \\
146 \\
\cdots\end{array}$ & $\begin{array}{l}110 \text { SDMN } \\
110 \text { SDMN } \\
\text { BEDROCK }\end{array}$ & $\begin{array}{l}\text { SAND } \\
\text { SDCL }\end{array}$ \\
\hline FLW & 40 & $\cdots$ & 32 & $\cdots$ & $\begin{array}{r}0 \\
33\end{array}$ & $\begin{array}{l}33 \\
\cdots\end{array}$ & $\begin{array}{l}110 \text { SDMN } \\
110 \text { SDMN }\end{array}$ & $\begin{array}{l}\text { SAND } \\
\text { CLAY }\end{array}$ \\
\hline FLW & 41 & $\cdots$ & 277 & $\cdots$ & $\begin{array}{r}0 \\
102\end{array}$ & $\begin{array}{r}102 \\
\cdots\end{array}$ & $\begin{array}{l}\text { 112TILL } \\
\text { BEDROCK }\end{array}$ & TILL GRAVEL, CLAY, HARDPAN \\
\hline FLW & 42 & $\cdots$ & 530 & $\cdots$ & $\begin{array}{l}0 \\
8\end{array}$ & $\begin{array}{r}8 \\
--\end{array}$ & $\begin{array}{l}110 \text { SDMN } \\
\text { BEDROCK }\end{array}$ & SDGL \\
\hline FLW & 43 & $\cdots$ & 45 & $\cdots$ & $\cdots$ & $\cdots$ & $110 \mathrm{SDMN}$ & SDGL \\
\hline FLW & 44 & $\cdots$ & 34 & - & $\cdots$ & $\cdots$ & 110 SDMN & SAND \\
\hline FLW & 45 & - & 33 & $-\cdot$ & $\cdots$ & $\cdot-$ & 110 SDMN & SAND \\
\hline FLW & 46 & $\cdots$ & 38 & $-\cdot$ & $\cdots$ & - & 110 SDMN & SAND \\
\hline FLW & 47 & $\cdots$ & 66 & $\cdots$ & $\begin{array}{r}0 \\
15\end{array}$ & $\begin{array}{l}15 \\
\cdots\end{array}$ & $\begin{array}{l}110 \text { SDMN } \\
110 \text { SDMN }\end{array}$ & $\begin{array}{l}\text { SDGL } \\
\text { SAND }\end{array}$ \\
\hline FLW & 48 & $\cdots$ & 65 & $\cdots$ & $\cdots$ & $\cdots$ & 110 SDMN & SDGL \\
\hline FLW & 49 & $\cdots$ & 35 & $\cdots$ & $\cdots$ & $-\cdot$ & $110 \mathrm{SDMN}$ & SDGL \\
\hline FLW & 50 & $\cdots$ & 362 & $\cdots$ & $\begin{array}{r}0 \\
35 \\
110\end{array}$ & $\begin{array}{r}35 \\
110 \\
\cdots\end{array}$ & $\begin{array}{l}110 \text { SDMN } \\
110 \text { SDMN } \\
\text { BEDROCK }\end{array}$ & $\begin{array}{l}\text { SAND } \\
\text { CLAY }\end{array}$ \\
\hline FLW & 51 & $\cdots$ & 47.6 & $\cdots$ & $\begin{array}{r}0 \\
22\end{array}$ & $\begin{array}{l}22 \\
-\end{array}$ & $\begin{array}{l}110 \mathrm{SDMN} \\
110 \mathrm{SDMN}\end{array}$ & $\begin{array}{l}\text { SAND } \\
\text { SDGL }\end{array}$ \\
\hline FLW & 52 & $\cdots$ & 242 & $\cdots$ & $\begin{array}{r}0 \\
106\end{array}$ & $\begin{array}{r}106 \\
\cdots\end{array}$ & $\begin{array}{l}110 \text { SDMN } \\
\text { BEDROCK }\end{array}$ & SDCL \\
\hline FLW & 53 & - & 282 & $\cdots$ & $\begin{array}{r}0 \\
85\end{array}$ & $\begin{array}{l}85 \\
\cdots\end{array}$ & $\begin{array}{l}110 \text { SDMN } \\
\text { BEDROCK }\end{array}$ & SAND \\
\hline
\end{tabular}


Table 2-1. Stratigraphic logs of wells and borings in the Saco and Ossipee River Basins, eastcentral New Hampshire--Continued

\begin{tabular}{|c|c|c|c|c|c|c|c|c|}
\hline $\begin{array}{l}\text { Loca } \\
\text { site } \\
\text { No. }\end{array}$ & & $\begin{array}{l}\text { Depth } \\
\text { drilled } \\
\text { (feet) }\end{array}$ & $\begin{array}{l}\text { Depth } \\
\text { of } \\
\text { well } \\
\text { (feet) }\end{array}$ & $\begin{array}{l}\text { Depth } \\
\text { to } \\
\text { refusal } \\
\text { (feet) }\end{array}$ & $\begin{array}{l}\text { Depth } \\
\text { to } \\
\text { top } \\
\text { (feet) }\end{array}$ & $\begin{array}{l}\text { Depth } \\
\text { to } \\
\text { bottom } \\
\text { (feet) }\end{array}$ & $\begin{array}{l}\text { Aquifer } \\
\text { code }\end{array}$ & Lithology \\
\hline & & \multicolumn{7}{|c|}{ FREGDOK - Continued } \\
\hline FLW & 54 & $\cdots$ & 325 & $\cdots$ & $\begin{array}{r}0 \\
62\end{array}$ & $\begin{array}{l}62 \\
-\cdot\end{array}$ & $\begin{array}{l}\text { 110SDMN } \\
\text { BEDROCK }\end{array}$ & SDGL \\
\hline FLW & 55 & $\cdots$ & 302 & $\cdots$ & $\begin{array}{r}0 \\
55\end{array}$ & 55 & $\begin{array}{l}110 \text { SDMN } \\
\text { BEDROCK }\end{array}$ & SDCL \\
\hline FLW & 56 & $\cdots$ & 702 & $\cdots$ & $\begin{array}{r}0 \\
40\end{array}$ & $\begin{array}{l}40 \\
\cdots\end{array}$ & $\begin{array}{l}\text { 112TILL } \\
\text { BEDROCK }\end{array}$ & TILL CLAY, HARDPAN \\
\hline FLW & 57 & $-\cdot$ & 152 & $\cdots$ & $\begin{array}{r}0 \\
30\end{array}$ & $\begin{array}{l}30 \\
--\end{array}$ & $\begin{array}{l}110 \text { SDMN } \\
\text { BEDROCK }\end{array}$ & SAND \\
\hline FLW & 58 & $\cdots$ & 302 & $\cdots$ & $\begin{array}{r}0 \\
60 \\
69\end{array}$ & $\begin{array}{l}60 \\
69 \\
\cdots\end{array}$ & $\begin{array}{l}110 \text { SDMN } \\
110 \text { SDMN } \\
\text { BEDROCK }\end{array}$ & $\begin{array}{l}\text { SAND } \\
\text { CLAY }\end{array}$ \\
\hline FLW & 59 & $\cdots$ & 400 & $\cdots$ & $\begin{array}{r}0 \\
45\end{array}$ & $\begin{array}{l}45 \\
--\end{array}$ & $\begin{array}{l}\text { 110SDMN } \\
\text { BEDROCK }\end{array}$ & SAND \\
\hline FLW & 60 & $\cdots$ & 142 & $\cdots$ & $\begin{array}{r}0 \\
42\end{array}$ & $\begin{array}{l}42 \\
--\end{array}$ & $\begin{array}{l}110 \text { SDMN } \\
\text { BEDROCK }\end{array}$ & CLAY \\
\hline FLW & 61 & $\cdots$ & 45 & $\cdots$ & $\cdots$ & $\cdots$ & 110 SDMN & SDGL \\
\hline FLW & 62 & $\cdots$ & 57 & $\cdots$ & $\begin{array}{r}0 \\
48\end{array}$ & $\begin{array}{l}48 \\
-\cdots\end{array}$ & $\begin{array}{l}110 \text { SDMN } \\
110 \text { SDMN }\end{array}$ & $\begin{array}{l}\text { SDCL } \\
\text { SAND }\end{array}$ \\
\hline FLW & 63 & $\cdots$ & 482 & $\cdots$ & $\begin{array}{r}0 \\
177 \\
195 \\
222\end{array}$ & $\begin{array}{r}177 \\
195 \\
222 \\
--\end{array}$ & $\begin{array}{l}110 \text { SDMN } \\
110 \text { SDMN } \\
112 \text { TILL } \\
\text { BEDROCK }\end{array}$ & $\begin{array}{l}\text { SAND } \\
\text { CLAY } \\
\text { TILE GRAVEL, HARDPAN }\end{array}$ \\
\hline FLW & 64 & $\cdots$ & 423 & $\cdots$ & $\begin{array}{r}0 \\
30\end{array}$ & $\begin{array}{l}30 \\
\cdots\end{array}$ & $\begin{array}{l}\text { 110SDMN } \\
\text { BEDROCK }\end{array}$ & SDGL \\
\hline FLW & 65 & $\cdots$ & 30 & $\cdots$ & $\begin{array}{r}0 \\
30\end{array}$ & 30 & $\begin{array}{l}110 \text { SDMN } \\
112 \mathrm{TILL}\end{array}$ & $\begin{array}{l}\text { SAND } \\
\text { TILL }\end{array}$ \\
\hline FLW & 66 & $\cdots$ & 51 & $\cdots$ & $\begin{array}{ll}-\cdot \\
51\end{array}$ & $\begin{array}{l}\cdots \\
\cdots\end{array}$ & $\begin{array}{l}110 \mathrm{SDMN} \\
110 \mathrm{SDMN}\end{array}$ & $\begin{array}{l}\text { SAND } \\
\text { SDGL }\end{array}$ \\
\hline FLW & 67 & $\cdots$ & 178 & $\cdots$ & 5 & $\cdots$ & BEDROCK & \\
\hline FLW & 68 & $\cdots$ & 803 & - & $\begin{array}{r}0 \\
40\end{array}$ & $\begin{array}{l}40 \\
\cdots\end{array}$ & $\begin{array}{l}\text { 112TILL } \\
\text { BEDROCK }\end{array}$ & TILL GRAVEL, HARDPAN \\
\hline FLW & 69 & $\cdots$ & 403 & $\cdots$ & $\begin{array}{r}0 \\
10\end{array}$ & $\begin{array}{l}10 \\
\cdots\end{array}$ & $\begin{array}{l}\text { 112TILL } \\
\text { BEDROCK }\end{array}$ & TILL SAND， GRAVEL， HARDPAN \\
\hline FLW & 70 & $\cdots$ & 800 & - & $\begin{array}{r}0 \\
30 \\
40\end{array}$ & $\begin{array}{l}30 \\
40 \\
\cdots\end{array}$ & $\begin{array}{l}110 \text { SDMN } \\
110 \text { SDMN } \\
\text { BEDROCK }\end{array}$ & $\begin{array}{l}\text { SAND } \\
\text { OTHR }\end{array}$ \\
\hline FLW & 71 & $\cdots$ & 335 & $\cdots$ & $\begin{array}{r}0 \\
38\end{array}$ & $\begin{array}{l}38 \\
\cdots\end{array}$ & $\begin{array}{l}\text { 110SDMN } \\
\text { BEDROCK }\end{array}$ & SAND \\
\hline FLW & 72 & $\cdots$ & 303 & $\cdots$ & $\begin{array}{r}0 \\
17\end{array}$ & $\begin{array}{l}17 \\
\cdots\end{array}$ & $\begin{array}{l}\text { 110SDMN } \\
\text { BEDROCK }\end{array}$ & SAND \\
\hline FLW & 73 & $\cdots$ & 503 & $\cdots$ & $\begin{array}{r}0 \\
15\end{array}$ & $\begin{array}{l}15 \\
-\cdot\end{array}$ & $\begin{array}{l}\text { 112TILL } \\
\text { BEDROCK }\end{array}$ & TILL \\
\hline FLW & 74 & $\cdots$ & 303 & $\cdots$ & $\begin{array}{r}0 \\
53\end{array}$ & $\begin{array}{l}53 \\
\cdots\end{array}$ & $\begin{array}{l}110 \text { SDMN } \\
\text { BEDROCK }\end{array}$ & SAND \\
\hline FLW & 75 & $\cdots$ & 43 & $\cdots$ & $\begin{array}{r}0 \\
24 \\
28 \\
43\end{array}$ & $\begin{array}{l}24 \\
28 \\
43 \\
-\cdots\end{array}$ & $\begin{array}{l}110 \text { SDMN } \\
110 \text { SDMN } \\
110 \text { SDMN } \\
112 \text { TILL }\end{array}$ & $\begin{array}{l}\text { SAND } \\
\text { CLAY } \\
\text { SAND } \\
\text { TILI }\end{array}$ \\
\hline FLW & 76 & $\cdots$ & 61 & - & $-\cdot$ & $-\cdot$ & $110 \mathrm{SDMN}$ & SDGL \\
\hline
\end{tabular}


Table 2-1. Stratigraphic logs of wells and borings in the Saco and Ossipee River Basins, eastcentral New Hampshire--Continued

\begin{tabular}{|c|c|c|c|c|c|c|c|c|}
\hline \multicolumn{2}{|c|}{$\begin{array}{l}\text { Local } \\
\text { site } \\
\text { no. }\end{array}$} & $\begin{array}{l}\text { Depth } \\
\text { drilled } \\
\text { (feet) }\end{array}$ & $\begin{array}{l}\text { Depth } \\
\text { of } \\
\text { well } \\
\text { (feet) }\end{array}$ & $\begin{array}{l}\text { Depth } \\
\text { to } \\
\text { refusal } \\
\text { (feet) }\end{array}$ & $\begin{array}{l}\text { Depth } \\
\text { to } \\
\text { top } \\
\text { (feet) }\end{array}$ & $\begin{array}{l}\text { Depth } \\
\text { to } \\
\text { bottom } \\
\text { (feet) }\end{array}$ & $\begin{array}{l}\text { Aquifer } \\
\text { code }\end{array}$ & Lithology \\
\hline & & \multicolumn{7}{|c|}{ FREEDOM- - Continued } \\
\hline FLW & 77 & -- & 802 & $\cdots$ & $\begin{array}{r}0 \\
14\end{array}$ & $\begin{array}{l}14 \\
-.\end{array}$ & $\begin{array}{l}\text { 112TILL } \\
\text { BEDROCK }\end{array}$ & TILL \\
\hline FLW & 78 & $\cdots$ & 1000 & $\cdots$ & $\begin{array}{r}\cdots \\
150\end{array}$ & $\begin{array}{r}-- \\
150 \\
--\end{array}$ & $\begin{array}{l}\text { 11OSDMN } \\
112 \text { TILL } \\
\text { BEDROCK }\end{array}$ & $\begin{array}{l}\text { SAND } \\
\text { TILL CLAY, HARDPAN }\end{array}$ \\
\hline FLW & 79 & - & 550 & $\cdots$ & $\begin{array}{r}0 \\
30 \\
78\end{array}$ & $\begin{array}{l}30 \\
78 \\
\cdots\end{array}$ & $\begin{array}{l}110 \text { SDMN } \\
110 \text { SDMN } \\
\text { BEDROCK }\end{array}$ & $\begin{array}{l}\text { SAND } \\
\text { CLAY }\end{array}$ \\
\hline FLW & 80 & - & 200 & $\cdots$ & $\begin{array}{r}0 \\
17\end{array}$ & $\begin{array}{l}17 \\
\cdots\end{array}$ & $\begin{array}{l}110 \text { SDMN } \\
\text { BEDROCK }\end{array}$ & CLAY \\
\hline FLW & 81 & $-\cdot$ & 377 & $-\cdot$ & $\begin{array}{r}0 \\
70 \\
106\end{array}$ & $\begin{array}{r}70 \\
106 \\
\cdots\end{array}$ & $\begin{array}{l}110 \text { SDMN } \\
112 \text { TILL } \\
\text { BEDROCK }\end{array}$ & $\begin{array}{l}\text { SAND } \\
\text { TILL SAND, CLAY, HARDPAN }\end{array}$ \\
\hline FLW & 82 & $-\cdot$ & 202 & $\cdots$ & $\begin{array}{r}0 \\
20 \\
60\end{array}$ & $\begin{array}{l}20 \\
60 \\
--\end{array}$ & $\begin{array}{l}110 \text { SDMN } \\
112 \text { TILL } \\
\text { BEDROCK }\end{array}$ & $\begin{array}{l}\text { SAND } \\
\text { TILL }\end{array}$ \\
\hline FLW & 83 & $-\cdot$ & 655 & $\cdots$ & $\begin{array}{r}0 \\
145\end{array}$ & $\begin{array}{r}145 \\
-.\end{array}$ & $\begin{array}{l}\text { 112TILL } \\
\text { BEDROCK }\end{array}$ & TILL \\
\hline FLW & 84 & $\cdots$ & 200 & $\cdots$ & $\begin{array}{r}0 \\
33\end{array}$ & $\begin{array}{l}33 \\
\cdots\end{array}$ & $\begin{array}{l}110 \text { SDMN } \\
\text { BEDROCK }\end{array}$ & SGVC \\
\hline FLW & 85 & $\cdots$ & 400 & - & $\begin{array}{r}0 \\
87\end{array}$ & $\begin{array}{l}87 \\
--\end{array}$ & $\begin{array}{l}110 \text { SDMN } \\
\text { BEDROCK }\end{array}$ & SDGL \\
\hline FLW & 86 & -- & 452 & $\cdots$ & $\begin{array}{r}0 \\
92\end{array}$ & $\begin{array}{l}92 \\
\ldots\end{array}$ & $\begin{array}{l}110 \text { SDMN } \\
\text { BEDROCK }\end{array}$ & SDCL \\
\hline FLW & 87 & $-\cdot$ & 46 & $\cdots$ & $\begin{array}{r}0 \\
30 \\
39\end{array}$ & $\begin{array}{l}30 \\
39 \\
-\cdot\end{array}$ & $\begin{array}{l}110 \text { SDMN } \\
112 \text { TILL } \\
110 \text { SDMN }\end{array}$ & $\begin{array}{l}\text { SAND } \\
\text { TILL } \\
\text { SDGL }\end{array}$ \\
\hline FLW & 88 & $\cdots$ & 463 & - & $\begin{array}{r}0 \\
25\end{array}$ & $\begin{array}{l}25 \\
-\cdot\end{array}$ & $\begin{array}{l}110 \text { SDMN } \\
\text { BEDROCK }\end{array}$ & SAND \\
\hline FLW & 89 & $\cdots$ & 502 & $\cdots$ & $\begin{array}{r}0 \\
38\end{array}$ & 38 & $\begin{array}{l}112 \text { TILL } \\
\text { BEDROCK }\end{array}$ & TILL SAND, HARDPAN \\
\hline FLW & 90 & $\cdots$ & 227 & -. & $\begin{array}{r}0 \\
32\end{array}$ & $\begin{array}{l}32 \\
-\end{array}$ & $\begin{array}{l}110 \text { SDMN } \\
\text { BEDROCK }\end{array}$ & SDCL \\
\hline FLW & 91 & $\cdots$ & 30 & $-\cdot$ & $\begin{array}{r}0 \\
30 \\
43\end{array}$ & $\begin{array}{l}30 \\
43 \\
-\cdot\end{array}$ & $\begin{array}{l}110 \text { SDMN } \\
110 \text { SDMN } \\
110 \text { SDMN }\end{array}$ & $\begin{array}{l}\text { SAND } \\
\text { SDCL } \\
\text { CLAY }\end{array}$ \\
\hline FLW & 92 & $\cdots$ & 39 & $\cdots$ & -. & $\cdots$ & $110 \mathrm{SDMN}$ & SAND \\
\hline FLW & 93 & - & 302 & $\cdots$ & $\begin{array}{r}0 \\
10\end{array}$ & $\begin{array}{l}10 \\
\cdots\end{array}$ & $\begin{array}{l}\text { 112TILL } \\
\text { BEDROCK }\end{array}$ & TILL \\
\hline FLW & 94 & $\cdots$ & 552 & $\cdots$ & $\begin{array}{r}0 \\
152\end{array}$ & $\begin{array}{r}152 \\
--\end{array}$ & $\begin{array}{l}110 \text { SDMN } \\
\text { BEDROCK }\end{array}$ & SDCL \\
\hline FLW & 95 & - & 1000 & $\cdots$ & $\begin{array}{r}0 \\
20\end{array}$ & $\begin{array}{l}20 \\
\cdots\end{array}$ & $\begin{array}{l}\text { 112TILL } \\
\text { BEDROCK }\end{array}$ & TILL SAND, GRAVEL, HARDPAN \\
\hline FLW & 96 & $\cdots$ & 45 & $-\cdot$ & $-\cdot$ & $-\cdot$ & 110 SDMN & SDGL \\
\hline FLW & 97 & $-\cdot$ & 34 & $-\cdot$ & $-\cdot$ & $\cdots$ & 110 SDMN & SAND \\
\hline FLW & 98 & - & 352 & .. & $\begin{array}{r}0 \\
50 \\
157\end{array}$ & $\begin{array}{r}50 \\
157 \\
-\end{array}$ & $\begin{array}{l}\text { 110SDMN } \\
112 \text { TILL } \\
\text { BEDROCK }\end{array}$ & $\begin{array}{l}\text { SAND } \\
\text { TILL CLAY, HARDPAN }\end{array}$ \\
\hline FLW & 99 & - & 202 & - & $\begin{array}{r}0 \\
12\end{array}$ & $\begin{array}{l}12 \\
-.\end{array}$ & $\begin{array}{l}112 \text { TILL } \\
\text { BEDROCK }\end{array}$ & TILL GRAVEL, CLAY, HARDPAN \\
\hline
\end{tabular}


Table 2-1. Stratigraphic logs of wells and borings in the Saco and Ossipee River Basins, eastcentral New Hampshire--Continued

\begin{tabular}{|c|c|c|c|c|c|c|c|c|}
\hline \multicolumn{2}{|c|}{$\begin{array}{l}\text { Local } \\
\text { site } \\
\text { No. }\end{array}$} & $\begin{array}{l}\text { Depth } \\
\text { drilled } \\
\text { (feet) }\end{array}$ & $\begin{array}{l}\text { Depth } \\
\text { of } \\
\text { well } \\
\text { (feet) }\end{array}$ & $\begin{array}{l}\text { Depth } \\
\text { to } \\
\text { refusal } \\
\text { (feet) }\end{array}$ & $\begin{array}{l}\text { Depth } \\
\text { to } \\
\text { top } \\
\text { (feet) }\end{array}$ & $\begin{array}{l}\text { Depth } \\
\text { to } \\
\text { bottom } \\
\text { (feet) }\end{array}$ & $\begin{array}{l}\text { Aquifer } \\
\text { code }\end{array}$ & Lithology \\
\hline & & \multicolumn{7}{|c|}{ FREEDOM - Continued } \\
\hline FLW & 100 & $\cdots$ & 24 & $\cdots$ & $\begin{array}{r}0 \\
25\end{array}$ & $\begin{array}{l}25 \\
\cdots\end{array}$ & $\begin{array}{l}110 \text { SDMN } \\
110 \text { SDMN }\end{array}$ & $\begin{array}{l}\text { SDGL } \\
\text { SDCL }\end{array}$ \\
\hline FLW & 101 & $-\cdot$ & 20 & $-\cdot$ & $\cdots$ & $-\cdot$ & $110 \mathrm{SDMN}$ & CLAY \\
\hline FLW & 102 & $-\cdot$ & 442 & $-\cdot$ & $\begin{array}{r}0 \\
200\end{array}$ & 200 & $\begin{array}{l}110 \text { SDMN } \\
\text { BEDROCK }\end{array}$ & SAND \\
\hline FLW & 103 & $\cdots$ & 417 & $\cdots$ & $\begin{array}{r}0 \\
22\end{array}$ & $\begin{array}{l}22 \\
\cdots\end{array}$ & $\begin{array}{l}\text { 110SDMN } \\
\text { BEDROCK }\end{array}$ & SAND \\
\hline FLW & 104 & $-\cdot$ & 501 & $-\cdot$ & $\begin{array}{r}0 \\
35 \\
71\end{array}$ & $\begin{array}{l}35 \\
71 \\
\cdots\end{array}$ & $\begin{array}{l}\text { 110SDMN } \\
\text { 112TILL } \\
\text { BEDROCK }\end{array}$ & $\begin{array}{l}\text { SDGL } \\
\text { TILL CLAY, HARDPAN }\end{array}$ \\
\hline FLW & 105 & - & 395 & - & $\begin{array}{r}0 \\
20\end{array}$ & $\begin{array}{l}20 \\
\cdots\end{array}$ & $\begin{array}{l}\text { 112TILL } \\
\text { BEDROCK }\end{array}$ & TILL \\
\hline FLW & 106 & - & 477 & $\cdots$ & $\begin{array}{r}0 \\
212\end{array}$ & $\begin{array}{c}212 \\
--\end{array}$ & $\begin{array}{l}\text { 112TILL } \\
\text { BEDROCK }\end{array}$ & TILL CLAY, HARDPAN \\
\hline FLW & 107 & - & 39 & $\cdots$ & $\begin{array}{r}0 \\
45\end{array}$ & $\begin{array}{l}45 \\
--\end{array}$ & $\begin{array}{l}110 \text { SDMN } \\
110 \text { SDMN }\end{array}$ & $\begin{array}{l}\text { SAND } \\
\text { CLAY }\end{array}$ \\
\hline FLW & 108 & - & 26 & $\cdots$ & $\cdots$ & $-\cdot$ & 110 SDMN & sGvc \\
\hline FLW & 109 & $-\cdot$ & 34 & $\cdots$ & $\begin{array}{r}0 \\
10 \\
28 \\
40\end{array}$ & $\begin{array}{l}10 \\
28 \\
40 \\
-\cdots\end{array}$ & $\begin{array}{l}110 \text { SDMN } \\
112 T I L L \\
110 \text { SDMN } \\
112 \text { TILL }\end{array}$ & $\begin{array}{l}\text { SAND } \\
\text { TILL SAND, HARDPAN } \\
\text { SDCL } \\
\text { TILL }\end{array}$ \\
\hline FLW & 110 & $\cdots$ & 282 & $\cdots$ & $\begin{array}{l}0 \\
5\end{array}$ & 5 & $\begin{array}{l}110 \text { SDMN } \\
\text { BEDROCK }\end{array}$ & OTHR \\
\hline FLW & 111 & -. & 102 & - & $\begin{array}{r}0 \\
18\end{array}$ & $\begin{array}{l}18 \\
\cdots\end{array}$ & $\begin{array}{l}110 \text { SDMN } \\
\text { BEDROCK }\end{array}$ & SAND \\
\hline FLW & 112 & -- & 182 & - & $\begin{array}{r}-1 \\
0 \\
92\end{array}$ & $\begin{array}{l}-- \\
92 \\
--\end{array}$ & $\begin{array}{l}\text { 112TILL } \\
112 T I L L \\
\text { BEDROCK }\end{array}$ & $\begin{array}{l}\text { TILL } \\
\text { TILL CLAY, HARDPAN }\end{array}$ \\
\hline FLW & 113 & - & 1000 & $\cdots$ & $\begin{array}{r}0 \\
27\end{array}$ & $\begin{array}{l}27 \\
--\end{array}$ & $\begin{array}{l}\text { 112TILL } \\
\text { BEDROCK }\end{array}$ & TILL GRAVEL, HARDPAN \\
\hline FLW & 114 & $\cdots$ & 302 & $-\cdot$ & $\begin{array}{r}0 \\
20 \\
50\end{array}$ & $\begin{array}{l}20 \\
50 \\
\cdots\end{array}$ & $\begin{array}{l}110 \text { SDMN } \\
112 \text { TILL } \\
\text { BEDROCK }\end{array}$ & $\begin{array}{l}\text { SAND } \\
\text { TILL }\end{array}$ \\
\hline FLW & 115 & $\cdots$ & 422 & - & $\begin{array}{r}0 \\
10 \\
150\end{array}$ & $\begin{array}{r}10 \\
150 \\
\cdots\end{array}$ & $\begin{array}{l}110 \text { SDMN } \\
112 \text { TILL } \\
\text { BEDROCK }\end{array}$ & $\begin{array}{l}\text { SAND } \\
\text { TILI }\end{array}$ \\
\hline FLW & 116 & $\cdots$ & 422 & -. & $\begin{array}{r}0 \\
10 \\
150\end{array}$ & $\begin{array}{r}10 \\
150 \\
--\end{array}$ & $\begin{array}{l}\text { 110SDMN } \\
\text { 112TILL } \\
\text { BEDROCK }\end{array}$ & $\begin{array}{l}\text { SAND } \\
\text { TILL }\end{array}$ \\
\hline FLW & 117 & $\cdots$ & 37 & $\cdots$ & $\cdots$ & $\cdots$ & 110 SDMN & SAND \\
\hline FLW & 118 & $-\cdot$ & 75 & $\cdots$ & $\cdots$ & $\cdots$ & $110 \mathrm{SDMN}$ & SDGL \\
\hline FLW & 119 & $\cdots$ & 424 & - & $\begin{array}{r}0 \\
50\end{array}$ & 50 & $\begin{array}{l}\text { 110SDMN } \\
\text { BEDROCK }\end{array}$ & SDGL \\
\hline FLW & 120 & $\cdots$ & 57 & $\cdots$ & $-\cdot$ & $-\cdot$ & $110 \mathrm{SDMN}$ & SDGL \\
\hline FLW & 121 & $\cdots$ & 441 & $\cdots$ & $\begin{array}{r}0 \\
135\end{array}$ & $\begin{array}{r}135 \\
--\end{array}$ & $\begin{array}{l}112 \text { TILL } \\
\text { BEDROCK }\end{array}$ & TILI \\
\hline FLW & 122 & $\cdots$ & 544 & $\cdots$ & $\begin{array}{l}0 \\
5\end{array}$ & $\begin{array}{l}5 \\
\cdots\end{array}$ & $\begin{array}{l}\text { 112TILL } \\
\text { BEDROCK }\end{array}$ & TILL \\
\hline
\end{tabular}


Table 2-1. Stratigraphic logs of wells and borings in the Saco and Ossipee River Basins, eastcentral New Hampshire--Continued

\begin{tabular}{|c|c|c|c|c|c|c|c|c|}
\hline $\begin{array}{l}\text { Loca } \\
\text { site } \\
\text { no. }\end{array}$ & & $\begin{array}{l}\text { Depth } \\
\text { drilled } \\
\text { (feet) }\end{array}$ & $\begin{array}{l}\text { Depth } \\
\text { of } \\
\text { well } \\
\text { (feet) }\end{array}$ & $\begin{array}{l}\text { Depth } \\
\text { to } \\
\text { refusal } \\
\text { (feet) }\end{array}$ & $\begin{array}{l}\text { Depth } \\
\text { to } \\
\text { top } \\
\text { (feet) }\end{array}$ & $\begin{array}{l}\text { Depth } \\
\text { to } \\
\text { bottom } \\
\text { (feet) }\end{array}$ & $\begin{array}{l}\text { Aquifer } \\
\text { code }\end{array}$ & Lithology \\
\hline & & \multicolumn{7}{|c|}{ FREEDOM - - Continued } \\
\hline FLW & 123 & $\cdots$ & 403 & $-\cdot$ & $\begin{array}{l}0 \\
5\end{array}$ & $\begin{array}{r}5 \\
--\end{array}$ & $\begin{array}{l}\text { 112TILL } \\
\text { BEDROCK }\end{array}$ & TILL \\
\hline FLW & 124 & - & 275 & $\cdots$ & $\begin{array}{r}0 \\
20\end{array}$ & 20 & $\begin{array}{l}\text { 112TILL } \\
\text { BEDROCK }\end{array}$ & TILL \\
\hline FLW & 125 & $\cdots$ & 502 & -. & $\begin{array}{l}0 \\
4\end{array}$ & $\begin{array}{c}4 \\
-.\end{array}$ & $\begin{array}{l}110 \text { SDMN } \\
\text { BEDROCK }\end{array}$ & SAND \\
\hline FLW & 126 & $\cdots$ & 282 & $\cdots$ & $\begin{array}{r}0 \\
15\end{array}$ & $\begin{array}{l}15 \\
--\end{array}$ & $\begin{array}{l}110 \text { SDMN } \\
\text { BEDROCK }\end{array}$ & SAND \\
\hline FLW & 127 & $\cdots$ & 377 & $\cdots$ & $\begin{array}{r}0 \\
21\end{array}$ & $\begin{array}{l}21 \\
-\cdots\end{array}$ & $\begin{array}{l}110 \text { SDMN } \\
\text { BEDROCK }\end{array}$ & SDCL \\
\hline FLW & 128 & $\cdots$ & 377 & $\cdots$ & $\begin{array}{r}0 \\
21\end{array}$ & $\begin{array}{l}21 \\
\cdots\end{array}$ & $\begin{array}{l}110 \text { SDMN } \\
\text { BEDROCK }\end{array}$ & SDCL \\
\hline FLW & 129 & $-\cdot$ & 302 & $\cdots$ & $\begin{array}{r}0 \\
48\end{array}$ & $\begin{array}{l}48 \\
-\cdots\end{array}$ & $\begin{array}{l}110 \text { SDMN } \\
\text { BEDROCK }\end{array}$ & SAND \\
\hline FLW & 130 & $\cdots$ & 360 & - & $\begin{array}{r}0 \\
58\end{array}$ & $\begin{array}{l}58 \\
--\end{array}$ & $\begin{array}{l}112 \text { TILL } \\
\text { BEDROCK }\end{array}$ & TILL SAND, CLAY, HARDPAN \\
\hline FLW & 131 & - & 35 & - & $\cdots$ & $\cdots$ & $\cdots$ & \\
\hline FLW & 132 & $-\cdot$ & 53 & - & $\cdots$ & $\cdots$ & 110 SDMN & SAND \\
\hline FLW & 133 & $\cdots$ & 61 & -. & $\begin{array}{r}0 \\
25\end{array}$ & $\begin{array}{l}25 \\
-\end{array}$ & $\begin{array}{l}110 \text { SDMN } \\
110 \text { SDMN }\end{array}$ & $\begin{array}{l}\text { SDGL } \\
\text { SAND }\end{array}$ \\
\hline FLW & 134 & -. & 73 & - & $\cdots$ & -. & 110 SDMN & SDGL \\
\hline FLW & 135 & - & 243 & $\cdots$ & $\begin{array}{r}0 \\
20\end{array}$ & $\begin{array}{l}20 \\
\cdots\end{array}$ & $\begin{array}{l}110 \text { SDMN } \\
\text { BEDROCK }\end{array}$ & SDGL \\
\hline FLW & 136 & - & 31 & $\cdots$ & $\cdots$ & $\cdots$ & $110 \mathrm{SDMN}$ & SDGL \\
\hline FLW & 137 & - & 39 & $\cdots$ & $\begin{array}{r}0 \\
5 \\
42\end{array}$ & $\begin{array}{r}5 \\
42 \\
--\end{array}$ & $\begin{array}{l}110 \text { SDMN } \\
110 \text { SDMN } \\
112 \text { TILL }\end{array}$ & $\begin{array}{l}\text { SDGL } \\
\text { SAND } \\
\text { TILL CLAY, HARDPAN }\end{array}$ \\
\hline FLW & 138 & -. & 1000 & - & $\begin{array}{r}0 \\
20\end{array}$ & $\begin{array}{l}20 \\
\cdots\end{array}$ & $\begin{array}{l}110 \text { SDMN } \\
\text { BEDROCK }\end{array}$ & CLAY \\
\hline FLW & 139 & $\cdots$ & 84 & $-\cdot$ & $\begin{array}{r}0 \\
25 \\
44 \\
46 \\
50 \\
60\end{array}$ & $\begin{array}{l}25 \\
44 \\
46 \\
50 \\
60 \\
--\end{array}$ & $\begin{array}{l}110 \mathrm{SDMN} \\
110 \mathrm{SDMN} \\
110 \mathrm{SDMN} \\
110 \mathrm{SDMN} \\
110 \mathrm{SDMN} \\
110 \mathrm{SDMN}\end{array}$ & $\begin{array}{l}\text { SDCL } \\
\text { SDGL } \\
\text { CLAY } \\
\text { SAND } \\
\text { SGVC } \\
\text { SDGL }\end{array}$ \\
\hline FLW & 140 & - & 427 & - & $\begin{array}{r}0 \\
200\end{array}$ & 200 & $\begin{array}{l}110 \text { SDMN } \\
\text { BEDROCK }\end{array}$ & CLAY \\
\hline FLW & 141 & -. & 327 & - & $\begin{array}{r}0 \\
50 \\
258\end{array}$ & $\begin{array}{r}50 \\
258 \\
-\cdot\end{array}$ & $\begin{array}{l}110 \text { SDMN } \\
110 \text { SDMN } \\
\text { BEDROCK }\end{array}$ & $\begin{array}{l}\text { SDGL } \\
\text { CLAY }\end{array}$ \\
\hline FLW & 142 & - & 202 & - & $\begin{array}{r}0 \\
20 \\
45\end{array}$ & $\begin{array}{l}20 \\
45 \\
--\end{array}$ & $\begin{array}{l}110 \text { SDMN } \\
110 \text { SDMN } \\
\text { BEDROCK }\end{array}$ & $\begin{array}{l}\text { SAND } \\
\text { CLAY }\end{array}$ \\
\hline FLW & 143 & . & 202 & - & $\begin{array}{r}0 \\
72\end{array}$ & $\begin{array}{l}72 \\
\ldots\end{array}$ & $\begin{array}{l}110 \text { SDMN } \\
\text { BEDROCK }\end{array}$ & SGVC \\
\hline FLW & 144 & $\cdots$ & 362 & $\cdots$ & $\begin{array}{r}0 \\
10\end{array}$ & $\begin{array}{l}10 \\
\cdots\end{array}$ & $\begin{array}{l}110 \text { SDMN } \\
\text { BEDROCK }\end{array}$ & SDCL \\
\hline FLW & 145 & $\cdots$ & 142 & $\cdots$ & $\begin{array}{r}0 \\
20\end{array}$ & $\begin{array}{l}20 \\
\cdots\end{array}$ & $\begin{array}{l}110 \text { SDMN } \\
\text { BEDROCK }\end{array}$ & SAND \\
\hline
\end{tabular}


Table 2-1. Stratigraphic logs of wells and borings in the Saco and Ossipee River Basins, eastcentrai New Hampshire--Continued

\begin{tabular}{|c|c|c|c|c|c|c|c|c|}
\hline \multicolumn{2}{|c|}{$\begin{array}{l}\text { Local } \\
\text { site } \\
\text { No. }\end{array}$} & $\begin{array}{l}\text { Depth } \\
\text { drilled } \\
\text { (feet) }\end{array}$ & $\begin{array}{l}\text { Depth } \\
\text { of } \\
\text { well } \\
\text { (feet) }\end{array}$ & $\begin{array}{l}\text { Depth } \\
\text { to } \\
\text { refusal } \\
\text { (feet) }\end{array}$ & $\begin{array}{l}\text { Depth } \\
\text { to } \\
\text { top } \\
\text { (feet) }\end{array}$ & $\begin{array}{l}\text { Depth } \\
\text { to } \\
\text { bottom } \\
\text { (feet) }\end{array}$ & $\begin{array}{l}\text { Aquifer } \\
\text { code }\end{array}$ & Lithology \\
\hline & & \multicolumn{7}{|c|}{ FREBDOM - - Continued } \\
\hline FLW & 146 & $\cdots$ & 352 & $\cdots$ & $\begin{array}{r}0 \\
55\end{array}$ & $\begin{array}{l}55 \\
--\end{array}$ & $\begin{array}{l}\text { 11OSDMN } \\
\text { BEDROCK }\end{array}$ & SDCL \\
\hline FLW & 147 & $\cdots$ & 43 & $\cdots$ & $\begin{array}{r}0 \\
10 \\
25 \\
43\end{array}$ & $\begin{array}{l}10 \\
25 \\
43 \\
-.\end{array}$ & $\begin{array}{l}110 S D M N \\
110 \text { SDMN } \\
110 \text { SDMN } \\
112 T I L L\end{array}$ & $\begin{array}{l}\text { SAND } \\
\text { SDCL } \\
\text { SDGL } \\
\text { TILL }\end{array}$ \\
\hline FLW & 148 & $\cdots$ & 273 & $\cdots$ & $\begin{array}{r}0 \\
23\end{array}$ & $\begin{array}{l}23 \\
\cdots\end{array}$ & $\begin{array}{l}110 \text { SDMN } \\
\text { BEDROCK }\end{array}$ & SAND \\
\hline FLW & 149 & $\cdots$ & 273 & $\cdots$ & $\begin{array}{r}0 \\
86\end{array}$ & $\begin{array}{l}86 \\
\cdots\end{array}$ & $\begin{array}{l}110 \text { SDMN } \\
\text { BEDROCK }\end{array}$ & SAND \\
\hline FLW & 150 & $\cdots$ & 322 & $\cdots$ & $\begin{array}{r}0 \\
22\end{array}$ & $\begin{array}{l}22 \\
\cdots\end{array}$ & $\begin{array}{l}\text { 112TILL } \\
\text { BEDROCK }\end{array}$ & TILL \\
\hline FLW & 151 & $\cdots$ & 475 & $-\cdot$ & $\begin{array}{r}0 \\
50\end{array}$ & $\begin{array}{l}50 \\
\ldots\end{array}$ & $\begin{array}{l}110 \text { SDMN } \\
\text { BEDROCK }\end{array}$ & SDCL \\
\hline FLW & 152 & $\cdots$ & 782 & $\cdots$ & $\begin{array}{r}0 \\
15\end{array}$ & $\begin{array}{l}15 \\
\cdots\end{array}$ & $\begin{array}{l}\text { 112TILL } \\
\text { BEDROCK }\end{array}$ & TILL \\
\hline FLW & 153 & $\cdots$ & 482 & $\cdots$ & $\begin{array}{l}0 \\
8\end{array}$ & $\begin{array}{r}8 \\
--\end{array}$ & $\begin{array}{l}\text { 112TILL } \\
\text { BEDROCK }\end{array}$ & TILL \\
\hline FLW & 154 & $\cdots$ & 960 & $\cdots$ & $\begin{array}{r}0 \\
240\end{array}$ & $\begin{array}{r}240 \\
-.\end{array}$ & $\begin{array}{l}\text { 112TILL } \\
\text { BEDROCK }\end{array}$ & TILL CLAY, HARDPAN \\
\hline$F L W$ & 155 & $\cdots$ & 140 & $\cdots$ & $\begin{array}{r}0 \\
50\end{array}$ & $\begin{array}{l}50 \\
\cdots\end{array}$ & $\begin{array}{l}110 \text { SDMN } \\
\text { BEDROCK }\end{array}$ & SDGL \\
\hline FLW & 156 & $\cdots$ & 442 & $\cdots$ & $\begin{array}{r}0 \\
13\end{array}$ & $\begin{array}{l}13 \\
\cdots\end{array}$ & $\begin{array}{l}110 \text { SDMN } \\
\text { BEDROCK }\end{array}$ & SDCL \\
\hline FLW & 157 & $\cdots$ & 420 & $\cdots$ & $\begin{array}{r}0 \\
50\end{array}$ & $\begin{array}{l}50 \\
--\end{array}$ & $\begin{array}{l}\text { 112TILL } \\
\text { BEDROCK }\end{array}$ & TILL CLAY, HARDPAN \\
\hline FLW & 158 & $\cdots$ & 442 & $\cdots$ & $\begin{array}{r}0 \\
25\end{array}$ & $\begin{array}{l}25 \\
\cdots\end{array}$ & $\begin{array}{l}110 \text { SDMN } \\
\text { BEDROCK }\end{array}$ & SAND \\
\hline FLW & 159 & $\cdots$ & 242 & $\cdots$ & $\begin{array}{r}0 \\
80\end{array}$ & $\begin{array}{l}80 \\
\cdots\end{array}$ & $\begin{array}{l}\text { 112TILL } \\
\text { BEDROCK }\end{array}$ & TILL \\
\hline FLW & 160 & $\cdots$ & 475 & - & $\begin{array}{r}0 \\
100\end{array}$ & $\begin{array}{c}100 \\
\ldots\end{array}$ & $\begin{array}{l}110 \text { SDMN } \\
\text { BEDROCK }\end{array}$ & SDGL \\
\hline FLW & 161 & $\cdots$ & 423 & $\cdots$ & $\begin{array}{r}0 \\
60 \\
120 \\
215\end{array}$ & $\begin{array}{r}60 \\
120 \\
215 \\
-\cdots\end{array}$ & $\begin{array}{l}112 \mathrm{TILL} \\
112 \mathrm{TILL} \\
110 \mathrm{SDMN} \\
\text { BEDROCK }\end{array}$ & $\begin{array}{l}\text { TILL SAND, HARDPAN } \\
\text { TILL } \\
\text { CLAY }\end{array}$ \\
\hline FLW & 162 & $\cdots$ & 360 & $\cdots$ & $\begin{array}{r}0 \\
160\end{array}$ & 160 & $\begin{array}{l}\text { 112TILL } \\
\text { BEDROCK }\end{array}$ & TILL \\
\hline FLW & 163 & $\cdots$ & 48 & $\cdots$ & $-\cdot$ & $\cdots$ & 110 SDMN & SDGL \\
\hline FLW & 164 & $\cdots$ & 1000 & $\cdots$ & $\begin{array}{r}0 \\
47\end{array}$ & $\begin{array}{l}47 \\
--\end{array}$ & $\begin{array}{l}110 \text { SDMN } \\
\text { BEDROCK }\end{array}$ & CLAY \\
\hline FLW & 165 & - & 802 & $\cdots$ & $\begin{array}{r}0 \\
35\end{array}$ & 35 & $\begin{array}{l}110 \text { SDMN } \\
\text { BEDROCK }\end{array}$ & SAND \\
\hline FLW & 166 & $\cdots$ & 502 & $\cdots$ & $\begin{array}{r}0 \\
120\end{array}$ & 120 & $\begin{array}{l}\text { 112TILL } \\
\text { BEDROCK }\end{array}$ & TILL CLAY, HARDPAN \\
\hline FLW & 167 & $\cdots$ & 400 & $\cdots$ & $\begin{array}{r}0 \\
135\end{array}$ & 135 & $\begin{array}{l}\text { 112TILL } \\
\text { BEDROCK }\end{array}$ & TILL CLAY, HARDPAN \\
\hline
\end{tabular}


Table 2-1. Stratlgraphic logs of wells and borings in the Saco and Ossipee River Basins, eastcentral New Hampshire--Continued

\begin{tabular}{|c|c|c|c|c|c|c|c|c|}
\hline $\begin{array}{l}\text { Loca } \\
\text { site } \\
\text { no. }\end{array}$ & & $\begin{array}{l}\text { Depth } \\
\text { drilled } \\
\text { (feet) }\end{array}$ & $\begin{array}{l}\text { Depth } \\
\text { of } \\
\text { well } \\
\text { (feet) }\end{array}$ & $\begin{array}{l}\text { Depth } \\
\text { to } \\
\text { refusal } \\
\text { (feet) }\end{array}$ & $\begin{array}{l}\text { Depth } \\
\text { to } \\
\text { top } \\
\text { (feet) }\end{array}$ & $\begin{array}{l}\text { Depth } \\
\text { to } \\
\text { bottom } \\
\text { (feet) }\end{array}$ & $\begin{array}{c}\text { Aquifer } \\
\text { code }\end{array}$ & Lithology \\
\hline & & \multicolumn{7}{|c|}{ FREBDOK- - Continued } \\
\hline FLW & 168 & - & 303 & -- & $\begin{array}{r}0 \\
25 \\
100 \\
180\end{array}$ & $\begin{array}{r}25 \\
100 \\
180 \\
--\end{array}$ & $\begin{array}{l}110 \text { SDMN } \\
112 \text { TILL } \\
110 \text { SDMN } \\
\text { BEDROCK }\end{array}$ & $\begin{array}{l}\text { SDGL } \\
\text { TILL } \\
\text { OTHR }\end{array}$ \\
\hline FLW & 169 & $\cdots$ & 242 & $\cdots$ & $\begin{array}{r}0 \\
20 \\
55\end{array}$ & $\begin{array}{l}20 \\
55 \\
-.\end{array}$ & $\begin{array}{l}110 \text { SDMN } \\
110 \text { SDMN } \\
\text { BEDROCK }\end{array}$ & $\begin{array}{l}\text { SAND } \\
\text { OTHR }\end{array}$ \\
\hline FLW & 170 & - & 242 & $\cdots$ & $\begin{array}{r}0 \\
155\end{array}$ & $\begin{array}{c}155 \\
\cdots\end{array}$ & $\begin{array}{l}110 \text { SDMN } \\
\text { BEDROCK }\end{array}$ & SDCL \\
\hline FLW & 171 & $\cdots$ & 505 & $\cdots$ & $\begin{array}{l}0 \\
3\end{array}$ & $\begin{array}{l}3 \\
-\cdots\end{array}$ & $\begin{array}{l}\text { 112TILL } \\
\text { BEDROCK }\end{array}$ & TILL \\
\hline FLW & 172 & $\cdots$ & 305 & $\cdots$ & $\begin{array}{r}0 \\
67\end{array}$ & $\begin{array}{l}67 \\
\cdots\end{array}$ & $\begin{array}{l}\text { 112TILL } \\
\text { BEDROCK }\end{array}$ & TILL SAND, GRAVEL， HARDPAN \\
\hline FLW & 173 & - & 302 & $\cdots$ & $\begin{array}{r}0 \\
38\end{array}$ & $\begin{array}{l}38 \\
\cdots\end{array}$ & $\begin{array}{l}\text { 112TILL } \\
\text { BEDROCK }\end{array}$ & TILL CLAY, HARDPAN \\
\hline FLW & 174 & $-\cdot$ & 302 & $-\cdot$ & $\begin{array}{r}0 \\
49\end{array}$ & $\begin{array}{l}49 \\
-\end{array}$ & $\begin{array}{l}110 \text { SDMN } \\
\text { BEDROCK }\end{array}$ & CLAY \\
\hline FLW & 175 & $\cdots$ & 220 & $\cdots$ & $\begin{array}{r}0 \\
26\end{array}$ & 26 & $\begin{array}{l}\text { 112TILL } \\
\text { BEDROCK }\end{array}$ & TILL \\
\hline FLW & 176 & -- & 220 & $\cdots$ & $\begin{array}{r}0 \\
39\end{array}$ & 39 & $\begin{array}{l}\text { 112TILL } \\
\text { BEDROCK }\end{array}$ & TILL CLAY, HARDPAN \\
\hline FLW & 177 & $\cdots$ & 523 & $-\cdot$ & $\begin{array}{r}0 \\
180\end{array}$ & 180 & $\begin{array}{l}110 \text { SDMN } \\
\text { BEDROCK }\end{array}$ & OTHR \\
\hline FLW & 178 & $-\cdot$ & 35 & - & - & $\cdots$ & 110 SDMN & SAND \\
\hline FLW & 179 & $\cdots$ & 482 & $-\cdot$ & $\begin{array}{l}0 \\
4\end{array}$ & $\begin{array}{l}4 \\
--\end{array}$ & $\begin{array}{l}\text { 110SDMN } \\
\text { BEDROCK }\end{array}$ & SAND \\
\hline FLW & 180 & - & 505 & - & $\begin{array}{l}0 \\
3\end{array}$ & $\begin{array}{c}3 \\
--\end{array}$ & $\begin{array}{l}\text { 110SDMN } \\
\text { BEDROCK }\end{array}$ & SAND \\
\hline FLW & 181 & $-\cdot$ & 630 & $\cdots$ & $\begin{array}{l}0 \\
7\end{array}$ & $\begin{array}{l}7 \\
-\end{array}$ & $\begin{array}{l}\text { 112TILL } \\
\text { BEDROCK }\end{array}$ & TILL \\
\hline FLW & 182 & $\cdots$ & 305 & $\cdots$ & $\begin{array}{r}0 \\
15\end{array}$ & $\begin{array}{l}15 \\
-\end{array}$ & $\begin{array}{l}110 \text { SDMN } \\
\text { BEDROCK }\end{array}$ & CLAY \\
\hline FLW & 183 & -. & 555 & -. & $\begin{array}{r}0 \\
17\end{array}$ & $\begin{array}{l}17 \\
-.\end{array}$ & $\begin{array}{l}110 \text { SDMN } \\
\text { BEDROCK }\end{array}$ & CLAY \\
\hline FLW & 184 & -- & 15 & - & $\cdots$ & - & $110 \mathrm{SDMN}$ & SAND \\
\hline FLW & 185 & - & 39 & - & - & $-\cdot$ & 110 SDMN & SDGL \\
\hline FLW & 186 & -. & 63 & $\cdots$ & $\cdots$ & -. & $110 \mathrm{SDMN}$ & SAND \\
\hline FLW & 187 & -. & 61 & - - & $\begin{array}{r}0 \\
220\end{array}$ & $\begin{array}{r}220 \\
-\end{array}$ & $\begin{array}{l}110 \text { SDMN } \\
\text { BEDROCK }\end{array}$ & SDGL \\
\hline FLW & 188 & - & 41 & $\cdots$ & $\begin{array}{r}0 \\
41\end{array}$ & $\begin{array}{l}41 \\
--\end{array}$ & $\begin{array}{l}110 \text { SDMN } \\
112 \text { TILL }\end{array}$ & $\begin{array}{l}\text { SAND } \\
\text { TILL }\end{array}$ \\
\hline FLW & 189 & - & 242 & $-\cdot$ & $\begin{array}{r}0 \\
38\end{array}$ & $\begin{array}{l}38 \\
\cdots\end{array}$ & $\begin{array}{l}110 \text { SDMN } \\
\text { BEDROCK }\end{array}$ & SAND \\
\hline FLW & 190 & - & 422 & . & $\begin{array}{r}0 \\
30\end{array}$ & $\begin{array}{l}30 \\
-.\end{array}$ & $\begin{array}{l}\text { 110SDMN } \\
\text { BEDROCK }\end{array}$ & SDCL \\
\hline FLW & 191 & - & 37 & -. & $\begin{array}{r}0 \\
40 \\
50\end{array}$ & $\begin{array}{l}40 \\
50 \\
-.\end{array}$ & $\begin{array}{l}110 \text { SDMN } \\
110 \text { SDMN } \\
110 \text { SDMN }\end{array}$ & $\begin{array}{l}\text { SDGL } \\
\text { SAND } \\
\text { CLAY }\end{array}$ \\
\hline
\end{tabular}


Table 2-1. Stratigraphic logs of wells and borings in the Saco and Ossipee River Basins, eastcentral New Hampshire--Continued

\begin{tabular}{|c|c|c|c|c|c|c|c|c|}
\hline $\begin{array}{l}\text { Loca } \\
\text { site } \\
\text { No. }\end{array}$ & & $\begin{array}{l}\text { Depth } \\
\text { drilled } \\
\text { (feet) }\end{array}$ & $\begin{array}{l}\text { Depth } \\
\text { of } \\
\text { well } \\
\text { (feet) }\end{array}$ & $\begin{array}{l}\text { Depth } \\
\text { to } \\
\text { refusal } \\
\text { (feet) }\end{array}$ & $\begin{array}{l}\text { Depth } \\
\text { to } \\
\text { top } \\
\text { (feet) }\end{array}$ & $\begin{array}{l}\text { Depth } \\
\text { to } \\
\text { bottom } \\
\text { (feet) }\end{array}$ & $\begin{array}{l}\text { Aquifer } \\
\text { code }\end{array}$ & Lithology \\
\hline \multicolumn{9}{|c|}{ FREEDOM - Cont inued } \\
\hline FLW & 192 & $\cdots$ & 39 & $\cdots$ & $\cdots$ & $\cdots$ & $110 \mathrm{SDMN}$ & SDGL \\
\hline FLW & 193 & $\cdots$ & 302 & $\cdots$ & $\begin{array}{r}0 \\
25\end{array}$ & $\begin{array}{l}25 \\
\cdots\end{array}$ & $\begin{array}{l}112 \text { TILL } \\
\text { BEDROCK }\end{array}$ & TILL SAND, CLAY, HARDPAN \\
\hline FLW & 194 & $\cdots$ & 200 & $\cdots$ & $\begin{array}{r}0 \\
40\end{array}$ & $\begin{array}{l}40 \\
\cdots\end{array}$ & $\begin{array}{l}110 \text { SDMN } \\
\text { BEDROCK }\end{array}$ & SGVC \\
\hline FLW & 195 & $\cdots$ & 220 & -- & $\begin{array}{r}0 \\
27\end{array}$ & $\begin{array}{l}27 \\
--\end{array}$ & $\begin{array}{l}\text { 112TILL } \\
\text { BEDROCK }\end{array}$ & TILL \\
\hline FLW & 196 & $\cdots$ & 260 & $\cdots$ & $\begin{array}{l}0 \\
9\end{array}$ & $\begin{array}{r}9 \\
\cdots\end{array}$ & $\begin{array}{l}110 \text { SDMN } \\
\text { BEDROCK }\end{array}$ & SDGL \\
\hline FLW & 197 & $\cdots$ & 525 & $\cdots$ & $\begin{array}{r}0 \\
85\end{array}$ & 85 & $\begin{array}{l}\text { 112TILL } \\
\text { BEDROCK }\end{array}$ & TILL \\
\hline FLW & 198 & $\cdots$ & 302 & $\cdots$ & $\begin{array}{r}0 \\
55\end{array}$ & $\begin{array}{l}55 \\
\cdots\end{array}$ & $\begin{array}{l}110 \mathrm{SDMN} \\
\text { BEDROCK }\end{array}$ & CLAY \\
\hline FLW & 199 & $\cdots$ & 1180 & $\cdots$ & $\begin{array}{r}0 \\
80\end{array}$ & $\begin{array}{l}80 \\
--\end{array}$ & $\begin{array}{l}110 \text { SDMN } \\
\text { BEDROCK }\end{array}$ & CLAY \\
\hline FLW & 200 & - & 302 & -- & $\begin{array}{r}0 \\
18\end{array}$ & $\begin{array}{l}18 \\
\cdots\end{array}$ & $\begin{array}{l}110 \text { SDMN } \\
\text { BEDROCK }\end{array}$ & CLAY \\
\hline \multicolumn{9}{|c|}{ HART'S LOCATION } \\
\hline KJW & 1 & $\cdots$ & 565 & $\cdots$ & $\begin{array}{r}0 \\
35 \\
108\end{array}$ & $\begin{array}{r}35 \\
108 \\
--\end{array}$ & $\begin{array}{l}110 \text { SDMN } \\
\text { BEDROCK } \\
\text { BEDROCK }\end{array}$ & SDGL \\
\hline KJW & 2 & $\cdots$ & 81 & $\cdots$ & $\begin{array}{r}0 \\
90\end{array}$ & $\begin{array}{l}90 \\
\cdots\end{array}$ & $\begin{array}{l}110 \text { SDMN } \\
\text { BEDROCK }\end{array}$ & SDGL \\
\hline HJB & 1 & 28 & $\cdots$ & 28 & $\begin{array}{r}0 \\
2 \\
28\end{array}$ & $\begin{array}{r}2 \\
28 \\
-\end{array}$ & $\begin{array}{l}112 \mathrm{SRFD} \\
112 \mathrm{SRFD} \\
112 \mathrm{SRFD}\end{array}$ & $\begin{array}{l}\text { GRVL } \\
\text { BLDR } \\
\text { TILL TILL WITH BOULDERS }\end{array}$ \\
\hline HJB & 2 & 14 & $\cdots$ & 14 & $\cdots$ & $\cdots$ & 112SRFD & $\begin{array}{l}\text { TILL GRAVELLY TILL AND BOULDERS } \\
\text { (STREAMBED) }\end{array}$ \\
\hline нJB & 3 & 13 & -. & 13 & - & $-\cdot$ & 112SRFD & GRVL GRAVEL WITH BOULDERS \\
\hline HJB & 4 & 22 & $\cdots$ & 22 & $\cdots$ & $\cdots$ & 112 SRFD & $\begin{array}{l}\text { SDGL SAND AND GRAVEL WITH } \\
\text { COBBLES }\end{array}$ \\
\hline HJB & 5 & 31 & $\cdots$ & $\cdots$ & $\begin{array}{r}0 \\
5 \\
17 \\
21\end{array}$ & $\begin{array}{r}5 \\
17 \\
21 \\
\cdots\end{array}$ & $\begin{array}{l}112 \text { SRFD } \\
112 \text { SRFD } \\
112 \text { SRFD } \\
112 \text { SRFD }\end{array}$ & $\begin{array}{l}\text { GRVL } \\
\text { BLDR } \\
\text { GRVL } \\
\text { ROCK }\end{array}$ \\
\hline HJB & 6 & 9 & $\cdots$ & 9 & $\cdots$ & $\cdots$ & $112 \mathrm{SRFD}$ & GRVL GRAVEL WITH BOULDERS \\
\hline \multicolumn{9}{|c|}{ JACKSON } \\
\hline JAB & 1 & 15 & $\cdots$ & 15 & $\cdots$ & $\cdots$ & $112 \mathrm{SRFD}$ & TILL TILL WITH BOULDERS \\
\hline JAB & 2 & 25 & $\cdots$ & 25 & .. & $\cdots$ & 112SRFD & $\begin{array}{l}\text { TILL TILL WITH SAND, SILT, AND } \\
\text { BOULDERS }\end{array}$ \\
\hline JAB & 3 & 8 & $\cdots$ & 8 & 0 & 4 & 112SRFD & $\begin{array}{l}\text { SDGL SAND AND GRAVEL WITH } \\
\text { BOULDERS }\end{array}$ \\
\hline JAW & 1 & 24 & 24 & -. & - & . & - & \\
\hline JAW & 2 & 250 & - & - & - & $\cdots$ & $\cdots$ & \\
\hline
\end{tabular}


Table 2-1. Stratigraphic logs of wells and borings in the Saco and Ossipee River Basins, eastcentral New Hampshire--Continued

\begin{tabular}{|c|c|c|c|c|c|c|c|c|}
\hline $\begin{array}{l}\text { Loca } \\
\text { site } \\
\text { no. }\end{array}$ & & $\begin{array}{l}\text { Depth } \\
\text { drilled } \\
\text { (feet) }\end{array}$ & $\begin{array}{l}\text { Depth } \\
\text { of } \\
\text { well } \\
\text { (feet) }\end{array}$ & $\begin{array}{l}\text { Depth } \\
\text { to } \\
\text { refusal } \\
\text { (feet) }\end{array}$ & $\begin{array}{l}\text { Depth } \\
\text { to } \\
\text { top } \\
\text { (feet) }\end{array}$ & $\begin{array}{l}\text { Depth } \\
\text { to } \\
\text { bottom } \\
\text { (feet) }\end{array}$ & $\begin{array}{l}\text { Aquifer } \\
\text { code }\end{array}$ & Lithology \\
\hline & & \multicolumn{7}{|c|}{ JACKSON- - Continued } \\
\hline JAW & 3 & $-\cdot$ & 205 & $-\cdot$ & $\begin{array}{r}0 \\
15\end{array}$ & $\begin{array}{l}15 \\
-\cdot\end{array}$ & $\begin{array}{l}110 \text { SDMN } \\
\text { BEDROCK }\end{array}$ & SDGL \\
\hline JAW & 4 & $\cdots$ & 394 & $\cdots$ & $\begin{array}{r}- \\
0 \\
130\end{array}$ & 130 & $\begin{array}{l}112 \mathrm{TILL} \\
110 \mathrm{SDMN} \\
\text { BEDROCK }\end{array}$ & $\begin{array}{l}\text { TILL } \\
\text { SDGL }\end{array}$ \\
\hline JAW & 5 & $\cdots$ & 125 & $\cdots$ & $\begin{array}{r}0 \\
24\end{array}$ & $\begin{array}{l}24 \\
\cdots\end{array}$ & $\begin{array}{l}110 \text { SDMN } \\
\text { BEDROCK }\end{array}$ & SDGL \\
\hline JAW & 6 & $\cdots$ & 184 & $\cdots$ & $\begin{array}{r}0 \\
50\end{array}$ & $\begin{array}{l}50 \\
--\end{array}$ & $\begin{array}{l}\text { 112TILL } \\
\text { BEDROCK }\end{array}$ & TILL \\
\hline JAW & 7 & $\cdots$ & 678 & $\cdots$ & $\begin{array}{l}0 \\
5\end{array}$ & 5 & $\begin{array}{l}110 \text { SDMN } \\
\text { BEDROCK }\end{array}$ & SDGL \\
\hline JAW & 8 & $\cdots$ & 438 & $\cdots$ & $\begin{array}{r}0 \\
195\end{array}$ & 195 & $\begin{array}{l}112 \text { TILL } \\
\text { BEDROCK }\end{array}$ & TILL \\
\hline JAW & 9 & $-\cdot$ & 405 & $\cdots$ & $\begin{array}{r}0 \\
250\end{array}$ & $\begin{array}{r}250 \\
\cdots\end{array}$ & $\begin{array}{l}112 \mathrm{TILL} \\
\text { BEDROCK }\end{array}$ & TILL \\
\hline JAW & 10 & $\cdots$ & 318 & $\cdots$ & $\begin{array}{r}0 \\
20\end{array}$ & $\begin{array}{l}20 \\
\cdots\end{array}$ & $\begin{array}{l}112 \text { TILL } \\
\text { BEDROCK }\end{array}$ & TILL \\
\hline JAW & 11 & $\cdots$ & 480 & $\cdots$ & $\begin{array}{r}0 \\
284\end{array}$ & $\begin{array}{r}284 \\
\cdots\end{array}$ & $\begin{array}{l}\text { 112TILL } \\
\text { BEDROCK }\end{array}$ & TILL GRAVEL, CLAY, HARDPAN \\
\hline JAW & 12 & $-\cdot$ & 275 & $\cdots$ & $\begin{array}{r}0 \\
92\end{array}$ & $\begin{array}{l}92 \\
-.\end{array}$ & $\begin{array}{l}112 \text { TILL } \\
\text { BEDROCK }\end{array}$ & TILL \\
\hline JAW & 13 & - & 502 & $\cdots$ & $\begin{array}{r}0 \\
20\end{array}$ & $\begin{array}{l}20 \\
-\cdot\end{array}$ & $\begin{array}{l}110 \text { SDMN } \\
\text { BEDROCK }\end{array}$ & OTHR \\
\hline JAW & 14 & $\cdots$ & 528 & $\cdots$ & $\begin{array}{r}0 \\
415\end{array}$ & $\begin{array}{r}415 \\
-.\end{array}$ & $\begin{array}{l}110 \text { SDMN } \\
\text { BEDROCK }\end{array}$ & OTHR \\
\hline JAW & 15 & - & 403 & $\cdots$ & $\begin{array}{r}0 \\
48\end{array}$ & $\begin{array}{l}48 \\
--\end{array}$ & $\begin{array}{l}\text { 112TILL } \\
\text { BEDROCK }\end{array}$ & TILL GRAVEL, HARDPAN \\
\hline JAW & 16 & $\cdots$ & 362 & $\cdots$ & $\begin{array}{r}0 \\
185\end{array}$ & 185 & $\begin{array}{l}112 T I L L \\
\text { BEDROCK }\end{array}$ & TILL \\
\hline JAW & 17 & - - & 377 & $\cdots$ & $\begin{array}{r}0 \\
105\end{array}$ & $\begin{array}{r}105 \\
\ldots\end{array}$ & $\begin{array}{l}112 \mathrm{TILL} \\
\text { BEDROCK }\end{array}$ & TILL \\
\hline JAW & 18 & $\cdots$ & 602 & $\cdots$ & 6 & $-\cdot$ & BEDROCK & \\
\hline JAW & 19 & $\cdots$ & 487 & $\cdots$ & $\begin{array}{r}0 \\
140\end{array}$ & $\begin{array}{r}140 \\
\ldots\end{array}$ & $\begin{array}{l}112 \text { TILL } \\
\text { BEDROCK }\end{array}$ & TILL \\
\hline JAW & 20 & $\cdots$ & 282 & $\cdots$ & $\begin{array}{r}0 \\
18\end{array}$ & $\begin{array}{l}18 \\
\cdots\end{array}$ & $\begin{array}{l}112 \mathrm{TILL} \\
\text { BEDROCK }\end{array}$ & TILL \\
\hline JAW & 21 & $\cdots$ & 442 & $\cdots$ & $\begin{array}{r}0 \\
70\end{array}$ & $\begin{array}{l}70 \\
\cdots\end{array}$ & $\begin{array}{l}112 T I L L \\
\text { BEDROCK }\end{array}$ & TILL \\
\hline JAW & 22 & - & 421 & $\cdots$ & $\begin{array}{r}0 \\
15\end{array}$ & $\begin{array}{l}15 \\
\cdots\end{array}$ & $\begin{array}{l}\text { 112TILL } \\
\text { BEDROCK }\end{array}$ & TILL \\
\hline JAW & 23 & - & 122 & - & $\begin{array}{r}0 \\
35\end{array}$ & $\begin{array}{l}35 \\
\cdots\end{array}$ & $\begin{array}{l}\text { 112TILL } \\
\text { BEDROCK }\end{array}$ & TILL \\
\hline JAW & 24 & - & 421 & $\cdots$ & $\begin{array}{r}0 \\
20\end{array}$ & $\begin{array}{l}20 \\
\cdots\end{array}$ & $\begin{array}{l}\text { 112TILL } \\
\text { BEDROCK }\end{array}$ & TILL \\
\hline JAW & 25 & - & 1280 & $\cdots$ & $\begin{array}{r}0 \\
220\end{array}$ & $\begin{array}{r}220 \\
-.\end{array}$ & $\begin{array}{l}\text { 112TILL } \\
\text { BEDROCK }\end{array}$ & TILL CLAY, HARDPAN \\
\hline JAW & 26 & $\cdots$ & 362 & - & $\begin{array}{r}0 \\
60\end{array}$ & $\begin{array}{l}60 \\
\ldots\end{array}$ & $\begin{array}{l}\text { 112TILL } \\
\text { BEDROCK }\end{array}$ & TILL \\
\hline
\end{tabular}


Table 2-1. Stratigraphic logs of wells and borings in the Saco and Ossipee River Basins, eastcentral New Hampshire--Continued

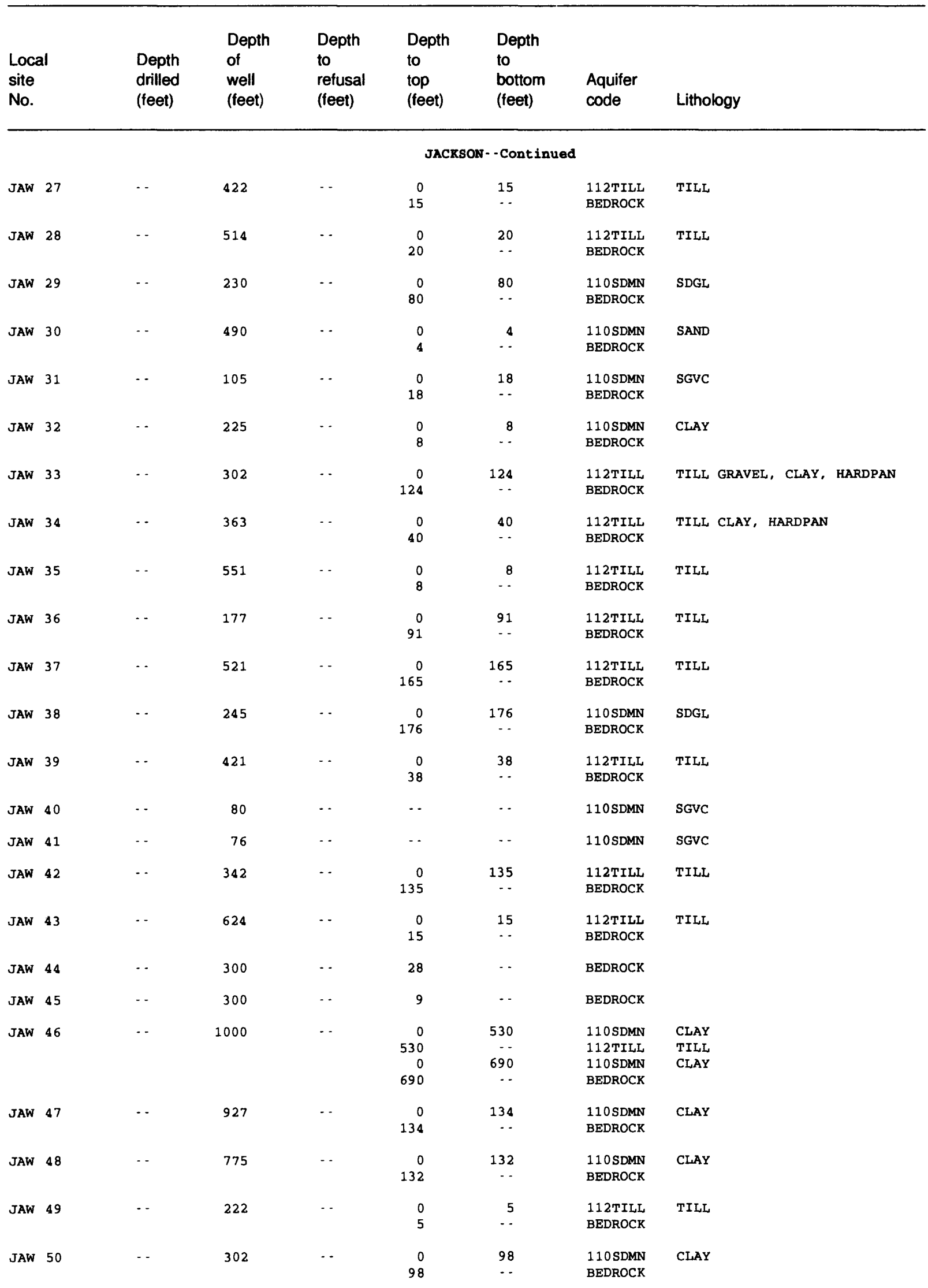


Table 2-1. Stratigraphic logs of wells and borings in the Saco and Ossipee River Basins, eastcentral New Hampshire--Continued

\begin{tabular}{|c|c|c|c|c|c|c|c|c|}
\hline \multicolumn{2}{|c|}{$\begin{array}{l}\text { Local } \\
\text { site } \\
\text { no. }\end{array}$} & $\begin{array}{l}\text { Depth } \\
\text { drilled } \\
\text { (feet) }\end{array}$ & $\begin{array}{l}\text { Depth } \\
\text { of } \\
\text { well } \\
\text { (feet) }\end{array}$ & $\begin{array}{l}\text { Depth } \\
\text { to } \\
\text { refusal } \\
\text { (feet) }\end{array}$ & $\begin{array}{l}\text { Depth } \\
\text { to } \\
\text { top } \\
\text { (feet) }\end{array}$ & $\begin{array}{l}\text { Depth } \\
\text { to } \\
\text { bottom } \\
\text { (feet) }\end{array}$ & $\begin{array}{c}\text { Aquifer } \\
\text { code }\end{array}$ & Lithology \\
\hline & & \multicolumn{7}{|c|}{ JACKSON- - Continued } \\
\hline JAW & 51 & $\cdots$ & 523 & $\cdots$ & $\begin{array}{r}0 \\
100\end{array}$ & $\begin{array}{r}100 \\
--\end{array}$ & $\begin{array}{l}110 \text { SDMN } \\
\text { BEDROCK }\end{array}$ & CLAY \\
\hline JAW & 52 & $\cdots$ & 504 & $-\cdot$ & $\begin{array}{r}0 \\
50\end{array}$ & $\begin{array}{l}50 \\
\cdots\end{array}$ & $\begin{array}{l}110 \text { SDMN } \\
\text { BEDROCK }\end{array}$ & CLAY \\
\hline JAW & 53 & $\cdots$ & 421 & $-\cdot$ & $\begin{array}{r}0 \\
100 \\
135\end{array}$ & $\begin{array}{r}100 \\
135 \\
-.\end{array}$ & $\begin{array}{l}\text { 112TILL } \\
110 \text { SDMN } \\
\text { BEDROCK }\end{array}$ & $\begin{array}{l}\text { TILL } \\
\text { CLAY }\end{array}$ \\
\hline JAW & 54 & $\cdots$ & 423 & - & $\begin{array}{r}0 \\
20\end{array}$ & $\begin{array}{l}20 \\
--\end{array}$ & $\begin{array}{l}\text { 112TILL } \\
\text { BEDROCK }\end{array}$ & TILL \\
\hline JAW & 55 & $\cdots$ & 305 & -- & $\begin{array}{r}0 \\
92\end{array}$ & $\begin{array}{l}92 \\
--\end{array}$ & $\begin{array}{l}\text { 110SDMN } \\
\text { BEDROCK }\end{array}$ & SGVC \\
\hline JAW & 56 & $\cdots$ & 805 & $-\cdot$ & $\begin{array}{r}0 \\
115\end{array}$ & $\begin{array}{r}115 \\
\cdots\end{array}$ & $\begin{array}{l}\text { 110SDMN } \\
\text { BEDROCK }\end{array}$ & SGVC \\
\hline JAW & 57 & - & 355 & $-\cdot$ & $\begin{array}{l}0 \\
7\end{array}$ & $\begin{array}{l}7 \\
--\end{array}$ & $\begin{array}{l}\text { 11OSDMN } \\
\text { BEDROCK }\end{array}$ & SDGL \\
\hline JAW & 58 & $\cdots$ & 423 & $\cdots$ & $\begin{array}{r}0 \\
40 \\
90\end{array}$ & $\begin{array}{l}40 \\
90 \\
--\end{array}$ & $\begin{array}{l}110 \text { SDMN } \\
112 \mathrm{TILL} \\
\text { BEDROCK }\end{array}$ & $\begin{array}{l}\text { SDGL } \\
\text { TILL CLAY, HARDPAN }\end{array}$ \\
\hline JAW & 59 & -- & 665 & $\cdots$ & $\begin{array}{r}0 \\
330\end{array}$ & $\begin{array}{r}330 \\
\cdots\end{array}$ & $\begin{array}{l}\text { 112TILL } \\
\text { BEDROCK }\end{array}$ & TILL \\
\hline JAW & 60 & $\cdots$ & 484 & - & $\begin{array}{r}0 \\
80 \\
140\end{array}$ & $\begin{array}{r}80 \\
140 \\
\cdots\end{array}$ & $\begin{array}{l}\text { BEDROCK } \\
112 T I L L \\
\text { BEDROCK }\end{array}$ & TILL \\
\hline JAW & 61 & -- & 102 & $\cdots$ & $\begin{array}{r}0 \\
10\end{array}$ & $\begin{array}{l}10 \\
\cdots\end{array}$ & $\begin{array}{l}\text { 112TILL } \\
\text { BEDROCK }\end{array}$ & TILL CLAY, HARDPAN \\
\hline JAW & 62 & - & 350 & $-\cdot$ & $\begin{array}{r}0 \\
80\end{array}$ & 80 & $\begin{array}{l}110 \text { SDMN } \\
\text { BEDROCK }\end{array}$ & CLAY \\
\hline JAW & 63 & $-\cdot$ & 240 & - & $\begin{array}{r}0 \\
10 \\
175 \\
210\end{array}$ & $\begin{array}{r}10 \\
175 \\
210 \\
--\end{array}$ & $\begin{array}{l}\text { 110SDMN } \\
112 T I L L \\
\text { BEDROCK } \\
\text { BEDROCK }\end{array}$ & $\begin{array}{l}\text { SDGL } \\
\text { TILL }\end{array}$ \\
\hline JAW & 64 & $\cdots$ & 302 & -- & $\begin{array}{r}0 \\
10 \\
100\end{array}$ & $\begin{array}{r}10 \\
100 \\
\cdots\end{array}$ & $\begin{array}{l}110 \text { SDMN } \\
112 T I L L \\
\text { BEDROCK }\end{array}$ & $\begin{array}{l}\text { SAND } \\
\text { TILL }\end{array}$ \\
\hline JAW & 65 & $\cdots$ & 605 & $\cdots$ & $\begin{array}{r}0 \\
100 \\
120\end{array}$ & $\begin{array}{r}100 \\
120 \\
-\cdot\end{array}$ & $\begin{array}{l}\text { 112TILL } \\
\text { BEDROCK } \\
\text { BEDROCK }\end{array}$ & TILL CLAY, HARDPAN \\
\hline JAW & 66 & $\cdots$ & 182 & - & $\begin{array}{r}0 \\
25\end{array}$ & $\begin{array}{l}25 \\
-\cdot\end{array}$ & $\begin{array}{l}\text { 112TILL } \\
\text { BEDROCK }\end{array}$ & TILL \\
\hline JAW & 67 & $\cdots$ & 521 & -- & $\begin{array}{r}0 \\
80 \\
140\end{array}$ & $\begin{array}{r}80 \\
140 \\
--\end{array}$ & $\begin{array}{l}112 T I L L \\
110 \text { SDMN } \\
\text { BEDROCK }\end{array}$ & $\begin{array}{l}\text { TILL } \\
\text { CLAY }\end{array}$ \\
\hline JAW & 68 & $\cdots$ & 255 & -- & $\begin{array}{r}0 \\
34 \\
61\end{array}$ & $\begin{array}{l}34 \\
61 \\
--\end{array}$ & $\begin{array}{l}\text { 112TILL } \\
\text { BEDROCK } \\
\text { BEDROCK }\end{array}$ & TILL CLAY, HARDPAN \\
\hline JAW & 69 & $\cdots$ & 584 & $\cdots$ & $\begin{array}{r}0 \\
120\end{array}$ & $\begin{array}{r}120 \\
\cdots\end{array}$ & $\begin{array}{l}\text { 112TILL } \\
\text { BEDROCK }\end{array}$ & TILL \\
\hline JAW & 70 & $\cdots$ & 422 & - & $\begin{array}{r}0 \\
50\end{array}$ & $\begin{array}{l}50 \\
\cdots\end{array}$ & $\begin{array}{l}\text { 112TILL } \\
\text { BEDROCK }\end{array}$ & TILL \\
\hline JAW & 71 & $-\cdot$ & 262 & $\cdots$ & $\begin{array}{r}0 \\
12\end{array}$ & $\begin{array}{l}12 \\
\cdots\end{array}$ & $\begin{array}{l}\text { 112TILL } \\
\text { BEDROCK }\end{array}$ & TILL \\
\hline
\end{tabular}


Table 2-1. Stratigraphic logs of wells and borings in the Saco and Ossipee River Basins, eastcentral New Hampshire--Continued

\begin{tabular}{|c|c|c|c|c|c|c|c|}
\hline $\begin{array}{l}\text { Local } \\
\text { site } \\
\text { No. }\end{array}$ & $\begin{array}{l}\text { Depth } \\
\text { drilled } \\
\text { (feet) }\end{array}$ & $\begin{array}{l}\text { Depth } \\
\text { of } \\
\text { well } \\
\text { (feet) }\end{array}$ & $\begin{array}{l}\text { Depth } \\
\text { to } \\
\text { refusal } \\
\text { (feet) }\end{array}$ & $\begin{array}{l}\text { Depth } \\
\text { to } \\
\text { top } \\
\text { (feet) }\end{array}$ & $\begin{array}{l}\text { Depth } \\
\text { to } \\
\text { bottom } \\
\text { (feet) }\end{array}$ & $\begin{array}{l}\text { Aquifer } \\
\text { code }\end{array}$ & Lithology \\
\hline
\end{tabular}

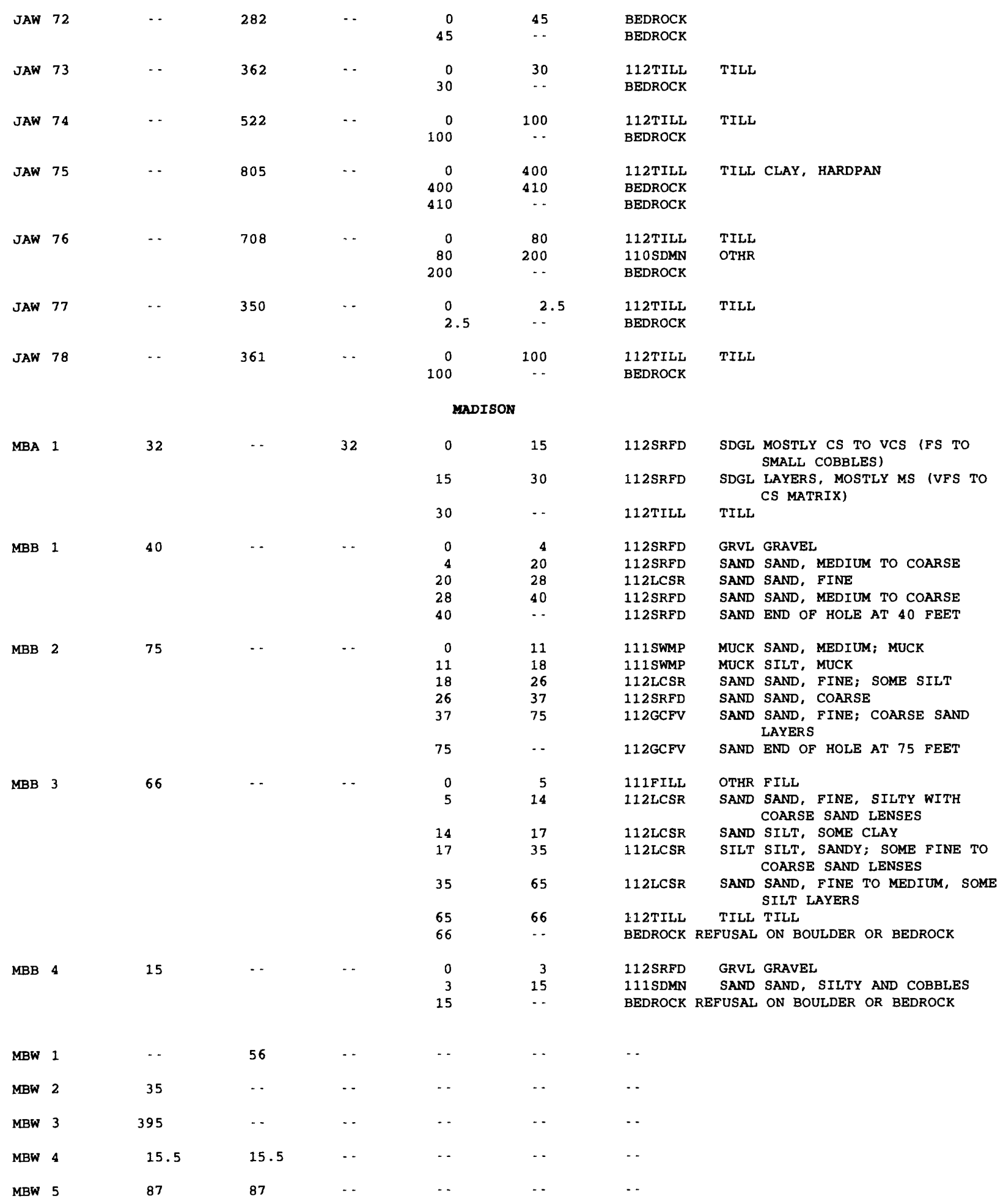


Table 2-1. Stratigraphic logs of wells and borings in the Saco and Ossipee River Basins, eastcentral New Hampshire--Continued

\begin{tabular}{|c|c|c|c|c|c|c|c|c|}
\hline \multicolumn{2}{|c|}{$\begin{array}{l}\text { Local } \\
\text { site } \\
\text { no. }\end{array}$} & $\begin{array}{l}\text { Depth } \\
\text { drilled } \\
\text { (feet) }\end{array}$ & $\begin{array}{l}\text { Depth } \\
\text { of } \\
\text { well } \\
\text { (feet) }\end{array}$ & $\begin{array}{l}\text { Depth } \\
\text { to } \\
\text { refusal } \\
\text { (feet) }\end{array}$ & $\begin{array}{l}\text { Depth } \\
\text { to } \\
\text { top } \\
\text { (feet) }\end{array}$ & $\begin{array}{l}\text { Depth } \\
\text { to } \\
\text { bottom } \\
\text { (feet) }\end{array}$ & $\begin{array}{l}\text { Aquifer } \\
\text { code }\end{array}$ & Lithology \\
\hline & & \multicolumn{7}{|c|}{ MADISON- - Continued } \\
\hline MBW & 6 & 55 & 55 & $\cdots$ & $\cdots$ & $\cdots$ & $-\cdot$ & \\
\hline MBW & 7 & 10.3 & 10.3 & $\cdots$ & $\cdots$ & $\cdots$ & $\cdots$ & \\
\hline \multirow[t]{4}{*}{ MBW } & 10 & 124 & 59 & $\cdots$ & 0 & 31 & 112SRFD & $\begin{array}{l}\text { SDGL C TO VCS, PEBBLES AND } \\
\text { COBBLES }\end{array}$ \\
\hline & & & & & 31 & 41 & 112SRFD & SAND M TO CS LAYERS \\
\hline & & & & & 41 & 93 & 112SRFD & SDGL $F$ TO VCS WITH PEBBLES \\
\hline & & & & & 93 & $\cdots$ & 112SRFD & SAND F TO VCS LAYERS \\
\hline MBW & 11 & $\cdots$ & 40 & $\cdots$ & 0 & 15 & $110 \mathrm{SDMN}$ & SDGL \\
\hline \multirow{3}{*}{ MBW } & & & & & & & & \\
\hline & 12 & $\cdots$ & 242 & $\cdots$ & $\begin{array}{r}0 \\
40\end{array}$ & $\begin{array}{l}40 \\
65\end{array}$ & $\begin{array}{l}110 \text { SDMN } \\
110 \text { SDMN }\end{array}$ & $\begin{array}{l}\text { SDGL } \\
\text { SAND }\end{array}$ \\
\hline & & & & & 65 & - & BEDROCK & \\
\hline \multirow[t]{2}{*}{ MBW } & 13 & - & 802 & - & 0 & 31 & $110 \mathrm{SDMN}$ & SAND \\
\hline & & & & & 31 & $-\cdot$ & BEDROCK & \\
\hline \multirow[t]{3}{*}{ MBW } & 14 & -. & 55.5 & $\cdots$ & 0 & 12 & 110 SDMN & SDGL \\
\hline & & & & & 12 & 32 & 110 SDMN & SAND \\
\hline & & & & & 32 & $-\cdot$ & $110 \mathrm{SDMN}$ & SAND \\
\hline MBW & 15 & - & 770 & $-\cdot$ & $\begin{array}{l}0 \\
8\end{array}$ & 8 & $\begin{array}{l}112 \text { TILL } \\
\text { BEDROCK }\end{array}$ & TILL \\
\hline \multirow[t]{2}{*}{ MBW } & 16 & -. & 55 & $\cdots$ & 0 & 15 & $110 \mathrm{SDMN}$ & SDGL \\
\hline & & & & & 15 & $\cdots$ & $110 \mathrm{SDMN}$ & SDGL \\
\hline \multirow[t]{3}{*}{ MBW } & 17 & $\cdots$ & 800 & - & 0 & 15 & 110SDMN & SAND $F$ \\
\hline & & & & & 15 & 50 & $110 \mathrm{SDMN}$ & SDGL \\
\hline & & & & & 50 & & BEDROCK & \\
\hline MBW & 18 & -. & 43 & $\cdots$ & -- & - & $110 \mathrm{SDMN}$ & SDGL \\
\hline MBW & 19 & - & 422 & $\cdots$ & $\begin{array}{r}0 \\
18\end{array}$ & $\begin{array}{l}18 \\
\cdots\end{array}$ & $\begin{array}{l}110 \text { SDMN } \\
\text { BEDROCK }\end{array}$ & SDGL \\
\hline \multirow[t]{3}{*}{ MBW } & 20 & - & 361 & -. & 0 & 10 & $110 \mathrm{SDMN}$ & SDGL \\
\hline & & & & & 10 & 35 & $110 \mathrm{SDMN}$ & SAND \\
\hline & & & & & 35 & $\cdots$ & BEDROCK & \\
\hline \multirow[t]{2}{*}{ MBW } & 21 & - - & 122 & $\cdots$ & 0 & 25 & 112TILL & TILL \\
\hline & & & & & 25 & - & BEDROCK & \\
\hline \multirow[t]{2}{*}{ MBW } & 22 & -. & 30 & - & 0 & 30 & 110 SDMN & SDGL \\
\hline & & & & & 30 & $\cdots$ & $112 \mathrm{TILL}$ & TILL \\
\hline MBW & 23 & - & 53 & $\cdots$ & - & - & 110 SDMN & SDGL \\
\hline MBW & 24 & -. & 53 & - & - & $\cdots$ & $110 \mathrm{SDMN}$ & SDGL \\
\hline \multirow[t]{3}{*}{ MBW } & 25 & -. & 33 & - & 0 & 30 & $110 \mathrm{SDMN}$ & SDGL \\
\hline & & & & & 30 & 42 & 110 SDMN & SDCL \\
\hline & & & & & 42 & $\therefore$ & $112 \mathrm{TILL}$ & TILL \\
\hline MBW & 26 & $\cdots$ & 401 & $\cdots$ & 0 & 55 & 110 SDMN & SDGL \\
\hline \multirow{2}{*}{ MBW } & 27 & .. & 301 & .. & 0 & 105 & 110 SDMN & SAND \\
\hline & 21 & & 301 & & 105 & 105 & BEDROCK & \\
\hline \multirow[t]{2}{*}{ MBW } & 28 & $\cdots$ & 443 & - & 0 & 45 & 110 SDMN & SDGL \\
\hline & & & & & 45 & $\cdots$ & BEDROCK & \\
\hline MBW & 29 & - & 52.5 & $\cdots$ & $\cdots$ & $\cdots$ & 110 SDMN & SDGL \\
\hline MBW & 30 & . & 58 & - & - & - & 110 SDMN & SDGL \\
\hline
\end{tabular}


Table 2-1. Stratigraphic logs of wells and borings in the Saco and Ossipee River Basins, eastcentral New Hampshire--Continued

\begin{tabular}{|c|c|c|c|c|c|c|c|c|}
\hline $\begin{array}{l}\text { Loca } \\
\text { site } \\
\text { No. }\end{array}$ & & $\begin{array}{l}\text { Depth } \\
\text { drilled } \\
\text { (feet) }\end{array}$ & $\begin{array}{l}\text { Depth } \\
\text { of } \\
\text { well } \\
\text { (feet) }\end{array}$ & $\begin{array}{l}\text { Depth } \\
\text { to } \\
\text { refusal } \\
\text { (feet) }\end{array}$ & $\begin{array}{l}\text { Depth } \\
\text { to } \\
\text { top } \\
\text { (feet) }\end{array}$ & $\begin{array}{l}\text { Depth } \\
\text { to } \\
\text { bottom } \\
\text { (feet) }\end{array}$ & $\begin{array}{l}\text { Aquifer } \\
\text { code }\end{array}$ & Lithology \\
\hline & & \multicolumn{7}{|c|}{ MADISON- - Continued } \\
\hline MBW & 31 & - & 114 & $\cdots$ & $\begin{array}{r}0 \\
208\end{array}$ & $\begin{array}{r}208 \\
--\end{array}$ & $\begin{array}{l}110 \text { SDMN } \\
\text { BEDROCK }\end{array}$ & SDGL \\
\hline MBW & 32 & $\cdots$ & 241 & $-\cdot$ & $\begin{array}{r}0 \\
40 \\
72\end{array}$ & $\begin{array}{l}40 \\
72 \\
\cdots\end{array}$ & $\begin{array}{l}\text { 110SDMN } \\
\text { 112TILL } \\
\text { BEDROCK }\end{array}$ & $\begin{array}{l}\text { SAND } \\
\text { TILL }\end{array}$ \\
\hline MBW & 33 & $\cdots$ & 57 & $\cdots$ & $-\cdot$ & $\cdots$ & $110 \mathrm{SDMN}$ & SDGL \\
\hline MBW & 34 & $\cdots$ & 363 & $\cdots$ & $\begin{array}{r}0 \\
15\end{array}$ & $\begin{array}{l}15 \\
\cdots\end{array}$ & $\begin{array}{l}110 \text { SDMN } \\
\text { BEDROCK }\end{array}$ & SDGL \\
\hline MBW & 35 & $-\cdot$ & 61 & $\cdots$ & $\cdots$ & $\cdots$ & 110 SDMN & SDGL \\
\hline MBW & 36 & $\cdots$ & 522 & $\cdots$ & $\begin{array}{r}0 \\
47\end{array}$ & $\begin{array}{l}47 \\
--\end{array}$ & $\begin{array}{l}110 \text { SDMN } \\
\text { BEDROCK }\end{array}$ & OTHR \\
\hline MBW & 37 & $\cdots$ & 59 & $-\cdot$ & $\begin{array}{r}0 \\
28 \\
59\end{array}$ & $\begin{array}{l}28 \\
59 \\
--\end{array}$ & $\begin{array}{l}110 \text { SDMN } \\
110 \text { SDMN } \\
112 \text { TILL }\end{array}$ & $\begin{array}{l}\text { SDGL } \\
\text { SAND } \\
\text { TILL SAND, HARDPAN }\end{array}$ \\
\hline MBW & 38 & $\cdots$ & 48 & $\cdots$ & $\cdots$ & $\cdots$ & $110 \mathrm{SDMN}$ & SDGL \\
\hline MBW & 39 & $\cdots$ & 503 & $\cdots$ & $\begin{array}{r}0 \\
35\end{array}$ & $\begin{array}{l}35 \\
--\end{array}$ & $\begin{array}{l}110 \text { SDMN } \\
\text { BEDROCK }\end{array}$ & SDGL \\
\hline MBW & 40 & $\cdots$ & 36 & $\cdots$ & $\cdots$ & $\cdots$ & $110 \mathrm{SDMN}$ & SDGL \\
\hline MBW & 41 & $\cdots$ & 63 & $\cdots$ & $\begin{array}{r}0 \\
20\end{array}$ & $\begin{array}{l}20 \\
\cdots\end{array}$ & $\begin{array}{l}110 \mathrm{SDMN} \\
110 \mathrm{SDMN}\end{array}$ & $\begin{array}{l}\text { SDGL } \\
\text { SAND }\end{array}$ \\
\hline MBW & 42 & - & 30.5 & $\cdots$ & $\begin{array}{r}0 \\
38\end{array}$ & $\begin{array}{l}38 \\
-\cdot\end{array}$ & $\begin{array}{l}110 \text { SDMN } \\
112 \mathrm{TILL}\end{array}$ & $\begin{array}{l}\text { SDGL } \\
\text { TILL }\end{array}$ \\
\hline MBW & 43 & $\cdots$ & 17 & $\cdots$ & $\cdots$ & $\cdots$ & $110 \mathrm{SDMN}$ & SAND \\
\hline MBW & 44 & $\cdots$ & 602 & $-\cdot$ & $\begin{array}{r}0 \\
93\end{array}$ & $\begin{array}{l}93 \\
-\cdot\end{array}$ & $\begin{array}{l}110 \text { SDMN } \\
\text { BEDROCK }\end{array}$ & CLAY \\
\hline MBW & 45 & $\cdots$ & 63 & $\cdots$ & $\cdots$ & $\cdots$ & $110 \mathrm{SDMN}$ & SDGL \\
\hline MBW & 46 & $\cdots$ & 362 & $\cdots$ & $\begin{array}{r}0 \\
60\end{array}$ & $\begin{array}{l}60 \\
\cdots\end{array}$ & $\begin{array}{l}110 \text { SDMN } \\
\text { BEDROCK }\end{array}$ & SDCL \\
\hline MBW & 47 & $\cdots$ & 57 & $\cdots$ & $\cdots$ & $\cdots$ & $110 \mathrm{SDMN}$ & SDGL \\
\hline MBW & 48 & -. & 282 & $\cdots$ & $\begin{array}{r}0 \\
41\end{array}$ & 41 & $\begin{array}{l}110 \text { SDMN } \\
\text { BEDROCK }\end{array}$ & SAND \\
\hline MBW & 49 & - & 642 & $-\cdot$ & $\begin{array}{r}0 \\
35 \\
48\end{array}$ & $\begin{array}{l}35 \\
48 \\
\cdots\end{array}$ & $\begin{array}{l}110 \text { SDMN } \\
110 \text { SDMN } \\
\text { BEDROCK }\end{array}$ & $\begin{array}{l}\text { SAND } \\
\text { SDGL }\end{array}$ \\
\hline MBW & 50 & - & 62 & $\cdots$ & $\cdots$ & $\cdots$ & $110 \mathrm{SDMN}$ & SDGL \\
\hline MBW & 51 & -- & 57 & $-\cdot$ & $\begin{array}{r}0 \\
58\end{array}$ & $\begin{array}{l}58 \\
\cdots\end{array}$ & $\begin{array}{l}110 \text { SDMN } \\
112 \text { TILL }\end{array}$ & $\begin{array}{l}\text { SDGL } \\
\text { TILL SAND, GRAVEL, HARDPAN }\end{array}$ \\
\hline MBW & 52 & $\cdots$ & 300 & .. & $\begin{array}{r}0 \\
31\end{array}$ & $\begin{array}{l}31 \\
--\end{array}$ & $\begin{array}{l}112 \text { TILL } \\
\text { BEDROCK }\end{array}$ & TILL SAND, CLAY, HARDPAN \\
\hline MBW & 53 & $\cdots$ & 175 & $\cdots$ & $\cdots$ & $\cdots$ & 110 SDMN & SDGL \\
\hline MBW & 54 & $\cdots$ & 241 & $\cdots$ & $\begin{array}{r}0 \\
99\end{array}$ & $\begin{array}{l}99 \\
-.\end{array}$ & $\begin{array}{l}110 \text { SDMN } \\
\text { BEDROCK }\end{array}$ & SDCL \\
\hline
\end{tabular}


Table 2-1. Stratigraphic logs of wells and borings in the Saco and Ossipee River Basins, eastcentral New Hampshire--ContInued

\begin{tabular}{|c|c|c|c|c|c|c|c|c|}
\hline \multicolumn{2}{|c|}{$\begin{array}{l}\text { Local } \\
\text { site } \\
\text { no. }\end{array}$} & $\begin{array}{l}\text { Depth } \\
\text { drilled } \\
\text { (feet) }\end{array}$ & $\begin{array}{l}\text { Depth } \\
\text { of } \\
\text { well } \\
\text { (feet) }\end{array}$ & $\begin{array}{l}\text { Depth } \\
\text { to } \\
\text { refusal } \\
\text { (feet) }\end{array}$ & $\begin{array}{l}\text { Depth } \\
\text { to } \\
\text { top } \\
\text { (feet) }\end{array}$ & $\begin{array}{l}\text { Depth } \\
\text { to } \\
\text { bottom } \\
\text { (feet) }\end{array}$ & $\begin{array}{l}\text { Aquifer } \\
\text { code }\end{array}$ & Lithology \\
\hline & & \multicolumn{7}{|c|}{ MADISON - - Continued } \\
\hline MBW & 55 & 57.5 & 49 & - - & $\begin{array}{r}0 \\
13\end{array}$ & $\begin{array}{l}13 \\
48\end{array}$ & $\begin{array}{l}112 \text { SRFD } \\
112 \text { SRFD }\end{array}$ & $\begin{array}{l}\text { SAND F TO MS SOME CS } \\
\text { SDGL LAYERS MOSTLY VCS (FS TO } \\
\text { PEBBLES) }\end{array}$ \\
\hline & & & & & $\begin{array}{l}48 \\
57\end{array}$ & $\begin{array}{l}57 \\
--\end{array}$ & $\begin{array}{l}\text { 112TILL } \\
\text { BEDROCK }\end{array}$ & $\begin{array}{l}\text { TILL } \\
\text { ROTTEN ROCK }\end{array}$ \\
\hline MBW & 56 & 89 & 69.1 & $\cdots$ & $\begin{array}{r}0 \\
30\end{array}$ & $\begin{array}{l}30 \\
68\end{array}$ & $\begin{array}{l}\text { 112SRFD } \\
112 \text { SRFD }\end{array}$ & $\begin{array}{l}\text { SDGL M TO VCS WITH PEBBLES } \\
\text { SAND LAYERS M TO F SOME CS AND } \\
\text { VFS }\end{array}$ \\
\hline & & & & & $\begin{array}{l}68 \\
84\end{array}$ & $\begin{array}{l}78 \\
--\end{array}$ & $\begin{array}{l}112 \text { SRFD } \\
112 \text { SRFD }\end{array}$ & $\begin{array}{l}\text { SDST VFS TO SILT LAYERS } \\
\text { SAND VFS TO FS LAYERS SOME MS }\end{array}$ \\
\hline MBW & 57 & 78 & 49 & $\cdots$ & $\begin{array}{r}0 \\
15\end{array}$ & $\begin{array}{l}15 \\
23\end{array}$ & $\begin{array}{l}112 \text { SRFD } \\
112 \text { SRFD }\end{array}$ & $\begin{array}{l}\text { SAND F TO VF } \\
\text { SAND LAYERS MOSTLY VCS (MS TO } \\
\text { GRAN) }\end{array}$ \\
\hline & & & & & 23 & 68 & 112SRFD & $\begin{array}{l}\text { SDGL LAYERS MATRIX MOSTLY VCS } \\
\text { CS (SOME VFS, FS, OR MS) }\end{array}$ \\
\hline & & & & & 68 & $\cdots$ & 112TILL & TILL \\
\hline MBW & 59 & $\cdots$ & 177 & $-\cdot$ & $\begin{array}{r}0 \\
30 \\
40 \\
50\end{array}$ & $\begin{array}{l}30 \\
40 \\
50 \\
-.\end{array}$ & $\begin{array}{l}110 \mathrm{SDMN} \\
110 \mathrm{SDMN} \\
112 \mathrm{TILL} \\
\text { BEDROCK }\end{array}$ & $\begin{array}{l}\text { SDGL } \\
\text { CLAY } \\
\text { TILL }\end{array}$ \\
\hline MBW & 60 & $\cdots$ & 453 & - & $\begin{array}{r}0 \\
12\end{array}$ & $\begin{array}{l}12 \\
-\cdots\end{array}$ & $\begin{array}{l}110 S D M N \\
\text { BEDROCK }\end{array}$ & SDGL \\
\hline MBW & 61 & $\cdots$ & 303 & $\cdots$ & $\begin{array}{r}0 \\
93\end{array}$ & $\begin{array}{l}93 \\
--\end{array}$ & $\begin{array}{l}\text { 112TILL } \\
\text { BEDROCK }\end{array}$ & TILL SAND, CLAY, HARDPAN \\
\hline MBW & 62 & - & 253 & $-\cdot$ & $\begin{array}{r}0 \\
80\end{array}$ & $\begin{array}{l}80 \\
-\cdot\end{array}$ & $\begin{array}{l}\text { 112TILL } \\
\text { BEDROCK }\end{array}$ & TILL CLAY, HARDPAN \\
\hline MBW & 63 & - & 203 & $-\cdot$ & $\begin{array}{r}0 \\
80\end{array}$ & 80 & $\begin{array}{l}\text { 112TILL } \\
\text { BEDROCK }\end{array}$ & TILL CLAY, HARDPAN \\
\hline MBW & 64 & $-\cdot$ & 753 & - & $\begin{array}{r}0 \\
10\end{array}$ & 10 & $\begin{array}{l}110 \text { SDMN } \\
\text { BEDROCK }\end{array}$ & CLAY \\
\hline MBW & 65 & - & 423 & -- & $\begin{array}{r}0 \\
40\end{array}$ & 40 & $\begin{array}{l}\text { 110SDMN } \\
\text { BEDROCK }\end{array}$ & SDGL \\
\hline MBW & 66 & $-\cdot$ & 252 & $\cdots$ & $\begin{array}{r}0 \\
27 \\
85\end{array}$ & $\begin{array}{l}27 \\
85 \\
-\cdots\end{array}$ & $\begin{array}{l}110 \text { SDMN } \\
110 \text { SDMN } \\
\text { BEDROCK }\end{array}$ & $\begin{array}{l}\text { SAND } \\
\text { CLAY }\end{array}$ \\
\hline MBW & 67 & -- & 60 & $-\cdot$ & $\begin{array}{r}0 \\
40\end{array}$ & $\begin{array}{l}40 \\
\cdots\end{array}$ & $\begin{array}{l}110 \text { SDMN } \\
110 \text { SDMN }\end{array}$ & $\begin{array}{l}\text { SAND } \\
\text { SDGL }\end{array}$ \\
\hline MBW & 68 & $-\cdot$ & 61 & - & $\begin{array}{r}0 \\
40\end{array}$ & $\begin{array}{l}40 \\
-\cdot\end{array}$ & $\begin{array}{l}110 \text { SDMN } \\
11.0 \text { SDMN }\end{array}$ & $\begin{array}{l}\text { SAND } \\
\text { SDGL }\end{array}$ \\
\hline MBW & 69 & $\cdots$ & 765 & -- & $\begin{array}{l}0 \\
7\end{array}$ & $\begin{array}{l}7 \\
\cdots\end{array}$ & $\begin{array}{l}110 \text { SDMN } \\
\text { BEDROCK }\end{array}$ & SDGL \\
\hline MBW & 70 & $\cdots$ & 17 & -- & 0 & $\cdots$ & 110SDMN & SDGL \\
\hline MBW & 71 & $-\cdot$ & 18 & $-\cdot$ & $\begin{array}{l}0 \\
7\end{array}$ & $\begin{array}{l}7 \\
-.\end{array}$ & $\begin{array}{l}110 \mathrm{SDMN} \\
110 \mathrm{SDMN}\end{array}$ & $\begin{array}{l}\text { SDGL } \\
\text { SAND }\end{array}$ \\
\hline MBW & 72 & $\cdots$ & 36 & $\cdots$ & 0 & $-\cdot$ & $110 \mathrm{SDMN}$ & SDGL \\
\hline MBW & 73 & - & 103 & $-\cdot$ & $\begin{array}{r}0 \\
21\end{array}$ & $\begin{array}{l}21 \\
\cdots\end{array}$ & $\begin{array}{l}110 \text { SDMN } \\
\text { BEDROCK }\end{array}$ & SGVC \\
\hline MBW & 74 & $-\cdot$ & 452 & $\cdots$ & $\begin{array}{r}0 \\
73\end{array}$ & $\begin{array}{l}73 \\
\cdots\end{array}$ & $\begin{array}{l}\text { 112TILL } \\
\text { BEDROCK }\end{array}$ & TILL CLAY, HARDPAN \\
\hline
\end{tabular}


Table 2-1. Stratigraphic logs of wells and borings in the Saco and Ossipee River Basins, eastcentral New Hampshire--Continued

\begin{tabular}{|c|c|c|c|c|c|c|c|c|}
\hline \multicolumn{2}{|c|}{$\begin{array}{l}\text { Local } \\
\text { site } \\
\text { No. }\end{array}$} & $\begin{array}{l}\text { Depth } \\
\text { drilled } \\
\text { (feet) }\end{array}$ & $\begin{array}{l}\text { Depth } \\
\text { of } \\
\text { well } \\
\text { (feet) }\end{array}$ & $\begin{array}{l}\text { Depth } \\
\text { to } \\
\text { refusal } \\
\text { (feet) }\end{array}$ & $\begin{array}{l}\text { Depth } \\
\text { to } \\
\text { top } \\
\text { (feet) }\end{array}$ & $\begin{array}{l}\text { Depth } \\
\text { to } \\
\text { bottom } \\
\text { (feet) }\end{array}$ & $\begin{array}{l}\text { Aquifer } \\
\text { code }\end{array}$ & Lithology \\
\hline & & \multicolumn{7}{|c|}{ MADISON-Continued } \\
\hline MBW & 75 & $\cdots$ & 803 & $\cdots$ & $\begin{array}{r}0 \\
15\end{array}$ & $\begin{array}{l}15 \\
--\end{array}$ & $\begin{array}{l}\text { 112TILL } \\
\text { BEDROCK }\end{array}$ & TILL GRAVEL, HARDPAN \\
\hline MBW & 76 & $\cdots$ & 12 & $\cdots$ & $\begin{array}{l}0 \\
5\end{array}$ & $\begin{array}{r}5 \\
--\end{array}$ & $\begin{array}{l}\text { 112TILL } \\
110 \mathrm{SDMN}\end{array}$ & $\begin{array}{l}\text { TILL CLAY, HARDPAN } \\
\text { SDGL }\end{array}$ \\
\hline MBW & 77 & - & 18 & - & $\begin{array}{r}0 \\
5 \\
12 \\
18\end{array}$ & $\begin{array}{r}5 \\
12 \\
18 \\
-\end{array}$ & $\begin{array}{l}112 \mathrm{TILL} \\
110 \mathrm{SDMN} \\
112 \mathrm{TILL} \\
\text { BEDROCK }\end{array}$ & $\begin{array}{l}\text { TILL } \\
\text { SDGL } \\
\text { TILL }\end{array}$ \\
\hline MBW & 78 & $\cdots$ & 62 & $\cdots$ & $\cdots$ & $\cdots$ & $110 \mathrm{SDMN}$ & SDGL \\
\hline MBW & 79 & $\cdots$ & 253 & $\cdots$ & $\begin{array}{r}0 \\
73\end{array}$ & $\begin{array}{l}73 \\
-.\end{array}$ & $\begin{array}{l}\text { 112TILL } \\
\text { BEDROCK }\end{array}$ & TILL SAND, CLAY, HARDPAN \\
\hline MBW & 80 & $\cdots$ & 175 & $\cdots$ & $\begin{array}{r}0 \\
80\end{array}$ & $\begin{array}{l}80 \\
\cdots\end{array}$ & $\begin{array}{l}110 \text { SDMN } \\
\text { BEDROCK }\end{array}$ & OTHR \\
\hline MBW & 81 & $\cdots$ & 402 & $\cdots$ & $\begin{array}{r}0 \\
125\end{array}$ & 125 & $\begin{array}{l}112 \text { TILL } \\
\text { BEDROCK }\end{array}$ & TILL CLAY, HARDPAN \\
\hline MBW & 82 & -- & 527 & $-\cdot$ & $\begin{array}{r}0 \\
80 \\
97\end{array}$ & $\begin{array}{l}80 \\
97 \\
--\end{array}$ & $\begin{array}{l}110 \text { SDMN } \\
112 \text { TILL } \\
\text { BEDROCK }\end{array}$ & $\begin{array}{l}\text { SAND } \\
\text { TILL GRAVEL, HARDPAN }\end{array}$ \\
\hline MBW & 83 & $\cdots$ & 327 & $\cdots$ & $\begin{array}{r}0 \\
80 \\
120\end{array}$ & $\begin{array}{r}80 \\
120\end{array}$ & $\begin{array}{l}\text { 110SDMN } \\
112 \text { TILL } \\
\text { BEDROCK }\end{array}$ & $\begin{array}{l}\text { SDGL } \\
\text { TILL }\end{array}$ \\
\hline MBW & 84 & - & 17 & $\cdots$ & $\begin{array}{r}0 \\
12\end{array}$ & $\begin{array}{l}12 \\
\cdots\end{array}$ & $\begin{array}{l}110 \text { SDMN } \\
110 \text { SDMN }\end{array}$ & $\begin{array}{l}\text { SDGL } \\
\text { SAND }\end{array}$ \\
\hline MBW & 85 & $\cdots$ & 20 & $\cdots$ & $\cdots$ & $\cdots$ & 112TILL & TILL \\
\hline MBW & 86 & - & 204 & - & $\begin{array}{r}0 \\
130\end{array}$ & $\begin{array}{c}130 \\
\cdots\end{array}$ & $\begin{array}{l}110 \text { SDMN } \\
\text { BEDROCK }\end{array}$ & SAND \\
\hline MBW & 87 & $\cdots$ & 322 & $\cdots$ & $\begin{array}{r}0 \\
80\end{array}$ & $\begin{array}{l}80 \\
\cdots\end{array}$ & $\begin{array}{l}\text { 110SDMN } \\
\text { BEDROCK }\end{array}$ & SDGL \\
\hline MBW & 88 & $\cdots$ & 101 & $\cdots$ & $\begin{array}{r}0 \\
15\end{array}$ & $\begin{array}{l}15 \\
\cdots\end{array}$ & $\begin{array}{l}\text { 112TILL } \\
\text { BEDROCK }\end{array}$ & TILL \\
\hline MBW & 89 & $\cdots$ & 361 & $\cdots$ & $\begin{array}{r}0 \\
45\end{array}$ & $\begin{array}{l}45 \\
--\end{array}$ & $\begin{array}{l}110 \text { SDMN } \\
\text { BEDROCK }\end{array}$ & SDGL \\
\hline MBW & 90 & $\cdots$ & 428 & $\cdots$ & $\begin{array}{l}0 \\
6\end{array}$ & $\begin{array}{c}6 \\
\cdots\end{array}$ & $\begin{array}{l}\text { 112TILL } \\
\text { BEDROCK }\end{array}$ & TILL \\
\hline MBW & 91 & $\cdots$ & 500 & $\cdots$ & $\begin{array}{l}0 \\
3\end{array}$ & $\begin{array}{l}3 \\
\cdots\end{array}$ & $\begin{array}{l}110 \text { SDMN } \\
\text { BEDROCK }\end{array}$ & SAND \\
\hline MBW & 92 & $\cdots$ & 638 & -. & $\begin{array}{r}0 \\
12\end{array}$ & $\begin{array}{l}12 \\
-\cdot\end{array}$ & $\begin{array}{l}110 \text { SDMN } \\
\text { BEDROCK }\end{array}$ & SAND \\
\hline MBW & 93 & $\cdots$ & 242 & $\cdots$ & $\begin{array}{l}0 \\
6\end{array}$ & $\begin{array}{c}6 \\
--\end{array}$ & $\begin{array}{l}112 \text { TILL } \\
\text { BEDROCK }\end{array}$ & TILL \\
\hline MBW & 94 & $\cdots$ & 322 & $\cdots$ & $\begin{array}{r}0 \\
20\end{array}$ & $\begin{array}{l}20 \\
--\end{array}$ & $\begin{array}{l}\text { 112TILL } \\
\text { BEDROCK }\end{array}$ & TILL \\
\hline MBW & 95 & .. & 282 & $\cdots$ & $\begin{array}{l}0 \\
3\end{array}$ & $\begin{array}{r}3 \\
--\end{array}$ & $\begin{array}{l}\text { 112TILL } \\
\text { BEDROCK }\end{array}$ & TILL \\
\hline MBW & 96 & $\cdots$ & 202 & $\cdots$ & $\begin{array}{r}0 \\
15\end{array}$ & $\begin{array}{l}15 \\
-.\end{array}$ & $\begin{array}{l}\text { 112TILL } \\
\text { BEDROCK }\end{array}$ & TILL \\
\hline MBW & 97 & $\cdots$ & 342 & $\cdots$ & $\begin{array}{r}0 \\
45\end{array}$ & $\begin{array}{l}45 \\
--\end{array}$ & $\begin{array}{l}\text { 112TILL } \\
\text { BEDROCK }\end{array}$ & TILL \\
\hline
\end{tabular}


Table 2-1. Stratigraphic logs of wells and borings in the Saco and Ossipee River Basins, eastcentral New Hampshire--Continued

\begin{tabular}{|c|c|c|c|c|c|c|c|c|}
\hline \multicolumn{2}{|c|}{$\begin{array}{l}\text { Local } \\
\text { site } \\
\text { no. }\end{array}$} & $\begin{array}{l}\text { Depth } \\
\text { drilled } \\
\text { (feet) }\end{array}$ & $\begin{array}{l}\text { Depth } \\
\text { of } \\
\text { well } \\
\text { (feet) }\end{array}$ & $\begin{array}{l}\text { Depth } \\
\text { to } \\
\text { refusal } \\
\text { (feet) }\end{array}$ & $\begin{array}{l}\text { Depth } \\
\text { to } \\
\text { top } \\
\text { (feet) }\end{array}$ & $\begin{array}{l}\text { Depth } \\
\text { to } \\
\text { bottom } \\
\text { (feet) }\end{array}$ & $\begin{array}{l}\text { Aquifer } \\
\text { code }\end{array}$ & Lithology \\
\hline & & \multicolumn{7}{|c|}{ MaDISON- - Continued } \\
\hline MBW & 98 & $\cdots$ & 334 & $\cdots$ & $\begin{array}{l}0 \\
7\end{array}$ & $\begin{array}{l}7 \\
.\end{array}$ & $\begin{array}{l}110 \text { SDMN } \\
\text { BEDROCK }\end{array}$ & SAND \\
\hline MBW & 99 & - & 322 & $\cdots$ & $\begin{array}{r}0 \\
135\end{array}$ & $\begin{array}{r}135 \\
-\cdot\end{array}$ & $\begin{array}{l}\text { 112TILL } \\
\text { BEDROCK }\end{array}$ & TILL \\
\hline MBW & 100 & $\cdots$ & 19 & - & $\begin{array}{l}0 \\
4\end{array}$ & $\begin{array}{r}4 \\
-\end{array}$ & $\begin{array}{l}110 \text { SDMN } \\
112 \text { TILL }\end{array}$ & $\begin{array}{l}\text { SDGL } \\
\text { TILL }\end{array}$ \\
\hline MBW & 101 & $\cdots$ & 600 & $\cdots$ & $\begin{array}{r}0 \\
40 \\
170\end{array}$ & $\begin{array}{r}40 \\
170 \\
\ldots\end{array}$ & $\begin{array}{l}110 \text { SDMN } \\
112 \text { TILL } \\
\text { BEDROCK }\end{array}$ & $\begin{array}{l}\text { SDGL } \\
\text { TILL SAND, HARDPAN }\end{array}$ \\
\hline MBW & 102 & - & 182 & $\cdots$ & $\begin{array}{r}0 \\
18\end{array}$ & $\begin{array}{l}18 \\
--\end{array}$ & $\begin{array}{l}\text { 112TILL } \\
\text { BEDROCK }\end{array}$ & TILL \\
\hline MBW & 103 & - & 377 & - & $\begin{array}{r}0 \\
42\end{array}$ & $\begin{array}{l}42 \\
\cdots\end{array}$ & $\begin{array}{l}110 \text { SDMN } \\
\text { BEDROCK }\end{array}$ & SDGL \\
\hline MBW & 104 & - & 323 & $-\cdot$ & $\begin{array}{r}0 \\
20\end{array}$ & $\begin{array}{l}20 \\
\cdots\end{array}$ & $\begin{array}{l}110 \text { SDMN } \\
\text { BEDROCK }\end{array}$ & SDGL \\
\hline MBW & 105 & $-\cdot$ & 221 & $\cdots$ & $\begin{array}{r}0 \\
28\end{array}$ & $\begin{array}{l}28 \\
\cdots\end{array}$ & $\begin{array}{l}\text { 112TILL } \\
\text { BEDROCK }\end{array}$ & TILL \\
\hline MBW & 106 & $\cdots$ & 32 & $-\cdot$ & $\begin{array}{r}0 \\
33\end{array}$ & $\begin{array}{l}33 \\
--\end{array}$ & $\begin{array}{l}110 \mathrm{SDMN} \\
112 \mathrm{TILL}\end{array}$ & $\begin{array}{l}\text { SDGL } \\
\text { TILL }\end{array}$ \\
\hline MBW & 107 & -. & 545 & $\cdots$ & $\begin{array}{r}0 \\
10\end{array}$ & $\begin{array}{l}10 \\
--\end{array}$ & $\begin{array}{l}\text { 112TILL } \\
\text { BEDROCK }\end{array}$ & TILL \\
\hline MBW & 108 & $-\cdot$ & 281 & $\cdots$ & $\begin{array}{r}0 \\
12\end{array}$ & $\begin{array}{l}12 \\
-.\end{array}$ & $\begin{array}{l}110 \text { SDMN } \\
\text { BEDROCK }\end{array}$ & SDGL \\
\hline MBW & 109 & $\cdots$ & 420 & - & $\begin{array}{r}0 \\
240\end{array}$ & $\begin{array}{c}240 \\
--\end{array}$ & $\begin{array}{l}\text { 112TILL } \\
\text { BEDROCK }\end{array}$ & TILL \\
\hline MBW & 110 & - & 281 & $\cdots$ & $\begin{array}{l}0 \\
4\end{array}$ & $\begin{array}{l}4 \\
-.\end{array}$ & $\begin{array}{l}\text { 112TILL } \\
\text { BEDROCK }\end{array}$ & $\operatorname{TILL}$ \\
\hline MBW & 111 & - & 213 & - & $\begin{array}{r}0 \\
15\end{array}$ & $\begin{array}{l}15 \\
--\end{array}$ & $\begin{array}{l}\text { 112TILL } \\
\text { BEDROCK }\end{array}$ & TILL \\
\hline MBW & 112 & $\cdots$ & 188 & - & $\begin{array}{r}0 \\
15\end{array}$ & $\begin{array}{l}-\cdot \\
\cdots\end{array}$ & $\begin{array}{l}\text { 112TILL } \\
\text { BEDROCK }\end{array}$ & TILL \\
\hline MBW & 113 & $\cdots$ & 323 & $-\cdot$ & $\begin{array}{r}0 \\
236\end{array}$ & 236 & $\begin{array}{l}\text { 112TILL } \\
\text { BEDROCK }\end{array}$ & TILL GRAVEL, HARDPAN \\
\hline MBW & 114 & - & 500 & - & $\begin{array}{l}0 \\
8\end{array}$ & $\begin{array}{c}8 \\
-.\end{array}$ & $\begin{array}{l}110 \text { SDMN } \\
\text { BEDROCK }\end{array}$ & SDGL \\
\hline MBW & 115 & $\cdots$ & 400 & $\cdots$ & $\begin{array}{l}0 \\
6\end{array}$ & $\begin{array}{c}6 \\
-.\end{array}$ & $\begin{array}{l}110 \text { SDMN } \\
\text { BEDROCK }\end{array}$ & SDGL \\
\hline MBW & 116 & - & 540 & - & $\begin{array}{r}0 \\
18\end{array}$ & $\begin{array}{l}18 \\
\cdots\end{array}$ & $\begin{array}{l}\text { 112TILL } \\
\text { BEDROCK }\end{array}$ & TILL \\
\hline MBW & 117 & - & 241 & - & $\begin{array}{l}0 \\
5\end{array}$ & $\begin{array}{l}5 \\
-.\end{array}$ & $\begin{array}{l}112 \text { TILL } \\
\text { BEDROCK }\end{array}$ & TILL \\
\hline MBW & 118 & - & 302 & $\cdots$ & $\begin{array}{r}0 \\
35\end{array}$ & $\begin{array}{l}35 \\
\cdots\end{array}$ & $\begin{array}{l}110 \text { SDMN } \\
\text { BEDROCK }\end{array}$ & SDGL \\
\hline MBW & 119 & $-\cdot$ & 424 & $-\cdot$ & $\begin{array}{r}0 \\
15\end{array}$ & $\begin{array}{l}15 \\
\cdots\end{array}$ & $\begin{array}{l}110 \text { SDMN } \\
\text { BEDROCK }\end{array}$ & SDGL \\
\hline MBW & 120 & $-\cdot$ & 424 & - & $\begin{array}{r}0 \\
40\end{array}$ & $\begin{array}{l}40 \\
--\end{array}$ & $\begin{array}{l}110 \text { SDMN } \\
\text { BEDROCK }\end{array}$ & SDGL \\
\hline
\end{tabular}


Table 2-1. Stratigraphic logs of wells and borings in the Saco and Ossipee River Basins, eastcentrai New Hampshire--Continued

\begin{tabular}{|c|c|c|c|c|c|c|c|c|}
\hline $\begin{array}{l}\text { Loca } \\
\text { site } \\
\text { No. }\end{array}$ & & $\begin{array}{l}\text { Depth } \\
\text { drilled } \\
\text { (feet) }\end{array}$ & $\begin{array}{l}\text { Depth } \\
\text { of } \\
\text { well } \\
\text { (feet) }\end{array}$ & $\begin{array}{l}\text { Depth } \\
\text { to } \\
\text { refusal } \\
\text { (feet) }\end{array}$ & $\begin{array}{l}\text { Depth } \\
\text { to } \\
\text { top } \\
\text { (feet) }\end{array}$ & $\begin{array}{l}\text { Depth } \\
\text { to } \\
\text { bottom } \\
\text { (feet) }\end{array}$ & $\begin{array}{l}\text { Aquifer } \\
\text { code }\end{array}$ & Lithology \\
\hline & & \multicolumn{7}{|c|}{ MaDIsos--Continued } \\
\hline MBW & 121 & $\cdots$ & 538 & $\cdots$ & $\begin{array}{r}0 \\
10\end{array}$ & 10 & $\begin{array}{l}\text { 112TILL } \\
\text { BEDROCK }\end{array}$ & TILL \\
\hline MBW & 122 & $\cdots$ & 543 & $-\cdot$ & $\begin{array}{r}0 \\
10\end{array}$ & 10 & $\begin{array}{l}\text { 112TILL } \\
\text { BEDROCK }\end{array}$ & TILL \\
\hline MBW & 123 & $\cdots$ & 443 & $\cdots$ & $\begin{array}{r}0 \\
30\end{array}$ & $\begin{array}{l}30 \\
--\end{array}$ & $\begin{array}{l}\text { 112TILL } \\
\text { BEDROCK }\end{array}$ & TILL \\
\hline MBW & 124 & $\cdots$ & 927 & $\cdots$ & $\begin{array}{r}0 \\
135\end{array}$ & $\begin{array}{c}135 \\
-.\end{array}$ & $\begin{array}{l}\text { 112TILL } \\
\text { BEDROCK }\end{array}$ & TILL \\
\hline MBW & 125 & $\cdots$ & 220 & $\cdots$ & $\begin{array}{r}0 \\
30 \\
107\end{array}$ & $\begin{array}{r}30 \\
107 \\
--\end{array}$ & $\begin{array}{l}110 \text { SDMN } \\
110 \text { SDMN } \\
\text { BEDROCK }\end{array}$ & $\begin{array}{l}\text { SDGL } \\
\text { OTHR }\end{array}$ \\
\hline MBW & 126 & $\cdots$ & 227 & $\cdots$ & $\begin{array}{r}0 \\
52\end{array}$ & $\begin{array}{l}52 \\
\cdots\end{array}$ & $\begin{array}{l}\text { 112TILL } \\
\text { BEDROCK }\end{array}$ & TILL GRAVEL, CLAY, HARDPAN \\
\hline MBW & 127 & $\cdots$ & 402 & $\cdots$ & $\begin{array}{r}0 \\
18\end{array}$ & $\begin{array}{l}18 \\
\cdots\end{array}$ & $\begin{array}{l}\text { 112TILL } \\
\text { BEDROCK }\end{array}$ & TILL GRAVEL, CLAY, HARDPAN \\
\hline MBW & 128 & $\cdots$ & 423 & $\cdots$ & $\begin{array}{r}0 \\
10\end{array}$ & $\begin{array}{l}10 \\
-\cdot\end{array}$ & $\begin{array}{l}\text { 112TILL } \\
\text { BEDROCK }\end{array}$ & TILL \\
\hline MBW & 129 & $-\cdot$ & 48 & $\cdots$ & 0 & $\cdots$ & $110 \mathrm{SDMN}$ & SDGL \\
\hline MBW & 130 & $\cdots$ & 152 & $\cdots$ & $\begin{array}{r}0 \\
22\end{array}$ & $\begin{array}{l}22 \\
\cdots\end{array}$ & $\begin{array}{l}\text { 112TILL } \\
\text { BEDROCK }\end{array}$ & TILL \\
\hline MBW & 131 & $-\cdot$ & 442 & $\cdots$ & 7 & $\cdots$ & BEDROCK & \\
\hline MBW & 132 & $\cdots$ & 342 & $\cdots$ & $\begin{array}{r}0 \\
205\end{array}$ & $\begin{array}{r}205 \\
-.\end{array}$ & $\begin{array}{l}\text { 112TILL } \\
\text { BEDROCK }\end{array}$ & TILL CLAY, HARDPAN \\
\hline MBW & 133 & $-\cdot$ & 20 & $\cdots$ & $\begin{array}{r}0 \\
9 \\
10 \\
20\end{array}$ & $\begin{array}{r}9 \\
10 \\
20 \\
--\end{array}$ & $\begin{array}{l}\text { 112TILL } \\
110 \text { SDMN } \\
112 \mathrm{TILL} \\
\text { BEDROCK }\end{array}$ & $\begin{array}{l}\text { TILL } \\
\text { SDGL } \\
\text { TILL }\end{array}$ \\
\hline MBW & 134 & - & 62 & $-\cdot$ & 0 & $\cdots$ & 110 SDMN & SDGL \\
\hline MBW & 135 & $-\cdot$ & 602 & $-\cdot$ & $\begin{array}{l}0 \\
5\end{array}$ & $\begin{array}{r}5 \\
\therefore\end{array}$ & $\begin{array}{l}\text { 112TILL } \\
\text { BEDROCK }\end{array}$ & TILL \\
\hline MBW & 136 & $\cdots$ & 545 & $-\cdot$ & $\begin{array}{r}0 \\
10\end{array}$ & $\begin{array}{l}10 \\
\cdots\end{array}$ & $\begin{array}{l}\text { 112TILL } \\
\text { BEDROCK }\end{array}$ & TILL \\
\hline MBW & 137 & $\cdots$ & 61 & - & - & $\cdots$ & $110 \mathrm{SDMN}$ & SDGL \\
\hline MBW & 138 & $-\cdot$ & 18 & -. & $\begin{array}{r}0 \\
37\end{array}$ & $\begin{array}{l}37 \\
-\end{array}$ & $\begin{array}{l}110 \mathrm{SDMN} \\
112 \mathrm{TILL}\end{array}$ & $\begin{array}{l}\text { SAND } \\
\text { TILL }\end{array}$ \\
\hline MBW & 139 & $\cdots$ & 242 & $\cdots$ & $\begin{array}{r}0 \\
40\end{array}$ & $\begin{array}{l}40 \\
\cdots\end{array}$ & $\begin{array}{l}110 \text { SDMN } \\
\text { BEDROCK }\end{array}$ & SDGL \\
\hline MBW & 140 & $-\cdot$ & 595 & $\cdots$ & 7 & $\cdots$ & BEDROCK & \\
\hline MBW & 141 & $-\cdot$ & 562 & $\cdots$ & $\begin{array}{r}0 \\
10\end{array}$ & $\begin{array}{l}10 \\
--\end{array}$ & $\begin{array}{l}\text { 112TILL } \\
\text { BEDROCK }\end{array}$ & TILL \\
\hline MBW & 142 & $\cdots$ & 540 & $\cdots$ & $\begin{array}{r}0 \\
10\end{array}$ & $\begin{array}{l}10 \\
\cdots\end{array}$ & $\begin{array}{l}\text { 112TILL } \\
\text { BEDROCK }\end{array}$ & TILL \\
\hline MBW & 143 & $\cdots$ & 462 & $\cdots$ & $\begin{array}{r}0 \\
25\end{array}$ & $\begin{array}{l}25 \\
\cdots\end{array}$ & $\begin{array}{l}\text { 112TILL } \\
\text { BEDROCK }\end{array}$ & TILL \\
\hline MBW & 144 & $-\cdot$ & 61 & - & $\begin{array}{r}0 \\
13\end{array}$ & $\begin{array}{l}13 \\
\cdots\end{array}$ & $\begin{array}{l}110 \text { SDMN } \\
110 \text { SDMN }\end{array}$ & $\begin{array}{l}\text { SDGL } \\
\text { SAND }\end{array}$ \\
\hline
\end{tabular}


Table 2-1. Stratigraphic logs of wells and borings in the Saco and Ossipee River Basins, eastcentral New Hampshire--Continued

\begin{tabular}{|c|c|c|c|c|c|c|c|c|}
\hline \multicolumn{2}{|c|}{$\begin{array}{l}\text { Local } \\
\text { site } \\
\text { no. }\end{array}$} & $\begin{array}{l}\text { Depth } \\
\text { drilled } \\
\text { (feet) }\end{array}$ & $\begin{array}{l}\text { Depth } \\
\text { of } \\
\text { well } \\
\text { (feet) }\end{array}$ & $\begin{array}{l}\text { Depth } \\
\text { to } \\
\text { refusal } \\
\text { (feet) }\end{array}$ & $\begin{array}{l}\text { Depth } \\
\text { to } \\
\text { top } \\
\text { (feet) }\end{array}$ & $\begin{array}{l}\text { Depth } \\
\text { to } \\
\text { bottom } \\
\text { (feet) }\end{array}$ & $\begin{array}{l}\text { Aquifer } \\
\text { code }\end{array}$ & Lithology \\
\hline & & \multicolumn{7}{|c|}{ MADIsON- - Continued } \\
\hline MBW & 145 & - & 423 & $\cdots$ & $\begin{array}{r}0 \\
15\end{array}$ & $\begin{array}{l}15 \\
\cdots\end{array}$ & $\begin{array}{l}110 \text { SDMN } \\
\text { BEDROCK }\end{array}$ & CLAY \\
\hline MBW & 146 & $\cdots$ & 223 & $-\cdot$ & $\begin{array}{r}0 \\
15\end{array}$ & 15 & $\begin{array}{l}\text { 112TILL } \\
\text { BEDROCK }\end{array}$ & TILL \\
\hline MBW & 147 & $-\cdot$ & 424 & $\cdots$ & $\begin{array}{r}0 \\
15\end{array}$ & $\begin{array}{l}15 \\
\cdots\end{array}$ & $\begin{array}{l}\text { 112TILI } \\
\text { BEDROCK }\end{array}$ & TILL \\
\hline MBW & 148 & $\cdots$ & 198 & $\cdots$ & $\begin{array}{r}0 \\
12\end{array}$ & $\begin{array}{l}12 \\
\cdots\end{array}$ & $\begin{array}{l}112 \text { TILL } \\
\text { BEDROCK }\end{array}$ & TILL \\
\hline MBW & 149 & $\cdots$ & 422 & $\cdots$ & $\begin{array}{r}0 \\
40\end{array}$ & $\begin{array}{l}40 \\
\cdots\end{array}$ & $\begin{array}{l}110 \text { SDMN } \\
\text { BEDROCK }\end{array}$ & SDGL \\
\hline MBW & 150 & $\cdots$ & 560 & $\cdots$ & $\begin{array}{r}0 \\
10 \\
18\end{array}$ & $\begin{array}{l}10 \\
18 \\
-.\end{array}$ & $\begin{array}{l}110 \text { SDMN } \\
110 \text { SDMN } \\
\text { BEDROCK }\end{array}$ & $\begin{array}{l}\text { SDGL } \\
\text { CLAY }\end{array}$ \\
\hline MBW & 151 & $-\cdot$ & 55.5 & $\cdots$ & $\cdots$ & $\cdots$ & $110 \mathrm{SDMN}$ & SDGL \\
\hline MBW & 152 & $\cdots$ & 377 & $\cdots$ & $\begin{array}{r}0 \\
130\end{array}$ & 130 & $\begin{array}{l}112 \mathrm{TILL} \\
\text { BEDROCK }\end{array}$ & TILL GRAVEL, CLAY, HARDPAN \\
\hline MBW & 153 & $-\cdot$ & 302 & $\cdots$ & $\begin{array}{r}0 \\
10\end{array}$ & $\begin{array}{l}10 \\
--\end{array}$ & $\begin{array}{l}110 \text { SDMN } \\
\text { BEDROCK }\end{array}$ & SAND \\
\hline MBW & 154 & $\cdots$ & 177 & $\cdots$ & $\begin{array}{r}0 \\
60\end{array}$ & $\begin{array}{l}60 \\
\cdots\end{array}$ & $\begin{array}{l}110 \text { SDMN } \\
\text { BEDROCK }\end{array}$ & SDCL \\
\hline MBW & 155 & $\cdots$ & 302 & $\cdots$ & $\begin{array}{r}0 \\
117\end{array}$ & 117 & $\begin{array}{l}\text { 110SDMN } \\
\text { BEDROCK }\end{array}$ & SAND \\
\hline MBW & 156 & $\cdots$ & 202 & $\cdots$ & $\begin{array}{r}0 \\
10\end{array}$ & $\begin{array}{l}10 \\
\cdots\end{array}$ & $\begin{array}{l}112 \text { TILL } \\
\text { BEDROCK }\end{array}$ & TILL CLAY, HARDPAN \\
\hline MBW & 157 & $\cdots$ & 876 & $\cdots$ & $\begin{array}{r}0 \\
21\end{array}$ & $\begin{array}{l}21 \\
\cdots\end{array}$ & $\begin{array}{l}\text { 112TILL } \\
\text { BEDROCK }\end{array}$ & TILL \\
\hline MBW & 158 & - & 502 & - & $\begin{array}{r}0 \\
15\end{array}$ & $\begin{array}{l}15 \\
\cdots\end{array}$ & $\begin{array}{l}112 \text { TILL } \\
\text { BEDROCK }\end{array}$ & TILL \\
\hline MBW & 159 & - & 303 & - & $\begin{array}{r}0 \\
30\end{array}$ & $\begin{array}{l}30 \\
\cdots\end{array}$ & $\begin{array}{l}110 \text { SDMN } \\
\text { BEDROCK }\end{array}$ & SDGL \\
\hline MBW & 160 & $\cdots$ & 424 & $\cdots$ & $\begin{array}{r}0 \\
18\end{array}$ & $\begin{array}{l}18 \\
\cdots\end{array}$ & $\begin{array}{l}\text { 110SDMN } \\
\text { BEDROCK }\end{array}$ & SDGL \\
\hline MBW & 161 & - & 1060 & $\cdots$ & $\begin{array}{r}0 \\
20\end{array}$ & 20 & $\begin{array}{l}\text { 110SDMN } \\
\text { BEDROCK }\end{array}$ & SAND \\
\hline MBW & 162 & - & 604 & - & $\begin{array}{r}0 \\
65\end{array}$ & $\begin{array}{l}65 \\
\cdots\end{array}$ & $\begin{array}{l}\text { 112TILL } \\
\text { BEDROCK }\end{array}$ & TILI \\
\hline MBW & 163 & $\cdots$ & 202 & $\cdots$ & $\begin{array}{r}0 \\
40 \\
80\end{array}$ & $\begin{array}{l}40 \\
80 \\
-\end{array}$ & $\begin{array}{l}110 \text { SDMN } \\
112 \text { TILL } \\
\text { BEDROCK }\end{array}$ & $\begin{array}{l}\text { SDGL } \\
\text { TILL }\end{array}$ \\
\hline MBW & 164 & $\cdots$ & 438 & - & $\begin{array}{r}0 \\
60 \\
75\end{array}$ & $\begin{array}{l}60 \\
75 \\
\cdots\end{array}$ & $\begin{array}{l}112 \mathrm{TILL} \\
110 \text { SDMN } \\
\text { BEDROCK }\end{array}$ & $\begin{array}{l}\text { TILL } \\
\text { CLAY }\end{array}$ \\
\hline MBW & 165 & -. & 303 & $\cdots$ & $\begin{array}{r}0 \\
60\end{array}$ & $\begin{array}{l}60 \\
\cdots\end{array}$ & $\begin{array}{l}112 \text { TILL } \\
\text { BEDROCK }\end{array}$ & TILL \\
\hline MBW & 166 & - & 263 & - & $\begin{array}{r}0 \\
50\end{array}$ & $\begin{array}{l}50 \\
\cdots\end{array}$ & $\begin{array}{l}\text { 112TILL } \\
\text { BEDROCK }\end{array}$ & TILL \\
\hline MBW & 167 & - & 164 & $\cdots$ & $\begin{array}{r}0 \\
20 \\
65\end{array}$ & $\begin{array}{l}20 \\
65\end{array}$ & $\begin{array}{l}112 \mathrm{TILI} \\
110 \mathrm{SDMN} \\
\text { BEDROCK }\end{array}$ & $\begin{array}{l}\text { TILL } \\
\text { CLAY }\end{array}$ \\
\hline
\end{tabular}


Table 2-1. Stratigraphic logs of wells and borings in the Saco and Ossipee River Basins, eastcentral New Hampshire--Continued

\begin{tabular}{|c|c|c|c|c|c|c|c|c|}
\hline \multicolumn{2}{|c|}{$\begin{array}{l}\text { Local } \\
\text { site } \\
\text { No. }\end{array}$} & $\begin{array}{l}\text { Depth } \\
\text { drilled } \\
\text { (feet) }\end{array}$ & $\begin{array}{l}\text { Depth } \\
\text { of } \\
\text { well } \\
\text { (feet) }\end{array}$ & $\begin{array}{l}\text { Depth } \\
\text { to } \\
\text { refusal } \\
\text { (feet) }\end{array}$ & $\begin{array}{l}\text { Depth } \\
\text { to } \\
\text { top } \\
\text { (feet) }\end{array}$ & $\begin{array}{l}\text { Depth } \\
\text { to } \\
\text { bottom } \\
\text { (feet) }\end{array}$ & $\begin{array}{l}\text { Aquifer } \\
\text { code }\end{array}$ & Lithology \\
\hline & & \multicolumn{7}{|c|}{ MADISON- - Continued } \\
\hline MBW & 168 & $\cdots$ & 545 & $-\cdot$ & $\begin{array}{r}0 \\
10\end{array}$ & $\begin{array}{l}10 \\
\cdots\end{array}$ & $\begin{array}{l}\text { 112TILL } \\
\text { BEDROCK }\end{array}$ & TILL \\
\hline MBW & 169 & $\cdots$ & 482 & $\cdots$ & $\begin{array}{r}0 \\
26\end{array}$ & $\begin{array}{l}26 \\
\cdots\end{array}$ & $\begin{array}{l}\text { 112TILL } \\
\text { BEDROCK }\end{array}$ & TILL CLAY, HARDPAN \\
\hline MBW & 170 & $\cdots$ & 402 & $\cdots$ & $\begin{array}{r}0 \\
210\end{array}$ & $\begin{array}{r}210 \\
\cdots\end{array}$ & $\begin{array}{l}\text { 110SDMN } \\
\text { BEDROCK }\end{array}$ & CLAY \\
\hline MBW & 171 & $\cdots$ & 202 & $\cdots$ & $\begin{array}{r}0 \\
55\end{array}$ & $\begin{array}{l}55 \\
\cdots\end{array}$ & $\begin{array}{l}110 \text { SDMN } \\
\text { BEDROCK }\end{array}$ & SGVC \\
\hline MBW & 172 & $-\cdot$ & 652 & $-\cdot$ & $\begin{array}{r}0 \\
85\end{array}$ & $\begin{array}{l}85 \\
\cdots\end{array}$ & $\begin{array}{l}112 \text { TILL } \\
\text { BEDROCK }\end{array}$ & TILL CLAY, HARDPAN \\
\hline MBW & 173 & $\cdots$ & 48 & $\cdots$ & $\begin{array}{r}0 \\
25 \\
39 \\
50\end{array}$ & $\begin{array}{l}25 \\
39 \\
50 \\
--\end{array}$ & $\begin{array}{l}110 \text { SDMN } \\
110 S D M N \\
110 S D M N \\
\text { BEDROCK }\end{array}$ & $\begin{array}{l}\text { SAND } \\
\text { CLAY } \\
\text { SDGL }\end{array}$ \\
\hline MBW & 174 & $-\cdot$ & 525 & $\cdots$ & $\begin{array}{r}0 \\
30\end{array}$ & $\begin{array}{l}30 \\
\cdots\end{array}$ & $\begin{array}{l}112 \text { TILL } \\
\text { BEDROCK }\end{array}$ & TILL \\
\hline MBW & 175 & $\cdots$ & 242 & $-\cdot$ & $\begin{array}{r}0 \\
30\end{array}$ & $\begin{array}{l}30 \\
\cdots\end{array}$ & $\begin{array}{l}\text { 112TILL } \\
\text { BEDROCK }\end{array}$ & TILL \\
\hline MBW & 176 & $-\cdot$ & 423 & $\cdots$ & $\begin{array}{r}0 \\
20\end{array}$ & $\begin{array}{l}20 \\
\cdots\end{array}$ & $\begin{array}{l}112 \text { TILL } \\
\text { BEDROCK }\end{array}$ & TILL \\
\hline MBW & 177 & $\cdots$ & 1200 & $\cdots$ & $\begin{array}{r}0 \\
55\end{array}$ & $\begin{array}{l}55 \\
--\end{array}$ & $\begin{array}{l}110 \text { SDMN } \\
\text { BEDROCK }\end{array}$ & CLAY \\
\hline MBW & 178 & $\cdots$ & 60 & - & $-\cdot$ & $-\cdot$ & $110 \mathrm{SDMN}$ & SDGL \\
\hline MBW & 179 & $-\cdot$ & 340 & $\cdots$ & $\begin{array}{r}0 \\
45\end{array}$ & $\begin{array}{l}45 \\
--\end{array}$ & $\begin{array}{l}\text { 112TILL } \\
\text { BEDROCK }\end{array}$ & TILL \\
\hline MBW & 180 & - & 423 & $\cdots$ & $\begin{array}{r}0 \\
160\end{array}$ & $\begin{array}{c}160 \\
-\cdot\end{array}$ & $\begin{array}{l}\text { 112TrLL } \\
\text { BEDROCK }\end{array}$ & TILL \\
\hline MBW & 181 & $\cdots$ & 420 & $\cdots$ & $\begin{array}{l}0 \\
8\end{array}$ & $\begin{array}{c}8 \\
--\end{array}$ & $\begin{array}{l}112 \text { TILL } \\
\text { BEDROCK }\end{array}$ & TILL \\
\hline MBW & 182 & -- & 402 & - & $\begin{array}{l}0 \\
2\end{array}$ & 2 & $\begin{array}{l}\text { 112TILL } \\
\text { BEDROCK }\end{array}$ & TILL \\
\hline MBW & 183 & $-\cdot$ & 602 & $\cdots$ & $\begin{array}{r}0 \\
10\end{array}$ & $\begin{array}{l}10 \\
\cdots\end{array}$ & $\begin{array}{l}110 \text { SDMN } \\
\text { BEDROCK }\end{array}$ & CLAY \\
\hline MBW & 184 & - & 20 & $-\cdot$ & $\begin{array}{l}\cdots \\
\cdots\end{array}$ & $\begin{array}{l}\cdots \\
\cdots\end{array}$ & $\begin{array}{l}110 S D M N \\
110 S D M N\end{array}$ & $\begin{array}{l}\text { SAND } \\
\text { SDGL }\end{array}$ \\
\hline MBW & 185 & $-\cdot$ & 504 & $-\cdot$ & $\begin{array}{r}0 \\
12\end{array}$ & $\begin{array}{l}12 \\
\cdots\end{array}$ & $\begin{array}{l}112 \text { TILL } \\
\text { BEDROCK }\end{array}$ & TILL \\
\hline MBW & 186 & - & 502 & $-\cdot$ & $\begin{array}{r}0 \\
35\end{array}$ & $\begin{array}{l}35 \\
\cdots\end{array}$ & $\begin{array}{l}\text { 112TILL } \\
\text { BEDROCK }\end{array}$ & TILL CLAY, HARDPAN \\
\hline MBW & 187 & -- & 302 & $-\cdot$ & $\begin{array}{r}0 \\
35\end{array}$ & $\begin{array}{l}35 \\
\cdots\end{array}$ & $\begin{array}{l}110 \text { SDMN } \\
\text { BEDROCK }\end{array}$ & CLAY \\
\hline MBW & 188 & $\cdots$ & 1050 & $\cdots$ & $\begin{array}{r}0 \\
80\end{array}$ & $\begin{array}{l}80 \\
\cdots\end{array}$ & $\begin{array}{l}\text { 112TILL } \\
\text { BEDROCK }\end{array}$ & TILL \\
\hline MBW & 189 & $\cdots$ & 345 & $\cdots$ & $\begin{array}{r}0 \\
65\end{array}$ & $\begin{array}{l}65 \\
\cdots\end{array}$ & $\begin{array}{l}110 \text { SDMN } \\
\text { BEDROCK }\end{array}$ & OTHR \\
\hline MBW & 190 & $\cdots$ & 1020 & $\cdots$ & $\begin{array}{r}0 \\
40 \\
50\end{array}$ & $\begin{array}{l}40 \\
50 \\
--\end{array}$ & $\begin{array}{l}110 \text { SDMN } \\
110 \text { SDMN } \\
\text { BEDROCK }\end{array}$ & $\begin{array}{l}\text { SDCL } \\
\text { OTHR ROTTEN ROCK }\end{array}$ \\
\hline
\end{tabular}


Table 2-1. Stratigraphic logs of wells and borings in the Saco and Ossipee River Basins, eastcentral New Hampshire--Continued

\begin{tabular}{|c|c|c|c|c|c|c|c|c|}
\hline \multicolumn{2}{|c|}{$\begin{array}{l}\text { Local } \\
\text { site } \\
\text { no. }\end{array}$} & $\begin{array}{l}\text { Depth } \\
\text { drilled } \\
\text { (feet) }\end{array}$ & $\begin{array}{l}\text { Depth } \\
\text { of } \\
\text { well } \\
\text { (feet) }\end{array}$ & $\begin{array}{l}\text { Depth } \\
\text { to } \\
\text { refusal } \\
\text { (feet) }\end{array}$ & $\begin{array}{l}\text { Depth } \\
\text { to } \\
\text { top } \\
\text { (feet) }\end{array}$ & $\begin{array}{l}\text { Depth } \\
\text { to } \\
\text { bottom } \\
\text { (feet) }\end{array}$ & $\begin{array}{l}\text { Aquifer } \\
\text { code }\end{array}$ & Lithology \\
\hline & & \multicolumn{7}{|c|}{ MaDISON - - Continued } \\
\hline MBW & 191 & $\cdots$ & 502 & $\cdots$ & $\begin{array}{r}0 \\
20 \\
85\end{array}$ & $\begin{array}{l}20 \\
85 \\
\cdots\end{array}$ & $\begin{array}{l}\text { 112TILL } \\
110 \text { SDMN } \\
\text { BEDROCK }\end{array}$ & $\begin{array}{l}\text { TILL } \\
\text { CLAY }\end{array}$ \\
\hline MBW & 192 & - & 422 & $\cdots$ & $\begin{array}{r}0 \\
100\end{array}$ & 100 & $\begin{array}{l}110 \text { SDMN } \\
\text { BEDROCK }\end{array}$ & OTHR \\
\hline MBW & 193 & $-\cdot$ & 422 & - & $\begin{array}{r}0 \\
95\end{array}$ & $\begin{array}{l}95 \\
-.\end{array}$ & $\begin{array}{l}\text { 112TILL } \\
\text { BEDROCK }\end{array}$ & TILL \\
\hline MBW & 194 & $\cdots$ & 242 & $-\cdot$ & $\begin{array}{r}- \\
0 \\
75\end{array}$ & $\begin{array}{l}-. \\
75 \\
--\end{array}$ & $\begin{array}{l}110 \text { SDMN } \\
110 \text { SDMN } \\
\text { BEDROCK }\end{array}$ & $\begin{array}{l}\text { SAND } \\
\text { SDCL }\end{array}$ \\
\hline MBW & 195 & $\cdots$ & 282 & - & $\begin{array}{r}0 \\
52\end{array}$ & $\begin{array}{l}52 \\
\cdots\end{array}$ & $\begin{array}{l}110 \text { SDMN } \\
\text { BEDROCK }\end{array}$ & SDGL \\
\hline MBW & 196 & -- & 17 & $\cdots$ & $\begin{array}{r}0 \\
28\end{array}$ & $\begin{array}{l}28 \\
\cdots\end{array}$ & $\begin{array}{l}110 \text { SDMN } \\
112 \text { TILL }\end{array}$ & $\begin{array}{l}\text { SDGL } \\
\text { TILL }\end{array}$ \\
\hline MBW & 197 & . & 16 & - & $\begin{array}{r}0 \\
10\end{array}$ & $\begin{array}{l}10 \\
--\end{array}$ & $\begin{array}{l}110 \text { SDMN } \\
112 \mathrm{TILL}\end{array}$ & $\begin{array}{l}\text { SDGL } \\
\text { TILL }\end{array}$ \\
\hline MBW & 198 & - & 303 & $\cdots$ & $\begin{array}{r}0 \\
15\end{array}$ & $\begin{array}{l}15 \\
\cdots\end{array}$ & $\begin{array}{l}110 \text { SDMN } \\
\text { BEDROCK }\end{array}$ & SDGL \\
\hline MBW & 199 & $-\cdot$ & 362 & - & $\begin{array}{r}0 \\
55\end{array}$ & $\begin{array}{l}55 \\
\cdots\end{array}$ & $\begin{array}{l}112 T I L L \\
\text { BEDROCK }\end{array}$ & TILL \\
\hline MBW & 200 & $\cdots$ & 327 & -- & $\begin{array}{r}0 \\
180\end{array}$ & $\begin{array}{r}180 \\
\ldots\end{array}$ & $\begin{array}{l}110 \text { SDMN } \\
\text { BEDROCK }\end{array}$ & OTHR \\
\hline MBW & 201 & $-\cdot$ & 177 & $\cdots$ & $\begin{array}{r}0 \\
18\end{array}$ & $\begin{array}{l}18 \\
-.\end{array}$ & $\begin{array}{l}\text { 112TILL } \\
\text { BEDROCK }\end{array}$ & TILL \\
\hline MBW & 202 & $-\cdot$ & 51 & - & $\begin{array}{r}0 \\
40\end{array}$ & $\begin{array}{l}40 \\
\ldots\end{array}$ & $\begin{array}{l}110 \text { SDMN } \\
\text { BEDROCK }\end{array}$ & SDGL \\
\hline MBW & 203 & $\cdots$ & 48 & $-\cdot$ & $\cdots$ & $\cdots$ & $110 \mathrm{SDMN}$ & SDGL \\
\hline MBW & 204 & $\cdots$ & 525 & - & $\begin{array}{l}0 \\
2\end{array}$ & $\begin{array}{r}2 \\
-\end{array}$ & $\begin{array}{l}\text { 112TILL } \\
\text { BEDROCK }\end{array}$ & TILL \\
\hline MBW & 205 & . & 190 & - & $\begin{array}{r}0 \\
40\end{array}$ & $\begin{array}{l}40 \\
-\cdots\end{array}$ & $\begin{array}{l}\text { 112TILL } \\
\text { BEDROCK }\end{array}$ & TILL \\
\hline MBW & 206 & - - & 164 & $\cdots$ & $\begin{array}{r}0 \\
50\end{array}$ & $\begin{array}{l}50 \\
\cdots\end{array}$ & $\begin{array}{l}\text { 112TILL } \\
\text { BEDROCK }\end{array}$ & TILL GRAVEL, HARDPAN \\
\hline MBW & 207 & $\cdots$ & 202 & - & $\begin{array}{r}0 \\
12\end{array}$ & $\begin{array}{l}12 \\
\cdots\end{array}$ & $\begin{array}{l}\text { 112TILL } \\
\text { BEDROCK }\end{array}$ & TILL \\
\hline MBW & 208 & - & 522 & - & $\begin{array}{r}0 \\
16\end{array}$ & $\begin{array}{l}16 \\
\cdots\end{array}$ & $\begin{array}{l}110 \text { SDMN } \\
\text { BEDROCK }\end{array}$ & SDGL \\
\hline MBW & 209 & - & 402 & - & $\begin{array}{r}0 \\
18\end{array}$ & $\begin{array}{l}18 \\
--\end{array}$ & $\begin{array}{l}110 \text { SDMN } \\
\text { BEDROCK }\end{array}$ & CLAY \\
\hline MBW & 210 & - & 20 & $-\cdot$ & $\begin{array}{r}0 \\
21\end{array}$ & $\begin{array}{l}21 \\
\cdots\end{array}$ & $\begin{array}{l}110 \mathrm{SDMN} \\
112 \mathrm{TILL}\end{array}$ & $\begin{array}{l}\text { SDGL } \\
\text { TILL SAND, HARDPAN }\end{array}$ \\
\hline MBW & 211 & - & 20 & -. & $\begin{array}{r}0 \\
2 \\
17\end{array}$ & $\begin{array}{r}2 \\
17 \\
\cdots\end{array}$ & $\begin{array}{l}110 \text { SDMN } \\
110 \text { SDMN } \\
\text { BEDROCK }\end{array}$ & $\begin{array}{l}\text { SAND } \\
\text { SDGL }\end{array}$ \\
\hline MBW & 212 & - & 19 & - & $\begin{array}{l}0 \\
3\end{array}$ & $\begin{array}{c}3 \\
--\end{array}$ & $\begin{array}{l}110 \mathrm{SDMN} \\
112 \mathrm{TILL}\end{array}$ & $\begin{array}{l}\text { SDGL } \\
\text { TILL }\end{array}$ \\
\hline MBW & 213 & - & 45 & $-\cdot$ & - & $-\cdot$ & $110 \mathrm{SDMN}$ & SAND \\
\hline MBW & 214 & - & 72 & - & 0 & 4 & $110 \mathrm{SDMN}$ & SDGL \\
\hline
\end{tabular}


Table 2-1. Stratigraphic logs of wells and borings in the Saco and Ossipee River Basins, eastcentral New Hampshire--Continued

\begin{tabular}{|c|c|c|c|c|c|c|c|c|}
\hline \multicolumn{2}{|c|}{$\begin{array}{l}\text { Local } \\
\text { site } \\
\text { No. }\end{array}$} & $\begin{array}{l}\text { Depth } \\
\text { drilled } \\
\text { (feet) }\end{array}$ & $\begin{array}{l}\text { Depth } \\
\text { of } \\
\text { well } \\
\text { (feet) }\end{array}$ & $\begin{array}{l}\text { Depth } \\
\text { to } \\
\text { refusal } \\
\text { (feet) }\end{array}$ & $\begin{array}{l}\text { Depth } \\
\text { to } \\
\text { top } \\
\text { (feet) }\end{array}$ & $\begin{array}{l}\text { Depth } \\
\text { to } \\
\text { bottom } \\
\text { (feet) }\end{array}$ & $\begin{array}{l}\text { Aquifer } \\
\text { code }\end{array}$ & Lithology \\
\hline & & \multicolumn{7}{|c|}{ MDIsON- - Continued } \\
\hline MBW & 215 & $\cdots$ & 300 & $\cdots$ & 4 & $\cdots$ & BEDROCK & \\
\hline MBW & 216 & $\cdots$ & 525 & $\cdots$ & $\begin{array}{r}0 \\
25\end{array}$ & $\begin{array}{l}25 \\
\cdots\end{array}$ & $\begin{array}{l}\text { 110SDMN } \\
\text { BEDROCK }\end{array}$ & OTHR \\
\hline MBW & 217 & $\cdots$ & 162 & -- & $\begin{array}{r}0 \\
180\end{array}$ & $\begin{array}{c}180 \\
-.\end{array}$ & $\begin{array}{l}110 \text { SDMN } \\
\text { BEDROCK }\end{array}$ & SDGL \\
\hline MBW & 218 & $\cdots$ & 525 & $\cdots$ & $\begin{array}{r}0 \\
47\end{array}$ & $\begin{array}{l}47 \\
-\cdot\end{array}$ & $\begin{array}{l}110 \text { SDMN } \\
\text { BEDROCK }\end{array}$ & SDGL \\
\hline MBW & 219 & $-\cdot$ & 37 & $\cdots$ & $\begin{array}{r}0 \\
38\end{array}$ & $\begin{array}{l}38 \\
-\end{array}$ & $\begin{array}{l}110 \text { SDMN } \\
112 \text { TILL }\end{array}$ & $\begin{array}{l}\text { SDGL } \\
\text { TILL }\end{array}$ \\
\hline MBW & 220 & $-\cdot$ & 323 & $\cdots$ & $\begin{array}{r}0 \\
25\end{array}$ & $\begin{array}{l}25 \\
-.\end{array}$ & $\begin{array}{l}110 \text { SDMN } \\
\text { BEDROCK }\end{array}$ & OTHR \\
\hline MBW & 221 & - & 122 & $-\cdot$ & $\begin{array}{r}0 \\
60\end{array}$ & $\begin{array}{l}60 \\
--\end{array}$ & $\begin{array}{l}110 \text { SDMN } \\
\text { BEDROCK }\end{array}$ & SDGL \\
\hline MBW & 222 & $\cdots$ & 322 & $\cdots$ & $\begin{array}{r}0 \\
175\end{array}$ & $\begin{array}{r}175 \\
-.\end{array}$ & $\begin{array}{l}110 \text { SDMN } \\
\text { BEDROCK }\end{array}$ & OTHR \\
\hline MBW & 223 & $-\cdot$ & 28 & $\cdots$ & $\begin{array}{r}0 \\
28\end{array}$ & $\begin{array}{l}28 \\
--\end{array}$ & $\begin{array}{l}110 \text { SDMN } \\
112 \text { TILL }\end{array}$ & $\begin{array}{l}\text { SDGL } \\
\text { TILL }\end{array}$ \\
\hline MBW & 224 & -- & 422 & $-\cdot$ & 78 & $-\cdot$ & BEDROCK & \\
\hline MBW & 225 & $\cdots$ & 223 & -- & $\begin{array}{r}0 \\
50\end{array}$ & $\begin{array}{l}50 \\
--\end{array}$ & $\begin{array}{l}110 \text { SDMN } \\
\text { BEDROCK }\end{array}$ & OTHR \\
\hline MBW & 226 & $-\cdot$ & 75 & - & $\cdots$ & $\cdots$ & 110 SDMN & SDGL \\
\hline MBW & 227 & - & 53 & -- & $\cdots$ & $-\cdot$ & 110 SDMN & SDGL \\
\hline MBW & 228 & -- & 49 & - & $\cdots$ & $-\cdot$ & 110 SDMN & SDGL \\
\hline MBW & 229 & $\cdots$ & 72 & $-\cdot$ & - & 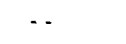 & 110 SDMN & SDGL \\
\hline MBW & 230 & $-\cdot$ & 526 & $-\cdot$ & $\begin{array}{r}0 \\
140\end{array}$ & $\begin{array}{r}140 \\
\cdots\end{array}$ & $\begin{array}{l}110 \text { SDMN } \\
\text { BEDROCK }\end{array}$ & OTHR \\
\hline MBW & 231 & $-\cdot$ & 362 & - & $\begin{array}{r}0 \\
50\end{array}$ & $\begin{array}{l}50 \\
\cdots\end{array}$ & $\begin{array}{l}110 \text { SDMN } \\
\text { BEDROCK }\end{array}$ & SDCL \\
\hline MBW & 232 & $-\cdot$ & 23 & $\cdots$ & $\cdots$ & -. & 112TILL & TILL GRAVEL, HARDPAN \\
\hline MBW & 233 & -- & 523 & - & $\begin{array}{r}0 \\
100\end{array}$ & $\begin{array}{r}100 \\
\cdots\end{array}$ & $\begin{array}{l}\text { 112TILL } \\
\text { BEDROCK }\end{array}$ & TILL \\
\hline MBW & 234 & $\cdots$ & 60 & -- & $\cdots$ & $\cdots$ & $110 \mathrm{SDMN}$ & SDGL \\
\hline MBW & 235 & - & 352 & $-\cdot$ & $\begin{array}{r}0 \\
205\end{array}$ & $\begin{array}{c}205 \\
\cdots\end{array}$ & $\begin{array}{l}\text { 112TILL } \\
\text { BEDROCK }\end{array}$ & TILL SAND, CLAY, HARDPAN \\
\hline MBW & 236 & $\cdots$ & 73 & $\cdots$ & - & $\cdots$ & 110 SDMN & SDGL \\
\hline MBW & 237 & -- & 63 & -- & $\begin{array}{r}0 \\
35\end{array}$ & $\begin{array}{l}35 \\
\cdots\end{array}$ & $\begin{array}{l}110 \mathrm{SDMN} \\
110 \mathrm{SDMN}\end{array}$ & $\begin{array}{l}\text { SDGL } \\
\text { SAND }\end{array}$ \\
\hline MBW & 238 & $\cdots$ & 952 & $-\cdot$ & $\begin{array}{r}0 \\
80 \\
140 \\
189\end{array}$ & $\begin{array}{r}80 \\
140 \\
189 \\
\cdots\end{array}$ & $\begin{array}{l}110 \mathrm{SDMN} \\
110 \mathrm{SDMN} \\
110 \mathrm{SDMN} \\
\text { BEDROCK }\end{array}$ & $\begin{array}{l}\text { SAND } \\
\text { SGVC } \\
\text { CLAY }\end{array}$ \\
\hline MBW & 239 & $\cdots$ & 803 & $\cdots$ & 5 & $\cdots$ & BEDROCK & \\
\hline MBW & 240 & -- & 200 & -- & $\begin{array}{r}0 \\
140\end{array}$ & 140 & $\begin{array}{l}110 \text { SDMN } \\
\text { BEDROCK }\end{array}$ & SDGL \\
\hline MBW & 241 & $\cdots$ & 73 & - & - & - & $110 \mathrm{SDMN}$ & SDGL \\
\hline
\end{tabular}


Table 2-1. Stratigraphic logs of wells and borings in the Saco and Ossipee River Basins, eastcentral New Hampshire--Continued

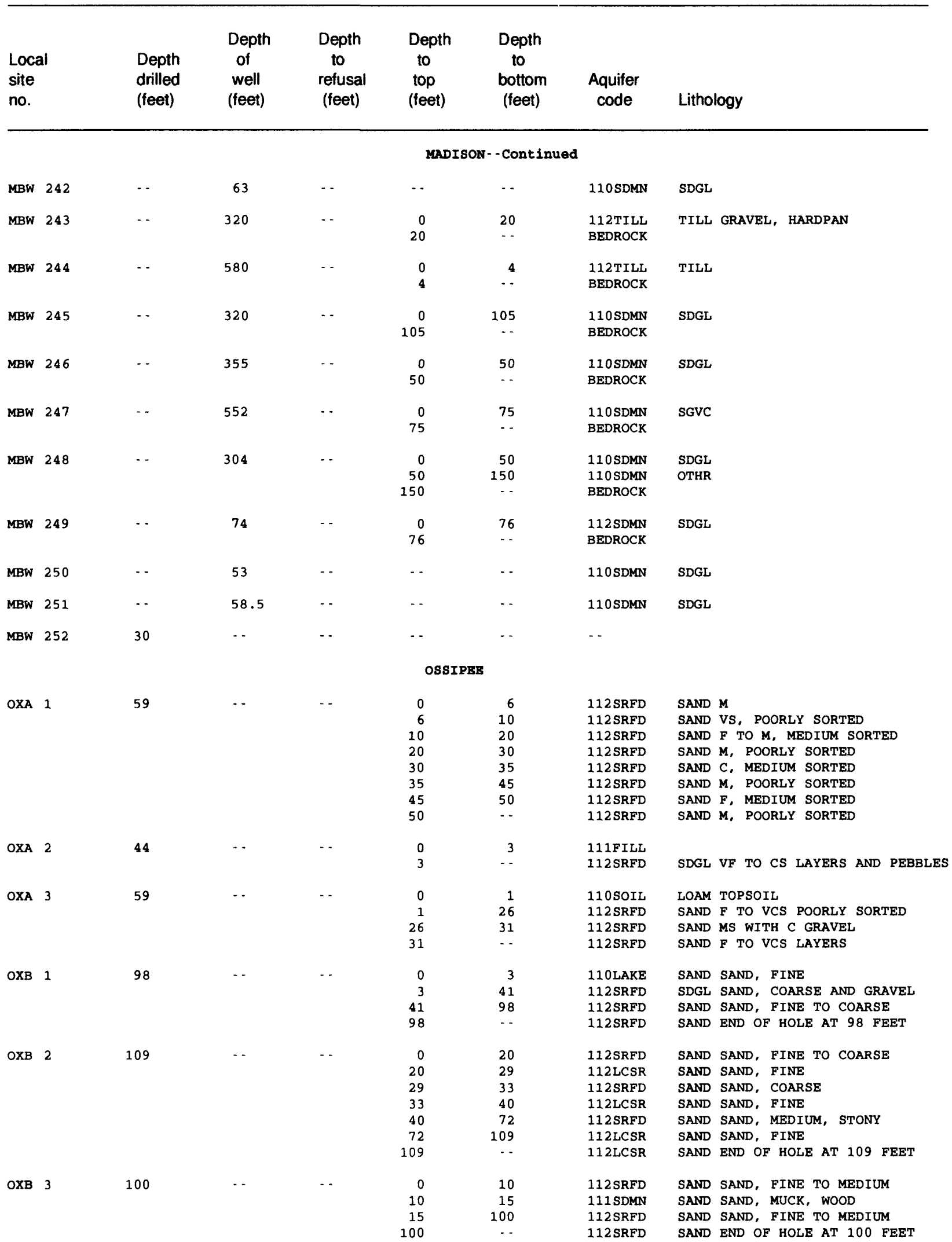


Table 2-1. Stratigraphic logs of wells and borings in the Saco and Ossipee River Basins, eastcentral New Hampshire--Continued

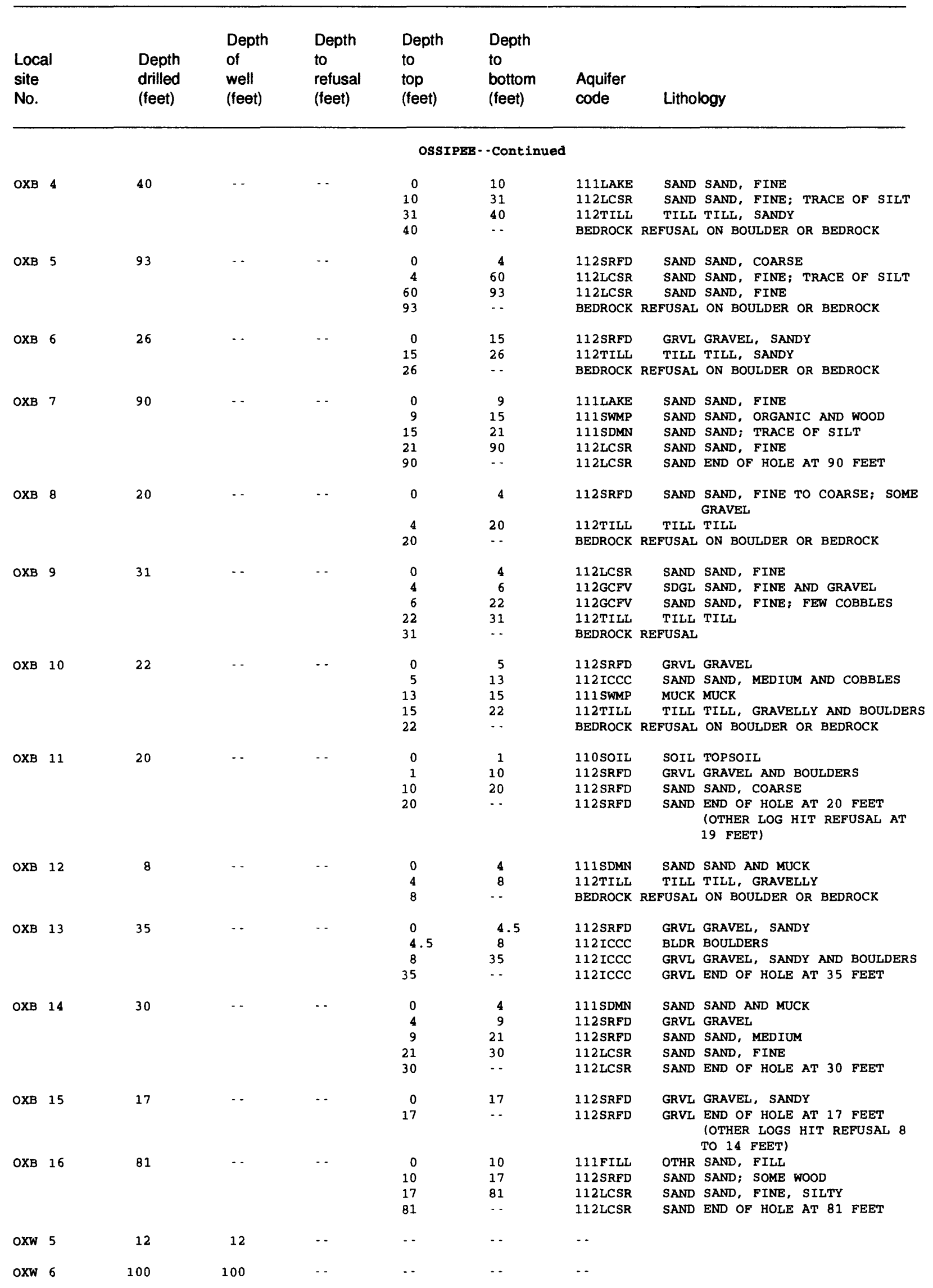


Table 2-1. Stratigraphic logs of wells and borings in the Saco and Osslpee Rlver BasIns, eastcentrai New Hampshire--Contlnued

\begin{tabular}{|c|c|c|c|c|c|c|c|c|}
\hline \multicolumn{2}{|c|}{$\begin{array}{l}\text { Local } \\
\text { site } \\
\text { no. }\end{array}$} & $\begin{array}{l}\text { Depth } \\
\text { drilled } \\
\text { (feet) }\end{array}$ & $\begin{array}{l}\text { Depth } \\
\text { of } \\
\text { well } \\
\text { (feet) }\end{array}$ & $\begin{array}{l}\text { Depth } \\
\text { to } \\
\text { refusal } \\
\text { (feet) }\end{array}$ & $\begin{array}{l}\text { Depth } \\
\text { to } \\
\text { top } \\
\text { (feet) }\end{array}$ & $\begin{array}{l}\text { Depth } \\
\text { to } \\
\text { bottom } \\
\text { (feet) }\end{array}$ & $\begin{array}{l}\text { Aquifer } \\
\text { code }\end{array}$ & Lithology \\
\hline & & \multicolumn{7}{|c|}{ OSSIPEE--Continued } \\
\hline oxw & 7 & 25 & 25 & $\cdots$ & - & - & $-\cdot$ & \\
\hline oxw & 8 & 40 & 40 & $-\cdot$ & - & - & - & \\
\hline oxw & 9 & 48 & 48 & $\cdots$ & - & - & - & \\
\hline oxw & 10 & 260 & 250 & $\cdots$ & - & - & $\cdots$ & \\
\hline oxw & 11 & 13 & 13 & $-\cdot$ & - & $-\cdot$ & - & \\
\hline oxw & 12 & 12.5 & 12.5 & $\cdots$ & $-\cdot$ & - & $\cdots$ & \\
\hline oxw & 13 & 9.8 & 9.8 & $\cdots$ & $-\cdot$ & $\cdots$ & $-\cdot$ & \\
\hline oxw & 15 & 18 & 18 & $\cdots$ & - & $\cdots$ & - & \\
\hline oxw & 16 & 17.5 & 17.5 & $\cdots$ & - & $\cdots$ & - & \\
\hline oxw & 18 & 15 & 15 & $\cdots$ & - & - & - & \\
\hline oxw & 19 & 20 & 20 & - & - & - & -. & \\
\hline oxw & 20 & 250 & $\cdots$ & - & - & $\cdots$ & - - & \\
\hline oxw & 22 & 20 & 20 & $\cdots$ & $\cdots$ & $\cdots$ & $-\cdot$ & \\
\hline oxw & 23 & 327 & $\cdots$ & $\cdots$ & - & - & $\cdots$ & \\
\hline oxw & 24 & 325 & - & - & - & $\cdots$ & - & \\
\hline oxw & 25 & 8.6 & 8.6 & $\cdots$ & - & $\cdots$ & - & \\
\hline $\mathrm{OXW}$ & 26 & 75 & 75 & - & - & - & - & \\
\hline $\mathrm{oxw}$ & 27 & 230 & - & - & - & - & - & \\
\hline oxw & 29 & 510 & -. & - & - & $\cdots$ & - & \\
\hline oXw & 30 & 554 & - & $\cdots$ & - & - & - & \\
\hline oxw & 31 & 15 & 15 & $\cdots$ & - & - & $\cdots$ & \\
\hline oxw & 32 & 69 & 69 & - & - & - & - & \\
\hline oxw & 33 & 25 & 25 & $\cdots$ & $\cdots$ & $\cdots$ & $\cdots$ & \\
\hline oxw & 34 & 114 & 69 & $\cdots$ & $\begin{array}{r}0 \\
15 \\
89\end{array}$ & $\begin{array}{l}15 \\
89 \\
--\end{array}$ & $\begin{array}{l}112 \mathrm{SRFD} \\
112 \mathrm{SRFD} \\
112 \mathrm{SRFD}\end{array}$ & $\begin{array}{l}\text { SDGL C TO CVS AND GRANUALS } \\
\text { SAND LAYERS FS TO VCS } \\
\text { SAND VFS TO FS WITH SILT }\end{array}$ \\
\hline oxw & 35 & 71 & 18.5 & 71 & $\begin{array}{r}0 \\
26 \\
68\end{array}$ & $\begin{array}{l}26 \\
68 \\
\cdots\end{array}$ & $\begin{array}{l}112 \text { SRFD } \\
112 \text { SRFD } \\
112 \text { TILL }\end{array}$ & $\begin{array}{l}\text { SDGL M TO VCS AND. PEBBLES } \\
\text { SDST LAYERS VF TO MS AND SILT } \\
\text { TILL }\end{array}$ \\
\hline OXW & 36 & 103 & 90 & 103 & $\begin{array}{l}0 \\
83 \\
98\end{array}$ & $\begin{array}{l}83 \\
98 \\
\cdots\end{array}$ & $\begin{array}{l}112 \mathrm{SRFD} \\
112 \mathrm{SRFD} \\
112 \mathrm{TILL}\end{array}$ & $\begin{array}{l}\text { SDGL } M \text { TO VCS, PEBBLES AND } \\
\text { COBBLES WITH SILT } \\
\text { SAND C TO VCS } \\
\text { TILL }\end{array}$ \\
\hline $\mathrm{OXW}$ & 37 & 94 & 49 & 94 & $\begin{array}{l}0 \\
23 \\
56.5 \\
78\end{array}$ & $\begin{array}{l}23 \\
56.5 \\
78 \\
\cdots\end{array}$ & $\begin{array}{l}112 \mathrm{SRFD} \\
112 \mathrm{SRFD} \\
112 \mathrm{GLCL} \\
112 \mathrm{SRFD}\end{array}$ & $\begin{array}{l}\text { SAND LAYERS OF C TO VCS } \\
\text { SDGL LAYERS C TO VCS AND PEBBLES } \\
\text { GRDS DIAMICTON SILT TO PEBBLES } \\
\text { SDGL CS TO } \\
\text { PEBBLES, SILTY, DIAMICTON } \\
\text { AND CS LAYERS }\end{array}$ \\
\hline
\end{tabular}


Table 2-1. Stratigraphic logs of wells and borings in the Saco and Ossipee River Basins, eastcentral New Hampshire--Contlnued

\begin{tabular}{|c|c|c|c|c|c|c|c|c|}
\hline \multicolumn{2}{|c|}{$\begin{array}{l}\text { Local } \\
\text { site } \\
\text { No. }\end{array}$} & $\begin{array}{l}\text { Depth } \\
\text { drilled } \\
\text { (feet) }\end{array}$ & $\begin{array}{l}\text { Depth } \\
\text { of } \\
\text { well } \\
\text { (feet) }\end{array}$ & $\begin{array}{l}\text { Depth } \\
\text { to } \\
\text { refusal } \\
\text { (feet) }\end{array}$ & $\begin{array}{l}\text { Depth } \\
\text { to } \\
\text { top } \\
\text { (feet) }\end{array}$ & $\begin{array}{l}\text { Depth } \\
\text { to } \\
\text { bottom } \\
\text { (feet) }\end{array}$ & $\begin{array}{l}\text { Aquifer } \\
\text { code }\end{array}$ & Lithology \\
\hline & & \multicolumn{7}{|c|}{ OSSIPEE $\cdot \cdot$ Continued } \\
\hline oxw & 38 & 115 & 115 & $\cdots$ & $\begin{array}{r}0 \\
25 \\
53\end{array}$ & $\begin{array}{l}25 \\
53 \\
97\end{array}$ & $\begin{array}{l}112 \text { SRFD } \\
112 \text { SRFD } \\
112 \text { SRFD }\end{array}$ & $\begin{array}{l}\text { SAND } M \text { OVER F TO VF } \\
\text { SDGL LAYERS M TO VCS AND PEBBLES } \\
\text { SDGL SILTY SAND AND } \\
\text { PEBBLES, LAYERS F TO CS TO } \\
\text { C TO VCS }\end{array}$ \\
\hline & & & & & $\begin{array}{r}97 \\
104\end{array}$ & $\begin{array}{r}104 \\
--\end{array}$ & $\begin{array}{l}112 \mathrm{GLCL} \\
112 \mathrm{SRFD}\end{array}$ & $\begin{array}{l}\text { GRDS DIAMICTON } \\
\text { SDGL MS TO C TO VCS AND PEBBLES }\end{array}$ \\
\hline oxw & 40 & $\cdots$ & 40 & $\cdot-$ & $\begin{array}{r}0 \\
20\end{array}$ & $\begin{array}{l}20 \\
\cdots\end{array}$ & $\begin{array}{l}110 \text { SDMN } \\
110 \text { SDMN }\end{array}$ & $\begin{array}{l}\text { SAND } \\
\text { SDGL }\end{array}$ \\
\hline oxw & 41 & $-\cdot$ & 503 & $\cdot-$ & $\begin{array}{r}0 \\
67\end{array}$ & $\begin{array}{l}67 \\
\cdots\end{array}$ & $\begin{array}{l}110 \text { SDMN } \\
\text { BEDROCK }\end{array}$ & SAND \\
\hline oxw & 42 & $\cdots$ & 585 & $\cdots$ & $\begin{array}{r}0 \\
35\end{array}$ & 35 & $\begin{array}{l}110 \text { SDMN } \\
\text { BEDROCK }\end{array}$ & SAND \\
\hline oxw & 43 & $\cdots$ & 753 & $\cdot-$ & $\begin{array}{r}0 \\
35\end{array}$ & $\begin{array}{l}35 \\
\cdots\end{array}$ & $\begin{array}{l}\text { 110SDMN } \\
\text { BEDROCK }\end{array}$ & SAND \\
\hline $\mathrm{OXW}$ & 44 & $\cdots$ & 72 & $\cdots$ & $\begin{array}{r}0 \\
10 \\
62 \\
87\end{array}$ & $\begin{array}{l}10 \\
62 \\
87 \\
-\end{array}$ & $\begin{array}{l}110 \text { SDMN } \\
110 \text { SDMN } \\
110 \text { SDMN } \\
\text { BEDROCK }\end{array}$ & $\begin{array}{l}\text { SDGL } \\
\text { SAND } \\
\text { SDGL }\end{array}$ \\
\hline oxw & 45 & $\cdots$ & 62 & $\cdots$ & $\begin{array}{r}0 \\
12 \\
53 \\
57 \\
89\end{array}$ & $\begin{array}{l}12 \\
53 \\
57 \\
89 \\
--\end{array}$ & $\begin{array}{l}110 \text { SDMN } \\
110 \text { SDMN } \\
110 \text { SDMN } \\
110 \text { SDMN } \\
\text { BEDROCK }\end{array}$ & $\begin{array}{l}\text { SDGL } \\
\text { SAND } \\
\text { SDGL } \\
\text { SAND }\end{array}$ \\
\hline oxw & 46 & $\cdots$ & 75.6 & $\cdots$ & $\begin{array}{r}0 \\
20 \\
35 \\
69 \\
89\end{array}$ & $\begin{array}{l}20 \\
35 \\
69 \\
89 \\
--\end{array}$ & $\begin{array}{l}110 \text { SDMN } \\
110 \text { SDMN } \\
110 \text { SDMN } \\
110 \text { SDMN } \\
\text { BEDROCK }\end{array}$ & $\begin{array}{l}\text { SAND } \\
\text { SDGL } \\
\text { SAND } \\
\text { SDGL }\end{array}$ \\
\hline oxw & 47 & $\cdots$ & 113 & $\cdots$ & $\cdots$ & $\cdot \cdot$ & $\cdots$ & \\
\hline oxw & 48 & $\cdots$ & 40 & $\cdots$ & -. & $\cdots$ & 110 SDMN & SAND \\
\hline oxw & 49 & $\cdots$ & 540 & $\cdots$ & $\begin{array}{r}0 \\
120\end{array}$ & 120 & $\begin{array}{l}\text { 112TILL } \\
\text { BEDROCK }\end{array}$ & TILL SAND, CLAY, HARDPAN \\
\hline oxw & 50 & $\cdots$ & 253 & $\cdots$ & $\begin{array}{r}0 \\
195\end{array}$ & $\begin{array}{r}195 \\
-\cdot\end{array}$ & $\begin{array}{l}\text { 112TILL } \\
\text { BEDROCK }\end{array}$ & TILL CLAY, HARDPAN \\
\hline $\mathrm{oxw}$ & 51 & - & 552 & $\cdots$ & $\begin{array}{r}0 \\
277\end{array}$ & $\begin{array}{r}277 \\
\cdots\end{array}$ & $\begin{array}{l}110 \text { SDMN } \\
\text { BEDROCK }\end{array}$ & SDCL \\
\hline OXW & 52 & $\cdots$ & 377 & $\cdots$ & $\begin{array}{r}0 \\
83\end{array}$ & $\begin{array}{l}83 \\
\cdots\end{array}$ & $\begin{array}{l}110 \text { SDMN } \\
\text { BEDROCK }\end{array}$ & SAND \\
\hline $\mathrm{oxw}$ & 53 & $\cdots$ & 800 & - & $\begin{array}{r}0 \\
120\end{array}$ & $\begin{array}{r}120 \\
\cdots\end{array}$ & $\begin{array}{l}\text { 112TILL } \\
\text { BEDROCK }\end{array}$ & TILL SAND, CLAY, HARDPAN \\
\hline oxw & 54 & $\cdots$ & 61 & $\cdots$ & - & $\cdots$ & $110 \mathrm{SDMN}$ & SAND \\
\hline oxw & 55 & $\cdots$ & 312 & $\cdots$ & $\begin{array}{r}0 \\
22\end{array}$ & $\begin{array}{l}22 \\
\cdots\end{array}$ & $\begin{array}{l}110 \text { SDMN } \\
\text { BEDROCK }\end{array}$ & SAND \\
\hline oxw & 56 & $\cdots$ & 237 & $\cdots$ & $\begin{array}{r}0 \\
25\end{array}$ & $\begin{array}{l}25 \\
--\end{array}$ & $\begin{array}{l}110 \text { SDMN } \\
\text { BEDROCK }\end{array}$ & SAND \\
\hline oxw & 57 & $\cdots$ & 32 & $\cdots$ & $\cdots$ & $\cdot-$ & $110 \mathrm{SDMN}$ & SAND \\
\hline oxw & 58 & $\cdots$ & 600 & $\cdots$ & $\begin{array}{r}0 \\
250\end{array}$ & $\begin{array}{r}250 \\
-\cdot\end{array}$ & $\begin{array}{l}110 \text { SDMN } \\
\text { BEDROCK }\end{array}$ & SAND \\
\hline
\end{tabular}


Table 2-1. Stratigraphic logs of wells and borings in the Saco and Ossipee River Basins, eastcentral New Hampshire--Continued

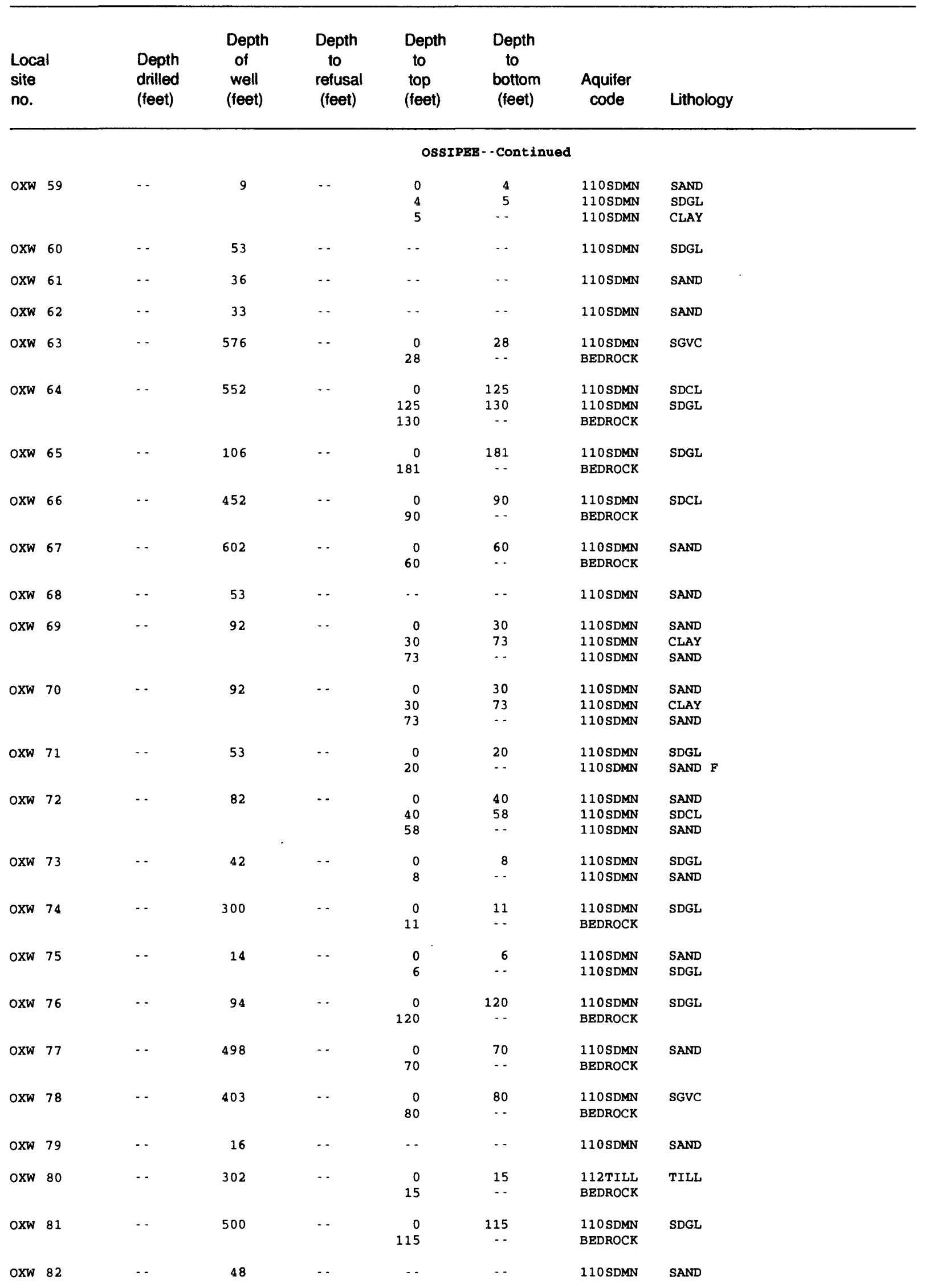


Table 2-1. Stratigraphic logs of wells and borings in the Saco and Ossipee River Basins, eastcentral New Hampshire--Continued

\begin{tabular}{|c|c|c|c|c|c|c|c|c|}
\hline \multicolumn{2}{|c|}{$\begin{array}{l}\text { Local } \\
\text { site } \\
\text { No. }\end{array}$} & $\begin{array}{l}\text { Depth } \\
\text { drilled } \\
\text { (feet) }\end{array}$ & $\begin{array}{l}\text { Depth } \\
\text { of } \\
\text { well } \\
\text { (feet) }\end{array}$ & $\begin{array}{l}\text { Depth } \\
\text { to } \\
\text { refusal } \\
\text { (feet) }\end{array}$ & $\begin{array}{l}\text { Depth } \\
\text { to } \\
\text { top } \\
\text { (feet) }\end{array}$ & $\begin{array}{l}\text { Depth } \\
\text { to } \\
\text { bottom } \\
\text { (feet) }\end{array}$ & $\begin{array}{l}\text { Aquifer } \\
\text { code }\end{array}$ & Lithology \\
\hline & & \multicolumn{7}{|c|}{ OSSIPEE - - Continued } \\
\hline oxw & 83 & $\cdots$ & 11 & - & $\begin{array}{r}0 \\
12\end{array}$ & 12 & $\begin{array}{l}110 \text { SDMN } \\
110 \text { SDMN }\end{array}$ & $\begin{array}{l}\text { SAND } \\
\text { SDCL }\end{array}$ \\
\hline oxw & 84 & $\cdots$ & 264 & $-\cdot$ & $\begin{array}{r}0 \\
60\end{array}$ & 60 & $\begin{array}{l}\text { 110SDMN } \\
\text { BEDROCK }\end{array}$ & SDGL \\
\hline oxw & 85 & - & 91 & $-\cdot$ & $\begin{array}{r}0 \\
132\end{array}$ & $\begin{array}{r}132 \\
--\end{array}$ & $\begin{array}{l}\text { 110SDMN } \\
\text { BEDROCK }\end{array}$ & SAND \\
\hline oxw & 86 & $\cdots$ & 39 & $\cdots$ & $\cdots$ & $\cdots$ & $110 \mathrm{SDMN}$ & SAND \\
\hline oxw & 87 & -- & 423 & $\cdots$ & $\begin{array}{r}0 \\
100\end{array}$ & $\begin{aligned} 100 \\
\ldots\end{aligned}$ & $\begin{array}{l}110 \text { SDMN } \\
\text { BEDROCK }\end{array}$ & SDGL \\
\hline oxw & 88 & $\cdots$ & 180 & $\cdots$ & $\begin{array}{r}0 \\
130\end{array}$ & $\begin{array}{r}130 \\
\ldots\end{array}$ & $\begin{array}{l}\text { 110SDMN } \\
\text { BEDROCK }\end{array}$ & SAND \\
\hline oxw & 89 & - & 690 & $\cdots$ & $\begin{array}{r}\cdots \\
72\end{array}$ & $\overline{72}$ & $\begin{array}{l}110 \text { SDMN } \\
110 \text { SDMN } \\
\text { BEDROCK }\end{array}$ & $\begin{array}{l}\text { SAND } \\
\text { CLAY }\end{array}$ \\
\hline oxw & 90 & -. & 41 & $\cdots$ & $\cdots$ & $\cdots$ & $110 \mathrm{SDMN}$ & SAND \\
\hline oxw & 91 & $\cdots$ & 50 & $\cdots$ & $\cdots$ & $\cdots$ & $110 \mathrm{SDMN}$ & SAND \\
\hline oxw & 92 & - & 93 & $\cdots$ & $\begin{array}{r}0 \\
30 \\
75\end{array}$ & $\begin{array}{l}30 \\
75 \\
\cdots\end{array}$ & $\begin{array}{l}110 \mathrm{SDMN} \\
110 \mathrm{SDMN} \\
110 \mathrm{SDMN}\end{array}$ & $\begin{array}{l}\text { SDCL } \\
\text { CLAY } \\
\text { SAND }\end{array}$ \\
\hline oxw & 93 & $\cdots$ & 63 & $\cdots$ & $\begin{array}{r}0 \\
20 \\
50 \\
85\end{array}$ & $\begin{array}{l}20 \\
50 \\
85 \\
-\cdots\end{array}$ & $\begin{array}{l}110 \text { SDMN } \\
110 \text { SDMN } \\
110 \text { SDMN } \\
110 \text { SDMN }\end{array}$ & $\begin{array}{l}\text { SAND } \\
\text { CLAY } \\
\text { SAND } \\
\text { SDGL }\end{array}$ \\
\hline oxw & 94 & . & 44 & $\cdots$ & $\begin{array}{r}0 \\
15\end{array}$ & $\begin{array}{l}15 \\
-\end{array}$ & $\begin{array}{l}110 \text { SDMN } \\
112 \text { TILL }\end{array}$ & $\begin{array}{l}\text { SDGL } \\
\text { TILL SAND, GRAVEL, HARDPAN }\end{array}$ \\
\hline oxw & 95 & $\cdots$ & 60 & $\cdots$ & $\cdots$ & $\cdots$ & $110 \mathrm{SDMN}$ & SAND \\
\hline oxw & 96 & $\cdots$ & 380 & $\cdots$ & $\begin{array}{r}0 \\
39\end{array}$ & $\begin{array}{l}39 \\
--\end{array}$ & $\begin{array}{l}\text { 110SDMN } \\
\text { BEDROCK }\end{array}$ & SAND \\
\hline oxw & 97 & $\cdots$ & 502 & $\cdots$ & $\begin{array}{r}0 \\
170^{\circ}\end{array}$ & 170 & $\begin{array}{l}\text { 110SDMN } \\
\text { BEDROCK }\end{array}$ & SDCL \\
\hline oxw & 98 & - & 894 & $\cdots$ & $\begin{array}{r}0 \\
89\end{array}$ & $\begin{array}{l}89 \\
\cdots\end{array}$ & $\begin{array}{l}110 \text { SDMN } \\
\text { BEDROCK }\end{array}$ & SDCL \\
\hline oxw & 99 & $-\cdot$ & 575 & $\cdots$ & $\begin{array}{r}0 \\
35 \\
80\end{array}$ & $\begin{array}{l}35 \\
80 \\
-\end{array}$ & $\begin{array}{l}110 \text { SDMN } \\
110 \text { SDMN } \\
\text { BEDROCK }\end{array}$ & $\begin{array}{l}\text { SAND } \\
\text { OTHR }\end{array}$ \\
\hline oxw & 100 & $\cdots$ & 202 & $\cdots$ & $\begin{array}{r}0 \\
72\end{array}$ & $\begin{array}{l}72 \\
\cdots\end{array}$ & $\begin{array}{l}110 \text { SDMN } \\
\text { BEDROCK }\end{array}$ & SDGL \\
\hline oxw & 101 & - & 802 & $\cdots$ & $\begin{array}{r}0 \\
85\end{array}$ & $\begin{array}{l}85 \\
--\end{array}$ & $\begin{array}{l}110 \text { SDMN } \\
\text { BEDROCK }\end{array}$ & CLAY \\
\hline oxw & 102 & $\cdots$ & 100 & $\cdots$ & $\cdots$ & $\cdots$ & $110 \mathrm{SDMN}$ & SAND \\
\hline oxw & 103 & $\cdots$ & 202 & - & $\begin{array}{r}0 \\
88\end{array}$ & 88 & $\begin{array}{l}\text { 110SDMN } \\
\text { BEDROCK }\end{array}$ & SDGL \\
\hline oxw & 104 & - & 140 & $\cdots$ & $\begin{array}{r}0 \\
20 \\
38\end{array}$ & $\begin{array}{l}20 \\
38 \\
-.\end{array}$ & $\begin{array}{l}110 \text { SDMN } \\
110 \text { SDMN } \\
\text { BEDROCK }\end{array}$ & $\begin{array}{l}\text { SAND } \\
\text { SDGL }\end{array}$ \\
\hline oxw & 105 & $\cdots$ & 352 & $\cdots$ & $\begin{array}{r}0 \\
210\end{array}$ & $\begin{array}{r}210 \\
\cdots\end{array}$ & $\begin{array}{l}\text { 110SDMN } \\
\text { BEDROCK }\end{array}$ & SGVC \\
\hline oxw & 106 & $\cdots$ & 315 & $\ldots$ & - & $\cdots$ & $\cdots$ & \\
\hline
\end{tabular}


Table 2-1. Stratigraphic logs of wells and borings in the Saco and Ossipee River Basins, eastcentral New Hampshire--Continued

\begin{tabular}{|c|c|c|c|c|c|c|c|c|}
\hline \multicolumn{2}{|c|}{$\begin{array}{l}\text { Local } \\
\text { site } \\
\text { no. }\end{array}$} & $\begin{array}{l}\text { Depth } \\
\text { drilled } \\
\text { (feet) }\end{array}$ & $\begin{array}{l}\text { Depth } \\
\text { of } \\
\text { well } \\
\text { (feet) }\end{array}$ & \multirow{3}{*}{$\begin{array}{c}\begin{array}{c}\text { Depth } \\
\text { to } \\
\text { refusal } \\
\text { (feet) }\end{array} \\
\\
\end{array}$} & \multirow{3}{*}{$\begin{array}{c}\begin{array}{c}\text { Depth } \\
\text { to } \\
\text { top } \\
\text { (feet) }\end{array} \\
\text { ossI } \\
0 \\
6\end{array}$} & \multirow{2}{*}{\multicolumn{2}{|c|}{$\begin{array}{c}\text { Depth } \\
\text { to } \\
\text { bottom } \\
\text { (feet) }\end{array}$}} & \multirow[t]{2}{*}{ Lithology } \\
\hline & & & & & & & & \\
\hline oxw & 107 & $\cdots$ & 51 & & & 6 & $\begin{array}{l}110 \mathrm{SDMN} \\
110 \mathrm{SDMN}\end{array}$ & $\begin{array}{l}\text { SDCL } \\
\text { SDGL }\end{array}$ \\
\hline oxw & 108 & $\cdots$ & 31 & $\cdots$ & $\begin{array}{r}0 \\
32\end{array}$ & $\begin{array}{l}32 \\
\cdots\end{array}$ & $\begin{array}{l}110 \text { SDMN } \\
110 \text { SDMN }\end{array}$ & $\begin{array}{l}\text { SDGL } \\
\text { SAND }\end{array}$ \\
\hline oxw & 109 & $\cdots$ & 84 & $\cdots$ & $\begin{array}{r}0 \\
20 \\
72\end{array}$ & $\begin{array}{l}20 \\
72 \\
-.\end{array}$ & $\begin{array}{l}110 \mathrm{SDMN} \\
110 \mathrm{SDMN} \\
110 \mathrm{SDMN}\end{array}$ & $\begin{array}{l}\text { SAND } \\
\text { CLAY } \\
\text { SAND }\end{array}$ \\
\hline oxw & 110 & $\cdots$ & 31 & $\cdots$ & -- & - & 110 SDMN & SDGL \\
\hline oxw & 111 & $-\cdot$ & 34 & $-\cdot$ & $-\cdot$ & $\cdots$ & $110 \mathrm{SDMN}$ & SAND \\
\hline oxw & 112 & - & 242 & $-\cdot$ & $\begin{array}{r}0 \\
138\end{array}$ & $\begin{array}{r}138 \\
--\end{array}$ & $\begin{array}{l}\text { 112TILL } \\
\text { BEDROCK }\end{array}$ & TILL SAND， GRAVEL， HARDPAN \\
\hline oxw & 113 & $\cdots$ & 421 & $\cdots$ & $\begin{array}{r}0 \\
40 \\
80 \\
140\end{array}$ & $\begin{array}{r}40 \\
80 \\
140 \\
--\end{array}$ & $\begin{array}{l}\text { 112TILL } \\
\text { 110SDMN } \\
\text { BEDROCK } \\
\text { BEDROCK }\end{array}$ & $\begin{array}{l}\text { TILL } \\
\text { CLAY }\end{array}$ \\
\hline oxw & 114 & $\cdots$ & 17 & $\cdots$ & $\begin{array}{r}0 \\
18 \\
25\end{array}$ & $\begin{array}{l}18 \\
25 \\
-\cdot\end{array}$ & $\begin{array}{l}\text { 110SDMN } \\
112 \text { TILL } \\
\text { BEDROCK }\end{array}$ & $\begin{array}{l}\text { SAND } \\
\text { TILL }\end{array}$ \\
\hline oxw & 115 & $\cdots$ & 54 & $\cdots$ & $\cdots$ & $\cdots$ & $=10$ SDMN & SDGL \\
\hline oxw & 116 & $-\cdot$ & 105 & $-\cdot$ & $\begin{array}{r}0 \\
88\end{array}$ & $\begin{array}{l}88 \\
\cdots\end{array}$ & $\begin{array}{l}\text { 112TILL } \\
\text { BEDROCK }\end{array}$ & TILL SAND, HARDPAN \\
\hline oxw & 117 & $\cdots$ & 1120 & $\cdots$ & $\begin{array}{r}0 \\
20\end{array}$ & $\begin{array}{l}20 \\
\cdots\end{array}$ & $\begin{array}{l}110 \text { SDMN } \\
\text { BEDROCK }\end{array}$ & SDGL \\
\hline oxw & 118 & $-\cdot$ & 25 & $\cdots$ & $\begin{array}{r}0 \\
5 \\
10\end{array}$ & $\begin{array}{r}5 \\
10 \\
\cdots\end{array}$ & $\begin{array}{l}110 \text { SDMN } \\
110 \text { SDMN } \\
110 \text { SDMN }\end{array}$ & $\begin{array}{l}\text { SDGL } \\
\text { SDGL } \\
\text { SAND }\end{array}$ \\
\hline oxw & 119 & $\cdots$ & 500 & $\cdots$ & $\begin{array}{r}0 \\
90\end{array}$ & 90 & $\begin{array}{l}110 \text { SDMN } \\
\text { BEDROCK }\end{array}$ & SAND \\
\hline oxw & 120 & $\cdots$ & 43 & $\cdots$ & $\begin{array}{r}0 \\
44\end{array}$ & $\begin{array}{l}44 \\
\cdots\end{array}$ & $\begin{array}{l}110 \text { SDMN } \\
112 \text { TILL }\end{array}$ & $\begin{array}{l}\text { SDGL } \\
\text { TILL GRAVEL, CLAY, HARDPAN }\end{array}$ \\
\hline oxw & 121 & $\cdots$ & 505 & $\cdots$ & $\begin{array}{r}0 \\
121\end{array}$ & 121 & $\begin{array}{l}110 \text { SDMN } \\
\text { BEDROCK }\end{array}$ & SDCL \\
\hline oxw & 122 & $\cdots$ & 515 & $\cdots$ & $\begin{array}{r}0 \\
120 \\
145\end{array}$ & $\begin{array}{r}120 \\
145 \\
\cdots\end{array}$ & $\begin{array}{l}110 \text { SDMN } \\
112 T I L L \\
\text { BEDROCK }\end{array}$ & $\begin{array}{l}\text { SAND } \\
\text { TILL CLAY, HARDPAN }\end{array}$ \\
\hline oxw & 123 & $\cdots$ & 585 & $\cdots$ & $\begin{array}{r}0 \\
35\end{array}$ & $\begin{array}{l}35 \\
--\end{array}$ & $\begin{array}{l}\text { 110SDMN } \\
\text { BEDROCK }\end{array}$ & SDGL \\
\hline oxw & 124 & $\cdots$ & 32 & $\cdots$ & $\begin{array}{r}0 \\
35\end{array}$ & 35 & $\begin{array}{l}110 \mathrm{SDMN} \\
110 \mathrm{SDMN}\end{array}$ & $\begin{array}{l}\text { SDGL } \\
\text { CLAY }\end{array}$ \\
\hline oxw & 125 & $-\cdot$ & 202 & - & $\begin{array}{r}0 \\
20 \\
45\end{array}$ & $\begin{array}{l}20 \\
45 \\
\cdots\end{array}$ & $\begin{array}{l}110 \text { SDMN } \\
110 \text { SDMN } \\
\text { BEDROCK }\end{array}$ & $\begin{array}{l}\text { SDGL } \\
\text { CLAY }\end{array}$ \\
\hline oxw & 126 & $\cdots$ & 202 & - & $\begin{array}{r}0 \\
14\end{array}$ & $\begin{array}{l}14 \\
-\end{array}$ & $\begin{array}{l}110 \text { SDMN } \\
\text { BEDROCK }\end{array}$ & SDGL \\
\hline oxw & 127 & $\cdots$ & 302 & $\cdots$ & $\begin{array}{r}0 \\
10\end{array}$ & $\begin{array}{l}10 \\
\cdots\end{array}$ & $\begin{array}{l}112 \text { TILL } \\
\text { BEDROCK }\end{array}$ & TILL GRAVEL, HARDPAN \\
\hline oxw & 128 & $\cdots$ & 553 & $\cdots$ & $\begin{array}{r}0 \\
80 \\
115\end{array}$ & $\begin{array}{r}80 \\
115 \\
-.\end{array}$ & $\begin{array}{l}110 \text { SDMN } \\
110 \text { SDMN } \\
\text { BEDROCK }\end{array}$ & $\begin{array}{l}\text { SAND } \\
\text { CLAYY }\end{array}$ \\
\hline
\end{tabular}


Table 2-1. Stratigraphic logs of wells and borings in the Saco and Ossipee River Basins, eastcentral New Hampshire--Continued

\begin{tabular}{|c|c|c|c|c|c|c|c|c|}
\hline \multicolumn{2}{|c|}{$\begin{array}{l}\text { Local } \\
\text { site } \\
\text { No. }\end{array}$} & $\begin{array}{l}\text { Depth } \\
\text { drilled } \\
\text { (feet) }\end{array}$ & $\begin{array}{l}\text { Depth } \\
\text { of } \\
\text { well } \\
\text { (feet) }\end{array}$ & $\begin{array}{l}\text { Depth } \\
\text { to } \\
\text { refusal } \\
\text { (feet) }\end{array}$ & $\begin{array}{l}\text { Depth } \\
\text { to } \\
\text { top } \\
\text { (feet) }\end{array}$ & $\begin{array}{l}\text { Depth } \\
\text { to } \\
\text { bottom } \\
\text { (feet) }\end{array}$ & $\begin{array}{l}\text { Aquifer } \\
\text { code }\end{array}$ & Lithology \\
\hline & & \multicolumn{7}{|c|}{ OSSIPER - - Continued } \\
\hline $\mathrm{oxw}$ & 129 & $-\cdot$ & 204 & - & $\begin{array}{r}0 \\
40 \\
65\end{array}$ & $\begin{array}{l}40 \\
65 \\
\cdots\end{array}$ & $\begin{array}{l}110 \text { SDMN } \\
110 \text { SDMN } \\
\text { BEDROCK }\end{array}$ & $\begin{array}{l}\text { SDGL } \\
\text { SAND }\end{array}$ \\
\hline $\mathrm{oxw}$ & 130 & $\cdots$ & 303 & $\cdots$ & $\begin{array}{r}0 \\
25\end{array}$ & $\begin{array}{l}25 \\
--\end{array}$ & $\begin{array}{l}110 \text { SDMN } \\
\text { BEDROCK }\end{array}$ & SAND \\
\hline oxw & 131 & $\cdots$ & 43.5 & $\cdots$ & $\begin{array}{r}0 \\
45\end{array}$ & $\begin{array}{l}45 \\
-\cdot\end{array}$ & $\begin{array}{l}110 \text { SDMN } \\
112 \text { TILL }\end{array}$ & $\begin{array}{l}\text { SAND } \\
\text { TILL }\end{array}$ \\
\hline oxw & 132 & $\cdots$ & 163 & $\cdots$ & $\begin{array}{r}0 \\
20 \\
30\end{array}$ & $\begin{array}{l}20 \\
30 \\
-.\end{array}$ & $\begin{array}{l}110 \text { SDMN } \\
112 \mathrm{TILL} \\
\text { BEDROCK }\end{array}$ & $\begin{array}{l}\text { SAND } \\
\text { TILL }\end{array}$ \\
\hline oxw & 133 & -- & 800 & $-\cdot$ & $\begin{array}{r}0 \\
38\end{array}$ & $\begin{array}{l}38 \\
\cdots\end{array}$ & $\begin{array}{l}110 \text { SDMN } \\
\text { BEDROCK }\end{array}$ & SDCL \\
\hline oxw & 134 & $\cdots$ & 302 & $\cdots$ & $\begin{array}{r}0 \\
169\end{array}$ & $\begin{array}{r}169 \\
\cdots\end{array}$ & $\begin{array}{l}\text { 112TILL } \\
\text { BEDROCK }\end{array}$ & ,TILL SAND, CLAY, HARDPAN \\
\hline oxw & 135 & -- & 253 & $\cdots$ & $\begin{array}{r}0 \\
40\end{array}$ & $\begin{array}{l}40 \\
--\end{array}$ & $\begin{array}{l}110 \text { SDMN } \\
\text { BEDROCK }\end{array}$ & SAND \\
\hline oxw & 136 & $\cdots$ & 58 & $\cdots$ & $\cdots$ & $\cdots$ & $110 \mathrm{SDMN}$ & SAND \\
\hline oxw & 137 & $\cdots$ & 362 & $\cdots$ & $\begin{array}{r}0 \\
30\end{array}$ & $\begin{array}{l}30 \\
--\end{array}$ & $\begin{array}{l}110 \text { SDMN } \\
\text { BEDROCK }\end{array}$ & SAND \\
\hline oxw & 138 & $\cdots$ & 493 & $\cdots$ & $\begin{array}{r}0 \\
160\end{array}$ & $\begin{array}{r}160 \\
\cdots\end{array}$ & $\begin{array}{l}110 \mathrm{SDMN} \\
\text { BEDROCK }\end{array}$ & SAND \\
\hline oxw & 139 & $-\cdot$ & 402 & - & $\begin{array}{r}0 \\
115\end{array}$ & $\begin{array}{r}115 \\
\ldots\end{array}$ & $\begin{array}{l}110 \text { SDMN } \\
\text { BEDROCK }\end{array}$ & SDCL \\
\hline oxw & 140 & . & 127 & $\cdots$ & $\begin{array}{r}0 \\
22\end{array}$ & 22 & $\begin{array}{l}110 \text { SDMN } \\
\text { BEDROCK }\end{array}$ & SAND \\
\hline oxw & 141 & -. & 252 & - & $\begin{array}{r}0 \\
40 \\
88\end{array}$ & $\begin{array}{l}40 \\
88 \\
-\end{array}$ & $\begin{array}{l}\text { 112TILL } \\
112 \mathrm{TILL} \\
\text { BEDROCK }\end{array}$ & $\begin{array}{l}\text { TILL GRAVEL, HARDPAN } \\
\text { TILL CLAY, HARDPAN }\end{array}$ \\
\hline$O X W$ & 142 & - & 113 & $\cdots$ & $\begin{array}{r}0 \\
13\end{array}$ & $\begin{array}{l}13 \\
\cdots\end{array}$ & $\begin{array}{l}110 \text { SDMN } \\
\text { BEDROCK }\end{array}$ & OTHR \\
\hline $\mathrm{OXW}$ & 143 & - & 145 & -. & $\begin{array}{r}0 \\
50\end{array}$ & $\begin{array}{l}50 \\
\cdots\end{array}$ & $\begin{array}{l}110 \mathrm{SDMN} \\
\text { BEDROCK }\end{array}$ & SAND \\
\hline OXW & 144 & $\cdots$ & 304 & - & $\begin{array}{r}0 \\
25\end{array}$ & $\begin{array}{l}25 \\
\cdots\end{array}$ & $\begin{array}{l}110 \text { SDMN } \\
\text { BEDROCK }\end{array}$ & SAND \\
\hline OXW & 145 & - & 652 & $\cdots$ & $\begin{array}{r}0 \\
195\end{array}$ & $\begin{array}{r}195 \\
\cdots\end{array}$ & $\begin{array}{l}110 \mathrm{SDMN} \\
\text { BEDROCK }\end{array}$ & SDCL \\
\hline $\mathrm{OXW}$ & 146 & - - & 442 & -. & $\begin{array}{r}0 \\
100 \\
190\end{array}$ & $\begin{array}{l}100 \\
190\end{array}$ & $\begin{array}{l}110 \text { SDMN } \\
112 \text { TILL } \\
\text { BEDROCK }\end{array}$ & $\begin{array}{l}\text { SDGL } \\
\text { TILL }\end{array}$ \\
\hline oxw & 147 & $\cdots$ & 302 & - & $\begin{array}{r}0 \\
100 \\
135\end{array}$ & $\begin{array}{c}100 \\
135 \\
\ldots\end{array}$ & $\begin{array}{l}110 \mathrm{SDMN} \\
112 \mathrm{TILL} \\
\text { BEDROCK }\end{array}$ & $\begin{array}{l}\text { SAND } \\
\text { TILL }\end{array}$ \\
\hline OXW & 148 & $\cdots$ & 177 & - & $\begin{array}{r}0 \\
48\end{array}$ & 48 & $\begin{array}{l}110 \mathrm{SDMN} \\
\text { BEDROCK }\end{array}$ & SDCL \\
\hline OXW & 149 & - & 177 & $\cdots$ & $\begin{array}{r}0 \\
90 \\
110\end{array}$ & $\begin{array}{r}90 \\
110 \\
\ldots\end{array}$ & $\begin{array}{l}110 \text { SDMN } \\
112 \mathrm{TILL} \\
\text { BEDROCK }\end{array}$ & $\begin{array}{l}\text { SDGL } \\
\text { TILL CLAY, HARDPAN }\end{array}$ \\
\hline oxw & 150 & - & 800 & $\cdots$ & $\begin{array}{r}0 \\
20\end{array}$ & 20 & $\begin{array}{l}110 \text { SDMN } \\
\text { BEDROCK }\end{array}$ & SDGL \\
\hline
\end{tabular}


Table 2-1. Stratigraphic logs of wells and borings in the Saco and Ossipee River Basins, eastcentral New Hampshire--Continued

\begin{tabular}{|c|c|c|c|c|c|c|c|c|}
\hline \multicolumn{2}{|c|}{$\begin{array}{l}\text { Local } \\
\text { site } \\
\text { no. }\end{array}$} & $\begin{array}{l}\text { Depth } \\
\text { drilled } \\
\text { (feet) }\end{array}$ & $\begin{array}{l}\text { Depth } \\
\text { of } \\
\text { well } \\
\text { (feet) }\end{array}$ & $\begin{array}{l}\text { Depth } \\
\text { to } \\
\text { refusal } \\
\text { (feet) }\end{array}$ & $\begin{array}{l}\text { Depth } \\
\text { to } \\
\text { top } \\
\text { (feet) }\end{array}$ & $\begin{array}{l}\text { Depth } \\
\text { to } \\
\text { bottom } \\
\text { (feet) }\end{array}$ & $\begin{array}{l}\text { Aquifer } \\
\text { code }\end{array}$ & Lithology \\
\hline & & \multicolumn{7}{|c|}{ OSSIPEB - - Continued } \\
\hline oxw & 151 & $-\cdot$ & 182 & $\cdots$ & $\begin{array}{r}0 \\
71\end{array}$ & $\begin{array}{l}71 \\
\cdots\end{array}$ & $\begin{array}{l}\text { 112TILL } \\
\text { BEDROCK }\end{array}$ & TILL SAND， GRAVEL， HARDPAN \\
\hline oxw & 152 & $\cdots$ & 422 & $\cdots$ & $\begin{array}{r}0 \\
100 \\
162\end{array}$ & $\begin{array}{r}100 \\
162 \\
\ldots\end{array}$ & $\begin{array}{l}110 \text { SDMN } \\
112 \text { TILL } \\
\text { BEDROCK }\end{array}$ & $\begin{array}{l}\text { SAND } \\
\text { TILL CLAY, HARDPAN }\end{array}$ \\
\hline oxw & 153 & $\cdots$ & 30 & $\cdots$ & $\begin{array}{r}0 \\
32\end{array}$ & $\begin{array}{l}32 \\
\cdots\end{array}$ & $\begin{array}{l}110 \text { SDMN } \\
110 \text { SDMN }\end{array}$ & $\begin{array}{l}\text { SAND } \\
\text { SDCL }\end{array}$ \\
\hline oxw & 154 & $\cdots$ & 162 & $\cdots$ & $\begin{array}{r}0 \\
125\end{array}$ & 125 & $\begin{array}{l}110 \text { SDMN } \\
\text { BEDROCK }\end{array}$ & SDGL \\
\hline oxw & 155 & $\cdots$ & 202 & $\cdots$ & $\begin{array}{r}0 \\
130\end{array}$ & $\begin{array}{r}130 \\
-.\end{array}$ & $\begin{array}{l}110 \text { SDMN } \\
\text { BEDROCK }\end{array}$ & OTHR \\
\hline oxw & 156 & $\cdot-$ & 545 & $-\cdot$ & $\begin{array}{r}0 \\
105\end{array}$ & $\begin{array}{r}105 \\
--\end{array}$ & $\begin{array}{l}110 \text { SDMN } \\
\text { BEDROCK }\end{array}$ & SAND \\
\hline oxw & 157 & $-\cdot$ & 700 & $\cdots$ & 0 & $\cdots$ & $\cdots$ & \\
\hline oxw & 158 & $-\cdot$ & 140 & $\cdots$ & $\begin{array}{r}0 \\
115\end{array}$ & $\begin{array}{r}115 \\
\cdots\end{array}$ & $\begin{array}{l}110 \text { SDMN } \\
\text { BEDROCK }\end{array}$ & SDGL \\
\hline oxw & 159 & $-\cdot$ & 252 & $\cdots$ & $\begin{array}{r}0 \\
16\end{array}$ & 16 & $\begin{array}{l}110 \text { SDMN } \\
\text { BEDROCK }\end{array}$ & SAND \\
\hline $\mathrm{oxw}$ & 160 & $-\cdot$ & 522 & $\cdots$ & $\begin{array}{r}0 \\
47\end{array}$ & $\begin{array}{l}47 \\
\cdots\end{array}$ & $\begin{array}{l}110 \text { SDMN } \\
\text { BEDROCK }\end{array}$ & SAND \\
\hline oxw & 161 & $\cdots$ & 502 & $\cdots$ & $\begin{array}{r}0 \\
122\end{array}$ & 122 & $\begin{array}{l}110 \text { SDMN } \\
\text { BEDROCK }\end{array}$ & SGVC \\
\hline oxw & 162 & $\cdot-$ & 303 & $\cdots$ & $\begin{array}{r}0 \\
120\end{array}$ & 120 & $\begin{array}{l}110 \text { SDMN } \\
\text { BEDROCK }\end{array}$ & SDGL \\
\hline oxw & 163 & $\cdots$ & 640 & $\cdots$ & $\begin{array}{r}0 \\
25\end{array}$ & $\begin{array}{l}25 \\
-.\end{array}$ & $\begin{array}{l}112 \text { TILL } \\
\text { BEDROCK }\end{array}$ & TILL GRAVEL, HARDPAN \\
\hline oxw & 164 & $\cdots$ & 112 & $\cdots$ & $\begin{array}{r}0 \\
95\end{array}$ & 95 & $\begin{array}{l}112 \mathrm{TILL} \\
110 \mathrm{SDMN}\end{array}$ & $\begin{array}{l}\text { TILL } \\
\text { SDGL }\end{array}$ \\
\hline $\mathrm{OXW}$ & 165 & $\cdots$ & 421 & $\cdots$ & $\begin{array}{r}0 \\
25\end{array}$ & $\begin{array}{l}25 \\
--\end{array}$ & $\begin{array}{l}110 \text { SDMN } \\
\text { BEDROCK }\end{array}$ & SDGL \\
\hline oxw & 166 & $\cdots$ & 46.5 & $\cdots$ & $\cdots$ & $\cdots$ & $110 \mathrm{SDMN}$ & SDCL \\
\hline oxw & 167 & $\cdots$ & 402 & $\cdots$ & $\begin{array}{r}0 \\
104\end{array}$ & 104 & $\begin{array}{l}110 \text { SDMN } \\
\text { BEDROCK }\end{array}$ & SDCL \\
\hline oxw & 168 & $\cdots$ & 602 & $\cdots$ & $\begin{array}{r}0 \\
80\end{array}$ & $\begin{array}{l}80 \\
-.\end{array}$ & $\begin{array}{l}112 \text { TILL } \\
\text { BEDROCK }\end{array}$ & TILL CLAY, HARDPAN \\
\hline oxw & 169 & $-\cdot$ & 39 & $\cdots$ & $\begin{array}{r}0 \\
12 \\
15\end{array}$ & $\begin{array}{l}12 \\
15 \\
--\end{array}$ & $\begin{array}{l}110 \text { SDMN } \\
110 \text { SDMN } \\
110 \text { SDMN }\end{array}$ & $\begin{array}{l}\text { SDGL } \\
\text { CLAY } \\
\text { SAND }\end{array}$ \\
\hline oxw & 170 & $\cdots$ & 353 & $\cdots$ & $\begin{array}{r}0 \\
177\end{array}$ & $\begin{array}{r}177 \\
-.\end{array}$ & $\begin{array}{l}110 \text { SDMN } \\
\text { BEDROCK }\end{array}$ & SDCL \\
\hline oxw & 171 & $-\cdot$ & 422 & $-\cdot$ & $\begin{array}{r}0 \\
75\end{array}$ & $\begin{array}{l}75 \\
\cdots\end{array}$ & $\begin{array}{l}112 T I L L \\
\text { BEDROCK }\end{array}$ & TILL \\
\hline oxw & 172 & $\cdots$ & 202 & $\cdots$ & $\begin{array}{r}0 \\
91\end{array}$ & 91 & $\begin{array}{l}110 \text { SDMN } \\
\text { BEDROCK }\end{array}$ & SDGL \\
\hline oxw & 173 & $\cdots$ & 880 & $\cdots$ & $\begin{aligned} 0 \\
19\end{aligned}$ & $\begin{array}{l}19 \\
\cdots\end{array}$ & $\begin{array}{l}110 \text { SDMN } \\
\text { BEDROCK }\end{array}$ & SDGL \\
\hline
\end{tabular}


Table 2-1. Stratigraphic logs of wells and borings in the Saco and Ossipee River Basins, eastcentral New Hampshire--Continued

\begin{tabular}{|c|c|c|c|c|c|c|c|c|}
\hline \multicolumn{2}{|c|}{$\begin{array}{l}\text { Local } \\
\text { site } \\
\text { No. }\end{array}$} & $\begin{array}{l}\text { Depth } \\
\text { drilled } \\
\text { (feet) }\end{array}$ & $\begin{array}{l}\text { Depth } \\
\text { of } \\
\text { well } \\
\text { (feet) }\end{array}$ & $\begin{array}{l}\text { Depth } \\
\text { to } \\
\text { refusal } \\
\text { (feet) }\end{array}$ & $\begin{array}{l}\text { Depth } \\
\text { to } \\
\text { top } \\
\text { (feet) }\end{array}$ & $\begin{array}{l}\text { Depth } \\
\text { to } \\
\text { bottom } \\
\text { (feet) }\end{array}$ & $\begin{array}{l}\text { Aquifer } \\
\text { code }\end{array}$ & Lithology \\
\hline & & \multicolumn{7}{|c|}{ OSSIPEE - - Continued } \\
\hline oxw & 174 & $-\cdot$ & 785 & $\cdots$ & $\begin{array}{r}0 \\
109\end{array}$ & 109 & $\begin{array}{l}\text { 110SDMN } \\
\text { BEDROCK }\end{array}$ & SAND \\
\hline oxw & 175 & $\cdots$ & 600 & $-\cdot$ & $\begin{array}{r}0 \\
60\end{array}$ & $\begin{array}{l}60 \\
\cdots\end{array}$ & $\begin{array}{l}110 \text { SDMN } \\
\text { BEDROCK }\end{array}$ & SAND \\
\hline oxw & 176 & $\cdots$ & 322 & $\cdots$ & $\begin{array}{r}0 \\
40\end{array}$ & $\begin{array}{l}40 \\
\cdots\end{array}$ & $\begin{array}{l}110 \text { SDMN } \\
\text { BEDROCK }\end{array}$ & SDGL \\
\hline oxw & 177 & $\cdots$ & 402 & - & $\begin{array}{r}0 \\
14\end{array}$ & $\begin{array}{l}14 \\
\cdots\end{array}$ & $\begin{array}{l}\text { 112TILL } \\
\text { BEDROCK }\end{array}$ & TILL \\
\hline oxw & 178 & $-\cdot$ & 39 & $\cdots$ & $\begin{array}{r}0 \\
2 \\
35 \\
43\end{array}$ & $\begin{array}{r}2 \\
35 \\
43 \\
\cdots\end{array}$ & $\begin{array}{l}110 \text { SDMN } \\
110 \text { SDMN } \\
110 \text { SDMN } \\
112 \text { TILL }\end{array}$ & $\begin{array}{l}\text { SDGL } \\
\text { CLAY } \\
\text { SDGL } \\
\text { TILL SAND, GRAVEL, HARDPAN }\end{array}$ \\
\hline oxw & 179 & $\cdots$ & 527 & $\cdots$ & $\begin{array}{r}0 \\
65\end{array}$ & $\begin{array}{l}65 \\
\cdots\end{array}$ & $\begin{array}{l}\text { 112TILL } \\
\text { BEDROCK }\end{array}$ & TILL SAND, CLAY, HARDPAN \\
\hline oxw & 180 & $\cdots$ & 63 & $\cdots$ & $\cdots$ & $\cdots$ & $110 \mathrm{SDMN}$ & SAND \\
\hline oxw & 181 & $\cdots$ & 542 & $\cdots$ & $\begin{array}{r}0 \\
40 \\
185\end{array}$ & $\begin{array}{r}40 \\
185 \\
-.\end{array}$ & $\begin{array}{l}112 \text { TILL } \\
110 \text { SDMN } \\
\text { BEDROCK }\end{array}$ & $\begin{array}{l}\text { TILL } \\
\text { CLAY }\end{array}$ \\
\hline oxw & 182 & -- & 440 & $\cdots$ & $\begin{array}{r}0 \\
50 \\
90\end{array}$ & $\begin{array}{l}50 \\
90\end{array}$ & $\begin{array}{l}110 \text { SDMN } \\
110 \text { SDMN } \\
\text { BEDROCK }\end{array}$ & $\begin{array}{l}\text { SAND } \\
\text { SDCL }\end{array}$ \\
\hline oxw & 183 & $-\cdot$ & 202 & $-\cdot$ & $\begin{array}{r}0 \\
60\end{array}$ & $\begin{array}{l}60 \\
\cdots\end{array}$ & $\begin{array}{l}110 \text { SDMN } \\
\text { BEDROCK }\end{array}$ & OTHR \\
\hline oxw & 184 & $\cdots$ & 352 & - & $\begin{array}{r}0 \\
172\end{array}$ & 172 & $\begin{array}{l}110 \text { SDMN } \\
\text { BEDROCK }\end{array}$ & CLAY \\
\hline oxw & 185 & $\cdots$ & 302 & $\cdots$ & $\begin{array}{r}0 \\
68\end{array}$ & $\begin{array}{l}68 \\
--\end{array}$ & $\begin{array}{l}110 \text { SDMN } \\
\text { BEDROCK }\end{array}$ & SDCL \\
\hline oxw & 186 & $\cdots$ & 410 & $\cdots$ & $\begin{array}{r}0 \\
60\end{array}$ & $\begin{array}{l}60 \\
--\end{array}$ & $\begin{array}{l}110 \text { SDMN } \\
\text { BEDROCK }\end{array}$ & SAND \\
\hline oxw & 187 & $\cdots$ & 502 & $\cdots$ & $\begin{array}{r}0 \\
108\end{array}$ & 108 & $\begin{array}{l}\text { 112TILL } \\
\text { BEDROCK }\end{array}$ & TILL SAND, HARDPAN \\
\hline oxw & 188 & - & 125 & $-\cdot$ & $\begin{array}{r}0 \\
76\end{array}$ & $\begin{array}{l}76 \\
--\end{array}$ & $\begin{array}{l}\text { 110SDMN } \\
\text { BEDROCK }\end{array}$ & SAND \\
\hline oxw & 189 & $\cdots$ & 200 & $\cdot-$ & $\begin{array}{r}0 \\
20\end{array}$ & 20 & $\begin{array}{l}\text { 112TILL } \\
\text { BEDROCK }\end{array}$ & TILL \\
\hline oxw & 190 & $\cdots$ & 30 & $\cdots$ & $\cdots$ & $\cdots$ & 110 SDMN & SAND \\
\hline oxw & 191 & $-\cdot$ & 302 & $\cdots$ & $\begin{array}{r}0 \\
50\end{array}$ & $\begin{array}{l}50 \\
\cdots\end{array}$ & $\begin{array}{l}110 \text { SDMN } \\
\text { BEDROCK }\end{array}$ & OTHR \\
\hline oxw & 192 & $-\cdot$ & 240 & $-\cdot$ & $\begin{array}{r}0 \\
46 \\
82\end{array}$ & $\begin{array}{l}46 \\
82 \\
-.\end{array}$ & $\begin{array}{l}110 \text { SDMN } \\
112 T I L L \\
\text { BEDROCK }\end{array}$ & $\begin{array}{l}\text { SAND } \\
\text { TILL CLAY, HARDPAN }\end{array}$ \\
\hline oxw & 193 & $\cdots$ & 304 & - & $\begin{array}{r}0 \\
80\end{array}$ & $\begin{array}{l}80 \\
-\cdots\end{array}$ & $\begin{array}{l}110 S D M N \\
\text { BEDROCK }\end{array}$ & OTHR \\
\hline oxw & 194 & $\cdots$ & 39 & $\cdots$ & $\begin{array}{r}0 \\
40\end{array}$ & $\begin{array}{l}40 \\
--\end{array}$ & $\begin{array}{l}110 \mathrm{SDMN} \\
112 \mathrm{TILL}\end{array}$ & $\begin{array}{l}\text { SDGL } \\
\text { TILL }\end{array}$ \\
\hline oxw & 195 & - & 405 & $-\cdot$ & $\begin{array}{r}0 \\
174\end{array}$ & 174 & $\begin{array}{l}110 \text { SDMN } \\
\text { BEDROCK }\end{array}$ & SDCL \\
\hline oxw & 196 & -- & 602 & $-\cdot$ & $\begin{array}{r}0 \\
70\end{array}$ & $\begin{array}{l}70 \\
--\end{array}$ & $\begin{array}{l}110 S D M N \\
\text { BEDROCK }\end{array}$ & SDCL \\
\hline
\end{tabular}


Table 2-1. Stratigraphic logs of wells and borings in the Saco and Ossipee River Basins, eastcentral New Hampshire--Continued

\begin{tabular}{|c|c|c|c|c|c|c|c|c|}
\hline \multicolumn{2}{|c|}{$\begin{array}{l}\text { Local } \\
\text { site } \\
\text { no. }\end{array}$} & $\begin{array}{l}\text { Depth } \\
\text { drilled } \\
\text { (feet) }\end{array}$ & $\begin{array}{l}\text { Depth } \\
\text { of } \\
\text { well } \\
\text { (feet) }\end{array}$ & $\begin{array}{l}\text { Depth } \\
\text { to } \\
\text { refusal } \\
\text { (feet) }\end{array}$ & $\begin{array}{l}\text { Depth } \\
\text { to } \\
\text { top } \\
\text { (feet) }\end{array}$ & $\begin{array}{l}\text { Depth } \\
\text { to } \\
\text { bottom } \\
\text { (feet) }\end{array}$ & $\begin{array}{l}\text { Aquifer } \\
\text { code }\end{array}$ & Lithology \\
\hline & & \multicolumn{7}{|c|}{ OSSIPEB - Continued } \\
\hline OXW & 197 & $\cdots$ & 402 & $\cdots$ & $\begin{array}{r}0 \\
39\end{array}$ & $\begin{array}{l}39 \\
--\end{array}$ & $\begin{array}{l}110 \text { SDMN } \\
\text { BEDROCK }\end{array}$ & SDGL \\
\hline oxw & 198 & $\cdots$ & 54 & $\cdots$ & $\cdots$ & $\cdots$ & 110 SDMN & SAND \\
\hline $\mathrm{oxw}$ & 199 & $\cdots$ & 403 & $\cdots$ & $\begin{array}{r}0 \\
117\end{array}$ & $\begin{array}{r}117 \\
\cdots\end{array}$ & $\begin{array}{l}110 \text { SDMN } \\
\text { BEDROCK }\end{array}$ & SDCL \\
\hline oxw & 200 & $\cdots$ & 25 & $\cdots$ & $\begin{array}{r}0 \\
5 \\
10 \\
11 \\
32\end{array}$ & $\begin{array}{r}5 \\
10 \\
11 \\
32 \\
\cdots\end{array}$ & $\begin{array}{l}110 \text { SDMN } \\
110 \text { SDMN } \\
110 \text { SDMN } \\
110 \text { SDMN } \\
110 \text { SDMN }\end{array}$ & $\begin{array}{l}\text { SDGL } \\
\text { SDGL } \\
\text { CLAY } \\
\text { SAND } \\
\text { SDCL }\end{array}$ \\
\hline $\mathrm{oxw}$ & 201 & $\cdots$ & 478 & $\cdots$ & $\begin{array}{r}0 \\
382\end{array}$ & $\begin{array}{r}382 \\
\cdots\end{array}$ & $\begin{array}{l}\text { 112TILL } \\
\text { BEDROCK }\end{array}$ & TILL GRAVEL, CLAY, HARDPAN \\
\hline OXW & 202 & $\cdots$ & 302 & $\cdots$ & $\begin{array}{r}0 \\
30 \\
180\end{array}$ & $\begin{array}{r}30 \\
180 \\
-.\end{array}$ & $\begin{array}{l}110 \text { SDMN } \\
110 \text { SDMN } \\
\text { BEDROCK }\end{array}$ & $\begin{array}{l}\text { SDGL } \\
\text { OTHR }\end{array}$ \\
\hline oxw & 203 & $\cdots$ & 328 & $\cdots$ & $\begin{array}{r}0 \\
50\end{array}$ & $\begin{array}{l}50 \\
\cdots\end{array}$ & $\begin{array}{l}\text { 112TILL } \\
\text { BEDROCK }\end{array}$ & TILL CLAY, HARDPAN \\
\hline $\mathrm{OXW}$ & 204 & $\cdots$ & 310 & $\cdots$ & $\begin{array}{r}0 \\
20 \\
90\end{array}$ & $\begin{array}{l}20 \\
90 \\
--\end{array}$ & $\begin{array}{l}110 \text { SDMN } \\
112 \text { TILL } \\
\text { BEDROCK }\end{array}$ & $\begin{array}{l}\text { SDGL } \\
\text { TILL }\end{array}$ \\
\hline oxw & 205 & $\cdots$ & 403 & $\cdots$ & $\begin{array}{r}0 \\
152\end{array}$ & $\begin{array}{r}152 \\
\cdots\end{array}$ & $\begin{array}{l}\text { I12TILL } \\
\text { BEDROCK }\end{array}$ & TILL SAND, CLAY, HARDPAN \\
\hline $\mathrm{OXW}$ & 206 & $\cdots$ & 300 & $\cdots$ & $\begin{array}{r}0 \\
15\end{array}$ & $\begin{array}{l}15 \\
\cdots\end{array}$ & $\begin{array}{l}110 \text { SDMN } \\
\text { BEDROCK }\end{array}$ & SAND \\
\hline OXW & 207 & $\cdots$ & 502 & $\cdots$ & $\begin{array}{r}0 \\
84\end{array}$ & $\begin{array}{l}84 \\
-\end{array}$ & $\begin{array}{l}\text { 112TILL } \\
\text { BEDROCK }\end{array}$ & TILL SAND, CLAY, HARDPAN \\
\hline oxw & 208 & $\cdots$ & 278 & - & $\begin{array}{r}0 \\
32\end{array}$ & $\begin{array}{l}32 \\
\cdots\end{array}$ & $\begin{array}{l}110 \text { SDMN } \\
\text { BEDROCK }\end{array}$ & SDGL \\
\hline OXW & 209 & $\cdots$ & 377 & $\cdots$ & $\begin{array}{r}0 \\
32\end{array}$ & $\begin{array}{l}32 \\
\cdots\end{array}$ & $\begin{array}{l}110 \text { SDMN } \\
\text { BEDROCK }\end{array}$ & SDGL \\
\hline oxw & 210 & $\cdots$ & 177 & $\cdots$ & $\begin{array}{r}0 \\
80\end{array}$ & $\begin{array}{l}80 \\
\cdots\end{array}$ & $\begin{array}{l}\text { 112TILL } \\
\text { BEDROCK }\end{array}$ & TILL CLAY, HARDPAN \\
\hline oxw & 211 & $\cdots$ & 202 & -. & $\begin{array}{r}0 \\
92\end{array}$ & $\begin{array}{l}92 \\
\cdots\end{array}$ & $\begin{array}{l}\text { 112TILL } \\
\text { BEDROCK }\end{array}$ & TILL CLAY, HARDPAN \\
\hline oxw & 212 & $\cdots$ & 552 & $\cdot$ & $\begin{array}{r}0 \\
136\end{array}$ & 136 & $\begin{array}{l}\text { 112TILL } \\
\text { BEDROCK }\end{array}$ & TILL GRAVEL, CLAY, HARDPAN \\
\hline oxw & 213 & $\cdots$ & 352 & $\cdots$ & $\begin{array}{r}0 \\
34\end{array}$ & $\begin{array}{l}34 \\
-\end{array}$ & $\begin{array}{l}110 \text { SDMN } \\
\text { BEDROCK }\end{array}$ & OTHR \\
\hline oxw & 214 & $\cdots$ & 12.5 & $\cdots$ & $\begin{array}{r}0 \\
13\end{array}$ & $\begin{array}{l}13 \\
\cdots\end{array}$ & $\begin{array}{l}110 \mathrm{SDMN} \\
112 \mathrm{TILL}\end{array}$ & $\begin{array}{l}\text { SDGL } \\
\text { TILL GRAVEL, HARDPAN }\end{array}$ \\
\hline oxw & 215 & $\cdots$ & 190 & $\cdots$ & $\begin{array}{r}0 \\
40\end{array}$ & $\begin{array}{l}40 \\
\cdots\end{array}$ & $\begin{array}{l}110 \text { SDMN } \\
110 \text { SDMN }\end{array}$ & $\begin{array}{l}\text { SAND } \\
\text { SDGL }\end{array}$ \\
\hline$O X W$ & 216 & $\cdots$ & 377 & $\cdots$ & $\begin{array}{r}0 \\
88\end{array}$ & $\begin{array}{l}88 \\
\cdots\end{array}$ & $\begin{array}{l}\text { 112TILL } \\
\text { BEDROCK }\end{array}$ & TILL CLAY, HARDPAN \\
\hline oXw & 217 & $-\cdot$ & 402 & $\cdots$ & $\begin{array}{r}0 \\
175\end{array}$ & 175 & $\begin{array}{l}112 \text { TILL } \\
\text { BEDROCK }\end{array}$ & TILL CLAY, HARDPAN \\
\hline oxw & 218 & $\cdots$ & 252 & $\cdots$ & $\begin{array}{r}0 \\
185\end{array}$ & 185 & $\begin{array}{l}\text { 112TILL } \\
\text { BEDROCK }\end{array}$ & TILL GRAVEL， HARDPAN \\
\hline
\end{tabular}


Table 2-1. Stratigraphic logs of wells and borings in the Saco and Ossipee River Basins, eastcentral New Hampshire--Continued

\begin{tabular}{|c|c|c|c|c|c|c|c|c|}
\hline \multicolumn{2}{|c|}{$\begin{array}{l}\text { Local } \\
\text { site } \\
\text { No. }\end{array}$} & $\begin{array}{l}\text { Depth } \\
\text { drilled } \\
\text { (feet) }\end{array}$ & $\begin{array}{l}\text { Depth } \\
\text { of } \\
\text { well } \\
\text { (feet) }\end{array}$ & $\begin{array}{l}\text { Depth } \\
\text { to } \\
\text { refusal } \\
\text { (feet) }\end{array}$ & $\begin{array}{l}\text { Depth } \\
\text { to } \\
\text { top } \\
\text { (feet) }\end{array}$ & $\begin{array}{l}\text { Depth } \\
\text { to } \\
\text { bottom } \\
\text { (feet) }\end{array}$ & $\begin{array}{l}\text { Aquifer } \\
\text { code }\end{array}$ & Lithology \\
\hline & & \multicolumn{7}{|c|}{ OSSIPES - - Continued } \\
\hline oxw & 219 & $-\cdot$ & 29 & - & $\begin{array}{r}0 \\
12 \\
21 \\
30\end{array}$ & $\begin{array}{l}12 \\
21 \\
30 \\
--\end{array}$ & $\begin{array}{l}110 \mathrm{SDMN} \\
110 \mathrm{SDMN} \\
110 \mathrm{SDMN} \\
110 \mathrm{SDMN}\end{array}$ & $\begin{array}{l}\text { SDGL } \\
\text { CLAY } \\
\text { SDGL } \\
\text { CLAY }\end{array}$ \\
\hline $\mathrm{oxw}$ & 220 & - & 328 & $\cdot-$ & $\begin{array}{r}0 \\
149\end{array}$ & $\begin{array}{r}149 \\
-.\end{array}$ & $\begin{array}{l}112 T I L L \\
\text { BEDROCK }\end{array}$ & TILL CLAY, HARDPAN \\
\hline oxw & 221 & $\cdots$ & 228 & $\cdots$ & $\begin{array}{r}0 \\
21\end{array}$ & $\begin{array}{l}21 \\
--\end{array}$ & $\begin{array}{l}110 \text { SDMN } \\
\text { BEDROCK }\end{array}$ & SDCL \\
\hline oxw & 222 & $\cdots$ & 252 & $-\cdot$ & $\begin{array}{r}0 \\
153\end{array}$ & 153 & $\begin{array}{l}110 \text { SDMN } \\
\text { BEDROCK }\end{array}$ & CLAY \\
\hline oxw & 223 & - & 425 & $\cdots$ & $\begin{array}{r}0 \\
89\end{array}$ & $\begin{array}{l}89 \\
-\cdot\end{array}$ & $\begin{array}{l}112 \text { TILL } \\
\text { BEDROCK }\end{array}$ & TILL GRAVEL, CLAY, HARDPAN \\
\hline oxw & 224 & $\cdots$ & 552 & - & $\begin{array}{r}0 \\
200 \\
250\end{array}$ & $\begin{array}{r}200 \\
250 \\
--\end{array}$ & $\begin{array}{l}110 \text { SDMN } \\
112 \text { TILL } \\
\text { BEDROCK }\end{array}$ & $\begin{array}{l}\text { CLAY } \\
\text { TILL }\end{array}$ \\
\hline oxw & 225 & $\cdots$ & 427 & - & $\begin{array}{r}0 \\
60\end{array}$ & $\begin{array}{l}60 \\
\cdots\end{array}$ & $\begin{array}{l}\text { 112TILL } \\
\text { BEDROCK }\end{array}$ & TILL CLAY, HARDPAN \\
\hline $\mathrm{oxw}$ & 226 & - & 142 & -. & $\begin{array}{r}0 \\
39\end{array}$ & $\begin{array}{l}39 \\
\cdots\end{array}$ & $\begin{array}{l}110 \mathrm{SDMN} \\
\text { BEDROCK }\end{array}$ & CLAY \\
\hline $\mathrm{oxw}$ & 227 & $\cdots$ & 402 & - & $\begin{array}{r}0 \\
117\end{array}$ & $\begin{array}{r}117 \\
\cdots\end{array}$ & $\begin{array}{l}110 \text { SDMN } \\
\text { BEDROCK }\end{array}$ & SAND \\
\hline oxw & 228 & $-\cdot$ & 160 & - & $\begin{array}{r}0 \\
30\end{array}$ & 30 & $\begin{array}{l}110 \text { SDMN } \\
\text { BEDROCK }\end{array}$ & OTHR \\
\hline oxw & 229 & $-\cdot$ & 14 & - & $\begin{array}{r}0 \\
12\end{array}$ & $\begin{array}{l}12 \\
\cdots\end{array}$ & $\begin{array}{l}110 \mathrm{SDMN} \\
\text { BEDROCK }\end{array}$ & SDGL \\
\hline oxw & 230 & $-\cdot$ & 344 & $\cdots$ & $\begin{array}{r}0 \\
80\end{array}$ & $\begin{array}{l}80 \\
-.\end{array}$ & $\begin{array}{l}\text { 112TILL } \\
\text { BEDROCK }\end{array}$ & TILL \\
\hline oxw & 231 & $-\cdot$ & 100 & $-\cdot$ & $\begin{array}{r}0 \\
212\end{array}$ & $\begin{array}{c}212 \\
\cdots\end{array}$ & $\begin{array}{l}110 S D M N \\
\text { BEDROCK }\end{array}$ & SDGL \\
\hline oxw & 232 & -. & 315 & $\cdots$ & $\begin{array}{r}0 \\
130\end{array}$ & $\begin{array}{c}130 \\
\ldots\end{array}$ & $\begin{array}{l}\text { 112TILL } \\
\text { BEDROCK }\end{array}$ & TILL \\
\hline oxw & 233 & - & 502 & $-\cdot$ & $\begin{array}{r}0 \\
100 \\
250 \\
375\end{array}$ & $\begin{array}{l}100 \\
250 \\
375\end{array}$ & $\begin{array}{l}112 \mathrm{TILL} \\
112 \mathrm{TILL} \\
112 \mathrm{TILL} \\
\text { BEDROCK }\end{array}$ & $\begin{array}{l}\text { TILL GRAVEL, HARDPAN } \\
\text { TILL } \\
\text { TILL GRAVEL, HARDPAN }\end{array}$ \\
\hline oxw & 234 & $-\cdot$ & 736 & $\cdots$ & $\begin{array}{r}0 \\
12\end{array}$ & $\begin{array}{l}12 \\
-.\end{array}$ & $\begin{array}{l}110 \text { SDMN } \\
\text { BEDROCK }\end{array}$ & SDGL \\
\hline oxw & 235 & $-\cdot$ & 402 & $\cdots$ & $\begin{array}{r}0 \\
150\end{array}$ & $\begin{array}{r}150 \\
\ldots\end{array}$ & $\begin{array}{l}\text { 112TILL } \\
\text { BEDROCK }\end{array}$ & TILL GRAVEL, HARDPAN \\
\hline oxw & 236 & $\cdots$ & 505 & $\cdots$ & $\begin{array}{r}0 \\
105\end{array}$ & $\begin{array}{r}105 \\
\ldots\end{array}$ & $\begin{array}{l}110 S D M N \\
\text { BEDROCK }\end{array}$ & SAND \\
\hline oxw & 237 & $\cdots$ & 322 & - & $\begin{array}{r}0 \\
20 \\
40 \\
175\end{array}$ & $\begin{array}{r}20 \\
40 \\
175 \\
\ldots\end{array}$ & $\begin{array}{l}112 \mathrm{TILL} \\
110 \mathrm{SDMN} \\
112 \mathrm{TILL} \\
\text { BEDROCK }\end{array}$ & $\begin{array}{l}\text { TILL } \\
\text { CLAY } \\
\text { TILL }\end{array}$ \\
\hline oxw & 238 & - & 621 & $\cdots$ & $\begin{array}{r}0 \\
50\end{array}$ & $\begin{array}{l}50 \\
\cdots\end{array}$ & $\begin{array}{l}\text { 112TILL } \\
\text { BEDROCK }\end{array}$ & TILL \\
\hline oxw & 239 & - & 421 & $\cdots$ & $\begin{array}{l}0 \\
4\end{array}$ & $\begin{array}{r}4 \\
-\end{array}$ & $\begin{array}{l}\text { 112TILL } \\
\text { BEDROCK }\end{array}$ & TILL \\
\hline
\end{tabular}


Table 2-1. Stratigraphic logs of wells and borings in the Saco and Ossipee River Basins, eastcentral New Hampshire--Continued

\begin{tabular}{|c|c|c|c|c|c|c|c|c|}
\hline \multicolumn{2}{|c|}{$\begin{array}{l}\text { Local } \\
\text { site } \\
\text { no. }\end{array}$} & $\begin{array}{l}\text { Depth } \\
\text { drilled } \\
\text { (feet) }\end{array}$ & $\begin{array}{l}\text { Depth } \\
\text { of } \\
\text { well } \\
\text { (feet) }\end{array}$ & $\begin{array}{l}\text { Depth } \\
\text { to } \\
\text { refusal } \\
\text { (feet) }\end{array}$ & $\begin{array}{l}\text { Depth } \\
\text { to } \\
\text { top } \\
\text { (feet) }\end{array}$ & $\begin{array}{l}\text { Depth } \\
\text { to } \\
\text { bottom } \\
\text { (feet) }\end{array}$ & $\begin{array}{l}\text { Aquifer } \\
\text { code }\end{array}$ & Lithology \\
\hline & & \multicolumn{7}{|c|}{ OSSIPEE - - Continued } \\
\hline oxw & 240 & - & 380 & $\cdots$ & $\begin{array}{r}0 \\
91\end{array}$ & 91 & $\begin{array}{l}\text { 110SDMN } \\
\text { BEDROCK }\end{array}$ & SDGL \\
\hline oxw & 241 & $\cdots$ & 365 & $\cdots$ & $\begin{array}{r}0 \\
12\end{array}$ & $\begin{array}{l}12 \\
\cdots\end{array}$ & $\begin{array}{l}\text { 112TILL } \\
\text { BEDROCK }\end{array}$ & TILL \\
\hline oxw & 242 & - & 565 & $\cdots$ & $\begin{array}{r}0 \\
44\end{array}$ & $\begin{array}{l}44 \\
\cdots\end{array}$ & $\begin{array}{l}\text { 112TILL } \\
\text { BEDROCK }\end{array}$ & TILL GRAVEL, HARDPAN \\
\hline oxw & 243 & -- & 325 & - & $\begin{array}{r}0 \\
33\end{array}$ & $\begin{array}{l}33 \\
-\end{array}$ & $\begin{array}{l}\text { 112TILL } \\
\text { BEDROCK }\end{array}$ & TILL GRAVEL, HARDPAN \\
\hline oxw & 244 & - & 302 & $\cdots$ & $\begin{array}{l}0 \\
5\end{array}$ & $\begin{array}{r}5 \\
--\end{array}$ & $\begin{array}{l}\text { 112TILL } \\
\text { BEDROCK }\end{array}$ & TILL \\
\hline oxw & 245 & $\cdots$ & 300 & -- & $\begin{array}{r}0 \\
14\end{array}$ & $\begin{array}{l}14 \\
\cdots\end{array}$ & $\begin{array}{l}\text { 112TILL } \\
\text { BEDROCK }\end{array}$ & TILL \\
\hline oxw & 246 & - & 902 & - & $\begin{array}{r}0 \\
15\end{array}$ & $\begin{array}{l}15 \\
-.\end{array}$ & $\begin{array}{l}\text { 112TILL } \\
\text { BEDROCK }\end{array}$ & TILL \\
\hline oxw & 247 & - & 302 & $\cdots$ & $\begin{array}{r}0 \\
131\end{array}$ & $\begin{array}{r}131 \\
\ldots\end{array}$ & $\begin{array}{l}110 \text { SDMN } \\
\text { BEDROCK }\end{array}$ & SGVC \\
\hline oxw & 248 & - & 423 & $\cdots$ & $\begin{array}{r}0 \\
70\end{array}$ & $\begin{array}{l}70 \\
\cdots\end{array}$ & $\begin{array}{l}\text { 110SDMN } \\
\text { BEDROCK }\end{array}$ & CLAY \\
\hline oxw & 249 & - & 262 & $\cdots$ & $\begin{array}{r}0 \\
159\end{array}$ & 159 & $\begin{array}{l}\text { 112TILL } \\
\text { BEDROCK }\end{array}$ & TILL SAND, GRAVEL, HARDPAN \\
\hline oxw & 250 & - & 363 & -- & $\begin{array}{r}0 \\
225\end{array}$ & $\begin{array}{r}225 \\
-\end{array}$ & $\begin{array}{l}\text { 112TILL } \\
\text { BEDROCK }\end{array}$ & TILL \\
\hline oxw & 251 & - & 482 & $\cdots$ & $\begin{array}{r}0 \\
11\end{array}$ & $\begin{array}{l}11 \\
\cdots\end{array}$ & $\begin{array}{l}110 \text { SDMN } \\
\text { BEDROCK }\end{array}$ & SDGL \\
\hline oxw & 252 & $-\cdot$ & 402 & -- & $\begin{array}{r}0 \\
160\end{array}$ & $\begin{array}{r}160 \\
\ldots\end{array}$ & $\begin{array}{l}110 \text { SDMN } \\
\text { BEDROCK }\end{array}$ & SDGL \\
\hline oxw & 253 & $\cdots$ & 302 & -- & $\begin{array}{r}0 \\
87\end{array}$ & $\begin{array}{l}87 \\
-.\end{array}$ & $\begin{array}{l}110 \text { SDMN } \\
\text { BEDROCK }\end{array}$ & SDGL \\
\hline $\mathrm{oxw}$ & 254 & $\cdots$ & 57 & $\cdots$ & - & - & 110 SDMN & SAND \\
\hline oxw & 255 & $-\cdot$ & 142 & $\cdots$ & $\ddot{10}$ & $\begin{array}{l}10 \\
-.\end{array}$ & $\begin{array}{l}\text { 112TILL } \\
\text { BEDROCK }\end{array}$ & TILL \\
\hline oxw & 256 & $\cdots$ & 62 & -. & - & - & $110 \mathrm{SDMN}$ & SDCL \\
\hline oxw & 257 & $\cdots$ & 86 & -. & $\cdots$ & - & $110 \mathrm{SDMN}$ & SAND \\
\hline oxw & 258 & -- & 277 & - & $\begin{array}{r}0 \\
55\end{array}$ & 55 & $\begin{array}{l}110 \text { SDMN } \\
\text { BEDROCK }\end{array}$ & SDCL \\
\hline oxw & 259 & .. & 500 & - & $\begin{array}{r}0 \\
95\end{array}$ & 95 & $\begin{array}{l}110 \text { SDMN } \\
\text { BEDROCK }\end{array}$ & SDGL \\
\hline OXW & 260 & . & 705 & $\cdots$ & $\begin{array}{l}0 \\
8\end{array}$ & 8 & $\begin{array}{l}\text { 112TILL } \\
\text { BEDROCK }\end{array}$ & TILL \\
\hline $\mathrm{OXW}$ & 261 & - & 220 & -. & $\begin{array}{r}0 \\
78\end{array}$ & $\begin{array}{l}78 \\
-\end{array}$ & $\begin{array}{l}110 \text { SDMN } \\
\text { BEDROCK }\end{array}$ & SDGL \\
\hline oxw & 262 & . & 650 & - & $\begin{array}{r}0 \\
101\end{array}$ & 101 & $\begin{array}{l}110 \text { SDMN } \\
\text { BEDROCK }\end{array}$ & OTHR \\
\hline oxw & 263 & $\cdots$ & 327 & $\cdots$ & $\begin{array}{r}0 \\
50\end{array}$ & $\begin{array}{l}50 \\
\ldots\end{array}$ & $\begin{array}{l}110 \text { SDMN } \\
\text { BEDROCK }\end{array}$ & SDGL \\
\hline
\end{tabular}


Table 2-1. Stratigraphic logs of weils and borings in the Saco and Ossipee River Basins, eastcentral New Hampshire--Continued

\begin{tabular}{|c|c|c|c|c|c|c|c|c|}
\hline \multicolumn{2}{|c|}{$\begin{array}{l}\text { Local } \\
\text { site } \\
\text { No. }\end{array}$} & $\begin{array}{l}\text { Depth } \\
\text { drilled } \\
\text { (feet) }\end{array}$ & $\begin{array}{l}\text { Depth } \\
\text { of } \\
\text { well } \\
\text { (feet) }\end{array}$ & $\begin{array}{l}\text { Depth } \\
\text { to } \\
\text { refusal } \\
\text { (feet) }\end{array}$ & $\begin{array}{l}\text { Depth } \\
\text { to } \\
\text { top } \\
\text { (feet) }\end{array}$ & $\begin{array}{l}\text { Depth } \\
\text { to } \\
\text { bottom } \\
\text { (feet) }\end{array}$ & $\begin{array}{l}\text { Aquifer } \\
\text { code }\end{array}$ & Lithology \\
\hline & & \multicolumn{7}{|c|}{ OSSIPBE - Continued } \\
\hline oxw & 264 & $\cdots$ & 203 & $\cdots$ & $\begin{array}{r}- \\
0 \\
80\end{array}$ & $\begin{array}{l}-\cdot \\
80 \\
--\end{array}$ & $\begin{array}{l}\text { 112TILL } \\
110 \text { SDMN } \\
\text { BEDROCK }\end{array}$ & $\begin{array}{l}\text { TILL GRAVEL, HARDPAN } \\
\text { CLAY }\end{array}$ \\
\hline oxw & 265 & $\cdots$ & 262 & $\cdots$ & $\begin{array}{r}0 \\
100\end{array}$ & 100 & $\begin{array}{l}\text { 112TILL } \\
\text { BEDROCK }\end{array}$ & TILL \\
\hline oxw & 266 & $\cdots$ & 294 & $\cdots$ & $\begin{array}{r}0 \\
10\end{array}$ & $\begin{array}{l}10 \\
\cdots\end{array}$ & $\begin{array}{l}112 \text { TILL } \\
\text { BEDROCK }\end{array}$ & TILL \\
\hline oxw & 267 & $\cdots$ & 342 & $\cdots$ & $\begin{array}{r}0 \\
74\end{array}$ & $\begin{array}{l}74 \\
\cdots\end{array}$ & $\begin{array}{l}110 \text { SDMN } \\
\text { BEDROCK }\end{array}$ & SDGL \\
\hline oxw & 268 & $\cdots$ & 177 & $\cdots$ & $\begin{array}{r}0 \\
85\end{array}$ & $\begin{array}{l}85 \\
\cdots\end{array}$ & $\begin{array}{l}\text { 112TILL } \\
\text { BEDROCK }\end{array}$ & TILL \\
\hline oxw & 269 & $\cdots$ & 603 & $\cdots$ & $\begin{array}{r}0 \\
12\end{array}$ & $\begin{array}{l}12 \\
-\cdot\end{array}$ & $\begin{array}{l}\text { 112TILL } \\
\text { BEDROCK }\end{array}$ & TILL \\
\hline oxw & 270 & $\cdots$ & 222 & $-\cdot$ & $\begin{array}{r}0 \\
50 \\
180\end{array}$ & $\begin{array}{r}50 \\
180\end{array}$ & $\begin{array}{l}110 \text { SDMN } \\
112 \text { TILL } \\
\text { BEDROCK }\end{array}$ & $\begin{array}{l}\text { SDGL } \\
\text { TILL GRAVEL, CLAY, HARDPAN }\end{array}$ \\
\hline oxw & 271 & - & 400 & $\cdots$ & $\begin{array}{l}0 \\
8\end{array}$ & $\begin{array}{l}8 \\
-\cdot\end{array}$ & $\begin{array}{l}110 \text { SDMN } \\
\text { BEDROCK }\end{array}$ & SDGL \\
\hline oxw & 272 & $\cdots$ & 14 & $\cdots$ & $\begin{array}{r}0 \\
15 \\
28\end{array}$ & $\begin{array}{l}15 \\
28 \\
--\end{array}$ & $\begin{array}{l}110 \text { SDMN } \\
112 \text { TILL } \\
\text { BEDROCK }\end{array}$ & $\begin{array}{l}\text { SDGL } \\
\text { TILL }\end{array}$ \\
\hline oxw & 273 & $-\cdot$ & 262 & $\cdots$ & $\begin{array}{r}0 \\
30 \\
130\end{array}$ & $\begin{array}{r}30 \\
130\end{array}$ & $\begin{array}{l}\text { 112TILL } \\
110 \text { SDMN } \\
\text { BEDROCK }\end{array}$ & $\begin{array}{l}\text { TILL } \\
\text { CLAY }\end{array}$ \\
\hline oxw & 274 & $-\cdot$ & 45 & -- & - & $\cdots$ & $110 \mathrm{SDMN}$ & SAND \\
\hline oxw & 275 & $-\cdot$ & 265 & $\cdots$ & $\begin{array}{r}0 \\
95\end{array}$ & $\begin{array}{l}95 \\
-\cdot\end{array}$ & $\begin{array}{l}\text { 112TILL } \\
\text { BEDROCK }\end{array}$ & TILL \\
\hline oxw & 276 & $\cdots$ & 28 & $\cdots$ & $\cdots$ & $-\cdot$ & $110 \mathrm{SDMN}$ & SDGL \\
\hline OXW & 277 & $\cdots$ & 300 & $\cdots$ & $\begin{array}{r}0 \\
20 \\
60 \\
120 \\
166\end{array}$ & $\begin{array}{r}20 \\
60 \\
120 \\
166 \\
\ldots\end{array}$ & $\begin{array}{l}110 \text { SDMN } \\
110 \text { SDMN } \\
110 \text { SDMN } \\
110 \text { SDMN } \\
\text { BEDROCK }\end{array}$ & $\begin{array}{l}\text { SAND } \\
\text { SDGL } \\
\text { CLAY } \\
\text { SDGL }\end{array}$ \\
\hline oxw & 278 & - & 277 & - & 2 & $\cdots$ & BEDROCK & \\
\hline oxw & 279 & $\cdots$ & 665 & - & $\begin{array}{r}0 \\
120\end{array}$ & 120 & $\begin{array}{l}110 \text { SDMN } \\
\text { BEDROCK }\end{array}$ & SDGL \\
\hline oxw & 280 & $-\cdot$ & 365 & -- & $\begin{array}{r}0 \\
15\end{array}$ & $\begin{array}{l}15 \\
\cdots\end{array}$ & $\begin{array}{l}110 \text { SDMN } \\
\text { BEDROCK }\end{array}$ & SDGL \\
\hline oxw & 281 & $\cdots$ & 142 & $\cdots$ & $\begin{array}{r}0 \\
29\end{array}$ & $\begin{array}{l}29 \\
-\end{array}$ & $\begin{array}{l}\text { 112TILL } \\
\text { BEDROCK }\end{array}$ & TILL \\
\hline oxw & 282 & $\cdots$ & 35 & . & - & $\cdots$ & $110 \mathrm{SDMN}$ & SAND \\
\hline oxw & 283 & - & 24 & $\cdots$ & $-\cdot$ & $\cdots$ & $110 \mathrm{SDMN}$ & SDGL \\
\hline oXw & 284 & - & 42 & - & $\begin{array}{c}0 \\
42.5\end{array}$ & $\begin{array}{l}42.5 \\
\cdots\end{array}$ & $\begin{array}{l}110 \text { SDMN } \\
112 \text { TILL }\end{array}$ & $\begin{array}{l}\text { SDGL } \\
\text { TILL CLAY, HARDPAN }\end{array}$ \\
\hline oxw & 285 & $\cdots$ & 88 & - & $\cdots$ & - & $110 \mathrm{SDMN}$ & SAND \\
\hline oxw & 286 & $\cdots$ & 48 & $\cdots$ & $\cdots$ & $\cdots$ & $110 \mathrm{SDMN}$ & SAND \\
\hline oxw & 287 & - & 88 & - & - & - & $110 \mathrm{SDMN}$ & SAND \\
\hline
\end{tabular}


Table 2-1. Stratigraphic logs of wells and borings in the Saco and Ossipee River Basins, eastcentral New Hampshire--Continued

\begin{tabular}{|c|c|c|c|c|c|c|c|c|}
\hline $\begin{array}{l}\text { Loca } \\
\text { site } \\
\text { no. }\end{array}$ & & $\begin{array}{l}\text { Depth } \\
\text { drilled } \\
\text { (feet) }\end{array}$ & $\begin{array}{l}\text { Depth } \\
\text { of } \\
\text { well } \\
\text { (feet) }\end{array}$ & $\begin{array}{l}\text { Depth } \\
\text { to } \\
\text { refusal } \\
\text { (feet) }\end{array}$ & $\begin{array}{l}\text { Depth } \\
\text { to } \\
\text { top } \\
\text { (feet) }\end{array}$ & $\begin{array}{l}\text { Depth } \\
\text { to } \\
\text { bottom } \\
\text { (feet) }\end{array}$ & $\begin{array}{l}\text { Aquifer } \\
\text { code }\end{array}$ & Lithology \\
\hline \multicolumn{9}{|c|}{ OSSIPEs - - Continued } \\
\hline oxw & 288 & $\cdots$ & 242 & $\cdots$ & $\begin{array}{r}0 \\
45\end{array}$ & 45 & $\begin{array}{l}\text { 112TILL } \\
\text { BEDROCK }\end{array}$ & TILL GRAVEL, HARDPAN \\
\hline oxw & 289 & $\cdots$ & 602 & $\cdots$ & $\begin{array}{r}0 \\
50\end{array}$ & $\begin{array}{l}50 \\
\cdots\end{array}$ & $\begin{array}{l}\text { 112TILL } \\
\text { BEDROCK }\end{array}$ & TILL SAND, HARDPAN \\
\hline oxw & 290 & $-\cdot$ & 202 & $\cdots$ & $\begin{array}{r}0 \\
10\end{array}$ & $\begin{array}{l}10 \\
\cdots\end{array}$ & $\begin{array}{l}\text { 112TILL } \\
\text { BEDROCK }\end{array}$ & TILL \\
\hline $\mathrm{OXw}$ & 291 & $\cdots$ & 302 & $\cdots$ & $\begin{array}{r}0 \\
85\end{array}$ & $\begin{array}{l}85 \\
-\cdot\end{array}$ & $\begin{array}{l}\text { 112TILL } \\
\text { BEDROCK }\end{array}$ & TILL \\
\hline OXW & 292 & $\cdots$ & 1280 & $\cdots$ & $\begin{array}{r}0 \\
220\end{array}$ & 220 & $\begin{array}{l}\text { 112TILL } \\
\text { BEDROCK }\end{array}$ & TILL \\
\hline oxw & 293 & $\cdots$ & 330 & $\cdots$ & $\begin{array}{r}0 \\
35\end{array}$ & $\begin{array}{l}35 \\
\cdots\end{array}$ & $\begin{array}{l}\text { 112TILL } \\
\text { BEDROCK }\end{array}$ & TILL GRAVEL, HARDPAN \\
\hline oxw & 294 & $\cdots$ & 280 & $\cdots$ & $\begin{array}{r}0 \\
134\end{array}$ & $\begin{array}{r}134 \\
\cdots\end{array}$ & $\begin{array}{l}110 \text { SDMN } \\
\text { BEDROCK }\end{array}$ & SGVC \\
\hline oxw & 295 & $\cdots$ & 660 & $-\cdot$ & $\begin{array}{r}0 \\
21\end{array}$ & $\begin{array}{l}21 \\
\cdots\end{array}$ & $\begin{array}{l}110 \text { SDMN } \\
\text { BEDROCK }\end{array}$ & SAND \\
\hline OXW & 296 & $\cdots$ & 502 & $\cdots$ & $\begin{array}{l}0 \\
7\end{array}$ & $\begin{array}{r}7 \\
--\end{array}$ & $\begin{array}{l}\text { 112TILL } \\
\text { BEDROCK }\end{array}$ & TILL \\
\hline oxw & 297 & $\cdots$ & 277 & $\cdots$ & $\begin{array}{r}0 \\
83\end{array}$ & $\begin{array}{l}83 \\
\cdots\end{array}$ & $\begin{array}{l}\text { 112TILL } \\
\text { BEDROCK }\end{array}$ & TILL CLAY, HARDPAN \\
\hline OXW & 298 & $\cdots$ & 43 & $\cdots$ & $\begin{array}{r}0 \\
45\end{array}$ & 45 & $\begin{array}{l}110 \mathrm{SDMN} \\
110 \mathrm{SDMN}\end{array}$ & $\begin{array}{l}\text { SAND } \\
\text { SDCL }\end{array}$ \\
\hline oxw & 299 & $\cdots$ & 362 & $\cdots$ & $\begin{array}{r}0 \\
40\end{array}$ & $\begin{array}{l}40 \\
\cdots\end{array}$ & $\begin{array}{l}\text { 112TILL } \\
\text { BEDROCK }\end{array}$ & TILL \\
\hline oxw & 300 & $\cdots$ & 477 & $\cdots$ & $\begin{array}{r}0 \\
80 \\
220\end{array}$ & $\begin{array}{r}80 \\
220 \\
--\end{array}$ & $\begin{array}{l}110 \text { SDMN } \\
110 \text { SDMN } \\
\text { BEDROCK }\end{array}$ & $\begin{array}{l}\text { SAND } \\
\text { CLAY }\end{array}$ \\
\hline oxw & 301 & $\cdots$ & 88 & $\cdots$ & 0 & $\cdots$ & 110 SDMN & SAND \\
\hline $\mathrm{OXW}$ & 302 & $\cdots$ & 39 & $\cdots$ & $\begin{array}{r}0 \\
20\end{array}$ & $\begin{array}{l}20 \\
\cdots\end{array}$ & $\begin{array}{l}110 \text { SDMN } \\
110 \text { SDMN }\end{array}$ & $\begin{array}{l}\text { SDCL } \\
\text { SAND }\end{array}$ \\
\hline $\mathrm{OXw}$ & 303 & $\cdots$ & 61 & $\cdots$ & $\begin{array}{r}0 \\
65\end{array}$ & $\begin{array}{l}65 \\
\cdots\end{array}$ & $\begin{array}{l}110 \mathrm{SDMN} \\
112 \mathrm{TILL}\end{array}$ & $\begin{array}{l}\text { SDGL } \\
\text { TILL }\end{array}$ \\
\hline \multicolumn{9}{|c|}{ SANDWICH } \\
\hline SEA & 1 & 35 & $\cdots$ & $\cdots$ & $\begin{array}{r}0 \\
12 \\
15 \\
23\end{array}$ & $\begin{array}{l}12 \\
15 \\
23 \\
-\end{array}$ & $\begin{array}{l}110 \text { SDMN } \\
112 \text { SRFD } \\
112 \text { SRFD } \\
112 \text { TILL }\end{array}$ & $\begin{array}{l}\text { SAND CS (MS TO GRANULES) } \\
\text { SDST LAYERS FS, VFS, SILT } \\
\text { GRDS SAND AND SILT, POORLY } \\
\text { SORTED, WITH PEBBLES } \\
\text { TILL GRAY, COMPACT }\end{array}$ \\
\hline SEB & 1 & 32 & $\cdots$ & $\cdots$ & $\begin{array}{r}0 \\
7 \\
32\end{array}$ & $\begin{array}{r}7 \\
32 \\
-\end{array}$ & $\begin{array}{l}112 \mathrm{LCSR} \\
112 \mathrm{TILL} \\
112 \mathrm{TILL}\end{array}$ & $\begin{array}{l}\text { SAND SAND, FINE } \\
\text { TILL TILL } \\
\text { TILL TILL; END OF HOLE }\end{array}$ \\
\hline SEB & 11 & 24 & $\cdots$ & 24 & $\cdots$ & $\cdots$ & $112 \mathrm{TILL}$ & $\begin{array}{l}\text { TILL LAYERS OF SILTY, CLAYEY AND } \\
\text { GRAVELLY TILL }\end{array}$ \\
\hline SEB & 12 & 39 & $\cdots$ & $\cdots$ & $\begin{array}{r}0 \\
6 \\
20\end{array}$ & $\begin{array}{r}6 \\
20 \\
-\cdot\end{array}$ & $\begin{array}{l}112 \text { SRFD } \\
112 \text { SRFD } \\
112 \text { SRFD }\end{array}$ & $\begin{array}{l}\text { SDGL } \\
\text { SAND CS } \\
\text { SAND FS WITH SILT AND STONES }\end{array}$ \\
\hline SEW & 3 & 165 & $\cdots$ & $\cdots$ & $\cdots$ & $\cdots$ & $\cdots$ & \\
\hline SEW & 4 & 10.4 & 10.4 & $\cdots$ & $\cdots$ & $\cdots$ & $\cdots$ & \\
\hline
\end{tabular}


Table 2-1. Stratigraphic logs of wells and borings in the Saco and Ossipee River Basins, eastcentral New Hampshire--Continued

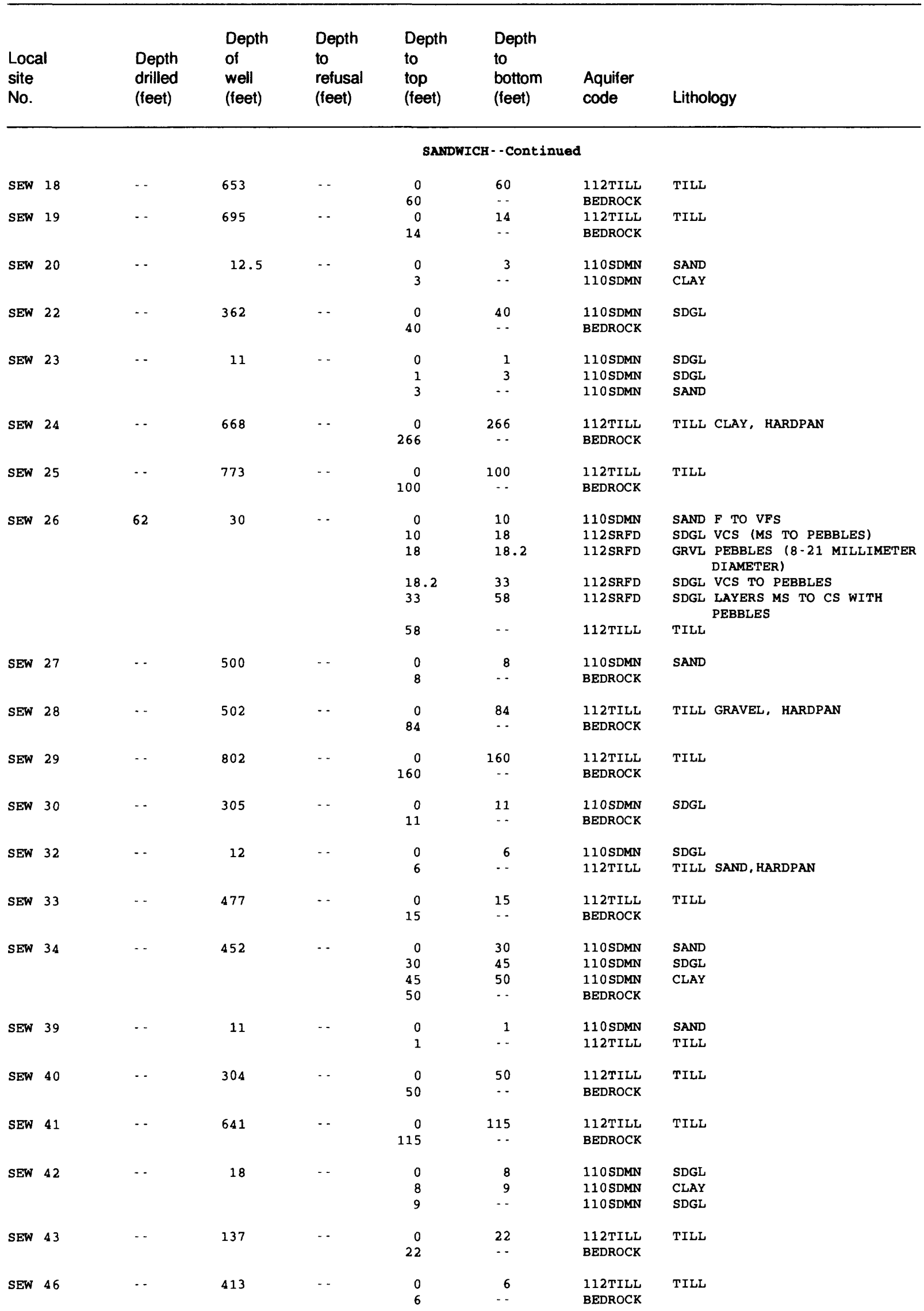


Table 2-1. Stratigraphic logs of wells and borings in the Saco and Ossipee River Basins, eastcentral New Hampshire--Continued

\begin{tabular}{|c|c|c|c|c|c|c|c|c|}
\hline \multicolumn{2}{|c|}{$\begin{array}{l}\text { Local } \\
\text { site } \\
\text { no. }\end{array}$} & $\begin{array}{l}\text { Depth } \\
\text { drilled } \\
\text { (feet) }\end{array}$ & $\begin{array}{l}\text { Depth } \\
\text { of } \\
\text { well } \\
\text { (feet) }\end{array}$ & $\begin{array}{l}\text { Depth } \\
\text { to } \\
\text { refusal } \\
\text { (feet) }\end{array}$ & $\begin{array}{l}\text { Depth } \\
\text { to } \\
\text { top } \\
\text { (feet) }\end{array}$ & $\begin{array}{l}\text { Depth } \\
\text { to } \\
\text { bottom } \\
\text { (feet) }\end{array}$ & $\begin{array}{l}\text { Aquifer } \\
\text { code }\end{array}$ & Lithology \\
\hline & & \multicolumn{7}{|c|}{ SANDWICH - Continued } \\
\hline SEW & 47 & - & 302 & $\cdots$ & $\begin{array}{r}0 \\
40 \\
60\end{array}$ & $\begin{array}{l}40 \\
60 \\
\cdots\end{array}$ & $\begin{array}{l}110 \text { SDMN } \\
110 \text { SDMN } \\
\text { BEDROCK }\end{array}$ & $\begin{array}{l}\text { SDCL } \\
\text { SGVC }\end{array}$ \\
\hline SEW & 48 & $\cdots$ & 11 & $-\cdot$ & $\begin{array}{l}0 \\
2\end{array}$ & $\begin{array}{l}2 \\
\therefore\end{array}$ & $\begin{array}{l}110 \text { SDMN } \\
112 \mathrm{TILL}\end{array}$ & $\begin{array}{l}\text { SDGL } \\
\text { TILL }\end{array}$ \\
\hline SEW & 51 & $\cdots$ & 204 & - & $\begin{array}{r}0 \\
15\end{array}$ & $\begin{array}{l}15 \\
-.\end{array}$ & $\begin{array}{l}\text { 112TILL } \\
\text { BEDROCK }\end{array}$ & TILL \\
\hline SEW & 54 & $-\cdot$ & 82 & $\cdots$ & $\begin{array}{l}0 \\
9\end{array}$ & $\begin{array}{r}9 \\
--\end{array}$ & $\begin{array}{l}\text { 112TILL } \\
\text { BEDROCK }\end{array}$ & TILL \\
\hline SEW & 56 & $\cdots$ & 423 & $\cdots$ & $\begin{array}{r}0 \\
60 \\
85\end{array}$ & $\begin{array}{l}60 \\
85 \\
--\end{array}$ & $\begin{array}{l}\text { 112TILL } \\
\text { 110SDMN } \\
\text { BEDROCK }\end{array}$ & $\begin{array}{l}\text { TILL } \\
\text { CLAY }\end{array}$ \\
\hline SEW & 57 & $-\cdot$ & 11 & $\cdots$ & $\begin{array}{l}0 \\
3\end{array}$ & $\begin{array}{l}3 \\
-\cdot\end{array}$ & $\begin{array}{l}110 \text { SDMN } \\
110 \text { SDMN }\end{array}$ & $\begin{array}{l}\text { SAND } \\
\text { SDGL }\end{array}$ \\
\hline SEW & 59 & $-\cdot$ & 290 & - & $\begin{array}{r}0 \\
80\end{array}$ & $\begin{array}{l}80 \\
\cdots\end{array}$ & $\begin{array}{l}\text { 112TILL } \\
\text { BEDROCK }\end{array}$ & TILL CLAY, HARDPAN \\
\hline SEW & 62 & $-\cdot$ & 873 & $\cdots$ & $\begin{array}{r}0 \\
42\end{array}$ & $\begin{array}{l}42 \\
--\end{array}$ & $\begin{array}{l}\text { 110SDMN } \\
\text { BEDROCK }\end{array}$ & SDGL \\
\hline SEW & 63 & - & 473 & $-\cdot$ & $\begin{array}{r}0 \\
81\end{array}$ & $\begin{array}{l}81 \\
-\cdot\end{array}$ & $\begin{array}{l}\text { 112TILL } \\
\text { BEDROCK }\end{array}$ & TILL CLAY, HARDPAN \\
\hline SEW & 64 & $-\cdot$ & 524 & - & $\begin{array}{r}0 \\
150\end{array}$ & $\begin{array}{r}150 \\
--\end{array}$ & $\begin{array}{l}\text { 112TILL } \\
\text { BEDROCK }\end{array}$ & TILL \\
\hline SEW & 65 & $\cdots$ & 400 & $\cdot-$ & $\begin{array}{r}0 \\
200\end{array}$ & 200 & $\begin{array}{l}110 \text { SDMN } \\
\text { BEDROCK }\end{array}$ & CLAY \\
\hline SEW & 73 & - & 233 & -. & $\begin{array}{r}0 \\
20\end{array}$ & $\begin{array}{l}20 \\
--\end{array}$ & $\begin{array}{l}\text { 112TILL } \\
\text { BEDROCK }\end{array}$ & TILL \\
\hline SEW & 74 & $\cdots$ & 424 & $\cdots$ & $\begin{array}{r}0 \\
100\end{array}$ & $\begin{array}{r}100 \\
\cdots\end{array}$ & $\begin{array}{l}\text { 112TILL } \\
\text { BEDROCK }\end{array}$ & TILL \\
\hline SEW & 75 & $-\cdot$ & 15 & $\cdots$ & $\begin{array}{c}0 \\
7 \\
10 \\
11.5\end{array}$ & $\begin{array}{l}7 \\
10 \\
11.5 \\
-.\end{array}$ & $\begin{array}{l}110 \mathrm{SDMN} \\
110 \mathrm{SDMN} \\
110 \mathrm{SDMN} \\
110 \mathrm{SDMN}\end{array}$ & $\begin{array}{l}\text { SAND } \\
\text { SDGL } \\
\text { SAND } \\
\text { SDGL }\end{array}$ \\
\hline SEW & 76 & $\cdots$ & 552 & - & $\begin{array}{r}0 \\
18\end{array}$ & $\begin{array}{l}18 \\
\cdots\end{array}$ & $\begin{array}{l}110 \text { SDMN } \\
\text { BEDROCK }\end{array}$ & CLAY \\
\hline SEW & 77 & - & 330 & .. & $\begin{array}{r}0 \\
90\end{array}$ & $\begin{array}{l}\cdots \\
\cdots\end{array}$ & $\begin{array}{l}\text { 110SDMN } \\
\text { BEDROCK }\end{array}$ & OTHR \\
\hline SEW & 81 & $\cdots$ & 400 & $\cdots$ & $\begin{array}{r}0 \\
60 \\
69\end{array}$ & $\begin{array}{l}60 \\
69 \\
\cdots\end{array}$ & $\begin{array}{l}\text { 112TILL } \\
110 \text { SDMN } \\
\text { BEDROCK }\end{array}$ & $\begin{array}{l}\text { TILL CLAY, HARDPAN } \\
\text { SDGL }\end{array}$ \\
\hline SEW & 82 & $\cdots$ & 503 & $\cdots$ & $\begin{array}{r}0 \\
50 \\
150 \\
194\end{array}$ & $\begin{array}{r}50 \\
150 \\
194 \\
--\end{array}$ & $\begin{array}{l}110 \text { SDMN } \\
112 \text { TILL } \\
110 \text { SDMN } \\
\text { BEDROCK }\end{array}$ & $\begin{array}{l}\text { SDGL } \\
\text { TILL CLAY, HARDPAN } \\
\text { CLAY }\end{array}$ \\
\hline SEW & 84 & $\cdots$ & 237 & - & $\begin{array}{r}0 \\
36\end{array}$ & $\begin{array}{l}36 \\
\cdots\end{array}$ & $\begin{array}{l}\text { 110SDMN } \\
\text { BEDROCK }\end{array}$ & SDGL \\
\hline SEW & 85 & $\cdots$ & 477 & $\cdots$ & $\begin{array}{r}0 \\
245\end{array}$ & 245 & $\begin{array}{l}\text { 112TILL } \\
\text { BEDROCK }\end{array}$ & TILL CLAY, HARDPAN \\
\hline SEW & 86 & $\cdots$ & 303 & $\cdots$ & $\begin{array}{r}0 \\
20 \\
85\end{array}$ & $\begin{array}{l}20 \\
85 \\
\cdots\end{array}$ & $\begin{array}{l}\text { 112TILL } \\
110 \text { SDMN } \\
\text { BEDROCK }\end{array}$ & $\begin{array}{l}\text { TILL } \\
\text { CLAY }\end{array}$ \\
\hline
\end{tabular}


Table 2-1. Stratigraphic logs of wells and borings in the Saco and Ossipee River Basins, eastcentral New Hampshire--Continued

\begin{tabular}{|c|c|c|c|c|c|c|c|c|}
\hline \multicolumn{2}{|c|}{$\begin{array}{l}\text { Local } \\
\text { site } \\
\text { No. }\end{array}$} & $\begin{array}{l}\text { Depth } \\
\text { drilled } \\
\text { (feet) }\end{array}$ & $\begin{array}{l}\text { Depth } \\
\text { of } \\
\text { well } \\
\text { (feet) }\end{array}$ & $\begin{array}{l}\text { Depth } \\
\text { to } \\
\text { refusal } \\
\text { (feet) }\end{array}$ & $\begin{array}{l}\text { Depth } \\
\text { to } \\
\text { top } \\
\text { (feet) }\end{array}$ & $\begin{array}{l}\text { Depth } \\
\text { to } \\
\text { bottom } \\
\text { (feet) }\end{array}$ & $\begin{array}{l}\text { Aquifer } \\
\text { code }\end{array}$ & Lithology \\
\hline & & \multicolumn{7}{|c|}{ SANDWICH - - Cont inued } \\
\hline SEW & 87 & $\cdots$ & 405 & $\cdots$ & $\begin{array}{r}0 \\
12\end{array}$ & $\begin{array}{l}12 \\
\cdots\end{array}$ & $\begin{array}{l}\text { 110SDMN } \\
\text { BEDROCK }\end{array}$ & SAND \\
\hline SEW & 88 & $\cdots$ & 154 & $\cdots$ & $\begin{array}{r}0 \\
90\end{array}$ & $\begin{array}{l}90 \\
\cdots\end{array}$ & $\begin{array}{l}\text { 112TILL } \\
\text { BEDROCK }\end{array}$ & TILL \\
\hline SEW & 89 & $\cdots$ & 462 & $\cdots$ & $\begin{array}{r}0 \\
20\end{array}$ & $\begin{array}{l}20 \\
-\cdots\end{array}$ & $\begin{array}{l}\text { 112TILL } \\
\text { BEDROCK }\end{array}$ & TILL \\
\hline SEW & 92 & $\cdots$ & 13 & $\cdots$ & $\begin{array}{l}\cdots \\
\cdots\end{array}$ & $\begin{array}{l}\cdots \\
\cdots\end{array}$ & $\begin{array}{l}\text { 110SDMN } \\
112 \mathrm{TILL}\end{array}$ & $\begin{array}{l}\text { SAND } \\
\text { TILL CLAY, HARDPAN }\end{array}$ \\
\hline SEW & 96 & $\cdots$ & 255 & $\cdots$ & $\begin{array}{l}0 \\
4\end{array}$ & $\begin{array}{r}4 \\
--\end{array}$ & $\begin{array}{l}\text { 112TILL } \\
\text { BEDROCK }\end{array}$ & TILL \\
\hline SEW & 97 & $\cdots$ & 530 & $\cdots$ & $\begin{array}{r}0 \\
45\end{array}$ & $\begin{array}{l}45 \\
\cdots\end{array}$ & $\begin{array}{l}\text { 112TILL } \\
\text { BEDROCK }\end{array}$ & TILL SAND, GRAVEL, HARDPAN \\
\hline SEW & 98 & $\cdots$ & 18 & $\cdots$ & $\begin{array}{l}\cdots \\
\cdots\end{array}$ & $\begin{array}{l}\cdots \\
\cdots\end{array}$ & $\begin{array}{l}\text { 110SDMN } \\
112 \mathrm{TILL}\end{array}$ & $\begin{array}{l}\text { CLAY } \\
\text { TILL }\end{array}$ \\
\hline SEW & 99 & $\cdots$ & 305 & $\cdots$ & $\begin{array}{r}0 \\
48\end{array}$ & $\begin{array}{l}48 \\
--\end{array}$ & $\begin{array}{l}\text { 112TILL } \\
\text { BEDROCK }\end{array}$ & TILL GRAVEL, CLAY, HARDPAN \\
\hline SEW & 101 & $\cdots$ & 520 & $\cdots$ & $\begin{array}{l}0 \\
8\end{array}$ & $\begin{array}{r}8 \\
--\end{array}$ & $\begin{array}{l}\text { 112TILL } \\
\text { BEDROCK }\end{array}$ & TILL \\
\hline SEW & 102 & $\cdots$ & 523 & $\cdots$ & $\begin{array}{r}0 \\
200 \\
230\end{array}$ & $\begin{array}{r}200 \\
230 \\
\cdots\end{array}$ & $\begin{array}{l}112 \text { TILL } \\
110 \text { SDMN } \\
\text { BEDROCK }\end{array}$ & $\begin{array}{l}\text { TILL } \\
\text { OTHR ROTTEN ROCK }\end{array}$ \\
\hline SEW & 103 & $\cdots$ & 12 & $\cdots$ & 0 & $\cdots$ & $110 \mathrm{SDMN}$ & SDGL \\
\hline SEW & 104 & $\cdots$ & 250 & $\cdots$ & $\begin{array}{l}0 \\
8\end{array}$ & $\begin{array}{c}8 \\
\cdots\end{array}$ & $\begin{array}{l}\text { 112TILL } \\
\text { BEDROCK }\end{array}$ & TILL \\
\hline SEW & 111 & 12.5 & 12.5 & $\cdots$ & $\cdots$ & $\cdots$ & $\cdots$ & \\
\hline SEW & 152 & $\cdots$ & 402 & $\cdots$ & $\begin{array}{r}0 \\
35\end{array}$ & $\begin{array}{l}35 \\
-\end{array}$ & $\begin{array}{l}\text { 112TILL } \\
\text { BEDROCK }\end{array}$ & TILL SAND, HARDPAN \\
\hline SEW & 153 & 12 & 12 & $\cdots$ & $-\cdot$ & $\cdots$ & $\cdots$ & \\
\hline SEW & 154 & 4 & 4 & $\cdots$ & $\cdots$ & $\cdots$ & $\cdots$ & \\
\hline SEW & 155 & 90 & $\cdots$ & $\cdots$ & $-\cdot$ & $\cdots$ & $\cdots$ & \\
\hline SEW & 156 & 700 & $\cdots$ & $\cdots$ & $\cdots$ & $\cdots$ & $\cdots$ & \\
\hline SEW & 158 & 10.6 & 10.6 & $\cdots$ & $\cdots$ & $-\cdot$ & $\cdots$ & \\
\hline & & & & & TAME & & & \\
\hline TAB & 1 & 24 & - & $-\cdot$ & $\begin{array}{r}0 \\
3 \\
8 \\
24\end{array}$ & $\begin{array}{r}3 \\
8 \\
24 \\
\cdots\end{array}$ & $\begin{array}{l}\text { 111SDMN } \\
112 \text { SRFD } \\
\text { 112TILL } \\
\text { BEDROCK }\end{array}$ & $\begin{array}{l}\text { OTHR FILL } \\
\text { GRVL GRAVEL } \\
\text { TILL TILL } \\
\text { REFUSAL ON BOULDER OR BEDROCK }\end{array}$ \\
\hline TAB & 2 & 51 & $\cdots$ & $\cdots$ & $\begin{array}{r}0 \\
7 \\
30 \\
51\end{array}$ & $\begin{array}{r}7 \\
30 \\
51 \\
-\end{array}$ & $\begin{array}{l}111 \text { SDMN } \\
112 \text { SRFD } \\
112 \mathrm{LCSR} \\
\text { BEDROCK }\end{array}$ & $\begin{array}{l}\text { SAND SAND AND MUCK } \\
\text { SAND SAND, GRAVELLY AND SILTY } \\
\text { SAND SAND, SILTY } \\
\text { REFUSAL ON BOULDER OR BEDROCK }\end{array}$ \\
\hline TAB & 3 & 23.5 & $\cdots$ & - & $\begin{array}{c}0 \\
23.5\end{array}$ & $\begin{array}{l}23.5 \\
\cdots\end{array}$ & $\begin{array}{l}\text { 112SRFD } \\
\text { BEDROCK }\end{array}$ & $\begin{array}{l}\text { SDGL SAND AND GRAVEL; SOME } \\
\text { BOULDERS } \\
\text { REFUSAL ON BOULDER OR BEDROCK }\end{array}$ \\
\hline TAB & 4 & 20 & $\cdots$ & $\cdots$ & $\begin{array}{r}0 \\
20\end{array}$ & 20 & $\begin{array}{l}\text { 112SRFD } \\
\text { BEDROCK }\end{array}$ & $\begin{array}{l}\text { GRVL GRAVEL, COARSE AND BOULDERS } \\
\text { REFUSAL }\end{array}$ \\
\hline
\end{tabular}


Table 2-1. Stratigraphic logs of welis and borings in the Saco and Ossipee River Basins, eastcentral New Hampshire--Continued

\begin{tabular}{|c|c|c|c|c|c|c|c|c|}
\hline \multicolumn{2}{|c|}{$\begin{array}{l}\text { Local } \\
\text { site } \\
\text { no. }\end{array}$} & $\begin{array}{l}\text { Depth } \\
\text { drilled } \\
\text { (feet) }\end{array}$ & $\begin{array}{l}\text { Depth } \\
\text { of } \\
\text { well } \\
\text { (feet) }\end{array}$ & $\begin{array}{l}\text { Depth } \\
\text { to } \\
\text { refusal } \\
\text { (feet) }\end{array}$ & $\begin{array}{l}\text { Depth } \\
\text { to } \\
\text { top } \\
\text { (feet) }\end{array}$ & $\begin{array}{l}\text { Depth } \\
\text { to } \\
\text { bottom } \\
\text { (feet) }\end{array}$ & $\begin{array}{l}\text { Aquifer } \\
\text { code }\end{array}$ & Lithology \\
\hline & & \multicolumn{7}{|c|}{ TAMWORTH - - Continued } \\
\hline TAB & 5 & 34.5 & - & $-\cdot$ & $\begin{array}{l}0 \\
15 \\
20 \\
26 \\
34.5\end{array}$ & $\begin{array}{l}15 \\
20 \\
26 \\
34.5 \\
\cdots\end{array}$ & $\begin{array}{l}112 \text { SRFD } \\
112 \text { SRFD } \\
112 \text { SRFD } \\
112 \text { TILL } \\
\text { BEDROCK }\end{array}$ & $\begin{array}{l}\text { SAND SAND, FINE TO MEDIUM } \\
\text { SAND SAND, FINE TO COARSE } \\
\text { SAND SAND, FINE TO MEDIUM } \\
\text { TILL TILL }\end{array}$ \\
\hline \multirow[t]{2}{*}{ TAB } & 6 & 42 & - & $\cdots$ & $\begin{array}{l}0 \\
2\end{array}$ & $\begin{array}{l}2 \\
5\end{array}$ & $\begin{array}{l}112 \text { SRFD } \\
112 \text { GCFV }\end{array}$ & $\begin{array}{l}\text { GRVL GRAVEL, LOAMY } \\
\text { SAND SAND, FINE TO MEDIUM; SOME } \\
\text { SILT }\end{array}$ \\
\hline & & & & & $\begin{array}{r}5 \\
10 \\
42\end{array}$ & $\begin{array}{l}10 \\
42 \\
\cdots\end{array}$ & $\begin{array}{l}112 \text { SRFD } \\
112 \text { TILL } \\
\text { BEDROCK }\end{array}$ & $\begin{array}{l}\text { GRVL GRAVEL } \\
\text { TILL TILL }\end{array}$ \\
\hline \multirow[t]{7}{*}{ TAB } & 7 & 67 & $\cdots$ & $\cdots$ & $\begin{array}{l}0 \\
9\end{array}$ & $\begin{array}{r}9 \\
20\end{array}$ & $\begin{array}{l}112 \mathrm{SRFD} \\
112 \mathrm{SRFD}\end{array}$ & $\begin{array}{l}\text { GRVL GRAVEL; SOME SILT } \\
\text { SAND SAND, FINE TO MEDIUM; SOME } \\
\text { COBBLES }\end{array}$ \\
\hline & & & & & 20 & 27 & $112 \mathrm{LCSR}$ & SAND SAND, FINE, SILTY \\
\hline & & & & & 27 & 37 & 112 SRFD & SAND SAND, FINE TO COARSE \\
\hline & & & & & 37 & 42 & $112 \mathrm{LCSR}$ & SAND SAND, FINE; SOME SILT \\
\hline & & & & & 42 & 60 & 112 SRFD & $\begin{array}{l}\text { SAND SAND, FINE TO MEDIUM; SOME } \\
\text { COARSE SAND }\end{array}$ \\
\hline & & & & & 60 & 67 & 112 SRFD & $\begin{array}{l}\text { SAND SAND, FINE TO MEDIUM; SOME } \\
\text { SILT }\end{array}$ \\
\hline & & & & & 67 & $-\cdot$ & 112SRFD & SAND END OF HOLE AT 67 FEET \\
\hline TAB & 8 & 15 & $\cdots$ & -- & $\begin{array}{l}0 \\
1\end{array}$ & $\begin{array}{l}1 \\
7\end{array}$ & $\begin{array}{l}110 \text { SOIL } \\
111 \text { SDMN }\end{array}$ & $\begin{array}{l}\text { SOIL TOPSOIL } \\
\text { GRVL GRAVEL, FINE, SILTY }\end{array}$ \\
\hline & & & & & 7 & 15 & 112 TILL & TILL TILL \\
\hline & & & & & 15 & $\cdots$ & BEDROCK & REFUSAL ON BOULDER OR BEDROCK \\
\hline TAB & 9 & 20 & $-\cdot$ & - & $\begin{array}{r}0 \\
10\end{array}$ & 10 & $\begin{array}{l}112 \text { SRFD } \\
112 \text { SRFD }\end{array}$ & $\begin{array}{l}\text { SDGL GRAVEL WITH BOULDERS } \\
\text { SAND MEDIUM TO COARSE SAND }\end{array}$ \\
\hline TAW & 1 & 190 & - & $\cdots$ & - & - & -. & \\
\hline TAW & 2 & 75 & $\cdots$ & - & - & $\cdots$ & $\cdots$ & \\
\hline TAW & 4 & 32 & 32 & - & - & - & - & \\
\hline TAW & 5 & 240 & - & - & - & - & - & \\
\hline TAW & 6 & 13.2 & 13.2 & - & - & $\cdots$ & - & \\
\hline TAW & 8 & 300 & $\cdots$ & . & - & - & - & \\
\hline TAW & 9 & 67 & 67 & - - & $\cdots$ & - & - & \\
\hline TAW & 10 & 46 & 46 & - - & - & - & - - & \\
\hline TAW & 12 & 30 & 30 & . & - & $\cdots$ & - & \\
\hline TAW & 13 & 8.8 & 8.8 & - & $\cdots$ & - & $\cdots$ & \\
\hline TAW & 14 & 15 & 15 & - & . & - & - & \\
\hline TAW & 15 & 25 & 25 & - & - & . & - & \\
\hline TAW & 16 & 20 & 20 & . & . & - & . & \\
\hline TAW & 17 & 32 & 32 & - & $\cdots$ & $\cdots$ & - & \\
\hline TAW & 18 & 1210 & $\cdots$ & - & . & $\cdots$ & $\cdots$ & \\
\hline TAW & 19 & 430 & - & - & - & $\cdots$ & - & \\
\hline TAW & 20 & 360 & - & - & - & $\cdots$ & - & \\
\hline TAW & 21 & 100 & 100 & $\cdots$ & $\cdots$ & $\cdots$ & - & \\
\hline TAW & 22 & 9 & 9 & - - & . & $\cdots$ & - & \\
\hline
\end{tabular}


Table 2-1. Stratigraphic logs of wells and borings in the Saco and Ossipee River Basins, eastcentral New Hampshire--Continued

\begin{tabular}{llllllll}
\hline & & Depth & Depth & Depth & Depth & & \\
Local & Depth & of & to & to & to & \\
site & drilled & well & refusal & top & bottom & Aquifer & \\
No. & (feet) & (feet) & (feet) & (feet) & (feet) & code & Lithology
\end{tabular}

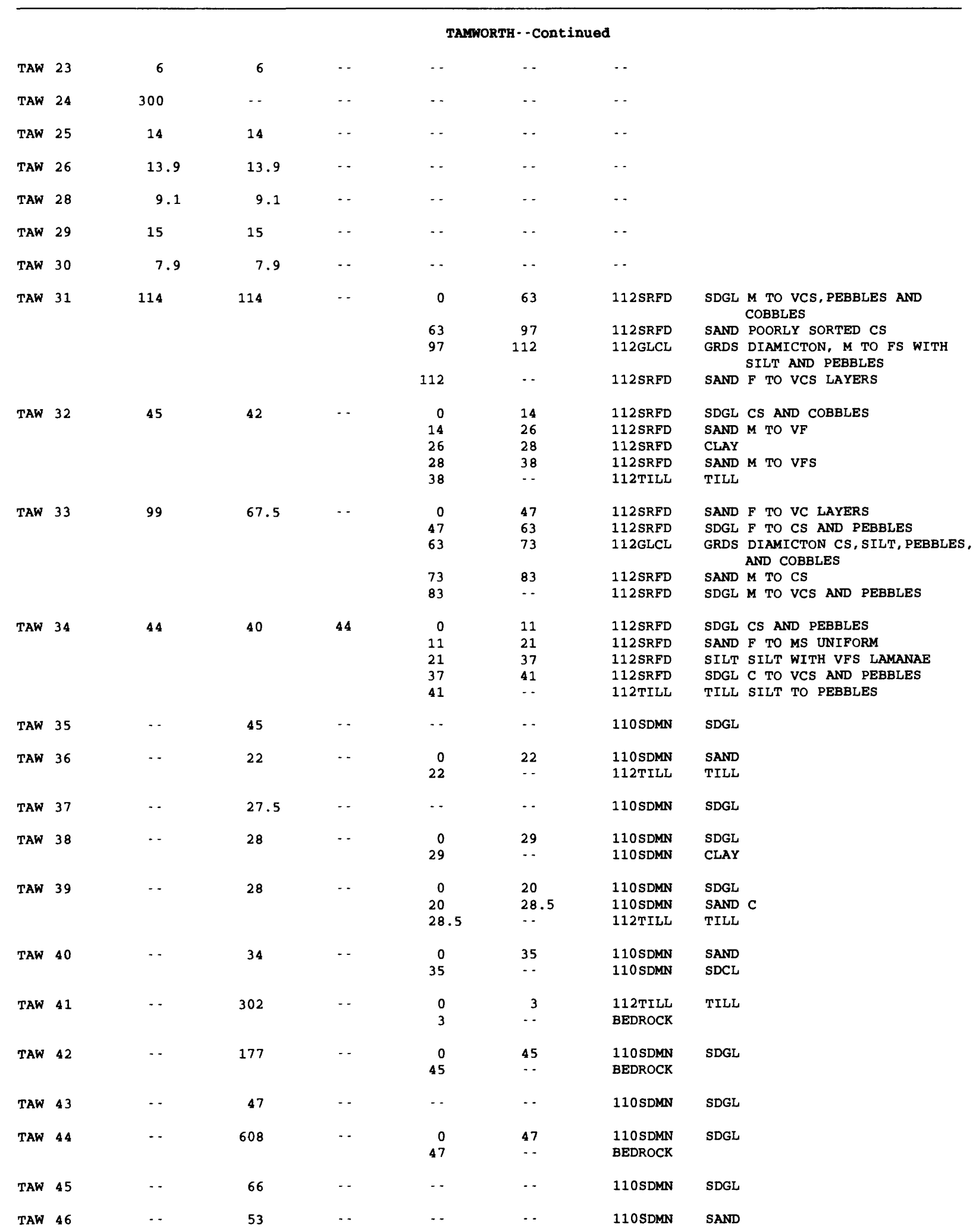


Table 2-1. Stratigraphic logs of wells and borings in the Saco and Ossipee River Basins, eastcentral New Hampshire--Continued

\begin{tabular}{|c|c|c|c|c|c|c|c|c|}
\hline \multicolumn{2}{|c|}{$\begin{array}{l}\text { Local } \\
\text { site } \\
\text { no. }\end{array}$} & $\begin{array}{l}\text { Depth } \\
\text { drilled } \\
\text { (feet) }\end{array}$ & $\begin{array}{l}\text { Depth } \\
\text { of } \\
\text { well } \\
\text { (feet) }\end{array}$ & $\begin{array}{l}\text { Depth } \\
\text { to } \\
\text { refusal } \\
\text { (feet) }\end{array}$ & $\begin{array}{l}\text { Depth } \\
\text { to } \\
\text { top } \\
\text { (feet) }\end{array}$ & $\begin{array}{l}\text { Depth } \\
\text { to } \\
\text { bottom } \\
\text { (feet) }\end{array}$ & $\begin{array}{l}\text { Aquifer } \\
\text { code }\end{array}$ & Lithology \\
\hline & & \multicolumn{7}{|c|}{ TAYWORTH - Continued } \\
\hline TAW & 47 & -- & 67 & $\cdots$ & -- & $\cdots$ & $110 \mathrm{SDMN}$ & SDGL \\
\hline TAW & 48 & $\cdots$ & 68 & $\cdots$ & -- & $-\cdot$ & $110 \mathrm{SDMN}$ & SDGL \\
\hline TAW & 49 & $\cdots$ & 88 & $\cdots$ & $\because 6$ & $\begin{array}{r}160 \\
--\end{array}$ & $\begin{array}{l}110 \text { SDMN } \\
\text { BEDROCK }\end{array}$ & SDGL \\
\hline TAW & 50 & $\cdots$ & 352 & $\cdots$ & $\begin{array}{r}0 \\
41 \\
270\end{array}$ & $\begin{array}{r}41 \\
270 \\
--\end{array}$ & $\begin{array}{l}\text { 110SDMN } \\
\text { 112TILL } \\
\text { BEDROCK }\end{array}$ & $\begin{array}{l}\text { SGVC } \\
\text { TILL CLAY, HARDPAN }\end{array}$ \\
\hline TAW & 51 & -- & 64 & $\cdots$ & $-\cdot$ & - & $110 \mathrm{SDMN}$ & SDGL \\
\hline TAW & 52 & $\cdots$ & 70 & $\cdots$ & $\cdots$ & $\cdots$ & $110 \mathrm{SDMN}$ & SDGL \\
\hline TAW & 53 & -- & 63 & -. & $\cdots$ & $\cdots$ & $110 \mathrm{SDMN}$ & SDGL \\
\hline TAW & 54 & - & 43 & $\cdots$ & $-\cdot$ & $\cdots$ & & \\
\hline TAW & 55 & - & 53 & $\cdots$ & $\cdots$ & - & $110 \mathrm{SDMN}$ & SDGL \\
\hline TAW & 56 & - & 502 & $\cdots$ & $\begin{array}{r}0 \\
132\end{array}$ & $\begin{array}{r}132 \\
--\end{array}$ & $\begin{array}{l}110 \text { SDMN } \\
\text { BEDROCK }\end{array}$ & SAND \\
\hline TAW & 57 & $\cdots$ & 400 & - & $\begin{array}{r}0 \\
104\end{array}$ & 104 & $\begin{array}{l}110 \text { SDMN } \\
\text { BEDROCK }\end{array}$ & SAND \\
\hline TAW & 58 & $\cdots$ & 120 & $\cdots$ & $\begin{array}{r}0 \\
85\end{array}$ & 85 & $\begin{array}{l}110 \text { SDMN } \\
\text { BEDROCK }\end{array}$ & SAND \\
\hline TAW & 59 & $-\cdot$ & 310 & $\cdots$ & $\begin{array}{r}0 \\
80\end{array}$ & $\begin{array}{l}80 \\
\cdots\end{array}$ & $\begin{array}{l}110 \text { SDMN } \\
\text { BEDROCK }\end{array}$ & SAND \\
\hline TAW & 60 & $\cdots$ & 802 & $\cdots$ & $\begin{array}{r}0 \\
15\end{array}$ & $\begin{array}{l}15 \\
\cdots\end{array}$ & $\begin{array}{l}110 \text { SDMN } \\
\text { BEDROCK }\end{array}$ & SAND \\
\hline TAW & 61 & -- & 28 & $\cdots$ & $\cdots$ & $\cdots$ & $110 \mathrm{SDMN}$ & SDGL \\
\hline TAW & 62 & -- & 700 & $\cdots$ & $\begin{array}{r}0 \\
13\end{array}$ & $\begin{array}{l}13 \\
\cdots\end{array}$ & $\begin{array}{l}110 \text { SDMN } \\
\text { BEDROCK }\end{array}$ & SGVC \\
\hline TAW & 63 & - & 13 & $\cdots$ & $\begin{array}{l}0 \\
2\end{array}$ & $\begin{array}{l}2 \\
\therefore\end{array}$ & $\begin{array}{l}\text { 112TILL } \\
110 \text { SDMN }\end{array}$ & $\begin{array}{l}\text { TILL } \\
\text { SDGL }\end{array}$ \\
\hline TAW & 64 & - & 39 & - & - & $\cdots$ & $110 \mathrm{SDMN}$ & SDGL \\
\hline TAW & 65 & $\cdots$ & 48 & $\cdots$ & $\cdots$ & $\cdots$ & $110 \mathrm{SDMN}$ & SDGL \\
\hline TAW & 66 & - & 500 & $\cdots$ & $\begin{array}{r}0 \\
103\end{array}$ & $\begin{array}{r}103 \\
\ldots\end{array}$ & $\begin{array}{l}110 \text { SDMN } \\
\text { BEDROCK }\end{array}$ & SAND \\
\hline TAW & 67 & $\cdots$ & 15 & $\cdots$ & $\begin{array}{r}0 \\
10\end{array}$ & $\begin{array}{l}10 \\
\cdots\end{array}$ & $\begin{array}{l}110 \text { SDMN } \\
110 \text { SDMN }\end{array}$ & $\begin{array}{l}\text { SDGL } \\
\text { SAND }\end{array}$ \\
\hline TAW & 68 & - & 12 & -- & $\cdots$ & $\cdots$ & $110 \mathrm{SDMN}$ & SAND \\
\hline TAW & 69 & $\cdots$ & 60 & $\cdots$ & $\cdots$ & $\cdots$ & $110 \mathrm{SDMN}$ & SAND \\
\hline TAW & 70 & -- & 342 & $\cdots$ & $\begin{array}{r}0 \\
52\end{array}$ & $\begin{array}{l}52 \\
--\end{array}$ & $\begin{array}{l}\text { 112TILL } \\
\text { BEDROCK }\end{array}$ & TILL GRAVEL, HARDPAN \\
\hline TAW & 71 & $\cdots$ & 702 & $\cdots$ & $\begin{array}{r}0 \\
42\end{array}$ & $\begin{array}{l}42 \\
\cdots\end{array}$ & $\begin{array}{l}\text { 112TILL } \\
\text { BEDROCK }\end{array}$ & TILL GRAVEL, HARDPAN \\
\hline TAW & 72 & $\cdots$ & 60 & - & $\cdots$ & - & $110 \mathrm{SDMN}$ & SDGL \\
\hline TAW & 73 & $\cdots$ & 402 & $\cdots$ & $\begin{array}{r}0 \\
50 \\
150 \\
215\end{array}$ & $\begin{array}{r}50 \\
150 \\
215 \\
-.\end{array}$ & $\begin{array}{l}110 \text { SDMN } \\
112 \text { TILL } \\
110 \text { SDMN } \\
\text { BEDROCK }\end{array}$ & $\begin{array}{l}\text { SAND } \\
\text { TILL } \\
\text { CLAY }\end{array}$ \\
\hline
\end{tabular}


Table 2-1. Stratigraphic logs of wells and borings in the Saco and Ossipee River Basins, eastcentral New Hampshire--Continued

\begin{tabular}{|c|c|c|c|c|c|c|c|}
\hline $\begin{array}{l}\text { Local } \\
\text { site } \\
\text { No. }\end{array}$ & $\begin{array}{l}\text { Depth } \\
\text { drilled } \\
\text { (feet) }\end{array}$ & $\begin{array}{l}\text { Depth } \\
\text { of } \\
\text { well } \\
\text { (feet) }\end{array}$ & $\begin{array}{l}\text { Depth } \\
\text { to } \\
\text { refusal } \\
\text { (feet) }\end{array}$ & $\begin{array}{l}\text { Depth } \\
\text { to } \\
\text { top } \\
\text { (feet) }\end{array}$ & $\begin{array}{l}\text { Depth } \\
\text { to } \\
\text { bottom } \\
\text { (feet) }\end{array}$ & $\begin{array}{l}\text { Aquifer } \\
\text { code }\end{array}$ & Lithology \\
\hline
\end{tabular}

\begin{tabular}{|c|c|c|c|c|c|c|c|c|}
\hline \multirow[b]{2}{*}{ TAW } & \multirow[b]{2}{*}{74} & \multirow[b]{2}{*}{ - - } & \multicolumn{6}{|c|}{ TAYWORTH - - Continued } \\
\hline & & & 274 & $\cdots$ & $\begin{array}{r}0 \\
60 \\
200\end{array}$ & $\begin{array}{r}60 \\
200 \\
--\end{array}$ & $\begin{array}{l}110 \text { SDMN } \\
\text { BEDROCK } \\
\text { BEDROCK }\end{array}$ & SDGL \\
\hline TAW & 75 & $\cdots$ & 1100 & $\cdots$ & $\begin{array}{r}0 \\
161\end{array}$ & 161 & $\begin{array}{l}110 \text { SDMN } \\
\text { BEDROCK }\end{array}$ & SDCL \\
\hline TAW & 76 & $\cdots$ & 63 & - & - & $\cdots$ & $110 \mathrm{SDMN}$ & SDGL \\
\hline TAW & 77 & $-\cdot$ & 23 & $-\cdot$ & $\begin{array}{r}0 \\
24\end{array}$ & $\begin{array}{l}24 \\
\cdots\end{array}$ & $\begin{array}{l}110 \mathrm{SDMN} \\
110 \mathrm{SDMN}\end{array}$ & $\begin{array}{l}\text { SDGL } \\
\text { SDCL }\end{array}$ \\
\hline TAW & 78 & $\cdots$ & 45 & $\cdots$ & -. & $\cdots$ & $110 \mathrm{SDMN}$ & SDGL \\
\hline TAW & 79 & $\cdots$ & 70 & - & $\begin{array}{r}0 \\
30\end{array}$ & $\begin{array}{l}30 \\
\cdots\end{array}$ & $\begin{array}{l}110 \mathrm{SDMN} \\
110 \mathrm{SDMN}\end{array}$ & $\begin{array}{l}\text { SAND } \\
\text { SDGL }\end{array}$ \\
\hline TAW & 80 & $\cdots$ & 602 & $-\cdot$ & $\begin{array}{r}0 \\
50\end{array}$ & $\begin{array}{l}50 \\
--\end{array}$ & $\begin{array}{l}110 \text { SDMN } \\
\text { BEDROCK }\end{array}$ & SDCL \\
\hline TAW & 81 & $\cdots$ & 60 & $\cdots$ & $\cdots$ & $\cdots$ & $110 \mathrm{SDMN}$ & SDGL \\
\hline TAW & 82 & -- & 577 & $\cdots$ & $\begin{array}{r}0 \\
25 \\
30 \\
40\end{array}$ & $\begin{array}{l}25 \\
30 \\
40 \\
-.\end{array}$ & $\begin{array}{l}110 \text { SDMN } \\
110 \text { SDMN } \\
110 \text { SDMN } \\
\text { BEDROCK }\end{array}$ & $\begin{array}{l}\text { SDCL } \\
\text { OTHR } \\
\text { CLAY }\end{array}$ \\
\hline TAW & 83 & $\cdots$ & 63 & $\cdots$ & $\cdots$ & $\cdots$ & $110 \mathrm{SDMN}$ & SDGL \\
\hline TAW & 84 & $\cdots$ & 69 & $\cdots$ & $\cdots$ & $\cdots$ & $110 \mathrm{SDMN}$ & SDGL \\
\hline TAW & 85 & $\cdots$ & 72 & $\cdots$ & -- & $\cdots$ & $110 \mathrm{SDMN}$ & SDGL \\
\hline TAW & 86 & $\cdots$ & 66 & $-\cdot$ & $\cdots$ & $\cdots$ & 110 SDMN & SDGL \\
\hline TAW & 87 & $-\cdot$ & 74 & $\cdots$ & $\cdots$ & $\cdots$ & $110 \mathrm{SDMN}$ & SDGL \\
\hline TAW & 88 & - & 363 & $-\cdot$ & $\begin{array}{r}0 \\
12\end{array}$ & 12 & $\begin{array}{l}110 \text { SDMN } \\
\text { BEDROCK }\end{array}$ & SDGL \\
\hline TAW & 89 & -. & 525 & $-\cdot$ & $\begin{array}{r}0 \\
55\end{array}$ & $\begin{array}{l}55 \\
\cdots\end{array}$ & $\begin{array}{l}110 \text { SDMN } \\
\text { BEDROCK }\end{array}$ & SDGL \\
\hline TAW & 90 & $\cdots$ & 48 & $\cdots$ & $\cdots$ & $\cdots$ & $110 \mathrm{SDMN}$ & SDGL \\
\hline TAW & 91 & $\cdots$ & 43 & $\cdots$ & - & $\cdots$ & $110 \mathrm{SDMN}$ & SDGL \\
\hline TAW & 92 & $\cdots$ & 15 & $\cdots$ & $\begin{array}{r}0 \\
52\end{array}$ & $\begin{array}{l}52 \\
\cdots\end{array}$ & $\begin{array}{l}110 \mathrm{SDMN} \\
112 \mathrm{TILL}\end{array}$ & $\begin{array}{l}\text { SDGL } \\
\text { TILL GRAVEL, HARDPAN }\end{array}$ \\
\hline TAW & 93 & $\cdots$ & 550 & - & $\begin{array}{r}0 \\
71\end{array}$ & $\begin{array}{l}71 \\
-\cdot\end{array}$ & $\begin{array}{l}110 \text { SDMN } \\
\text { BEDROCK }\end{array}$ & SDGL \\
\hline TAW & 94 & $\cdots$ & 68 & $\cdots$ & $\cdots$ & $\cdots$ & $110 \mathrm{SDMN}$ & SDGL \\
\hline TAW & 95 & $-\cdot$ & 82 & $\cdots$ & $\begin{array}{r}0 \\
45\end{array}$ & $\begin{array}{l}45 \\
-\end{array}$ & $\begin{array}{l}110 \text { SDMN } \\
\text { BEDROCK }\end{array}$ & SDGL \\
\hline TAW & 96 & $\cdots$ & 40 & $\cdots$ & $\cdots$ & $\cdots$ & $110 \mathrm{SDMN}$ & SAND \\
\hline TAW & 97 & $\cdots$ & 135 & - & $\begin{array}{r}0 \\
130\end{array}$ & $\begin{array}{r}130 \\
\cdots\end{array}$ & $\begin{array}{l}110 \text { SDMN } \\
110 \text { SDMN }\end{array}$ & $\begin{array}{l}\text { SAND } \\
\text { SDGL }\end{array}$ \\
\hline TAW & 98 & $\cdots$ & 453 & $\cdots$ & $\begin{array}{r}-\cdot \\
0 \\
226\end{array}$ & $\begin{array}{r}-. \\
226 \\
-.\end{array}$ & $\begin{array}{l}110 \text { SDMN } \\
112 \text { TILL } \\
\text { BEDROCK }\end{array}$ & $\begin{array}{l}\text { OTHR } \\
\text { TILL CLAY, HARDPAN }\end{array}$ \\
\hline TAW & 99 & - & 28 & $\cdots$ & $\begin{array}{r}0 \\
21 \\
28\end{array}$ & $\begin{array}{l}21 \\
28 \\
\cdots\end{array}$ & $\begin{array}{l}110 \mathrm{SDMN} \\
110 \mathrm{SDMN} \\
112 \mathrm{TILL}\end{array}$ & $\begin{array}{l}\text { SDGL } \\
\text { SAND } \\
\text { TILL }\end{array}$ \\
\hline
\end{tabular}


Table 2-1. Stratigraphic logs of wells and borings in the Saco and Ossipee River Basins, eastcentral New Hampshire--Continued

\begin{tabular}{|c|c|c|c|c|c|c|c|}
\hline $\begin{array}{l}\text { Local } \\
\text { site } \\
\text { no. }\end{array}$ & $\begin{array}{l}\text { Depth } \\
\text { drilled } \\
\text { (feet) }\end{array}$ & $\begin{array}{l}\text { Depth } \\
\text { of } \\
\text { well } \\
\text { (feet) }\end{array}$ & $\begin{array}{l}\text { Depth } \\
\text { to } \\
\text { refusal } \\
\text { (feet) }\end{array}$ & $\begin{array}{l}\text { Depth } \\
\text { to } \\
\text { top } \\
\text { (feet) }\end{array}$ & $\begin{array}{l}\text { Depth } \\
\text { to } \\
\text { bottom } \\
\text { (feet) }\end{array}$ & $\begin{array}{l}\text { Aquifer } \\
\text { code }\end{array}$ & Lithology \\
\hline
\end{tabular}

\begin{tabular}{|c|c|c|c|c|c|c|c|c|}
\hline \multirow[b]{2}{*}{ TAW } & \multirow[b]{2}{*}{100} & \multicolumn{7}{|c|}{ TAMWORTH - - Continued } \\
\hline & & $\cdots$ & 12 & $\cdots$ & $\begin{array}{l}0 \\
2\end{array}$ & 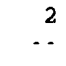 & $\begin{array}{l}\text { 110SDMN } \\
\text { 112TILL }\end{array}$ & $\begin{array}{l}\text { SDGL } \\
\text { TILL }\end{array}$ \\
\hline TAW & 101 & $\cdots$ & 678 & $\cdots$ & $\begin{array}{r}0 \\
14\end{array}$ & $\begin{array}{l}14 \\
\cdots\end{array}$ & $\begin{array}{l}\text { 112TILL } \\
\text { BEDROCK }\end{array}$ & TILL GRAVEL, HARDPAN \\
\hline TAW & 102 & $\cdots$ & 803 & $\cdots$ & $\begin{array}{r}0 \\
12\end{array}$ & $\begin{array}{l}12 \\
\cdots\end{array}$ & $\begin{array}{l}110 \text { SDMN } \\
\text { BEDROCK }\end{array}$ & OTHR \\
\hline TAW & 103 & $\cdots$ & 319 & $\cdots$ & $\begin{array}{l}0 \\
5\end{array}$ & $\begin{array}{c}5 \\
\therefore\end{array}$ & $\begin{array}{l}\text { 112TILL } \\
\text { BEDROCK }\end{array}$ & TILL \\
\hline TAW & 104 & $\cdots$ & 304 & $\cdots$ & $\begin{array}{r}0 \\
50\end{array}$ & $\begin{array}{l}50 \\
\cdots\end{array}$ & $\begin{array}{l}110 \text { SDMN } \\
\text { BEDROCK }\end{array}$ & SAND \\
\hline TAW & 105 & -- & 12 & $-\cdot$ & - & $\cdots$ & 112TILL & TILL \\
\hline TAW & 106 & $\cdots$ & 66 & $\cdots$ & $\cdots$ & $\cdots$ & 110SDMN & SDGL \\
\hline TAW & 107 & $\cdots$ & 502 & $\cdots$ & $\begin{array}{r}0 \\
22\end{array}$ & $\begin{array}{l}22 \\
\cdots\end{array}$ & $\begin{array}{l}110 \text { SDMN } \\
\text { BEDROCK }\end{array}$ & SDGL \\
\hline TAW & 108 & $-\cdot$ & 800 & $\cdots$ & $\begin{array}{r}0 \\
70 \\
185\end{array}$ & $\begin{array}{r}70 \\
185 \\
-\cdot\end{array}$ & $\begin{array}{l}\text { 112TILL } \\
112 T I L L \\
\text { BEDROCK }\end{array}$ & $\begin{array}{l}\text { TILL GRAVEL, CLAY, HARDPAN } \\
\text { TILL CLAY, HARDPAN }\end{array}$ \\
\hline TAW & 109 & $\cdots$ & 300 & $\cdots$ & $\begin{array}{r}0 \\
70 \\
185\end{array}$ & $\begin{array}{r}70 \\
185 \\
-.\end{array}$ & $\begin{array}{l}110 \text { SDMN } \\
112 \text { TILL } \\
\text { BEDROCK }\end{array}$ & $\begin{array}{l}\text { SGVC } \\
\text { TILL CLAY, HARDPAN }\end{array}$ \\
\hline TAW & 110 & $\cdots$ & 527 & $\cdots$ & $\begin{array}{r}0 \\
50\end{array}$ & $\begin{array}{l}50 \\
\cdots\end{array}$ & $\begin{array}{l}110 \text { SDMN } \\
\text { BEDROCK }\end{array}$ & SDCL \\
\hline TAW & 111 & $\cdots$ & 378 & $\cdots$ & $\begin{array}{r}0 \\
210\end{array}$ & $\begin{array}{r}210 \\
-\end{array}$ & $\begin{array}{l}\text { 110SDMN } \\
\text { BEDROCK }\end{array}$ & SGVC \\
\hline TAW & 112 & $\cdots$ & 20 & $\cdots$ & $\begin{array}{r}0 \\
25\end{array}$ & $\begin{array}{l}25 \\
\cdots\end{array}$ & $\begin{array}{l}\text { 110SDMN } \\
112 \text { TILL }\end{array}$ & $\begin{array}{l}\text { SDGL } \\
\text { TILL }\end{array}$ \\
\hline TAW & 113 & - & 33 & $\cdots$ & $\begin{array}{r}0 \\
12\end{array}$ & $\begin{array}{l}12 \\
\cdots\end{array}$ & $\begin{array}{l}110 \text { SDMN } \\
110 \text { SDMN }\end{array}$ & $\begin{array}{l}\text { SDGL } \\
\text { SAND }\end{array}$ \\
\hline TAW & 114 & $\cdots$ & 71 & $\cdots$ & $\cdots$ & $\cdots$ & 110 SDMN & SDGL \\
\hline TAW & 115 & $\cdots$ & 84 & $\cdots$ & $\begin{array}{r}0 \\
60\end{array}$ & $\begin{array}{l}60 \\
\cdots\end{array}$ & $\begin{array}{l}110 \text { SDMN } \\
110 \text { SDMN }\end{array}$ & $\begin{array}{l}\text { SAND } \\
\text { SDGL }\end{array}$ \\
\hline TAW & 116 & $\cdots$ & 650 & $\cdots$ & $\begin{array}{r}0 \\
40\end{array}$ & $\begin{array}{l}40 \\
\cdots\end{array}$ & $\begin{array}{l}\text { 112TILL } \\
\text { BEDROCK }\end{array}$ & TILL SAND, CLAY, HARDPAN \\
\hline TAW & 117 & $\cdots$ & 453 & $\cdots$ & $\begin{array}{r}0 \\
10\end{array}$ & 10 & $\begin{array}{l}\text { 112TILL } \\
\text { BEDROCK }\end{array}$ & TILL \\
\hline TAW & 118 & $\cdots$ & 527 & $\cdots$ & $\begin{array}{l}0 \\
8\end{array}$ & $\begin{array}{l}8 \\
--\end{array}$ & $\begin{array}{l}\text { 112TILL } \\
\text { BEDROCK }\end{array}$ & TILL \\
\hline TAW & 119 & $\cdots$ & 66 & $\cdots$ & $\cdots$ & $\cdots$ & 110 SDMN & SDGL \\
\hline TAW & 120 & $\cdots$ & 66 & $\cdots$ & $\cdots$ & $\cdots$ & 110SDMN & SDGL \\
\hline TAW & 121 & $\cdots$ & 65.5 & $\cdots$ & $\cdots$ & $\cdots$ & $110 \mathrm{SDMN}$ & SAND \\
\hline TAW & 122 & $\cdots$ & 627 & $\cdots$ & $\begin{array}{r}0 \\
26\end{array}$ & $\begin{array}{l}26 \\
\cdots\end{array}$ & $\begin{array}{l}110 S D M N \\
\text { BEDROCK }\end{array}$ & SDGL \\
\hline TAW & 123 & $\cdots$ & 577 & $\cdots$ & $\begin{array}{r}0 \\
47 \\
138\end{array}$ & $\begin{array}{r}47 \\
138 \\
\cdots\end{array}$ & $\begin{array}{l}110 \text { SDMN } \\
112 \text { TILL } \\
\text { BEDROCK }\end{array}$ & $\begin{array}{l}\text { SDGL } \\
\text { TILL CLAY, HARDPAN }\end{array}$ \\
\hline TAW & 124 & $\cdots$ & 427 & $\cdots$ & $\begin{array}{r}0 \\
194\end{array}$ & $\begin{array}{r}194 \\
\cdots\end{array}$ & $\begin{array}{l}\text { 112TILL } \\
\text { BEDROCK }\end{array}$ & TILL CLAY, HARDPAN \\
\hline
\end{tabular}


Table 2-1. Stratigraphic logs of wells and borings in the Saco and Ossipee River Basins, eastcentral New Hampshire--Continued

\begin{tabular}{|c|c|c|c|c|c|c|c|c|}
\hline $\begin{array}{l}\text { Loce } \\
\text { site } \\
\text { No. }\end{array}$ & & $\begin{array}{l}\text { Depth } \\
\text { drilled } \\
\text { (feet) }\end{array}$ & $\begin{array}{l}\text { Depth } \\
\text { of } \\
\text { well } \\
\text { (feet) }\end{array}$ & $\begin{array}{l}\text { Depth } \\
\text { to } \\
\text { refusal } \\
\text { (feet) }\end{array}$ & $\begin{array}{l}\text { Depth } \\
\text { to } \\
\text { top } \\
\text { (feet) }\end{array}$ & $\begin{array}{l}\text { Depth } \\
\text { to } \\
\text { bottom } \\
\text { (feet) }\end{array}$ & $\begin{array}{l}\text { Aquifer } \\
\text { code }\end{array}$ & Lithology \\
\hline & & \multicolumn{7}{|c|}{ TAYWORTH - - Continued } \\
\hline TAW & 125 & $\cdots$ & 800 & $\cdots$ & $\begin{array}{r}0 \\
30 \\
35 \\
100\end{array}$ & $\begin{array}{r}30 \\
35 \\
100 \\
\ldots\end{array}$ & $\begin{array}{l}112 \text { TILL } \\
110 \text { SDMN } \\
112 \text { TILL } \\
\text { BEDROCK }\end{array}$ & $\begin{array}{l}\text { TILL } \\
\text { SAND } \\
\text { TILL CLAY, HARDPAN }\end{array}$ \\
\hline TAW & 126 & $\cdots$ & 727 & $\cdots$ & $\begin{array}{r}0 \\
10\end{array}$ & $\begin{array}{l}10 \\
-\cdot\end{array}$ & $\begin{array}{l}\text { 112TILL } \\
\text { BEDROCK }\end{array}$ & TILL CLAY, HARDPAN \\
\hline TAW & 127 & - & 227 & $\cdots$ & $\begin{array}{r}0 \\
17\end{array}$ & $\begin{array}{l}17 \\
\therefore\end{array}$ & $\begin{array}{l}110 \text { SDMN } \\
\text { BEDROCK }\end{array}$ & SDGL \\
\hline TAW & 128 & $\cdots$ & 253 & -. & $\begin{array}{r}0 \\
180\end{array}$ & $\begin{array}{r}180 \\
-.\end{array}$ & $\begin{array}{l}\text { 112TILL } \\
\text { BEDROCK }\end{array}$ & TILL CLAY, HARDPAN \\
\hline TAW & 129 & $\cdots$ & 66 & $\cdots$ & $-\cdot$ & $\cdots$ & $\cdots$ & \\
\hline TAW & 130 & $\cdots$ & 875 & $\cdots$ & $\begin{array}{r}0 \\
20\end{array}$ & $\begin{array}{l}20 \\
-.\end{array}$ & $\begin{array}{l}110 \mathrm{SDMN} \\
\text { BEDROCK }\end{array}$ & SDGL \\
\hline TAW & 131 & $\cdots$ & 425 & $\cdots$ & $\begin{array}{r}0 \\
30\end{array}$ & $\begin{array}{l}30 \\
--\end{array}$ & $\begin{array}{l}\text { 112TILL } \\
\text { BEDROCK }\end{array}$ & TILL \\
\hline TAW & 132 & $\cdots$ & 752 & $\cdots$ & $\begin{array}{l}0 \\
8\end{array}$ & $\begin{array}{l}8 \\
-\end{array}$ & $\begin{array}{l}112 \text { TILL } \\
\text { BEDROCK }\end{array}$ & TILL \\
\hline TAW & 133 & $-\cdot$ & 452 & $\cdots$ & $\begin{array}{r}0 \\
25\end{array}$ & $\begin{array}{l}25 \\
--\end{array}$ & $\begin{array}{l}110 \text { SDMN } \\
\text { BEDROCK }\end{array}$ & SDGL \\
\hline TAW & 134 & $\cdots$ & 202 & $\cdots$ & $\begin{array}{r}0 \\
24\end{array}$ & 24 & $\begin{array}{l}110 \text { SDMN } \\
\text { BEDROCK }\end{array}$ & SAND \\
\hline TAW & 135 & $\cdots$ & 66 & $\cdots$ & $\cdots$ & $\cdots$ & $110 \mathrm{SDMN}$ & SDGL \\
\hline TAW & 136 & -- & 66 & $\cdots$ & $\cdots$ & $-\cdot$ & 110 SDMN & SDGL \\
\hline TAW & 137 & $\cdots$ & 67 & $\cdots$ & $\cdots$ & $\cdots$ & $110 \mathrm{SDMN}$ & SDGL \\
\hline TAW & 138 & $\cdots$ & 66 & $\cdots$ & $\cdots$ & $\cdots$ & 110 SDMN & SDGL \\
\hline TAW & 139 & $\cdots$ & 66 & $\cdots$ & $\cdots$ & $\cdots$ & $110 \mathrm{SDMN}$ & SDGL \\
\hline TAW & 140 & $\cdots$ & 66 & $\cdots$ & $\cdots$ & $\cdots$ & 110 SDMN & SDGL \\
\hline TAW & 141 & - & 45 & - & $\begin{array}{r}0 \\
25\end{array}$ & $\begin{array}{l}25 \\
-.\end{array}$ & $\begin{array}{l}110 \mathrm{SDMN} \\
110 \mathrm{SDMN}\end{array}$ & $\begin{array}{l}\text { SDGL } \\
\text { SAND }\end{array}$ \\
\hline TAW & 142 & $\cdots$ & 310 & $\cdots$ & $\begin{array}{r}0 \\
160\end{array}$ & $\begin{array}{r}160 \\
\cdots\end{array}$ & $\begin{array}{l}\text { 112TILL } \\
\text { BEDROCK }\end{array}$ & TILL CLAY, HARDPAN \\
\hline TAW & 143 & -- & 440 & $\cdots$ & $\begin{array}{r}0 \\
10\end{array}$ & $\begin{array}{l}10 \\
\cdots\end{array}$ & $\begin{array}{l}\text { 112TILL } \\
\text { BEDROCK }\end{array}$ & TILL \\
\hline TAW & 144 & $\cdots$ & 52.5 & - & $\cdots$ & $\cdots$ & $110 \mathrm{SDMN}$ & SAND \\
\hline TAW & 145 & $\cdots$ & 62 & $\cdots$ & $-\cdot$ & $\cdots$ & $110 \mathrm{SDMN}$ & SDGL \\
\hline TAW & 146 & $\cdots$ & 63 & $\cdots$ & $\cdots$ & $\cdots$ & 110 SDMN & SDGL \\
\hline TAW & 147 & -. & 67.5 & $\cdots$ & $\cdots$ & $\cdots$ & $110 \mathrm{SDMN}$ & SDGL \\
\hline TAW & 148 & $\cdots$ & 63 & $\cdots$ & $\cdots$ & $\cdots$ & 110 SDMN & SDGL \\
\hline TAW & 149 & $-\cdot$ & 63 & - & -. & $\cdots$ & 110 SDMN & SDGL \\
\hline TAW & 150 & $\cdots$ & 502 & $-\cdot$ & $\begin{array}{r}0 \\
95\end{array}$ & $\begin{array}{l}95 \\
-.\end{array}$ & $\begin{array}{l}\text { 112TILL } \\
\text { BEDROCK }\end{array}$ & TILL SAND, CLAY, HARDPAN \\
\hline TAW & 151 & $\cdots$ & 242 & $\cdots$ & $\begin{array}{r}0 \\
100 \\
180\end{array}$ & $\begin{array}{r}100 \\
180 \\
-.\end{array}$ & $\begin{array}{l}\text { 112TILL } \\
\text { 110SDMN } \\
\text { BEDROCK }\end{array}$ & $\begin{array}{l}\text { TILL } \\
\text { CLAY }\end{array}$ \\
\hline
\end{tabular}


Table 2-1. Stratigraphic logs of wells and borings in the Saco and Ossipee River Basins, eastcentral New Hampshire--Continued

\begin{tabular}{|c|c|c|c|c|c|c|c|c|}
\hline \multicolumn{2}{|c|}{$\begin{array}{l}\text { Local } \\
\text { site } \\
\text { no. }\end{array}$} & $\begin{array}{l}\text { Depth } \\
\text { drilled } \\
\text { (feet) }\end{array}$ & $\begin{array}{l}\text { Depth } \\
\text { of } \\
\text { well } \\
\text { (feet) }\end{array}$ & $\begin{array}{l}\text { Depth } \\
\text { to } \\
\text { refusal } \\
\text { (feet) }\end{array}$ & $\begin{array}{l}\text { Depth } \\
\text { to } \\
\text { top } \\
\text { (feet) }\end{array}$ & $\begin{array}{l}\text { Depth } \\
\text { to } \\
\text { bottom } \\
\text { (feet) }\end{array}$ & $\begin{array}{l}\text { Aquifer } \\
\text { code }\end{array}$ & Lithology \\
\hline & & \multicolumn{7}{|c|}{ TAMarORTH - - Continued } \\
\hline TAW & 152 & $\cdots$ & 425 & $\cdots$ & $\begin{array}{l}0 \\
5\end{array}$ & $\begin{array}{r}5 \\
--\end{array}$ & $\begin{array}{l}\text { 112TILL } \\
\text { BEDROCK }\end{array}$ & TILL GRAVEL, HARDPAN \\
\hline TAW & 153 & - & 602 & -- & $\begin{array}{r}0 \\
115\end{array}$ & $\begin{array}{r}115 \\
-\cdot\end{array}$ & $\begin{array}{l}110 \text { SDMN } \\
\text { BEDROCK }\end{array}$ & SDCL \\
\hline TAW & 154 & - & 31 & - & $\begin{array}{r}0 \\
33\end{array}$ & 33 & $\begin{array}{l}110 \text { SDMN } \\
112 \text { TILL }\end{array}$ & $\begin{array}{l}\text { SAND } \\
\text { TILL }\end{array}$ \\
\hline TAW & 155 & $\cdots$ & 31 & $\cdots$ & $\begin{array}{r}0 \\
33\end{array}$ & $\begin{array}{l}33 \\
-\cdot\end{array}$ & $\begin{array}{l}110 \text { SDMN } \\
112 \text { TILL }\end{array}$ & $\begin{array}{l}\text { SAND } \\
\text { TILL }\end{array}$ \\
\hline TAW & 156 & $\cdots$ & 67 & $\cdots$ & -- & $\cdots$ & 110 SDMN & SDGL \\
\hline TAW & 157 & $\cdots$ & 680 & $\cdots$ & $\begin{array}{r}0 \\
160\end{array}$ & $\begin{array}{c}160 \\
\ldots\end{array}$ & $\begin{array}{l}\text { 112TILL } \\
\text { BEDROCK }\end{array}$ & TILL \\
\hline TAW & 158 & $\cdots$ & 49 & $\cdots$ & $\begin{array}{r}0 \\
35 \\
50\end{array}$ & $\begin{array}{l}35 \\
50 \\
\ldots\end{array}$ & $\begin{array}{l}110 \text { SDMN } \\
110 \text { SDMN } \\
\text { BEDROCK }\end{array}$ & $\begin{array}{l}\text { SAND } \\
\text { SDGL }\end{array}$ \\
\hline TAW & 159 & $\cdots$ & 762 & $\cdots$ & $\cdots$ & $-\cdot$ & - & \\
\hline TAW & 160 & $-\cdot$ & 908 & $\cdots$ & $\begin{array}{l}0 \\
4\end{array}$ & $\begin{array}{c}4 \\
--\end{array}$ & $\begin{array}{l}110 \text { SDMN } \\
\text { BEDROCK }\end{array}$ & SDGL \\
\hline TAW & 161 & $-\cdot$ & 1030 & $\cdots$ & $\begin{array}{r}0 \\
10\end{array}$ & 10 & $\begin{array}{l}110 \text { SDMN } \\
\text { BEDROCK }\end{array}$ & SDGL \\
\hline TAW & 162 & $-\cdot$ & 201 & $\cdots$ & $\begin{array}{l}0 \\
5\end{array}$ & $\begin{array}{c}5 \\
\cdots\end{array}$ & $\begin{array}{l}110 \text { SDMN } \\
\text { BEDROCK }\end{array}$ & SDGL \\
\hline TAW & 163 & $\cdots$ & 66 & $-\cdot$ & - & $\cdots$ & 110 SDMN & SDGL \\
\hline TAW & 164 & $\cdots$ & 65 & $\cdots$ & $\cdots$ & $\cdots$ & 110 SDMN & SDGL \\
\hline TAW & 165 & $\cdots$ & 30 & $\cdots$ & $-\cdot$ & $\cdots$ & 110 SDMN & SDGL \\
\hline TAW & 166 & $-\cdot$ & 25 & $\cdots$ & $\cdots$ & $\cdots$ & $110 \mathrm{SDMN}$ & SDGL \\
\hline TAW & 167 & $\cdots$ & 952 & $\cdots$ & $\begin{array}{r}0 \\
60 \\
105\end{array}$ & $\begin{array}{r}60 \\
105 \\
\cdots\end{array}$ & $\begin{array}{l}\text { 110SDMN } \\
112 \text { TILL } \\
\text { BEDROCK }\end{array}$ & $\begin{array}{l}\text { SDGL } \\
\text { TILL CLAY, HARDPAN }\end{array}$ \\
\hline TAW & 168 & - & 560 & $\cdots$ & $\begin{array}{r}0 \\
40\end{array}$ & $\begin{array}{l}40 \\
\cdots\end{array}$ & $\begin{array}{l}112 \text { TILL } \\
\text { BEDROCK }\end{array}$ & TILL GRAVEL, CLAY, HARDPAN \\
\hline TAW & 169 & $-\cdot$ & 66 & $\cdots$ & -- & $\cdots$ & 110 SDMN & SDGL \\
\hline TAW & 170 & $\cdots$ & 23 & $-\cdot$ & $\begin{array}{r}0 \\
12\end{array}$ & $\begin{array}{l}12 \\
\cdots\end{array}$ & $\begin{array}{l}110 \text { SDMN } \\
110 \text { SDMN }\end{array}$ & $\begin{array}{l}\text { SDGL } \\
\text { SDGL }\end{array}$ \\
\hline TAW & 171 & $\cdot-$ & 421 & $\cdots$ & $\begin{array}{r}0 \\
50 \\
60 \\
80\end{array}$ & $\begin{array}{l}50 \\
60 \\
80 \\
\cdots\end{array}$ & $\begin{array}{l}110 \text { SDMN } \\
110 \mathrm{SDMN} \\
112 \mathrm{TILL} \\
\text { BEDROCK }\end{array}$ & $\begin{array}{l}\text { SAND } \\
\text { SDGL } \\
\text { TILL }\end{array}$ \\
\hline TAW & 172 & $-\cdot$ & 400 & $\cdots$ & $\begin{array}{r}0 \\
126\end{array}$ & $\begin{array}{r}126 \\
\cdots\end{array}$ & $\begin{array}{l}110 \text { SDMN } \\
\text { BEDROCK }\end{array}$ & SAND \\
\hline TAW & 173 & $-\cdot$ & 66 & $\cdots$ & $\cdots$ & $\cdots$ & 110 SDMN & SDGL \\
\hline TAW & 174 & $\cdots$ & 75 & $\cdots$ & $\cdots$ & $\cdots$ & $110 \mathrm{SDMN}$ & SDGL \\
\hline TAW & 175 & $\cdots$ & 600 & $\cdots$ & $\begin{array}{r}0 \\
238\end{array}$ & $\begin{array}{r}238 \\
\cdots\end{array}$ & $\begin{array}{l}\text { 112TILL } \\
\text { BEDROCK }\end{array}$ & TILL GRAVEL, HARDPAN \\
\hline TAW & 176 & $\cdots$ & 66 & $\cdots$ & $\cdots$ & $\cdots$ & 110 SDMN & SAND \\
\hline TAW & 177 & - & 66 & .. & -. & - & $110 \mathrm{SDMN}$ & SDGL \\
\hline
\end{tabular}


Table 2-1. Stratigraphic logs of wells and borings in the Saco and Osslpee River Basins, eastcentral New Hampshire--Continued

\begin{tabular}{|c|c|c|c|c|c|c|c|c|}
\hline \multicolumn{2}{|c|}{$\begin{array}{l}\text { Local } \\
\text { site } \\
\text { No. }\end{array}$} & $\begin{array}{l}\text { Depth } \\
\text { drilled } \\
\text { (feet) }\end{array}$ & $\begin{array}{l}\text { Depth } \\
\text { of } \\
\text { well } \\
\text { (feet) }\end{array}$ & $\begin{array}{l}\text { Depth } \\
\text { to } \\
\text { refusal } \\
\text { (feet) }\end{array}$ & $\begin{array}{l}\text { Depth } \\
\text { to } \\
\text { top } \\
\text { (feet) }\end{array}$ & $\begin{array}{l}\text { Depth } \\
\text { to } \\
\text { bottom } \\
\text { (feet) }\end{array}$ & $\begin{array}{l}\text { Aquifer } \\
\text { code }\end{array}$ & Lithology \\
\hline & & \multicolumn{7}{|c|}{ TAMWORTH - - Continued } \\
\hline TAW & 178 & $-\cdot$ & 67.5 & $\cdots$ & $\cdots$ & $\cdots$ & 110 SDMN & SAND \\
\hline TAW & 179 & -- & 102 & $-\cdot$ & $\begin{array}{r}0 \\
62\end{array}$ & $\begin{array}{l}62 \\
\cdots\end{array}$ & $\begin{array}{l}\text { 112TILL } \\
\text { BEDROCK }\end{array}$ & TILL SAND, GRAVEL, HARDPAN \\
\hline TAW & 180 & - & 202 & $-\cdot$ & $\begin{array}{r}0 \\
89\end{array}$ & $\begin{array}{l}89 \\
\cdots\end{array}$ & $\begin{array}{l}110 \text { SDMN } \\
\text { BEDROCK }\end{array}$ & SAND \\
\hline TAW & 181 & $\cdots$ & 361 & $\cdots$ & $\begin{array}{r}0 \\
160\end{array}$ & $\begin{array}{r}160 \\
--\end{array}$ & $\begin{array}{l}\text { 112TILL } \\
\text { BEDROCK }\end{array}$ & TILL \\
\hline TAW & 182 & $-\cdot$ & 227 & 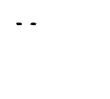 & $\begin{array}{r}0 \\
70 \\
77\end{array}$ & $\begin{array}{l}70 \\
77 \\
-.\end{array}$ & $\begin{array}{l}110 \text { SDMN } \\
110 \text { SDMN } \\
\text { BEDROCK }\end{array}$ & $\begin{array}{l}\text { SGVC } \\
\text { CLAY }\end{array}$ \\
\hline TAW & 183 & - & 377 & - & $\begin{array}{r}0 \\
117\end{array}$ & $\begin{array}{r}117 \\
-.\end{array}$ & $\begin{array}{l}\text { 112TILL } \\
\text { BEDROCK }\end{array}$ & TILL GRAVEL, CLAY, HARDPAN \\
\hline TAW & 184 & $-\cdot$ & 43 & $-\cdot$ & $-\cdot$ & $-\cdot$ & 110 SDMN & SAND \\
\hline TAW & 185 & $\cdots$ & 54 & $\cdots$ & $\cdots$ & $\cdots$ & 110 SDMN & SDGL \\
\hline TAW & 186 & $-\cdot$ & 66 & - & - & -. & $110 \mathrm{SDMN}$ & SDGL \\
\hline TAW & 187 & - & 72 & - & $\cdots$ & - & 110 SDMN & SDGL \\
\hline TAW & 188 & -. & 64 & -. & - & - - & $110 \mathrm{SDMN}$ & SDGL \\
\hline TAW & 189 & $\cdots$ & 402 & -. & $\begin{array}{r}0 \\
70\end{array}$ & $\begin{array}{l}70 \\
\cdots\end{array}$ & $\begin{array}{l}\text { 112TILL } \\
\text { BEDROCK }\end{array}$ & TILL \\
\hline TAW & 190 & - & 68 & $\cdots$ & - & $\cdots$ & $110 \mathrm{SDMN}$ & SDGL \\
\hline TAW & 191 & $\cdots$ & 68 & $\cdots$ & -. & - & 110 SDMN & SDGL \\
\hline TAW & 192 & $\cdots$ & 577 & $\cdots$ & $\begin{array}{r}0 \\
60 \\
105\end{array}$ & $\begin{array}{r}60 \\
105\end{array}$ & $\begin{array}{l}110 \text { SDMN } \\
110 \text { SDMN } \\
\text { BEDROCK }\end{array}$ & $\begin{array}{l}\text { SDGL } \\
\text { SDCL }\end{array}$ \\
\hline TAW & 193 & $\cdots$ & 277 & $\cdots$ & $\begin{array}{r}\cdots \\
0 \\
220\end{array}$ & 220 & $\begin{array}{l}110 \text { SDMN } \\
110 \text { SDMN } \\
\text { BEDROCK }\end{array}$ & $\begin{array}{l}\text { SGVC } \\
\text { CLAY }\end{array}$ \\
\hline TAW & 194 & - & 12 & .. & . & - & 112TILL & TILL \\
\hline TAW & 195 & - - & - & $\cdots$ & $\begin{array}{r}0 \\
55\end{array}$ & $\begin{array}{l}55 \\
-\cdot\end{array}$ & $\begin{array}{l}110 \text { SDMN } \\
\text { BEDROCK }\end{array}$ & SDCL \\
\hline TAW & 196 & $-\cdot$ & 230 & - & $\begin{array}{l}0 \\
9\end{array}$ & 9 & $\begin{array}{l}\text { 112TILL } \\
\text { BEDROCK }\end{array}$ & TILL \\
\hline TAW & 197 & $\cdots$ & 64 & $\cdots$ & $\cdots$ & $\cdots$ & 110 SDMN & SDGL \\
\hline TAW & 198 & $\cdots$ & 66 & $\cdots$ & $\cdots$ & -. & 110 SDMN & SDGL \\
\hline TAW & 199 & $\cdots$ & 423 & - & $\begin{array}{r}0 \\
190\end{array}$ & 190 & $\begin{array}{l}\text { 112TILL } \\
\text { BEDROCK }\end{array}$ & TILL \\
\hline TAW & 200 & $\cdots$ & 30 & $\cdots$ & $\begin{array}{r}0 \\
30\end{array}$ & 30 & $\begin{array}{l}110 \text { SDMN } \\
110 \text { SDMN }\end{array}$ & $\begin{array}{l}\text { SAND } \\
\text { SDCL }\end{array}$ \\
\hline TAW & 201 & - & 322 & $\cdots$ & $\begin{array}{r}0 \\
200\end{array}$ & $\begin{array}{r}200 \\
--\end{array}$ & $\begin{array}{l}\text { 112TILL } \\
\text { BEDROCK }\end{array}$ & TILL \\
\hline TAW & 202 & - & 550 & $\cdots$ & $\begin{array}{l}0 \\
7\end{array}$ & $\begin{array}{l}7 \\
\cdots\end{array}$ & $\begin{array}{l}\text { 112TILL } \\
\text { BEDROCK }\end{array}$ & TILL \\
\hline TAW & 203 & - & 68 & - & -. & - & 110 SDMN & SDGL \\
\hline TAW & 204 & . & 24 & -. & $\begin{array}{l}0 \\
5\end{array}$ & $\begin{array}{r}5 \\
--\end{array}$ & $\begin{array}{l}110 \text { SDMN } \\
110 \text { SDMN }\end{array}$ & $\begin{array}{l}\text { SGVC } \\
\text { SDGL }\end{array}$ \\
\hline
\end{tabular}


Table 2-1. Stratigraphic logs of wells and borings in the Saco and Ossipee River Basins, eastcentral New Hampshire--Continued

\begin{tabular}{|c|c|c|c|c|c|c|c|c|}
\hline \multicolumn{2}{|c|}{$\begin{array}{l}\text { Local } \\
\text { site } \\
\text { no. }\end{array}$} & $\begin{array}{l}\text { Depth } \\
\text { drilled } \\
\text { (feet) }\end{array}$ & $\begin{array}{l}\text { Depth } \\
\text { of } \\
\text { well } \\
\text { (feet) }\end{array}$ & $\begin{array}{l}\text { Depth } \\
\text { to } \\
\text { refusal } \\
\text { (feet) }\end{array}$ & $\begin{array}{l}\text { Depth } \\
\text { to } \\
\text { top } \\
\text { (feet) }\end{array}$ & $\begin{array}{l}\text { Depth } \\
\text { to } \\
\text { bottom } \\
\text { (feet) }\end{array}$ & $\begin{array}{l}\text { Aquifer } \\
\text { code }\end{array}$ & Lithology \\
\hline & & \multicolumn{7}{|c|}{ TAYWORTH - Continued } \\
\hline TAW & 205 & $\cdots$ & 360 & $\cdots$ & $\begin{array}{r}0 \\
78\end{array}$ & $\begin{array}{l}78 \\
-\end{array}$ & $\begin{array}{l}110 \text { SDMN } \\
\text { BEDROCK }\end{array}$ & SDGL \\
\hline TAW & 206 & $\cdots$ & 67 & $\cdots$ & $\cdots$ & $\cdots$ & $110 \mathrm{SDMN}$ & SDGL \\
\hline TAW & 207 & $\cdots$ & 85 & $\cdots$ & -- & $\cdots$ & $110 \mathrm{SDMN}$ & SDGL \\
\hline TAW & 208 & $\cdots$ & 83 & -. & $\cdots$ & -- & $110 \mathrm{SDMN}$ & SDGL \\
\hline TAW & 209 & $-\cdot$ & 352 & $\cdots$ & $\begin{array}{r}0 \\
40\end{array}$ & $\begin{array}{l}40 \\
-\end{array}$ & $\begin{array}{l}110 \text { SDMN } \\
\text { BEDROCK }\end{array}$ & OTHR \\
\hline TAW & 210 & $\cdots$ & 502 & $\cdots$ & $\begin{array}{r}0 \\
65\end{array}$ & $\begin{array}{l}65 \\
\cdots\end{array}$ & $\begin{array}{l}110 \text { SDMN } \\
\text { BEDROCK }\end{array}$ & CLAY \\
\hline TAW & 211 & $\cdots$ & 902 & $\cdots$ & $\begin{array}{r}0 \\
60\end{array}$ & $\begin{array}{l}60 \\
--\end{array}$ & $\begin{array}{l}110 \text { SDMN } \\
\text { BEDROCK }\end{array}$ & SDCL \\
\hline TAW & 212 & $\cdots$ & 652 & $\cdots$ & $\begin{array}{r}0 \\
15\end{array}$ & $\begin{array}{l}15 \\
\cdots\end{array}$ & $\begin{array}{l}110 \text { SDMN } \\
\text { BEDROCK }\end{array}$ & SDGL \\
\hline TAW & 213 & $\cdots$ & 1160 & $\cdots$ & $\begin{array}{r}0 \\
50\end{array}$ & $\begin{array}{l}50 \\
\cdots\end{array}$ & $\begin{array}{l}110 \text { SDMN } \\
\text { BEDROCK }\end{array}$ & SDCL \\
\hline TAW & 214 & $\cdots$ & 442 & $\cdots$ & $\begin{array}{r}0 \\
132\end{array}$ & 132 & $\begin{array}{l}110 \text { SDMN } \\
\text { BEDROCK }\end{array}$ & CLAY \\
\hline TAW & 215 & $\cdots$ & 322 & $\cdots$ & $\begin{array}{r}0 \\
52\end{array}$ & $\begin{array}{l}52 \\
-.\end{array}$ & $\begin{array}{l}110 \text { SDMN } \\
\text { BEDROCK }\end{array}$ & SDGL \\
\hline TAW & 216 & $\cdots$ & 69 & $\cdots$ & $\cdots$ & $\cdots$ & $110 \mathrm{SDMN}$ & SDGL \\
\hline TAW & 217 & $\cdots$ & 72 & $\cdots$ & $\cdots$ & $\cdots$ & $110 \mathrm{SDMN}$ & SDGL \\
\hline TAW & 218 & $\cdots$ & 260 & $-\cdot$ & $\begin{array}{r}0 \\
185\end{array}$ & $\begin{array}{r}185 \\
\cdots\end{array}$ & $\begin{array}{l}\text { 112TILL } \\
\text { BEDROCK }\end{array}$ & TILL GRAVEL, CLAY, HARDPAN \\
\hline TAW & 219 & $-\cdot$ & 79 & - & $\cdots$ & $\cdots$ & $110 \mathrm{SDMN}$ & SDGL \\
\hline TAW & 220 & $\cdots$ & 70 & $-\cdot$ & $\cdots$ & $\cdots$ & $110 \mathrm{SDMN}$ & SDGL \\
\hline TAW & 221 & - & 66 & $\cdots$ & $\cdots$ & - & 110 SDMN & SDGL \\
\hline TAW & 222 & $\cdots$ & 504 & $\cdots$ & $\begin{array}{r}0 \\
60 \\
95 \\
129\end{array}$ & $\begin{array}{r}60 \\
95 \\
129\end{array}$ & $\begin{array}{l}110 \text { SDMN } \\
112 \mathrm{TILL} \\
110 \mathrm{SDMN} \\
\text { BEDROCK }\end{array}$ & $\begin{array}{l}\text { SDGL } \\
\text { TILL } \\
\text { OTHR ROTTEN ROCK }\end{array}$ \\
\hline TAW & 223 & $\cdots$ & 525 & - & $\begin{array}{r}0 \\
50 \\
110\end{array}$ & $\begin{array}{r}50 \\
110 \\
\cdots\end{array}$ & $\begin{array}{l}110 \text { SDMN } \\
110 \text { SDMN } \\
\text { BEDROCK }\end{array}$ & $\begin{array}{l}\text { SDGL } \\
\text { OTHR ROTTEN ROCK }\end{array}$ \\
\hline TAW & 224 & $\cdots$ & 524 & - & $\begin{array}{r}0 \\
60 \\
100\end{array}$ & $\begin{array}{r}60 \\
100 \\
-\cdots\end{array}$ & $\begin{array}{l}110 \text { SDMN } \\
110 \text { SDMN } \\
\text { BEDROCK }\end{array}$ & $\begin{array}{l}\text { SDGL } \\
\text { OTHR ROTTEN ROCK }\end{array}$ \\
\hline TAW & 225 & - & 624 & $\cdots$ & $\begin{array}{r}0 \\
96\end{array}$ & $\begin{array}{l}96 \\
-.\end{array}$ & $\begin{array}{l}110 \text { SDMN } \\
\text { BEDROCK }\end{array}$ & SGVC \\
\hline TAW & 226 & $\cdots$ & 120 & $\cdots$ & $\begin{array}{r}0 \\
60\end{array}$ & $\begin{array}{l}60 \\
\cdots\end{array}$ & $\begin{array}{l}110 \text { SDMN } \\
\text { BEDROCK }\end{array}$ & SAND \\
\hline TAW & 227 & $\cdots$ & 66 & $\cdots$ & $\cdots$ & $-\cdot$ & $110 \mathrm{SDMN}$ & SDGL \\
\hline TAW & 228 & $\cdots$ & 13 & $\cdots$ & $\begin{array}{l}0 \\
3 \\
7\end{array}$ & $\begin{array}{r}3 \\
7 \\
--\end{array}$ & $\begin{array}{l}110 \mathrm{SDMN} \\
110 \mathrm{SDMN} \\
110 \mathrm{SDMN}\end{array}$ & $\begin{array}{l}\text { SAND } \\
\text { SDGL } \\
\text { SAND }\end{array}$ \\
\hline TAW & 229 & $\cdots$ & 66 & - & $\cdots$ & $\cdots$ & $110 \mathrm{SDMN}$ & SDGL \\
\hline
\end{tabular}


Table 2-1. Stratigraphic logs of wells and borings in the Saco and Ossipee River Basins, eastcentral New Hampshire--Continued

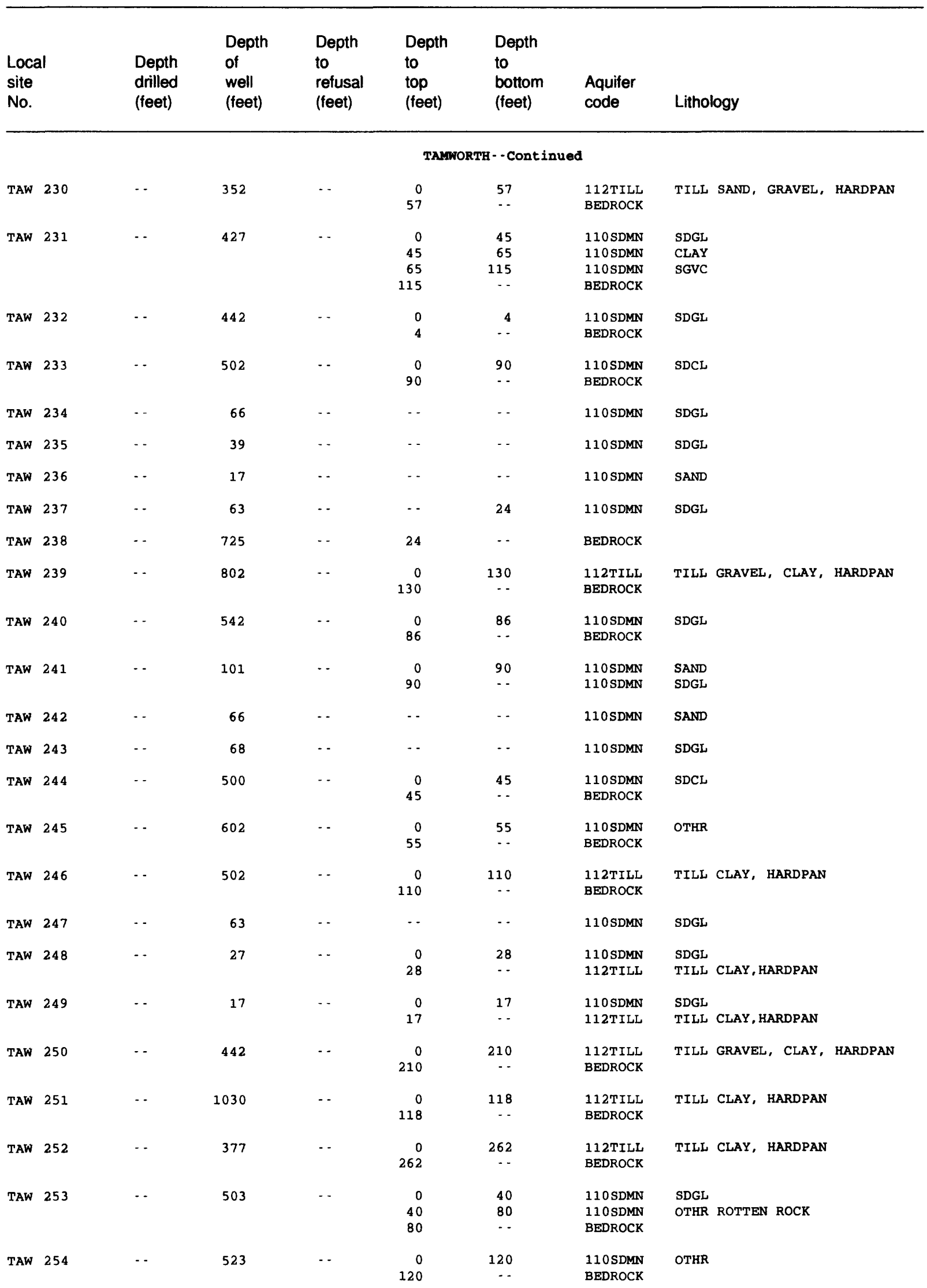


Table 2-1. Stratigraphic logs of wells and borings in the Saco and Ossipee River Basins, eastcentral New Hampshire--Continued

\begin{tabular}{|c|c|c|c|c|c|c|c|c|}
\hline \multicolumn{2}{|c|}{$\begin{array}{l}\text { Local } \\
\text { site } \\
\text { no. }\end{array}$} & $\begin{array}{l}\text { Depth } \\
\text { drilled } \\
\text { (feet) }\end{array}$ & $\begin{array}{l}\text { Depth } \\
\text { of } \\
\text { well } \\
\text { (feet) }\end{array}$ & $\begin{array}{l}\text { Depth } \\
\text { to } \\
\text { refusal } \\
\text { (feet) }\end{array}$ & $\begin{array}{l}\text { Depth } \\
\text { to } \\
\text { top } \\
\text { (feet) }\end{array}$ & $\begin{array}{l}\text { Depth } \\
\text { to } \\
\text { bottom } \\
\text { (feet) }\end{array}$ & $\begin{array}{l}\text { Aquifer } \\
\text { code }\end{array}$ & Lithology \\
\hline & & \multicolumn{7}{|c|}{ TAYWORTH - - Continued } \\
\hline TAW & 255 & $\cdots$ & 382 & $\cdots$ & $\begin{array}{r}0 \\
45\end{array}$ & $\begin{array}{l}45 \\
--\end{array}$ & $\begin{array}{l}110 \text { SDMN } \\
\text { BEDROCK }\end{array}$ & SDGL \\
\hline TAW & 256 & $\cdots$ & 47 & $\cdots$ & $\cdots$ & -- & $110 \mathrm{SDMN}$ & SDGL \\
\hline TAW & 257 & $\cdots$ & 36 & $\cdots$ & $\begin{array}{r}0 \\
38\end{array}$ & $\begin{array}{l}38 \\
\cdots\end{array}$ & $\begin{array}{l}110 \mathrm{SDMN} \\
112 \mathrm{TILL}\end{array}$ & $\begin{array}{l}\text { SDGL } \\
\text { TILL }\end{array}$ \\
\hline TZW & 37 & $\cdots$ & 652 & - & $\begin{array}{l}0 \\
2\end{array}$ & $\because$ & $\begin{array}{l}\text { 112TILL } \\
\text { BEDROCK }\end{array}$ & TILL \\
\hline TZW & 73 & $\cdots$ & 13 & $\cdots$ & $\begin{array}{l}\cdots \\
\cdots\end{array}$ & $\cdots$ & $\begin{array}{l}110 \mathrm{SDMN} \\
112 \mathrm{TILL}\end{array}$ & $\begin{array}{l}\text { CLAY } \\
\text { TILL }\end{array}$ \\
\hline TZW & 96 & $\cdots$ & 331 & $\cdots$ & $\begin{array}{r}0 \\
43\end{array}$ & $\begin{array}{l}\cdots \\
\cdots\end{array}$ & $\begin{array}{l}\text { 110 SDMN } \\
\text { BEDROCK }\end{array}$ & SDCL \\
\hline TZW & 134 & $\cdots$ & 380 & $\cdots$ & $\begin{array}{r}0 \\
40\end{array}$ & $\begin{array}{l}\cdots \\
\cdots\end{array}$ & $\begin{array}{l}\text { 112TILL } \\
\text { BEDROCK }\end{array}$ & TILL GRAVEL, CLAY, HARDPAN \\
\hline TZW & 159 & $\cdots$ & 502 & $\cdots$ & $\ddot{198}$ & $\cdots$ & $\begin{array}{l}\text { 112TILL } \\
\text { BEDROCK }\end{array}$ & TILL CLAY, HARDPAN \\
\hline TZW & 160 & $\cdots$ & 230 & $\cdots$ & $\begin{array}{l}-\cdot \\
52\end{array}$ & $\cdots$ & $\begin{array}{l}\text { I10SDMN } \\
\text { BEDROCK }\end{array}$ & SGVC \\
\hline TZW & 165 & $\cdots$ & 753 & $\cdots$ & $\ddot{28}$ & $\cdots$ & $\begin{array}{l}110 \text { SDMN } \\
\text { BEDROCK }\end{array}$ & CLAY \\
\hline \multicolumn{9}{|c|}{ WAKBFIBLD } \\
\hline WAA & 3 & 8.5 & $\cdots$ & 8.5 & $\begin{array}{l}0 \\
6\end{array}$ & $\begin{array}{r}6 \\
-.\end{array}$ & $\begin{array}{l}\text { 112SRFD } \\
\text { 112TILL }\end{array}$ & $\begin{array}{l}\text { SAND MS OVER SILTY SAND } \\
\text { TILL COMPACT COBBLES AND SANDS }\end{array}$ \\
\hline WAB & 1 & 30 & $\cdots$ & $\cdots$ & $\begin{array}{r}0 \\
6 \\
18 \\
20 \\
25 \\
30\end{array}$ & $\begin{array}{r}6 \\
18 \\
20 \\
25 \\
30 \\
\cdots\end{array}$ & $\begin{array}{l}111 \mathrm{SDMN} \\
112 \mathrm{SRFD} \\
112 \mathrm{LCSR} \\
112 \mathrm{SRFD} \\
112 \mathrm{TILL} \\
-\end{array}$ & $\begin{array}{l}\text { SAND AND MUCK } \\
\text { GRVL } \\
\text { SILT } \\
\text { GRVL } \\
\text { TILL } \\
\text { REFUSAL ON BOULDER OR BEDROCK }\end{array}$ \\
\hline WAB & 2 & 19 & $\cdots$ & $\cdots$ & $\begin{array}{r}0 \\
19\end{array}$ & 19 & $\begin{array}{l}112 \mathrm{SRFD} \\
-\end{array}$ & $\begin{array}{l}\text { GRVL SOME BOULDERS } \\
\text { REFUSAL ON BOULDER OR BEDROCK }\end{array}$ \\
\hline WAW & 3 & 253 & $\cdots$ & $\cdots$ & $\begin{array}{r}0 \\
15\end{array}$ & $\begin{array}{l}\cdots \\
\cdots\end{array}$ & $\begin{array}{l}\text { 112TrLL } \\
\text { BEDROCK }\end{array}$ & TILL \\
\hline WAW & 5 & 13 & $\cdots$ & $\cdots$ & $\begin{array}{r}0 \\
13\end{array}$ & $\begin{array}{l}13 \\
\cdots\end{array}$ & $\begin{array}{l}110 \text { SDMN } \\
112 \mathrm{TILL}\end{array}$ & $\begin{array}{l}\text { SDGL SAND AND GRAVEL } \\
\text { TILL END OF HOLE } 18 \text { FEET }\end{array}$ \\
\hline WAW & 6 & 360 & $\cdots$ & $\cdots$ & $\begin{array}{r}0 \\
60 \\
179\end{array}$ & $\begin{array}{r}60 \\
179 \\
--\end{array}$ & $\begin{array}{l}\text { 110SDMN } \\
\text { 112TILL } \\
\text { BEDROCK }\end{array}$ & $\begin{array}{l}\text { SAND } \\
\text { TILL }\end{array}$ \\
\hline WAW & 7 & 66 & $\cdots$ & $\cdots$ & $\begin{array}{r}0 \\
25 \\
35\end{array}$ & $\begin{array}{l}25 \\
35 \\
\cdots\end{array}$ & $\begin{array}{l}110 \mathrm{SDMN} \\
110 \mathrm{SDMN} \\
110 \mathrm{SDMN}\end{array}$ & $\begin{array}{l}\text { SDGL SAND, COARSE; GRAVEL } \\
\text { SAND MEDIUM } \\
\text { SAND FINE; END OF HOLE } 70 \text { FEET }\end{array}$ \\
\hline WAW & 8 & 303 & $\cdots$ & $\cdots$ & $\ddot{15}$ & $\begin{array}{l}\cdots \\
\cdots\end{array}$ & $\begin{array}{l}\text { 110SDMN } \\
\text { BEDROCK }\end{array}$ & SAND \\
\hline WAW & 9 & 303 & $\cdots$ & $\cdots$ & 43 & $\cdots$ & BEDROCK & \\
\hline WAW & 10 & 985 & $\cdots$ & -. & 43 & - & BEDROCK & \\
\hline WAW & 12 & 700 & - & $\cdots$ & $\begin{array}{r}0 \\
113\end{array}$ & $\begin{array}{l}113 \\
\cdots\end{array}$ & $\begin{array}{l}110 \text { SDMN } \\
\text { BEDROCK }\end{array}$ & SDCL SAND, CLAY \\
\hline WAW & 13 & 800 & $\cdots$ & $\cdots$ & 92 & $\cdots$ & BEDROCK & \\
\hline WAW & 15 & 110 & $\cdots$ & $\cdots$ & $\cdots$ & - & & \\
\hline
\end{tabular}


Table 2-1. Stratigraphic logs of wells and borings in the Saco and Ossipee River Basins, eastcentral New Hampshire--Continued

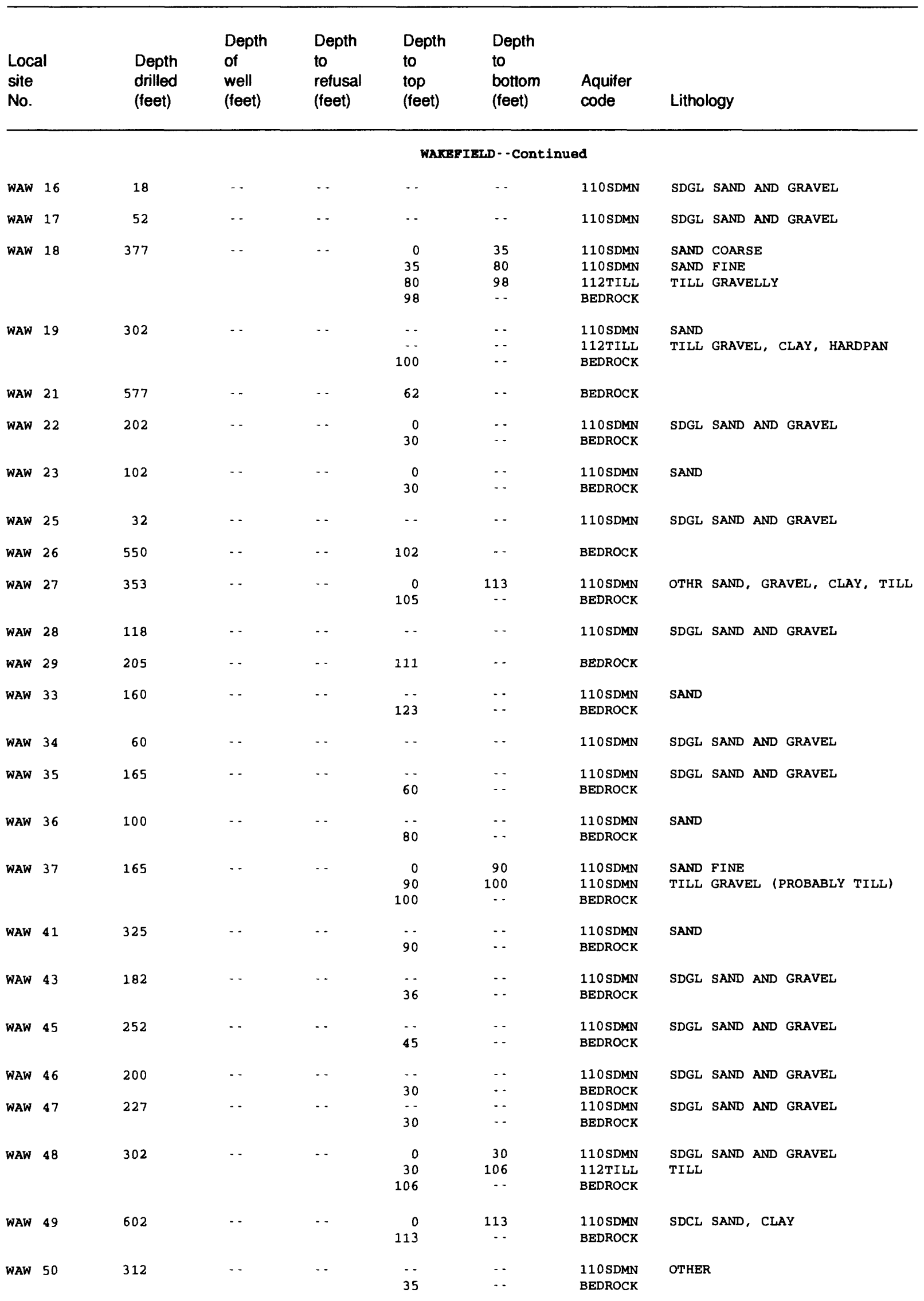


Table 2-1. Stratigraphic logs of wells and borings in the Saco and Ossipee River Basins, eastcentral New Hampshire--Continued

\begin{tabular}{|c|c|c|c|c|c|c|c|c|}
\hline \multicolumn{2}{|c|}{$\begin{array}{l}\text { Local } \\
\text { site } \\
\text { no. }\end{array}$} & $\begin{array}{l}\text { Depth } \\
\text { drilled } \\
\text { (feet) }\end{array}$ & $\begin{array}{l}\text { Depth } \\
\text { of } \\
\text { well } \\
\text { (feet) }\end{array}$ & $\begin{array}{l}\text { Depth } \\
\text { to } \\
\text { refusal } \\
\text { (feet) }\end{array}$ & $\begin{array}{l}\text { Depth } \\
\text { to } \\
\text { top } \\
\text { (feet) }\end{array}$ & $\begin{array}{l}\text { Depth } \\
\text { to } \\
\text { bottom } \\
\text { (feet) }\end{array}$ & $\begin{array}{l}\text { Aquiter } \\
\text { code }\end{array}$ & Lithology \\
\hline & & \multicolumn{7}{|c|}{ WAKEFIELD - Continued } \\
\hline WAW & 51 & 377 & $\cdots$ & $\cdots$ & $\overline{80}$ & $\begin{array}{l}-\cdot \\
\cdots\end{array}$ & $\begin{array}{l}110 \text { SDMN } \\
\text { BEDROCK }\end{array}$ & SDGL SAND AND GRAVEL \\
\hline WAW & 52 & 352 & $\cdots$ & $\cdots$ & $\overline{17}$ & $\cdots$ & $\begin{array}{l}\text { 110SDMN } \\
\text { BEDROCK }\end{array}$ & SAND \\
\hline WAW & 54 & 93 & $\cdots$ & $\cdots$ & $\ddot{9}$ & $\begin{array}{l}\cdots \\
\cdots\end{array}$ & $\begin{array}{l}\text { 110SDMN } \\
\text { BEDROCK }\end{array}$ & SDGL SAND AND GRAVEL \\
\hline WAW & 97 & $\cdots$ & 352 & $\cdots$ & $\begin{array}{r}0 \\
17\end{array}$ & $\begin{array}{l}17 \\
--\end{array}$ & $\begin{array}{l}110 \text { SDMN } \\
\text { BEDROCK }\end{array}$ & SAND \\
\hline WAW & 99 & $\cdots$ & 220 & $\cdots$ & $\begin{array}{l}0 \\
8\end{array}$ & $\begin{array}{c}8 \\
-\end{array}$ & $\begin{array}{l}110 \text { SDMN } \\
\text { BEDROCK }\end{array}$ & SDGL \\
\hline WAW & 100 & $\cdots$ & 422 & $\cdots$ & $\begin{array}{r}0 \\
26 \\
43 \\
81\end{array}$ & $\begin{array}{l}26 \\
43 \\
81\end{array}$ & $\begin{array}{l}110 \text { SDMN } \\
110 \text { SDMN } \\
112 \text { TILL } \\
\text { BEDROCK }\end{array}$ & $\begin{array}{l}\text { SAND } \\
\text { SDGL } \\
\text { TILL SAND, HARDPAN }\end{array}$ \\
\hline WAW & 101 & $\cdots$ & 427 & $\cdots$ & $\begin{array}{r}0 \\
60 \\
158\end{array}$ & $\begin{array}{r}60 \\
158 \\
--\end{array}$ & $\begin{array}{l}\text { 110SDMN } \\
112 \mathrm{TILL} \\
\text { BEDROCK }\end{array}$ & $\begin{array}{l}\text { SDGL } \\
\text { TILL GRAVEL, CLAY, HARDPAN }\end{array}$ \\
\hline WAW & 102 & $\cdots$ & 252 & $\cdots$ & $\begin{array}{r}0 \\
60\end{array}$ & $\begin{array}{l}60 \\
\cdots\end{array}$ & $\begin{array}{l}\text { I10SDMN } \\
\text { BEDROCK }\end{array}$ & SAND \\
\hline WAW & 103 & $\cdots$ & 277 & $\cdots$ & 45 & $\cdots$ & BEDROCK & \\
\hline WAW & 104 & $\cdots$ & 552 & $\cdots$ & $\begin{array}{r}0 \\
18\end{array}$ & $\begin{array}{l}18 \\
\cdots\end{array}$ & $\begin{array}{l}\text { 112TILL } \\
\text { BEDROCK }\end{array}$ & TILL \\
\hline WAW & 105 & $\cdots$ & 300 & $\cdots$ & $\begin{array}{r}0 \\
22\end{array}$ & 22 & $\begin{array}{l}110 \text { SDMN } \\
\text { BEDROCK }\end{array}$ & SAND \\
\hline WAW & 106 & $\cdots$ & 142 & $\cdots$ & $\begin{array}{r}0 \\
75\end{array}$ & $\begin{array}{l}75 \\
\cdots\end{array}$ & $\begin{array}{l}110 \text { SDMN } \\
\text { BEDROCK }\end{array}$ & SDGL \\
\hline WAW & 107 & $\cdots$ & 800 & $\cdots$ & $\begin{array}{r}0 \\
62\end{array}$ & 62 & $\begin{array}{l}110 \text { SDMN } \\
\text { BEDROCK }\end{array}$ & SAND \\
\hline WAW & 108 & $\cdots$ & 322 & $\cdots$ & $\begin{array}{r}0 \\
32\end{array}$ & $\begin{array}{l}32 \\
--\end{array}$ & $\begin{array}{l}\text { 110SDMN } \\
\text { BEDROCK }\end{array}$ & SDCL \\
\hline WAW & 109 & $\cdots$ & 402 & $\cdots$ & $\begin{array}{r}0 \\
17\end{array}$ & $\begin{array}{l}17 \\
--\end{array}$ & $\begin{array}{l}110 \text { SDMN } \\
\text { BEDROCK }\end{array}$ & SDGL \\
\hline WAW & 110 & - & 222 & $\cdots$ & $\begin{array}{r}0 \\
15\end{array}$ & $\begin{array}{l}15 \\
-\cdot\end{array}$ & $\begin{array}{l}110 \text { SDMN } \\
\text { BEDROCK }\end{array}$ & SAND \\
\hline WAW & 111 & $\cdots$ & 242 & $\cdots$ & $\begin{array}{r}0 \\
58\end{array}$ & $\begin{array}{l}58 \\
\cdots\end{array}$ & $\begin{array}{l}110 \text { SDMN } \\
\text { BEDROCK }\end{array}$ & SDGL \\
\hline WAW & 112 & $\cdots$ & 162 & $\cdots$ & $\begin{array}{r}0 \\
66\end{array}$ & $\begin{array}{l}66 \\
\cdots\end{array}$ & $\begin{array}{l}110 \text { SDMN } \\
\text { BEDROCK }\end{array}$ & SAND \\
\hline WAW & 113 & $\cdots$ & 260 & $\cdots$ & $\begin{array}{r}0 \\
23\end{array}$ & $\begin{array}{l}23 \\
-\cdot\end{array}$ & $\begin{array}{l}\text { 110SDMN } \\
\text { BEDROCK }\end{array}$ & SDGL \\
\hline WAW & 114 & $\cdots$ & 100 & $\cdots$ & $\begin{array}{r}0 \\
13\end{array}$ & $\begin{array}{l}13 \\
\cdots\end{array}$ & $\begin{array}{l}110 \text { SDMN } \\
\text { BEDROCK }\end{array}$ & SAND \\
\hline WAW & 115 & $\cdots$ & 142 & $\cdots$ & $\begin{array}{r}0 \\
23\end{array}$ & $\begin{array}{l}23 \\
--\end{array}$ & $\begin{array}{l}110 \text { SDMN } \\
\text { BEDROCK }\end{array}$ & SDGL \\
\hline WAW & 116 & - & 402 & $\cdots$ & $\begin{array}{r}0 \\
43\end{array}$ & $\begin{array}{l}43 \\
\cdots\end{array}$ & $\begin{array}{l}110 \text { SDMN } \\
\text { BEDROCK }\end{array}$ & SDGL \\
\hline WAW & 117 & $\cdots$ & 280 & $-\cdot$ & $\begin{array}{r}0 \\
116\end{array}$ & $\begin{array}{r}116 \\
\ldots\end{array}$ & $\begin{array}{l}110 \text { SDMN } \\
\text { BEDROCK }\end{array}$ & SAND \\
\hline
\end{tabular}


Table 2-1. Stratigraphic logs of wells and borings in the Saco and Osslpee River Basins, eastcentral New Hampshire--Continued

\begin{tabular}{|c|c|c|c|c|c|c|c|c|}
\hline \multicolumn{2}{|c|}{$\begin{array}{l}\text { Local } \\
\text { site } \\
\text { No. }\end{array}$} & $\begin{array}{l}\text { Depth } \\
\text { drilled } \\
\text { (feet) }\end{array}$ & $\begin{array}{l}\text { Depth } \\
\text { of } \\
\text { well } \\
\text { (feet) }\end{array}$ & $\begin{array}{l}\text { Depth } \\
\text { to } \\
\text { refusal } \\
\text { (feet) }\end{array}$ & $\begin{array}{l}\text { Depth } \\
\text { to } \\
\text { top } \\
\text { (feet) }\end{array}$ & $\begin{array}{l}\text { Depth } \\
\text { to } \\
\text { bottom } \\
\text { (feet) }\end{array}$ & $\begin{array}{l}\text { Aquifer } \\
\text { code }\end{array}$ & Lithology \\
\hline & & \multicolumn{7}{|c|}{ WAKBPIBLD - Continued } \\
\hline WAW & 118 & - & 300 & $\cdots$ & $\begin{array}{r}0 \\
40 \\
47\end{array}$ & $\begin{array}{l}40 \\
47 \\
\cdots\end{array}$ & $\begin{array}{l}110 \text { SDMN } \\
110 \text { SDMN } \\
\text { BEDROCK }\end{array}$ & $\begin{array}{l}\text { SAND } \\
\text { SDGL }\end{array}$ \\
\hline WAW & 119 & $-\cdot$ & 362 & $\cdots$ & $\begin{array}{r}0 \\
88\end{array}$ & $\begin{array}{l}88 \\
--\end{array}$ & $\begin{array}{l}110 \text { SDMN } \\
\text { BEDROCK }\end{array}$ & SDCL \\
\hline WAW & 120 & $\cdots$ & 82 & $\cdots$ & $\begin{array}{r}0 \\
36\end{array}$ & $\begin{array}{l}36 \\
-\end{array}$ & $\begin{array}{l}110 \text { SDMN } \\
\text { BEDROCK }\end{array}$ & SDGL \\
\hline WAW & 121 & - & 482 & $\cdots$ & $\begin{array}{l}0 \\
5\end{array}$ & $\begin{array}{r}5 \\
--\end{array}$ & $\begin{array}{l}110 \text { SDMN } \\
\text { BEDROCK }\end{array}$ & SAND \\
\hline WAW & 122 & -. & 252 & $\cdots$ & $\begin{array}{r}0 \\
60\end{array}$ & $\begin{array}{l}60 \\
\cdots\end{array}$ & $\begin{array}{l}110 \text { SDMN } \\
\text { BEDROCK }\end{array}$ & SAND \\
\hline WAW & 123 & - & 102 & $\cdots$ & $\begin{array}{r}0 \\
43\end{array}$ & $\begin{array}{l}43 \\
--\end{array}$ & $\begin{array}{l}110 \text { SDMN } \\
\text { BEDROCK }\end{array}$ & SAND \\
\hline WAW & 124 & $\cdots$ & 202 & $\cdots$ & $\begin{array}{r}0 \\
50\end{array}$ & $\begin{array}{l}50 \\
\cdots\end{array}$ & $\begin{array}{l}110 \text { SDMN } \\
\text { BEDROCK }\end{array}$ & SDGL \\
\hline WAW & 125 & - & 482 & $\cdots$ & $\begin{array}{r}0 \\
105\end{array}$ & $\begin{array}{r}105 \\
\ldots\end{array}$ & $\begin{array}{l}110 \text { SDMN } \\
\text { BEDROCK }\end{array}$ & SAND \\
\hline WAW & 126 & $-\cdot$ & 503 & $\cdots$ & $\begin{array}{r}0 \\
50\end{array}$ & $\begin{array}{l}50 \\
\cdots\end{array}$ & $\begin{array}{l}110 \text { SDMN } \\
\text { BEDROCK }\end{array}$ & SDGL \\
\hline WAW & 127 & -- & 282 & $\cdots$ & $\begin{array}{r}0 \\
80\end{array}$ & $\begin{array}{l}80 \\
\cdots\end{array}$ & $\begin{array}{l}110 \text { SDMN } \\
\text { BEDROCK }\end{array}$ & SDCL \\
\hline WAW & 128 & - & 162 & $\cdots$ & $\begin{array}{r}0 \\
20\end{array}$ & $\begin{array}{l}20 \\
\cdots\end{array}$ & $\begin{array}{l}110 \text { SDMN } \\
\text { BEDROCK }\end{array}$ & SGVC \\
\hline WAW & 129 & $-\cdot$ & 362 & - & $\begin{array}{r}0 \\
30\end{array}$ & $\begin{array}{l}30 \\
-.\end{array}$ & $\begin{array}{l}110 \text { SDMN } \\
\text { BEDROCK }\end{array}$ & SDGL \\
\hline WAW & 130 & $\cdots$ & 445 & $\cdots$ & $\begin{array}{r}0 \\
49\end{array}$ & $\begin{array}{l}49 \\
--\end{array}$ & $\begin{array}{l}110 \text { SDMN } \\
\text { BEDROCK }\end{array}$ & SDGL \\
\hline WAW & 131 & . & 283 & - & $\begin{array}{r}0 \\
30\end{array}$ & $\begin{array}{l}30 \\
\cdots\end{array}$ & $\begin{array}{l}110 \text { SDMN } \\
\text { BEDROCK }\end{array}$ & SDGL \\
\hline WAW & 132 & -- & 135 & $\cdots$ & $\begin{array}{r}0 \\
124\end{array}$ & 124 & $\begin{array}{l}110 \text { SDMN } \\
\text { BEDROCK }\end{array}$ & SDGL \\
\hline WAW & 133 & -- & 122 & - & $\begin{array}{r}0 \\
74\end{array}$ & $\begin{array}{l}74 \\
--\end{array}$ & $\begin{array}{l}110 \text { SDMN } \\
\text { BEDROCK }\end{array}$ & SDGL \\
\hline WAW & 134 & $-\cdot$ & 300 & $\cdots$ & $\begin{array}{r}0 \\
115\end{array}$ & $\begin{array}{r}115 \\
\cdots\end{array}$ & $\begin{array}{l}110 \text { SDMN } \\
\text { BEDROCK }\end{array}$ & SGVC \\
\hline WAW & 135 & - & 462 & . & $\begin{array}{r}0 \\
115\end{array}$ & $\begin{array}{c}115 \\
-.\end{array}$ & $\begin{array}{l}110 \text { SDMN } \\
\text { BEDROCK }\end{array}$ & OTHR \\
\hline WAW & 136 & - & 342 & - & $\begin{array}{r}0 \\
99\end{array}$ & $\begin{array}{l}99 \\
\cdots\end{array}$ & $\begin{array}{l}110 \text { SDMN } \\
\text { BEDROCK }\end{array}$ & SAND \\
\hline WAW & 137 & $\cdots$ & 502 & - & $\begin{array}{r}0 \\
20 \\
40 \\
55\end{array}$ & $\begin{array}{l}20 \\
40 \\
55 \\
-\cdot\end{array}$ & $\begin{array}{l}110 \text { SDMN } \\
110 \text { SDMN } \\
110 \text { SDMN } \\
\text { BEDROCK }\end{array}$ & $\begin{array}{l}\text { SAND } \\
\text { PEAT } \\
\text { SDGL }\end{array}$ \\
\hline WAW & 138 & - & 25 & -- & $\cdots$ & $\cdots$ & 110 SDMN & SDGL \\
\hline WAW & 139 & $\cdots$ & 70 & $\cdots$ & $\begin{array}{r}0 \\
25 \\
65\end{array}$ & $\begin{array}{l}25 \\
65 \\
\cdots\end{array}$ & $\begin{array}{l}110 \text { SDMN } \\
110 \text { SDMN } \\
110 \text { SDMN }\end{array}$ & $\begin{array}{l}\text { SDGL } \\
\text { SDCL } \\
\text { SDGL }\end{array}$ \\
\hline WAW & 140 & - & 602 & - & $\begin{array}{r}0 \\
50 \\
88\end{array}$ & $\begin{array}{l}50 \\
88 \\
\cdots\end{array}$ & $\begin{array}{l}110 \text { SDMN } \\
110 \text { SDMN } \\
\text { BEDROCK }\end{array}$ & $\begin{array}{l}\text { SDGL } \\
\text { SGVC }\end{array}$ \\
\hline
\end{tabular}


Table 2-1. Stratigraphic logs of wells and borings in the Saco and Ossipee River Basins, eastcentral New Hampshire--Continued

\begin{tabular}{|c|c|c|c|c|c|c|c|c|}
\hline $\begin{array}{l}\text { Loca } \\
\text { site } \\
\text { no. }\end{array}$ & & $\begin{array}{l}\text { Depth } \\
\text { drilled } \\
\text { (feet) }\end{array}$ & $\begin{array}{l}\text { Depth } \\
\text { of } \\
\text { well } \\
\text { (feet) }\end{array}$ & $\begin{array}{l}\text { Depth } \\
\text { to } \\
\text { refusal } \\
\text { (feet) }\end{array}$ & $\begin{array}{l}\text { Depth } \\
\text { to } \\
\text { top } \\
\text { (feet) }\end{array}$ & $\begin{array}{l}\text { Depth } \\
\text { to } \\
\text { bottom } \\
\text { (feet) }\end{array}$ & $\begin{array}{l}\text { Aquifer } \\
\text { code }\end{array}$ & Lithology \\
\hline & & \multicolumn{7}{|c|}{ WAKRFIBLD - - Continued } \\
\hline WAW & 141 & $-\cdot$ & 304 & $-\cdot$ & $\begin{array}{r}0 \\
20 \\
60\end{array}$ & $\begin{array}{l}20 \\
60 \\
-\end{array}$ & $\begin{array}{l}110 \text { SDMN } \\
110 \text { SDMN } \\
\text { BEDROCK }\end{array}$ & $\begin{array}{l}\text { SDGL } \\
\text { CLAY }\end{array}$ \\
\hline WAW & 142 & $\cdots$ & 424 & - & $\begin{array}{r}0 \\
40\end{array}$ & $\begin{array}{l}40 \\
--\end{array}$ & $\begin{array}{l}110 \mathrm{SDMN} \\
\text { BEDROCK }\end{array}$ & SDCL \\
\hline WAW & 143 & $\cdots$ & 140 & $\cdots$ & 125 & $\cdots$ & BEDROCK & \\
\hline WAW & 144 & $-\cdot$ & 120 & - & 110 & - & BEDROCK & \\
\hline WAW & 145 & $\cdots$ & 421 & $\cdots$ & $\begin{array}{r}0 \\
10\end{array}$ & 10 & $\begin{array}{l}\text { 112TILL } \\
\text { BEDROCK }\end{array}$ & TILL \\
\hline WAW & 146 & - & 225 & $\cdots$ & $\begin{array}{r}0 \\
87\end{array}$ & $\begin{array}{l}87 \\
\cdots\end{array}$ & $\begin{array}{l}110 \text { SDMN } \\
\text { BEDROCK }\end{array}$ & SDGL \\
\hline WAW & 147 & $-\cdot$ & 45 & $\cdots$ & $-\cdot$ & $\cdots$ & 110 SDMN & SAND \\
\hline WAW & 149 & 10.7 & 10.7 & $\cdots$ & $-\cdot$ & $-\cdot$ & $-\cdot$ & \\
\hline WAW & 150 & 14.9 & 14.9 & $\cdots$ & $-\cdot$ & $-\cdot$ & $-\cdot$ & \\
\hline WAW & 151 & 15.7 & 15.7 & $\cdots$ & -. & - & $\cdots$ & \\
\hline WAW & 152 & 9.5 & 9.5 & - & -. & $\cdots$ & - & \\
\hline WAW & 153 & 8 & 8 & $\cdots$ & $\cdots$ & $\cdots$ & $\cdots$ & \\
\hline WAW & 154 & 22 & 22 & - & $-\cdot$ & $\cdots$ & $\cdots$ & \\
\hline WAW & 155 & 80 & - & $\cdots$ & $\cdots$ & $\cdots$ & $\cdots$ & \\
\hline WAW & 156 & 11.3 & 11.3 & $\cdots$ & - & -. & $\cdots$ & \\
\hline WAW & 157 & 78 & 49 & 78 & $\begin{array}{r}7 \\
13 \\
77\end{array}$ & $\begin{array}{l}13 \\
77 \\
--\end{array}$ & $\begin{array}{l}112 \mathrm{SRFD} \\
112 \mathrm{SRFD} \\
112 \mathrm{TILL}\end{array}$ & $\begin{array}{l}\text { SAND VF OVER CS } \\
\text { SAND LAYERS M TO VFS } \\
\text { TILL }\end{array}$ \\
\hline \multicolumn{9}{|c|}{ WOLFBBORO } \\
\hline WRW & 28 & $\cdots$ & 518 & $\cdots$ & $\begin{array}{r}0 \\
80\end{array}$ & $\begin{array}{l}80 \\
\cdots\end{array}$ & $\begin{array}{l}\text { 112TILL } \\
\text { BEDROCK }\end{array}$ & TILL \\
\hline WRW & 38 & $\cdots$ & 128 & $\cdots$ & $\begin{array}{r}0 \\
18\end{array}$ & $\begin{array}{l}18 \\
\ldots\end{array}$ & $\begin{array}{l}\text { 112TILL } \\
\text { BEDROCK }\end{array}$ & TILL \\
\hline WRW & 50 & - & 402 & $\cdots$ & $\begin{array}{r}0 \\
19\end{array}$ & $\begin{array}{l}19 \\
--\end{array}$ & $\begin{array}{l}110 \text { SDMN } \\
\text { BEDROCK }\end{array}$ & SAND \\
\hline WRW & 63 & - & 304 & $\cdots$ & $\begin{array}{l}0 \\
4\end{array}$ & $\begin{array}{r}4 \\
--\end{array}$ & $\begin{array}{l}110 \mathrm{SDMN} \\
\text { BEDROCK }\end{array}$ & SAND \\
\hline WRW & 65 & -. & 182 & $\cdots$ & $\begin{array}{r}0 \\
42\end{array}$ & $\begin{array}{l}42 \\
\cdots\end{array}$ & $\begin{array}{l}\text { 112TILL } \\
\text { BEDROCK }\end{array}$ & TILL \\
\hline WRW & 98 & $\cdots$ & 202 & $\cdots$ & $\begin{array}{r}0 \\
22\end{array}$ & $\begin{array}{l}22 \\
-.\end{array}$ & $\begin{array}{l}\text { 112TILL } \\
\text { BEDROCK }\end{array}$ & TILL \\
\hline WRW & 135 & $\cdots$ & 322 & - & $\begin{array}{r}0 \\
74\end{array}$ & $\begin{array}{l}74 \\
\ldots\end{array}$ & $\begin{array}{l}\text { 112TILL } \\
\text { BEDROCK }\end{array}$ & TILL \\
\hline WRW & 140 & $\cdots$ & 125 & $\cdots$ & $\begin{array}{r}0 \\
30\end{array}$ & $\begin{array}{l}30 \\
\cdots\end{array}$ & $\begin{array}{l}110 \text { SDMN } \\
\text { BEDROCK }\end{array}$ & SAND \\
\hline WRW & 144 & $\cdots$ & 702 & $\cdots$ & $\begin{array}{r}0 \\
28\end{array}$ & $\begin{array}{l}28 \\
--\end{array}$ & $\begin{array}{l}\text { 112TILL } \\
\text { BEDROCK }\end{array}$ & TILL CLAY, HARDPAN \\
\hline WRW & 156 & $-\cdot$ & 142 & $\cdots$ & $\begin{array}{c}0 \\
70.5\end{array}$ & 70.5 & $\begin{array}{l}110 \text { SDMN } \\
\text { BEDROCK }\end{array}$ & CLAY \\
\hline
\end{tabular}


Table 2-1. Stratigraphic logs of wells and borings in the Saco and Ossipee River Basins, eastcentrai New Hampshire--Continued

\begin{tabular}{|c|c|c|c|c|c|c|c|}
\hline $\begin{array}{l}\text { Local } \\
\text { site } \\
\text { No. }\end{array}$ & $\begin{array}{l}\text { Depth } \\
\text { drilled } \\
\text { (feet) }\end{array}$ & $\begin{array}{l}\text { Depth } \\
\text { of } \\
\text { well } \\
\text { (feet) }\end{array}$ & $\begin{array}{l}\text { Depth } \\
\text { to } \\
\text { refusal } \\
\text { (feet) }\end{array}$ & $\begin{array}{l}\text { Depth } \\
\text { to } \\
\text { top } \\
\text { (feet) }\end{array}$ & $\begin{array}{l}\text { Depth } \\
\text { to } \\
\text { bottom } \\
\text { (feet) }\end{array}$ & $\begin{array}{l}\text { Aquifer } \\
\text { code }\end{array}$ & Lithology \\
\hline \multicolumn{8}{|c|}{ COOS COUNTY } \\
\hline \multicolumn{8}{|c|}{ CARROLL } \\
\hline CFB 7 & 8 & $\cdots$ & 8 & $\begin{array}{l}0 \\
4 \\
8\end{array}$ & $\begin{array}{r}4 \\
8 \\
-\end{array}$ & $\begin{array}{l}\text { 112SRFD } \\
112 \mathrm{TILL} \\
112 \mathrm{TILL}\end{array}$ & $\begin{array}{l}\text { SDGL SAND AND GRAVEL } \\
\text { TILL TILL, SANDY AND BOULDERS } \\
\text { TILL REFUSAL }\end{array}$ \\
\hline
\end{tabular}




\section{APPENDIX 3. Geohydrologic sections interpreted from seismic-refraction data, east-central \\ New Hampshire}



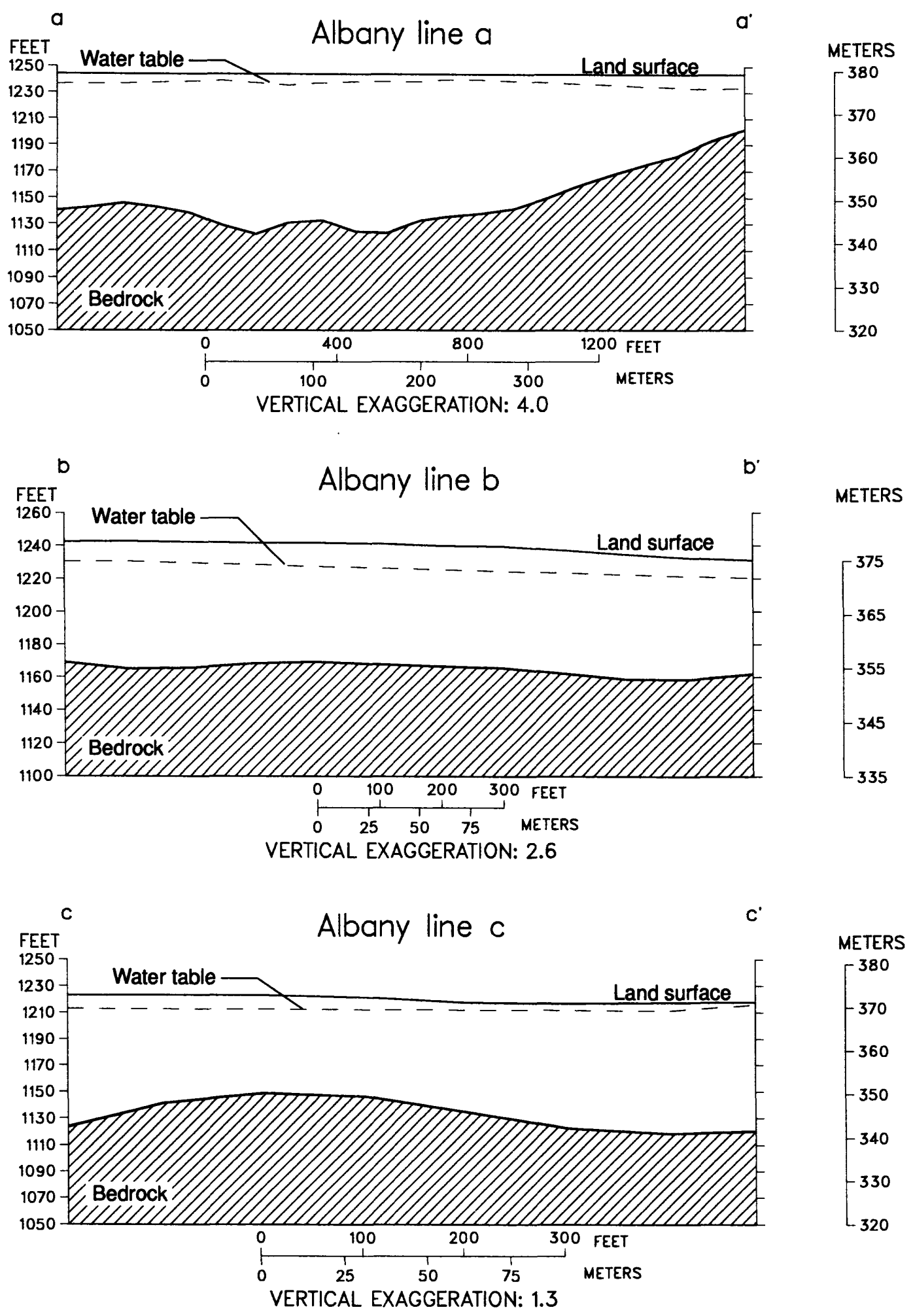

Figure 3-1. Geohydrologic sections interpreted from seismic-refraction data for Albany lines a-a', b-b', and c-c' (locations shown on plate 1). 


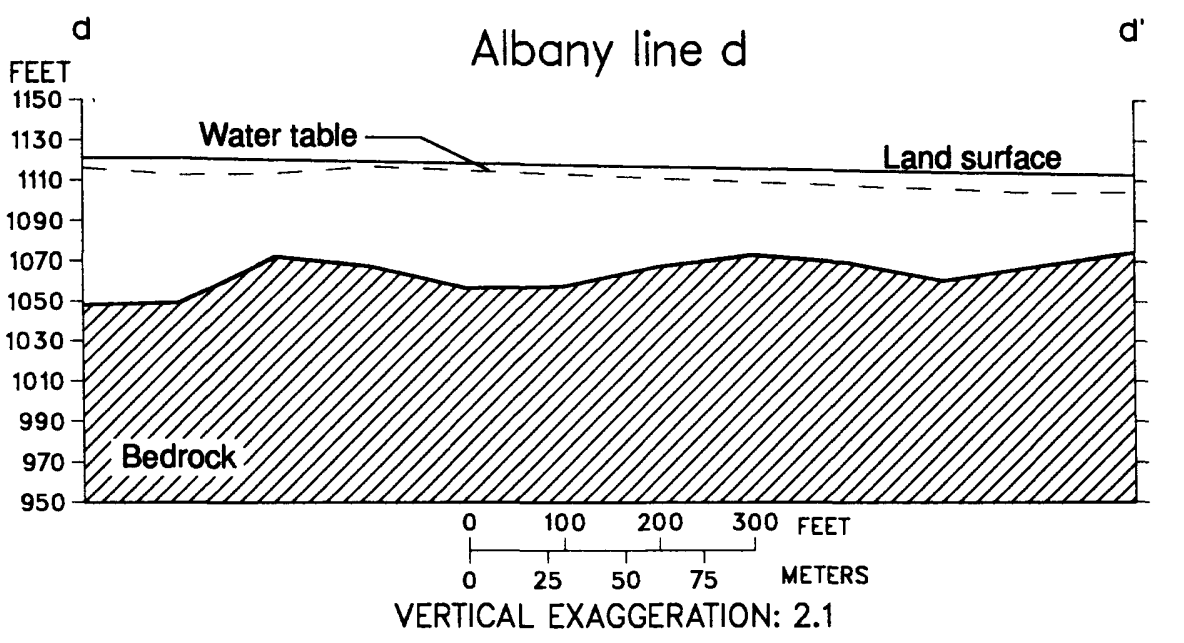

METERS
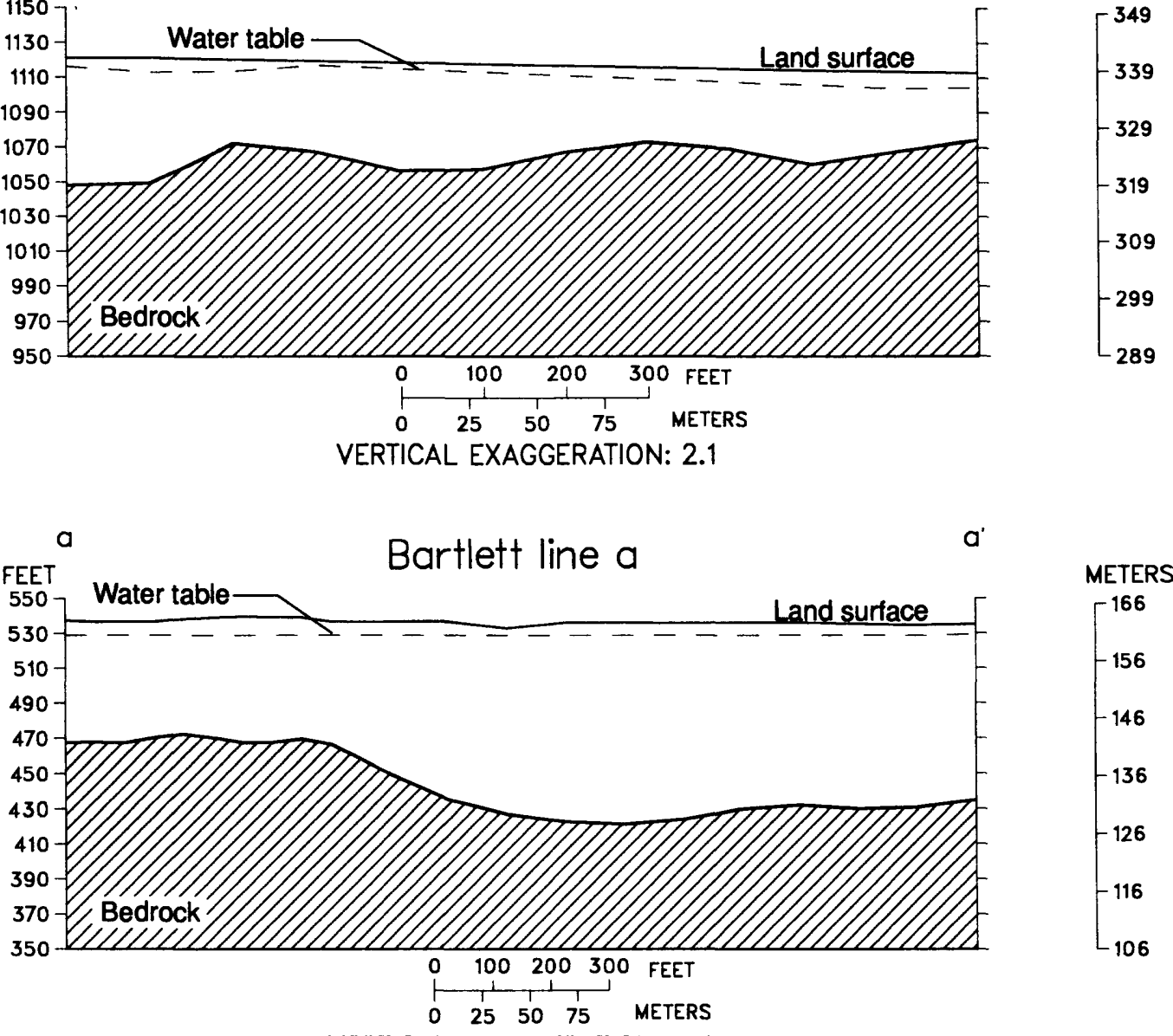

VERTICAL EXAGGERATION: 3.0

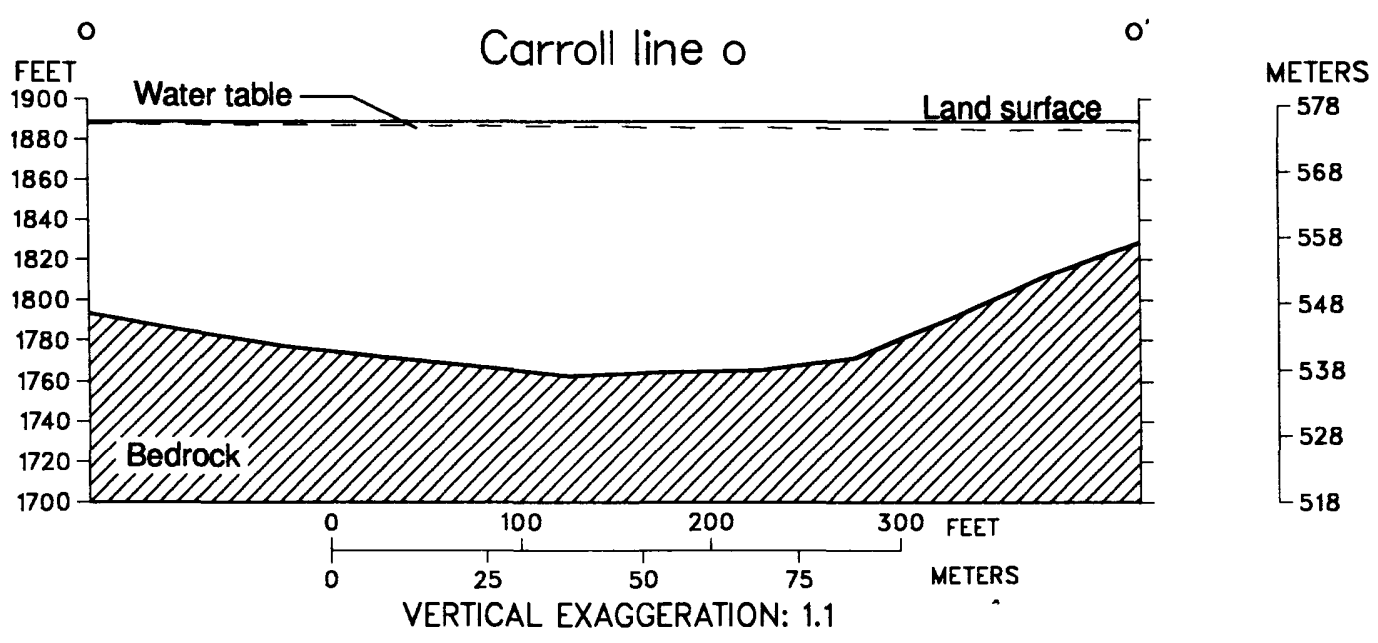

Figure 3-2. Geohydrologic sections interpreted from seismic-refraction data for Albany line d-d', Bartlett line a-a', and Carroll line o-o' (locations shown on plate 1). 

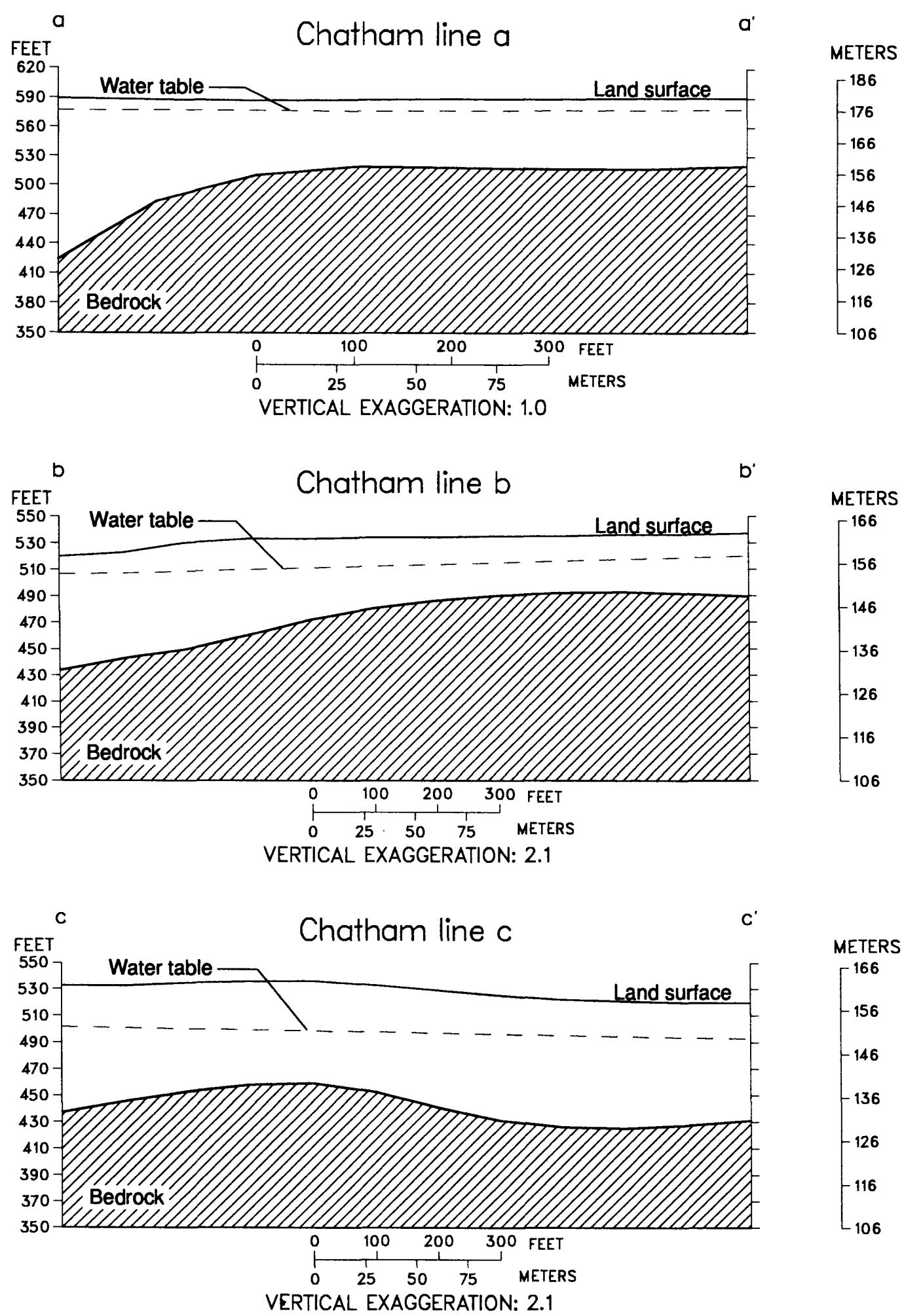

Figure 3-3. Geohydrologic sections interpreted from seismic-refraction data for Chatham lines aa', b-b', and c-c' (locations shown on plate 1). 


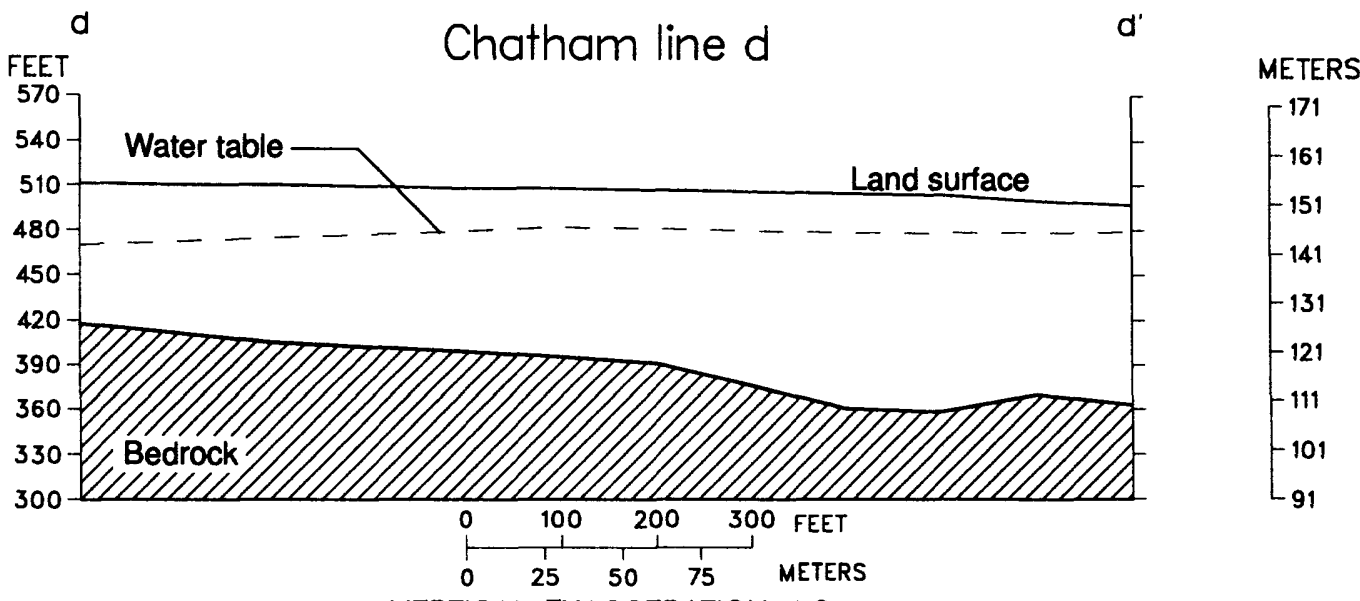

VERTICAL EXAGGERATION: 1.6
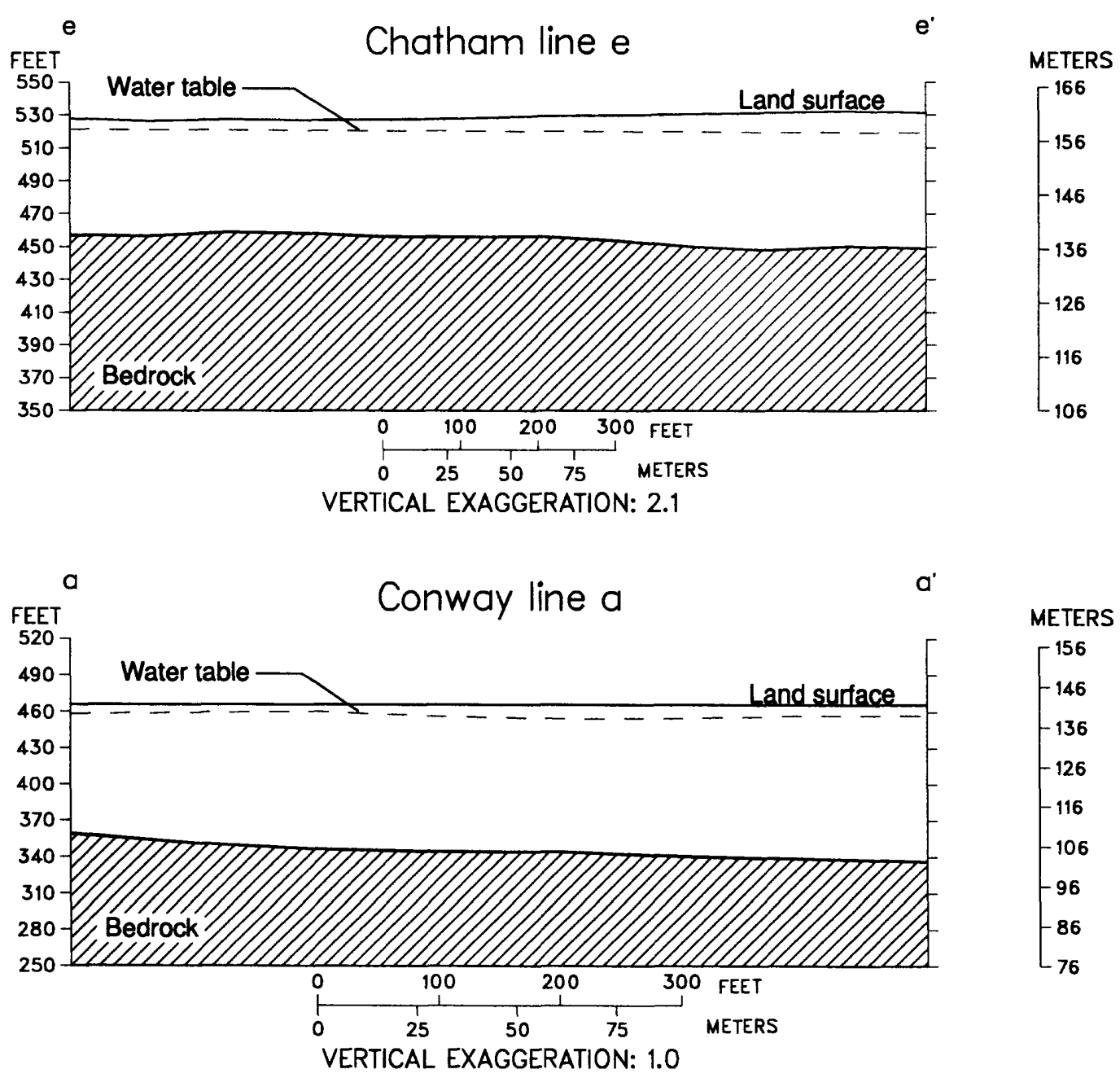

Figure 3-4. Geohydrologic sections interpreted from seismic-refraction data for Chatham lines dd' and e-e', and Conway line a-a' (locations shown on plate 1). 

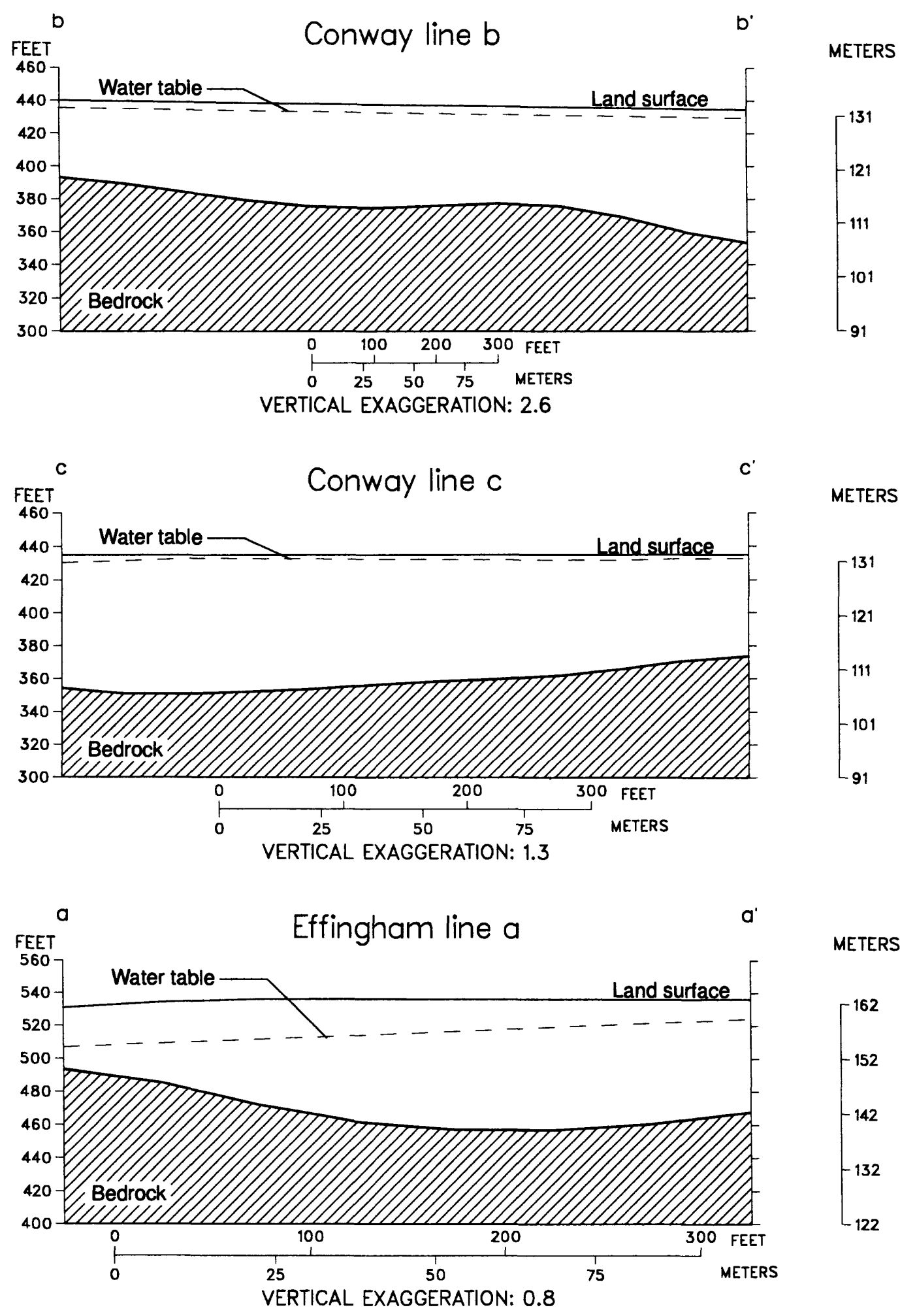

Figure 3-5. Geohydrologic sections interpreted from seismic-refraction data for Conway lines bb', and c-c', and Effingham line a-a' (locations shown on plates 1 and 2). 

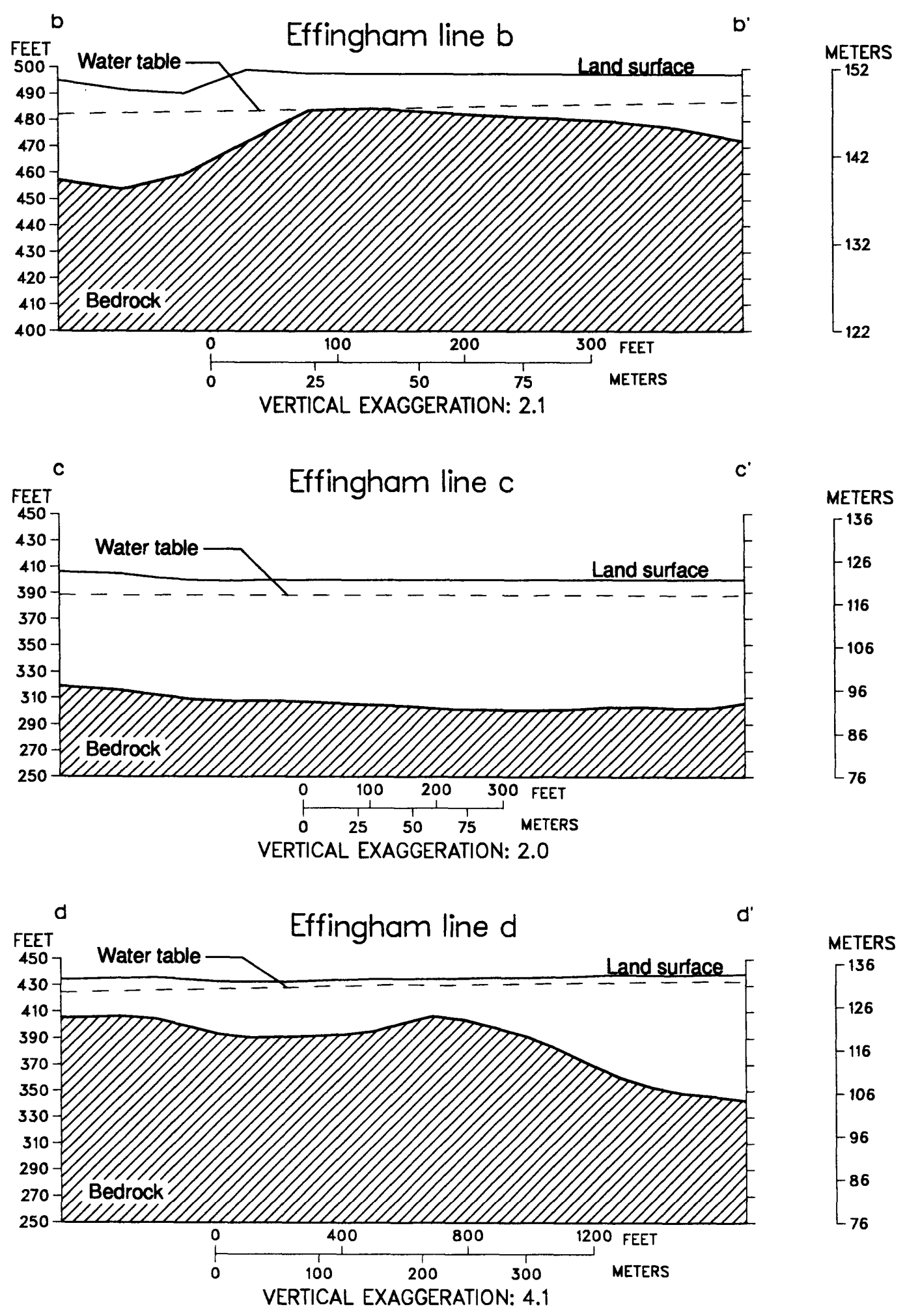

Figure 3-6. Geohydrologic sections interpreted from seismic-refraction data for Effingham lines b-b', c-c', and d-d' (locations shown on plate 2). 


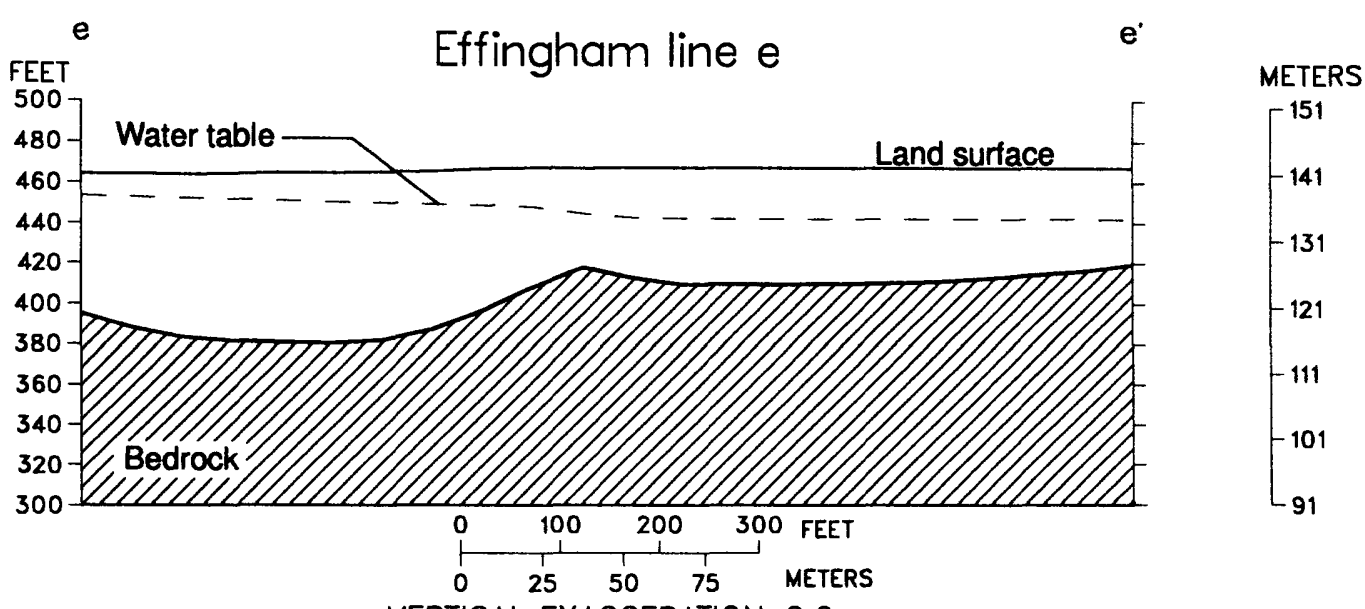

VERTICAL EXAGGERATION: 2.0
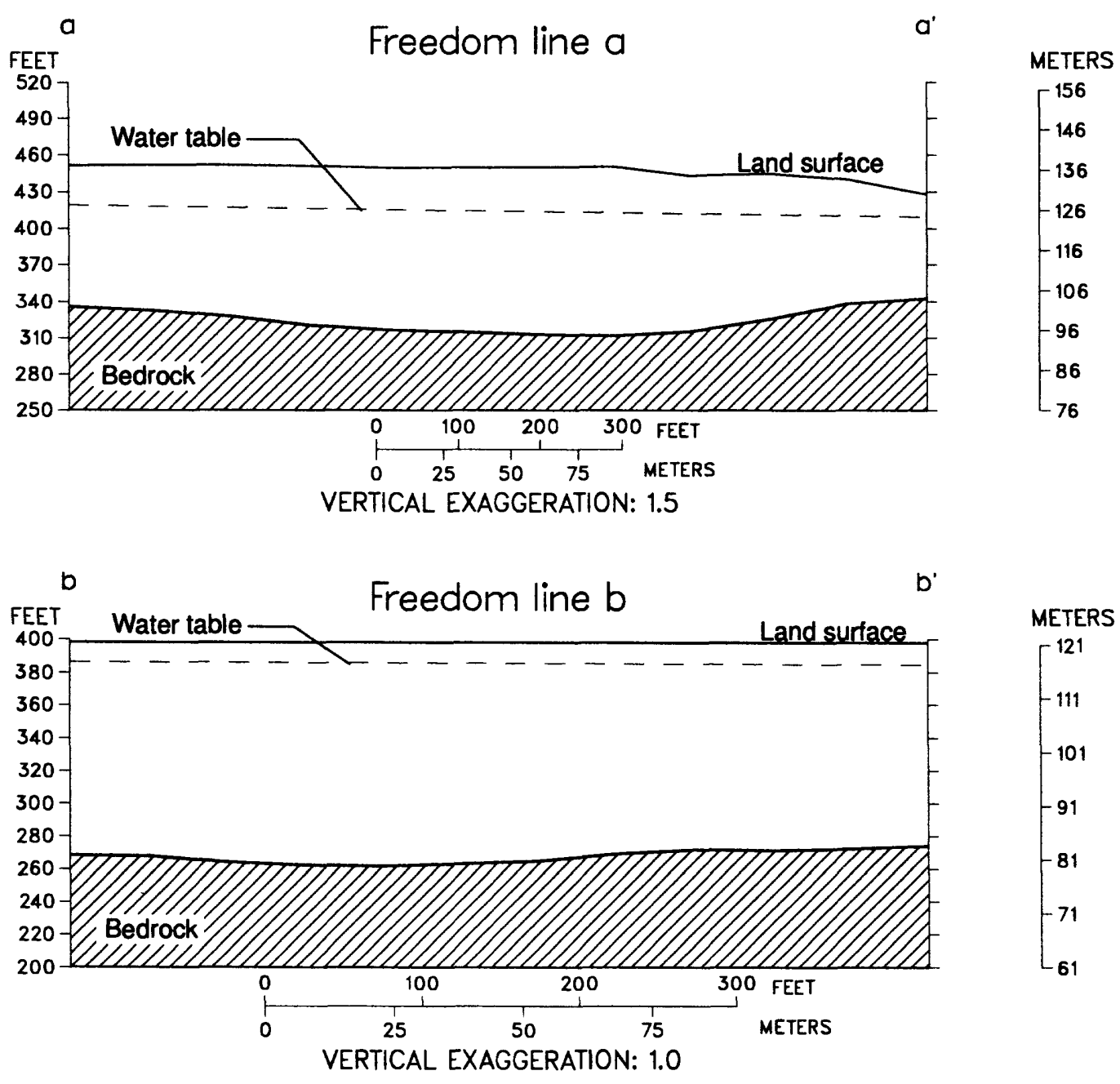

Figure 3-7. Geohydrologic sections interpreted from seismic-refraction data for Effingham line e$\mathrm{e}^{\prime}$, and Freedom line $\mathrm{a}-\mathrm{a}$ ' and b-b' (locations shown on plate 2). 


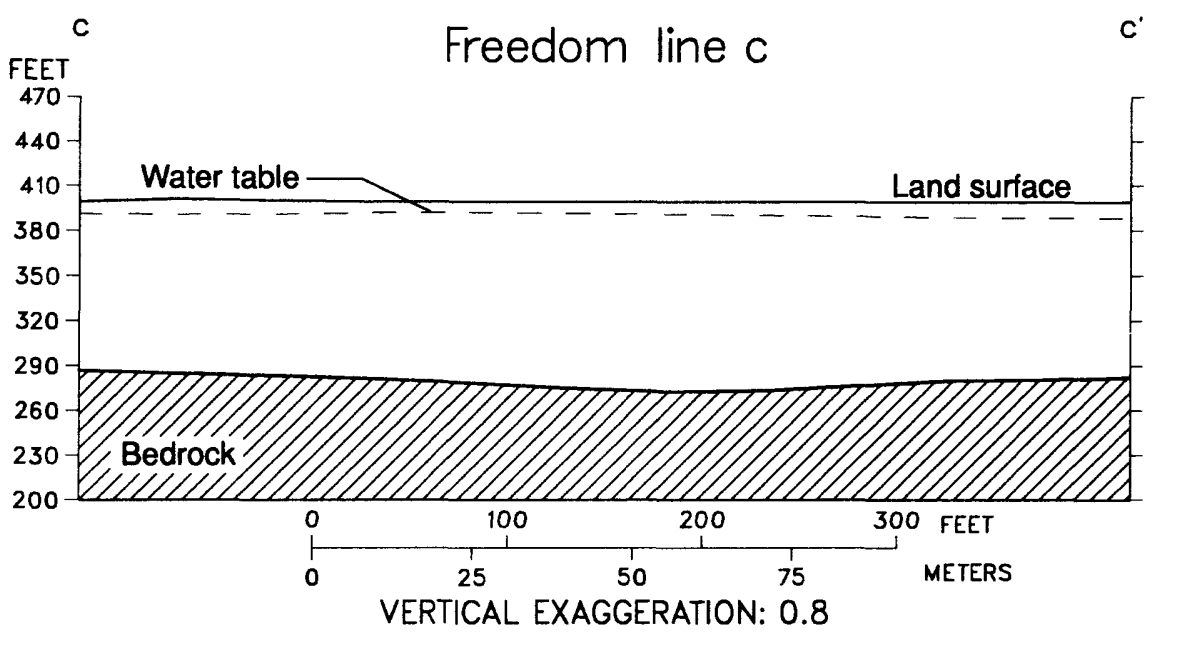

METERS
$\begin{aligned} & -141 \\ & -131 \\ & -121 \\ & -111 \\ & -101 \\ & -91 \\ & -81 \\ & -71 \\ & 61\end{aligned}$
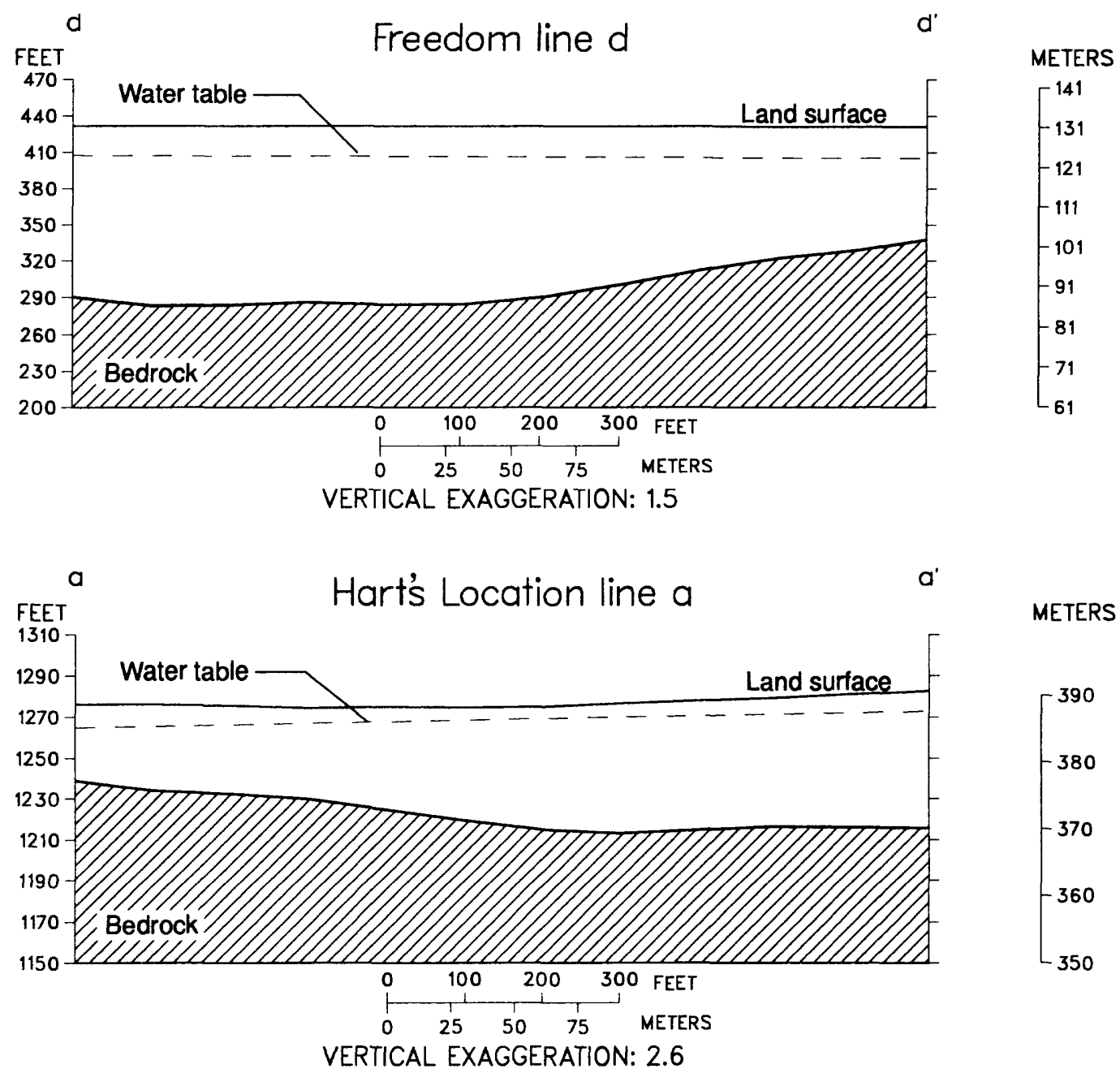

Figure 3-8. Geohydrologic sections interpreted from seismic-refraction data for Freedom lines cc' and d-d', and Hart's Location line a-a' (locations shown on plates 1 and 2). 

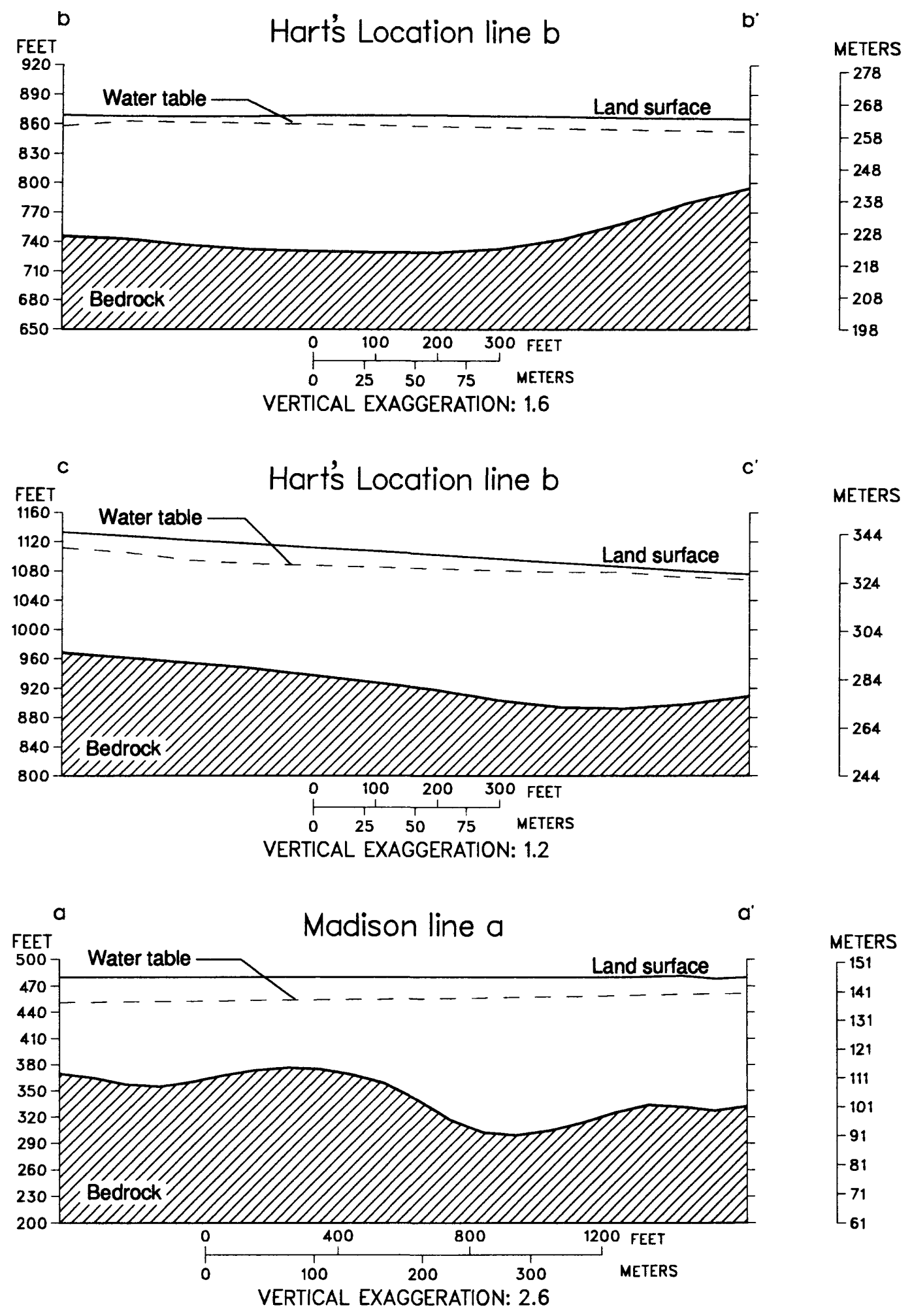

Figure 3-9. Geohydrologic sections interpreted from seismic-refraction data for Hart's Location lines b-b' and c-c', and Madison line a-a' (locations shown on plates land 2). 

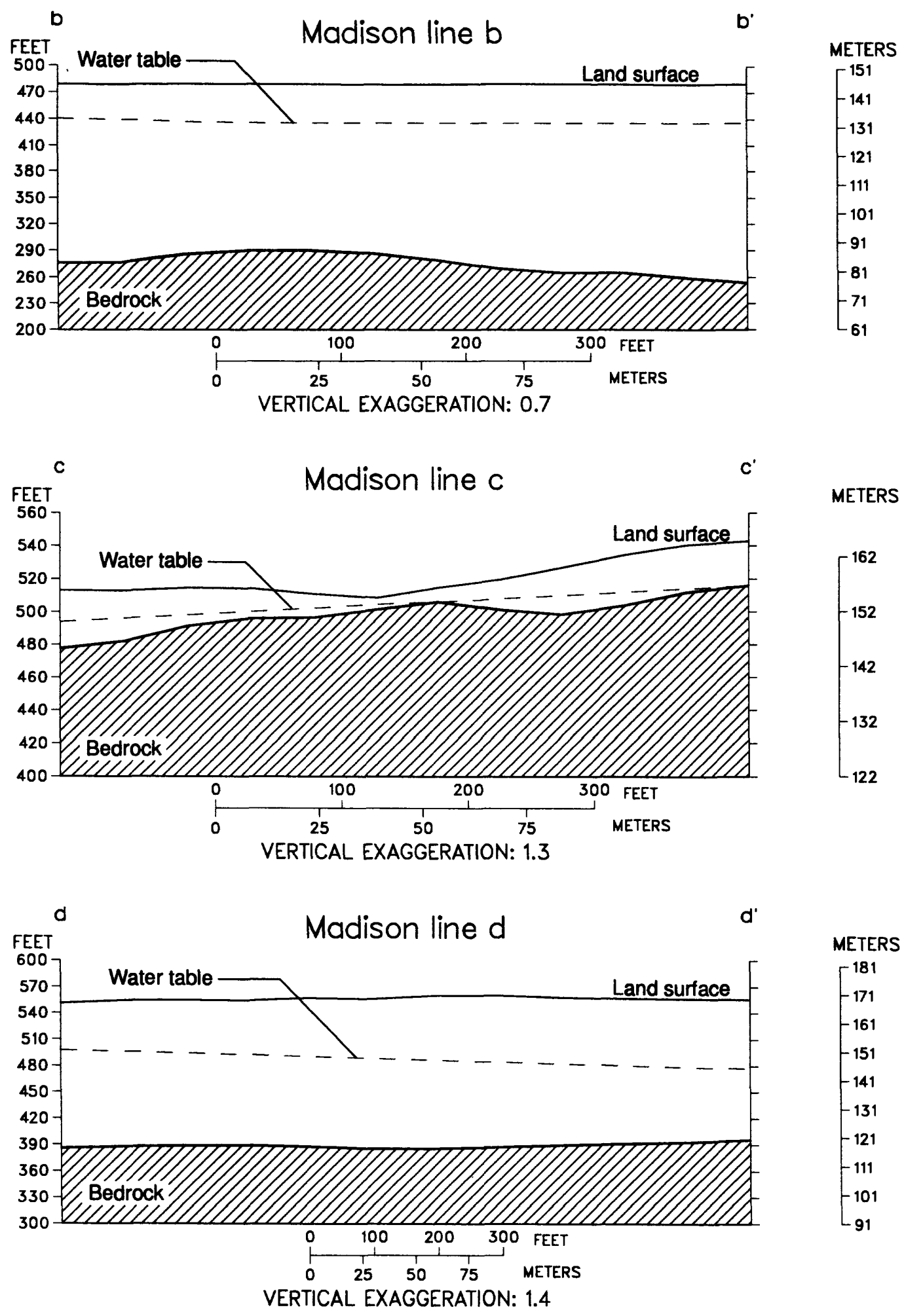

Figure 3-10. Geohydrologic sections interpreted from seismic-refraction data for Madison lines b-b', c-c', and d-d' (locations shown on plate 2). 


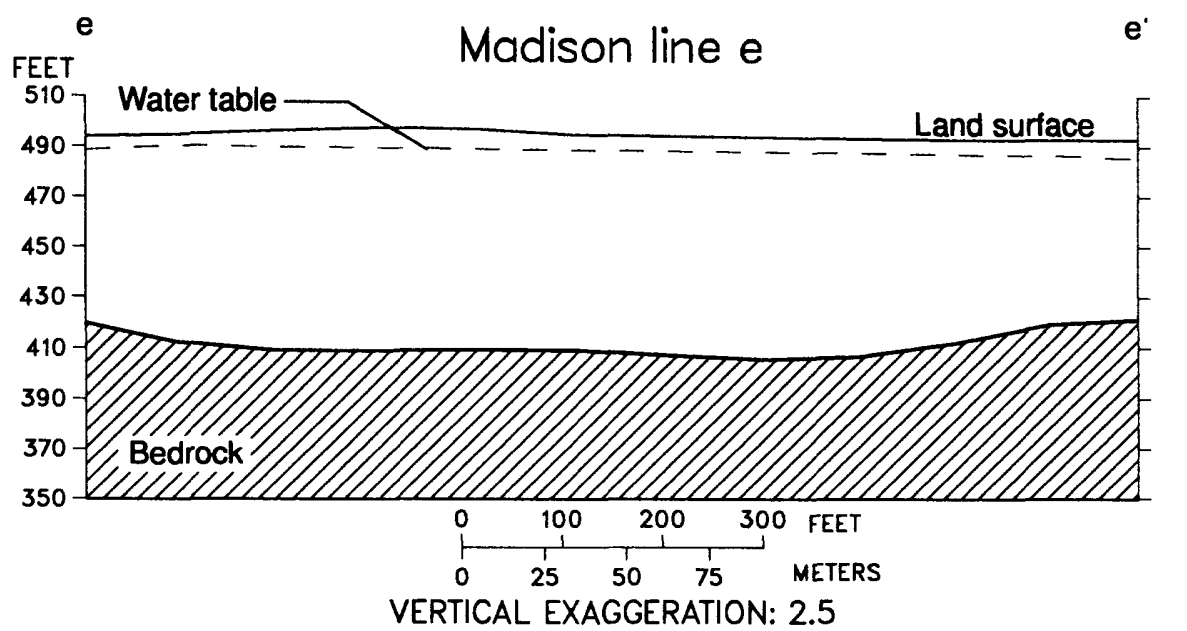

METERS
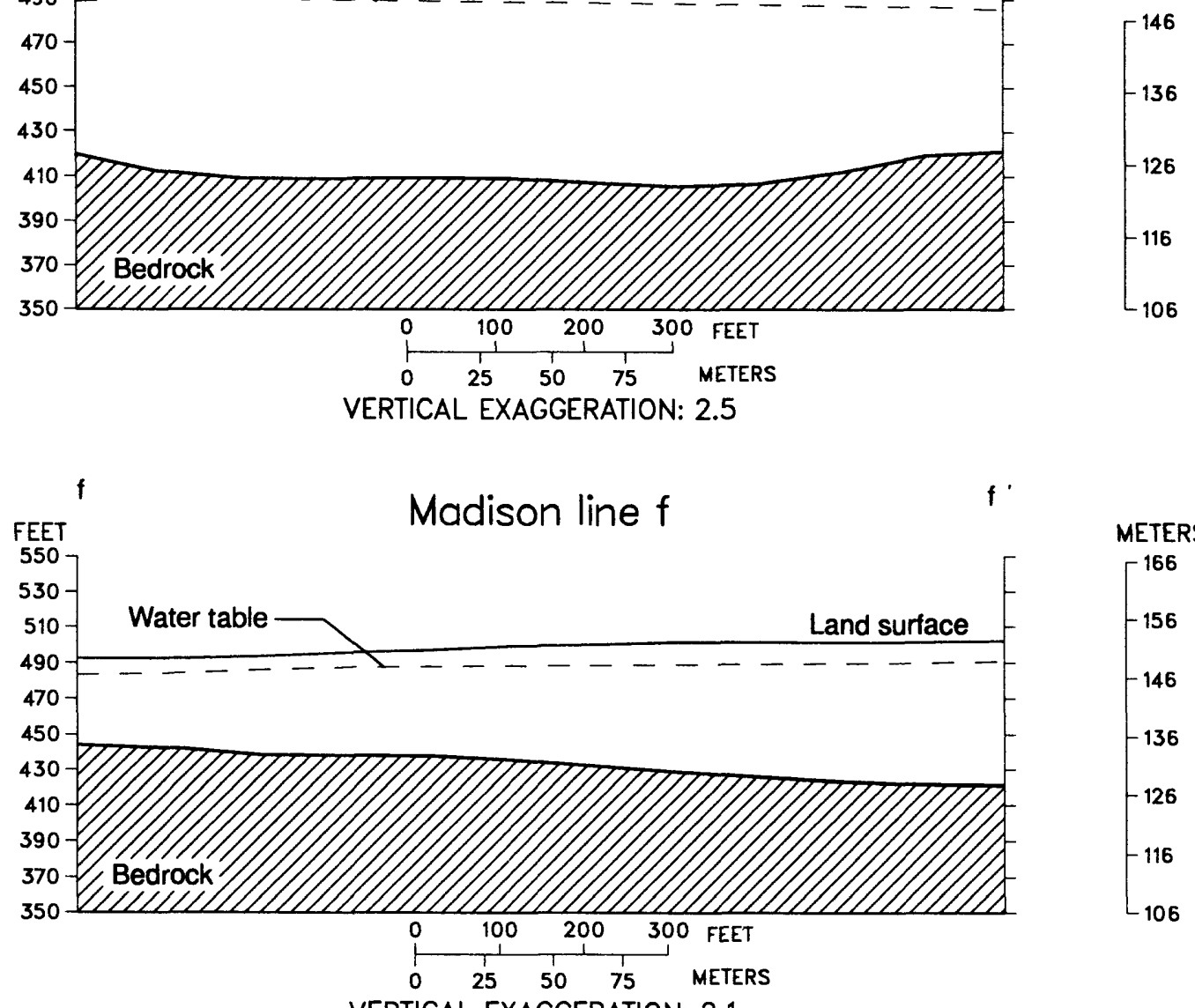

METERS

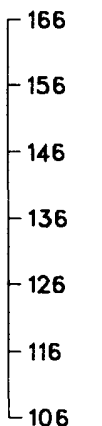

VERTICAL EXAGGERATION: 2.1

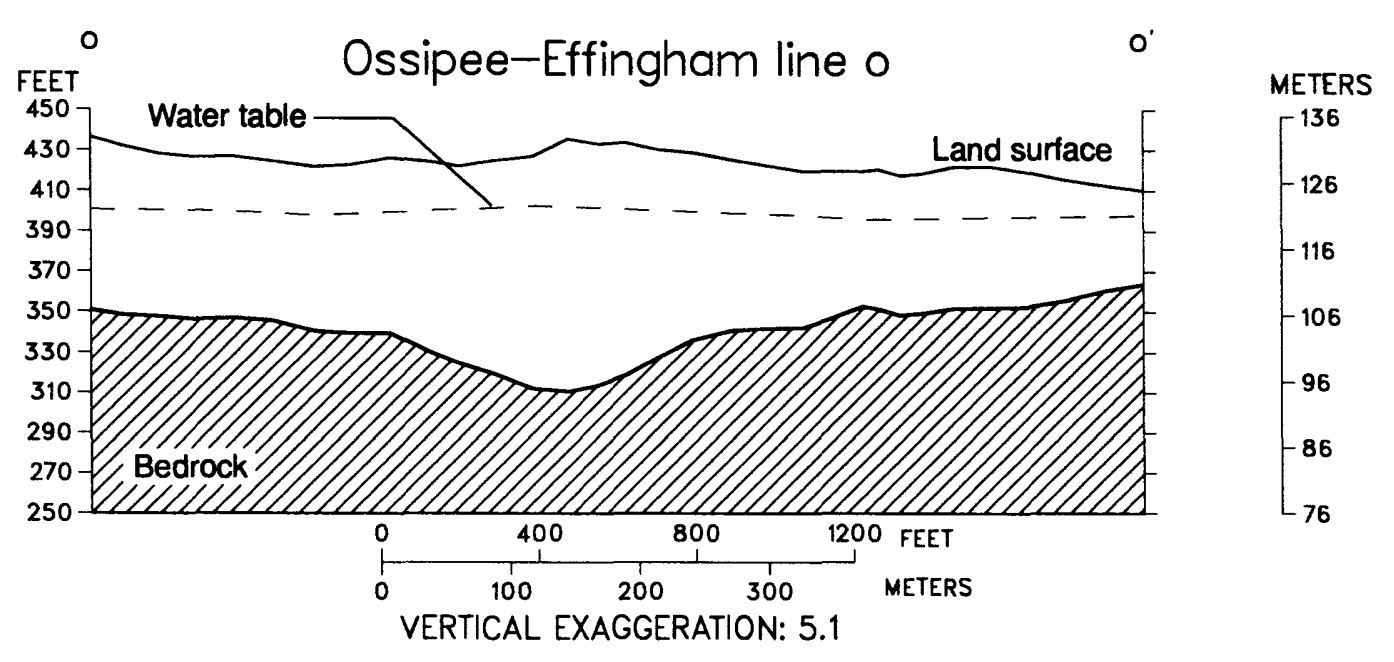

Figure 3-11. Geohydrologic sections interpreted from seismic-refraction data for Madison lines ee' and f-f', and Ossipee-Effingham line o-o' (locations shown on plate 2). 

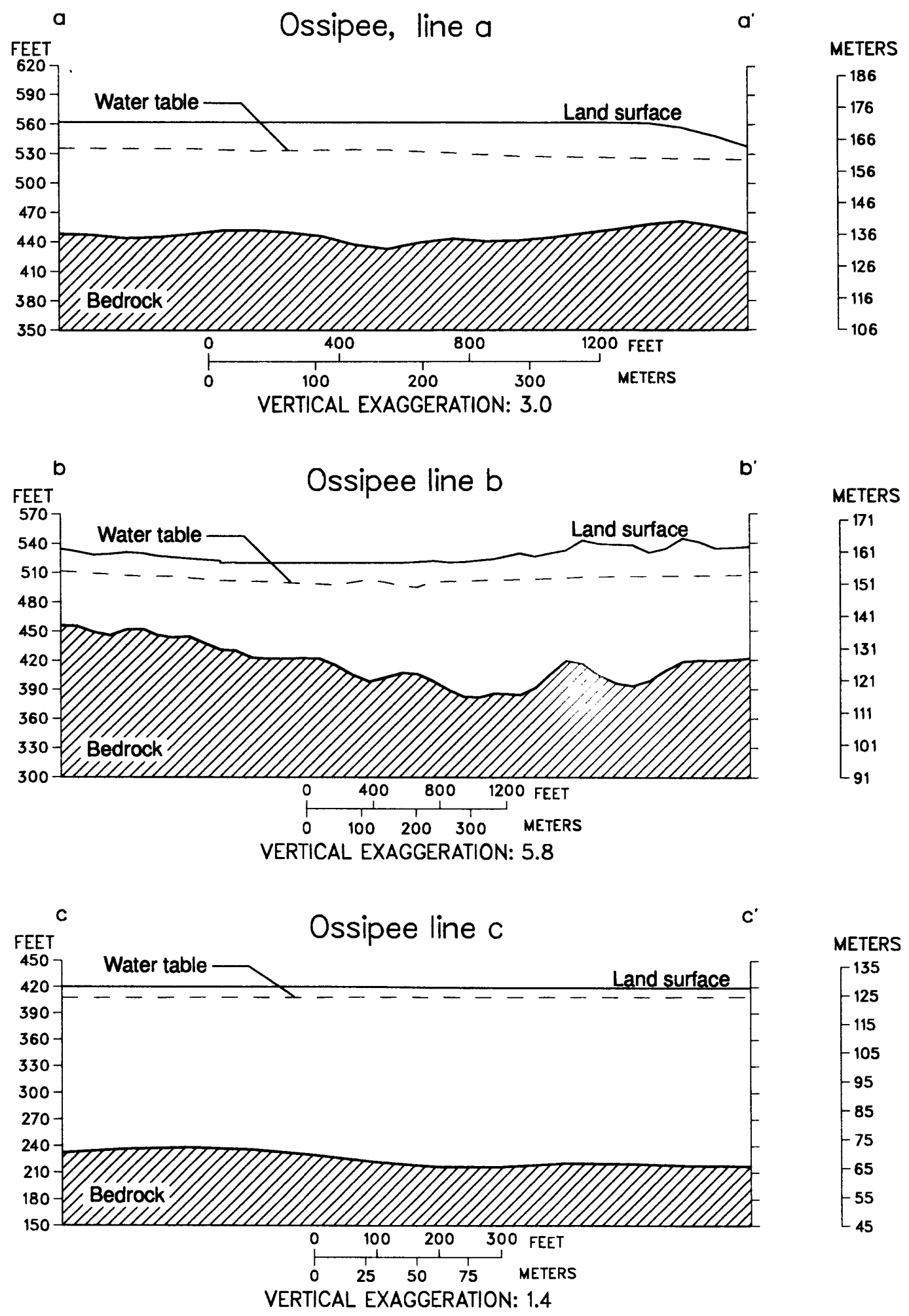

Figure 3-12. Geohydrologic sections interpreted from seismic-refraction data for Ossipee lines aa', b-b', and c-c' (locations shown on plate 2). 

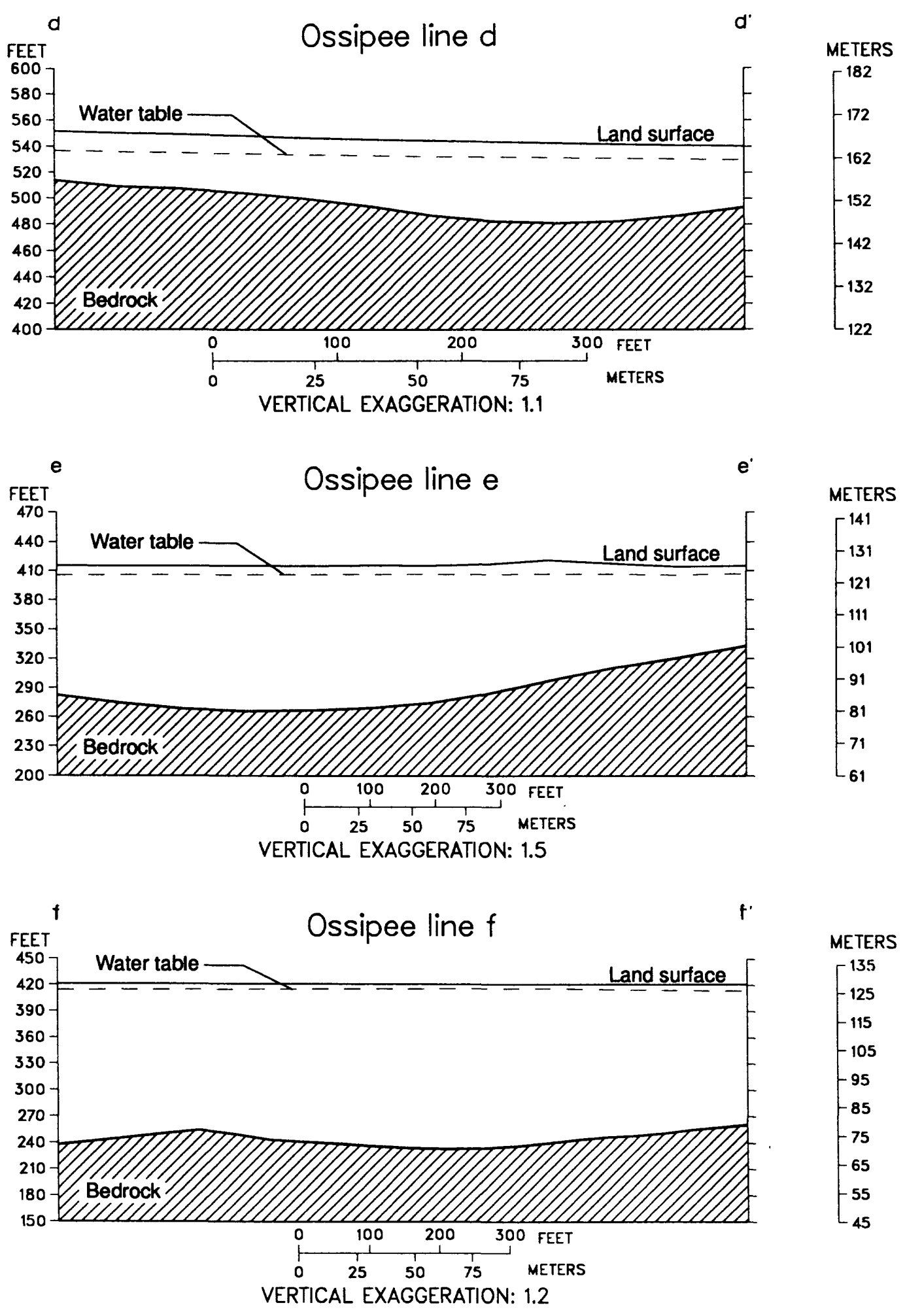

Figure 3-13. Geohydrologic sections interpreted from seismic-refraction data for Ossipee lines dd', e-e', and f-f' (locations shown on plate 2). 

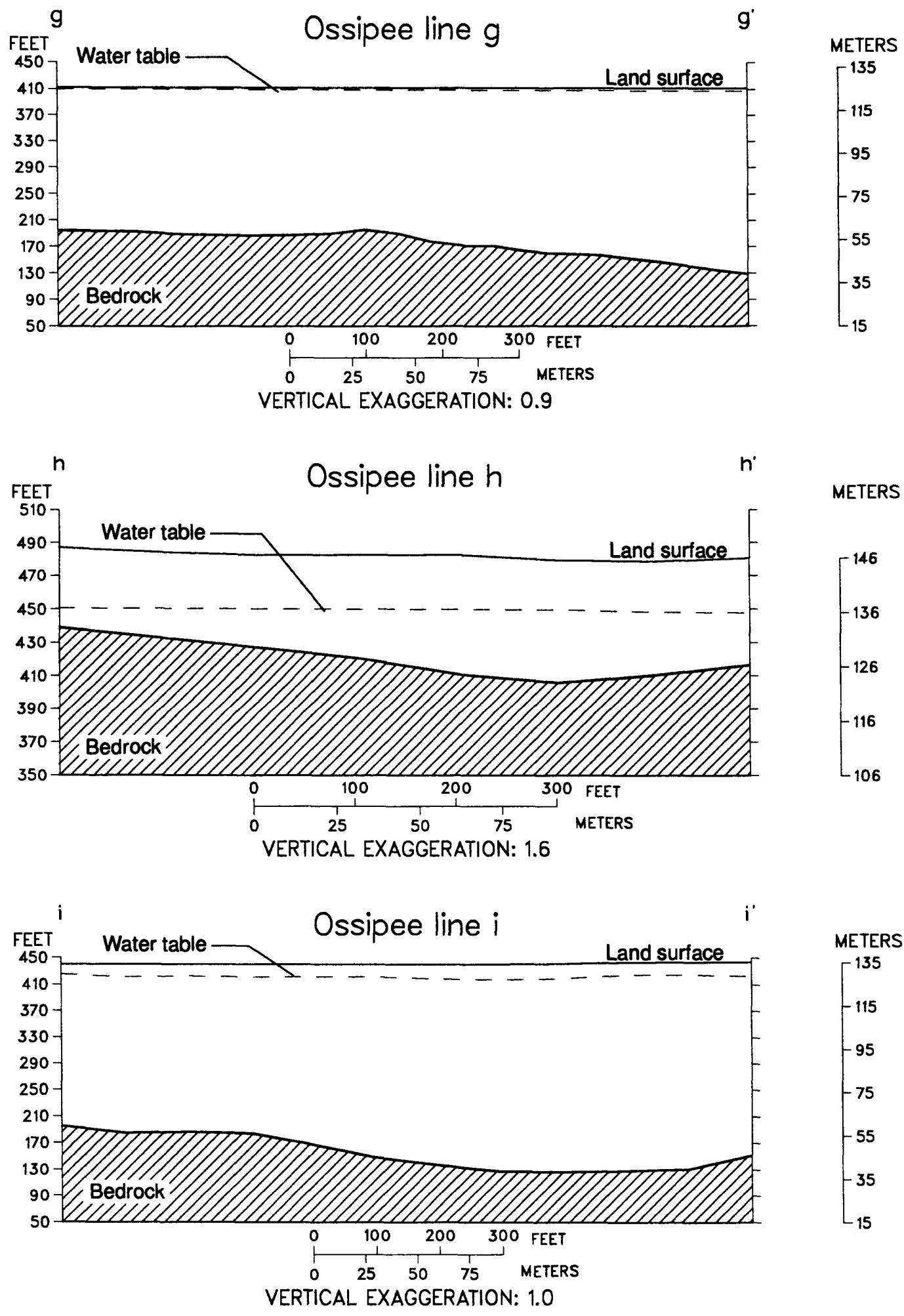

Figure 3-14. Geohydrologic sections interpreted from seismic-refraction data for Ossipee lines g$g^{\prime}, h-h '$, and i-i' (locations shown on plate 2). 

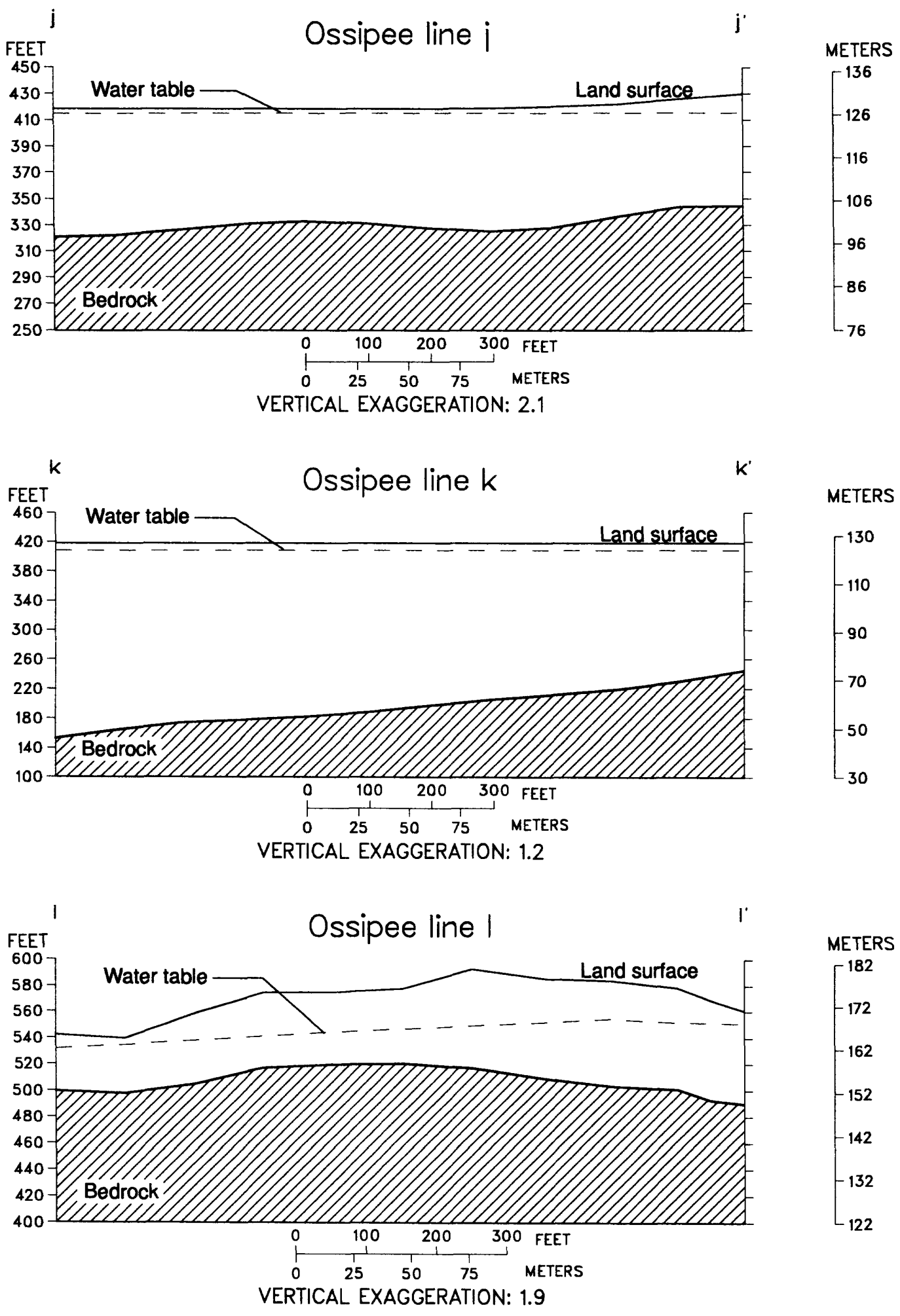

Figure 3-15. Geohydrologic sections interpreted from seismic-refraction data for Ossipee lines $\mathrm{j}-\mathrm{j}^{\prime}, \mathrm{k}-\mathrm{k}^{\prime}$, and l-l' (locations shown on plate 2). 

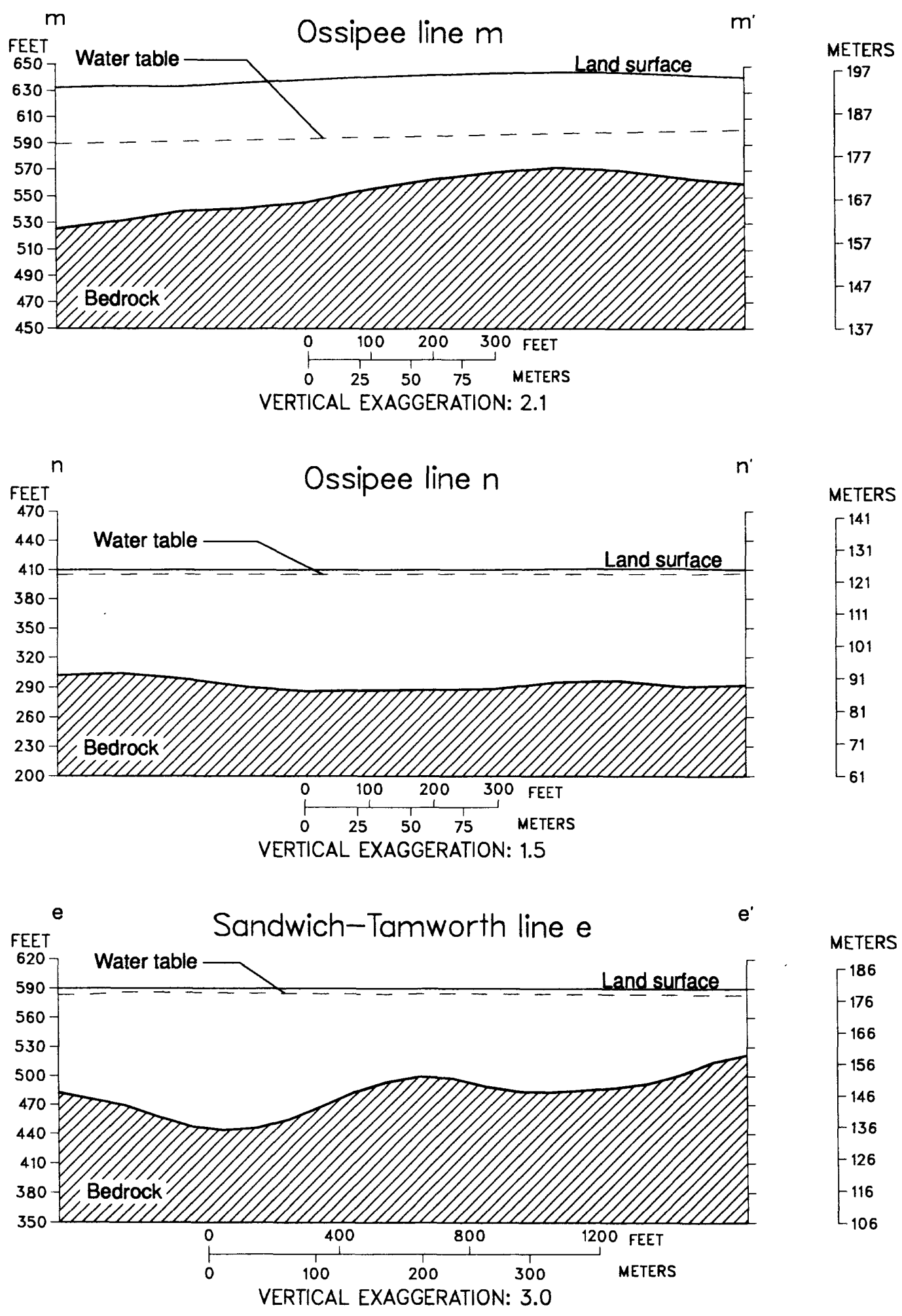

Figure 3-16. Geohydrologic sections interpreted from seismic-refraction data for Ossipee lines mm' and n-n', and Sandwich-Tamworth line e-e' (locations shown on plate 2). 

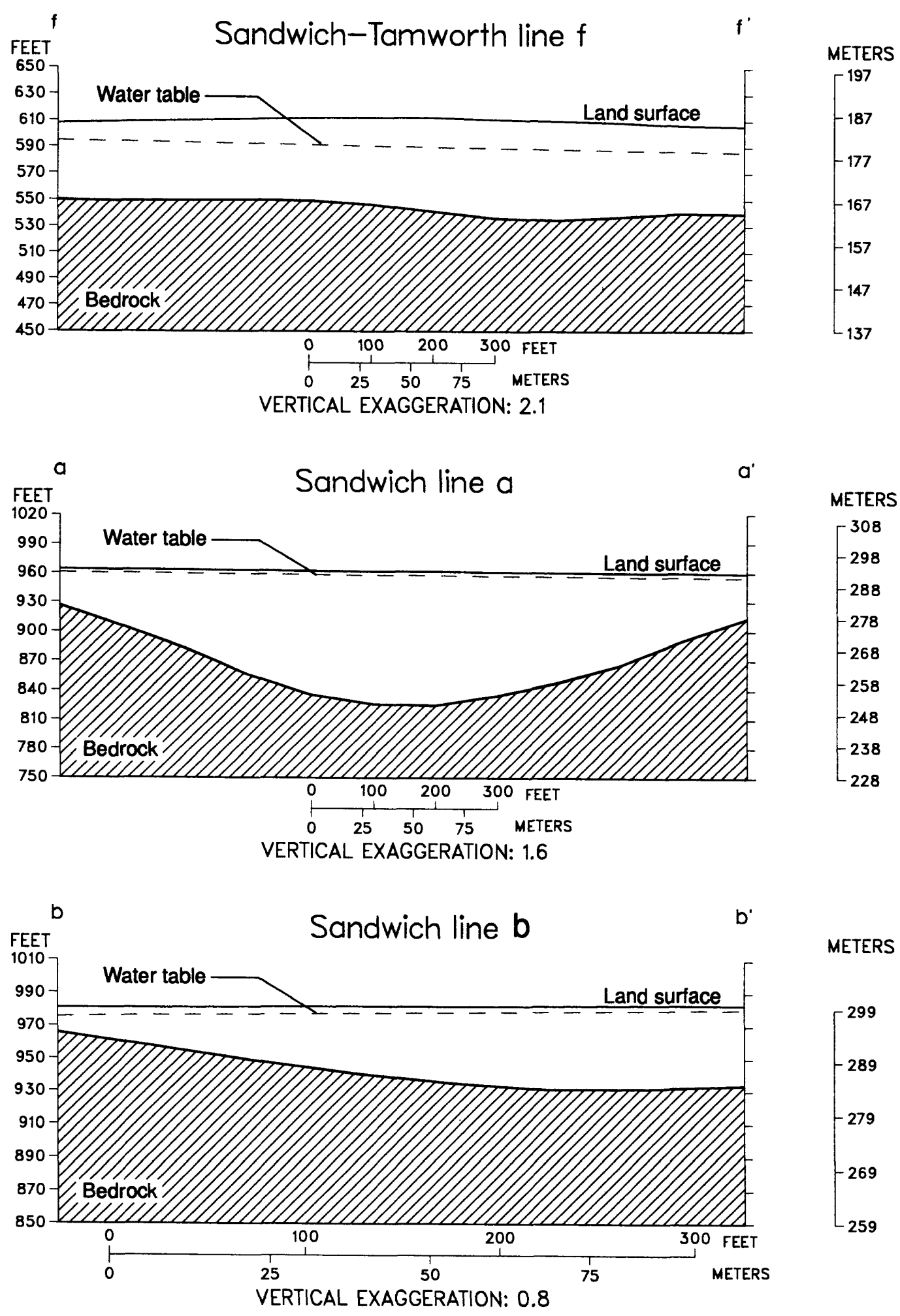

Figure 3-17. Geohydrologic sections interpreted from seismic-refraction data for Sandwich-Tamworth line f-f', Sandwich lines a-a' and b-b' (locations shown on plate 2). 


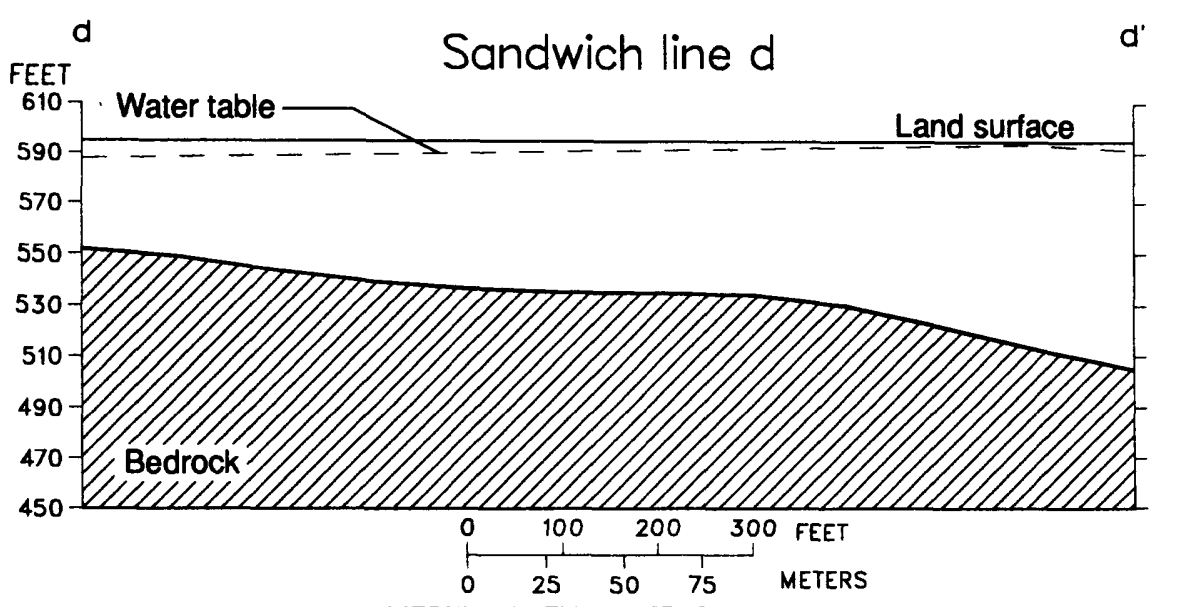

METERS

VERTICAL EXAGGERATION: 2.6
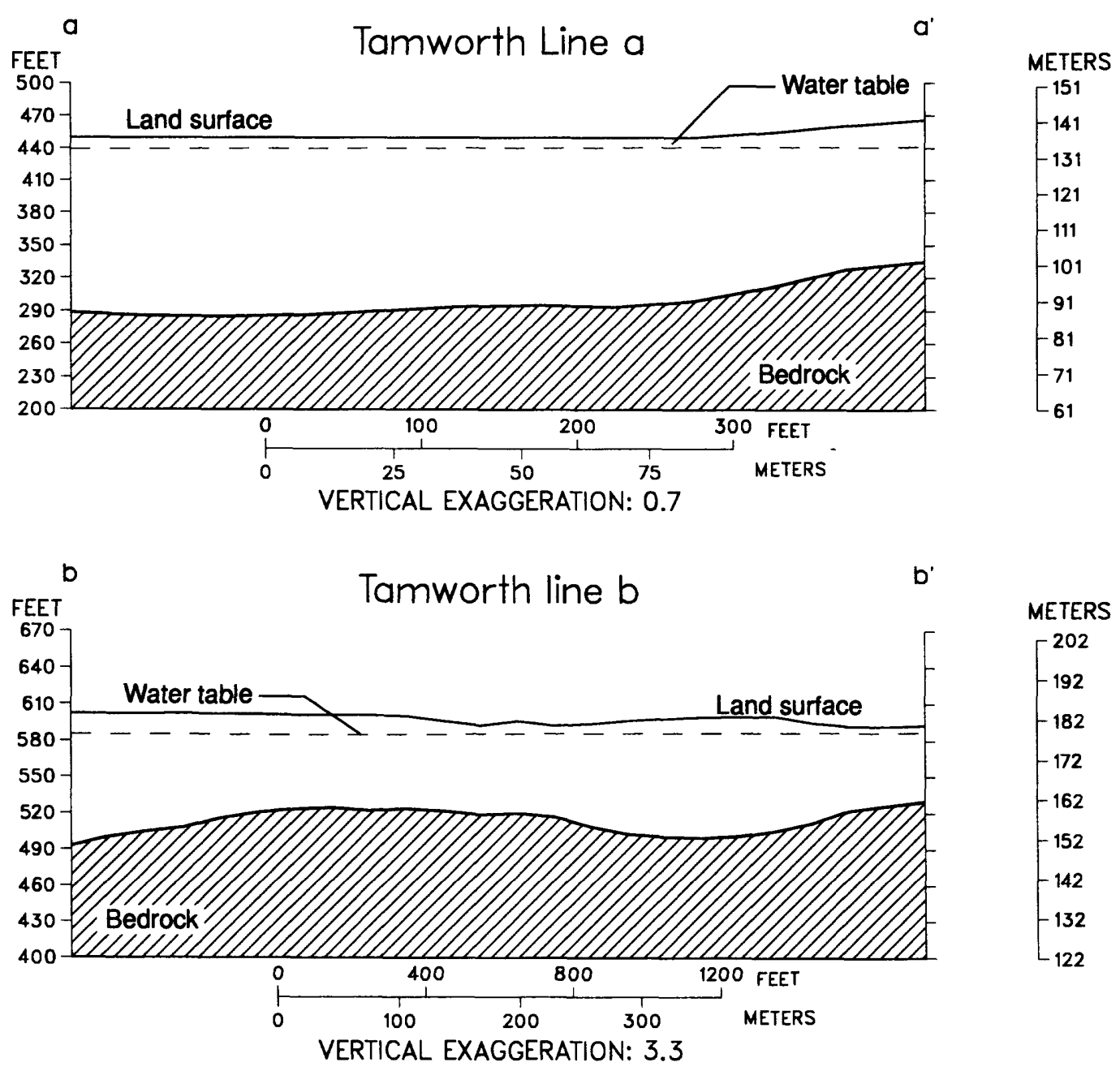

Figure 3-18. Geohydrologic sections interpreted from seismic-refraction data for Sandwich line d-d', Tamworth lines a-a' and b-b' (locations shown on plate 2). 

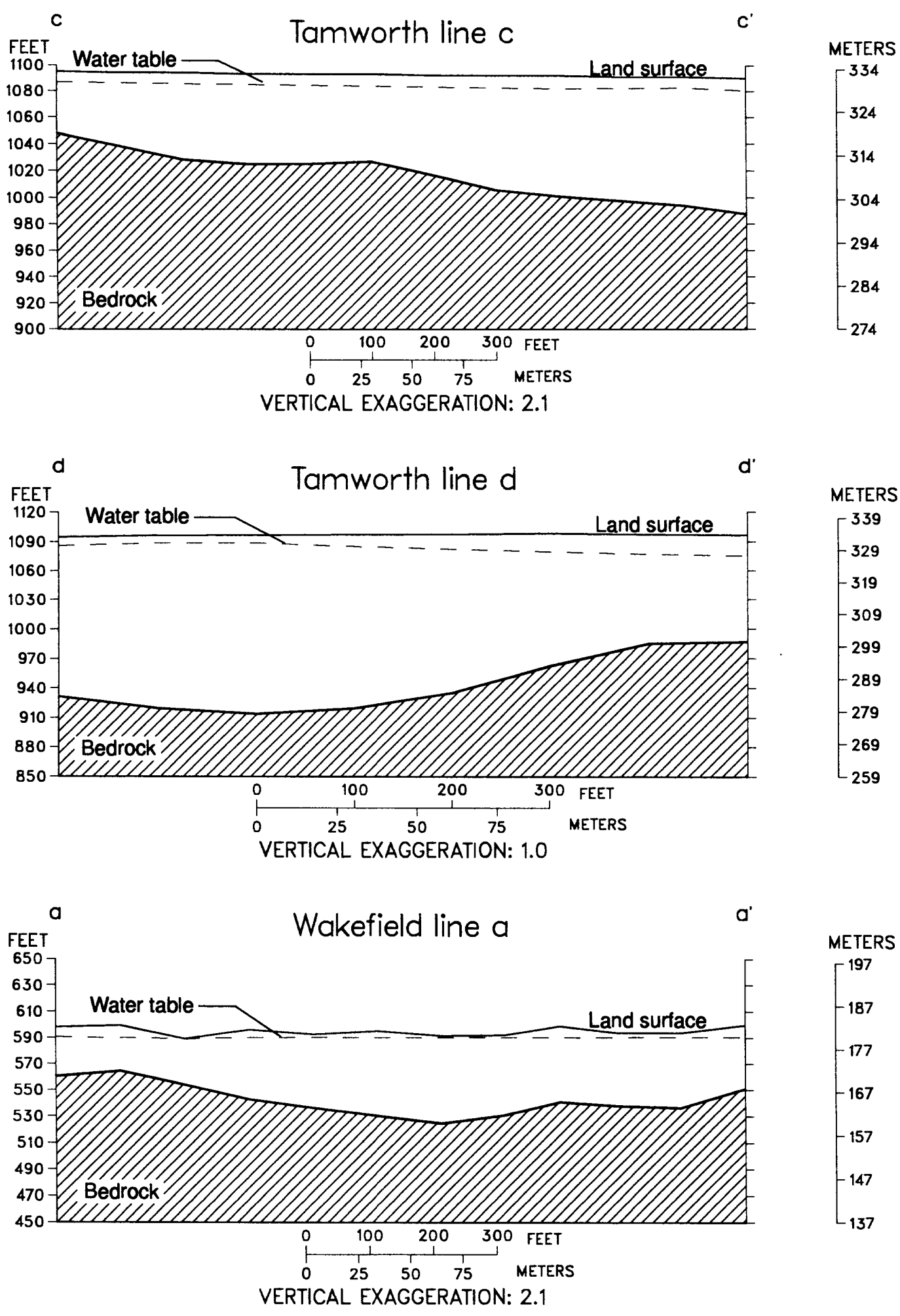

Figure 3-19. Geohydrologic sections interpreted from seismic-refraction data for Tamworth lines c-c', d-d' and Wakefield line a-a' (locations shown on plate 2). 

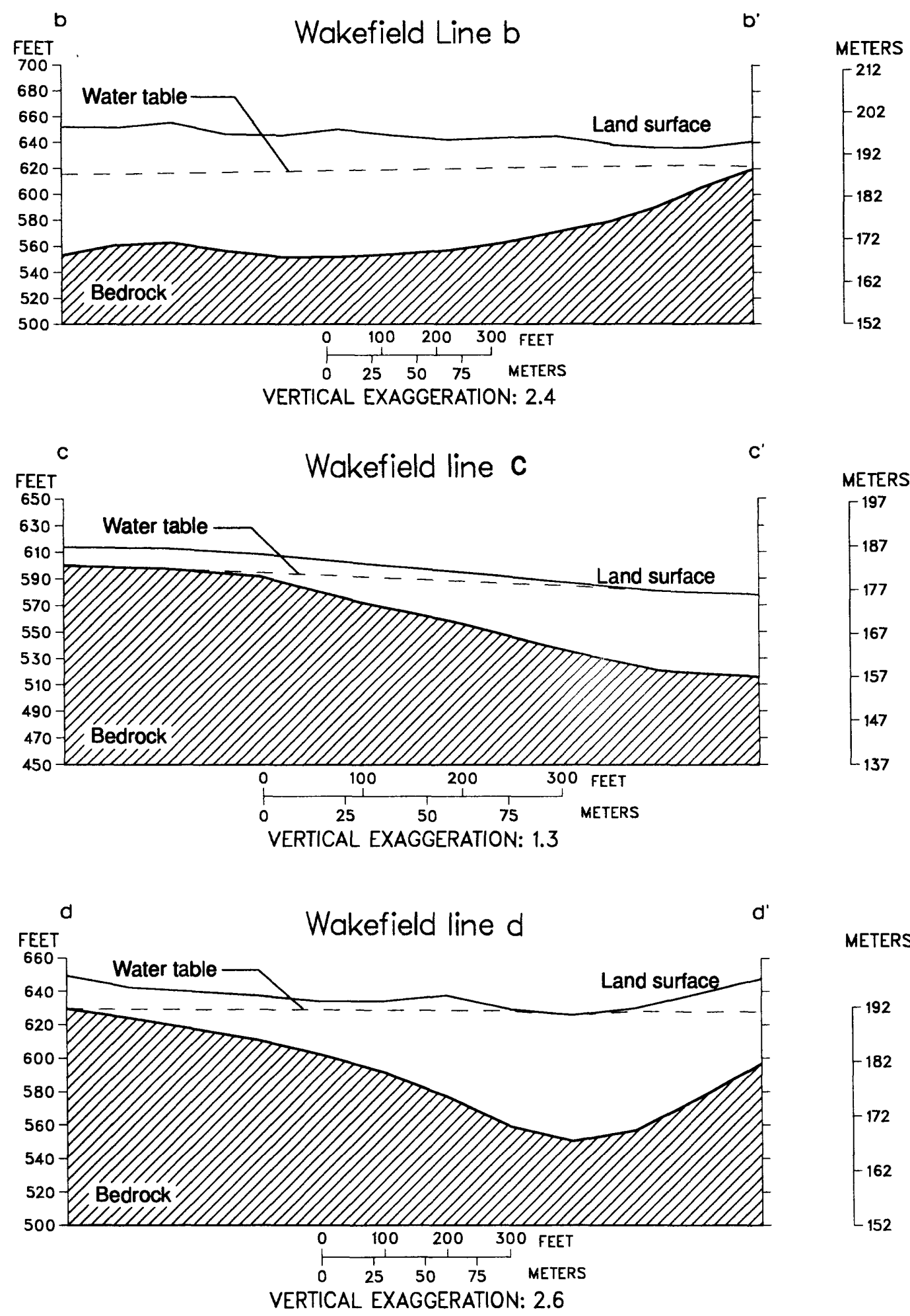

Figure 3-20. Geohydrologic sections interpreted from seismic-refraction data for Wakefield lines b-b', c-c', and d-d' (locations shown on plate 2). 


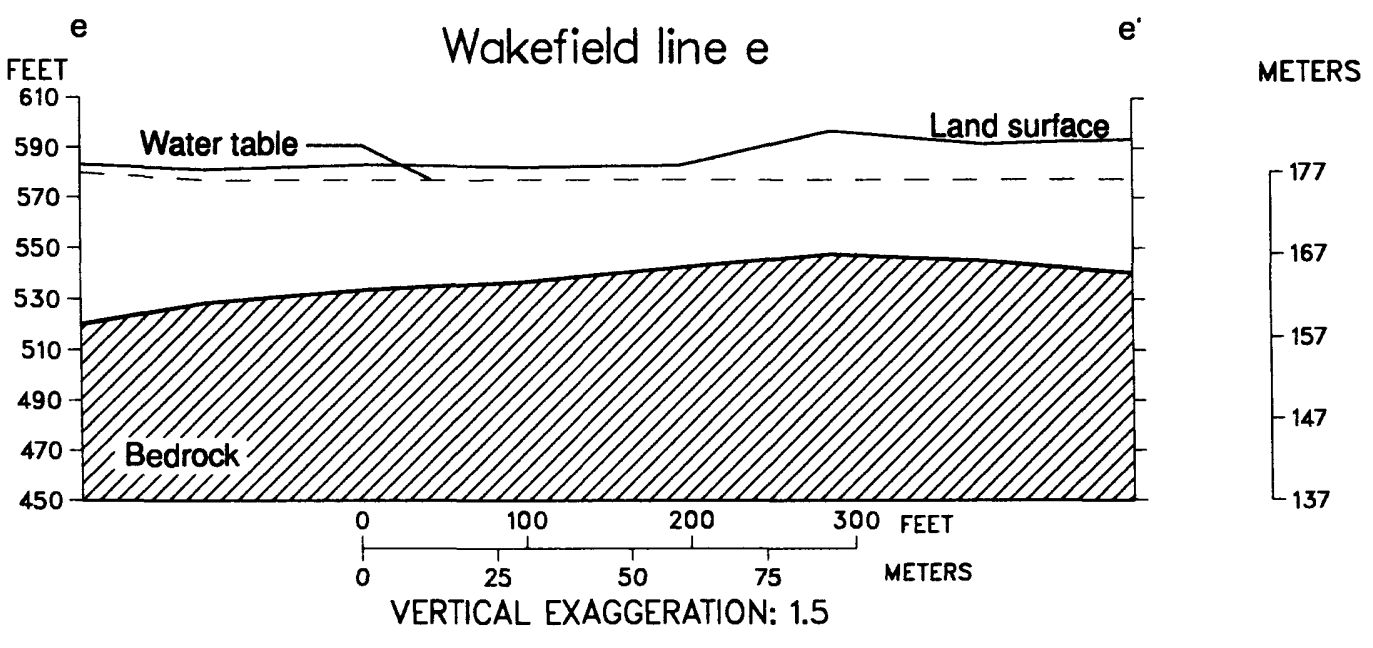

Figure 3-21. Geohydrologic sections interpreted from seismic-refraction data for Wakefield line e-e' (locations shown on plate 2). 


\section{APPENDIX 4. Low streamflow measurements at miscellaneous sites in the Saco and Ossipee River Basins, east-central New Hampshire}


Table 4-1. Low streamflow measurements at miscellaneous sites in the Saco and Ossipee River Basins, east-central New Hampshire

$\left[{ }^{\prime}\right.$ ' ", degree, minute, second; mm-dd-yy, month-day-year; $\mathrm{ft}^{3} / \mathrm{s}$, cubic foot per second]

\begin{tabular}{|c|c|c|c|c|c|}
\hline \multirow{2}{*}{$\begin{array}{l}\text { Name of brook or river } \\
\text { (reference number on } \\
\text { plates } 1 \text { and 2) }\end{array}$} & \multirow[b]{2}{*}{ Tributary to } & \multirow{2}{*}{$\begin{array}{c}\text { Latitude } \\
0,1 "\end{array}$} & \multirow{2}{*}{$\begin{array}{c}\text { Longitude } \\
0, " 1\end{array}$} & \multicolumn{2}{|c|}{ Measurements } \\
\hline & & & & $\begin{array}{c}\text { Date } \\
\text { (mm-dd-yy) }\end{array}$ & $\begin{array}{c}\text { Discharge } \\
\left(\mathrm{ft}^{3} / \mathrm{s}\right)\end{array}$ \\
\hline Saco River (1) & Atlantic Ocean & 440455 & 711831 & $07-22-91$ & 23.3 \\
\hline Albany Brook (2) & Saco River & 440427 & 711804 & $07-22-91$ & .57 \\
\hline Stony Brook (3) & Saco River & 440443 & 711420 & $07-22-91$ & .04 \\
\hline Stony Brook (4) & Saco River & 440456 & 711418 & $07-22-91$ & .08 \\
\hline East Branch Saco River (5) & Saco River & 440627 & 710832 & $07-22-91$ & 3.98 \\
\hline East Branch Saco River (6) & Saco River & 440602 & 710859 & $07-22-91$ & 3.52 \\
\hline East Branch Saco River (7) & Saco River & 440554 & 710932 & $07-22-91$ & 2.52 \\
\hline East Branch Saco River (8) & Saco River & 440550 & 710939 & $07-22-91$ & .16 \\
\hline East Branch Saco River (9) & Saco River & 440543 & 710954 & $07-22-91$ & .59 \\
\hline Saco River (10) & Atlantic Ocean & 440541 & 710950 & $07-22-91$ & 47.7 \\
\hline Saco River (11) & Atlantic Ocean & 440403 & 710855 & $07-22-91$ & 58.5 \\
\hline Lucy Brook (12) & Saco River & 440412 & 711023 & $07-22-91$ & .61 \\
\hline Lucy Brook (13) & Saco River & 440415 & 711012 & $07-22-91$ & .61 \\
\hline Lucy Brook (14) & Saco River & 440402 & 710933 & $07-22-91$ & .32 \\
\hline Saco River (15) & Atlantic Ocean & 440215 & 710821 & $07-22-91$ & 63.5 \\
\hline Saco River (16) & Atlantic Ocean & 440022 & 710700 & $07-22-91$ & 72.7 \\
\hline White Lot Brook (17) & Black Brook & 440203 & 710144 & $07-22-91$ & .28 \\
\hline White Lot Brook (18) & Black Brook & 440153 & 710138 & $07-22-91$ & .20 \\
\hline Pine River (19) & Ossipee Lake & 433851 & 710254 & $07-22-91$ & 2.56 \\
\hline Youngs Brook (20) & Pine River & 433935 & 710413 & $07-22-91$ & 2.21 \\
\hline Pine River (21) & Ossipee Lake & 434010 & 710427 & $07-22-91$ & 3.38 \\
\hline Pine River (22) & Ossipee Lake & 434103 & 710447 & $07-22-91$ & 8.46 \\
\hline Poland Brook (23) & Pine River & 434104 & 710453 & $07-22-91$ & .08 \\
\hline Wilkinson Brook (24) & Pine River & 434310 & 710147 & $07-22-91$ & .15 \\
\hline Wilkinson Brook (25) & Pine River & 434331 & 710423 & $07-22-91$ & 3.27 \\
\hline Pine River (26) & Ossipee Lake & 434436 & 710520 & $07-22-91$ & 10.5 \\
\hline Whiteface River (27) & Cold River & 435255 & 712335 & $07-22-91$ & 2.43 \\
\hline Bearcamp River (28) & Ossipee Lake & 434929 & 711839 & $07-22-91$ & \\
\hline Cold Brook (29) & Bearcamp River & 434857 & 711750 & $07-22-91$ & .34 \\
\hline Mill Brook (30) & Swift River & 435119 & 711830 & $07-22-91$ & .60 \\
\hline Mill Brook (31) & Swift River & 435022 & 711551 & $07-22-91$ & 2.40 \\
\hline Swift River (32) & Bearcamp River & 435330 & 711758 & $07-22-91$ & 11.1 \\
\hline Swift River (33) & Bearcamp River & 435050 & 711601 & $07-22-91$ & 10.7 \\
\hline Bearcamp River (34) & Ossipee Lake & 434920 & 711245 & $07-22-91$ & 25.2 \\
\hline Lovell River (35) & Ossipee Lake & 434642 & 710925 & $07-22-91$ & 1.24 \\
\hline West Branch River (36) & Ossipee Lake & 435051 & 711019 & $07-22-91$ & 1.97 \\
\hline
\end{tabular}


APPENDIX 5. Chemical analysis of the groundwater samples from the Saco and Ossipee River Basins, east-central, New Hampshire 
Table 5-1. Chemical analysis of ground-water samples from the Saco and Ossipee River Basins, east-central New Hampshire

[ ${ }^{\circ}$ ' ", degree, minute, second; $\mathrm{ft}$, foot; $\mu \mathrm{S} / \mathrm{cm}$, microsiemens per centimeter at 25 degrees Celsius; ${ }^{\circ} \mathrm{C}$, degrees Celsius; $\mathrm{mg} / \mathrm{L}$, milligrams per liter; $\mu \mathrm{g} / \mathrm{L}$, micrograms per liter; <, less than; --, no data available]

\begin{tabular}{|c|c|c|c|c|c|c|c|c|c|}
\hline \multicolumn{2}{|c|}{$\begin{array}{l}\text { Local site } \\
\text { number }\end{array}$} & Date & Time & $\begin{array}{c}\text { Latitude } \\
\circ, " 1\end{array}$ & $\begin{array}{l}\text { Longitude } \\
\circ, " 1\end{array}$ & $\begin{array}{l}\text { Depth of } \\
\text { well, total } \\
\text { (ft) }\end{array}$ & $\begin{array}{l}\text { Altitude } \\
\text { of land } \\
\text { surface } \\
\text { datum } \\
\text { (ft above } \\
\text { sea level) }\end{array}$ & $\begin{array}{l}\text { Specific } \\
\text { con- } \\
\text { duct- } \\
\text { ance } \\
(\mu \mathrm{S} / \mathrm{cm})\end{array}$ & $\begin{array}{c}\mathrm{pH}, \\
\text { field } \\
\text { measure- } \\
\text { ment } \\
\text { (standard } \\
\text { units) }\end{array}$ \\
\hline $\mathrm{ADW}$ & 13 & $08-27-92$ & 1700 & 435524 & 0711326 & 52.4 & 625 & 68 & 6.1 \\
\hline ADW & 14 & $09-01-92$ & 1330 & 435948 & 0712203 & 79.5 & 1,250 & 158 & 6.8 \\
\hline $\mathrm{ADW}$ & 15 & $09-01-92$ & 1430 & 435948 & 0712203 & 18.0 & 1,250 & 44 & 5.3 \\
\hline $\mathrm{ADW}$ & 16 & 09-01-92 & 1130 & 435936 & 0712104 & 49.5 & 1,250 & 54 & 5.9 \\
\hline CKW & 9 & 09-03-92 & 1230 & 441452 & 0710051 & 30.0 & 535 & 16 & 5.9 \\
\hline CKW & 10 & $09-03-92$ & 1430 & 441357 & 0710050 & 60.0 & 510 & 25 & 5.8 \\
\hline CKW & 11 & 09-03-92 & 1700 & 441004 & 0710040 & 59.0 & 530 & 1,190 & 6.5 \\
\hline CWW 6 & 66 & $08-28-92$ & 1330 & 435815 & 0710823 & 50.0 & 465 & 40 & 5.9 \\
\hline CWW & 67 & 09-04-92 & 1115 & 440423 & 0710100 & 69.0 & 430 & 50 & 6.6 \\
\hline CWW 7 & 70 & $09-04-92$ & 1015 & 440430 & 0710111 & 19.0 & 435 & 60 & 5.6 \\
\hline EFW & 9 & 09-02-92 & 1700 & 434608 & 0710640 & 74.5 & 420 & 24 & 6.0 \\
\hline FLW & 25 & $09-05-92$ & 1130 & 434856 & 0710940 & 39.5 & 415 & 21 & 5.5 \\
\hline MBW 1 & 10 & $08-28-92$ & 1100 & 435054 & 0711132 & 59.0 & 480 & 61 & 5.6 \\
\hline OXS & 1 & $09-06-92$ & 1315 & 434709 & 0711139 & -- & 590 & 42 & 6.2 \\
\hline OXS & 5 & $09-06-92$ & 1500 & 433909 & 0710350 & -- & 575 & 39 & 6.4 \\
\hline OXW & 34 & $08-26-92$ & 1330 & 434916 & 0711054 & 69.0 & 450 & 48 & 6.1 \\
\hline OXW & 35 & $09-05-92$ & 1430 & 434344 & 0710904 & 18.5 & 510 & 62 & 6.1 \\
\hline OXW & 36 & 09-04-92 & 1800 & 434653 & 0710638 & 90.0 & 420 & 152 & 5.9 \\
\hline OXW & 38 & $09-05-92$ & 1600 & 434221 & 0710515 & 114.7 & 550 & 35 & 6.4 \\
\hline SES & 1 & 09-06-92 & 1030 & 435238 & 0712401 & -- & 900 & 88 & 7.3 \\
\hline SEW & 26 & $08-27-92$ & 1100 & 434959 & 0712042 & 30.0 & 590 & 28 & 5.8 \\
\hline TAS & 1 & $09-06-92$ & 1730 & 435038 & 0711528 & -- & 450 & 64 & 5.7 \\
\hline TAW & 31 & $08-27-92$ & 1330 & 435016 & 0711406 & 114.0 & 450 & 26 & 5.7 \\
\hline TAW & 32 & $09-02-92$ & 1400 & 435005 & 0711945 & 42.0 & 600 & 40 & 7.1 \\
\hline TAW & 33 & $08-27-92$ & 1530 & 435000 & 0711337 & 67.5 & 450 & 53 & 5.8 \\
\hline TAW & 34 & $08-26-92$ & 1700 & 435013 & 0711521 & 40.0 & 450 & 46 & 6.6 \\
\hline WAW 2 & 23 & $09-05-92$ & 1800 & 433820 & 0710319 & 49.0 & 620 & 24 & - \\
\hline MBW 5 & 57 & 09-04-92 & 1530 & 435715 & 0710913 & 49.0 & 470 & 124 & 6.3 \\
\hline $\mathrm{MBW} 5$ & 55 & $09-04-92$ & 1400 & 435642 & 0710914 & 49.0 & 475 & 70 & 5.8 \\
\hline
\end{tabular}


Table 5-1. Chemical analysis of ground-water samples from the Saco and Ossipee River Basins, east-central New Hampshire--Continued

\begin{tabular}{|c|c|c|c|c|c|c|c|c|c|c|}
\hline $\begin{array}{l}\text { Local } \\
\text { numb }\end{array}$ & & $\begin{array}{c}\text { Temperature } \\
\text { of water } \\
\left({ }^{\circ} \mathrm{C}\right)\end{array}$ & $\begin{array}{c}\text { Oxygen, } \\
\text { dissolved } \\
(\mathrm{mg} / \mathrm{L})\end{array}$ & $\begin{array}{c}\text { Hardness } \\
\text { total } \\
(\mathrm{mg} / \mathrm{L} \text { as } \\
\left.\mathrm{CaCO}_{3}\right)\end{array}$ & $\begin{array}{c}\text { Acidity } \\
\text { (mg/L } \\
\text { as } \mathrm{H} \text { ) }\end{array}$ & $\begin{array}{c}\text { Calcium } \\
\text { dissolved } \\
(\mathrm{mg} / \mathrm{L} \\
\text { as } \mathrm{Ca})\end{array}$ & $\begin{array}{l}\text { Magne- } \\
\text { sium, } \\
\text { dissolved } \\
(\mathrm{mg} / \mathrm{L} \\
\text { as } \mathrm{Mg})\end{array}$ & $\begin{array}{c}\text { Sodium, } \\
\text { dissolved } \\
(\mathrm{mg} / \mathrm{L} \\
\text { as } \mathrm{Na})\end{array}$ & $\begin{array}{l}\text { Sodium } \\
\text { percent }\end{array}$ & $\begin{array}{c}\text { Potassium, } \\
\text { dissolved } \\
\text { (mg/L } \\
\text { as } \mathrm{K})\end{array}$ \\
\hline ADW & 13 & 9.5 & 4.9 & 15 & 0.1 & 5.1 & 0.56 & 6.2 & 45 & 0.90 \\
\hline ADW & 14 & 7.5 & 4.6 & 33 & $<.1$ & 11 & 1.3 & 17 & 52 & 1.5 \\
\hline $\mathrm{ADW}$ & 15 & 7.5 & 10.4 & 3 & .1 & 1.2 & .11 & 5.6 & 75 & .40 \\
\hline $\mathrm{ADW}$ & 16 & 7.5 & 9.9 & 14 & .1 & 5.1 & .35 & 3.8 & 35 & 1.0 \\
\hline CKW & 9 & 7.0 & 11.5 & 4 & .1 & 1.3 & .14 & 1.8 & 47 & .40 \\
\hline CKW & 10 & 7.5 & 9.5 & 7 & .3 & 2.0 & .47 & 3.3 & 48 & .60 \\
\hline CKW & 11 & 7.5 & 10.0 & 18 & .2 & 5.6 & .97 & 220 & 96 & 3.5 \\
\hline CWW & 66 & 9.5 & 8.5 & 8 & .3 & 2.4 & .38 & 3.6 & 47 & 1.0 \\
\hline CWW & 67 & 9.5 & 7.9 & 25 & .1 & 5.5 & 2.7 & 4.6 & 26 & 2.6 \\
\hline CWW & 70 & 9.0 & 2.0 & 20 & .9 & 6.0 & 1.3 & 4.4 & 28 & 3.6 \\
\hline EFW & 9 & 8.0 & 11.7 & 5 & .2 & 1.7 & .21 & 1.9 & 42 & .50 \\
\hline FLW & 25 & 8.5 & 11.6 & 4 & $<.1$ & 1.2 & .14 & 4.6 & 72 & .30 \\
\hline MBW & 10 & 10.0 & 10.7 & 7 & $<.1$ & 2.2 & .27 & 8.3 & 71 & .60 \\
\hline OXS & 1 & 11.5 & -- & 13 & .1 & 4.5 & .40 & 5.4 & 47 & .50 \\
\hline OXS & 5 & 10.0 & -- & 12 & $<.1$ & 3.8 & .62 & 5.2 & 47 & .40 \\
\hline OXW & 34 & 9.5 & 1.8 & 12 & .1 & 3.7 & .61 & 3.0 & 34 & .90 \\
\hline OXW & 35 & 9.5 & 11.7 & 12 & .2 & 4.2 & .41 & 7.0 & 52 & 1.3 \\
\hline OXW & 36 & 8.5 & 1.8 & 25 & .8 & 7.6 & 1.4 & 12 & 50 & 1.2 \\
\hline OXW & 38 & 8.0 & 11.4 & 10 & .1 & 3.2 & .55 & 2.7 & 35 & .60 \\
\hline SES & 1 & 9.0 & -- & 29 & $<.1$ & 8.0 & 2.2 & 7.2 & 34 & 1.3 \\
\hline SEW & 26 & 14.5 & 1.8 & 7 & $<.1$ & 2.0 & .37 & 1.7 & 34 & .50 \\
\hline TAS & 1 & 11.5 & -- & 13 & .1 & 4.2 & .49 & 6.1 & 49 & 1.1 \\
\hline TAW & 31 & 7.5 & 0 & 7 & $<.1$ & 2.1 & .32 & 1.5 & 32 & .40 \\
\hline TAW & 32 & 10.0 & 7.8 & 3 & $<.1$ & .80 & .29 & 3.7 & 64 & 1.1 \\
\hline TAW & 33 & 9.5 & 1.7 & 10 & .3 & 3.3 & .35 & 2.7 & 37 & .40 \\
\hline TAW & 34 & 9.0 & 5.4 & 14 & $<.1$ & 4.3 & .70 & 3.1 & 32 & .80 \\
\hline WAW & 23 & 8.5 & 11.8 & 6 & $<.1$ & 1.8 & .26 & 2.4 & 45 & .70 \\
\hline MBW & 57 & 10.5 & 10.1 & 30 & $<.1$ & 10 & 1.1 & 9.4 & 40 & 1.4 \\
\hline MBW & 55 & 10.0 & 11.2 & 22 & .2 & 7.3 & .82 & 4.2 & 29 & .90 \\
\hline
\end{tabular}


Table 5-1. Chemical analysis of ground-water samples from the Saco and Ossipee River Basins, east-central New Hampshire--Continued

\begin{tabular}{|c|c|c|c|c|c|c|c|c|c|c|}
\hline \multicolumn{2}{|c|}{$\begin{array}{c}\text { Local site } \\
\text { number }\end{array}$} & $\begin{array}{c}\text { Sulfate } \\
\text { dissolved } \\
(\mathrm{mg} / \mathrm{L} \\
\left.\text { as } \mathrm{SO}_{4}\right)\end{array}$ & $\begin{array}{c}\text { Chloride, } \\
\text { dissolved } \\
(\mathrm{mg} / \mathrm{L} \\
\text { as } \mathrm{Cl})\end{array}$ & $\begin{array}{c}\text { Flouride, } \\
\text { dissolved } \\
\text { (mg/L } \\
\text { as F) }\end{array}$ & $\begin{array}{c}\text { Silica } \\
\text { dissolved } \\
(\mathrm{mg} / \mathrm{L} \text { as } \\
\left.\mathrm{SiO}_{2}\right)\end{array}$ & $\begin{array}{l}\text { Solids, } \\
\text { residue at } \\
180^{\circ} \mathrm{C} \\
\text { dissolved } \\
(\mathrm{mg} / \mathrm{L})\end{array}$ & $\begin{array}{l}\text { Solids, } \\
\text { sum of } \\
\text { consti- } \\
\text { tuents, } \\
\text { dissolved } \\
(\mathrm{mg} / \mathrm{L})\end{array}$ & $\begin{array}{l}\text { Nitrogen, } \\
\text { nitrite } \\
\text { dissolved } \\
\text { (mg/L } \\
\text { as } \mathrm{N})\end{array}$ & $\begin{array}{c}\text { Nitrogen, } \\
\mathrm{NO}_{2}+\mathrm{NO}_{3} \\
\text { dissolved } \\
(\mathrm{mg} / \mathrm{L} \\
\text { as } \mathrm{N})\end{array}$ & $\begin{array}{c}\text { Nitrogen, } \\
\text { ammonia } \\
\text { dissolved } \\
(\mathrm{mg} / \mathrm{L} \\
\text { as } \mathrm{N})\end{array}$ \\
\hline $\mathrm{ADW}$ & 13 & 3.2 & 7.6 & 1.2 & 15 & 46 & 49 & -- & -- & -- \\
\hline ADW & 14 & 2.6 & 23 & 2.9 & 16 & 89 & 97 & $<.010$ & .110 & .010 \\
\hline ADW & 15 & 3.3 & 6.4 & .40 & 8.9 & 31 & 30 & $<.010$ & .180 & .020 \\
\hline $\mathrm{ADW}$ & 16 & 2.5 & 1.8 & 2.1 & 15 & 39 & 43 & $<.010$ & .420 & .010 \\
\hline CKW & 9 & 1.9 & .30 & $<.10$ & 6.4 & 11 & 18 & $<.010$ & .310 & $<.010$ \\
\hline CKW & 10 & 3.7 & .40 & .10 & 9.3 & 24 & 26 & $<.010$ & .120 & $<.010$ \\
\hline CKW & 11 & 55 & 300 & .20 & 11 & 602 & 612 & $<.010$ & .140 & .010 \\
\hline CWW & 66 & 2.0 & 3.5 & .40 & 14 & 37 & 35 & -- & -- & -- \\
\hline CWW & 67 & 2.5 & .50 & 1.6 & 40 & 67 & 80 & $<.010$ & .200 & .010 \\
\hline CWW & 70 & 6.4 & 1.1 & .10 & 10 & 39 & 48 & $<.010$ & .620 & $<.010$ \\
\hline EFW & 9 & 1.6 & .60 & $<.10$ & 11 & 18 & 24 & $<.010$ & $<.050$ & .010 \\
\hline FLW & 25 & .30 & 3.3 & .20 & 8.8 & 21 & 23 & $<.010$ & $<.050$ & $<.010$ \\
\hline MBW & 10 & 1.1 & 13 & $<.10$ & 11 & 52 & 41 & -- & -- & -- \\
\hline OXS & 1 & 2.8 & .40 & 1.2 & 16 & 33 & 42 & $<.010$ & $<.050$ & $<.010$ \\
\hline OXS & 5 & 1.7 & 3.5 & .10 & 11 & 32 & 35 & $<.010$ & .081 & .020 \\
\hline OXW & 34 & 2.9 & 2.3 & .80 & 17 & 34 & 42 & -- & -- & -- \\
\hline OXW & 35 & 1.4 & 10 & $<.10$ & 12 & 39 & 49 & $<.010$ & .730 & $<.010$ \\
\hline OXW & 36 & 1.2 & 32 & .20 & 17 & 95 & 87 & $<.010$ & $<.050$ & .090 \\
\hline OXW & 38 & 1.0 & 2.2 & .20 & 13 & 33 & 33 & $<.010$ & $<.050$ & .010 \\
\hline SES & 1 & 5.2 & .70 & 1.3 & 20 & 58 & 70 & $<.010$ & .280 & $<.010$ \\
\hline SEW & 26 & 4.0 & 1.0 & .10 & 7.8 & 17 & 22 & -- & -- & -- \\
\hline TAS & 1 & 3.5 & 9.8 & .20 & 9.5 & 39 & 45 & $<.010$ & 1.30 & $<.010$ \\
\hline TAW & 31 & 2.9 & .50 & $<.10$ & 7.5 & 19 & 21 & -- & -- & -- \\
\hline TAW & 32 & 3.4 & .40 & .20 & 11 & 29 & 30 & $<.010$ & .180 & .020 \\
\hline TAW & 33 & 2.3 & 1.4 & $<.10$ & 16 & 33 & 41 & -- & -- & -- \\
\hline TAW & 34 & 4.7 & .40 & .70 & 18 & 38 & 43 & -- & -- & -- \\
\hline WAW & 23 & $<.10$ & .60 & $<.10$ & 15 & 23 & -- & $<.010$ & $<.050$ & $<.010$ \\
\hline MBW & 57 & 2.3 & 26 & 1.3 & 16 & 84 & 78 & $<.010$ & .210 & .010 \\
\hline MBW & 55 & 5.1 & 8.5 & .40 & 11 & 50 & 51 & $<.010$ & 1.30 & $<.010$ \\
\hline
\end{tabular}


Table 5-1. Chemical analysis of ground-water samples from the Saco and Ossipee River Basins, east-central New Hampshire--Continued

\begin{tabular}{|c|c|c|c|c|c|c|c|c|c|}
\hline $\begin{array}{l}\text { Local s } \\
\text { numb }\end{array}$ & & $\begin{array}{l}\text { Nitrogen, } \\
\text { ammonia + } \\
\text { organic } \\
\text { dissolved }\end{array}$ & $\begin{array}{l}\text { Phosphorus } \\
\text { dissolved } \\
\text { (mg/L as P) }\end{array}$ & $\begin{array}{l}\text { Phosphorus, } \\
\text { ortho, } \\
\text { dissolved }\end{array}$ & $\begin{array}{c}\text { Barium, } \\
\text { dissolved } \\
\text { (mg/L as } \mathrm{Ba})\end{array}$ & $\begin{array}{l}\text { Beryllium, } \\
\text { dissolved } \\
(\mu \mathrm{g} / \mathrm{L}\end{array}$ & $\begin{array}{l}\text { Cadmium, } \\
\text { dissolved } \\
(\mu \mathrm{g} / \mathrm{L}\end{array}$ & $\begin{array}{l}\text { Cobalt, } \\
\text { dissolved } \\
(\mu \mathrm{g} / \mathrm{L}\end{array}$ & $\begin{array}{c}\text { Copper, } \\
\text { dissolved } \\
(\mu \mathrm{g} / \mathrm{L}\end{array}$ \\
\hline $\mathrm{ADW}$ & 13 & -- & -- & -- & 7 & $<0.5$ & $<1.0$ & $<3$ & $<10$ \\
\hline $\mathrm{ADW}$ & 14 & $<0.20$ & 0.020 & $<0.010$ & 10 & $<.5$ & $<1.0$ & $<3$ & $<10$ \\
\hline ADW & 15 & $<.20$ & $<.010$ & $<.010$ & 8 & .9 & $<1.0$ & $<3$ & $<10$ \\
\hline ADW & 16 & $<.20$ & $<.010$ & $<.010$ & 7 & .7 & $<1.0$ & $<3$ & $<10$ \\
\hline $\mathrm{CKW}$ & 9 & $<.20$ & .010 & $<.010$ & 7 & $<.5$ & 1.0 & $<3$ & $<10$ \\
\hline CKW & 10 & $<.20$ & .020 & $<.010$ & 6 & $<.5$ & 1.0 & $<3$ & $<10$ \\
\hline CKW & 11 & $<.20$ & .020 & $<.010$ & 30 & $<.5$ & 4.0 & 4 & $<10$ \\
\hline CWW & 66 & -- & -- & -- & 6 & $<.5$ & $<1.0$ & $<3$ & $<10$ \\
\hline CWW & 67 & $<.20$ & .170 & $<.010$ & 40 & $<.5$ & $<1.0$ & $<3$ & $<10$ \\
\hline CWW & 70 & $<.20$ & .050 & $<.010$ & 15 & $<.5$ & $<1.0$ & $<3$ & $<10$ \\
\hline EFW & 9 & $<.20$ & $<.010$ & $<.010$ & 6 & $<.5$ & $<1.0$ & $<3$ & $<10$ \\
\hline FLW & 25 & $<.20$ & .010 & $<.010$ & 11 & $<.5$ & $<1.0$ & $<3$ & $<10$ \\
\hline MBW & 10 & -- & -- & -- & 6 & $<.5$ & $<1.0$ & $<3$ & $<10$ \\
\hline OXS & 1 & $<.20$ & .010 & $<.010$ & 10 & $<.5$ & 1.0 & $<3$ & $<10$ \\
\hline OXS & 5 & $<.20$ & .020 & $<.010$ & 9 & $<.5$ & $<1.0$ & $<3$ & $<10$ \\
\hline OXW & 34 & -- & -- & -- & 6 & $<.5$ & $<1.0$ & 3 & $<10$ \\
\hline OXW & 35 & $<.20$ & .020 & $<.010$ & 7 & $<.5$ & $<1.0$ & $<3$ & $<10$ \\
\hline OXW & 36 & $<.20$ & .010 & $<.010$ & 17 & .8 & 1.0 & $<3$ & $<10$ \\
\hline OXW & 38 & $<.20$ & .010 & .010 & 6 & $<.5$ & $<1.0$ & $<3$ & $<10$ \\
\hline SES & 1 & $<.20$ & .050 & .040 & 7 & $<.5$ & $<1.0$ & $<3$ & $<10$ \\
\hline SEW & 26 & -- & -- & -- & 11 & $<.5$ & $<1.0$ & $<3$ & $<10$ \\
\hline TAS & 1 & $<.20$ & .020 & $<.010$ & 18 & .6 & $<1.0$ & $<3$ & $<10$ \\
\hline TAW & 31 & -- & -- & -- & 5 & $<.5$ & $<1.0$ & $<3$ & $<10$ \\
\hline TAW & 32 & $<.20$ & $<.010$ & .010 & 5 & $<.5$ & $<1.0$ & $<3$ & $<10$ \\
\hline TAW & 33 & -- & -- & -- & 7 & .7 & 1.0 & $<3$ & $<10$ \\
\hline TAW & 34 & -- & -- & -- & 6 & $<.5$ & $<1.0$ & $<3$ & $<10$ \\
\hline WAW & 23 & $<.20$ & .010 & $<.010$ & 6 & $<.5$ & $<1.0$ & $<3$ & $<10$ \\
\hline MBW & 57 & $<.20$ & $<.010$ & $<.010$ & 7 & $<.5$ & $<1.0$ & $<3$ & $<10$ \\
\hline MBW & 55 & $<.20$ & $<.010$ & $<.010$ & 6 & .6 & $<1.0$ & $<3$ & $<10$ \\
\hline
\end{tabular}


Table 5-1. Chemical analysis of ground-water samples from the Saco and Ossipee River Basins. east-central New Hampshire--Continued

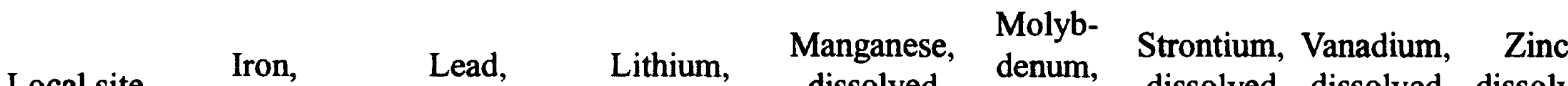
Local site dissolved dissolved dissolved dissolved dissolved dissolved dissolved dissolved
number

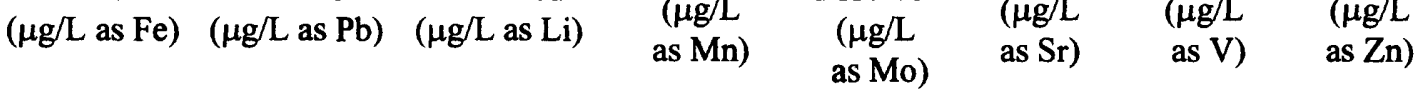

\begin{tabular}{|c|c|c|c|c|c|c|c|c|c|}
\hline $\mathrm{ADW}$ & 13 & 3 & $<10$ & $<4$ & 2 & $<10$ & 22 & $<6$ & 5 \\
\hline $\mathrm{ADW}$ & 14 & $<3$ & $<10$ & 6 & 24 & 10 & 58 & $<6$ & $<3$ \\
\hline $\mathrm{ADW}$ & 15 & $<3$ & $<10$ & $<4$ & 9 & $<10$ & 9 & $<6$ & $<3$ \\
\hline $\mathrm{ADW}$ & 16 & $<3$ & $<10$ & 4 & 20 & $<10$ & 19 & $<6$ & 3 \\
\hline CKW & 9 & 9 & $<10$ & $<4$ & 16 & $<10$ & 12 & $<6$ & $<3$ \\
\hline CKW & 10 & 5 & $<10$ & $<4$ & 48 & $<10$ & 20 & $<6$ & 4 \\
\hline CKW & 11 & 9 & $<10$ & $<4$ & 330 & $<10$ & 79 & $<6$ & 6 \\
\hline CWW & 66 & 5 & $<10$ & $<4$ & 77 & $<10$ & 16 & $<6$ & 4 \\
\hline CWW & 67 & 7,100 & $<10$ & 17 & 500 & $<10$ & 32 & 10 & 50 \\
\hline CWW & 70 & 9 & $<10$ & $<4$ & 48 & $<10$ & 63 & $<6$ & 11 \\
\hline EFW & 9 & 4 & $<10$ & $<4$ & 1 & $<10$ & 17 & $<6$ & 6 \\
\hline FLW & 25 & $<3$ & $<10$ & $<4$ & 15 & $<10$ & 10 & $<6$ & $<3$ \\
\hline MBW & 10 & 4 & $<10$ & $<4$ & 1 & $<10$ & 22 & $<6$ & $<3$ \\
\hline OXS & 1 & 25 & $<10$ & $<4$ & 720 & $<10$ & 33 & $<6$ & 13 \\
\hline OXS & 5 & 3 & $<10$ & $<4$ & 8 & $<10$ & 29 & $<6$ & $<3$ \\
\hline OXW & 34 & 890 & $<10$ & $<4$ & 370 & $<10$ & 30 & $<6$ & 5 \\
\hline OXW & 35 & $<3$ & $<10$ & $<4$ & $<1$ & $<10$ & 81 & $<6$ & $<3$ \\
\hline OXW & 36 & 8,700 & $<10$ & $<4$ & 260 & $<10$ & 80 & $<6$ & $<3$ \\
\hline OXW & 38 & $<3$ & $<10$ & $<4$ & $<1$ & $<10$ & 27 & $<6$ & 4 \\
\hline SES & 1 & $<3$ & $<10$ & 7 & $<1$ & $<10$ & 56 & $<6$ & $<3$ \\
\hline SEW & 26 & 83 & $<10$ & $<4$ & 63 & $<10$ & 22 & $<6$ & 3 \\
\hline TAS & 1 & 4 & $<10$ & $<4$ & 10 & $<10$ & 42 & $<6$ & 10 \\
\hline TAW & 31 & $<3$ & $<10$ & $<4$ & 1 & $<10$ & 15 & $<6$ & $<3$ \\
\hline TAW & 32 & 19 & $<10$ & $<4$ & 26 & $<10$ & 8 & $<6$ & 4 \\
\hline TAW & 33 & 5,300 & $<10$ & $<4$ & 62 & $<10$ & 25 & $<6$ & 16 \\
\hline TAW & 34 & 51 & $<10$ & 5 & 4 & $<10$ & 28 & $<6$ & $<3$ \\
\hline WAW & 23 & $<3$ & $<10$ & $<4$ & 5 & $<10$ & 19 & $<6$ & 4 \\
\hline MBW & 57 & 5 & $<10$ & 8 & 28 & $<10$ & 41 & $<6$ & 5 \\
\hline MBW & 55 & $<3$ & $<10$ & $<4$ & 36 & $<10$ & 38 & $<6$ & 4 \\
\hline
\end{tabular}

